\author{
SWANSEA UNIVERSITY \\ COLLEGE OF SCIENCE \\ BIOSCIENCES DEPARTMENT
}

\title{
Accounting for mixotrophy within microbial food webs
}

by

Suzana Gonçalves Leles

Submitted to Swansea University in fulfilment of the requirements for the Degree of Doctor of Philosophy 



\section{Abstract}

Protist plankton contribute to both primary and secondary production in the oceans fuelling life within pelagic food webs. Despite commonly perceived as 'phytoplankton' or 'zooplankton', most protist plankton are mixotrophs through the combination of photoautotrophy and phagotrophy within a single cell. Nevertheless, we lack a clear understanding of their biogeography and impact on ecosystem functioning. The aims of this thesis were: i) to investigate the biogeography of mixotrophs according to their functional diversity across oceanic biomes and to evaluate how it relates to environmental variability; ii) to explore the competitive outcomes between mixotrophs and their auto- or hetero- trophic counterparts and the effect of mixotrophy on ecosystem functioning; and iii) to investigate the seasonal succession of protist trophic strategies and the vertical distribution of mixotrophs within a coastal temperate sea. These aims were explored utilizing global databases, long-term monitoring datasets, and numerical models of plankton food webs across different spatio-temporal scales. Mixotrophs were found to be ubiquitous in the global oceans; however, different types displayed different distributions. Among non-constitutive mixotrophs, those that host prey populations dominate within oligotrophic seas while those that steal prey plastids dominate high-biomass systems. In turn, global databases were strongly biased by size, taxonomy, and oceanic biome, failing to represent the importance of smaller constitutive mixotrophs. The modelling studies showed that mixotrophs control nutrient regulation, trophic transfer, and the microbial loop. Size was an important trait determining the success of mixotrophs with an innate capacity for photosynthesis while the specificity of prey from which acquired phototrophs can photosynthesize affected their success. Model and data showed that mixotrophy is a persistent trait over the seasonal cycle and throughout the water column within coastal temperate seas. These findings significantly change our understanding of the functioning of marine food webs and biogeochemical cycling in the oceans, underscoring the need to integrate mixotrophy within marine ecology research. 


\section{Declaration and statements}

\section{DECLARATION}

This work has not previously been accepted in substance for any degree and is not being concurrently submitted in candidature for any degree.

Signed (candidate)

Date

\section{STATEMENT 1}

This thesis is the result of my own investigations, except where otherwise stated. Where correction services have been used, the extent and nature of the correction is clearly marked in a footnote(s).

Other sources are acknowledged by footnotes giving explicit references. A bibliography is appended.

Signed (candidate)

Date

\section{STATEMENT 2}

I hereby give consent for my thesis, if accepted, to be available for photocopying and for inter-library loan, and for the title and summary to be made available to outside organisations.

Signed (candidate)

Date 


\section{Contents}

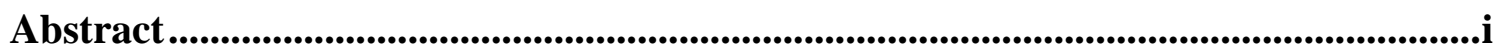

Declaration and statements ........................................................................................ ii

Acknowledgements........................................................................................................................ vii

Abbreviations ........................................................................................................................ viii

Chapter 1 General Introduction..................................................................................1

1.1. The ocean and the plankton .............................................................................. 2

1.2. Mixotrophic functional diversity ……………………………………………. 4

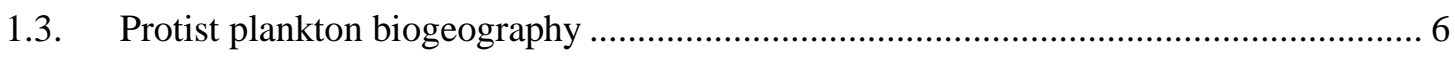

1.4. Plankton models: from cells to ecosystems ……………………………………. 7

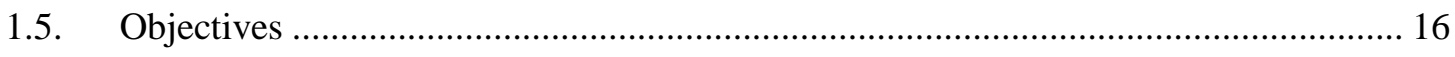

Chapter 2 Methods - Biogeographic analyses ........................................................................18

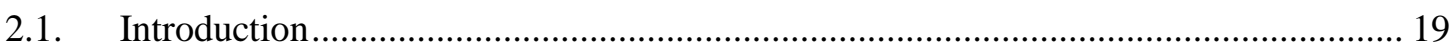

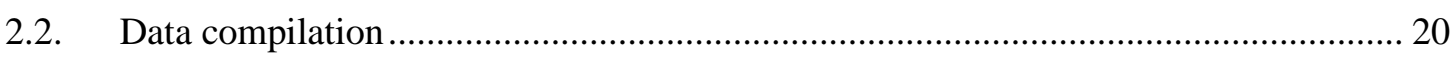

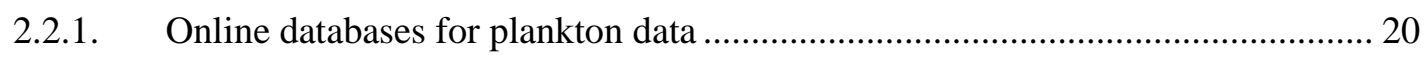

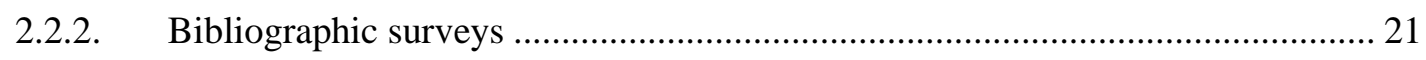

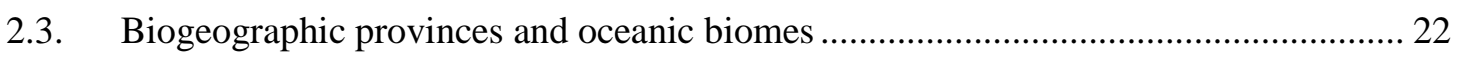

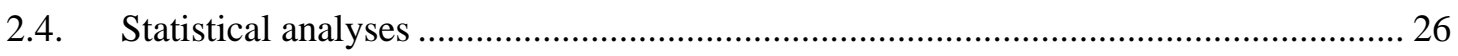

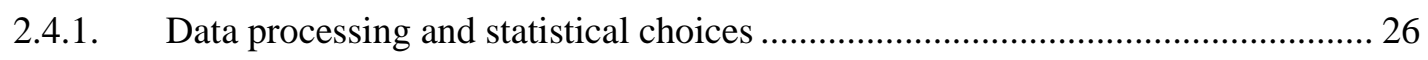

2.4.2. Comparison of means............................................................................... 27

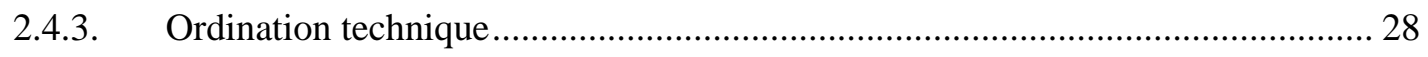

2.4.4. Relationship between variables.................................................................. 29

Chapter 3 Biogeography of non-constitutive mixotrophs ...............................................32

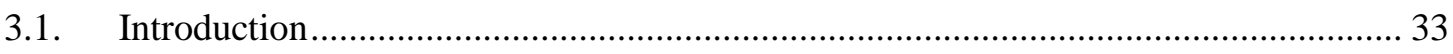

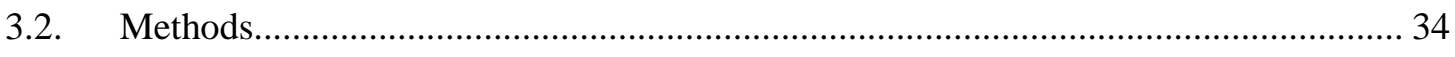

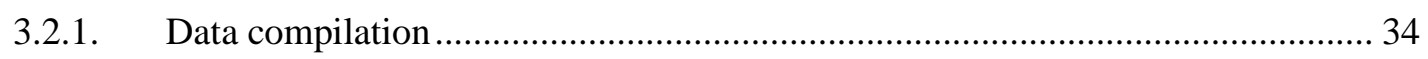

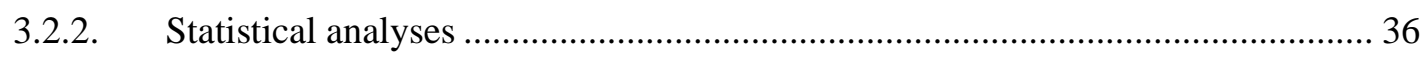

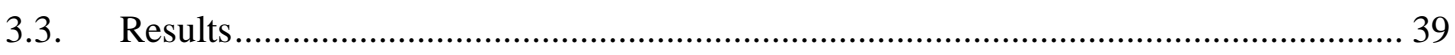

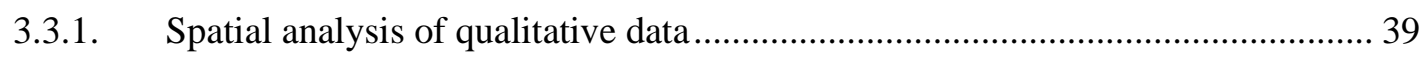

3.3.2. Spatio-temporal analysis of quantitative data ……………………………..... 43

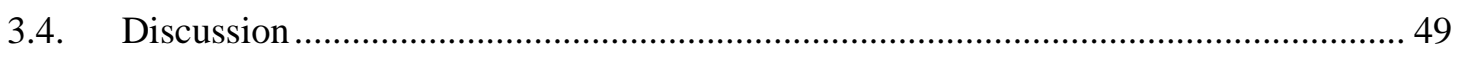

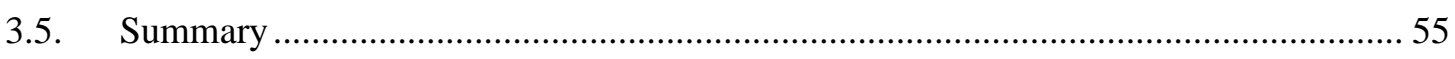


Chapter 4 Biogeography of constitutive mixotrophs ..........................................................56

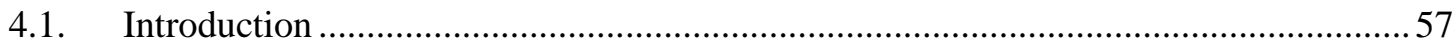

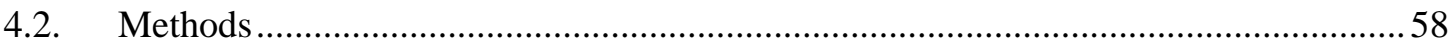

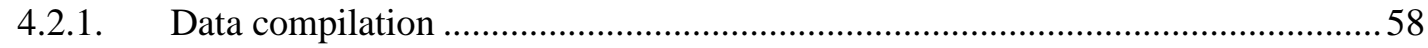

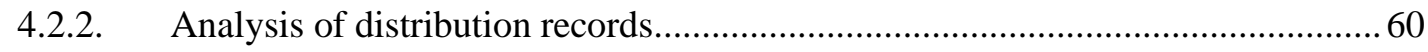

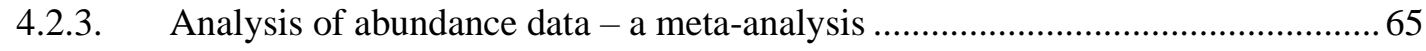

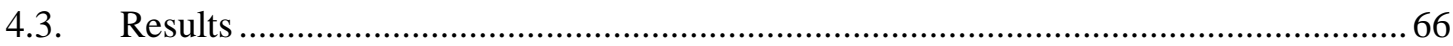

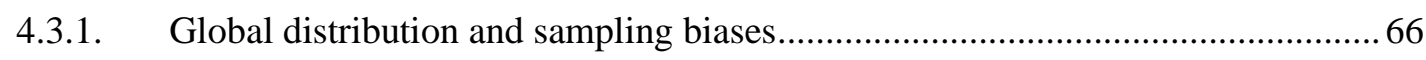

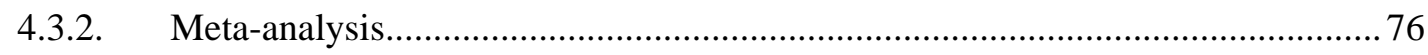

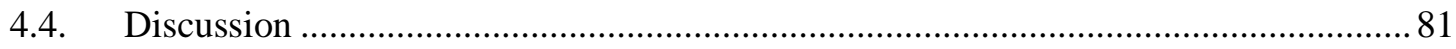

4.4.1. Biases associated to the identification of $\mathrm{CM}$ species ....................................... 82

4.4.2. Traditional sampling techniques and sampling biases ...................................... 82

4.4.3. Effect of environmental variability in the distribution of nano-CMs ................... 83

4.4.4. Ecological significance of nano-CMs as bacterivores across the oceans .............. 84

4.4.5. Microbial biogeography and the advent of molecular data ................................. 85

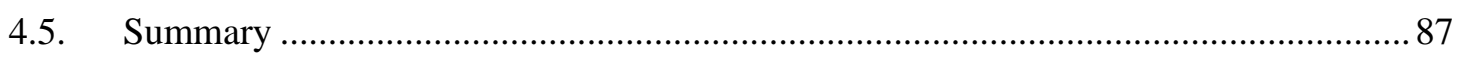

Chapter 5 Methods - Marine ecosystem modelling ...................................................88

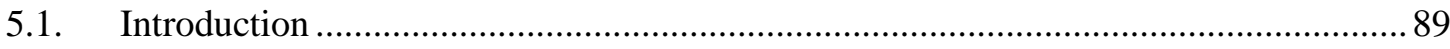

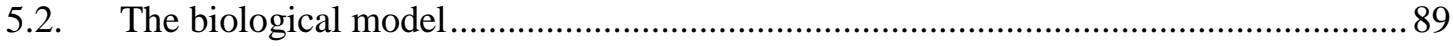

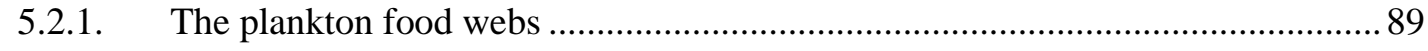

5.2.2. Nutrients, dissolved, and particulate organic matter .........................................95

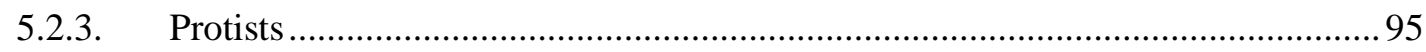

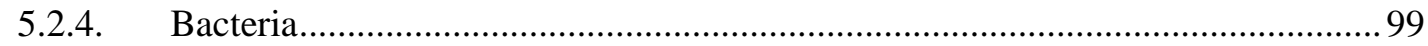

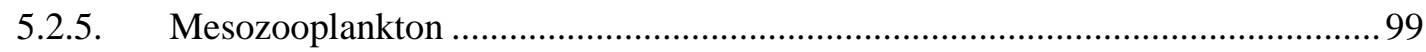

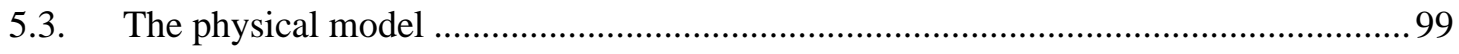

5.4. Model coupling, code accessibility, and other tools................................................ 101

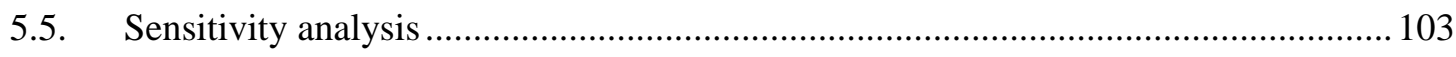

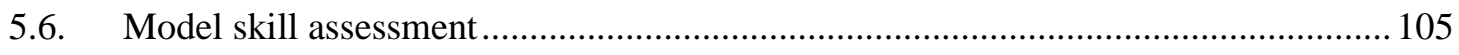

Chapter 6 Mixotrophic functional diversity within oceanic food-webs .................107

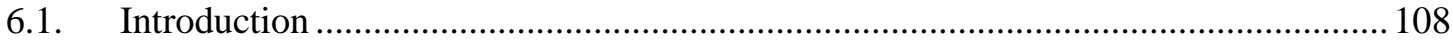

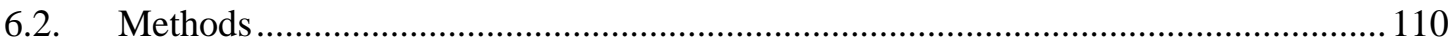

6.2.1. Validating silicon dynamics for diatoms ..................................................... 110

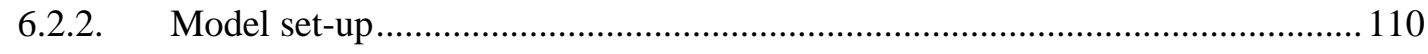

6.2.3. Sensitivity analyses for the mixotrophic food web ........................................ 116 
6.3. Results.

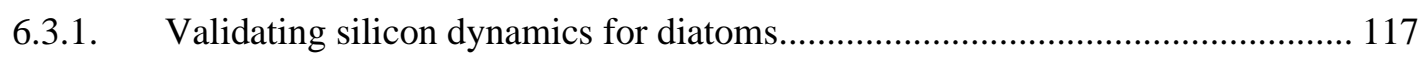

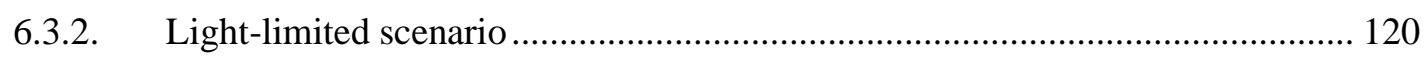

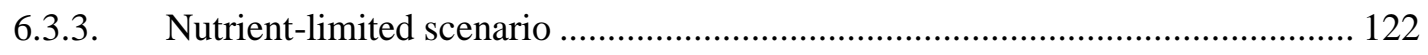

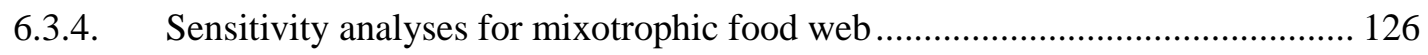

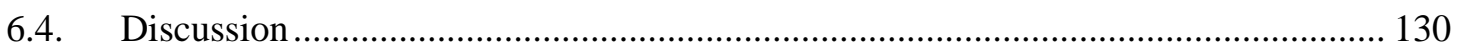

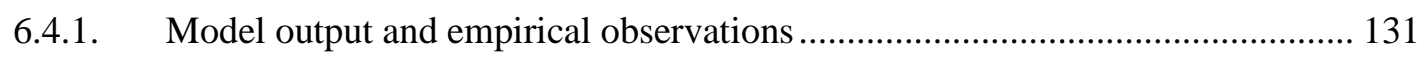

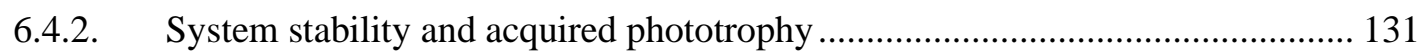

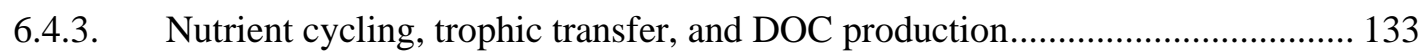

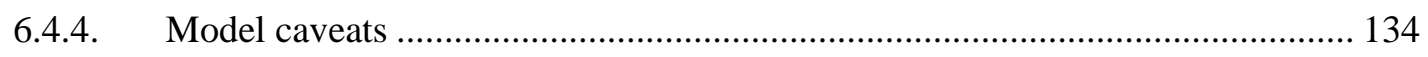

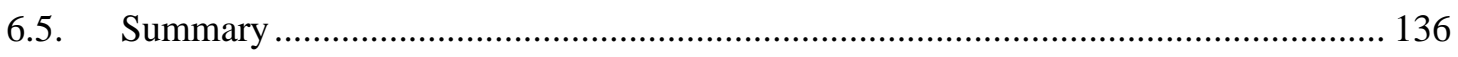

\section{Chapter 7 Seasonal succession of protist trophic strategies in a coastal temperate}

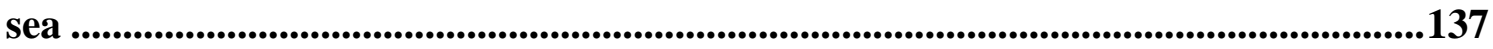

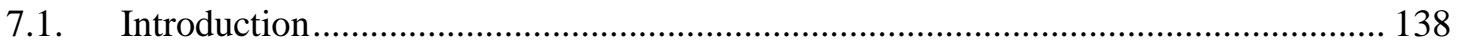

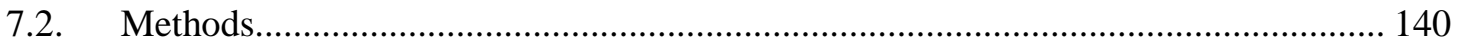

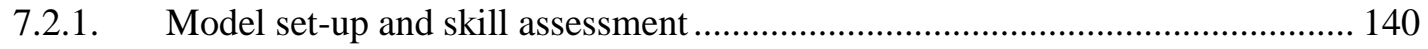

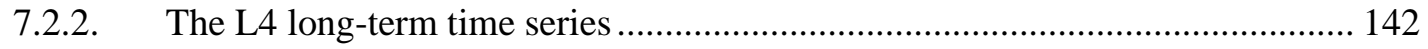

7.2.3. Assigning plankton taxa to functional types .................................................. 145

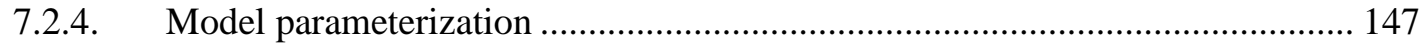

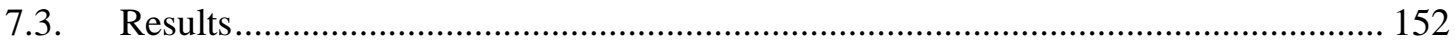

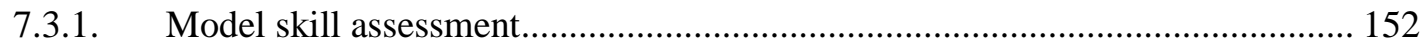

7.3.2. Physico-chemical characterization of the model............................................... 160

7.3.3. Modelling the seasonal succession of protist trophic strategies.......................... 161

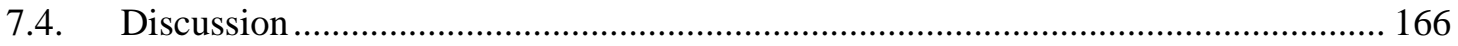

7.4.1. The seasonal succession of protist trophic strategies ......................................... 166

7.4.2. Mixotrophy is a persistent trait in temperate seas ............................................ 172

7.4.3. Different mixotrophic populations across depth? ............................................ 175

7.4.4. The dynamics of mixotrophic dinoflagellates at L4 ...................................... 177

7.4.5. Caveats: model vs observational data at L4 .................................................. 178

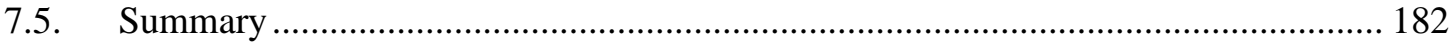

Chapter 8 General Discussion .................................................................................183

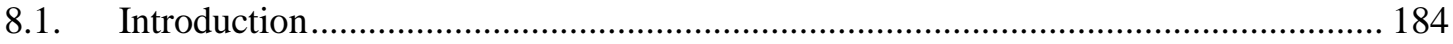

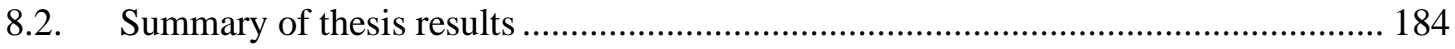

8.2.1. Biogeography of mixotrophs across the global oceans .................................... 184

8.2.2. Mixotrophy, ecosystem functioning, and community composition .................... 186 
8.2.3. Seasonal succession of protists in a coastal temperate sea ................................ 187

8.2.4. On the persistence of different protist functional types in the oceans ................. 189

8.3. Overview of findings in the context of the current literature .................................... 189

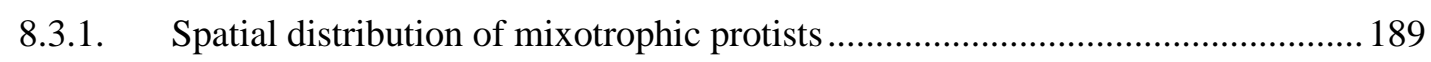

8.3.2. Temporal distribution of mixotrophic protists.................................................. 191

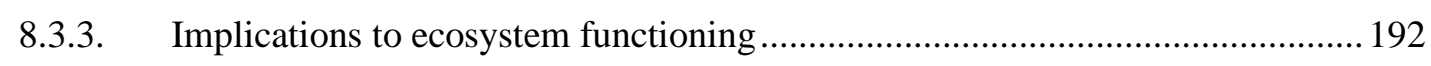

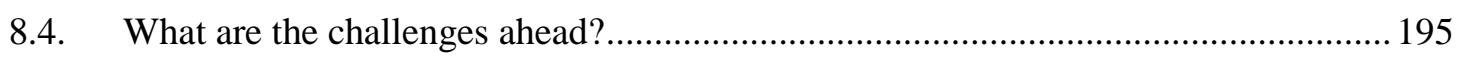

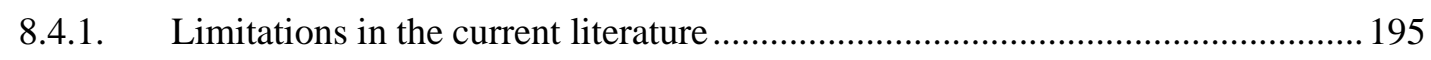

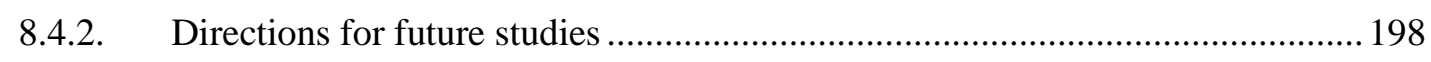

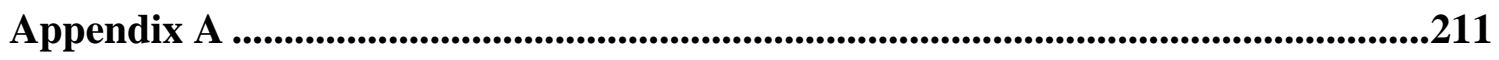

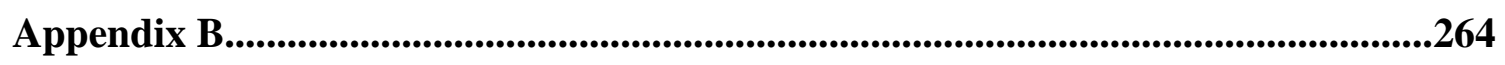

Appendix C ............................................................................................................................295

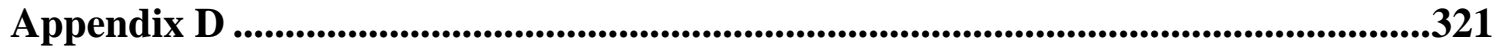

Appendix E..........................................................................................................................327

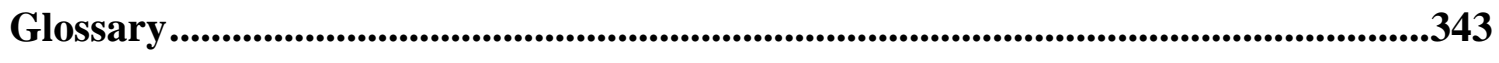

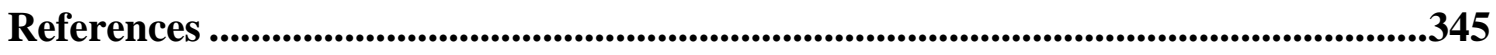




\section{Acknowledgements}

I would like to start acknowledging my supervisors Kevin Flynn, Aditee Mitra, Luca Polimene, Jorn Bruggeman, and Jerry Blackford. Thank you for giving me the opportunity to pursuit my dream within the field of marine ecosystem modelling. Without their knowledge, experience, and support this work would not have come to pass. Thank you for always being there.

To Kevin and Aditee, I am very grateful for all the advice and for the extraordinary opportunity to work with researchers worldwide. I am indebted for the feedback on plankton physiology and the numerous contributions on how to translate these into numerical models. To Luca, Jorn, and Jerry, I am thankful for hosting me at the Plymouth Marine Laboratory (PML), which was always a very pleasant experience, and for the endless encouragement and advice. Thank you for providing me with the platform and data to conduct my modelling investigations; big thanks to Jorn who helped me to go (smoothly!) through this process.

I could not forget to thank all my co-authors. I was fortunate to count with the advice of a team of experts in the field of mixotrophic plankton - Diane Stoecker, Per Juel Hansen, and Albert Calbet just to name a few. At PML, Stefano Ciavatta guided me on model sensitivity analyses - thank you for sharing your knowledge and your good humour. I am also indebted with the ecosystem modelling group at PML for the valuable feedback and with Angus Atkinson and Elaine Fileman for the insightful discussions about plankton dynamics at L4 station.

To my role 'modellers', Aditee Mitra, Helen Powley, Sevrine Sailley, and Susan Kay, thank you for being brilliant women that gave me inspiration throughout my modelling journey. To the incredible women who taught me in the very beginning - Gleyci Moser and Gisela Figueiredo - thank you for the science and for the continued friendship.

My $\mathrm{PhD}$ was fuelled by unique and passionate people that I've met along the way - Jess, Szymon, Dania, Pipo, Teja, David, Marta, Raul, Dimitra, Sara, André, Deiene, Matt, Nahaa, Diego, Helen, Mike, Ben, Frances. Including the awesome Brazilian crew - Laura, Igor, Waldir, Larissa. Thank you all for sharing your joy - Lana, Toby, Rohan and Bebo (the mascots).

To my family, thank you for all the love and support 'O céu é o limite.' - Marly, Paula, Alcides, Edu, Cris, Moki, and Nick. To him, Pietro, thank you for being such an amazing partner and for boarding on this adventure with me.

Finally, this work would have not been possible without the financial support of the Brazilian government through CNPq (Conselho Nacional de Desenvolvimento Científico e Tecnológico) and the program Science Without Borders. 


\section{Abbreviations}

$C M s:$ constitutive mixotrophs

$D I N$ : dissolved inorganic nitrogen

$D I P:$ dissolved inorganic phosphorus

$D O C$ : dissolved organic carbon

eSNCMs: endosymbiotic specialist non-constitutive mixotrophs

ERSEM: European Regional Seas Ecosystem Model

$G G E$ : gross growth efficiency

GNCMs: generalist non-constitutive mixotrophs

$G P P$ : gross primary productivity

$H A B s$ : harmful algal blooms

MesoZ (or MZ): mesozooplankton

Micro-A: autotrophic microflagellates

Micro-CMs: constitutive mixotrophic microflagellates

MicroZ (or $m Z$ ): heterotrophic microzooplankton

$M L D:$ mixed layer depth

Nano-A: autotrophic nanoflagellates

Nano-CMs: constitutive mixotrophic nanoflagellates

NanoZ (or HNF): heterotrophic nanoflagellates/nanozooplankton

NCMs: non-constitutive mixotrophs

$N P P$ : net primary productivity

OBIS: Ocean Biogeographic Information System

PAR: photosynthetically active radiation

PFT: plankton functional type

PicoP: picophytoplankton

pSNCMs: plastidic specialist non-constitutive mixotrophs

$r D O C$ : recalcitrant DOC 


\section{Chapter 1}

General Introduction 


\subsection{The ocean and the plankton}

The ocean is the largest continuous ecosystem on Earth. It comprises $71 \%$ of the planet's surface, > 96\% of total water volume, and has an average depth of $3.8 \mathrm{~km}$ (Schmitt, 2018). The very high capacity to absorb, accumulate, and transport the heat supplied by the sun grants the ocean a key role in the global climate. It is estimated that the ocean has over 1,000 times the heat capacity of the atmosphere (Schmitt, 2018). The ocean is also the main reservoir of carbon in the climate system (Le Quéré et al., 2018) and it is estimated to be absorbing 30\% of anthropogenic carbon (Fischer et al., 2010). Marine microorganisms consume $\mathrm{CO}_{2}$ and produce oxygen - half of the primary production in the planet takes place in the ocean (Field et al., 1998). The marine ecosystem is as important as forests but contrary to the large and slow growing primary producers on land, the main primary producers in the ocean are microscopic plankton and have short generation times. The plankton are passively drifting organisms that contribute to both primary and secondary production in the oceans, mediate key pathways and transformations in ocean biogeochemical cycling and fuel marine food webs (Hutchins et al., 2009). Since plankton communities are extremely diverse (Margalef, 1978; Sournia, 1982; Reynolds, 1988), it is important to identify key descriptors (traits; see Glossary) that differentiate their ecological roles in the ocean (McGill et al., 2006; Litchman et al., 2007; Barnett et al., 2007; Menden-Deuer and Kiørboe, 2016).

The diversity of forms and function among plankton can be defined based on individual's morphology, physiology, behaviour, or life-history (Litchman and Klausmeier, 2008; Barnett et al., 2007). Cell size, for instance, affects a series of physiological rates and ecological functions and can vary over eight orders of magnitude in cell length (Sieburth et al., 1978; Finkel et al., 2010). Traits associated with resource acquisition are also particularly important to understand the impact of plankton in nutrient cycling in the oceans. Traditionally, plankton has been divided based on the autotrophyheterotrophy dichotomy (Riley, 1946; Steele, 1959). However, this split neglects that many organisms combine both nutritional modes (Selosse et al., 2017). Mixotrophy is an intermediate nutritional strategy in which both heterotrophy and autotrophy co-occur within a single organism (Flynn et al., 2013). This is particularly the case among protist 
plankton; most protist classes are mixotrophs except for diatoms (Flynn et al., 2013). In this thesis, mixotrophy is defined as the combination of phototrophy and phagotrophy in a single cell (Mitra et al., 2016). Osmotrophy was not considered by Mitra et al. because almost all phototrophs require external sources for vitamins and, therefore, can acquire nutrients from dissolved organic matter (Croft et al., 2006; Burkholder et al., 2008). Assigning mixotrophy within plankton food webs (Fig. 1.1) has the potential to change the way we understand the functioning of the lower trophic levels within pelagic food webs (Mitra et al., 2014b; Stoecker et al., 2017). Nevertheless, we still lack a clear understanding of its impact in plankton trophodynamics and biogeochemical cycling in the oceans.

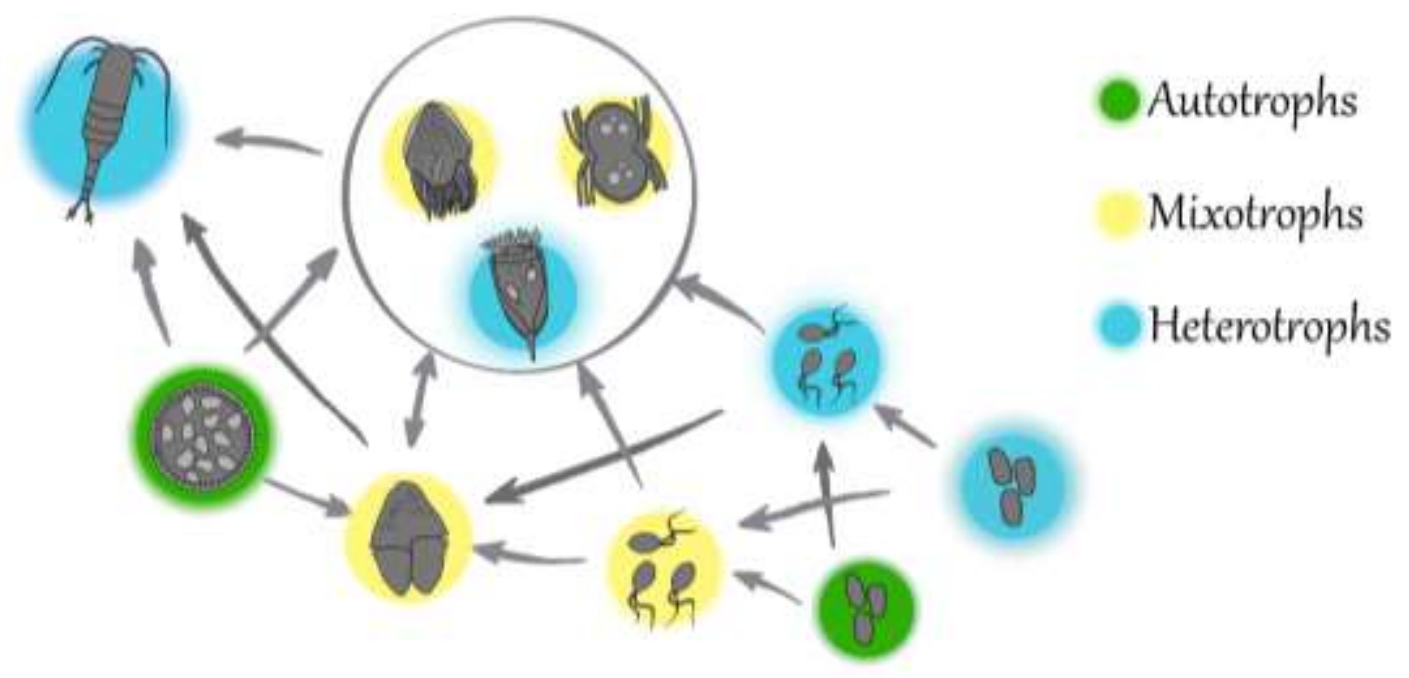

Figure 1.1 Conceptual plankton food web highlighting autotrophs (green), heterotrophs (blue), and mixotrophs (yellow) of different sizes. Autotrophs rely on inorganic nutrients and carbon for growth, heterotrophs rely on organic compounds, and mixotrophs combine both phototrophy and phagotrophy. Arrows indicate the ingestion of prey. Organisms represented from the left to the right: autotrophs are diatoms and picophytoplankton, mixotrophs are ciliates (upper schematics), dinoflagellates, and nanoflagellates, and heterotrophs are copepods, ciliates, nanoflagellates, and bacteria. Schematic courtesy by Gabriele Brambilla and Eduardo Gonçalves Martins. 


\subsection{Mixotrophic functional diversity}

Considering the high genetic breadth across marine protists, it is not surprising that mixotrophy can be expressed in different ways (Jones, 1997; Stoecker et al., 2009; Jeong et al., 2010c; Johnson et al., 2011; Flynn et al., 2013; Mitra et al., 2016). The diverse mixotrophic forms adopted by protists can be expected to confer them the ability to thrive in different ecosystems with, potentially, different impacts on ecosystem functioning (Mitra et al., 2016; Stoecker et al., 2017). But how is it possible to identify a mixotroph in the first place? Experimental investigations are necessary to verify if a species can engage on both phototrophy and phagotrophy (e.g., Stoecker et al., 1988; Caron et al., 1993; Caron et al., 1995). For example, in the late 80s, microscopic analyses of field samples revealed ciliates with intact chloroplasts inside their cells separated from the feeding vacuoles, suggesting that these were mixotrophs (Stoecker et al., 1987); in fact, photosynthetic activity was verified experimentally among several ciliate species (Stoecker et al., 1987; Stoecker et al., 1988; Stoecker et al., 1989). Meanwhile, species commonly perceived as strictly phototrophic were found to be mixotrophic and their ecological importance as bacterivores was first revealed through feeding experiments using natural assemblages from lakes (Bird and Kalf, 1986). Since then, several studies have been documenting mixotrophy among diverse protist taxa as well as its ecological significance within marine systems (Zubkob and Tarran, 2008; Hartmann et al., 2012; McManus et al., 2012; Stoecker et al., 2014; Jang et al., 2017; Gomes et al., 2018).

Once assigning that a species is mixotrophic, it is important to verify experimentally whether the species always display mixotrophic characteristics or not (i.e., obligate vs facultative behaviour, respectively) and how this trait varies according to environmental conditions. Obligate mixotrophs rely on both phototrophy and phagotrophy to support growth while facultative mixotrophs can survive through either photo- or phago- trophic nutrition (Stoecker et al., 2017). Current evidence supports that most phytoflagellates and dinoflagellates are facultative mixotrophs (reviewed in Jeong et al., 2010b; Hansen, 2011), while acquired phototrophs are obligate mixotrophs (reviewed in Stoecker et al., 2009). Consequently, determining mixotrophy in field samples through species identification is more challenging for the former than for the latter. Furthermore, 
mixotrophy is a plastic trait and its contribution to the total carbon budgets of mixotrophs can vary according to environmental conditions. (Stoecker and Michaels, 1991; Caron et al., 1993; Skovgaard, 1996, 2000; Johnson and Stoecker, 2005; Adolf et al., 2006; Princiotta et al., 2016; Schoener and McManus, 2017; Terrado et al., 2017). On top of that, species respond differently to environmental variability (Stoecker and Michaels, 1991; Johnson and Stoecker, 2005; Terrado et al., 2017). Therefore, in order to investigate the impact of mixotrophy on ecosystem functioning, it is important to consider mixotrophic functional diversity.

In this thesis, mixotrophic functional diversity is defined according to Mitra et al. (2016) (Fig. 1.2; see also Glossary). A basic distinction is made between mixotrophs which possess their own photosystems and those which must acquire phototrophic capacity from their prey; these are named constitutive and non-constitutive mixotrophs, respectively (Fig. 1.2). Constitutive mixotrophs (CMs) are thus akin to unicellular algae that can feed on other organisms (Burkholder et al., 2008; Jeong et al., 2010c; Hansen, 2011; Glibert et al., 2016) and are often referred as 'phytoplankton' (Flynn et al., 2013). In turn, non-constitutive mixotrophs (NCMs) lack an inherent capacity to maintain the photosynthetic machinery and, therefore, must acquire it from time to time through the engulfment of photosynthetic prey (Laval-Peuto and Febvre, 1986; Gustafson et al., 2000; Kim et al., 2012); these are often referred to as 'protozooplankton' (Flynn et al., 2013). Experiments performed with cultured strains show that phototrophic capabilities can either be acquired from diverse or specific prey (e.g., Gustafson et al., 2000; McManus et al., 2012; Wang et al., 2016), thus, a distinction is made between generalist (GNCMs) and specialist (SNCMs) non-constitutive mixotrophs, respectively (Fig. 1.2). In addition, another split is made among SNCMs since carbon fixation can be mediated by plastid sequestration (pSNCMs) or by endosymbiosis (eSNCMs) (Fig. 1.2). Culture methods in combination with molecular tools revealed that different eukaryotes (including foraminiferans, Acantharia, and dinoflagellates) host populations of symbionts from specific prey (e.g., Shaked and de Vargas 2006; Decelle et al., 2012; Wang et al., 2016); this is probably related to the high investment needed to maintain a symbiotic relationship (Dittami et al., 2019). 
Constitutive Mixotrophs (CMs)

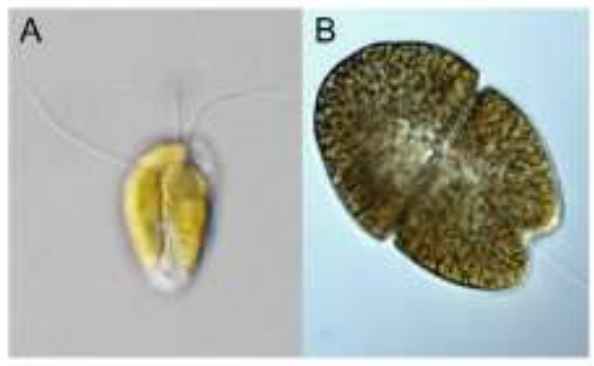

plastidic Specialist Non-Constitutive Mixotrophs (pSNCMs)

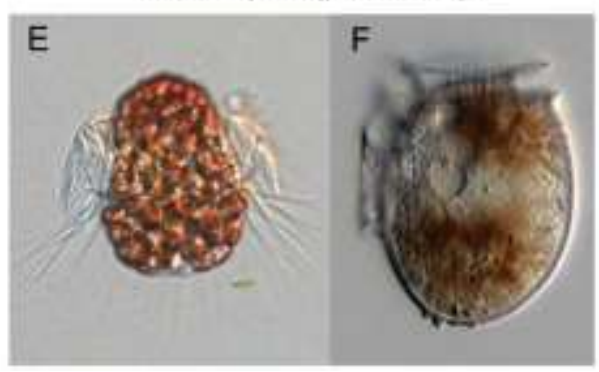

Generalist Non-Constitutive Mixotrophs

(GNCMs)

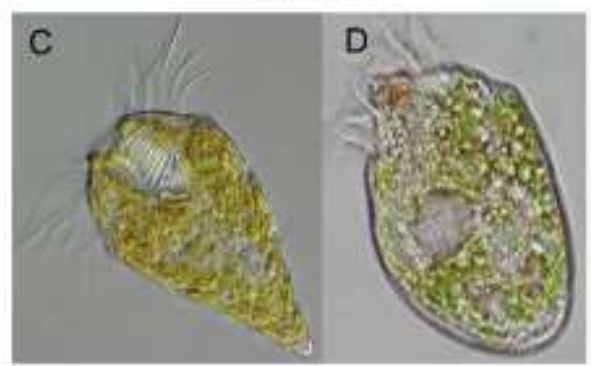

endosymbiotic Specialist Non-Constitutive Mixotrophs (eSNCMs)

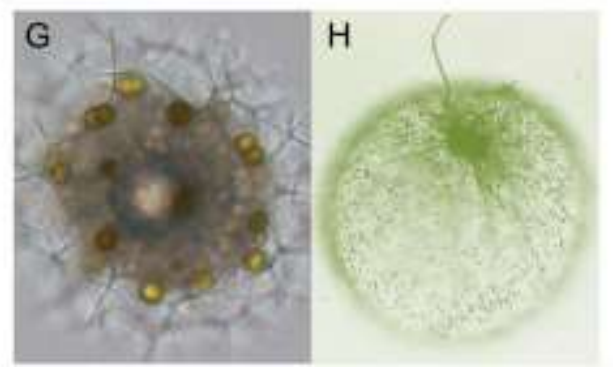

Figure 1.2 Protist genus examples within each mixotrophic functional group as defined by Mitra et al. (2016), as following (size as length): A) Prymnesium (12 $\mu \mathrm{m})$, B) Akashiwo $(100 \mu \mathrm{m})$, C) Laboea $(100 \mu \mathrm{m})$, D) Strombidium $(50 \mu \mathrm{m})$, E) Mesodinium $(25 \mu \mathrm{m}), \mathrm{F})$ Dinophysis $(40 \mu \mathrm{m}), \mathrm{G})$ Sphaerozoum $(200 \mu \mathrm{m})$, and H) Noctiluca $(500 \mu \mathrm{m})$. Photos from Leles et al. (2017) except for A and B, credits to Gert Hansen (nordicmicroalgae.org) and Kim Stark (kingcounty.gov), respectively.

\subsection{Protist plankton biogeography}

Biogeography is the study of the distribution of organisms across spatial and temporal scales. While this discipline has long been applied to understand the distribution of macroorganisms, it was not until the $21^{\text {st }}$ century that attention has been placed towards microorganisms (Dolan, 2005; Foissner 2006; Martiny et al., 2006; Ramette and Tiedje, 2007; Caron, 2009). One of the reasons for the lack of interest in microbial biogeography was the belief that 'everything is everywhere' - statement usually attributed to Beijerinck (1913). This statement was largely based on the inverse relationship between organism size and population size, and the relatively large capacity of dispersal among microscopic organisms (Finlay and Clarke, 1999; Fenchel and Finlay, 2003). However, at any location, physical, chemical, and biological factors will influence the relative abundance of microbes (inclusive of archaea, bacteria, fungi, and protists). In fact, it is argued that 
environmental selection (environmental factors) is more relevant than historical factors (past selection, drift, dispersal, and/or mutation) to determine microbial biogeography (Dolan 2005; Fenchel and Finlay, 2003). Nevertheless, these processes are not mutually exclusive and can confound our ability to interpret their distribution (Caron, 2009; Hanson et al., 2012).

Technical advancements such as the development of molecular techniques, the availability of distribution data in online databases, and mathematical simulations have boosted marine biogeography (Fuhrman et al., 2008; Bosch et al, 2017; Costello et al., 2017; Boltovskoy and Correa, 2017). Any biogeographic pattern will depend on the taxonomic breadth defined for the analysis; sometimes a pattern is not observed due to a lack of taxonomic resolution (Caron, 2009; Hanson et al., 2012). A key aspect of protist biogeography is the enormous phylogenetic breath of species which the term 'protist' encompasses and the application of multiple species concepts to these taxa (Caron, 2009; de Vargas et al., 2015). It is possible to interpret protist biogeography considering taxonspecific attributes or traits (Barton et al., 2013b). So far, biogeographic patterns have been described for different protist groups in the oceans, such as ciliates, diatoms, and radiolarians (e.g., Foissner et al., 2008; Cermeño and Falkowski, 2009; Biard et al., 2016; Malviya et al., 2016; Boltovskoy and Correa, 2017). Little attention has been given to investigate the biogeography of protists with explicit reference to mixotrophy (Leles et al., 2017; Leles et al., 2019; Faure et al., 2019).

\subsection{Plankton models: from cells to ecosystems}

Ecologists study how multiple processes interact over space and time to produce complex biological phenomena. The interactions between living organisms and the environment will define nutrient cycles and energy flows within an ecosystem. To investigate ecosystem functioning, different approaches are necessary (Flynn and McGillicuddy, 2018). Field studies, for instance, are subjected to all processes taking place in the natural environment and, as an output, provide data on physico-chemical and community characterization. The interpretation of such studies is commonly based on curve-fitting techniques (correlative models; see Glossary) to assess the correspondence between observed patterns and causal factors; however, the mechanisms behind the 
relationship investigated are not addressed directly (Gotelli et al., 2009; Coelho et al., 2018). Laboratory studies provide biochemical and physiological understanding but assume controlled abiotic conditions of selected biotic complexity (Flynn and McGillicuddy, 2018). Mechanistic models (see Glossary), in turn, offer an approach in which the complexity of the system can be defined a priori, and both field and laboratory data can be combined to explain nature (Gotelli et al., 2009; Coelho et al., 2018; Flynn and McGillicuddy, 2018).

Models are simplifications of real systems. Conceptual models (see Glossary) are akin to schematics (e.g., Fig. 1.1), being used in ecology to represent flow diagrams and trophic interactions and helping the formulation of ideas and the identification of strengths and weakness in knowledge (Flynn and McGillicuddy, 2018). Quantitative information is only possible once conceptual models are translated into numerical models. These are useful tools to synthesize knowledge, test hypothesis, make predictions, guide future investigations, and are widely applied to simulate plankton physiology and ocean biogeochemistry (e.g., Fasham et al., 1990; Flynn, 2001; Allen et al., 2004; Banas, 2011; Doron et al., 2013; Terseleer et al., 2014; Ghyoot et al., 2017a; Leles et al., 2018). Defining the form of the response curve between the driver and consequence is often the first step in converting a conceptual model into a numerical one (Flynn and McGillicuddy, 2018).

The choice of the response curves (see Glossary) used within plankton models are key to describe plankton physiology and differ in the level of mechanistic understanding (Franks, 2009). A classic example is the utilization of Michaelis-Menten type formulations (Eq. 1.1) to describe transport, uptake, and growth by phototrophs and ingestion of prey by zooplankton despite its several limitations (Flynn, 1998; Gentleman et al., 2003; Mitra et al., 2014b; Flynn, 2010; Flynn et al., 2018):

$$
\mathrm{V}=\mathrm{V}_{\max } \frac{\mathrm{S}}{\mathrm{S}+\mathrm{K}}
$$

where $\mathrm{V}$ is the process rate, $\mathrm{V}_{\max }$ is the maximum rate, $\mathrm{S}$ is the substrate concentration, and $\mathrm{K}$ is the half-saturation constant. This formulation, for instance, assumes that phytoplankton growth and zooplankton ingestion are solely determined by the external resource concentrations. In reality, phytoplankton growth is strongly dependent on the 
internal nutrient status of the cell (Droop, 1968; Flynn, 2001) and zooplankton respond to prey quality, i.e., the stoichiometric status of the prey (Mitra and Flynn, 2005). These are just two examples of features that can be incorporated into plankton models based on biological understanding. Other examples are the effect of cell size, light/dark cycles, photoacclimation, temperature, prey selectivity, satiation feedback, and variable assimilation efficiency on plankton growth dynamics (Flynn, 2001; Mitra, 2006; Mitra and Flynn, 2006; Banas, 2011; Bonachela et al., 2011; Montagnes and Fenton, 2012; Terseleer et al., 2014; Flynn et al., 2018).

Plankton models are usually based on the mass conservation principle and their structure and complexity differ mainly according to: i) the functional types described and their interactions, ii) the nutrients described and the elemental composition among plankton (fixed vs variable stoichiometry), iii) the platform describing the physical environment (from 0D to 3D models), and iv) the response curves within each functional type (Franks, 2002; Leles et al., 2016; Lindemann et al., 2017; Flynn and McGillicuddy, 2018). Differences may be related to the questions being addressed (which may require different models) and to a lack of the level of detail of how mass is transformed and allocated within an ecosystem. These are usually conferred to the compromise between the level of mechanistic understanding, availability of empirical data, and (critically) computational load (Anderson, 2005; Flynn, 2005; Le Quéré, 2006).

Since plankton organisms are key to the functioning of marine ecosystems and global biogeochemical cycles, it is necessary to link plankton ecology to ecosystem processes (Menden-Deuer and Kiørboe, 2016). The pioneering modelling studies by Riley (1946) and Steele (1959) described plankton trophodynamics (in nitrogen units) through the 'nutrient-phytoplankton-zooplankton' (NPZ) model type. Since then, variations of this structure include the explicit representation of bacteria, detritus and the partitioning of nitrogen in inorganic and organic forms (Fasham et al., 1990; Druon and Le Fèvre, 1999), the split of plankton in different functional types and/or size classes (Painting et al., 1993; Baretta-Bekker et al., 1995; Baird and Suthers, 2007), and the representation of other limiting nutrients (Baretta et al., 1995). The European Regional Seas Ecosystem Model (ERSEM), for instance, was formulated in the early 1990s and has evolved over 
the years, being now the main model of shelf-sea applications within the UK and other regions (Baretta et al., 1995; Blackford et al., 2004; Butenschön et al., 2016). ERSEM resolves the major chemical elements in the sea, i.e., carbon, nitrogen, phosphorus, and silicate (organic and inorganic forms), accounting for variable stoichiometry among plankton.

ERSEM and other models have shown reasonable skill in reproducing regional long-term monitoring datasets and global features of plankton dynamics (Blackford et al., 2004; Lancelot et al., 2005; Lewis et al., 2006; Kishi et al., 2007; Allen et al., 2007; Follows and Dutkiewicz, 2011; Yool et al., 2011). It is noteworthy, however, that ecosystem models have different purposes which drive their pros and cons and, ultimately, these models face a series of limitations (Blackford et al., 2010). Most ecosystem models are coupled to a physical representation of the ocean and are, thus, also subjected to the uncertainties of the physical model (Sinha et al., 2010). On the other extreme, there are challenges to link end-to-end ecosystem models to plankton models, particularly zooplankton (Fulton, 2010; Mitra et al., 2014a), although the scientific community has been progressing on this field (Everett et al., 2017). Another issue is related to the development and implementation of robust methods that allow to assess model performance (Friedrichs et al., 2009; Jolliff et al., 2009; Stow et al., 2009). Finally, and perhaps the biggest concern related to plankton models, their parameterisation; certain parameter values are extremely difficult to measure and/or are often obtained through laboratory experiments which are limited to certain species and, therefore, might not represent the behaviour of natural communities (Schartau et al., 2017). For this reason, some modelling approaches assume model parameters to follow a distribution rather than having a single fixed value (Bruggeman and Kooijman, 2007). There is, therefore, a tradeoff between model uncertainty (linked to parameterisation) and the level of mechanistic understanding within models (Blackford et al., 2010; Schartau et al., 2017).

Nevertheless, the structure of ecosystem models is limited by the poorly resolved ecology and remaining uncertainties in physiology (Franks, 2009; Flynn, 2010; Allen and Polimene, 2011). One might then argue that research efforts should be channelled towards experimental investigations (instead of building more elaborate models). 
Although prior information is needed to build a mechanistic model, modelling is not the final step in the scientific method. In fact, models are also used to guide new laboratory studies and to generate new hypotheses to be tested in the field (e.g., Flynn and McGillicuddy, 2018). In other words, mechanistic models can guide future investigations by challenging our current understanding of a system. Therefore, despite the uncertainties in physiology, complex mechanistic models are valuable tools to advance our understanding of biological systems. Acknowledging mixotrophy when modelling plankton communities is one example.

Models describing mixotrophy differ in many aspects; their differences are summarised in Tables 1.1 and 1.2 in chronological order. First, a comparison is given with respect to the functional types (based on the mixotrophic functional classification by Mitra et al. (2016)), nutrients, physical set-up and model application (Table 1.1). Second, a list of features described by the mixotrophic models is compared amongst the studies (Table 1.2). The functional types described in the models vary from including a single mixotrophic type from more 'complete' descriptions of the plankton food web, accounting for strict phototrophs, mixotrophs, strict heterotrophs, and decomposers (Table 1.1). The number of functional types also vary, but most of the studies represented constitutive mixotrophs (Table 1.1). Studies that aimed to model nonconstitutive mixotrophs but did not describe plastid acquisition and retention end up functioning as CMs (Stickney et al., 2000; Zhang et al., 2003; Hood et al., 2006; see also Table 1.1). Some studies explicitly represented $n$ constitutive mixotrophs through a continuum of mixotrophic strategies, from primarily phototrophs to primarily phagotrophs, and/or through a continuum of size classes (Table 1.1). Allometric-scaled models account for a diverse community in terms of size classes; however, these assume an inverse relationship between phototrophic potential and cell size (Ward and Follows, 2016; Chakraborty et al., 2017). In reality, large mixotrophic Rhizaria function mainly as phototrophs (de Vargas et al., 2015) and mixotrophic nanoflagellates can be primarily phagotrophs (Lie et al., 2018).

Nearly $40 \%$ of the models were based on a single limiting nutrient (mainly $\mathrm{N}$ ) while the remaining were based on two nutrients $(\mathrm{C}$ and $\mathrm{N} ; 19 \%)$ or three nutrients $(\mathrm{C}, \mathrm{N}$, 
and P; 31\%); whenever diatoms were described by the model, silicate was also included (Table 1.1). Models differ greatly in all aspects except for the physical set-up and application (Table 1.1). Most studies were performed utilizing zero-dimensional (OD) models which accounts for time variation but neglects any spatial variation (Table 1.1). From the 26 studies evaluated, only four simulated plankton dynamics vertically in the water column (1D models) and only one simulated plankton dynamics using a 3D global model. 
Table 1.1 Models describing mixotrophy in the literature including functional type ( $\mathrm{P}$-phototroph, $\mathrm{M}$-mixotroph, Z-heterotroph, Bbacteria, $n$ size-classes and/or levels of mixotrophy), mixotrophic type (OSM-osmotrophy), nutrient (Nut-generic nutrient, C-carbon, $\mathrm{N}$-nitrogen, $\mathrm{P}-$ phosphorus, $\mathrm{Si}$-silicate), platform, and application. Mixotrophic type is given according to Mitra et al. (2016).

\begin{tabular}{|c|c|c|c|c|c|}
\hline Reference & Functional type & Mixotrophic type & Nutrient & Platform & Application \\
\hline Thingstad et al. (1996) & $1 \mathrm{P}, 1 \mathrm{M}, 1 \mathrm{Z}, 1 \mathrm{~B}$ & $\mathrm{CMs}$ & Nut & OD & ecology theory \\
\hline Baretta-Bekker et al. (1998) & $3 \mathrm{P}, 1 \mathrm{M}, 3 \mathrm{Z}, 1 \mathrm{~B}$ & nano-CMs + OSM & $\mathrm{C}, \mathrm{N}, \mathrm{P}, \mathrm{Si}$ & OD & mesocosm simulations \\
\hline Stickney et al. (2000) & $1 \mathrm{P}, 1 \mathrm{M}, 1 \mathrm{Z}$ & CMs (3 levels) & $\mathrm{N}$ & OD & ecology theory \\
\hline Kooijman et al. (2002) & $1 \mathrm{M}$ & OSM & $\mathrm{C}, \mathrm{N}$ & $0 \mathrm{D} / 1 \mathrm{D}$ & ecology theory \\
\hline Zhang et al. (2003) & $1 \mathrm{P}, 1 \mathrm{M}, 1 \mathrm{Z}$ & $\mathrm{CMs}$ & $\mathrm{N}$ & $0 \mathrm{D}$ & flask simulations \\
\hline Jost et al. (2004) & $1 \mathrm{P}, 1 \mathrm{M}, 1 \mathrm{Z}$ & micro-CMs & Nut & $0 \mathrm{D}$ & ecology theory \\
\hline Hammer and Pitchford (2005) & $1 \mathrm{P}, 1 \mathrm{M}$ & $\mathrm{CMs}$ & Nut & $0 \mathrm{D}$ & ecology theory \\
\hline Troost et al. (2005) & $1 \mathrm{M}$ & OSM & $\mathrm{C}, \mathrm{N}$ & $1 \mathrm{D}$ & evolutionary theory \\
\hline Hood et al. (2006) & $2 \mathrm{P}, 1 \mathrm{M}, 2 \mathrm{Z}$ & $\mathrm{CMs}$ & $\mathrm{N}$ & OD & ecology theory \\
\hline Bruggeman (2009) & $1 \mathrm{M}$ & $\mathrm{CMs}$ & $\mathrm{C}, \mathrm{N}$ & $1 \mathrm{D}$ & ecology theory \\
\hline Flynn and Mitra (2009) & $1 \mathrm{P}, 1 \mathrm{M}, 1 \mathrm{~B}$ & CMs, GNCMs, pSNCMs & $\mathrm{C}, \mathrm{N}, \mathrm{P}$ & OD & ecology theory \\
\hline Mitra and Flynn (2010) & $1 \mathrm{P}, 1 \mathrm{M}, 1 \mathrm{~B}$ & CMs, GNCMs, pSNCMs & $\mathrm{C}, \mathrm{N}, \mathrm{P}$ & OD & ecology theory \\
\hline Ward et al. (2011) & $1 \mathrm{P}, n \mathrm{M}, 1 \mathrm{Z}, 1 \mathrm{~B}$ & CMs ( $n$ levels) & $\mathrm{N}$ & OD & ecology theory \\
\hline Våge et al. (2013) & $n \mathrm{P}, n \mathrm{M}, n \mathrm{Z}$ & CMs $(n$ levels $+n$ sizes $)+$ OSM & $\mathrm{P}$ & OD & ecology theory \\
\hline Flynn and Hansen (2013) & $1 \mathrm{P}, 1 \mathrm{M}$ & CMs, GNCMs & $\mathrm{C}, \mathrm{N}, \mathrm{P}$ & $0 \mathrm{D}$ & ecology theory \\
\hline Mitra et al. (2014) & $1 \mathrm{P}, 1 \mathrm{M}, 2 \mathrm{Z}, 1 \mathrm{~B}$ & nano-CMs & $\mathrm{C}, \mathrm{N}, \mathrm{P}$ & $0 \mathrm{D}$ & ecology theory \\
\hline Cropp and Norbury (2015) & $1 \mathrm{P}, 2 \mathrm{M}, 1 \mathrm{Z}$ & CMs (2 levels) & Nut & $0 \mathrm{D}$ & ecology theory \\
\hline Mitra et al. (2016) & $1 \mathrm{P}, 1 \mathrm{M}, 1 \mathrm{~B}$ & CMs, GNCMs & $\mathrm{C}, \mathrm{N}, \mathrm{P}$ & OD & ecology theory \\
\hline Ward and Follows (2016) & $n \mathrm{M}$ & CMs ( $n$ levels $+n$ sizes $)$ & $\mathrm{C}, \mathrm{N}, \mathrm{P}$ & $3 \mathrm{D}$ & field simulations \\
\hline Berge et al. (2017) & $1 \mathrm{M}$ & $\mathrm{CMs}$ & $\mathrm{C}, \mathrm{N}$ & $1 \mathrm{D}$ & ecology theory \\
\hline Chakraborty et al. (2017) & $n \mathrm{M}$ & CMs ( $n$ levels $+n$ sizes $)$ & $\mathrm{C}, \mathrm{N}$ & $0 \mathrm{D}$ & ecology theory \\
\hline Ghyoot et al. (2017a) & $1 \mathrm{M}, 1 \mathrm{~B}$ & nano-CMs (2 levels), GNCMs & $\mathrm{C}, \mathrm{N}, \mathrm{P}$ & OD & ecology theory \\
\hline Ghyoot et al. (2017b) & $1 \mathrm{P}, 3 \mathrm{M}, 1 \mathrm{Z}, 1 \mathrm{~B}$ & OSM, nano-CMs, GNCMs & $\mathrm{C}, \mathrm{N}, \mathrm{P}, \mathrm{Si}$ & OD & field simulations \\
\hline Lin et al. (2018) & $1 \mathrm{P}, 1 \mathrm{M}$ & $\mathrm{CMs}$ & $\mathrm{C}, \mathrm{N}, \mathrm{P}$ & OD & flask simulations \\
\hline Edwards (2019) & $1 \mathrm{P}, n \mathrm{M}, 1 \mathrm{Z}, 1 \mathrm{~B}$ & nano-CMs ( $n$ levels) & $\mathrm{N}$ & $0 \mathrm{D}$ & ecology theory \\
\hline
\end{tabular}


The mechanisms explicitly described also vary greatly among the models (Table 1.2). The first models have described mixotrophy through the addition of phototrophy and phagotrophy, i.e., summing up the formulations used in phytoplankton and zooplankton models and neglecting any feedback between these nutritional modes (Table 1.2). Models that include a more complex description of the physical environment (Table 1.1) are also largely based on simple descriptions of plankton physiology and may lack the description of important features such as variable stoichiometry, photoacclimation, intraguild predation, prey selectivity, and variable assimilation efficiency (Table 1.2). Mixotrophy is a synergistic interaction, in which different mechanisms can act regulating both nutritional modes (Flynn and Mitra, 2009; Stoecker et al., 2017). Simpler descriptions of mixotrophy might provide realistic biomass values, but metabolic rates differ greatly from more complex models (Mitra and Flynn, 2010). The model by Flynn and Mitra (2009) is a comprehensive mechanistic construct of mixotrophy, allowing for the representation of mixotrophic functional diversity, from constitutive to non-constitutive forms (Mitra et al., 2016). This construct imposes a large number of parameters which are not always well constrained by the data; nevertheless, its features (Table 1.2) can enable a more robust understanding of the impact of mixotrophs within marine ecosystems and, therefore, this model was chosen to be used in this thesis. Finally, only four studies compared simulations with empirical data and only one compared simulated mixotrophic dynamics with observational data from long-term monitoring time series (Ghyoot et al., 2017b). Future studies should aim to validate mixotrophy against laboratory and/or field observations.

Mechanistic models designed to understand complex biological processes are valuable and needed in ecology and evolutionary biology (Coelho et al., 2018). There is a lot to be learned from ecosystem models that assign mixotrophy: what are the competitive outcomes within plankton communities under different environmental conditions? How these affect ecosystem properties? Does mixotrophic functional diversity matters? What is the relevance of mixotrophy across different spatial and temporal scales? This thesis aimed to answer these questions with the hope to, ultimately, guide future studies that will keep advancing mixotrophic research and plankton ecology and, hence, improving our understanding of the functioning of marine ecosystems. 
Table 1.2 Features of models describing mixotrophy in the literature; $\Delta$-variable, AE-assimilation efficiency, *linked to stoichiometry.

\begin{tabular}{|c|c|c|c|c|c|c|c|c|c|c|}
\hline \multirow[b]{2}{*}{ Reference } & \multicolumn{10}{|c|}{ Model feature } \\
\hline & light & $\begin{array}{c}\Delta \\
\mathrm{C}: \mathrm{Chl}\end{array}$ & $\begin{array}{c}\Delta \\
\mathrm{C}: \mathrm{N}: \mathrm{P}\end{array}$ & $\begin{array}{l}\text { phototrophy } \\
\text { phagotrophy } \\
\text { feedbacks }\end{array}$ & $\begin{array}{l}\text { internal } \\
\text { nutrient } \\
\text { cycling* }\end{array}$ & $\begin{array}{l}\text { intraguild } \\
\text { predation }\end{array}$ & $\begin{array}{c}\text { prey } \\
\text { selection }\end{array}$ & $\begin{array}{l}\text { satiation } \\
\text { feedback }\end{array}$ & $\begin{array}{c}\Delta \\
\mathrm{AE}\end{array}$ & $\begin{array}{c}\text { validation } \\
\text { against } \\
\text { data }\end{array}$ \\
\hline \multicolumn{11}{|l|}{ Thingstad et al. (1996) } \\
\hline Baretta-Bekker et al. (1998) & $\mathrm{x}$ & & $\mathrm{x}$ & & $\mathrm{x}$ & $\mathrm{x}$ & $\mathrm{x}$ & & & \\
\hline Stickney et al. (2000) & $\mathrm{x}$ & & & & & $\mathrm{x}$ & & & & \\
\hline Kooijman et al. (2002) & $\mathrm{x}$ & & & & & & & & & \\
\hline Zhang et al. (2003) & & & & & & & & & & $\mathrm{x}$ \\
\hline \multicolumn{11}{|l|}{ Jost et al. (2004) } \\
\hline \multicolumn{11}{|l|}{ Hammer and Pitchford (2005) } \\
\hline Troost et al. (2005) & $\mathrm{x}$ & & & & & & & & & \\
\hline Hood et al. (2006) & $\mathrm{x}$ & & & & & & $\mathrm{X}$ & & & \\
\hline Bruggeman (2009) & $\mathrm{x}$ & & & $\mathrm{x}$ & & & & & & \\
\hline Flynn and Mitra (2009) & $\mathrm{X}$ & $\mathrm{X}$ & $\mathrm{X}$ & $\mathrm{X}$ & $\mathrm{X}$ & & & $\mathrm{X}$ & $\mathrm{X}$ & \\
\hline Mitra and Flynn (2010) & $\mathrm{x}$ & $\mathrm{x}$ & $\mathrm{x}$ & $\mathrm{x}$ & $\mathrm{x}$ & & & $\mathrm{x}$ & $\mathrm{x}$ & \\
\hline \multicolumn{11}{|l|}{ Ward et al. (2011) } \\
\hline Flynn and Hansen (2013) & $\mathrm{X}$ & $\mathrm{x}$ & $\mathrm{X}$ & $\mathrm{X}$ & $\mathrm{X}$ & & & $\mathrm{X}$ & $\mathrm{x}$ & \\
\hline Våge et al. (2013) & & & & & & $\mathrm{X}$ & $\mathrm{X}$ & & & \\
\hline Mitra et al. (2014) & $\mathrm{X}$ & $\mathrm{X}$ & $\mathrm{X}$ & $\mathrm{X}$ & $\mathrm{X}$ & $\mathrm{x}$ & $\mathrm{X}$ & $\mathrm{X}$ & $\mathrm{X}$ & \\
\hline \multicolumn{11}{|l|}{ Cropp and Norbury (2015) } \\
\hline Mitra et al. (2016) & $\mathrm{X}$ & $\mathrm{X}$ & $\mathrm{X}$ & $\mathrm{X}$ & $\mathrm{X}$ & $\mathrm{X}$ & $\mathrm{X}$ & $\mathrm{X}$ & $\mathrm{X}$ & \\
\hline Ward and Follows (2016) & $\mathrm{x}$ & $\mathrm{x}$ & $\mathrm{x}$ & & $\mathrm{x}$ & & $\mathrm{x}$ & & $\mathrm{x}$ & $\mathrm{x}$ \\
\hline Berge et al. (2017) & $\mathrm{x}$ & & & $\mathrm{x}$ & & & & & & \\
\hline Chakraborty et al. (2017) & $\mathrm{X}$ & & & $\mathrm{X}$ & & & $\mathrm{X}$ & & & \\
\hline Ghyoot et al. (2017a) & $\mathrm{x}$ & & $\mathrm{x}$ & $\mathrm{x}$ & $\mathrm{x}$ & & & & & \\
\hline Ghyoot et al. (2017b) & $\mathrm{x}$ & & $\mathrm{x}$ & $\mathrm{x}$ & $\mathrm{x}$ & & & & & $\mathrm{x}$ \\
\hline Lin et al. (2018) & $\mathrm{X}$ & $\mathrm{X}$ & $\mathrm{X}$ & $\mathrm{X}$ & $\mathrm{X}$ & & & $\mathrm{X}$ & $\mathrm{X}$ & $\mathrm{X}$ \\
\hline Edwards (2019) & $\mathrm{x}$ & & & & & & & & & \\
\hline
\end{tabular}




\subsection{Objectives}

The overall objective of this thesis was to investigate the biogeography of mixotrophs across the global oceans and their impact on ecosystem functioning. The role of mixotrophic functional diversity in determining biogeographic patterns and competitive outcomes was tested across both spatial and temporal scales. The specific aims of this thesis are outlined below:

Aim 1: to investigate the biogeography of mixotrophs according to their functional diversity across oceanic biomes and to evaluate how it relates to environmental variability.

The global biogeography of mixotrophs was assessed using online databases and examining the current literature (Chapter 2). Analyses were performed to verify if functional diversity shapes the biogeographic patterns of mixotrophs (Chapters 3 and 4). Their biogeography was evaluated across different oceanic biomes and seasons and the effect of different environmental variables was tested (Chapters 3 and 4). The sampling biases associated with size, taxonomy, and oceanic region hindering the biogeography of constitutive mixotrophs were also evaluated (Chapter 4).

Aim 2: to explore the competitive outcomes between mixotrophs and their auto-or heterotrophic counterparts and the effect of mixotrophy on ecosystem functioning.

The role of constitutive mixotrophic nanoflagellates as bacterivores and their success relative to heterotrophic competitors were first assessed through a meta-analysis (Chapter 4). Then, a conceptual plankton food web model was developed spanning from bacteria to mesozooplankton and including different mixotrophic functional types (Chapter 5). Chemostat modelling experiments were performed to test for the effect of mixotrophy on community composition and ecosystem functioning under different light and nutrient regimes (Chapter 6). Three ecosystem properties were targeted: ammonium regeneration, trophic transfer efficiency, and production of dissolved organic carbon (DOC). 
Aim 3: to investigate the seasonal succession of protist trophic strategies and the vertical distribution of mixotrophic populations within a coastal temperate sea.

The modelling framework developed in Chapter 5 was validated against observational data for the Western English Channel. The incorporation of realistic environmental variability allowed the investigation of the succession of protists over the seasonal cycle and throughout the water column (Chapter 7). The relative success of the different protist functional types was evaluated considering bottom-up and top-down processes. In addition, the balance between phototrophy and phagotrophy within mixotrophs was evaluated for the different mixotrophs included in the model (Chapter 7). Data compiled in Chapters 3 and 4 were also used for comparisons with modelling results. 


\section{Chapter 2}

Methods - Biogeographic analyses 


\subsection{Introduction}

The global biogeography of the various mixotrophic functional types was investigated through the compilation of information from online databases and the literature. This chapter describes the general methods applied to the biogeographic analyses conducted for NCMs (Chapter 3; Leles et al., 2017) and CMs (Chapter 4; Leles et al., 2019). Data compilation was mostly guided by species name since few studies specifically targeted mixotrophy. Accordingly, prior to the examination of the datasets, a list containing all protist species previously reported to be mixotrophs was built; mixotrophic species were identified experimentally by displaying both phototrophic and phagotrophic nutrition. This approach is then an approximation of the 'true' pool of mixotrophic protists since mixotrophy among several species of marine protists remains to be investigated. AlgaeBase (http://www.algaebase.org/) and WoRMS (http://www.marinespecies.org/) were used to account for species synonyms and basionyms when carrying out data compilation. This methodology (based merely on species name) does not account for the fact that the expression of mixotrophy can vary across space and time. While acquired phototrophs are obligate mixotrophs, constitutive forms might not always display phototrophic and phagotrophic nutrition. Only studies which specifically targeted phagotrophy among constitutive mixotrophs (as reviewed by Beisner et al., 2019) allowed the investigation of how mixotrophy vary with environmental conditions (Chapter 4.2.1 and 4.2.3).

Both qualitative (presence) and quantitative (biomass/numeric abundance) data were obtained from online databases and the literature. Details on how quantitative data were obtained are given in Chapters 3.2.1 and 4.2.1. The Longhurst biogeographic classification of the ocean was adopted to map the global distribution of mixotrophs (Longhurst, 2007). Coordinates corresponding to the locations where mixotrophic species were recorded were aligned with Longhurst's biogeographic provinces. 


\subsection{Data compilation}

\subsubsection{Online databases for plankton data}

Three online databases were initially examined for the analyses: the Ocean Biogeographic Information System (OBIS; http://www.iobis.org/), the Coastal \& Oceanic Plankton Ecology, Production, \& Observation Database (COPEPOD; http://www.st.nmfs.noaa.gov/copepod/), and the Continuous Plankton Recorder (CPR; https://www.cprsurvey.org) database. OBIS is a project under IOC-UNESCO's International Oceanographic Data and Information (IODE) programme. It connects 500 institutions from 56 countries providing over 45 million observations spanning bacteria to whales across the global oceans. COPEPOD is a global plankton database managed by the National Marine Fisheries Service (NMFS - 'NOAA Fisheries') with over 400,000 observations of planktonic organisms. The CPR consists on a towed instrument $(270 \mu \mathrm{m}$ mesh size) designed to capture plankton samples at $10 \mathrm{~m}$ depth over vast areas of the ocean utilising ships of opportunity. It is operated by the Sir Alister Hardy Foundation for Ocean Science (SAHFOS), being incorporated to the Marine Biological Association in April 2018. Thus, contrary to the previous databases which present a collection of data from different surveys, the CPR is a single continuous lmonitoring programme. Quantitative data can be obtained from COPEPOD and CPR but not through OBIS; the later consists only of distribution records.

Qualitative data were obtained through OBIS since the datasets are integrated and can be accessed by different entities, including species name. Despite providing quantitative data, the COPEPOD and CPR databases were identified as not suitable for this study because they target larger plankton members. COPEPOD database has the potential to provide taxonomic data for mixotrophic protists but it is still in development (COPEPEDIA). In its current form, COPEPOD did not provide any additional qualitative data further to those compiled from OBIS (interrogated on $16^{\text {th }}$ March 2017). In turn, CPR is particularly suitable for sampling mesozooplankton and larger ( $>250 \mu \mathrm{m}$ size) robust phytoplankton (Richardson et al., 2006), ruling out many mixotrophic species within the smallest plankton members (Zubkov and Tarran, 2008). Indeed, less than 20 mixotrophic species (all dinoflagellates) were identified on the online CPR list of available taxa ('Data 
Request Form'; interrogated on $16^{\text {th }}$ March 2017). The CPR data are also not suitable at a global scale since it is mainly used to sample plankton in the Northwest European shelf and in the Northeast and Northwest Atlantic. Thus, distribution records used in Chapters 3 and 4 were obtained through OBIS on $16^{\text {th }}$ March 2017 and on $20^{\text {th }}$ January 2018, respectively. Over 110,000 and 230,000 records were obtained for the analysis of the global biogeography of NCMs and CMs, respectively, including a total screen for 150 species (Chapters 3.2.2 and 4.2.2). OBIS possess its own quality control system in place for data, securing that data come from credible sources and applying a series of technical controls (e.g., species name misspellings, names not recognized, mapping errors) every time the data is crawled again from its source. On top of that, the data compiled for this thesis were checked independently for points on land, taxon matching, and depth values.

\subsubsection{Bibliographic surveys}

The electronic database ISI Web of Science was accessed for the bibliographic surveys within Chapter 3 and 4 on $16^{\text {th }}$ March 2017 and on $20^{\text {th }}$ January 2018, respectively. The surveys were conducted to obtain quantitative data on numeric abundance/biomass of mixotrophic protists from studies which specifically targeted mixotrophs (applying same methods) and to complement the OBIS survey. Different sets of keywords were used in the search for the biogeography of CMs and NCMs (details in Chapters 3.2.1 and 4.2.1, respectively). Over 200 studies were evaluated; for each study, latitude and longitude, period of the year (month and season), and sampling depth were recorded. Temporal resolution was represented according to seasonality: 21/06-15/09 (Boreal summer/Austral winter), 21/09-15/12 (Boreal autumn/Austral spring), 21/12-15/03 (Boreal winter/Austral summer), and 21/03-15/06 (Boreal spring/Austral autumn). Seasons were defined based on the equinoxes (day and night of equal length) and solstices (longest and shortest day of the year), assumed to happen on the $21^{\text {st }}$ of September/March, and June/December, respectively. Seasonal variability is particularly important within temperate and polar seas, in which gradients of environmental factors are well marked and play an important role in plankton dynamics (Sommer et al., 2012). Seasonality is not as well marked within tropical seas and certain coastal systems in which rainfall and riverine discharge may play a major role; therefore, care was taken to interpret temporal patterns among different locations. Data were recorded for different depths whenever possible; a single depth- 
integrated value was obtained otherwise. Sampling depths among studies ranged from surface to the local observed thermocline. Nitrate and phosphate concentrations, as well as the abundance of heterotrophic bacteria and/or cyanobacteria, were also extracted from the studies used in the meta-analysis of mixotrophic nanoflagellates (Chapter 4.2.3). Whenever possible, the proportion of mixotrophs and heterotrophic counterparts relative to the total population was estimated (e.g., mixotrophic ciliates vs strict heterotrophic ciliates). Where required, data from published figures were extracted using the free version of GetData Graph Digitizer (http://getdata-graph-digitizer.com/).

\subsection{Biogeographic provinces and oceanic biomes}

The biogeographic analyses were oriented by the division of the ocean into subsets defined by 54 biogeographic provinces according to Longhurst (2007) (Table 2.1 and Fig. 2.1). Longhurst's division of the ocean was based on the assumption that pelagic biogeography is dictated by seasonal and spatial variation of primary production, which, in turn, is mirrored by physical forcing (e.g., light, wind, temperature). Provinces were then defined according to observed discontinuities in physical processes and analysis of satellite images of surface chlorophyll and in situ chlorophyll depth profiles (Longhurst et al., 1995). This classification, however, assumed that the boundaries between the provinces are fixed and defined by rectangular grids (Fig. 2.1). In reality, the boundaries are influenced by changing environmental conditions at a global scale (Reygondeau et al., 2013). Notwithstanding, Longhurst's classification is a mark in marine biogeography that was possible with the advent of satellite products (Priede, 2014). Since then, it has been widely applied in oceanography and ecology and the spatial distribution of processes and species have been shown to match Longhurst's provinces (e.g., Corbineau et al., 2008; Demarcq et al., 2012; Burridge et al., 2017). In this context, it is relevant to investigate the distribution of mixotrophic plankton using Longhurst's classification.

At least one record was necessary to assume that mixotrophs occurred in any province. Grids corresponding to Longhurst's provinces used in the maps were obtained from http://www.marineregions.org/. Longhurst used the net primary productivity (NPP) obtained from SeaWIFS data from 1997 to 2002 to define the different biogeographic provinces (Longhurst, 2007). In order to quantitatively relate the distribution of 
mixotrophs with Longhurst's provinces in this thesis, the relationship between the NPP data used on his work and the biomass/numeric abundance of mixotrophs obtained here through the bibliographic surveys were investigated. In Chapter 3, the NPP data were processed so that global means and global variances were obtained for each province (Appendix A, Fig. A.1); these metrics allow an approximation to the overall nutrient load and system variability, respectively, within each province (Leles et al., 2017). The NPP and the mixotrophic datasets were then merged based on the different biogeographic provinces, identified in the mixotrophic dataset through the corresponding geographic coordinates from which estimates were obtained. In Chapter 4, the NPP and the mixotrophic datasets were merged based on the corresponding geographic coordinates as well as on the sampling month (Appendix B, Tables B.2 and B.3).

To allow the statistical comparison of means across major regions in the oceans, Longhurst's provinces were grouped into seven principal biomes: Coastal Seas, Equatorial Upwelling, Mediterranean Sea, Oligotrophic Gyres, Polar Seas, Temperate Seas, and Coastal Upwelling (Table 2.1 and Fig. 2.1). These biomes align in part with those defined by Longhurst (Longhurst et al., 1995; Longhurst, 2007), but expand his classification explicitly describing upwelling regions and the Mediterranean Sea. As per the biogeographic provinces, the biomes were defined based on primary production and physical forcing. Seasonality is the main characteristic of both Polar Seas and Temperate Seas biomes, but the former is subjected to the effect of melting ice while the second is related to wind stress (Longhurst et al., 1995). The Mediterranean Sea biome is also subjected to seasonality but differs from the others because it has limited exchange of water with outer oceans (Pinardi et al., 1997). In turn, the Oligotrophic Gyres biome is characterized by a shallow pycnocline of high stability and overall low primary production (Longhurst et al., 1995). In the Coastal Seas biome, the generic oceanic circulation is modified by coastal topography and wind coastal regime (Longhurst et al., 1995); coastal upwelling systems were defined separately since these support high primary production (Acha et al., 2004). Finally, equatorial upwelling regions were also considered as a separate biome; upwelling events in these regions are related to the Intertropical Convergence Zone, promoting highly productive regions within the open oceans (Acha et al., 2004). 
Table 2.1 Longhurst's biogeographic provinces following Fig. 2.1; these were grouped into seven principal biomes according to primary production and physical forcing.

\begin{tabular}{|c|c|c|c|}
\hline Oceanic Basin & Code & Province & Biome \\
\hline \multirow{2}{*}{ Antarctic } & ANTA & Antarctic & Polar Seas \\
\hline & APLR & Austral & Polar Seas \\
\hline \multirow[t]{4}{*}{ Arctic } & ARCT & Atlantic Arctic & Polar Seas \\
\hline & BERS & North Pacific Epicontinent & Polar Seas \\
\hline & BPLR & Boreal & Polar Seas \\
\hline & SARC & Atlantic Subarctic & Polar Seas \\
\hline \multirow{19}{*}{ Atlantic } & BENG & Benguela Current & Coastal Upwelling \\
\hline & BRAZ & Brazil Current & Coastal Seas \\
\hline & CNRY & Eastern Canary & Coastal Seas \\
\hline & EAFR & Eastern Africa & Coastal Seas \\
\hline & FKLD & Southwest Atlantic Shelves & Coastal Seas \\
\hline & GUIA & Guianas & Coastal Seas \\
\hline & GUIN & Guinea Current & Coastal Upwelling \\
\hline & NECS & Northeast Atlantic Shelves & Coastal Seas \\
\hline & NWCS & Northwest Atlantic Shelves & Coastal Seas \\
\hline & GFST & Gulf Stream & Temperate Seas \\
\hline & MEDI & Mediterranean Sea, Black Sea & Mediterranean Sea \\
\hline & NADR & North Atlantic Drift & Temperate Seas \\
\hline & NAST (E) & North Atlantic Subtropical Gyre (East) & Temperate Seas \\
\hline & NAST (W) & North Atlantic Subtropical Gyre (West) & Temperate Seas \\
\hline & CARB & Caribbean & Oligotrophic Gyres \\
\hline & ETRA & Eastern Tropical Atlantic & Equatorial Upwelling \\
\hline & NATR & North Atlantic Tropical Gyre & Oligotrophic Gyres \\
\hline & SATL & South Atlantic Gyre & Oligotrophic Gyres \\
\hline & WTRA & Western Tropical Atlantic & Oligotrophic Gyres \\
\hline \multirow[t]{7}{*}{ Indian } & ARAB & Northwestern Arabian Upwelling & Coastal Upwelling \\
\hline & AUSW & Australia-Indonesia & Coastal Seas \\
\hline & INDE & Eastern India & Coastal Seas \\
\hline & INDW & Western India & Coastal Seas \\
\hline & REDS & Red Sea, Persian Gulf & Coastal Seas \\
\hline & ISSG & Indian South Subtropical Gyre & Oligotrophic Gyres \\
\hline & MONS & Indian Monsoon Gyres & Oligotrophic Gyres \\
\hline \multirow[t]{20}{*}{ Pacific } & ALSK & Alaska Downwelling & Coastal Seas \\
\hline & AUSE & East Australia & Coastal Seas \\
\hline & CALC & California Current & Coastal Upwelling \\
\hline & CAMR & Central America & Coastal Upwelling \\
\hline & $\mathrm{CHIN}$ & China Sea & Coastal Seas \\
\hline & HUMB & Humboldt Current & Coastal Upwelling \\
\hline & NEWZ & New Zealand & Coastal Upwelling \\
\hline & SUND & Sunda-Arafura Shelves & Coastal Seas \\
\hline & KURO & Kuroshio Current & Temperate Seas \\
\hline & NPPF & North Pacific Transition Zone & Temperate Seas \\
\hline & PSAG (E) & Pacific Subarctic Gyre (East) & Temperate Seas \\
\hline & PSAG (W) & Pacific Subarctic Gyre (West) & Temperate Seas \\
\hline & TASM & Tasman Sea & Coastal Seas \\
\hline & $\mathrm{ARCH}$ & Archipelagic Deep Basins & Oligotrophic Gyres \\
\hline & NPTG (E) & North Pacific Tropical Gyre (East) & Oligotrophic Gyres \\
\hline & NPTG $(\mathrm{W})$ & North Pacific Tropical Gyre (West) & Oligotrophic Gyres \\
\hline & PEQD & Pacific Equatorial Divergence & Equatorial Upwelling \\
\hline & PNEC & North Pacific Equatorial Countercurrent & Equatorial Upwelling \\
\hline & SPSG & South Pacific Subtropical Gyre & Oligotrophic Gyres \\
\hline & WARM & Western Pacific Warm Pool & Oligotrophic Gyres \\
\hline
\end{tabular}



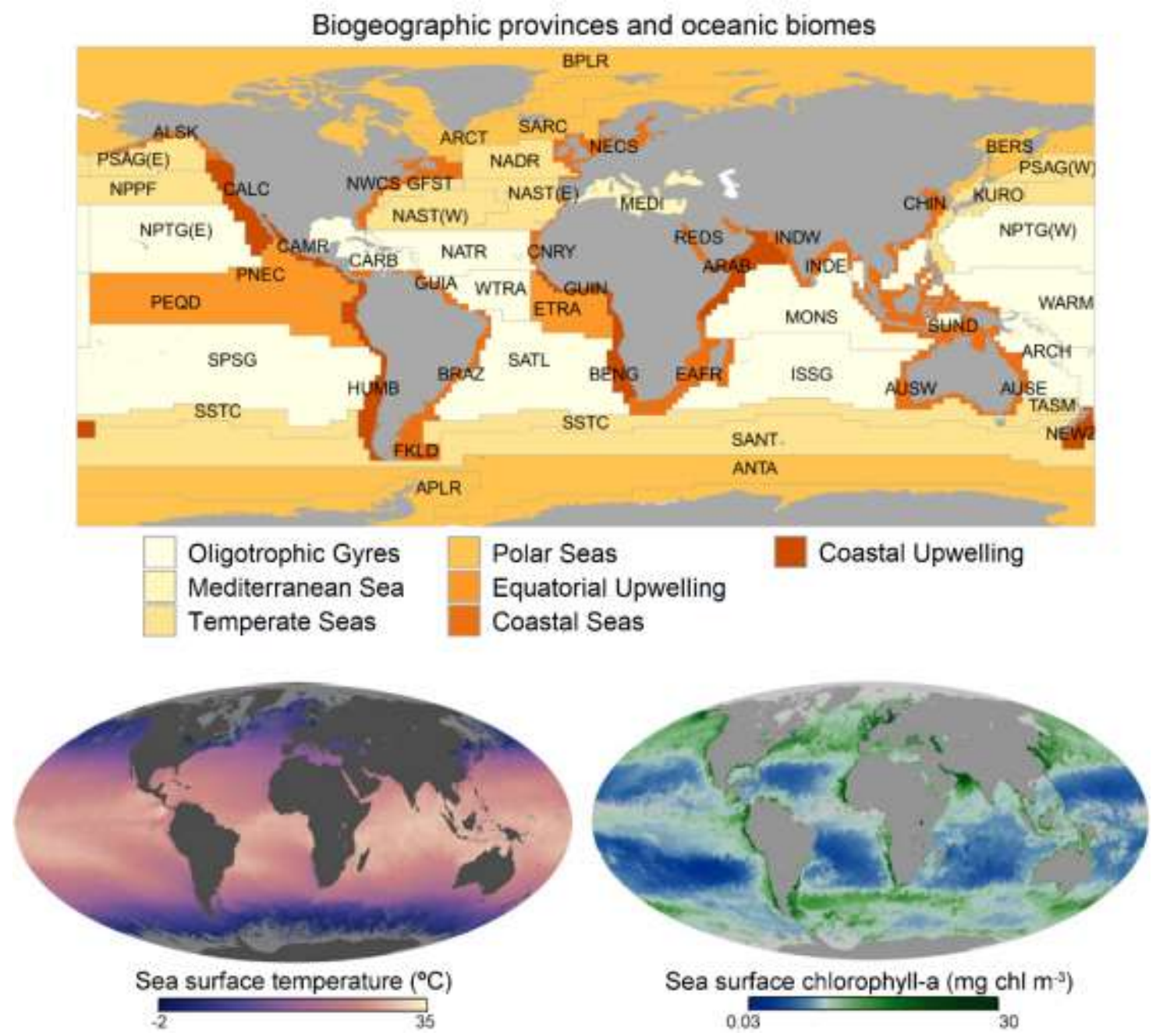

Figure 2.1 Longhurst division of the ocean in 54 biogeographic provinces (Longhurst, 2007) and the seven oceanic biomes defined in this thesis according to physical forcing and primary production (upper panel). Colours highlight the different biomes; from lighter to darker colours: Oligotrophic Gyres, Mediterranean Sea, Temperate Seas, Polar Seas, Equatorial Upwelling, Coastal Seas, and Coastal Upwelling biomes. Provinces abbreviations are given in Table 2.1. Averaged sea surface temperature (lower left panel) and chlorophyll-a (lower right panel) from satellite products are given as proxies for physical forcing and primary production and are not directly linked to Longhurst's work (source: MODIS (NASA); https://earthobservatory.nasa.gov). 


\subsection{Statistical analyses}

\subsubsection{Data processing and statistical choices}

Data were tabulated in Microsoft Office Excel (version 2010) or directly in R (version 3.4.3; R Core Team, 2016) for data processing and analysis. The packages (and functions) within $\mathrm{R}$ used for data processing and for statistical analyses will be referred herein between quotation marks ("). OBIS data were accessed through the 'robis' package; detailed guidelines can be found online (https://obis.org/manual/accessr/). Distribution records for each species were then obtained using the 'occurrence' function within 'robis'. The function 'readOGR' within the package 'rgdal' was used to read the shapefile containing the Longhurst's biogeographic provinces. The 'dplyr' package was then used to process the occurrence data and retrieve the geographic coordinates within each biogeographic province. Records with possible spatial errors, such as data points located in land, were excluded from the analysis. All figures summarizing data from the biogeographic analyses were created in $\mathrm{R}$ using the packages: 'ggplot2', 'maps', 'gridExtra', 'RColorBrewer', and 'scales'.

Statistical analyses were also conducted in R. Different statistical tests (or techniques) were applied to the biogeographic analyses. Data were interrogated in order to choose the most suitable tests (or techniques) to perform: i) comparison of means; ii) ordination; and iii) to investigate the relationship between variables (Fig. 2.2). Details for each of these analyses are given in the following sections, respectively. 


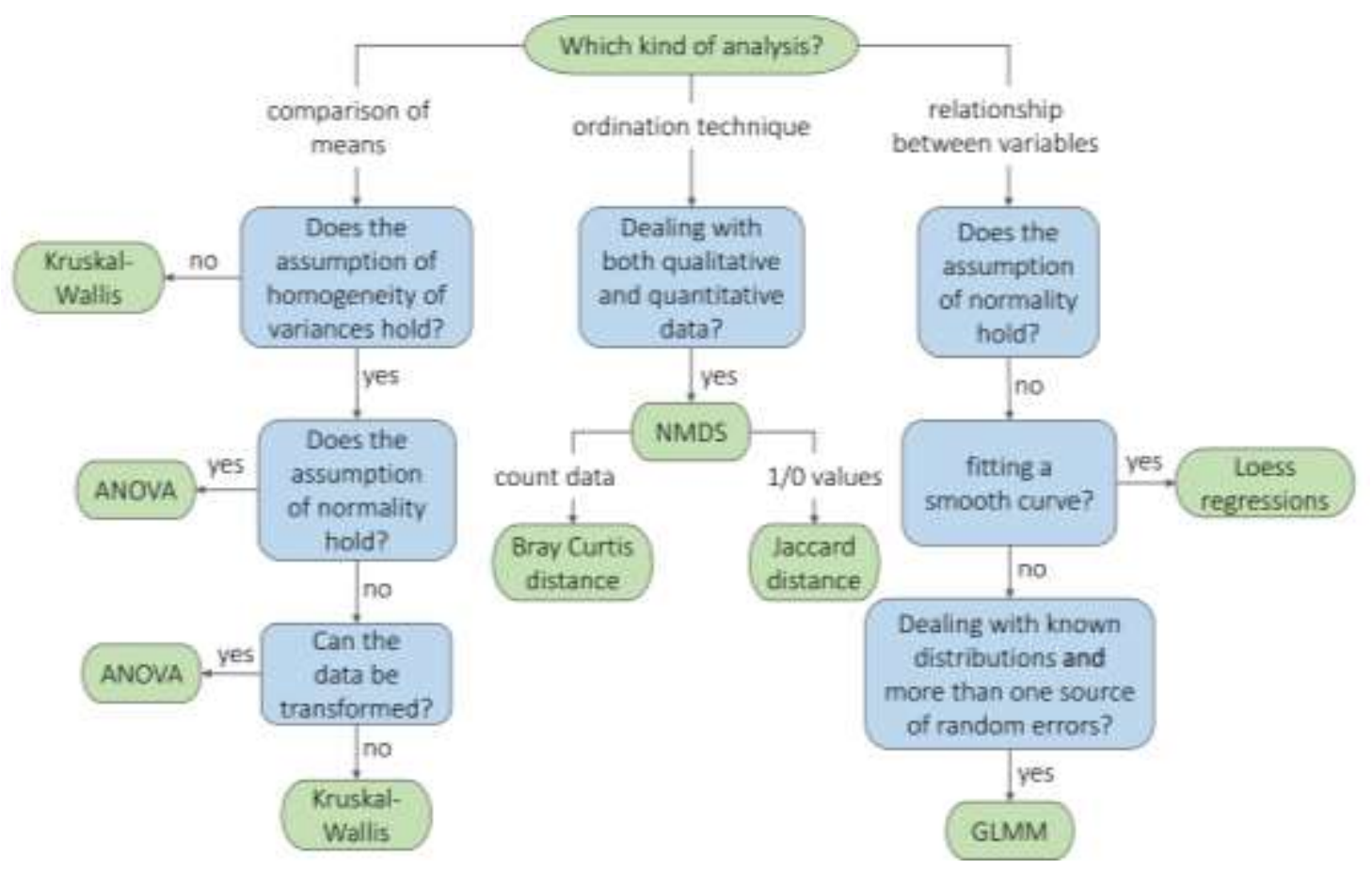

Fig. 2.2 Flowchart showing the different interrogations applied to the biogeographic data to perform comparison of means, ordination, and to investigate the relationship between variables.

\subsubsection{Comparison of means}

The comparison of means (or median) from different groups was performed through analysis of variance (ANOVA) or through Kruskal-Wallis tests (results reported as $\mathrm{F}=$ test statistic, $\mathrm{p}=$ significant value and $\mathrm{H}=$ test statistic, $\mathrm{p}=$ significant value, respectively) (Fig. 2.2). Residuals and residual variance were checked for normal distribution and homogeneity, respectively, through graphical visualization (Gotelli and Ellison, 2012). ANOVA was used if both assumptions were met (Fig. 2.2). Data were transformed using $\log (x+1)$ if necessary; however, if data transformation was not sufficient to meet the assumption of normality, a rank-based non-parametric KruskalWallis test was performed instead (Fig. 2.2; Gotelli and Ellison, 2012). Post-hoc tests were performed if results were significant to identify which means were different from each other; Tukey's and Dunn's post hoc-tests were performed for ANOVA and KruskalWallis test, respectively (Dunn, 1961; Gotelli and Ellison, 2012). The Dunn's post-hoc test gives Z-scores for each pairwise comparison as an output. Overall, a Z-score shows how many standard deviations (above or below) from the mean a data point is. Here, the 
Dunn's test uses the mean rankings of the outcome in each group from the preceding Kruskal-Wallis test. Positive values indicate that the mean of the reference group is higher than the mean of the second group while the opposite holds for negative values. ANOVA was conducted through the 'Im', 'anova', and 'TukeyHSD' functions in the 'vegan' package while Kruskal-Wallis tests were performed using the 'dunn.test' function in 'dunn.test' package.

\subsubsection{Ordination technique}

Non-metric multidimensional scaling (NMDS) was performed to explore the dissimilarities between species according to their distribution across the global oceans (Fig. 2.2). As for any other ordination technique, the NMDS firstly ordinates the objects in full-dimensional space and then represents them in a few dimensions (Legendre and Legendre 1998). It can be applied to any distance measure and to qualitative or quantitative data (Fig. 2.2). The NMDS is particularly used to plot dissimilar objects far apart in the ordination space and similar objects close to each other (Legendre and Legendre 1998). Contrary to other ordination methods based on eigenvector values, NMDS axes are arbitrary because it does not maximize the variability associated to individual axes; thus, plots can be rotated, centered, or inverted. A matrix 'species vs site' based on qualitative (presence/absence) or quantitative (number of occurrences) data and on the 54 Longhurst's biogeographic provinces was built to perform the analyses. To facilitate the interpretation of NMDS results, the 54 Longhurst's biogeographic provinces were later aligned within the 7 principal biomes described in the previous section. The Jaccard and Bray Curtis distances were used for datasets composed of presence/absence values and for count data, respectively (Fig. 2.2), and different random starting configurations were performed. The goodness-of-fit of the analysis is measured through the stress, a value between 0 and 1; as a rule of thumb, stress values $\leq 0.05$ indicate good fit and stress values $\geq 0.2$ indicates that results are arbitrary (Legendre and Legendre, 1998). The aim of the analysis is to use as few dimensions as possible to, ultimately, plot the results in a two-dimensional space; however, there is a trade-off between the number of dimensions and the stress value (Legendre and Legendre, 1998). Therefore, the choice of the number of dimensions must be made considering also the stress value. Ordination was performed using the 'metaMDS' function in the 'vegan' package. 


\subsubsection{Relationship between variables}

Loess regressions were used to explore the relationship between the relative biomass of mixotrophs and NPP within the different provinces (Chapter 3.3.2). This is a non-parametric method for fitting a smooth curve between two variables in which the linearity assumptions of conventional regression methods are relaxed (Fig. 2.2; Cleveland, 1979). It uses local weighted regression to fit a smooth curve through points in a scatter plot. The term 'local' is used because the fitting of a data point $x$ is weighted toward the data nearest to $x$. The proportion of observations used for local regression is assigned through a span parameter that varies between 0 and 1 . This parameter describes the tradeoff between bias (i.e., difference between the predicted and observed values) and variance (i.e., variability of prediction for a given data point). Parameter values closer to 1 assign a greater proportion of observations to the local regression increasing the bias whilst decreasing the variance and, thus, results in overfitting (Cleveland, 1979). Here, the span parameter was equal to 1 due to the limited availability of data and because the aim of the analysis was to obtain a general pattern instead of predicting the data at hand; standard error bands were given for all loess regressions. Loess regressions were plotted using the 'stat_smooth' function in the 'ggplot2' package.

Generalized linear mixed models (GLMMs) were used for the meta-analysis of actively feeding mixotrophic nanoflagellates across different oceanic systems (details are given in Chapter 4.2.3). GLMMs are a class of statistical models which describe the relationship between a response variable (i.e., outcome) and the explanatory variables (i.e., covariates) which have been measured along with the response (Bates et al., 2015). This statistical analysis is preferred over traditional methods, such as multiple linear regression, because the assumptions of normality and homogeneity of variance may be violated in the later (Fig. 2.2). GLMMs combine the characteristics of generalized linear models and mixed models (Bolker, 2007); they can be used to describe data that are not normally distributed and they account for random effects (Bolker et al., 2009). GLMMs are a good candidate if the distribution of the outcome is known and if more than one source of random errors is identified (Fig. 2.2). This class of statistical model do not assume a linear relationship between the response variable and the explanatory variables; instead, they allow any non-linear relationship that has a linearizing transformation 
through the 'link' function (Bolker, 2007). GLMMs can handle non-normal errors of the exponential family; different distributions can be specified through the link function (e.g., $\log$ link for Poisson distribution).

Covariates can be added to the statistical model as fixed or as random effects. Any continuous covariate must be assigned as a fixed effect; however, categorical covariates can be considered as fixed or as random effects. The difference here is that, as a fixed effect, the variability associated to each of the levels of the covariate are estimated, while only the overall variability associated to the covariate is estimated if assigning the covariate as a random effect. In practical terms, a random effect saves up degrees of freedom and function as an additional residual term. Usually, a minimum of 5 levels and at least 10 observations per level are needed to assign a covariate as a random effect (Bolker et al., 2009). Random effects can be added to the statistical model through random intercepts or random intercepts and random slopes. The first assumes that the different levels of the random effect have different intercepts, but same slopes, while the second would allow the random effect to have a different effect on the outcome for each level. However, care must be taken to avoid correlation between variables; maintaining a random intercept (and constant slopes) avoids correlation problems because observations that come from different groups are uncorrelated (Bolker et al., 2009).

The Poisson distribution assumes a variance to mean ratio of 1 . However, in many cases, count data spans from several orders of magnitude and the group variances increase with the mean far more than expected, indicating that the data is overdispersed. A random effect at the level of observation can then be included in a GLMM to account for overdispersion (Gelman and Hill, 2007; Harrison, 2014). This mechanism assigns the extra-Poisson variation in the outcome using a random effect with a unique level for every data point. This technique was applied to the GLMMs if overdispersion was detected.

The output of a GLMM provide estimates for the parameters associated with each fixed effect (e.g., differences between treatments and interactions) and standard deviations of the random effects. Parameter estimates in a GLMM are not obtained using least squares (unless dealing with normal response variables); maximum likelihood equations solved by interactive methods are used instead (Bolker et al., 2009). Different statistical 
techniques can be applied to estimate the parameters in a GLMM; here Laplace approximations were used (Tuerlinckx et al., 2006). This technique approximates the true GLMM likelihood by assuming that its distribution (of the likelihood, not the distribution of the data) is approximately normal (Bolker et al., 2009). This method was chosen because it works well even if standard deviations associated with random effects are large and because it allows likelihood-based inference (Bolker et al., 2009). Here, the significance of each term (fixed and random effects) in the GLMMs was tested through the likelihood ratio test (LRT). The LRT is a statistical test used to compare the difference between two statistical models, one of which is a special case of the other, using the Chisquare distribution. The test provides the ratio between the log likelihood of the two models and a $\mathrm{p}$-value (results reported as LR $=$ likelihood ratio, $\mathrm{p}=$ significant value). GLMMs were performed through the function 'glmer' in the 'Ime4' package. Inspection of estimates for the random effects were performed graphically and their significance was tested using the function 'anova'. Model inference was performed through the 'glmm_funs' package (Bolker et al., 2009). 


\section{Chapter 3}

\section{Biogeography of non-constitutive mixotrophs}

\section{Part of this chapter has been published as:}

Leles, S.G., Mitra, A., Flynn, K.J., Stoecker, D.K., Hansen, P.J., Calbet, A., McManus, G.B., Sanders, R.W., CAron, D.A., Not, F., HallegraefF, G.M., Pitta, P., Raven, J.A., Johnson, M.D., Glibert, P.M. \& VÅge, S. (2017) Oceanic protists with different forms of acquired phototrophy display contrasting biogeographies and abundance. Proceedings of The Royal Society B 284, 20170664. DOI: $10.1098 / \mathrm{rspb} .2017 .0664$ 


\subsection{Introduction}

Microzooplankton (i.e., protists $20-200 \mu \mathrm{m}$ perceived as strict phagotrophs) play a key role within pelagic marine food webs (Calbet and Landry, 2004; Schmoker et al., 2013). They are major grazers of phytoplankton across different oceanic systems, linking the primary production to copepods (Calbet and Landry, 2004; Schmoker et al., 2013). Within oligotrophic seas, the microbial loop envisions protist predatory activity as the main route for nutrient regeneration which, in turn, supports the primary production (Azam et al., 1983; Jiao et al., 2014). Despite usually perceived as strict phagotrophs, many species of microzooplankton are non-constitutive mixotrophs (NCMs) displaying acquired phototrophy (Stoecker et al., 2009; Johnson et al., 2011; Flynn et al., 2013; Mitra et al., 2016). Thus, their ecological relevance may be greater than previously anticipated. In fact, NCMs can dominate the microzooplankton assemblage at times in the ocean (Crawford, 1989; Dierssen et al., 2015).

Acquired phototrophy occur among ciliates, dinoflagellates, radiolarians, and foraminiferans through different evolutionary pathways, i.e., kleptoplasty and endosymbiosis (Chapter 1.2). In addition, various degree of control over the acquired phototrophic machinery are observed among species (Stoecker et al., 2009; Johnson et al., 2011a; Mitra et al., 2016; Moeller and Johnson, 2018). Thus, one may expect different forms of acquired phototrophy to occur and/or dominate in different oceanic systems. Although previous studies have investigated the distribution and abundance of protists within microzooplankton across the oceans, few have assigned the role of acquired phototrophy (Tarangkoon et al., 2010; Harrison et al., 2011; Biard et al., 2016). Acquired phototrophy is an overlooked trait when investigating the biogeography and ecology of protists. Most field studies reporting the abundance of microzooplankton do not distinguish between the photosynthetic and the non-photosynthetic forms (e.g., Davidson et al., 2010; Jayalakshmi et al., 2015; Yang et al., 2016; Cáceres et al., 2017). Similarly, most marine ecosystem models describe microzooplankton as a strict-heterotrophic functional group (e.g., Sinha et al., 2010; Yool et al., 2013; Butenschön et al., 2016). To date, only one study has explored the role of NCMs within biogeochemical models (Ghyoot et al., 2017b). 
The aim of this work was to investigate, for the first time, the biogeography of NCMs in the global oceans. It is hypothesised that protists with different forms of acquired phototrophy dominate in different oceanic systems due to differences in the degree of control of acquired phototrophy. Both qualitative and quantitative data were compiled from online databases and literature to investigate the spatio-temporal distribution of acquired phototrophs in the sunlit ocean. The findings presented herein show that mixotrophic functional diversity is an important factor determining the biogeography of NCMs in the global oceans. In addition, this study quantitatively estimates the proportion of microzooplankton which are mixotrophic through the acquisition of phototrophy, providing a robust dataset to guide the inclusion of acquired phototrophy in future modelling studies seeking to investigate the role of NCMs within marine ecosystems.

\subsection{Methods}

\subsubsection{Data compilation}

A global analysis of field data for the different groups of NCMs was conducted across the global oceans (Chapter 2.2). A total of 72 NCM species were identified through reference to Stoecker et al. (2009). The different forms of acquired phototrophy were grouped following the classification of Mitra et al. (2016), into GNCMs, pSNCMs, and eSNCMs (Table 3.1; see also Chapter 1.2 and Abbreviations for acronyms). Data compilation was conducted on $16^{\text {th }}$ March 2017. The set of keywords used in the bibliographic survey included "microzooplankton OR protozooplankton OR dinoflagellate OR ciliate OR foraminifera OR radiolaria OR rhizaria" followed by “mixotrophy OR plastid OR symbiont OR symbiosis OR green" and "abundance OR composition OR biomass" and "ocean OR marine". Over 110,000 records were obtained from OBIS (Table 3.1). The bibliographic survey added nearly 1,000 records to the analysis from over 180 articles (Appendix A, Table A.1).

Regarding the quantitative data (biomass/numeric abundance), the bibliographic survey targeted works from which it was possible to estimate the contribution of mixotrophs to the total microzooplankton assemblage in the sunlit ocean. Estimating the contribution of heterotrophic dinoflagellates engaging in acquired phototrophy to the total dinoflagellate biomass/numeric abundance is particularly challenging because they are 
difficult to distinguish from strict autotrophic dinoflagellates or constitutive mixotrophs (Stoecker et al., 1996). In contrast, it is relatively easy to separate groups of mixotrophic ciliates and mixotrophic Rhizaria.

Quantitative data for mixotrophic ciliates were obtained from $>45$ articles within the sunlit ocean (Appendix A, Table A.2). The articles comprised works which specifically targeted mixotrophy; these included field work that undertook analysis using epifluorescence microscopy (thus enabling identification of ciliates with algal plastids) and works which did not specifically target mixotrophy but reported data on microzooplankton species composition. However, there is a likely underestimation of mixotrophy using this methodology because not all ciliate species are identified down to species level. Ciliates are obligate mixotrophs and, therefore, always display mixotrophic characteristics (Stoecker et al., 2009). Their identification through epifluorescence microscopy or flow cytometry is possible because, differently from their heterotrophic counterparts, they retain intact chloroplasts scattered around their cell and these can be distinguished from feeding vacuoles (Stoecker et al., 2014). This method is robust and has been applied in several studies that aimed to estimate the proportion of mixotrophic ciliates relative to the total ciliate assemblage (e.g., Bernard and Rassoulzadegan, 1994; Chiang et al., 2003; Christaki et al., 2008; Stoecker et al., 2014; Haraguchi et al., 2018).

In contrast, the relative contribution of mixotrophic Rhizaria (eSNCMs) to the planktonic assemblage was estimated from a work which used a non-destructive in situ imaging system within the topmost $100 \mathrm{~m}$ (Biard et al., 2016). The Rhizaria data are publicly available in PANGAEA (https://doi.pangaea.de/10.1594/PANGAEA.858156). Among the Rhizaria groups investigated, all Acantharia and Collodaria were considered mixotrophic. It is noteworthy that the absolute biomasses of mixotrophic ciliates and mixotrophic Rhizaria were not directly comparable because they were estimated in different units ( $\mu \mathrm{g} \mathrm{C} \mathrm{L}^{-1}$ and $\mathrm{mg} \mathrm{C} \mathrm{m}^{-2}$, respectively). Biomass estimates from the different studies were obtained measuring the size of the cells, obtaining the cell biovolume, and later converting it to carbon biomass using carbon conversion factors from the literature (e.g., Menden-Deuer and Lessard, 2000). As for ciliates, Rhizaria are obligate mixotrophs and, therefore, always display mixotrophic characteristics (Stoecker et al., 2009). 


\subsubsection{Statistical analyses}

Over 110,000 records were obtained for 56 out of 72 species that comprised the initial list (Table 3.1). A presence-absence matrix based on Longhurst's biogeographic provinces was constructed to explore the dissimilarities between the NCM species using the NMDS technique (Chapter 2.4.3). At least one record was necessary to assume that mixotrophs occurred in any province. The number of records available for each species was highly variable (Table 3.1). Species for which datasets were not considered robust enough to represent their distribution over the globe were excluded; only species with a minimum of 30 records were included in the analysis. This cut-off limit was chosen to maximise the number of GNCMs (i.e., Strombidium capitatum, Strombidium oculatum, Strombidium reticulatum) evaluated. In addition, species with over 10,000 records were randomly subsampled down to a maximum of 2,000 records to decrease sampling bias among species. After filtering the dataset according to the above criteria, a total of 38 species were selected for analysis. The distance matrix used in the NMDS analysis was calculated using the Jaccard distance as the dataset was composed by $1 / 0$ values. To facilitate the interpretation of NMDS results, the 54 Longhurst's biogeographic provinces were aligned within the 7 principal biomes described in Chapter 2.3.

To explore the spatial distribution of NCMs quantitatively, the relationship between the relative biomass (\%) of NCMs and NPP within each province (Chapter 2.3) were investigated through loess regressions (Chapter 2.4.4). In addition to this analysis, the absolute and the relative biomass of mixotrophic oligotrich ciliates (GNCMs), Mesodinium (pSNCMs), and mixotrophic Rhizaria (eSNCMs) was compared across different oceanic biomes and seasons through two-way ANOVAs (Chapter 2.4.2). If necessary, absolute biomass data were transformed using $\log (x+1)$ to meet the assumptions of normality (Chapter 2.4.2). It is noteworthy, however, that data points were not homogenously distributed across biomes, nor across seasons. The complete seasonal cycle was available for all functional groups only in the Coastal Seas and Mediterranean Sea biomes. The seasonal progression of mixotrophic Rhizaria (eSNCMs) was also available in the Oligotrophic Gyres and Equatorial Upwelling biomes. No data were available for the Coastal Upwelling biome. 
Table 3.1 Number of occurrence records for protistan microzooplankton species with acquired phototrophy (NCMs) obtained through OBIS survey. Species selected for the analysis are highlighted in bold.

\begin{tabular}{|c|c|c|}
\hline Functional type & Species name & Number of records \\
\hline GNCM & Laboea strobila & 2754 \\
\hline GNCM & Laboea pulchra & no data \\
\hline GNCM & Cyrtostrombidium spp. & 3 \\
\hline GNCM & Strombidium acutum & 342 \\
\hline GNCM & Strombidium capitatum & 47 \\
\hline GNCM & Strombidium chlorophilum & 1 \\
\hline GNCM & Strombidium conicum & 2014 \\
\hline GNCM & Strombidium crassulum & 101 \\
\hline GNCM & Strombidium delicatissimum & 574 \\
\hline GNCM & Strombidium elegans & 1 \\
\hline GNCM & Strombidium oculatum & 34 \\
\hline GNCM & Strombidium purpureum & 1 \\
\hline GNCM & Strombidium reticulatum & 44 \\
\hline GNCM & Strombidium stylifer & 1 \\
\hline GNCM & Pseudotontonia cornuta & 23 \\
\hline GNCM & Tontonia appendiculariformis & 8 \\
\hline GNCM & Tontonia gracillima & 208 \\
\hline GNCM & Tontonia ovalis & 201 \\
\hline GNCM & Tontonia poopsia & 2 \\
\hline GNCM & Tontonia simplicidens & no data \\
\hline pSNCM & Mesodinium rubrum (= Myrionecta rubra) & 20533 \\
\hline pSNCM & Dinophysis acuminata & 21250 \\
\hline pSNCM & Dinophysis fortii & 497 \\
\hline pSNCM & Dinophysis infundibulus & 63 \\
\hline pSNCM & Dinophysis mitra & 182 \\
\hline pSNCM & Amphidinium latum & 13 \\
\hline pSNCM & Amphidinium poecilochrom & 1 \\
\hline pSNCM & Amylax triacantha & 1363 \\
\hline pSNCM & Cryptoperidiniopsis spp. & 6 \\
\hline pSNCM & Kleptodinium spp. & no data \\
\hline pSNCM & Pfiesteria piscicida & 3 \\
\hline eSNCM & Dinothrix paradoxa & 3 \\
\hline eSNCM & Durinskia baltica & 20 \\
\hline eSNCM & Durinskia capensis & no data \\
\hline eSNCM & Galeidinium rugatum & no data \\
\hline eSNCM & Kryptoperidinium foliaceum & 169 \\
\hline eSNCM & Noctiluca scintillans & 17120 \\
\hline eSNCM & Peridinium quinquecorne & 688 \\
\hline eSNCM & Histioneis spp. & 121 \\
\hline eSNCM & Amphisolenia spp. & 1552 \\
\hline eSNCM & Triposolenia spp. & 20 \\
\hline eSNCM & Ornithocercus spp. & 3148 \\
\hline eSNCM & Acanthmetra pellucida & no data \\
\hline eSNCM & Amphilonche elongata & 30 \\
\hline eSNCM & Lithoptera mulleri & no data \\
\hline eSNCM & Didymocyrtis tetrathalamus & 186 \\
\hline eSNCM & Spongodrymus spp. & 1 \\
\hline eSNCM & Tetrapetalon elegans & no data \\
\hline eSNCM & Spongostaurus spp. & no data \\
\hline
\end{tabular}


Table 3.1 continued

$\begin{array}{llc}\text { eSNCM } & \text { Dictyocoryne truncatum } & \mathbf{5 4} \\ \text { eSNCM } & \text { Spongodrymus spp. } & 1 \\ \text { eSNCM } & \text { Thallassolampe margarodes } & \text { no data } \\ \text { eSNCM } & \text { Spongodiscus biconcavus } & \text { no data } \\ \text { eSNCM } & \text { Acanthosphaera } & \mathbf{2 1 6} \\ \text { eSNCM } & \text { Actinomma } \text { spp. } & \mathbf{4 2 8} \\ \text { eSNCM } & \text { Plegmosphaera } \text { spp. } & \mathbf{8 0} \\ \text { eSNCM } & \text { Haxacontium } & \text { no data } \\ \text { eSNCM } & \text { Spongotrochus } & \mathbf{1 5 5} \\ \text { eSNCM } & \text { Collozoum } \text { spp. } & \mathbf{2 1 6} \\ \text { eSNCM } & \text { Collosphaera } \text { spp. } & \mathbf{2 3 9} \\ \text { eSNCM } & \text { Sphaerozoum } \text { spp. } & \mathbf{3 8} \\ \text { eSNCM } & \text { Acrosphaera spinosa } & \mathbf{9 6} \\ \text { eSNCM } & \text { Thalassicolla } \text { spp. } & 3 \\ \text { eSNCM } & \text { Pterocorys } \text { zancleus } & \mathbf{9 4} \\ \text { eSNCM } & \text { Pterocanium muelleri } & \text { no data } \\ \text { eSNCM } & \text { Heliodiscus } \text { spp. } & \mathbf{9 3} \\ \text { eSNCM } & \text { Phorticium pylonium } & \mathbf{4 5} \\ \text { eSNCM } & \text { Ceratospyris hyperborea } & \text { no data } \\ \text { eSNCM } & \text { Androcyclas gamphonyca } & \text { no data } \\ \text { eSNCM } & \text { Globigerinella siphonifera } & \mathbf{2 3 8 6 3} \\ \text { eSNCM } & \text { Globorotalia merardii } & \mathbf{1 6 4 2 9} \\ \text { eSNCM } & \text { Pulleniatina obliqueloculata } & \text { no data }\end{array}$




\subsection{Results}

\subsubsection{Spatial analysis of qualitative data}

The analysis revealed that acquired phototrophy is ubiquitous in the global oceans; however, the biogeography of the three functional groups differed markedly (Fig. 3.1). While the eSNCMs were observed to be widely distributed, GNCMs and pSNCMs were more restricted spatially (Fig. 3.1). Indeed, each of the three major plankton taxa within the eSNCM functional grouping (Dinoflagellates, Radiolaria, and Foraminifera) had a wider distribution than the GNCMs and the pSNCMs (Fig. 3.2).

The NMDS analysis revealed species clustered together according to the NCM functional groups (Fig. 3.3). Notably, pSNCMs were positioned between the GNCMs and eSNCMs; certain pSNCMs species (e.g., Amylax triacantha and Dinophysis mitra) were closer to the GNCMs, while others (e.g., Mesodinium rubrum and Dinophysis acuminata) were closer to the eSNCMs. Spatially, while GNCMs were mainly associated with the Temperate Seas, Polar Seas, and Mediterranean Sea biomes, eSNCMs were primarily associated with the Oligotrophic Gyres, Coastal Upwelling, and Equatorial Upwelling biomes (Fig. 3.3). The eSNCM distribution was observed to follow two distinctly diverse patterns. One group composed mainly by Rhizarian species of the class Polycystina (e.g., Acrosphaera spinosa and Dictyocoryne truncatum) was closely related to the Equatorial Upwelling biome (Fig. 3.3). The second group was mainly composed by cosmopolitan species of foraminiferans and dinoflagellates (e.g., Globigerinella siphonifera, Globorotalia merardii, Ornithocercus spp., and Amphisolenia spp.) and occurred in the intersection between most biomes (Fig. 3.3). The Coastal Sea biome was not associated with any single functional group; all the NCM functional groups occurred at least in one biogeographic province within this biome. 

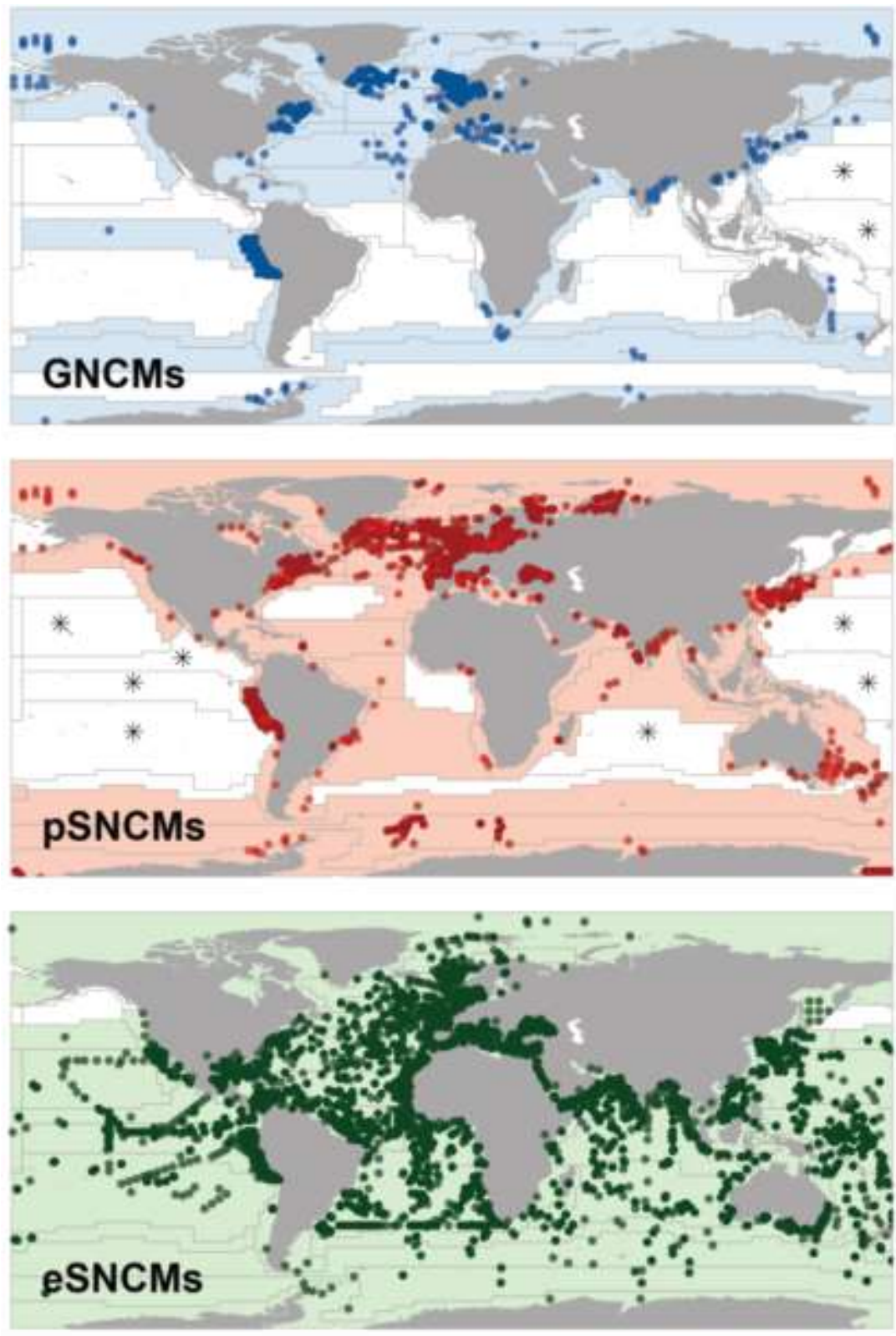

Figure 3.1 Global distribution of protists with acquired phototrophy. Functional groups identify protists which acquire plastids from a variety of prey (GNCMs; blue), or from specific prey (pSNCMs; red), or enslave entire specific autotrophic prey as symbionts (eSNCMs; green). In the maps, symbols correspond to the exact location where mixotrophic species/taxa were found (> 110,000 records mostly from OBIS). Overlaid is a grid that represents biogeographic provinces. Colour-cast provinces indicate the presence of NCMs and white provinces correspond to absence. Provinces marked with* indicate that studies conducted in these areas did not record the presence of mixotrophic species; unmarked white provinces indicate a lack of field studies providing information on acquired phototrophy among microzooplankton. At least one record was necessary to assume that mixotrophs occurred in any province. 

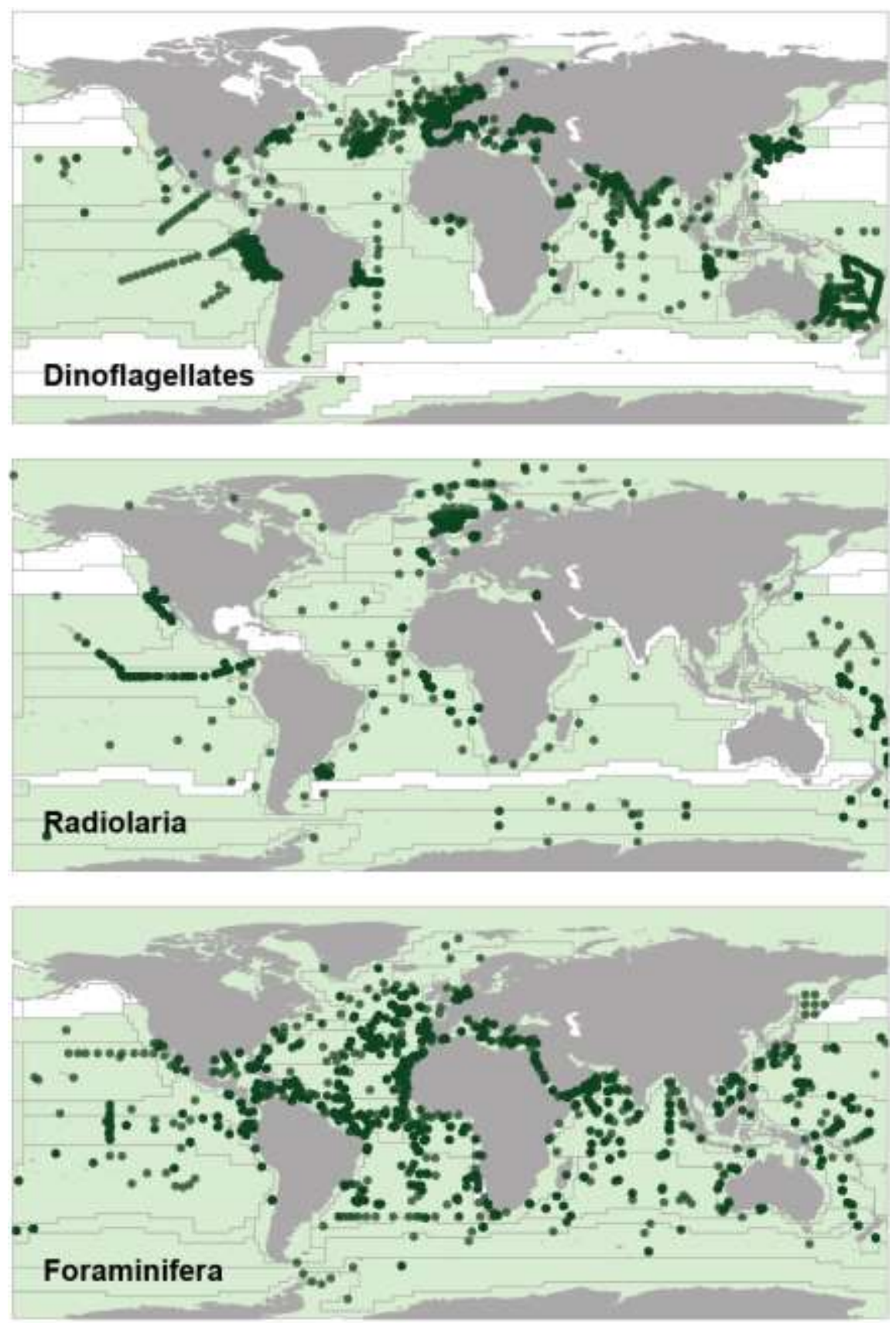

Figure 3.2 Global distribution of protists which enslave entire autotrophic prey (eSNCMs: dinoflagellates, Radiolaria, and Foraminifera). Symbols correspond to the exact location where mixotrophic species/taxa were found (over 60,000 records). Overlaid is a grid that represents Longhurst's biogeographic provinces. Colour-cast provinces indicate the presence of NCMs and white provinces correspond to absence and/or lack of data. At least one record was necessary to assume that mixotrophs occurred in any province. 


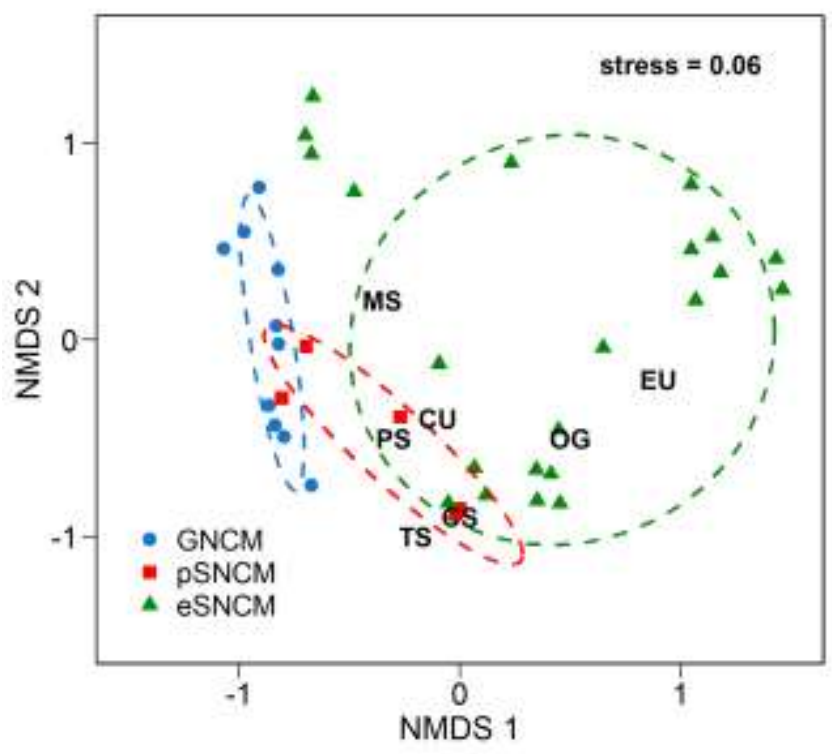

Figure 3.3 Results from the NMDS analysis showing the ordination of species and biomes in a two-dimensional space. Species were classified between GNCMs, pSNCMs, and eSNCMs. Each symbol represents a NCM species; different symbols and dashed ellipses (at $80 \%$ confidence interval) represent different functional groups. The different biomes are: MS, Mediterranean Sea; PS, Polar Seas; TS, Temperate Seas; CS, Coastal Seas; OG, Oligotrophic Gyres; CU, Coastal Upwelling; EU, Equatorial Upwelling (the positions of the 7 biomes were obtained from the centers of the ellipsoids were derived from Fig 3.4).

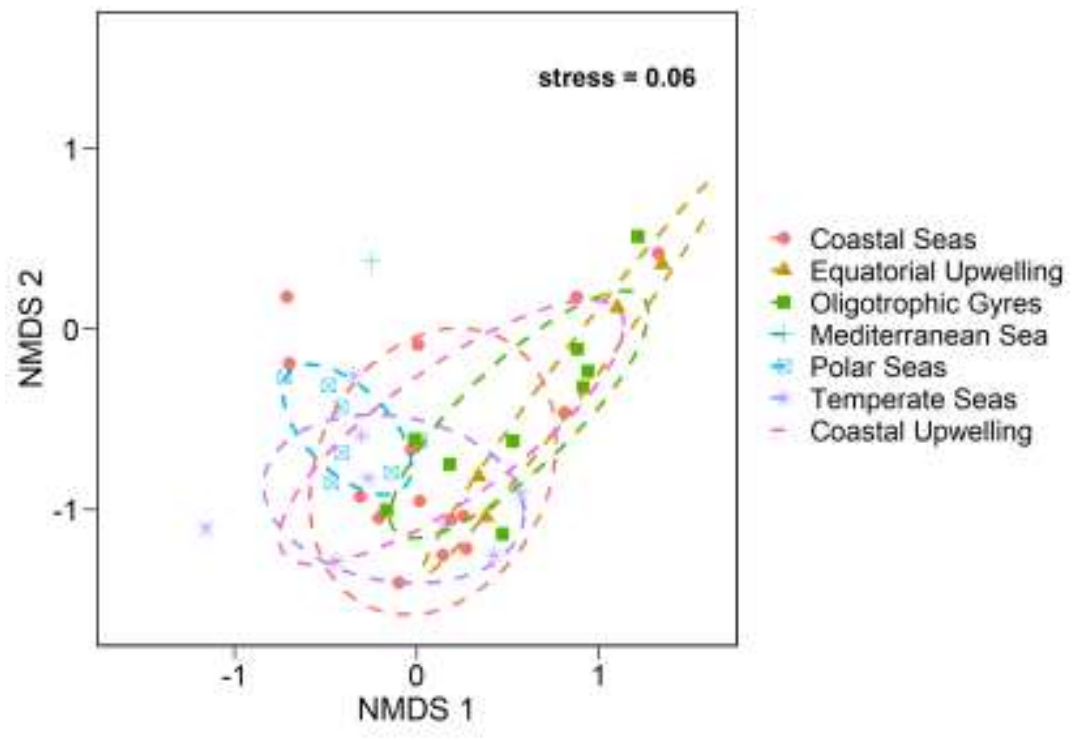

Figure 3.4 Additional results from the NMDS analysis showing the distribution of biogeographic provinces in a two-dimensional space and their grouping in seven larger biomes. Each dot represents a biogeographic province and symbols represent the different biomes; the dashed ellipses represent the biome clusters at $80 \%$ confidence interval. 


\subsubsection{Spatio-temporal analysis of quantitative data}

The quantitative data analysis revealed that the biomass contribution of acquired phototrophy varied across biomes according to the functional groups in a similar fashion as their presence/absence (Tables 3.2 and 3.3). The biomass contribution of mixotrophic ciliates increased towards more productive and more variable systems, while the opposite pattern was observed for mixotrophic Rhizaria (Fig. 3.5).

The absolute biomass of GNCMs was highest during summer within the Mediterranean Sea biome (Fig. 3.6, upper panel), making up 70\% of total ciliate biomass (Fig. 3.7, upper panel). The absolute pSNCM biomass (represented by Mesodinium) was highest during spring in the Coastal Seas biome (Fig. 3.6, middle panel), encompassing up to $80 \%$ of total ciliate biomass (Fig. 3.7, middle panel). While the lowest absolute biomass values of GNCMs and pSNCMs were observed within the Equatorial Upwelling biome, mixotrophic Rhizaria (eSNCMs) were most abundant in this biome, specifically during autumn (Fig. 3.6, lower panel). In turn, lower absolute biomass values were observed within the Coastal Seas and Mediterranean Sea biomes among mixotrophic Rhizaria (Fig. 3.6, lower panel); they contributed up to $65 \%$ of total Rhizaria biomass within these regions (Fig. 3.7, lower panel).

The ANOVAs (Table 3.4) suggest that the combined effect of biome and season on Mesodinium (relative biomass) and mixotrophic Rhizaria (absolute and relative biomass) was significant. The ANOVA of relative biomass of GNCMs showed a significant effect only when considering seasonality. However, it is difficult to separate the effects of season and biome since biomes are somehow delimited by latitude; thus, different biomes have different seasonalities. While there is a paucity of data for GNCMs and Mesodinium within certain oceanic regions and/or periods of the year (Table 3.4), ANOVAs were carried out to gain a basic understanding of the impact of seasonality and/or oceanic biomes on their biogeographies. 
Table 3.2 Mean ( \pm SE) biomass of GNCMs (i.e., mixotrophic oligotrich ciliates) and the pSNCM Mesodinium over seasons and across biomes in comparison with the biomass of heterotrophic ciliates within the sunlit ocean (see Table A.2 for details regarding sampling depth amongst the different studies). Note that the studies which estimated GNCMs biomass are not necessarily the same as those that estimated Mesodinium biomass (Table A.2); $\mathrm{n}$ - number of observationsa, nd - not determined.

\begin{tabular}{lcccccc}
\hline & \multicolumn{3}{c}{ Ciliate biomass $\left(\boldsymbol{\mu g} \mathbf{C ~ L ~ L}^{-1}\right)$} & \multicolumn{3}{c}{ Ciliate biomass $\left(\boldsymbol{\mu g} \mathbf{C ~ L ~}^{-\mathbf{1}}\right)$} \\
& GNCMs & Heterotrophs & $\mathrm{n}$ & Mesodinium & Heterotrophs & $\mathrm{n}$ \\
\hline Season & & & & & & \\
winter & $0.7 \pm 0.1$ & $4.7 \pm 1.3$ & 4 & $2.1 \pm 1.1$ & $4.7 \pm 1.3$ & 4 \\
spring & $5.2 \pm 2.0$ & $7.1 \pm 3.1$ & 15 & $4.1 \pm 2.3$ & $6.2 \pm 2.8$ & 17 \\
summer & $8.4 \pm 3.4$ & $4.9 \pm 1.4$ & 12 & $5.5 \pm 3.6$ & $5.3 \pm 1.4$ & 12 \\
autumn & $3.0 \pm 1.6$ & $6.0 \pm 2.0$ & 5 & $1.0 \pm 0.3$ & $5.0 \pm 1.9$ & 6 \\
Biome & & & & & & \\
Coastal Seas & $3.6 \pm 1.8$ & $5.2 \pm 1.7$ & 8 & $10.9 \pm 4.7$ & $4.9 \pm 1.4$ & 11 \\
& $0.02 \pm$ & & & & & \\
Equatorial Upwelling & 0.001 & $0.1 \pm 0.06$ & 2 & 0 & $0.1 \pm 0.06$ & 2 \\
Mediterranean Sea & $8.1 \pm 2.7$ & $8.3 \pm 2.6$ & 17 & $1.4 \pm 0.4$ & $7.8 \pm 2.5$ & 18 \\
Oligotrophic Gyres & nd & nd & nd & $0.005 \pm 0.001$ & $0.1 \pm 0.001$ & 2 \\
Polar Seas & $6.5 \pm 2.4$ & $6.4 \pm 1.6$ & 4 & $1.4 \pm 0.9$ & $7.0 \pm 2.2$ & 3 \\
Temperate Seas & $0.9 \pm 0.2$ & $1.0 \pm 0.4$ & 4 & $0.006 \pm 0.006$ & $0.6 \pm 0.2$ & 3 \\
\hline
\end{tabular}

Table 3.3 Mean ( \pm SE) biomass of mixotrophic Rhizaria over seasons and across biomes in comparison with the biomass of heterotrophic Rhizaria within the topmost $100 \mathrm{~m}$; $\mathrm{n}$ indicates number of observations.

\begin{tabular}{lccc}
\hline & \multicolumn{2}{c}{ Rhizaria biomass $\mathbf{( m g ~ C ~ ~ ^ { - 2 } )}$} & \\
& Mixotrophs & Heterotrophs & $\mathrm{n}$ \\
\hline Season & & & \\
winter & $376 \pm 141$ & $295 \pm 88$ & 33 \\
spring & $291 \pm 82$ & $110 \pm 39$ & 97 \\
summer & $86 \pm 13$ & $38 \pm 5$ & 244 \\
autumn & $341 \pm 50$ & $713 \pm 138$ & 255 \\
Biome & & & \\
Coastal Seas & $205 \pm 51$ & $1544 \pm 307$ & 109 \\
Equatorial Upwelling & $1457 \pm 427$ & $956 \pm 259$ & 11 \\
Mediterranean Sea & $93 \pm 13$ & $40.7 \pm 4.8$ & 252 \\
Oligotrophic Gyres & $400 \pm 61$ & $95 \pm 18.7$ & 216 \\
Polar Seas & $5 \pm 2$ & $45 \pm 26$ & 34 \\
Temperate Seas & $65 \pm 48$ & $61 \pm 39$ & 7 \\
\hline
\end{tabular}



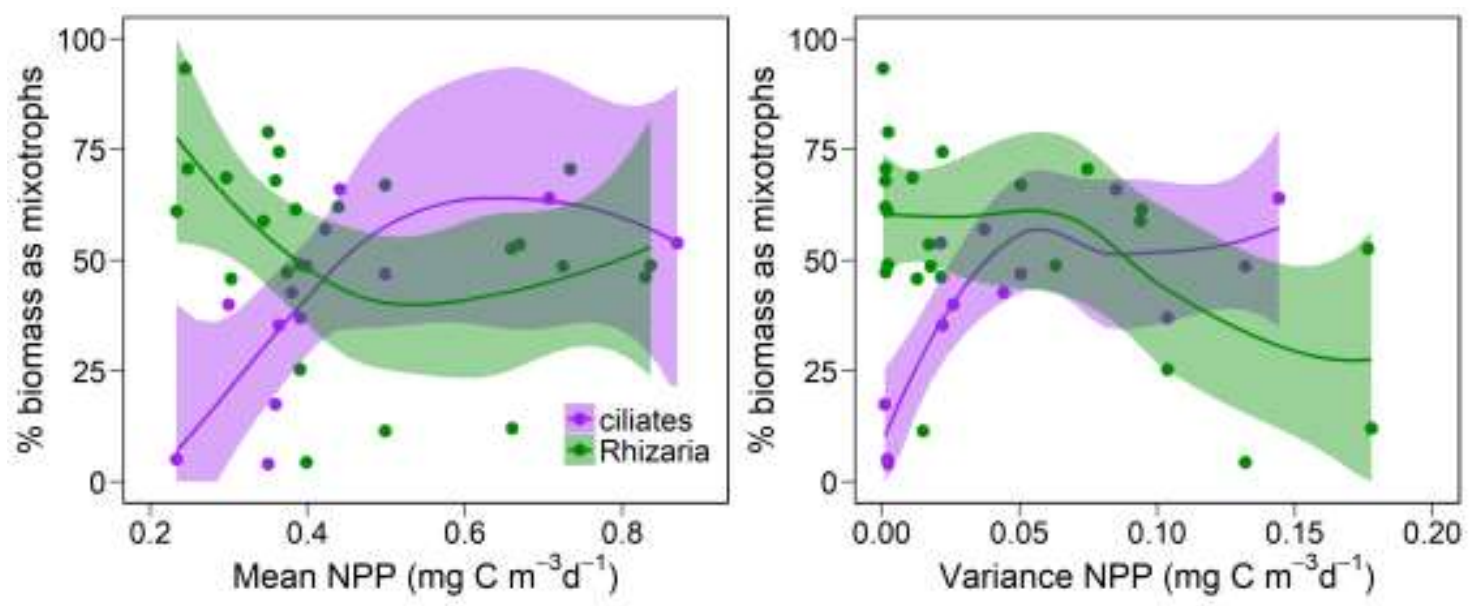

Figure 3.5 Relative contribution of mixotrophs (\% biomass) as a function of annual NPP average (left panel) and variance (right panel) as proxies for nutrient load and system variability, respectively (Chapter 2.3; Appendix A, Fig. A.1). Contribution of mixotrophic ciliate biomass (GNCMs + the pSNCM Mesodinium; purple) is plotted relative to total ciliate biomass while contribution of mixotrophic Rhizarian biomass (green) is presented relative to total Rhizarian biomass. Each symbol represents a biogeographic province; loess regressions were fitted to data using $\mathrm{R}$ package ggplot2; 95\% confidence intervals shown. 

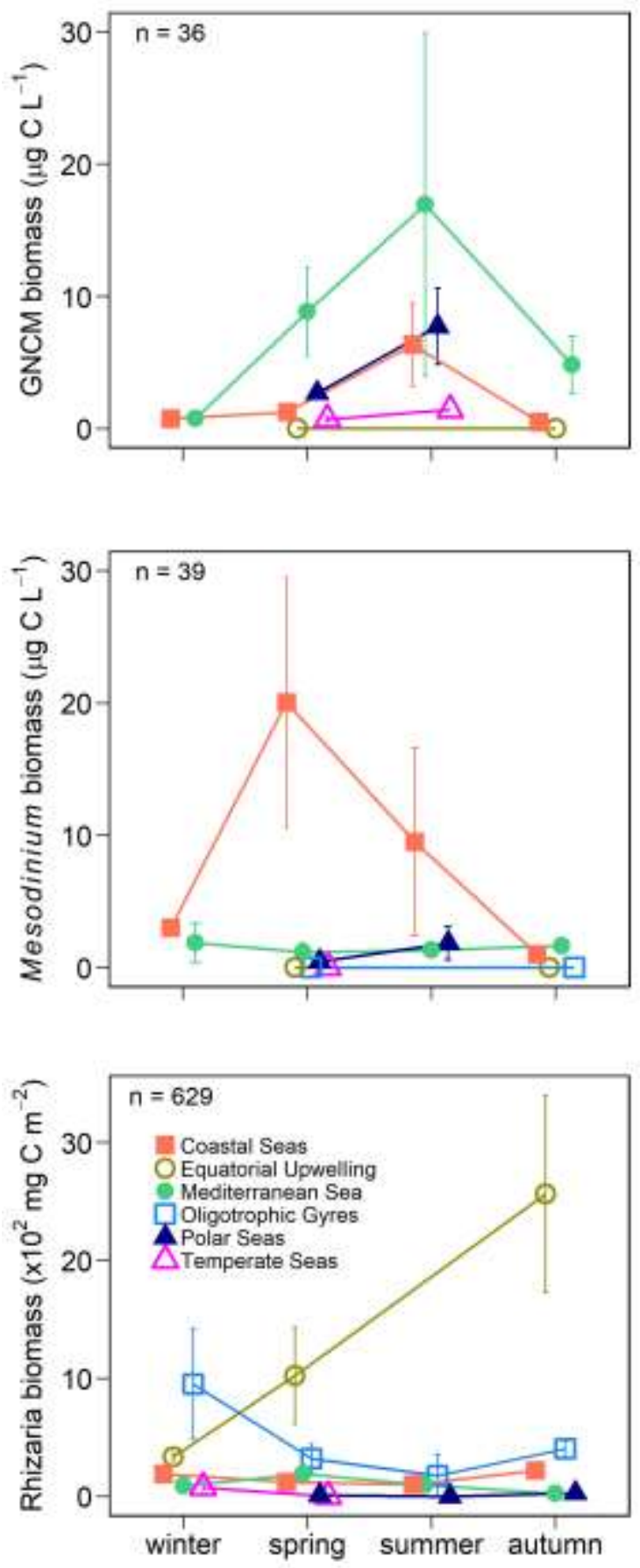

Figure 3.6 Global spatial and temporal distribution of protists with acquired phototrophy. Upper panel, GNCM ciliates; middle panel, pSNCM Mesodinium spp.; lower panel, eSNCM Rhizaria. Seasonal biomass for each group is shown across different biomes (note that biomass unit in the lower panel differ from the other panels); n, indicates the total number of observations used. No published data were available for the Coastal Upwelling biome. 


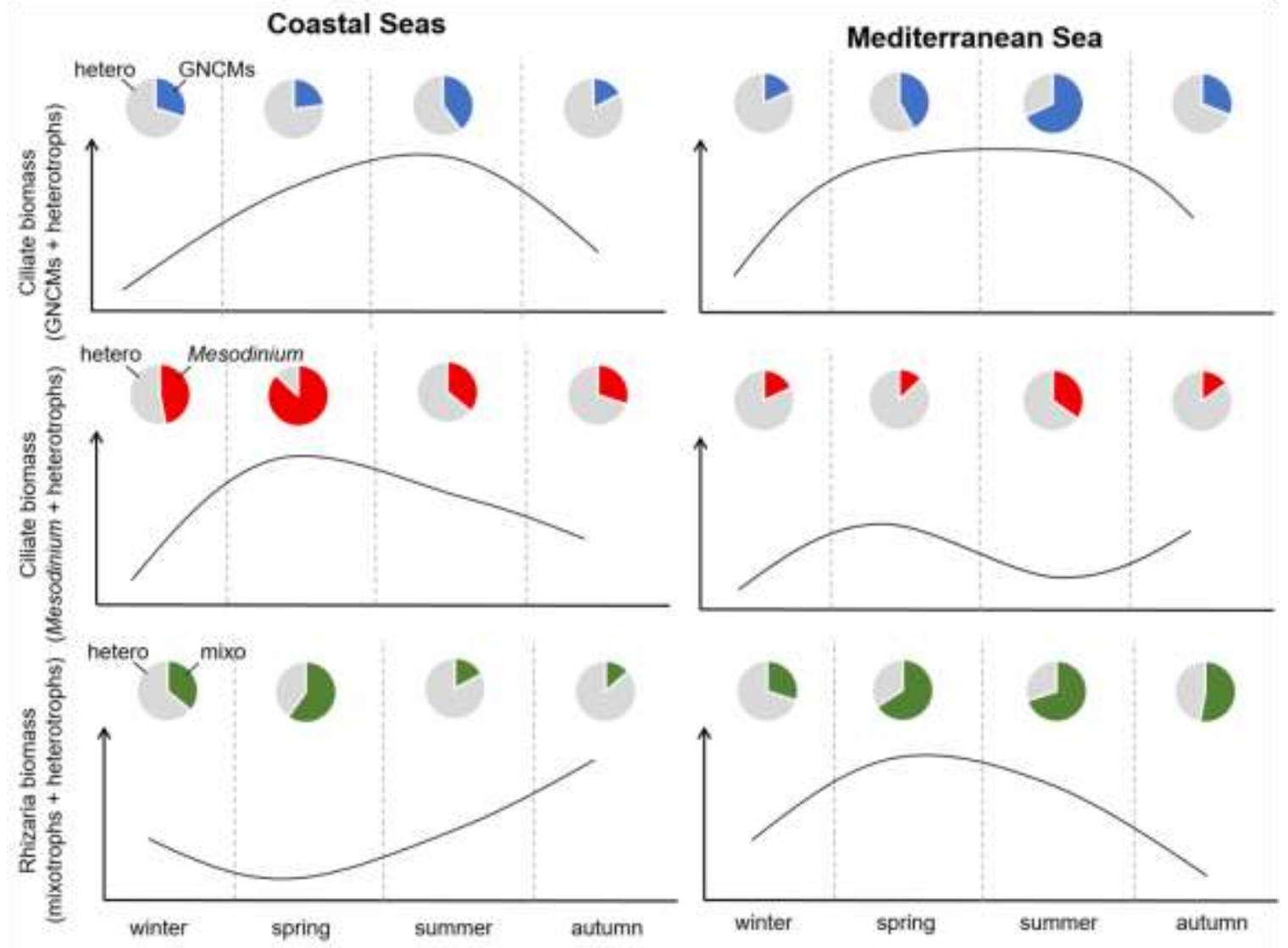

Figure 3.7 Schematics for selected biomes (Coastal Seas and Mediterranean Sea) comparing the seasonal progression of the relative contribution of mixotrophs (blue, GNCMs; red, pSNCM Mesodinium; green, eSNCM Rhizaria) versus heterotrophs (grey) to the total biomass (heterotrophs + mixotrophs) within each functional group. Solid lines indicate the temporal variability for the total biomass over the seasonal cycle. 
Table 3.4 Two-way ANOVA statistical analysis within 95\% confidence interval on biomass (relative and absolute) data for GNCMs, Mesodinium (pSNCM), and mixotrophic Rhizaria (eSNCMs).

\begin{tabular}{|c|c|c|c|c|c|c|}
\hline Dependent Variable & Factor & $\begin{array}{c}\text { Degrees of } \\
\text { freedom }\end{array}$ & $\begin{array}{c}\text { Sum of } \\
\text { squares }\end{array}$ & $\begin{array}{c}\text { Mean } \\
\text { square }\end{array}$ & f-value & p-value \\
\hline \multirow{4}{*}{$\begin{array}{l}\text { Relative contribution of GNCMs to } \\
\text { total ciliate assemblage } \\
\text { (GNCMs + heterotrophic ciliates) }\end{array}$} & Season & 3 & 4,290 & 1,430 & 3 & $<0.05$ \\
\hline & Biome & 4 & 2,154 & 538 & 1.2738 & 0.31 \\
\hline & Season $\times$ Biome & 6 & 1,116 & 185 & 0.4399 & 0.84 \\
\hline & Residuals & 21 & 8,878 & 422 & & \\
\hline \multirow{4}{*}{$\begin{array}{l}\text { Relative contribution of Mesodinium to } \\
\text { total ciliate assemblage } \\
\text { (Mesodinium + heterotrophic ciliates) }\end{array}$} & Season & 3 & 1,875 & 625 & 2.0657 & 0.13 \\
\hline & Biome & 5 & 10,044 & 2,008 & 6.6365 & $<0.001$ \\
\hline & Season $\times$ Biome & 6 & 6,912 & 1,152 & 3.8063 & $<0.01$ \\
\hline & Residuals & 24 & 7,264 & 302 & & \\
\hline \multirow{4}{*}{$\begin{array}{l}\text { Relative contribution of mixotrophic } \\
\text { Rhizaria to total Rhizaria assemblage } \\
\text { (mixotrophic Rhizaria + heterotrophic Rhizaria) }\end{array}$} & Season & 3 & 55,331 & 18,444 & 15.6696 & $<0.0001$ \\
\hline & Biome & 5 & 223,689 & 44,738 & 38.0086 & $<0.0001$ \\
\hline & Season $\times$ Biome & 11 & 57,739 & 5,249 & 4.4595 & $<0.0001$ \\
\hline & Residuals & 609 & 716,820 & 1,177 & & \\
\hline \multirow[t]{4}{*}{ Absolute biomass of GNCMs } & Season & 3 & 6 & 2 & 2 & 0.18 \\
\hline & Biome & 4 & 8 & 2.0028 & 1.8098 & 0.16 \\
\hline & Season $\times$ Biome & 6 & 1 & 0.11043 & 0.0998 & 0.99 \\
\hline & Residuals & 21 & 23 & 1.1067 & & \\
\hline \multirow{4}{*}{ Absolute biomass of Mesodinium } & Season & 3 & 2 & 0.668 & 0.8156 & 0.49 \\
\hline & Biome & 5 & 12 & 2 & 2.8822 & $<0.05$ \\
\hline & Season $\times$ Biome & 6 & 4 & 1 & 0.8352 & 0.55 \\
\hline & Residuals & 24 & 20 & 0.8189 & & \\
\hline \multirow[t]{4}{*}{ Absolute biomass of mixotrophic Rhizaria } & Season & 3 & 63 & 21 & 4.8636 & $<0.01$ \\
\hline & Biome & 5 & 437 & 87 & 20.358 & $<0.0001$ \\
\hline & Season $\times$ Biome & 11 & 103 & 9 & 2.1785 & $<0.05$ \\
\hline & Residuals & 609 & 2,616 & 2 & & \\
\hline
\end{tabular}




\subsection{Discussion}

Mixotrophic plankton with acquired phototrophy are prevalent across all ocean biomes, from polar to tropical regions, and from coastal to oceanic environments, in both Hemispheres (Fig. 3.1). However, the spatio-temporal distribution patterns of the three functional types (i.e., GNCMs, pSNCMs, and eSNCMs) differed markedly. There was no obvious latitudinal constraint on the occurrence of GNCMs and pSNCMs (Fig. 3.1), but most records corresponded to coastal environments. There were very few occurrence records within open oceans, particularly within oligotrophic gyres (Stoecker et al., 1996; Rychert et al., 2014; Stoecker et al., 2017). In contrast, eSNCMs were present across all latitudinal and coastal-oceanic gradients (Tarangkoon et al., 2010; Biard et al., 2016; Stoecker et al., 2017), with Radiolaria and Foraminifera dominating oceanic waters at low latitudes $\left(0^{\circ}-30^{\circ}\right.$ North and South) (Fig. 3.1). Quantitatively, the relative contribution of GNCMs and pSNCMs to total ciliate assemblage (abundance and biomass) was lower in low latitudes (Fig. 3.8) while the contribution of mixotrophic Rhizaria to total Rhizaria assemblage has been observed to decrease towards the higher latitudes (Biard et al., 2016).

Protists with acquired phototrophy occur within an ecological continuum from species that rely on sequestered chloroplasts but have low control over these (GNCMs), species that sequester chloroplasts and other plastids, such as prey nuclei, having higher control over the acquired phototrophic machinery (pSNCMs), to species that host entire populations of their prey through endosymbiosis (eSNCMs) and, therefore, are primarily phototrophic (Stoecker et al., 2009; Stoecker et al., 2017). The results reported here suggest that this continuum, GNCMs - pSNCMs - eSNCMs, is reflected in their biogeography (Fig. 3.3). In fact, evidence is also given for species within a single functional type. In the NMDS analysis, pSNCM species with lower physiological control over their acquired plastids (i.e., A. triacantha and D. mitra) were positioned closer to the GNCMs group while those with a higher level of control of acquired phototrophy (i.e., $M$. rubrum and D. acuminata) were closer to the eSNCMs group (Fig. 3.3) (Wisecaver and Hackett, 2010; Nishitani et al., 2012; Myung et al., 2013; Kim et al., 2014; HattenrathLehmann and Gober, 2015). 
While both GNCMs and pSNCMs share biogeographies that are similar and somewhat restricted to neritic regions, temporal and spatial differences can be drawn between them. Within temperate seas, GNCMs tend to dominate after the phytoplankton bloom, particularly in summer, under stratified water column conditions, while pSNCMs are more commonly encountered during spring, on nutrient-replete conditions (Fig. 3.6, upper and middle panels). Mesodinium also thrive in upwelling zones, largely due to their high rates of phototrophic growth in nutrient rich waters (Johnson, 2011a; Hansen et al., 2013). In contrast, GNCMs are usually outnumbered by heterotrophic ciliates (mainly tintinnids) in upwelling regions (Chang, 1990). The striking dominance of eSNCMs amongst mixotrophs in less productive ecosystems with low abiotic variability (Fig. 3.5), such as within the oligotrophic gyres, underscores the importance of resource partitioning between organisms and symbiosis in these low nutrient environments. The scarcity of resources in these areas are commonly expected to favour smaller plankton members; however, symbiosis promotes the growth of eSNCMs and thus helps to explain the anomalous preponderance of large cells in these systems (Selosse et al., 2017). The limited success of GNCMs in these conditions could be attributed to the low availability of prey which is detrimental to growth of the GNCMs which rely on a near-constant supply of prey for acquired phototrophy as well as for essential nutrients (Stoecker et al., 2009; Stoecker et al., 2017) (Fig. 3.5).

Acquired phototrophy among microzooplankton has been typically neglected in field and modelling studies; NCMs have hitherto not been considered a major component of the microzooplankton. According to this analysis, when the average contribution of mixotrophs was calculated across temporal and spatial scales, the mixotrophic ciliates (GNCMs + the pSNCM Mesodinium) contribute $\sim 45 \%$ to the total ciliate numeric abundance, and $\sim 40 \%$ of total ciliate biomass (Fig. 3.9). A previous study estimated that $\sim 30 \%$ of the numeric abundance of marine oligotrich ciliates globally were mixotrophic (i.e., GNCMs) (Dolan and Pérez, 2000). Amongst the eSNCMs, nearly half of total Rhizaria biomass is comprised of mixotrophic taxa across the spatial-temporal scales investigated in the present analysis (Fig. 3.10). Importantly, this analysis reveals great variation not only in the presence of mixotrophs but also on which mixotroph functional group dominates which biome during specific seasons. 
Understanding the biogeography of acquired phototrophs is crucial to investigate their potential impact (positive or negative) in different regions in the oceans. In the upper water column of pelagic environments, GNCMs can act shortening and, thus, increasing the efficiency of energy transfer along pelagic food webs (Stoecker et al., 2009). In the open ocean, eSNCMs are giant photosynthetic protists with high potential to contribute to vertical carbon flux, influencing the biological carbon pump (Biard et al., 2016). On the other hand, many pSNCMs and eSNCMs form extensive blooms in eutrophic coastal regions. For instance, blooms of the pSNCM ciliate Mesodinium spp. are of particularly concern because they can deteriorate water quality and are the source of the phototrophic capability of the toxicogenic pSNCM, Dinophysis spp. (Herfort et al., 2012; Kim et al., 2012). In turn, Dinophysis, an organism colloquially considered as an alga rather than a microzooplankter with acquired phototrophy, causes Diarrhetic Shellfish Poisoning, which can be responsible for closures of shellfish aquaculture operations (Reguera et al., 2012; Mafra et al., 2014). In the Arabian Sea, shifts from diatom blooms to those of the eSNCM "green Noctiluca" are circumstantially associated with hypoxia, and may adversely affect fisheries in a coastal ecosystem supporting 120 million people (Gomes et al., 2014).

The present analysis revealed that the functional diversity observed among NCMs is reflected on their biogeography, with different forms dominating in different systems. These findings have implications to understand how different forms of acquired phototrophy have the potential to impact marine food webs and the cycling of materials through different routes. Thus, it is important that NCMs functionality is incorporated in future conceptual and mathematical models in all spatial and temporal scales, particularly recognising that nearly half of the traditionally labelled 'microzooplankton' are in fact acquired phototrophs within the sunlit ocean (Figs. 3.9 and 3.10). 

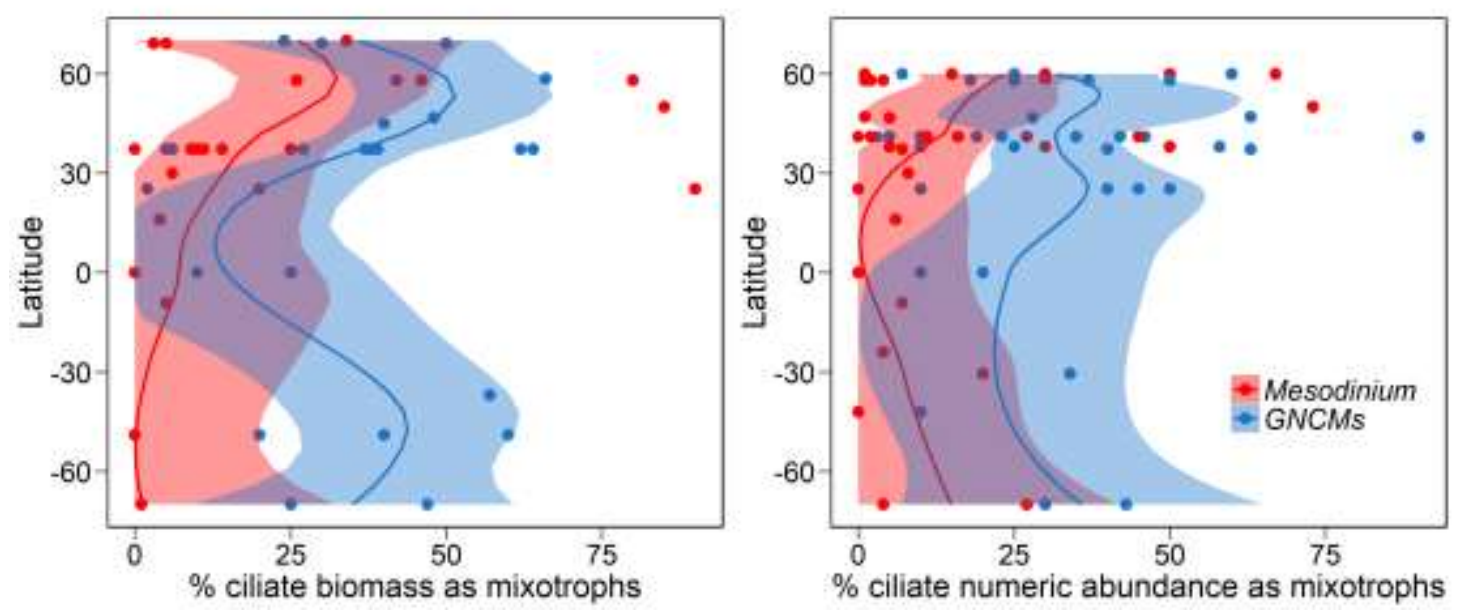

Figure 3.8 Latitudinal gradients for the relative contribution of mixotrophic ciliates to total ciliate biomass (left) and to total ciliate numeric abundance (right). GNCMs are indicated in blue and red-pigmented Mesodinium spp. in red. Loess regressions were fitted to data using R package ggplot2; 95\% confidence intervals are shown. Note that data were unequally distributed across latitude and period of the year. 

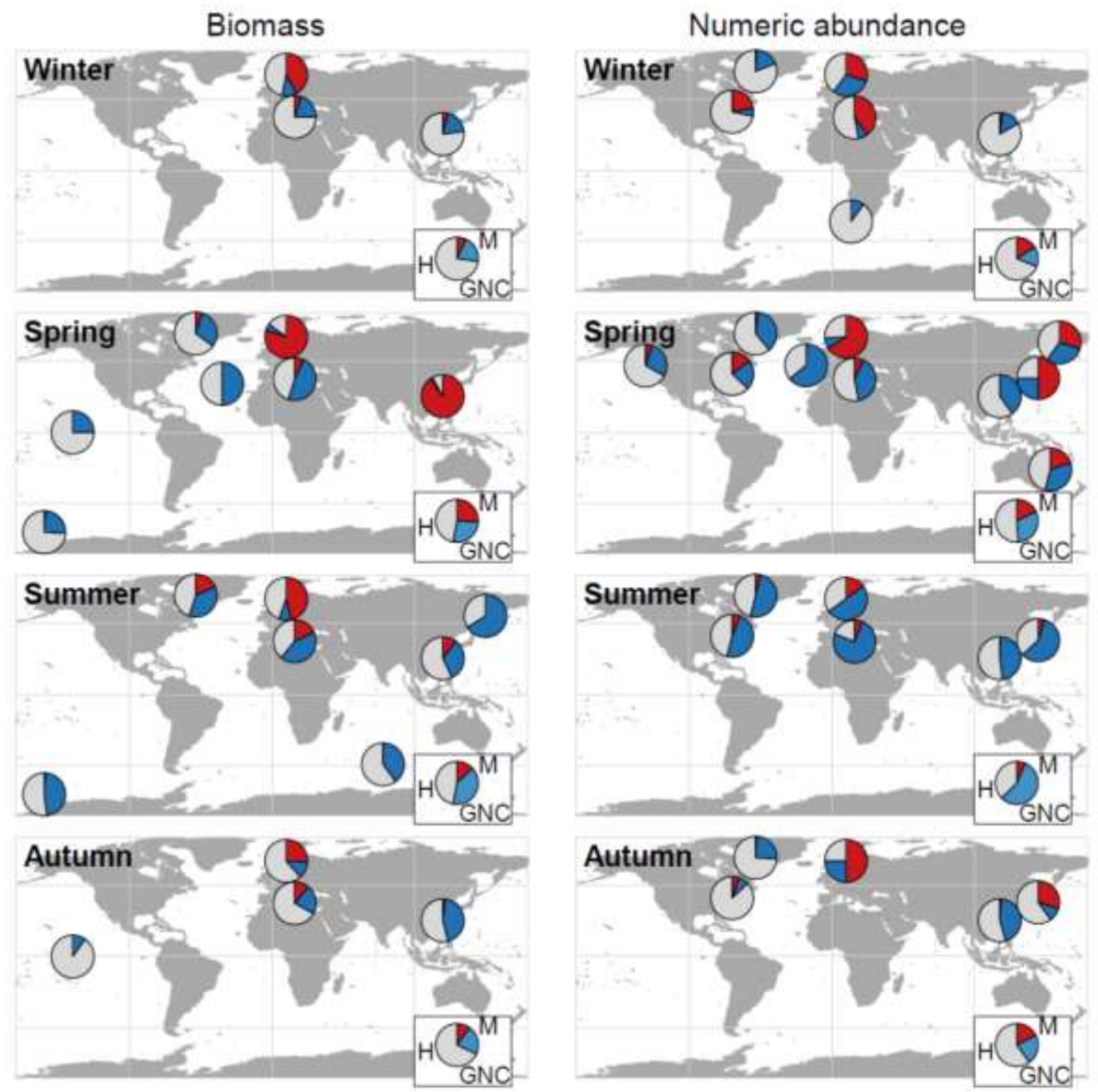

Figure 3.9 Seasonal relative contribution of GNCMs and Mesodinium to total ciliate biomass (left panels) and numeric abundance (right panels) over the globe. Pies highlighted within rectangles represent global means. GNC: GNCMs, M: pSNCM Mesodinium spp., and $\mathrm{H}$ : heterotrophic ciliates. 

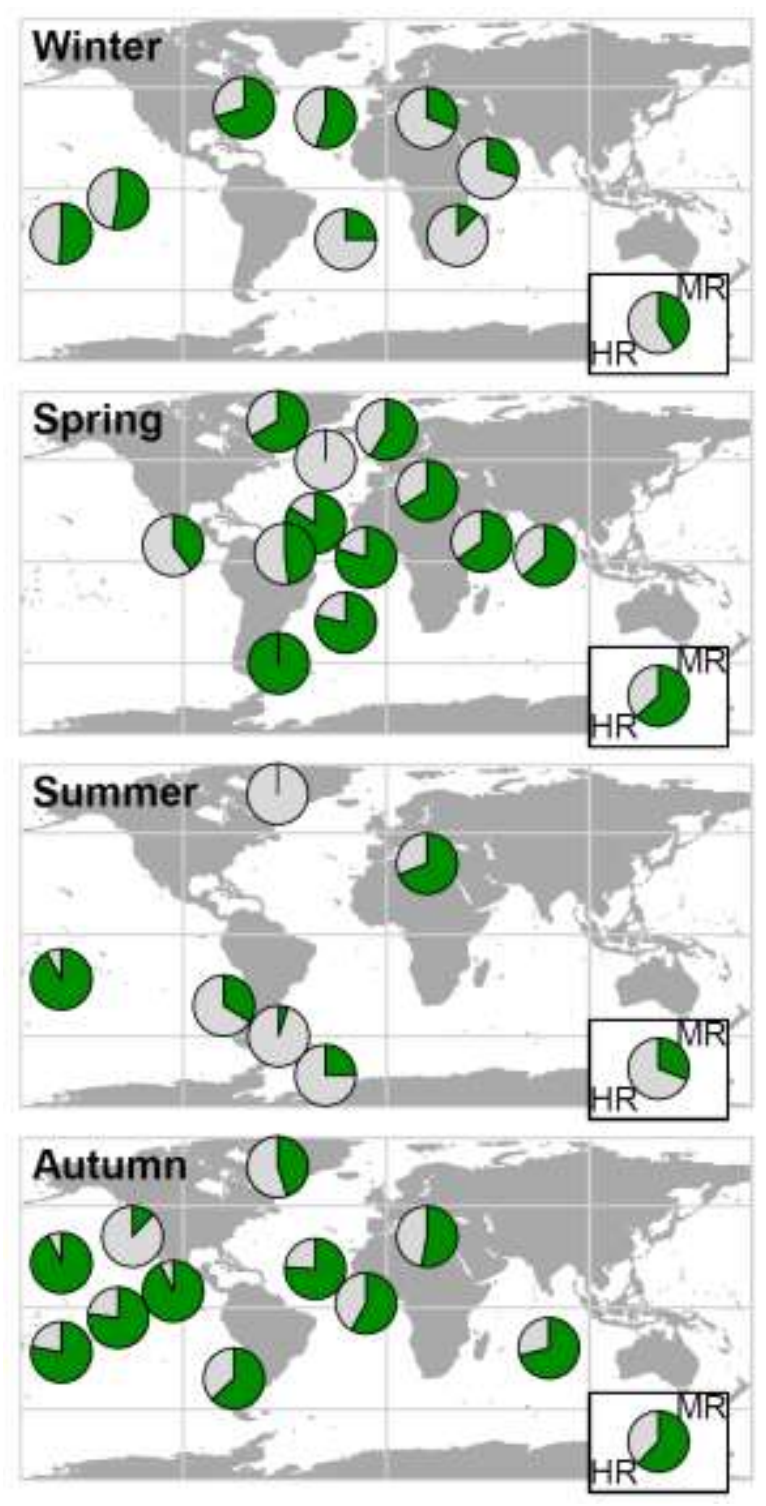

Figure 3.10 Seasonal relative contribution of mixotrophic Rhizaria (Collodaria and Acantharia) to total Rhizaria biomass over the globe. Pies highlighted within rectangles represent global means. MR: mixotrophic Rhizaria and HR: heterotrophic Rhizaria. 


\subsection{Summary}

This study revealed NCMs to be ubiquitous in the sunlit ocean and to comprise nearly half of the traditionally labelled "microzooplankton". Different forms of acquired phototrophy displayed contrasting biogeographies. Protists that rely on stolen chloroplasts were found to dominate in systems with higher productivity and abiotic variability, while protists harbouring endosymbionts were found to dominate in mature systems, mainly within lower latitudes and open seas. The importance of NCMs varied over the production cycle and different seasonalities were observed for each NCM functional group across different oceanic biomes. These findings call attention for the potential impact of acquired phototrophy within pelagic marine food webs and to biogeochemical cycling, warning to the recognition of diverse forms of mixotrophy. 


\section{Chapter 4}

\section{Biogeography of constitutive mixotrophs}

\section{Part of this chapter has been published as:}

Leles, S.G., Mitra, A., Flynn, K.J., Tillmann, U., Stoecker, D., JeOng, H.J., Burkholder, J., Hansen, P.J., Caron, D.A., Glibert, P.M., HallegraefF, G., RAVEN, J.A., SANDERS, R.W., \& ZuBKOV, M. (2019) Sampling bias misrepresents the biogeographical significance of constitutive mixotrophs across global oceans. Global Ecology and Biogeography, 1-11. DOI: 10.1111/geb.12853 


\subsection{Introduction}

Constitutive mixotrophs (CMs) comprise a wide range of size-classes and occur among different taxonomic groups within a continuum of mixotrophic strategies (Jeong et al., 2010c; Hansen, 2011; Granéli et al., 2012; Mitra et al., 2016; Glibert and Burford, 2017). These include from picoeukaryotic prasinophytes such as Micromonas to dinoflagellates larger than $200 \mu \mathrm{m}$ in cell length such as those within the genus Tripos (McKie-Krisberg and Sanders, 2014; Jacobson and Anderson, 1996). CMs also occur within a continuum of mixotrophic strategies, from primarily phototrophic to primarily phagotrophic species (Jeong et al., 2010c; Hansen, 2011; McKie-Krisberg et al., 2015; Lie et al., 2018). The diversity of forms and function promotes the occurrence of CMs across a range of environmental conditions (Zubkov and Tarran, 2008; Gast et al., 2014; Gomes et al., 2014). In Chapter 3, different biogeographic patterns were found for different functional groups of NCMs (Leles et al., 2017). Similarly, different CMs may be expected to have different distributions in the oceans.

Constitutive mixotrophs within the nanoplankton spectrum have been found to be important members of plankton communities within oligotrophic open seas, being major bacterivores at times (Zubkov and Tarran, 2008; Hartmann et al., 2012). CMs feed on bacteria whilst releasing dissolved organic matter and, therefore, are expected to play an important role within the microbial loop (Mitra et al., 2014b). Their importance, however, does not seem to be restricted to mature systems (e.g., oligotrophic seas and summer conditions within temperate seas). Within the polar seas, for example, the grazing impact by CMs on bacterial populations is similar to those reported for lower latitudes during periods of continuous daylight (Moorthi et al., 2009; Gast et al., 2014). Furthermore, several species of CMs thrive in eutrophic coastal seas and are responsible for the formation of harmful algal blooms (HABs) (Burkholder et al., 2008; Glibert and Burford, 2017). Many HABs species are dinophytes which act as bacterivores and as grazers of protist populations within the nano- and micro- plankton size spectrum (Jeong et al., 2010c; Hansen, 2011). Nevertheless, we still lack a clear understanding of the effects of different environmental factors on the distribution of CMs. This lack of knowledge is partially due to the difficulty of capturing and quantifying the abundance of CMs in the 
field (Anderson et al., 2017). In fact, it is not a common practice to identify species that are likely functionally mixotrophic in plankton surveys (Beisner et al., 2019). In addition, sampling can also be biased due to methodological artefacts that can underestimate the abundance of smaller size classes or more fragile groups (Gifford and Caron, 2000; Rutten et al., 2005; Biard et al., 2016).

In this context, the aim of this chapter is to investigate, for the first time, the global biogeography of CMs. Two questions are investigated: i) do CMs of different sizes and taxonomic groups have different biogeographic patterns? and ii) what are the effects of environmental variability on the distribution of CMs? The biogeography of CMs is first interrogated through the examination of online databases (OBIS) and analyses were based on the Longhurst's biogeographic classification of the ocean (Chapter 2.3). It is hypothesised, however, that OBIS underestimate the global distribution of CMs within the smaller size classes due to the traditional sampling techniques applied in plankton studies (Rose et al., 2004; Zarauz and Irigoien, 2008). To verify this hypothesis, data from studies which specifically targeted constitutive mixotrophs were compiled from the literature and compared against data from OBIS. The dataset derived from the studies which targeted CMs was then used to test the hypothesis that their distribution is expected to change with the trophic status of the system (i.e., from oligotrophic to eutrophic conditions) and throughout the water column. In the light of their biogeography and ecological importance, it is argued that a common and accurate sampling protocol is needed to correctly assign CMs in the field.

\subsection{Methods}

\subsubsection{Data compilation}

Distribution records for different species of CMs were compiled globally through OBIS (Chapter 2.2.1). Evidence for the ingestion of particles (live or dead) was required to include protist species in this study (Appendix B, Table B.1). The cosmopolitan coccolithophore Emiliania huxleyi, which has been previously suggested to take up particles, was excluded from the analysis because its mixotrophic potential is limited to the cell cycle form that is not recognised as the calcified coccolith-bearing morph (Rokitta et al., 2011). The list comprised 78 species across the different taxonomic taxa of 
cryptophytes, chrysophytes, haptophytes, prasinophytes, raphidophytes, dinophytes, chlorarachniophytes, and synchromophytes (Appendix B, Table B.1).

OBIS data are expected to provide a more robust picture of the global distribution of CMs in the microplankton spectrum (20-200 $\mu \mathrm{m})$ because smaller cells are not always identified down to species level on field surveys. Thus, the published literature was also investigated through the consulting of ISI Web of Science database on $20^{\text {th }}$ January 2018 (Chapter 2.2.2) to obtain records from studies which specifically targeted CMs in the nanoplankton spectrum $(2-20 \mu \mathrm{m})$. These studies reported in situ measurements of the abundance of actively feeding nano-CMs or the relative contribution of these to the total nanoflagellate bacterivory (methodological details are given below). The set of keywords used in the bibliographic survey included "nanoflagellates OR phytoplankton" followed by "mixotrophy OR phagotrophy OR bacterivory" and "ocean OR marine". A total of 21 studies were found (Appendix B, Tables B.2 and B.3). Geographic coordinates were retrieved from each study to compare the global distribution of nano-CMs obtained through the consultancy of OBIS versus through studies which specifically targeted these organisms.

Quantitative data for nano-CMs were also obtained from these studies. Obtaining quantitative estimates of numeric abundance (cells per water volume) and/or bacterivory impact of CMs is a non-trivial task (Anderson et al., 2017). These are commonly determined for the nanoplankton spectrum incubating samples of seawater collected in the field with fluorescent tracer particles (Safi and Hall, 1999; Gast et al., 2014; Sato et al., 2017). Different trophic groups are then identified through epifluorescence microscopy or through flow cytometric cell sorting combined with autofluorescence (Hartmann et al., 2012) and rarely identified down to genus/species level (Unrein et al., 2014). The nanoCMs are identified as nanoflagellates containing both chlorophyll and ingested particles, nanoflagellates containing chlorophyll but not ingested particles are classified as strictly autotrophs (ANF), and cells not-containing chlorophyll are classified as strictly heterotrophs (HNF). Epifluorescence microscopy allows the identification of nano-CMs through both the fluorescence derived from consumed fluorescently labelled bacterial (FLB) tracers and their simultaneous red chloroplast autofluorescence (Beisner et al., 
2019). Flow cytometry with fluorescence-activated cell sorting is another way that allows the separation of protists for which FLB have been detected with autofluorescence (Beisner et al., 2019). It is noteworthy that this methodology underestimates the abundance of nano-CMs while overestimating the abundance of ANF because CMs are not necessarily feeding all the time. Thus, this dataset only considers nano-CMs which were feeding at a certain location and time across the global oceans. From the 21 studies which provided quantitative data, 12 studies provided 167 estimates of the abundance of nano-CMs and 15 studies reported 87 estimates of their relative contribution to total nanoflagellate bacterivory (Appendix B, Table B.3).

To test the effects of environmental factors on the distribution of nano-CMs across the oceans, potential explanatory variables were also obtained. The variables were: net primary productivity (NPP; mg C m $\mathrm{m}^{-3}$ day $^{-1}$ ), sampling depth, measurements of nutrients concentration (nitrate and phosphate), and bacterial abundance (Appendix B, Tables B.2 and B.3). NPP data were obtained following Longhurst's biogeographic provinces (Chapter 2.3). All other explanatory variables were extracted from the original publications whenever possible. If source (raw) data were not reported in the publications, the authors were contacted to verify data availability. Publications were also classified according to sampling strategy between 'spatio-temporal' or 'temporal' to differentiate between studies in which sampling was conducted over time but at several stations, and studies in which sampling was conducted over time but at a fixed station, respectively. Furthermore, the abundance data for HNF, as well as the relative contribution of nanoCMs to total nanoflagellate bacterivory, were also extracted (Appendix B, Tables B.2 and B.3).

\subsubsection{Analysis of distribution records}

The biogeography of CMs was investigated according to taxonomy and size across different biogeographic provinces and oceanic biomes (Chapter 2.3) using the data obtained through OBIS. Over 230,000 records from 178 cruises were obtained from OBIS for 51 out of 78 species that comprised the initial list (Table 4.1). Species were grouped in taxonomic groups and among five size-classes (Table 4.1). Species size (length) was obtained from the literature (Tomas, 1997; Hoppenrath and Leander, 2007; Berge et al., 
2008a; Nézan and Chomérat, 2009; Yoo et al., 2010; Kang et al., 2011; Lim et al., 2015; Jang et al., 2017; Ok et al., 2017) and/or from the consultation of online repositories (http://nordicmicroalgae.org; http://www.sccap.dk; http://www.marinespecies.org). Global distribution maps were generated to visualize the presence of each taxonomic group within each size-class according to the biogeographic provinces.

The sampling locations from all 178 cruises included in the analysis were also mapped in order to compare the biogeographic patterns with the total sampling effort. It is noteworthy that it was not assumed $a$ priori that all taxonomic groups should contain individuals within all size-classes; indeed, that is not the case. The literature was examined to identify size class boundaries within each taxonomic group. For example, there are no known species within prasinophytes, chrysophytes, and haptophytes which are $>15 \mu \mathrm{m}$ in size (measured across the major cell axis). Likewise, all known raphidophyte species are $>10 \mu \mathrm{m}$. While there are suggestions that marine dinoflagellates $<5 \mu \mathrm{m}$ are present in the oceans, this information is derived from initial sequence data only (Moon-van der Staay et al., 2001; Lin et al., 2006); thus, these were not included in the present analysis. Dinophytes were, therefore, not assumed to occur within the $<5 \mu \mathrm{m}$ size-class.

The potential biases associated with size, taxonomy, and location were evaluated. The mean number of records was obtained for each size class among the oceanic biomes. Since the assumptions of normality were not met, Kruskal-Wallis rank tests were used to test for differences between size-classes or biomes (Chapter 2.4.2). Post-hoc nonparametric tests (Dunn's test) were performed to identify which pairs of size-classes or biomes were significantly different from each other (Chapter 2.4.2). The same procedure was performed to evaluate the potential bias related to taxonomy, grouping species according to taxonomy to test for any differences between taxonomic groups and biomes.

Non-metric multidimensional scaling (NMDS) analysis was conducted to visualize the interplay between size and taxonomy at species level (Chapter 2.4.3). To perform this analysis, a matrix 'species vs site' containing the number of records obtained for each species within each biogeographic province was built. The NMDS technique ordinates the species based on their dissimilarities; thus, if there are any differences among size-classes or taxonomic groups, species within the same size class or taxonomic group 
are expected to be closer to each other relative to other species. Similarly, the spatial distribution range of any species can be drawn from this analysis by its proximity to the different provinces and/or oceanic biomes. The distance matrix used in the NMDS analysis was calculated using the Bray-Curtis distance as the dataset was composed by count data (Chapter 2.4.3). It is noteworthy, however, that this analysis could not separate the effect of sampling biases from the differences associated to the 'true' biogeographic patterns of the different species; therefore, the data obtained from OBIS was compared against data retrieved from studies which specifically targeted mixotrophic protists (Appendix B, Table B.2). 
Table 4.1 Number of records retrieved from OBIS for each species included in the analysis; taxonomic group and size class indicated for each species.

\begin{tabular}{|c|c|c|c|}
\hline Group & Current species name & Length $(\mu \mathrm{m})$ & OBIS records \\
\hline Haptophyte & Chrysochromulina leadbeateri & $<5$ & no data \\
\hline Prasinophyte & Mantoniella antarctica & $<5$ & no data \\
\hline Prasinophyte & Micromonas spp. & $<5$ & 390 \\
\hline Prasinophyte & Mantoniella squamata & $<5$ & 53 \\
\hline Chrysophyte & Sulcochrysis biplastida & $<5$ & no data \\
\hline Haptophyte & Chrysochromulina brevifilum & $5-10$ & 31 \\
\hline Haptophyte & Haptolina ericina & $5-10$ & 87 \\
\hline Haptophyte & Haptolina hirta & $5-10$ & 56 \\
\hline Chrysophyte & Dinobryon spp. & $5-10$ & 8894 \\
\hline Chrysophyte & Ochromonas sp. & $5-10$ & 1423 \\
\hline Prasinophyte & Pyramimonas tychotreta & $5-10$ & no data \\
\hline Dinophyceae & Symbiodinium sp. & $5-10$ & 151 \\
\hline Dinophyceae & Yihiella yeosuensis & $5-10$ & no data \\
\hline Chrysophyte & Ochromonas monicis & $5-10$ & no data \\
\hline Chrysophyte & Ochromonas olivacea & $5-10$ & no data \\
\hline Chlorarachniophyte & Cryptochlora perforans & $5-10$ & no data \\
\hline Chlorarachniophyte & Lotharella oceanica & $5-10$ & no data \\
\hline Haptophyte & Prymnesium polylepis & $10-15$ & 111 \\
\hline Cryptophyte & Geminigera cryophila & $10-15$ & 1 \\
\hline Dinophyceae & Karlodinium veneficum & $10-15$ & 1439 \\
\hline Dinophyceae & Heterocapsa rotundata & $10-15$ & 8773 \\
\hline Dinophyceae & Paragymnodinium shiwhaense & $10-15$ & no data \\
\hline Dinophyceae & Prorocentrum cordatum & $10-15$ & 13869 \\
\hline Haptophyte & Prymnesium parvum & $10-15$ & 4 \\
\hline Prasinophyte & Pyramimonas gelidicola & $10-15$ & 15 \\
\hline Cryptophyte & Teleaulax amphioxeia & $10-15$ & no data \\
\hline Chrysophyte & Dinobryon balticum & $10-15$ & 3751 \\
\hline Dinophyceae & Biecheleria cincta & $10-15$ & no data \\
\hline Prasinophyte & Cymbomonas tetramitiformis & $10-15$ & 32 \\
\hline Dinophyceae & Amphidinium carterae & $15-20$ & 179 \\
\hline Dinophyceae & Heterosigma akashiwo & $15-20$ & 1298 \\
\hline Dinophyceae & Karlodinium armiger & $15-20$ & no data \\
\hline Dinophyceae & Prorocentrum donghaiense & $15-20$ & no data \\
\hline Dinophyceae & Prorocentrum triestinum & $15-20$ & 4666 \\
\hline Raphidophyceae & Fibrocapsa japonica & $15-20$ & 259 \\
\hline Synchromophyte & Synchroma grande & $15-20$ & no data \\
\hline Dinophyceae & Akashiwo sanguinea & $>20$ & 4056 \\
\hline Dinophyceae & Alexandrium affine & $>20$ & 98 \\
\hline Dinophyceae & Alexandrium andersonii & $>20$ & 47 \\
\hline Dinophyceae & Alexandrium catenella & $>20$ & 3142 \\
\hline
\end{tabular}


Table 4.1 continued

\begin{tabular}{|c|c|c|c|}
\hline Dinophyceae & Alexandrium minutum & $>20$ & 2902 \\
\hline Dinophyceae & Alexandrium ostenfeldii & $>20$ & 971 \\
\hline Dinophyceae & Alexandrium pohangense & $>20$ & no data \\
\hline Dinophyceae & Alexandrium pseudogonyaulax & $>20$ & 473 \\
\hline Raphidophyceae & Chattonella spp. & $>20$ & 1877 \\
\hline Dinophyceae & Margalefidinium polikrikoides & $>20$ & 65 \\
\hline Dinophyceae & Fragilidium duplocampanaeforme & $>20$ & no data \\
\hline Dinophyceae & Fragilidium mexicanum & $>20$ & no data \\
\hline Dinophyceae & Fragilidium subglobosum & $>20$ & 207 \\
\hline Dinophyceae & Gambierdiscus toxicus & $>20$ & 2 \\
\hline Dinophyceae & Gonyaulax diegensis & $>20$ & 127 \\
\hline Dinophyceae & Gonyaulax polygramma & $>20$ & 3462 \\
\hline Dinophyceae & Gonyaulax spinifera & $>20$ & 7333 \\
\hline Dinophyceae & Gymnodinium aureolum & $>20$ & 752 \\
\hline Dinophyceae & Gymnodinium catenatum & $>20$ & 388 \\
\hline Dinophyceae & Gymnodinium impudicum & $>20$ & 30 \\
\hline Dinophyceae & Levanderina fissa & $>20$ & 243 \\
\hline Dinophyceae & Barrufeta resplendens & $>20$ & 2 \\
\hline Dinophyceae & Heterocapsa circularisquama & $>20$ & 1 \\
\hline Dinophyceae & Heterocapsa triquetra & $>20$ & 8347 \\
\hline Dinophyceae & Karenia brevis & $>20$ & 442 \\
\hline Dinophyceae & Karenia mikimotoi & $>20$ & 6807 \\
\hline Dinophyceae & Lingulodinium polyedra & $>20$ & 2622 \\
\hline Dinophyceae & Tripos furca & $>20$ & 75924 \\
\hline Dinophyceae & Tripos longipes & $>20$ & 28733 \\
\hline Dinophyceae & Ostreopsis lenticularis & $>20$ & 9 \\
\hline Dinophyceae & Ostreopsis ovata & $>20$ & 7 \\
\hline Dinophyceae & Ostreopsis siamensis & $>20$ & no data \\
\hline Dinophyceae & Polykrikos hartmannii & $>20$ & 253 \\
\hline Dinophyceae & Prorocentrum belizeanum & $>20$ & 1 \\
\hline Dinophyceae & Prorocentrum lima & $>20$ & 1575 \\
\hline Dinophyceae & Prorocentrum micans & $>20$ & 23876 \\
\hline Dinophyceae & Scrippsiella acuminata & $>20$ & 8934 \\
\hline Dinophyceae & Takayama helix & $>20$ & 1 \\
\hline Dinophyceae & Tripos hexacanthus & $>20$ & 106 \\
\hline Dinophyceae & Tripos candelabrum & $>20$ & 4522 \\
\hline Dinophyceae & Polykrikos lebourae & $>20$ & 1079 \\
\hline
\end{tabular}




\subsubsection{Analysis of abundance data - a meta-analysis}

A meta-analysis was conducted to evaluate the effects of environmental factors on the abundance of actively feeding nano-CMs across the global oceans. GLMMs were chosen to perform the meta-analysis (Chapter 2.4.4). Considering the nature of the outcome (abundance of nano-CMs; count data), a Poisson distribution was chosen. The dataset was built from observational studies which encompass variation among regions (different latitudes and depths) and time (different periods of the year); thus, more than one source of random error was identified. The potential fixed and random effects (covariates) to be included in the analysis were then evaluated.

NPP data were included in the analysis since it is a measure of the trophic status of the system and allows a direct link to Longhurst's biogeographic provinces (Chapter 2.3); this covariate was included in the statistical model as a fixed effect. Measurements of nutrients concentration were not included in the analysis because data were not available for most of the studies that estimated the abundance of nano-CMs (Appendix B, Table B.3). To test for the effect of prey availability, bacterial abundance was included in the GLMM as another fixed effect. Both fixed effects comprise continuous variables of different units which vary greatly in magnitude. Sampling depth and publication ID (i.e., categories identifying the different studies included in the metaanalysis) were considered as potential random effects. Sampling depth was included as a random effect to account for vertical variability within the water column; this covariate was composed of 5 levels: surface, sub-surface, deep chlorophyll maximum (DCM), thermocline, and 'integrated' (i.e., when data were given as the average over the water column). Despite the non-independence of multiple entries extracted from the same study, publication ID was not included in the GLMM as a random effect. The data were analysed

a priori and variability was mainly related to spatial and temporal differences which were already embedded in the analysis within the NPP and bacterial abundance datasets because values were obtained for a particular region and period of the year.

Thus, the 'full' GLMM comprised NPP and bacterial abundance as well as their interaction as fixed effects and a random intercept at the depth level assuming Poisson distribution. Although different effects of NPP, bacterial abundance, and their interaction 
on the abundance of nano-CMs can be expected within each level of depth (corresponding to random slopes within the GLMM), it was not possible to account for this variability with the available dataset because there was a strong possibility that variables would be correlated. However, simple exponential fits were applied to visualise how the abundance on nano-CMs varies with depth; exponential fits were chosen vs linear fits since the assumptions of homogeneity of variances and normal distribution were not met and the data could be approximated by the Poisson distribution. The GLMM converged using Laplace approximation, but the residuals indicated overdispersion; thus, the statistical model was re-fitted adding a random effect at the level of observation (Chapter 2.4.4). The random effect term had non-zero variance and there was no possibility of correlations since only a single random effect was fitted at each level. The significance of all terms (fixed and random effects) was tested comparing the 'full' model with reduced models (represented by the deletion of single terms) through likelihood ratio tests (Chapter 2.4.4). Histograms of the data ( $n=164$ entries) revealed a right skewed distribution with just a few values on the right side of the histogram. Over $96 \%$ of the observations comprised abundance values between $0-700$ cells $\mathrm{ml}^{-1}$; only 6 observations were found between $700-2300$ cells $\mathrm{ml}^{-1}$. These 6 values were considered as potential outliers; therefore, the analysis was repeated excluding these to evaluate if they could affect the results.

Finally, the ecological relevance of nano-CMs in the global oceans was also investigated evaluating i) the abundance ratio between nano-CMs and HNF and ii) the relative contribution of nano-CMs to total nanoflagellate bacterivory across the different oceanic biomes (Chapter 2.3). Kruskal-Wallis tests were performed to test for differences among oceanic biomes (Chapter 2.4.2).

\subsection{Results}

\subsubsection{Global distribution and sampling biases}

The global distributions of CMs across the different biogeographic provinces were mapped according to size and taxonomy (Fig. 4.1). Nearly 50\% of the constitutive mixotrophic species were larger than $20 \mu \mathrm{m}$; except for two raphidophytes, all the species larger than $20 \mu \mathrm{m}$ belonged to dinophytes. A lack of data across the different size classes, particularly for other CM groups, is indicated by the "non-coloured" (white) 
biogeographic maps in Fig. 4.1. In order to compare these biogeographic patterns with the total sampling effort, the sampling locations from all 178 cruises included in the analysis were also mapped (Fig 4.2). From this it can be seen which biogeographic provinces were visited at different points in time and space but were not associated with the presence of a CM group (white biogeographic provinces in Fig 4.1). 


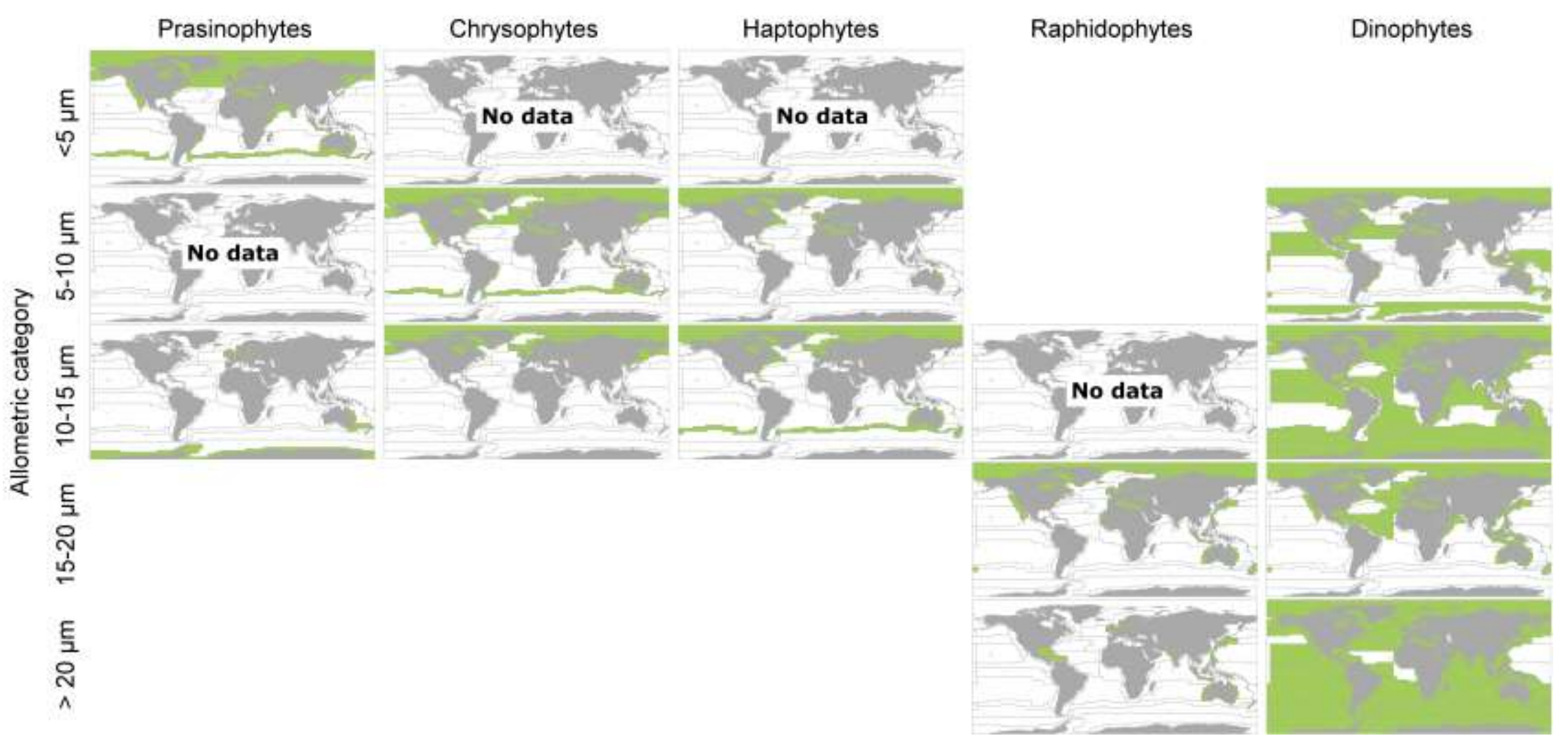

Fig. 4.1 Global distributions of CMs across Longhurst's biogeographic provinces. Distribution maps are shown for different groups (prasinophytes, raphidophytes, haptophytes, chrysophytes, and dinophytes) across different size classes (maximum cell dimension: < 5 $\mu \mathrm{m} ; 5-10 \mu \mathrm{m} ; 10-15 \mu \mathrm{m} ; 15-20 \mu \mathrm{m} ;>20 \mu \mathrm{m})$. Green provinces indicate the presence of CMs while white provinces indicate no data. Absence of maps (i.e., white spaces) indicate nonexistence of any species within these groups. At least one record was necessary to assume that mixotrophs occurred in any province. 


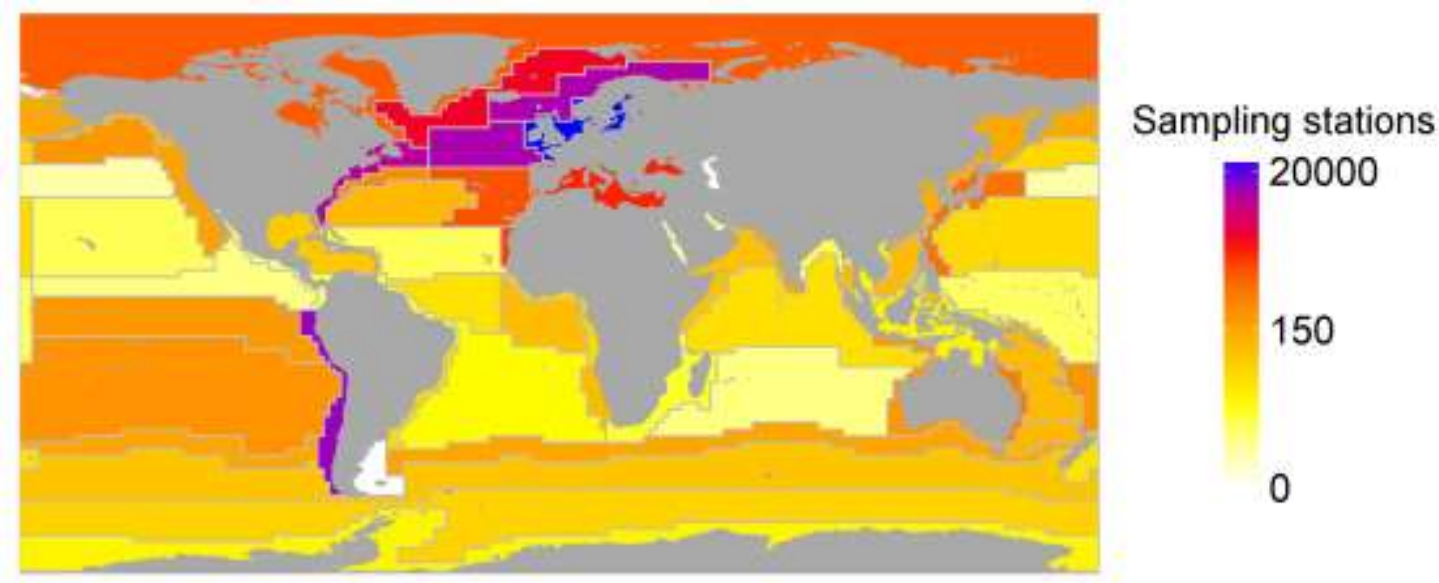

Fig. 4.2 Total sampling effort considering the pool of species selected for the present analysis. In total, 178 OBIS datasets were accessed. This map indicates how many times a biogeographic province was "visited" at a point in time and space. Only one biogeographic province ("Falklands"; white province in the map) had zero sampling stations.

The potential biases relating to size, taxonomy, and location were tested using quantitative data (Figs. 4.3 and 4.4). The number of records obtained within the different biomes differed significantly among size-classes $(H=11.8, p=0.02)$. Post-hoc comparisons confirmed that sampling effort was higher for species $>20 \mu \mathrm{m}$, except when comparing this group with species within 10-15 $\mu \mathrm{m}$ (Fig 4.3, Table 4.2). Differences were also found when comparing taxonomic groups $(H=12.2, p=0.02)$. The number of records obtained for dinophytes within the different biomes was much higher than that retrieved for any other group; no difference was found among other groups (Fig 4.4, Table 4.3). Differences between biomes were also tested for each dataset (i.e., grouped by size or by taxonomy, as per Figs. 4.3 and 4.4) and a significant difference was found in both analyses ( $\mathrm{H}=13.6, \mathrm{p}=0.02$; and $\mathrm{H}=11.2, \mathrm{p}=0.05$, respectively). Fewer records were obtained within oligotrophic gyres and equatorial regions when compared to other biomes and no difference was found between other biomes (Tables 4.4 and 4.5). 


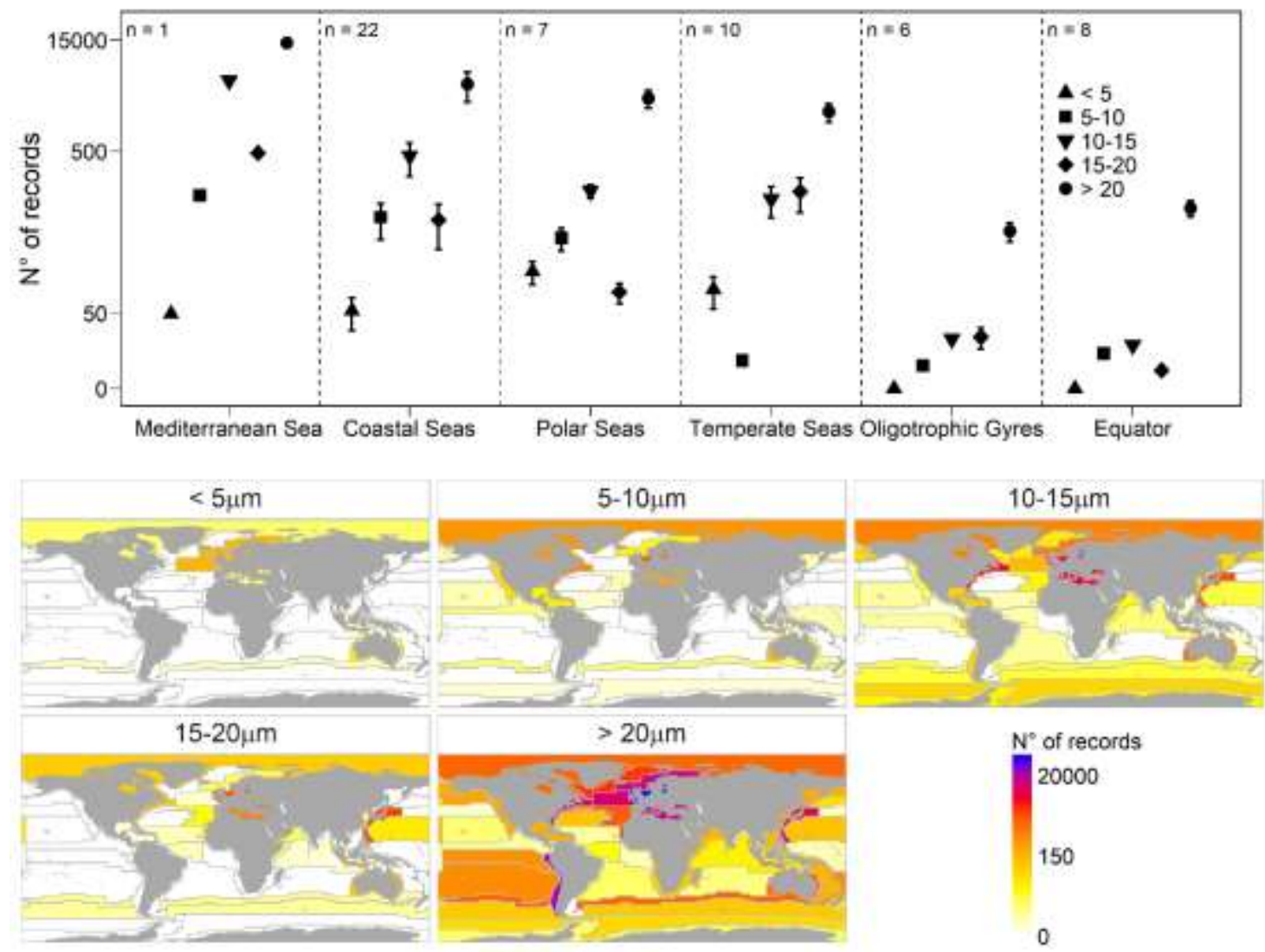

Fig 4.3 Number of records for CMs within different size-classes ( $\mu \mathrm{m}$, length) across different biomes (upper panel) and across different biogeographic provinces (lower panel; maps). Note that number of records was plotted on a log-scale. In the upper panel: n, number of biogeographic provinces within each biome and error bars indicate standard error. At least one record was necessary to assume that mixotrophs occurred in any province.

Table 4.2 Post-hoc pairwise comparisons by Dunn's test ( $Z$ scores) for size-classes.

\begin{tabular}{lllll}
\hline & $<\mathbf{5} \boldsymbol{\mu m}$ & $>\mathbf{2 0} \boldsymbol{\mu m}$ & $\mathbf{1 0 - 1 5} \boldsymbol{\mu m}$ & $\mathbf{1 5 - 2 0} \boldsymbol{\mu m}$ \\
\hline$>20 \mu \mathrm{m}$ & $-3.11^{* * *}$ & & & \\
$10-15 \mu \mathrm{m}$ & $-1.97^{*}$ & 1.15 & & \\
$15-20 \mu \mathrm{m}$ & -1.21 & $1.90^{*}$ & 0.75 & \\
$5-10 \mu \mathrm{m}$ & -0.59 & $2.52^{* *}$ & 1.38 & 0.62 \\
\hline$* \mathrm{p}<0.05, * * \mathrm{p}<0.01, * * * \mathrm{p}<0.001$ & &
\end{tabular}




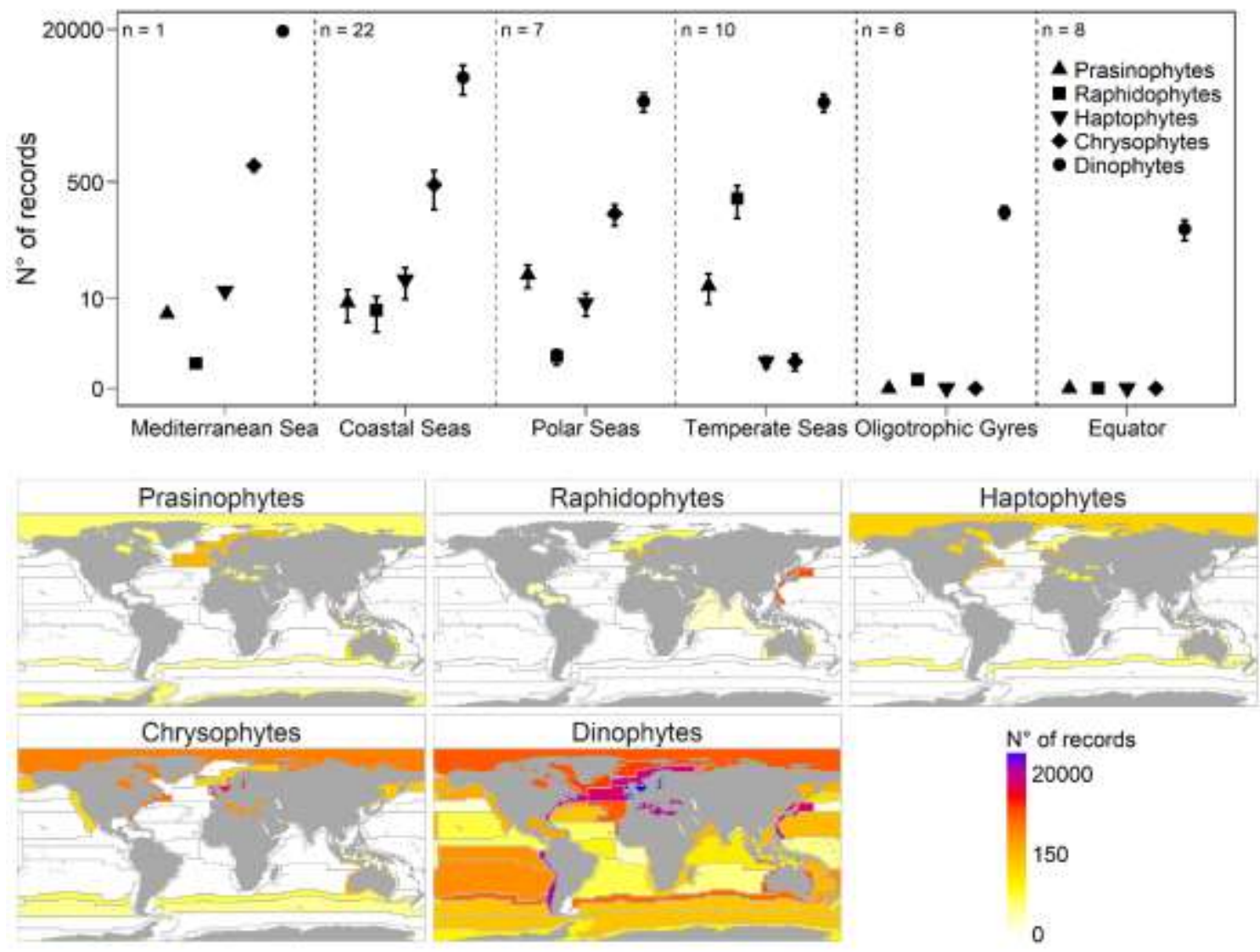

Fig 4.4 Number of records for CMs within different taxonomic groups across different biomes (upper panel) and across different biogeographic provinces (lower panel; maps). Note that number of records was plotted on a log-scale. In the upper panel: n, number of biogeographic provinces within each biome and error bars indicate standard error.

Table 4.3 Post-hoc pairwise comparisons using Dunn's test ( $Z$ scores) for taxonomic groups.

\begin{tabular}{lllll}
\hline & Chrysophytes & Dinophytes & Haptophytes & Prasinophytes \\
\hline Dinophytes & $-2.18^{*}$ & & & \\
Haptophytes & 0.74 & $2.92^{* *}$ & & \\
Prasinophytes & 0.59 & $2.77^{* *}$ & -0.15 & \\
Raphidophytes & 0.68 & $2.85^{* *}$ & -0.07 & 0.08 \\
\hline
\end{tabular}

$* \mathrm{p}<0.05, * * \mathrm{p}<0.01$ 
Table 4.4 Post-hoc pairwise comparisons using Dunn's test ( $Z$ scores) for biomes (averaged by size-classes).

\begin{tabular}{llllll}
\hline & Coastal & Equator & Mediterranean & Oligotrophic & Polar \\
\hline Equatorial Upwelling & $2.21^{*}$ & & & & \\
Mediterranean Sea & -0.61 & $-2.82^{* *}$ & & & \\
Oligotrophic Gyres & $2.14^{*}$ & -0.07 & $2.75^{* *}$ & & \\
Polar Seas & 0.25 & $-1.96^{*}$ & 0.86 & $-1.89^{*}$ & \\
Temperate Seas & 0.43 & $-1.78^{*}$ & 1.04 & $-1.71^{*}$ & 0.2 \\
\hline
\end{tabular}

$* \mathrm{p}<0.05, * * \mathrm{p}<0.01$

Table 4.5 Post-hoc pairwise comparisons by Dunn's test (Z scores) for biomes (averaged by taxonomic groups).

\begin{tabular}{llllll}
\hline & Coastal & Equator & Mediterranean & Oligotrophic & Polar \\
\hline Equatorial Upwelling & $2.28^{*}$ & & & & \\
Mediterranean Sea & 0.23 & $-2.04^{*}$ & & & \\
Oligotrophic Gyres & $2.20^{*}$ & -0.07 & $1.97^{*}$ & & \\
Polar Seas & 0.14 & $-2.13^{*}$ & -0.09 & $-2.06^{*}$ & \\
Temperate Seas & 0.45 & $-1.82^{*}$ & 0.22 & $-1.75^{*}$ & 0.3 \\
\hline
\end{tabular}

$* \mathrm{p}<0.05$

The relationship between different size classes and different taxonomic groups at species level was explored through the NMDS analysis (Fig 4.5). This analysis revealed dinophyte species within the larger size class (> $20 \mu \mathrm{m})$ present in different biomes thus displaying a broader geographic distribution compared to other species (Fig 4.5). The central position of the biomes in the NMDS plot (Fig. 4.5) indicates a large overlap between the different biogeographic provinces (Fig. 4.6) which, in turn, is associated to the presence of cosmopolitan species with a large number of records (Table 4.1). The different colours in this figure allow the reader to discern that different taxonomic groups have different distribution patterns. Through reference to the different symbols (Fig 4.5), it is clear that size and taxonomy are not independent and most species of dinophytes are $>20 \mu \mathrm{m}$ while most species within other groups are $<20 \mu \mathrm{m}$. However, even among dinophytes there were outliers indicating certain species $>20 \mu \mathrm{m}$ with a more limited 
distribution; Alexandrium andersonii and Fragilidium subglobosum are examples of large dinophytes ordinated closer to species belonging to other taxonomic groups, such as the haptophyte Prymnesium polylepis and the prasinophyte Cymbomonas tetramitiformis. It is noteworthy, however, that this analysis cannot separate the effects of sampling biases from the 'true' biogeographic patterns of the different species.

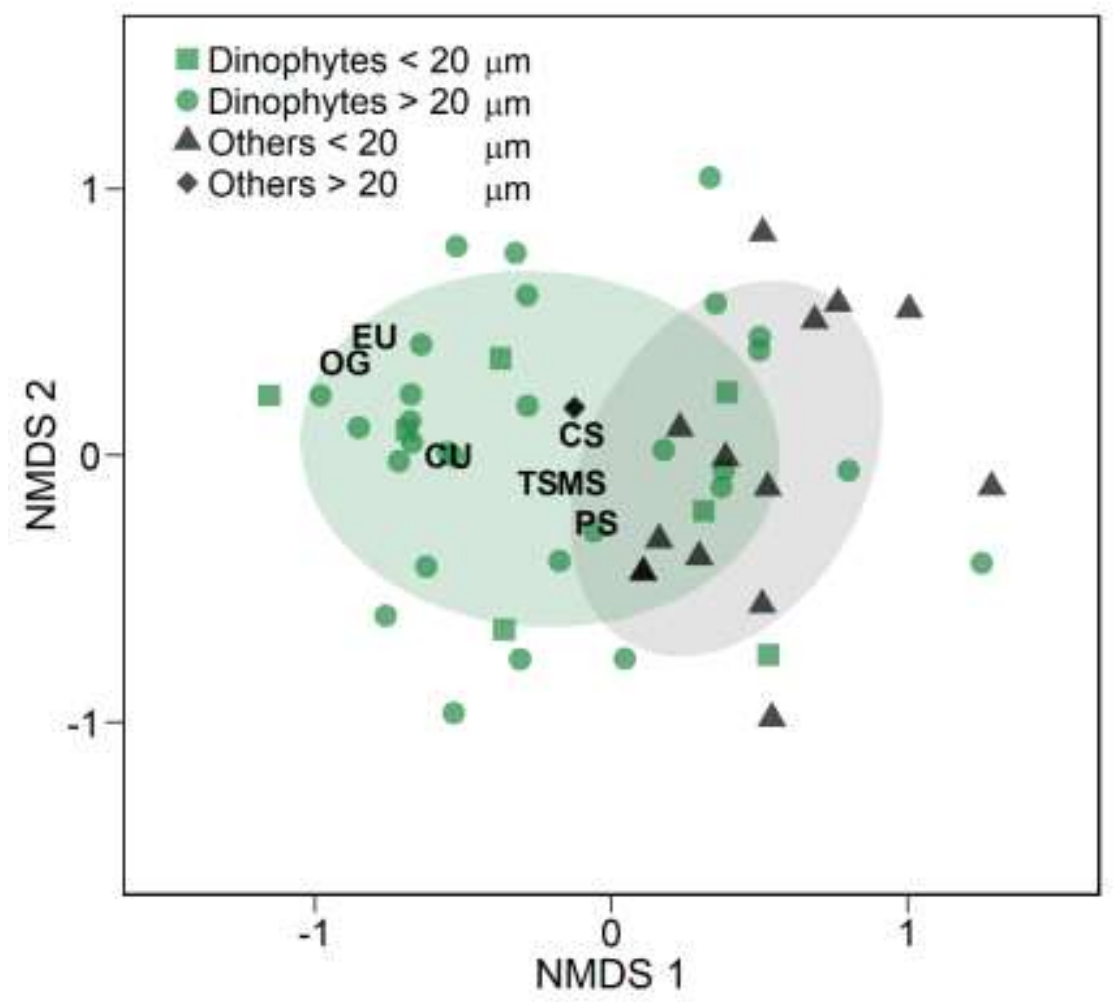

Fig 4.5 NMDS ordination of CM species based on the number of records observed within each biogeographic province; biomes were primarily derived from provinces (Fig 4.6). Each symbol represents a species which was classified according to taxonomy and size. Ellipses are shown at $75 \%$ confidence interval and were used to define dinophytes (green) and other groups (grey). The different biomes are: MS, Mediterranean Sea; PS, Polar Seas; TS, Temperate Seas; CS, Coastal Seas; OG, Oligotrophic Gyres; EU, Equatorial Upwelling (see also Fig. 4.6). 


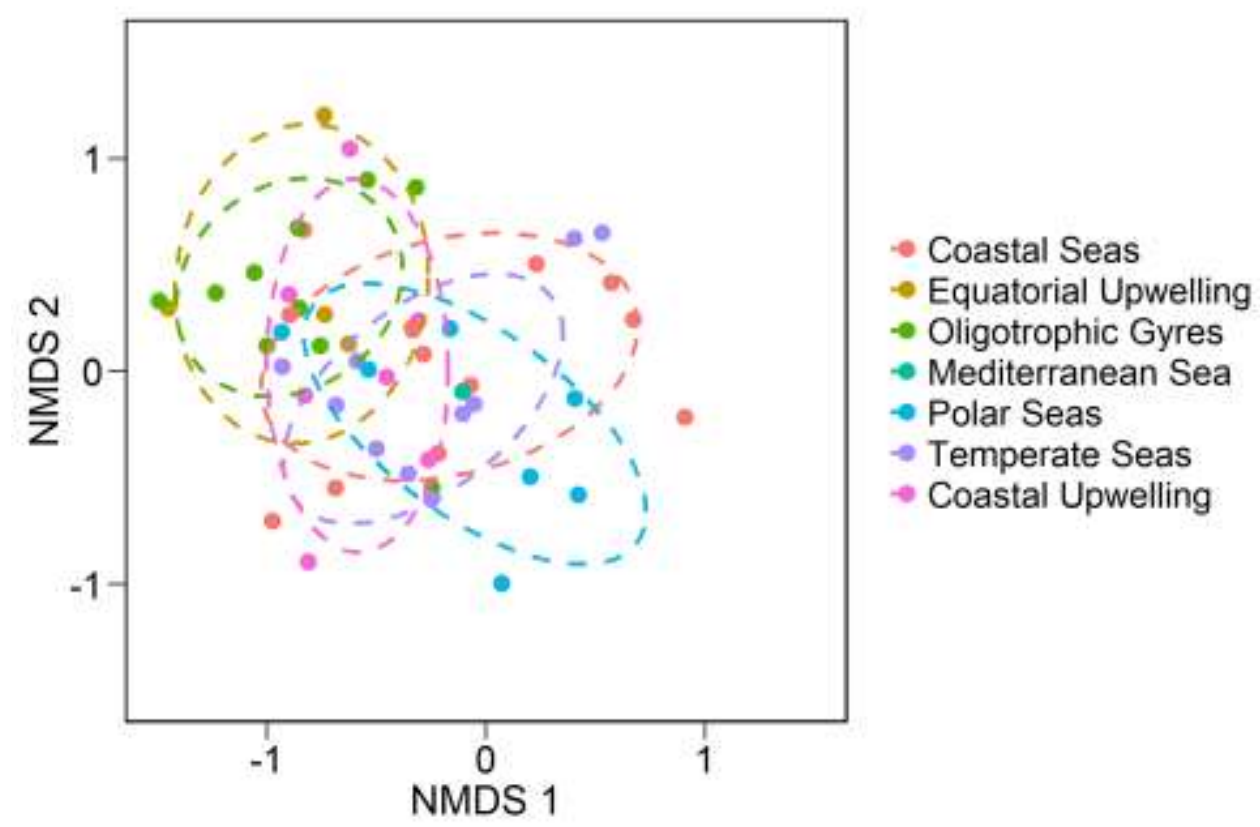

Fig 4.6 Additional results from the NMDS analysis (Fig 4.5) showing the distribution of biogeographic provinces in a two-dimensional space and their grouping in six larger biomes. Matrix input corresponds to the number of records for each CM species observed within each biogeographic province (same as in Fig. 4.5); the Coastal Upwelling biome was not represented due to a lack of data. Each dot represents a biogeographic province and colours represent the different biomes; the dashed ellipses represent the biome clusters at $75 \%$ confidence interval.

The results showed that non-dinophyte species $<20 \mu \mathrm{m}$ were the least represented group of CMs. To verify the under-representation of these groups within global databases, and thus the incomplete status of global distribution data, maps were plotted for the data available for these groups from OBIS and from studies that specifically targeted these organisms. Interestingly, the maps look quite different from each other (Fig 4.7). Despite the lower number of records, the distribution traced back from bacterivory studies showed nano-CMs to have a wider distribution across the oceans than the distribution drawn from global databases. One could argue that the distribution of nano-CMs is more limited to coastal regions and to temperate and polar seas analysing the map obtained by the later (Fig 4.7, upper panel). However, their true distribution largely encompasses open oceans including regions close to the Equator and within oligotrophic gyres in the Atlantic and the Pacific Oceans (Fig 4.7, lower panel). 

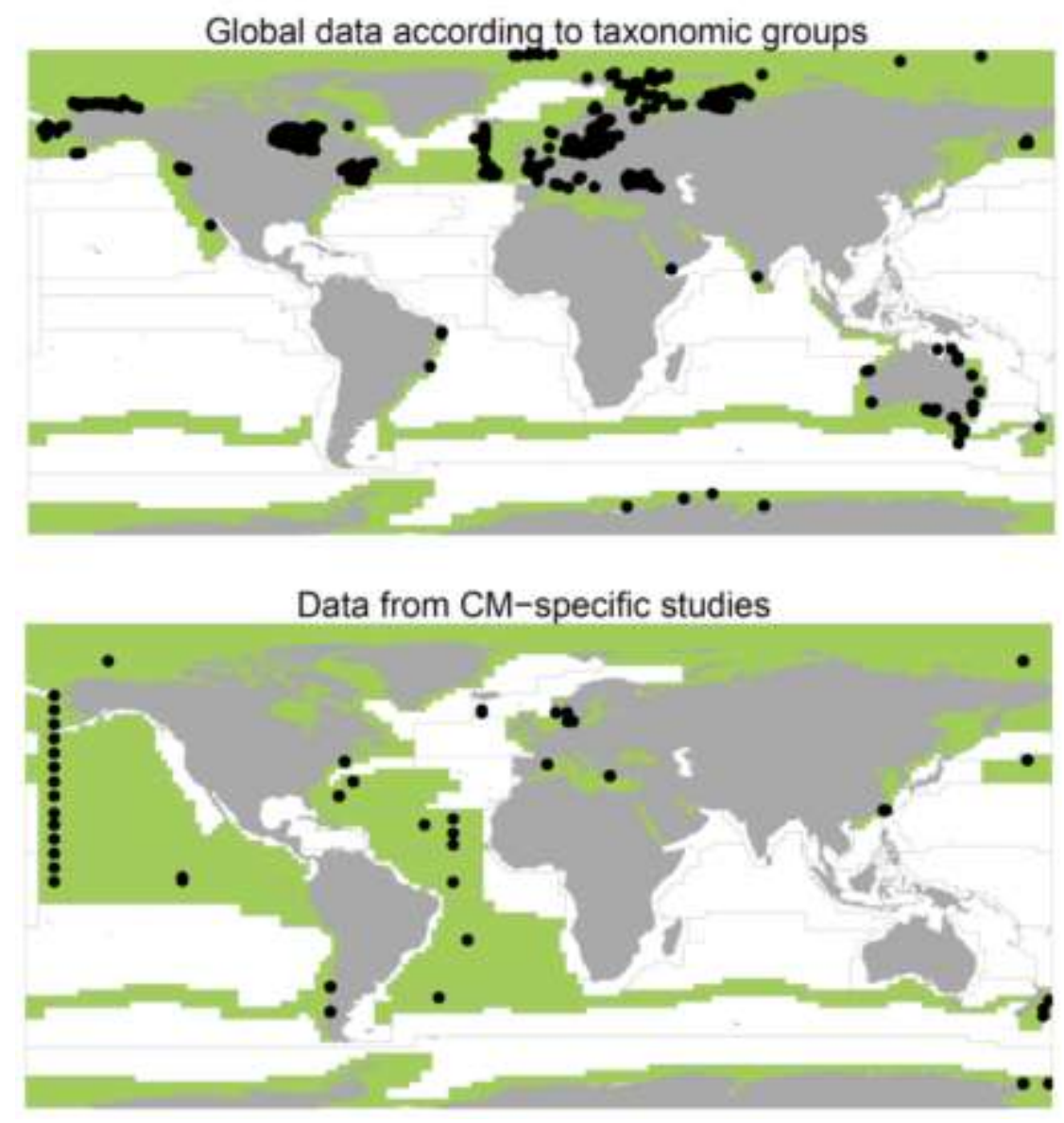

Fig 4.7 Global distribution of nano-CMs. Records obtained from OBIS (upper panel) and records obtained from protist nanoplankton bacterivory studies (lower panel). Symbols correspond to the exact location where nano-CMs were found. Green provinces indicate the presence of nano-CMs while white provinces correspond to absence due to lack of data; the grid indicate Longhurst's biogeographic provinces. At least one record was necessary to assume that mixotrophs occurred in any province. 


\subsubsection{Meta-analysis}

NPP, bacterial abundance, and depth were associated with variation in the abundance of nano-CMs in the global oceans (Table 4.6). These results were also consistent once outliers were excluded from the analysis (Table 4.7). The likelihood ratio tests (LRT) confirmed that all effects included in the GLMMs were significant (Tables 4.6 and 4.7). Overall, the results indicated that the abundance of nano-CMs increases exponentially with NPP (Fig 4.8); however, the interpretation of this relationship is complex since the effect of NPP varied across different depth levels and the interaction between NPP and bacterial abundance was significant (Table 4.6). Fig 4.8 shows how the abundance of nano-CMs relates to all covariates included in the GLMM (in their original units). Simple exponential fits applied to each depth level showed that the abundance of nano-CMs increases with NPP from surface waters to the DCM (Fig 4.8); in turn, the abundance of nano-CMs was relatively constant and independent from NPP within the thermocline and for measurements averaged over the water column (Fig 4.8). However, it was not possible to include this variation (random slopes among depth levels) in the GLMMs due to insufficient data and to correlation between variables. Furthermore, these results must be interpreted taking into account the interaction between NPP and bacterial abundance (Fig 4.8). Fig 4.9 clearly shows that the effect of NPP varies within different ranges of bacterial abundance. The abundance of nano-CMs increases exponentially with NPP for bacterial abundances lower than $1 \times 10^{7}$ cells $\mathrm{mL}^{-1}$ (Fig 4.9); in turn, no effect was observed once bacterial abundances were higher than $1 \times 10^{7}$ cells $\mathrm{mL}^{-1}$ (Fig 4.9).

Table 4.6 Estimates of fixed effects given by the GLMM of nano-CMs abundance (outcome) with depth as a random effect. The fixed effects included in the model were: net primary productivity (NPP), bacterial abundance (BA), and the interaction between these terms. LRT - likelihood ratio test; SE - standard error; SD - standard deviation.

\begin{tabular}{llcccc}
\hline Covariates & & LR & p-value LRT & Estimate & SE \\
\hline \multirow{3}{*}{ Fixed effects } & Intercept & & & 4.04 & 0.24 \\
& NPP & 68.00 & $<0.0001$ & 0.87 & 0.09 \\
& BA & 11.10 & 0.0009 & 0.34 & 0.10 \\
& NPP : BA & 10.78 & 0.001 & -0.39 & 0.12 \\
Random effect & depth & LR & p-value LRT & SD & \\
\hline
\end{tabular}


Table 4.7 Estimates of fixed effects given by the GLMM of nano-CMs abundance (outcome) with depth as a random effect excluding potential outliers. The fixed effects included in the model were: net primary productivity (NPP), bacterial abundance (BA), and the interaction between these terms. LRT - likelihood ratio test; SE - standard error; $\mathrm{SD}$ - standard deviation.

\begin{tabular}{llcccc}
\hline Covariates & & LR & p-value LRT & Estimate & SE \\
\hline \multirow{3}{*}{ Fixed effects } & Intercept & & & 4.00 & 0.22 \\
& NPP & 48.85 & $<0.0001$ & 0.75 & 0.10 \\
& BA & 10.42 & 0.001 & 0.32 & 0.10 \\
& NPP : BA & 14.64 & 0.0001 & -0.45 & 0.11 \\
Random effects & depth & LR & p-value LRT & SD & \\
\hline
\end{tabular}

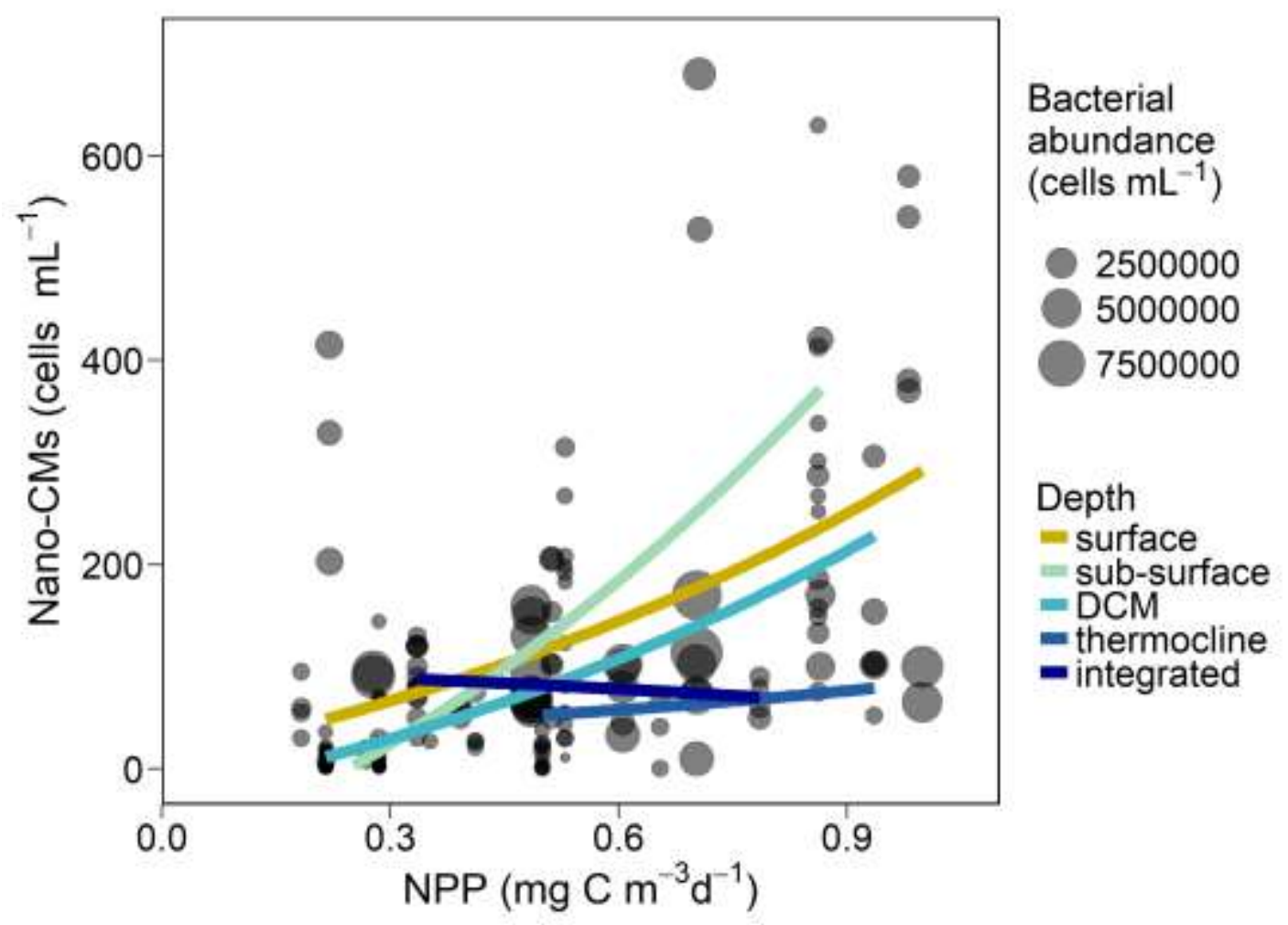

Fig 4.8 Abundance of nano-CMs along a gradient of net primary productivity (NPP). Bacterial abundance is given proportionally to the size of the dots. The lines represent the trends observed within different depth levels assuming that the abundance of nano-CMs increases exponentially with NPP; note, however, that random slopes among depth levels were not included in the GLMM. 


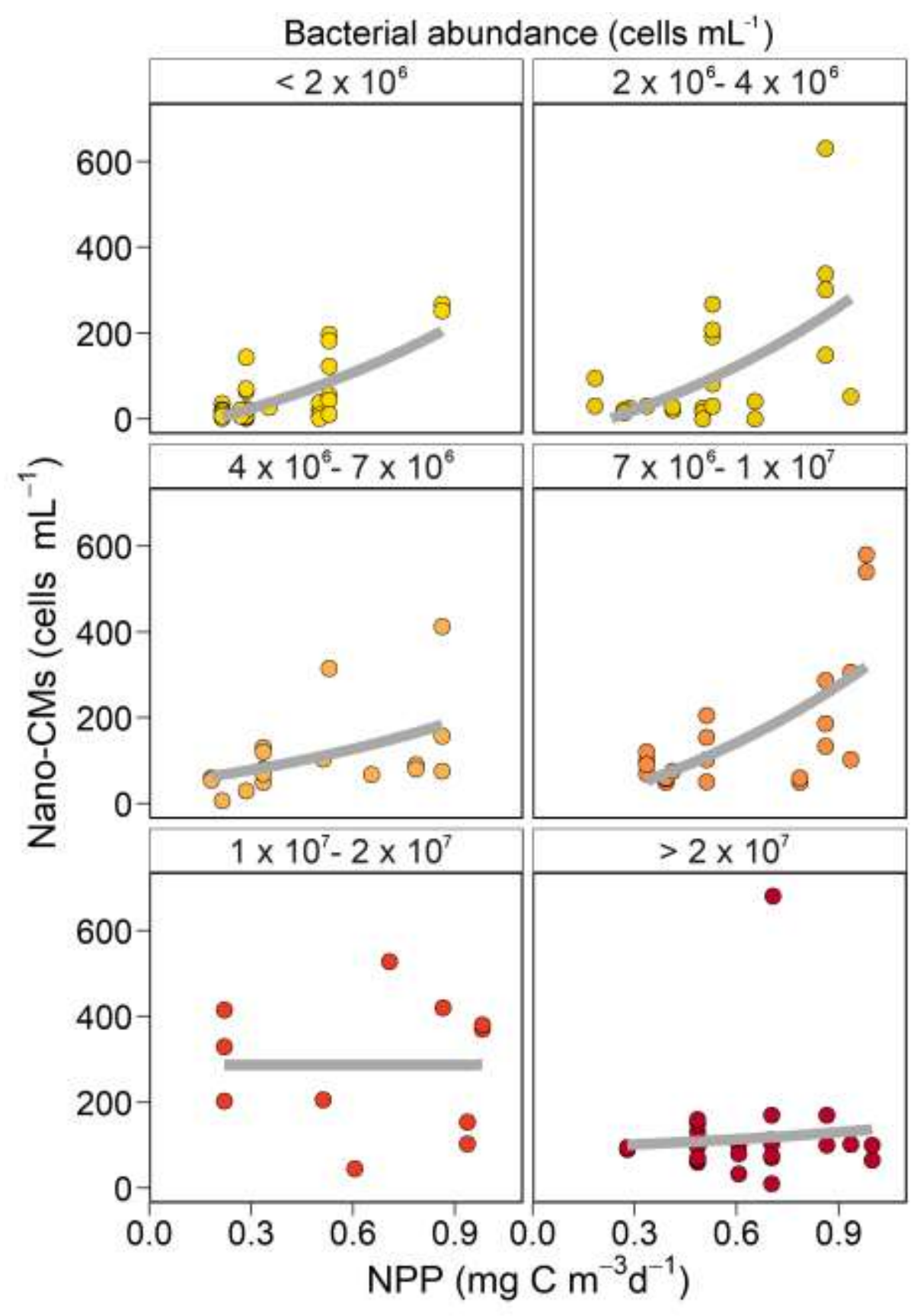

Fig 4.9 Abundance of nano-CMs along a gradient of net primary productivity (NPP) within different ranges of bacterial abundance (cells $\mathrm{mL}^{-1}$ ). The lines represent the trends within different ranges of bacterial abundance assuming that the abundance of nano-CMs increases exponentially with NPP; note that for bacterial abundances higher than $1 \times 10^{7}$ cells $\mathrm{mL}^{-1}$, no exponential trend was observed. 
The overall abundance of nano-CMs was lower than the abundance of HNF across all oceanic biomes (Fig 4.10, upper panel) and a significant difference was found when comparing their abundance ratio across the biomes $(\mathrm{H}=58.9, \mathrm{p}<0.05)$. Pairwise comparisons showed that the mean nano-CMs:HNF abundance ratio was higher (but also highly variable) among the Coastal Seas, Equatorial Upwelling, and Polar Seas biomes relative to the other biomes (Table 4.8). No significant difference was found among the other biomes (Table 4.8). Despite their low contribution in terms of abundance, nano-CMs contributed on average to half of the total nanoflagellate bacterivory across the global oceans (Fig 4.10, lower panel). The contribution of nano-CMs to total bacterivory also varied across the oceanic biomes $(\mathrm{H}=28.8, \mathrm{p}<0.05)$, being lower within the Coastal Upwelling biome followed by the Polar Seas biome (Table 4.9); but note the high variability within this biome. No difference was found among the other biomes (Table 4.9). 

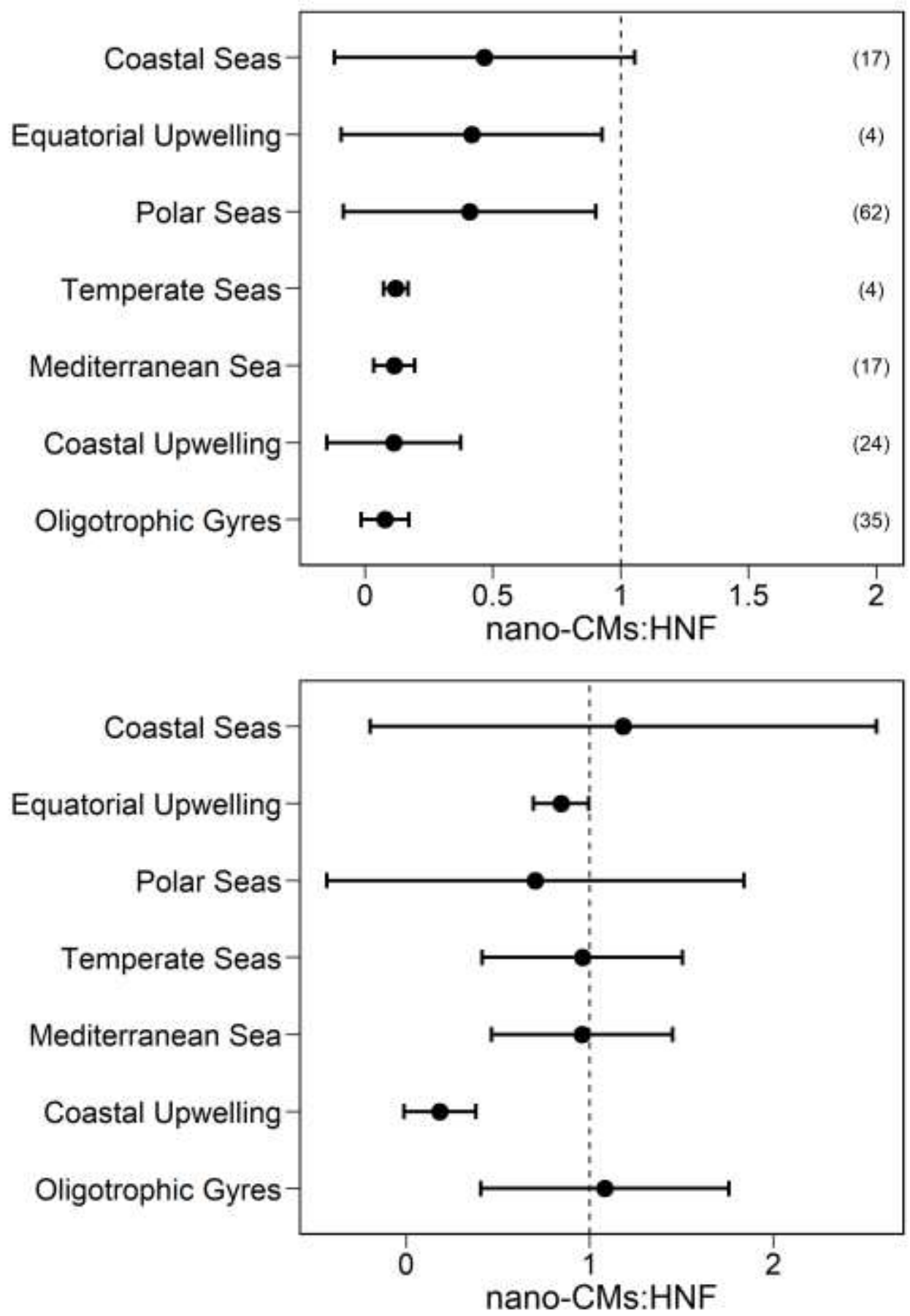

Fig 4.10 Abundance (upper panel) and bacterivory (lower panel) ratios between nanoCMs and strict heterotrophic nanoflagellates (HNF) across the different oceanic biomes. The dashed lines indicate the ratio in which the abundance (or bacterivory impact) of both groups is equal. Data were obtained from 21 studies. Error bars represent standard deviation; number of observations is given on the right side of the upper panel. 
Table 4.8 Post-hoc pairwise comparisons using Dunn's test ( $Z$ scores) for the abundance ratio of nano-CMs and HNF across the oceanic biomes; $\mathrm{CU}$ - Coastal Upwelling.

\begin{tabular}{lllllll}
\hline & $\begin{array}{l}\text { Coastal } \\
\text { Seas } \\
(\mathbf{C S})\end{array}$ & $\begin{array}{l}\text { Equatorial } \\
\text { Upwelling } \\
(\text { EU) }\end{array}$ & $\begin{array}{l}\text { Mediterra- } \\
\text { nean Sea } \\
\text { (MS) }\end{array}$ & $\begin{array}{l}\text { Polar } \\
\text { Oligotrophic } \\
\text { Gyres (OG) }\end{array}$ & $\begin{array}{l}\text { Seas } \\
\text { (PS) }\end{array}$ & $\begin{array}{l}\text { Temperate } \\
\text { Seas (TS) }\end{array}$ \\
\hline EU & 0.16 & & & & & \\
MS & $2.58^{*}$ & 1.43 & & & & \\
OG & $4.79 * *$ & $2.51^{*}$ & 1.79 & & & \\
PS & 0.72 & 0.20 & $-2.51^{*}$ & $-5.76^{* *}$ & & \\
TS & 1.35 & 0.93 & -0.25 & -1.26 & 1.07 & \\
CU & $4.70^{* *}$ & $2.59^{*}$ & 1.91 & 0.28 & $5.37^{* *}$ & 1.37 \\
\hline$* p<0.05, * * p<0.01$ & & & & &
\end{tabular}

Table 4.9 Post-hoc pairwise comparisons using Dunn's test ( $Z$ scores) for the relative contribution of nano-CMs to total nanoflagellate bacterivory across the oceanic biomes; CU - Coastal Upwelling

\begin{tabular}{lllllll}
\hline & $\begin{array}{l}\text { Coastal } \\
\text { Seas } \\
(\mathbf{C S})\end{array}$ & $\begin{array}{l}\text { Equatorial } \\
\text { Upwelling } \\
(\text { EU) }\end{array}$ & $\begin{array}{l}\text { Mediterra- } \\
\text { nean Sea } \\
(\mathbf{M S})\end{array}$ & $\begin{array}{l}\text { Polar } \\
\text { Oligotrophic } \\
\text { Gyres (OG) }\end{array}$ & $\begin{array}{l}\text { Seas } \\
\text { (PS) }\end{array}$ & $\begin{array}{l}\text { Temperate } \\
\text { Seas (TS) }\end{array}$ \\
\hline EU & -0.13 & & & & & \\
MS & -0.31 & -0.09 & & & & \\
OG & -0.38 & -0.14 & -0.07 & & & \\
PS & $2.28 *$ & 1.62 & $2.37 *$ & $2.39 *$ & -1.95 & \\
TS & -0.16 & -0.004 & 0.10 & 0.16 & $2.12^{*}$ & $3.44 * *$ \\
CU & $3.98^{* *}$ & $2.94 * *$ & $3.96 * *$ & $3.94 * *$ & &
\end{tabular}

\subsection{Discussion}

Distribution records derived from online databases revealed strong biases related with size, taxonomy, and oceanic biome in the biogeography of CMs across the global oceans (Figs. 4.1-4.7). This is particularly associated to traditional sampling procedures which fails to properly represent the mixotrophic potential of protist species and misrepresents the smallest members of planktonic communities. Nevertheless, other lines of evidence indicate that CMs were ubiquitous in the global oceans and hence should be considered when evaluating the biogeography and the ecology of plankton in marine systems. 


\subsubsection{Biases associated to the identification of $\mathrm{CM}$ species}

First, to access the global databases, the analysis needed to be based on species name. However, it is truly difficult to define which species are undeniably CMs due to acute methodological problems (Anderson et al., 2017). Only species which have been previously reported to ingest particles (live or dead) were included in the analysis. Evidence of mixotrophy among phytoplankton is mainly obtained through feeding experiments and examining one species at a time can be exhaustive. Despite the recognition that mixotrophy is present among all phytoplankton groups except for diatoms (Flynn et al., 2013; Stoecker et al., 2017), so far evidence of mixotrophy was obtained for fewer than 150 species (Tables 3.1 and 4.1). Thus, the list of protist species used here is likely to be a subset of the true set of CMs present in the oceans. Second, this list is biased towards species and/or groups which have been extensively investigated, for example, because can be more easily cultivated. For instance, from the 78 species included in the analysis, 51 species were dinophytes, even though the importance of mixotrophy is well recognized among other taxonomic groups (Gast et al., 2014; Unrein et al., 2014). Nevertheless, the results presented here represent the first attempt to document the biogeography of CMs.

\subsubsection{Traditional sampling techniques and sampling biases}

Biased sampling methods can give us a distorted picture of plankton composition across the oceans (e.g., Biard et al., 2016). The dataset examined here was strongly biased towards larger cells and/or certain taxonomic groups. This limitation is in part explained by traditional sampling and preservation methods which underestimate the abundance of smaller size fractions or more fragile groups within the plankton (Gifford and Caron, 2000; Edwards et al., 2006). Microscopic counts can underestimate the abundance of smaller size classes due to difficulties associated to their identification (Rose et al., 2004; Rutten et al., 2005). In addition, microscopic analyses usually require the utilization of fixatives, such as Lugol, but preservatives can underestimate the abundance of protists, not only within the microplankton, but also within the nanoplankton due to their aggregation into larger particles or to their disintegration into smaller units (Stoecker et al., 1994; Zarauz and Irigoien, 2008). The CPR is another important example; despite being widely applied to investigate plankton ecology, it is particularly suitable for 
sampling mesozooplankton and larger (> $250 \mu \mathrm{m}$ size) robust phytoplankton due to the mesh size of CPR silks (Edwards et al., 2006; Richardson et al., 2006), neglecting most CMs $<20 \mu \mathrm{m}$. The CPR cannot capture data for the majority of the non-diatom protist species, an exception being very large dinoflagellates such as CMs within the genus Tripos.

Given the changes in our understanding of marine ecology, with recognition of the increasing importance of the microbial loop and mixotrophic protists, the CPR requires augmentation with a device specifically designed for sampling small protists. Alternative methods, such as flow cytometry, not only speed sample processing time but also provide more accurate estimates of smaller size fractions of the plankton (Rose et al., 2004; Rutten et al., 2005). Small protists have been shown to be abundant and to play major role as bacterivorous in the oceans (Zubkov and Tarran, 2008; Hartmann et al., 2013). Furthermore, despite the impression given by Figs. 4.1-4.4 that dinophytes are more widespread than other nano-CMs (as given by presence/absence data), dinophytes were found to be minor contributors to the total abundance and total bacterivory of nano-CMs within oligotrophic oceans (Unrein et al., 2014). The present study showed that global databases failed to recognize the ubiquity of nano-CMs across the global oceans, particularly among non-dinophyte groups and in the open oceans (Fig 4.7).

\subsubsection{Effect of environmental variability in the distribution of nano-CMs}

The results from the meta-analysis revealed that the abundance of nano-CMs increases exponentially towards more productive systems (Fig. 4.8); however, this increase is less evident in deeper waters and absent when evaluating their abundance averaged over the water column. In deeper waters, this is related to the attenuation of light throughout the water column, enhancing light-limitation among phototrophic cells. Similarly, in highly productive systems, the high concentration of cells and organic matter increase light attenuation throughout the water column (Schanz, 1985; McMahon et al., 1992), potentially masking any effect of NPP when integrating the abundance of nanoCMs over depth. Although it is difficult to infer the mechanisms behind the relationship between nano-CMs abundance in the surface vs integrated water column, it is possible that this relationship is also related to the carrying capacity of the system and the main 
factors limiting growth. It can be hypothesized that the carrying capacity of the population in surface waters increases towards more productive systems while the same is not observed when values are integrated over the water column due to light limitation and other ecological interactions (such as competition with HNF) that can act limiting the growth of nano-CMs. It is also noteworthy that bacterial abundance and the interaction between NPP and bacterial abundance were found to be important to determine the abundance of nano-CMs (Table 4.6). The exponential increase in the abundance of nanoCMs with NPP was not observed for bacterial abundances higher than $10^{7}$ cells $\mathrm{mL}^{-1}$ (Fig. 4.9). The combined effects of NPP and prey availability are crucial to better understand the distribution of nano-CMs since these do not need to feed all the time (Stoecker et al., 2017) but these could not be investigated in here.

\subsubsection{Ecological significance of nano-CMs as bacterivores across the oceans}

Here, the ecological significance of nano-CMs as bacterivores was compared to their heterotrophic competitors of same size (HNF). The meta-analysis revealed that nanoCMs are present in lower numbers relative to their counterparts whilst having an important role controlling bacterial populations, not only in oligotrophic seas, but in the global oceans (Fig. 4.10). Overall, the mean abundance of nano-CMs across the different oceanic biomes was half or lower of that of HNF, with higher variability observed within more productive systems (Fig 4.10, upper panel). Despite their lower abundance, nearly half of total nanoflagellate bacterivory was attributed to nano-CMs worldwide (Fig 4.10, lower panel).

Estimates of bacterial ingestion rates by nano-CMs are usually obtained considering the total abundance of plastidic nanoflagellates, i.e., nano-CMs + ANF (e.g., Zubkov and Tarran, 2008). This methodology envisions that, despite their low ingestion rates, nano-CMs can be major bacterivores due to their high abundance in the oceans. However, not all plastidic nanoflagellates are always actively feeding in the oceans (Anderson et al., 2017). When explicitly accounting for the proportion of actively feeding cells it is possible to conclude that, despite the low abundance of nano-CMs, they can act as major bacterivores due to their high ingestion rates (Anderson et al., 2017). Nevertheless, it seems contradictory to assume that mixotrophic nanoflagellates have 
higher ingestion rates compared to their heterotrophic counterparts unless mixotrophic cells were larger in size. A previous study found that three mixotrophic nanoflagellates ingested bacteria at rates equal to or higher than a heterotrophic nanoflagellate (McKieKrisberg et al., 2015) but further investigations are needed to corroborate these mechanisms. Another factor that could explain the low abundance-high bacterivory pattern could be a higher top-down control on nano-CMs compared to HNF because the former could be positively selected by their common predators.

Studies which specifically target nano-CMs also have their own limitations. Quantitative estimates of abundance are challenging because current methods can only account for mixotrophs which were actively feeding by the time of the sampling/experiment (Safi and Hall, 1999; Gast et al., 2014; Sato et al., 2017). Estimates of bacterivory rates are also problematic because are based on the assumptions that bacterivory remains constant over short time scales and that community ingestion rates can be approximated to the average ingestion rate of all feeding individuals (Anderson et al., 2017). In reality, feeding is expected to vary over the diel cycle (Tsai et al., 2009) and only a small proportion of the total assemblage of CMs will be actively feeding by the time of the experiment (Christaki et al., 1999; Anderson et al., 2017). Furthermore, these studies usually group all nano-CMs in a single group, without providing taxonomic information (Gast et al., 2014; Unrein et al., 2014). Thus, while different mixotrophic communities were expected across the different studies analysed here due to their different spatio-temporal scales, it was not possible to fully explore this aspect due to a knowledge gap.

\subsubsection{Microbial biogeography and the advent of molecular data}

Recent advances in metagenomics and computational analysis allow the identification of the taxonomic and genomic content of marine communities and the investigation of their functional potential (Sunagawa et al., 2015). Nevertheless, such datasets could not be included in the present analysis. While sequence datasets offer a powerful approach to provide molecular taxonomy in the future, protistologists likely have decades of work ahead of them to match sequence data against morphology/physiology (Caron and $\mathrm{Hu}, 2019$ ). For the sequence datasets, one of the most problematic issues is 
converting the data into species information (Bucklin et al., 2016; Leray \& Knowlton, 2016; Caron and $\mathrm{Hu}, 2019)$. Within the arena of molecular analysis itself, there are different methodologies for obtaining sequence information, so that forming operational taxonomic units (OTUs) from those data are far from standardized (e.g., Callahan et al., 2017).

In addition, most mixotrophic protists fall into often closely-related groups that are composed of a mixture of species with different nutritional modes, i.e., autotrophic, heterotrophic, and mixotrophic. Although metagenomics may offer a promising pathway to identify mixotrophs (e.g. Rokitta et al., 2011; Yelton et al., 2016), it remains to be fully resolved how to use the potential of metagenomics to differentiate mixotrophs from their auto- or hetero- trophic counterparts (Santoferrara and McManus, 2017). Further, OTUs far outnumber the number of morphologically-described or nutritionally-described species that have been sequenced (Caron and $\mathrm{Hu}, 2019$ ); there is presently no way of differentiating the mixotrophs among the many OTUs typically recorded in molecular datasets. Meanwhile, experimental studies present increasing evidence that organisms that we used to view as strict-phytoplankton are in fact mixotrophs (e.g., Hoppenrath and Leander, 2007; Berge et al., 2008a; Nézan and Chomérat, 2009; Yoo et al., 2010; Kang et al., 2011; Lim et al., 2015; Jang et al., 2017; Ok et al., 2017). 


\subsection{Summary}

CMs were found to be ubiquitous in the global oceans; however, global databases were strongly biased by size, taxonomy, and biome. Distribution records were biased towards species larger than $20 \mu \mathrm{m}$, which comprised mainly dinophytes. While global databases recorded the distribution of nano-CMs limited to coastal regions and to temperate and polar seas, studies which specifically targeted these organisms revealed their presence to be much wider, including equatorial regions and oligotrophic gyres. Such biases likely have occurred due to a failure to capture and correctly identify these organisms in routine sampling protocols. The meta-analysis showed that the abundance of nano-CMs tends to increase exponentially with NPP from surface waters to the deep chlorophyll maximum; however, this relationship depends on bacteria availability and is weakened throughout the water column. Finally, despite the overall lower abundance compared to their heterotrophic counterparts, nano-CMs contributed to nearly half of total nanoflagellate bacterivory within different oceanic biomes.

\section{Acknowledgements}

I would like to acknowledge Ruth Anderson, Mitsuhide Sato, Cristian Vargas, Manuela Hartmann, An-Yi Tsai, Michael Stukel, and Julie Hall which gently provided clarifications and/or supplementary data to be used in the meta-analysis. 


\section{Chapter 5}

\section{Methods - Marine ecosystem modelling}




\subsection{Introduction}

The impact of mixotrophy within the lower trophic levels of marine food webs was investigated through ecosystem modelling. This chapter describes the general methods applied to build the modelling frameworks used in Chapters 6 and 7. In brief, a flexible protist model that enables the representation of different planktonic groups, from strict autotrophs to strict heterotrophs, was incorporated into ERSEM. Model equations and parameters descriptions are given in full in Appendix C. Parameterization and constant values applied to the different modelling experiments are given in Chapters 6 and 7 , respectively. The modelling experiments were designed to compare two plankton food webs that differ only in the presence of mixotrophy. In Chapter 6 , the food webs were simulated through chemostat-like modelling experiments assuming a constant dilution rate. Sensitivity analyses were performed to evaluate how selected model outputs are affected by the choice of parameter values and model initial conditions. In Chapter 7 , the food web models were simulated using a more complex representation of the physical environment and validated against biogeochemical observations. For this purpose, a onedimensional model representing the vertical structure of the water column was applied at one specific site (L4 station) located in the Western English Channel. Observations obtained at L4 station (Chapter 7.2.2) were used to investigate the succession of protist trophic strategies over the seasonal cycle and throughout the water column.

\subsection{The biological model}

\subsubsection{The plankton food webs}

Two plankton food webs (named here as 'non-mixotrophic' and 'mixotrophic' food webs) that differ only in the presence of mixotrophy were designed to perform the modelling experiments (Fig. 5.1). The community structure of the non-mixotrophic food web was defined following the conceptual framework of ERSEM (Baretta-Bekker et al., 1995; Butenschön et al., 2016). This non-mixotrophic food web comprised eight functional groups that differ mainly in size and trophic strategy (Fig. 5.1): four phototrophs (picophytoplankton, nanoflagellates, microflagellates, and diatoms), three predators (nanoflagellates, microzooplankton, and mesozooplankton), and one decomposer (heterotrophic bacteria). Here, heterotrophic nanoflagellates feed on pico- 
and nano- sized prey, microzooplankton feed on pico-, nano-, and micro- sized prey, and mesozooplankton feed on nano- and micro- sized prey (Fig. 5.1). Intraguild predation was allowed among all predators due to its importance in plankton trophodynamics (e.g., Hansen et al., 1994; Jeong et al., 2010b). Feeding processes are described by Eqs. 42-57 (Appendix C).

In the mixotrophic food web, nanoflagellates and microflagellates (previously perceived as strict autotrophs) were allowed to feed on diverse prey items, as supported by evidence from the literature (e.g., Zubkov and Tarran, 2008; Jeong et al., 2010; Hansen, 2011; Unrein et al., 2014). These constitutive mixotrophs are called hereafter as nanoCMs and micro-CMs, respectively. They can access the same prey as their heterotrophic competitors of same size (heterotrophic nanoflagellates and microzooplankton, respectively; Fig. 5.1 and Table 5.1). One might argue that not all species of phototrophic nanoflagellates and microflagellates are mixotrophic; however, the main objective here was to contrast the traditional modelling approach, which consider all species to be strictly phototrophic, with the mixotrophic paradigm that consider most species to be mixotrophic (Flynn et al., 2013). This comparison can be viewed as a baseline to investigate the impact of mixotrophy on trophodynamics. Notwithstanding, the framework used here considers competition between mixotrophic groups and strict autotrophs, which are represented by diatoms and picophytoplankton in the model.

In turn, the microzooplankton group was divided into strict heterotrophic species and NCMs in the mixotrophic model; estimates from Chapter 3 suggest that $40-60 \%$ of total microzooplankton can acquire phototrophic potential (Leles et al., 2017). They share the same prey items and were assumed not to feed on each other (Fig. 5.1). While the latter is not expected to hold for real systems, this assumption was necessary to allow their persistence in the model, as revealed by initial numerical experiments. Therefore, the model accounts for competition for prey among microzooplankton but ignores the fact that they can have a negative impact on each other through predation. Among NCMs, the conceptual framework included GNCMs, such as oligotrich ciliates, which have lower control over the acquired phototrophic machinery but can obtain it from diverse prey items, and SNCMs, such as Mesodinium rubrum, which have higher control over the 
acquired phototrophic machinery but rely on specific prey (Mitra et al., 2016). SNCMs must obtain the phototrophic potential by feeding on nano-CMs, while GNCMs can also obtain it feeding on micro-CMs (Stoecker et al., 1988-1989; Gustafson et al., 2000; Johnson et al., 2007; McManus et al., 2012). Intraguild predation was allowed within each mixotroph functional type (Fig. 5.1; references within Table 5.1). The model did not include eSCNMs because their physiology is largely unknown (Biard et al., 2016) and the model lacks an appropriate description of endosymbiosis.

The model resolves the major chemical elements in the ocean, i.e., carbon, nitrogen, phosphorus, and silicate, both in organic and inorganic forms (Chapter 5.2.2), accounting for variable stoichiometry within plankton groups (except for within mesozooplankton where $\mathrm{C}: \mathrm{N}: \mathrm{P}$ was held constant). Protist functional groups were described by a general plankton model that develops from the previous work by Flynn and Mitra (2009) and Mitra et al. (2016); details are given in Chapter 5.2.3. Nutrient pools and the bacteria and mesozooplankton submodels correspond to those of ERSEM (Butenschön et al., 2016) and are described further below (Chapter 5.2.4 and 5.2.5). Overall, plankton growth dynamics result from the balance of gains through uptake of nutrients and assimilation into organic compounds and losses through respiration, excretion (nonassimilated material) and/or release of excess of nutrients (linked to stoichiometric regulation), predation, and non-predatory mortality (e.g., viral lysis) (Appendix C, Eqs. 1-6). All state variables have units of element concentration (e.g., $\mathrm{mg} \mathrm{C} \mathrm{m}^{-3}$ ). 


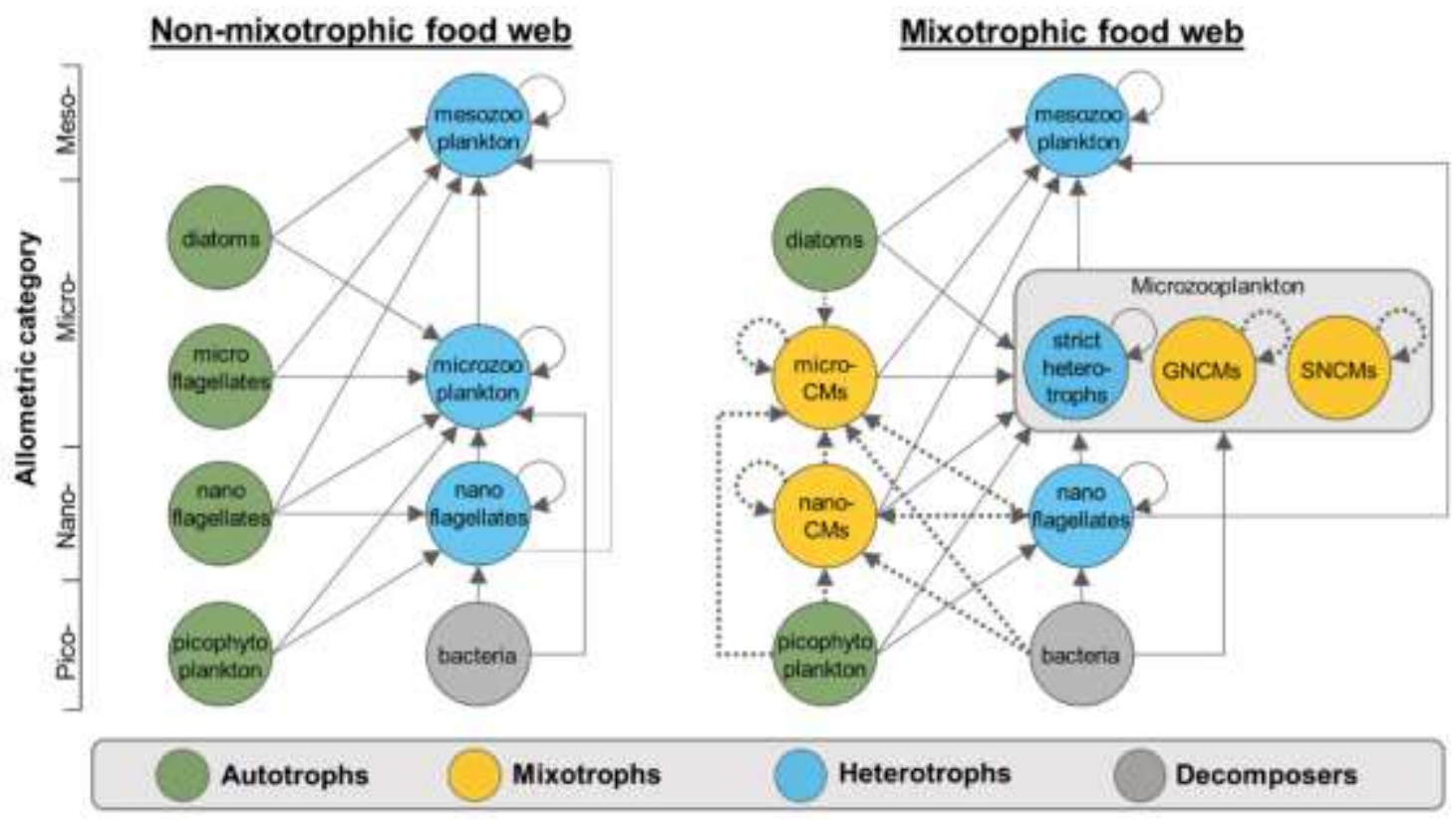

Figure 5.1 Conceptual frameworks of the plankton food webs used on Chapters 6 and 7. Food webs differ only in the presence of mixotrophs. Arrows indicate trophic interactions; dotted arrows correspond to new interactions associated to mixotrophy. Please refer to the Abbreviations section for acronyms. 
Table 5.1 Minimum $\left(S_{\min }\right)$, maximum $\left(S_{\max }\right)$, and optimum prey sizes $\left(S_{\text {opt }}\right)$ in $\mu \mathrm{m}$ ESD for different mixotrophic species (data compiled from the literature). Mixotroph size is also given in $\mu \mathrm{m}$ ESD. These values were used to guide the maximum (potential) prey size spectrum among the different mixotrophic functional types included in the model.

\begin{tabular}{|c|c|c|c|c|c|c|}
\hline Group & Mixotrophic species & Size & $\mathbf{S}_{\max }$ & $\mathbf{S}_{\min }$ & $\mathbf{S}_{\text {opt }}$ & Ref. \\
\hline nano-CMs & Yihiella yeosuensis & 8.0 & nd & nd & 5.6 & 1 \\
\hline nano-CMs & Natural populations of dinoflagellates & 15.8 & 6.7 & 3.4 & nd & 2 \\
\hline nano-CMs & Natural populations of flagellates & 6.1 & nd & 0.9 & nd & 2 \\
\hline nano-CMs & Teleaulax amphioxeia & 5.6 & 1.0 & 0.9 & nd & 3 \\
\hline nano-CMs & Karlodinium veneficum & 10.0 & 20.0 & 4.3 & 6.5 & $4-7$ \\
\hline nano-CMs & Ansanella granifera & 10.5 & 5.6 & 0.9 & 5.6 & 8 \\
\hline nano-CMs & Gymnodinium smaydae & 20.0 & 22.8 & 4.8 & 5.8 & 9 \\
\hline nano-CMs & Symbiodinium voratum & 11.1 & 11.5 & 0.5 & n.d & 10 \\
\hline nano-CMs & Paragymnodinium shiwhaense & 12.4 & 11.0 & 4.8 & 9.7 & 11 \\
\hline nano-CMs & Prorocentrum donghaiense & 13.3 & 12.1 & 1.0 & 5.6 & $12-13$ \\
\hline nano-CMs & Biecheleria cincta & 8.5 & 12.6 & 4.8 & 11.5 & 14 \\
\hline nano-CMs & Heterocapsa triquetra & 15.0 & 12.1 & 1.0 & nd & $12-13$ \\
\hline nano-CMs & Heterocapsa rotundata & 5.8 & 5.0 & 1.0 & nd & 15 \\
\hline nano-CMs & Heterosigma akashiwo & 11.0 & nd & 1.0 & nd & $15-17$ \\
\hline nano-CMs & Prorocentrum minimum & 12.1 & 11.0 & 1.0 & nd & 15 \\
\hline micro-CMs & Alexandrium minutum & 16.7 & nd & 1.0 & nd & 15 \\
\hline micro-CMs & Gymnodinium impudicum & 17.8 & 11.0 & 1.0 & nd & 15 \\
\hline micro-CMs & Gymnodinium aureolum & 19.4 & 11.5 & 0.9 & nd & 18 \\
\hline micro-CMs & Karlodinium armiger & 15.0 & 31.4 & 5.6 & 13.0 & $19-20$ \\
\hline micro-CMs & Karenia brevis & 20.3 & nd & 1.0 & nd & 15 \\
\hline micro-CMs & Fibrocapsa japonica & 20.5 & 1.2 & 0.2 & nd & 21 \\
\hline micro-CMs & Scrippsiella trochoidea & 22.8 & 12.1 & 1.0 & nd & 15 \\
\hline micro-CMs & Cochlodinium polykrikoides & 25.9 & 11.0 & 4.8 & 5.6 & 22 \\
\hline micro-CMs & Prorocentrum micans & 26.6 & 15.0 & 1.0 & nd & $12-13$ \\
\hline micro-CMs & Takayama helix & 27.4 & 67.4 & 15.0 & 31.2 & 23 \\
\hline micro-CMs & Alexandrium tamarense & 28.1 & 12.1 & 1.0 & nd & 15 \\
\hline micro-CMs & Akashiwo sanguinea & 30.8 & 15.0 & 1.0 & nd & 15 \\
\hline micro-CMs & Karenia mikimotoi & 30.0 & 4.9 & 0.5 & nd & 24 \\
\hline micro-CMs & Gonyaulax polygramma & 25.0 & 17.0 & 4.8 & 5.6 & $12-13$ \\
\hline micro-CMs & Alexandrium catenella & 32.6 & nd & 1.0 & nd & 15 \\
\hline micro-CMs & Chattonella ovata/subsalsa & 40.0 & nd & 1.0 & nd & $15 ; 25$ \\
\hline micro-CMs & Lingulodinium polyedrum & 38.2 & 28.1 & 1.0 & nd & $12-13$ \\
\hline micro-CMs & Fragilidium subglobosum & 50.0 & 40.0 & 24.2 & nd & 26 \\
\hline micro-CMs & Fragilidium mexicanum & 54.5 & 37.9 & 12.9 & 37.9 & 27 \\
\hline
\end{tabular}


Table 5.1 continued

\begin{tabular}{lllllll} 
SNCMs & Dinophysis acuminata & 25.0 & nd & nd & 17.0 & 28 \\
SNCMs & Mesodinium rubrum & 21.9 & 5.3 & 1.0 & 4.2 & $29-31$ \\
SNCMs & Pfiesteria piscicida & 13.5 & 27.4 & nd & nd & 32 \\
SNCMs & Fragilidium duplocampanaeforme & 38.6 & 52.0 & 31.2 & nd & 33 \\
SNCMs & Gymnodinium graciletum & 9.6 & 10.3 & 6.6 & 8.5 & 34 \\
SNCMs & Amylax triacantha & 27.5 & 20.5 & nd & nd & 35 \\
GNCMs & Strombidium reticulatum & 41.0 & 7.0 & 1.5 & 7.0 & 36 \\
GNCMs & Strombidium vestitum & 25.0 & 6.0 & 1.5 & 2.0 & 36 \\
GNCMs & Laboea strobila & 62.0 & 9.7 & 1.1 & 2.9 & 37 \\
GNCMs & Strombidium conicum & 49.0 & nd & nd & 5.4 & 37 \\
GNCMs & Lohmaniella oviformis & 25.0 & 11.2 & 1.4 & 5.6 & 38 \\
GNCMs & Strombidium sp. & 20.0 & 5.6 & 1.4 & 2.8 & 38 \\
GNCMs & Strombidium sp. & 25.0 & 9.8 & 1.4 & 5.6 & 38 \\
GNCMs & Strombidium conicum & 40.0 & 25.2 & 2.8 & 4.2 & 38 \\
\hline
\end{tabular}

${ }^{1}$ Jang et al., 2017; ${ }^{2}$ Havskum and Hansen, $1997 ;{ }^{3}$ Yoo et al., 2017; ${ }^{4} \mathrm{Li}$ et al., $1999 ;{ }^{5} \mathrm{Place}$ et al., 2012; ${ }^{6}$ Adolf et al., 2006; ${ }^{7}$ Calbet et al., 2011; ${ }^{8}$ Lee et al., 2014b; ${ }^{9}$ Lee et al., 2014a; $;{ }^{10}$ Jeong et al., 2012; ${ }^{11}$ Yoo et al., 2010b; ${ }^{12}$ Jeong et al., 2005a; ${ }^{13}$ Jeong et al., 2005b; ${ }^{14} \mathrm{Kang}$ et al., 2011; ${ }^{15}$ Jeong et al., 2010b; ${ }^{16}$ Seong et al., 2006; ${ }^{17}$ Nygaard and Tobiesen, $1993 ;{ }^{18}$ Jeong et al., 2010a; ${ }^{19}$ Berge et al., 2008a; ${ }^{20}$ Berge et al., 2008b; ${ }^{21}$ Jeong et al., 2011; ${ }^{22}$ Jeong et al., 2004; ${ }^{23}$ Jeong et al., 2016; ${ }^{24}$ Zhang et al., 2011; ${ }^{25}$ Jeong et al., 2010b; ${ }^{26} \mathrm{Hansen}$ and Nielsen, 1997; ${ }^{27} \mathrm{Jeong}$ et al., 1999; ${ }^{28} \mathrm{Kim}$ et al., 2008a; ${ }^{29}$ Yih et al., 2004; ${ }^{30}$ Myung et al., 2006; ${ }^{31}$ Yoo et al., 2015; ${ }^{32}$ Ok et al., 2017; ${ }^{33}$ Park and Kim, 2010; ${ }^{34}$ Jakobsen et al., 2000; ${ }^{35}$ Park et al., 2013; ${ }^{36}$ Jonsson, 1986; ${ }^{37}$ Jonsson, 1987; ${ }^{38}$ Kivi and Setala, 1995 


\subsubsection{Nutrients, dissolved, and particulate organic matter}

Nutrient pools (Appendix C, Eqs. 159-169) were divided between inorganics (nitrate, ammonium, phosphate, silicate, dissolved inorganic carbon), dissolved organic matter (DOM), and detrital particulate organic matter (POM). DOM was divided between labile and semi-labile assuming that the former is rapidly consumed by bacteria and that the latter is more resistant to microbial degradation (Hansell, 2013). Detrital POM was divided in three size-classes assuming that mesozooplankton can scavenge only on the medium size fraction. The chemical and the biological components of the food web model interact through the uptake of inorganics and the formation and recycling of organics, as described below for protists, bacteria, and mesozooplankton.

\subsubsection{Protists}

The main modifications and/or additions applied to the protist model with respect to Flynn and Mitra (2009) are described below; equations can be found in Appendix C. The model was developed in four main aspects:

i) The uptake of silicon (Eqs. 26-32) was implemented to simulate diatoms following Flynn (2006). The representation of diatoms differs from that of other phototrophic protists due to their physiological requirement for silicon to build their frustules. In turn, silicon uptake differs fundamentally from that of nitrogen and phosphorus because the external nutrient concentration, instead of the internal (nutrient quota) concentration, ultimately affects growth. Consequently, quota models are inappropriate for silicon (Si) dynamics; any factor limiting growth other than $\mathrm{Si}$ in the model leads to enhanced accumulation of Si (Flynn and Martin-Jézéquel, 2000).

ii) The allometric description of predation (Eqs. 44-51) was implemented as described by Flynn and Mitra (2016). This formulation simulates the kinetics of prey capture and ingestion relating prey abundance and encounter rates to a prey-selection function controlled by satiation. In the model, prey selection is controlled by the total prey size spectrum accessible by the predator and its optimal prey size; capture is then minimum on both extremes of the prey size spectrum increasing linearly towards the optimal prey size (Flynn, 2018). This approach is very similar to the Gaussian predation kernel (defined by a normal distribution), but it has the additional benefit of being defined directly by the observable lower and upper prey size limits. 
iii) Acquired phototrophy (Eq. 41) was modified so that kleptochloroplasts are not digested but lost over time at a constant rate (e.g., Schoener and McManus, 2012).

iv) All model equations (Eqs. 1-6) were modified so that state variables were expressed in units of element quantity per water volume (instead of nutrient ratios relative to the core biomass of the protist as in the base model by Flynn and Mitra) to allow model coupling with ERSEM submodels.

The general protist model has the potential to simulate any protist from strict autotrophs to strict heterotrophs, including CMs and NCMs. The uptake of inorganics (Eqs. 8-32), photoacclimation (Eqs. 80-83), prey ingestion (Eqs. 42-57), and acquired phototrophy (Eqs. 38 and 41) can be enabled/disabled accordingly.

In the food webs (Fig. 5.1), strict autotrophs can photoacclimate through the synthesis of chlorophyll (Eqs. 80-83), take up ammonium, nitrate, phosphorus, and silicon if diatoms (Eqs. 8-32), release labile DOC during photosynthesis and due to nonpredatory mortality (Eq. 164), and release dissolved inorganic carbon (DIC) and excess of inorganic nutrients (ammonium and phosphate) as part of respiration and stoichiometric regulation, respectively (Eqs. 97-112) (Fig. 5.2). Parameter values controlling light harvesting and nutrient uptake defined the differences between strict autotrophs in the model (Sathyendranath et al., 2009; Bouman et al., 2018). On top of these, CMs can engage in phagotrophy (Eqs. 42-57), re-assimilate inorganic nutrients released by breaking down their prey (Eqs. 97-112), and excrete the non-assimilated material as labile DOM (Eq. 164) (Fig. 5.2). CMs must acquire a critical proportion of growth through photosynthesis and phagotrophy can be down-regulated if enough carbon is provided through phototrophy (Hansen, 2011) (Eqs. 58-72). The model assumed that the internal re-assimilation of nutrients depends on the stoichiometric status of the mixotroph $(\mathrm{N}$ or $\mathrm{P}$ stress). It was also assumed that $\mathrm{CMs}$ have lower maximum growth rates $\left(\mu_{\max }\right)$ compared to their heterotrophic competitors (Fischer et al., 2017). In addition to the differences related to light harvesting and nutrient uptake, nano-CMs and micro-CMs differ in their predation impact, with the former selecting pico-sized prey and having a narrower prey size spectrum and the latter selecting for nano-sized prey (Table 5.1). 
Strict heterotrophs assimilate organics through predation (Eqs. 42-57) and release labile DOM due to non-assimilated material and non-predatory mortality (Eq. 164) and DIC through respiration (Eq. 163) (Fig. 5.2). The same processes were applied to NCMs, but these can fix inorganic carbon through acquired phototrophy (but do not photoacclimate; Eqs. 38 and 41), take up external inorganic nutrients (only SNCMs; Eqs. 8-25), and re-assimilate inorganic nutrients internally (Eqs. 97-112) (Fig. 5.2). Heterotrophic nanoflagellates select pico-size prey and have a narrower prey size spectrum while microzooplankton and NCMs select for nano-sized prey. NCMs were assumed to have the same $\mu_{\max }$ as their heterotrophic counterparts, to select autotrophic prey, and to be positively selected by mesozooplankton compared to strict heterotrophic microzooplankton (Dolan and Pérez, 2000; Broglio et al., 2004; Figueiredo et al., 2007; Schoener and McManus, 2017). Among NCMs, GNCMs retain chloroplasts from their prey while SNCMs can also retain other cellular components and largely rely on photosynthesis to obtain carbon; thus, SNCMs were assumed to rely on photosynthesis for a critical proportion of growth while GNCMs were not (Stoecker et al., 2009). In addition, SNCMs have better control over the acquired phototrophic machinery compared to GNCMs (Stoecker et al., 2009); thus, the model assumes lower loss rate of kleptochloroplasts for the former. 

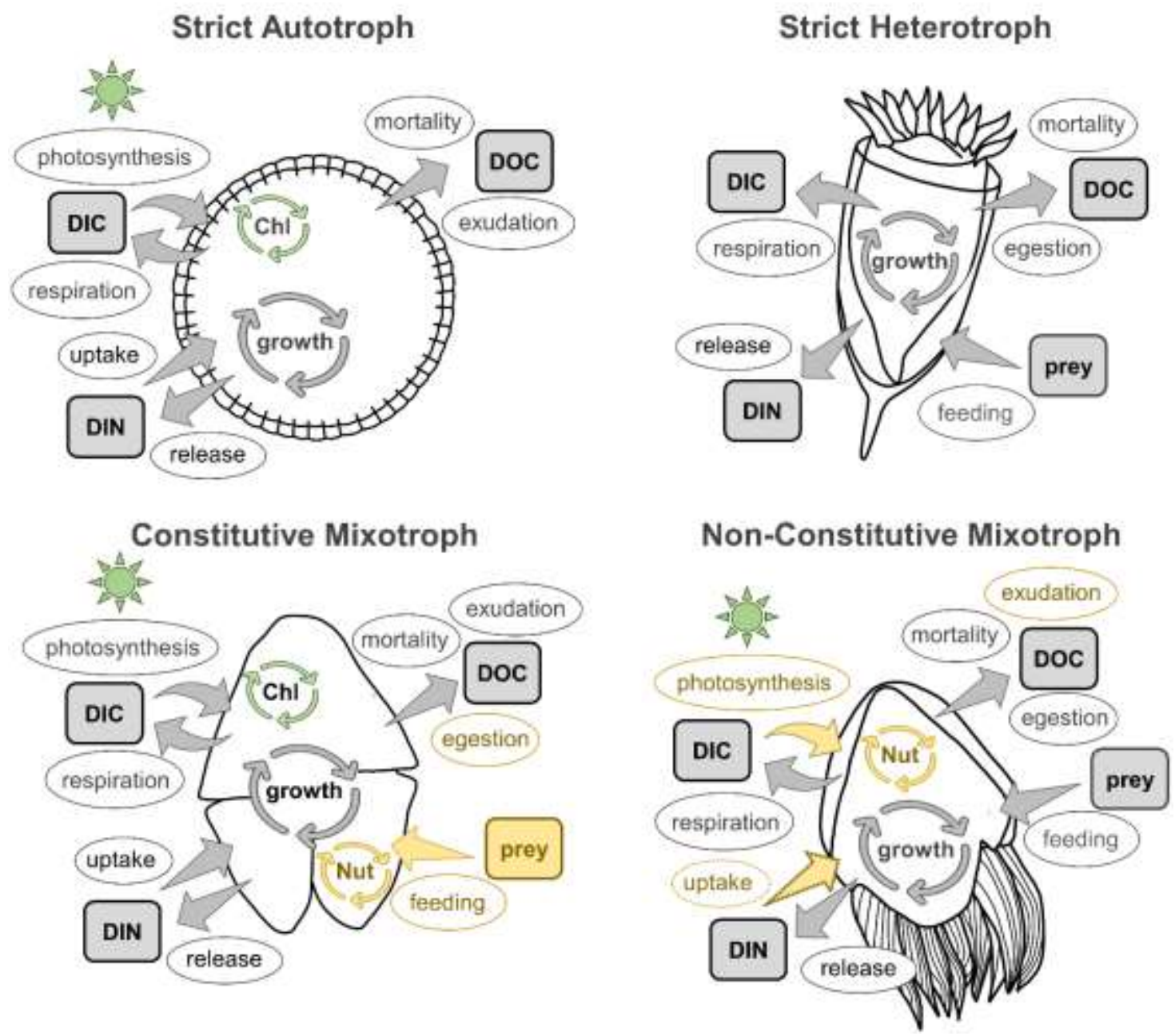

Figure 5.2 Schematics illustrating the processes influencing four main protist functional types (strict autotrophs, strict heterotrophs, constitutive mixotrophs-CMs, and nonconstitutive mixotrophs-NCMs) in the plankton food web model. Highlighted in yellow are the additional features to the strict autotroph or to the strict heterotroph to represent $\mathrm{CMs}$ or NCMs, respectively. Arrows and circles denote the processes and rectangular boxes the pools of dissolved inorganic carbon (DIC), dissolved inorganic nutrients (DIN), dissolved organic carbon (DOC), and prey. The recycling symbols denote growth, chlorophyll synthesis (Chl), and internal re-assimilation of inorganic nutrients (Nut). NCMs cannot photoacclimate, acquired chloroplasts are diluted over time, and the uptake of DIN is enabled only when simulating specialist forms. 


\subsubsection{Bacteria}

Following ERSEM, bacteria (Eqs. 116-118; Fig. 1.1) were assumed to consume all forms of particulate and dissolved organic matter and to take up or release inorganic nutrients depending on the quality (i.e., $\mathrm{N}$ and $\mathrm{P}$ relative content) of the organic matter (Eqs. 119-128); parameter values are given within Chapters 6 and 7. Bacteria thus compete with phytoplankton for inorganic nutrients when organic substrates are nutrient depleted. Bacteria were assumed to release any carbon in excess to their physiological requirement (which is regulated by an 'optimal' cellular carbon to nutrient ratio) as semilabile DOC (Eq. 167). Recalcitrant DOC was also produced by the release of capsular material (Stoderegger and Herndl, 1998) which was assumed to be a fixed proportion of the carbon uptake (Eq. 167). Overall these two fluxes imply that bacteria (especially when feeding on carbon-rich substrates) change the quality of DOM, increasing the proportion of recalcitrant DOC with respect to the labile forms. This mechanism is consistent with the microbial carbon pump concept (Jiao et al., 2010; Polimene et al., 2017).

\subsubsection{Mesozooplankton}

The mesozooplankton model (Eq. 138-158; Fig. 1.1) assumes a fixed internal nutrient to carbon ratio and the ability to scavenge on particulate organic matter (Butenschön et al., 2016); parameter values are given within Chapters 6 and 7. The predation function from ERSEM was modified according to Flynn and Mitra (2016) to be consistent with that used in the protist model but through a simpler description; clearance rate is prey specific and was defined by the biomass of prey multiplied by the slope of the relationship between the abundance of prey and capture (Eqs. 139-143). Prey preference was based on size and depends on functional type. Mesozooplankton release excess nutrients as ammonium and phosphate and contribute both to the pool of dissolved and particulates through mortality and excretion, e.g., faecal pellets (Eqs. 144-158).

\subsection{The physical model}

The plankton food webs were first simulated through a zero-dimensional model, which considers time variation but neglects any spatial variation (Chapter 6). It is a good modelling practice to investigate model behaviour through zero-dimensional models before adding further complexities to the physical environment (Hearn, 2008). This 
approach is robust once the 'boxes' represent systems which are physically distinct, and which internal processes are more relevant than the interactions between the systems (Hearn, 2008). Here, the zero-dimensional model is akin to a chemostat modelling experiment. This set up implies that plankton biomass is homogeneous within a 'box' subjected to a constant dilution rate, which acts bringing inorganic nutrients and washing out residual nutrients and other dissolved and particulate organics (including plankton) from the system. This construct is thus akin to a mixed layer environment which is subjected to an input of nutrients from a steady deeper layer, i.e., which does not accumulate properties over time (Fasham et al., 1990). Mesozooplankton are not subjected to dilution because they are assumed to actively keep themselves within the mixed layer. Specific configurations applied to the zero-dimensional model are given in Chapter 6.2.2.

To investigate the seasonal succession of protists, the plankton food webs were coupled to a 1D physical model of the water column (Chapter 7). One-dimensional models provide a platform to account for vertical mixing within biogeochemical models where lateral gradients can be neglected or prescribed. In addition, this approach allows the incorporation of realistic environmental variability (i.e., temperature, light, and mixing). Here, the General Ocean Turbulence Model (GOTM) was used (Burchard et al., 1999). GOTM computes solutions for the 1D version of the transport equations of momentum, salt, and heat. A key feature of GOTM is the vast number of well-tested turbulence models that have been implemented in the code and the extensive and comprehensive documentation available in the literature (Burchard et al., 1999). In fact, the first developments of GOTM started back in the 1990s. Since then, GOTM has been applied to investigate air-sea fluxes, surface and mixed-layer dynamics, stratification processes in shelf seas, and many other processes. This model has been applied in diverse oceanic regimes within the Atlantic, Pacific, and Indian Oceans, and in enclosed seas such as the North Sea and Baltic Sea (Allen et al., 2004; Holt and Umlauf, 2008; Drushka et al., 2016; Leeuwen et al., 2016; Karagali et al., 2017).

Here, the k- $\varepsilon$ model was used to describe turbulence which is controlled by the transfer of heat and momentum across the ocean surface and by tidal current (https://gotm.net). This model was chosen because it is derived from hydrodynamical 
equations, being able to simulate transport processes quite accurately with a high temporal resolution and being ideal for investigations of transport within the mixed layer (Goudsmit et al., 2002). When coupled to a biogeochemical model, GOTM is responsible for computing mixing intensity (or vertical diffusivity) and apply it to all pelagic biogeochemical state variables. The generic partial differential equation that governs a single tracer (T) within the biogeochemical model is described below (Eq. 5.1).

$$
\frac{\partial}{\partial \mathrm{z}} \mathrm{T}=\frac{\partial}{\partial \mathrm{z}}\left(\mathrm{K}_{\mathrm{z}} \frac{\partial}{\partial \mathrm{z}} \mathrm{T}\right)-\frac{\partial}{\partial \mathrm{z}}(\mathrm{wT})+\mathrm{f}
$$

in which $\mathrm{K}_{\mathrm{z}}$ is the vertical diffusivity $\left(\mathrm{m}^{2} \mathrm{~s}^{-1}\right)$, w is the sinking velocity $\left(\mathrm{m} \mathrm{s}^{-1}\right)$, and $\mathrm{f}$ is the net result of all local sources and sinks. For specific configurations applied to GOTM, please refer to Chapter 7.2.1.

\subsection{Model coupling, code accessibility, and other tools}

The plankton food web model was implemented in the open source Fortran-based Framework for Aquatic Biogeochemical Models (FABM), an open platform (freely available at http://fabm.net) through which different models or sub-models may be coupled in a single framework (Fig. 5.3; Bruggeman and Bolding, 2014). A key feature of this framework is to represent the hydrodynamic and the biogeochemical models in separated modules, with FABM in between; this allows to swap one or the other without affecting other parts of the coupled model (Fig. 5.3). The hydrodynamic model stores the physical variables and handles advection, diffusion, time integration and input/output, while the biogeochemical model provides the names and units of nutrient pools and biological variables as well as sink and source terms (Fig. 5.3). Since most of hydrodynamic models are written in Fortran, FABM is Fortran-based requiring all models to be written in Fortran to allow the coupling. 
ERSEM and GOTM are written in Fortran 2008 and are distributed under the open-source GNU Lesser General Public License through a gitlab server, being freely available upon registration through the http://www.shelfseasmodelling.org website. Detailed guidelines on how to obtain FABM, ERSEM, and GOTM are given in https://gitlab.ecosystem-modelling.pml.ac.uk/edge/ersem. In order to build and install these, it is necessary to have CMake 2.8.8 or later installed, a supported Fortran 2003 compiler, and a version of the NetCDF library. FABM and the NetCDF library should be compiled using the same compiler; here, the gfortran compiler was used. The released versions used in this thesis are as following: ERSEM (16.06), GOTM (v5.3), and FABM (v0.95.3).

The original modelling work describing the general protist model (Flynn and Mitra, 2009) was implemented in PowerSim Constructor v 2.51 (Isdalst $\varnothing$, Norway); thus, it was necessary to develop for this thesis a version of this model in Fortran through FABM to allow model coupling. The specific guidelines used to create the protist model through FABM can be found online at http://fabm.net in the sub-section 'Developing a new biogeochemical model'. The model requires two external dependencies, i.e., temperature and PAR, and several external state variables (such as nutrient pools and prey concentrations). Since the units of the protist model are not the same used by FABM, care was taken to convert the units of external variables, returned in $\mathrm{mmol} \mathrm{X} \mathrm{m}^{-3}$, to $\mathrm{mg} \mathrm{X} \mathrm{m}^{-}$ ${ }^{3}$, in which $\mathrm{X}$ can be $\mathrm{C}, \mathrm{N}, \mathrm{P}$, or Si. Similarly, units were converted back once setting the fluxes used to compute the ordinary differential equations of the external state variables.

Model output processing and graphical visualization were performed in R making use of several packages, such as: 'netcdf4', 'plyr', 'dplyr', 'zoo', 'ggplot2', and 'gridExtra'. 


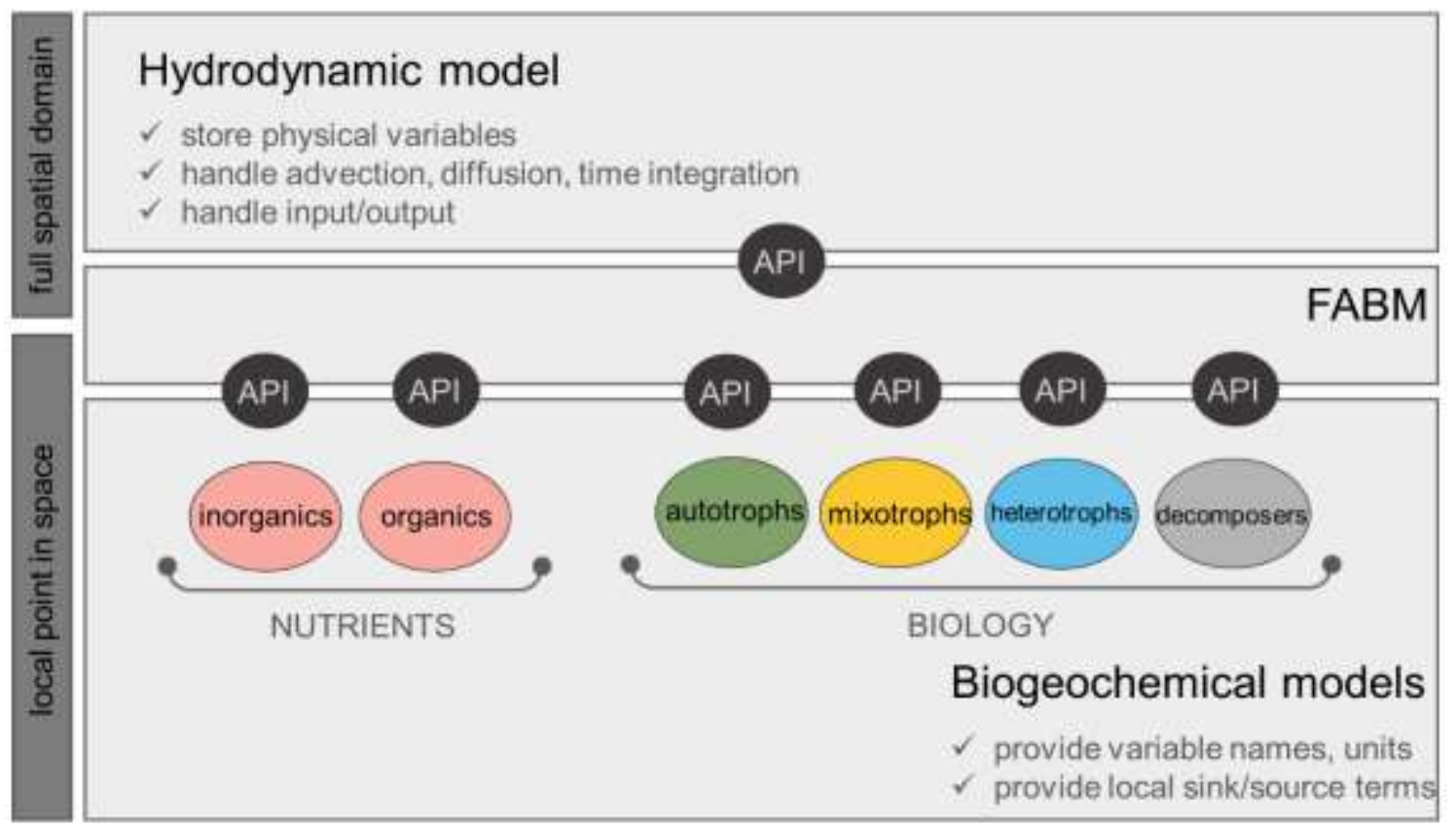

Figure 5.3 FABM provides an interface between the hydrodynamic and the biogeochemical models. API - application programming interfaces. Schematic based on Fig. 1 by Bruggeman and Bolding (2014).

\subsection{Sensitivity analysis}

Sensitivity analysis provides a measure of robustness of model assumptions, helps to identify elements within the model that require further measurements or investigations, and indicates if certain structures of the model can be simplified or need fixing. It can be defined as "The study of how uncertainty in the output of a model (numerical or otherwise) can be apportioned to different sources of uncertainty in the model input" (Saltelli et al., 2004). Model output is defined here as any variable that has its derivative computed over time and model input as any constant parameter and/or initial conditions of the simulation. For simplicity, model input will be referred herein as input parameter. The sensitivity analysis can approach one input parameter at a time or several parameters at once. Briefly, it consists in analysing the variation of the model output once different values are assigned to the input parameter(s), identifying those from which a target model output is most sensible to. 
The food web model used here is composed by sub-models previously published in the literature, in which sensitivity analyses for single parameters have been undertaken previously (Flynn and Mitra, 2009; Blackford et al., 2004; Butenschön et al., 2016). Taking into consideration the complexity associated with marine ecosystem models, due to the high number of state variables and free parameters, a sensitivity analysis of multiple input parameters can be extremely informative. Thus, this technique was performed here for the zero-dimensional model (Chapter 6). However, including all information in a single sensitivity analysis can lead to inconclusive results (Saltelli et al., 2008). It is responsibility of the modeller to define a clear objective for the sensitivity analysis. After defining the objective of the analysis, the targeted model output(s) are identified followed by the input parameters that are expected to be relevant to determine model output(s) variation. An interesting aspect of the sensitivity analysis is that it is very unlikely that all input parameters will be influential or non-influential, in turn, a few input parameters often account for most of the variation observed in model output, even if several input parameters are included in the analysis (Saltelli et al., 2008).

Different methods can be applied to conduct a sensitivity analysis. In Chapter 6, an approach based on the Monte Carlo ensemble technique was used to rank the importance of the input parameters at steady-state (Saltelli et al., 2008; Sankar et al., 2018). The method generates a number $n$ of realizations based on the probability density functions of $m$ input factors $\mathrm{x}_{\mathrm{i}}$ (i.e., model input parameters), assumed to be uniformly distributed and independent from each other. Each realization produces a vector containing values randomly sampled from the distributions of all input parameters. Each vector of parameters is then used to run a model simulation and compute the output $y$. The output of $n$ realizations and model runs is subsequently represented by a multiple linear regression (Eq. 5.2):

$$
y=b_{0}+\sum_{i=1}^{m} b_{i} x_{i}+\text { residuals }
$$

The standardized regression coefficients ( $\beta_{i}$ computed from $b_{i}$; Eq. 5.3) were used as global sensitivity indices of the input factors (Saltelli et al., 2008): 


$$
\beta_{\mathrm{i}}=\frac{\mathrm{b}_{\mathrm{i}} \sigma_{\mathrm{xi}}}{\sigma_{\mathrm{y}}}
$$

where $\sigma_{\mathrm{xi}}$ and $\sigma_{\mathrm{y}}$ are the standard deviations of the realizations of the input factor $\mathrm{x}_{\mathrm{i}}$ and of the model output y, respectively. Thus, each parameter included in the analysis is associated to a sensitivity coefficient which indicates whether an increase in the value of the parameter has a positive or negative effect on the targeted output (i.e., increase or decrease the output value, respectively). Since the validity of the results depends on the fraction of the model output variability that is explained by the multiple linear regression (Saltelli et al., 2000), the overall fraction of explained variance $\left(\mathrm{R}^{2}\right)$ and the significance of the standardized regression coefficients $\left(\beta_{\mathrm{i}}\right)$ were estimated (Chapter 6).

\subsection{Model skill assessment}

Model skill is defined here as the use of quantitative metrics and graphical approaches to assess how well the model fit the observations (Stow et al., 2009); i.e., akin to model validation. Model predictions and corresponding observations were compared for a single variable through time-series plots, as well as applying univariate comparison metrics, following Stow et al. (2003). In total, 3 metrics were used to capture different aspects of model performance (Eqs. 5.4-5.6). The correlation coefficient of the model predictions and observations (r; Eq. 5.4) measures the tendency of predictions and observations to vary together and can range from -1 to 1 , with negative values indicating that they vary inversely (Stow et al., 2009). However, even if the correlation is near to one, the values may not match each other and may differ by a consistent factor. Therefore, other metrics were used to check for this pattern, i.e., the root mean squared error (RMSE; Eq. 5.5) and the average error (AE; Eq. 5.6). RMSE and AE values near zero indicate a close match between predictions and observations. While the average error can be biased because negative and positive differences can cancel each other, the RMSE account for this feature considering the magnitude rather than the direction of the difference.

$$
r=\frac{\sum_{i=1}^{n}\left(O_{i}-\overline{0}\right) \cdot\left(P_{i}-\bar{P}\right)}{\sqrt{\sum_{i=1}^{n}\left(O_{i}-\overline{0}\right)^{2} \cdot \sum_{i=1}^{n}\left(P_{i}-\bar{P}\right)^{2}}}
$$




$$
\begin{array}{r}
\text { RMSE }=\sqrt{\frac{\sum_{i=1}^{n}\left(P_{i}-O_{i}\right)^{2}}{n}} \\
A E=\frac{\sum_{i=1}^{n}\left(P_{i}-O_{i}\right)}{n}=\bar{P}-\overline{0}
\end{array}
$$

where $n$ is the number of observations, $\mathrm{O}_{\mathrm{i}}$ is the ith of $n$ observations, $\mathrm{P}_{\mathrm{i}}$ is the ith of $n$ predictions, and $\overline{\mathrm{O}}$ and $\overline{\mathrm{P}}$ are the averages of observations and predictions, respectively.

Model skill was assessed in Chapter 6 to evaluate if silicon dynamics were correctly implemented in the protist model and in Chapter 7 to evaluate how well the model fit the observations at L4 station. Due to the complexity of the biogeochemical models used in Chapter 7, target diagrams were performed to visually summarize different aspects of the models. Target diagrams allow the exploration of relationships between the statistics metrics described above in a single plot (Jolliff et al., 2009). The target diagrams were constructed plotting the normalized $\mathrm{AE}$ ( $\mathrm{AE}^{*}$; Eq. 5.7) in the y-axis, the normalized and unbiased RMSE (RMSE*; Eqs. 5.8 and 5.9) in the x-axis, and the correlation coefficient (r; Eq. 5.4) through a colour scaling. The metrics were normalized by the standard deviation of the observations $\left(\sigma_{0}\right)$. The unbiased RMSE is utilized because the bias is already accounted within $\mathrm{AE}^{*}$. In addition, it is multiplied by the sign of the deviation difference to allow the utilization of the negative space of the Cartesian coordinate space (Jolliff et al., 2009). In Eq. 5.8, $\sigma_{P}$ is the standard deviation of the predictions.

$$
\begin{aligned}
& \mathrm{AE}^{*}=\frac{(\overline{\mathrm{P}}-\overline{\mathrm{O}})}{\sigma_{\mathrm{O}}} \\
& \operatorname{RMSE}^{\mathrm{ub}}=\sqrt{\frac{\sum_{\mathrm{i}=1}^{\mathrm{n}}\left(\left(\mathrm{P}_{\mathrm{i}}-\overline{\mathrm{P}}\right)-\left(\mathrm{O}_{\mathrm{i}}-\overline{\mathrm{O}}\right)\right)^{2}}{\mathrm{n}}} \cdot \operatorname{sign}\left(\sigma_{\mathrm{P}}-\sigma_{\mathrm{O}}\right), \\
& \text { in which: } \sigma_{\mathrm{P}}=\sqrt{\frac{\sum\left(\mathrm{P}_{\mathrm{i}}-\overline{\mathrm{P}}\right)^{2}}{\mathrm{n}}} \text { and } \sigma_{\mathrm{O}}=\sqrt{\frac{\sum\left(\mathrm{O}_{\mathrm{i}}-\overline{\mathrm{O}}\right)^{2}}{\mathrm{n}}} \\
& \operatorname{RMSE}^{*}=\frac{\mathrm{RMSE}^{\mathrm{ub}}}{\sigma_{\mathrm{O}}}
\end{aligned}
$$




\section{Chapter 6}

\section{Mixotrophic functional diversity within oceanic food-webs}

Part of this chapter has been published as:

Leles, S.G., Polimene, L., Bruggeman, J., Blackford, J., Ciavatta, S., Mitra, A. \& FLYNN, K.J. (2018) Modelling mixotrophic functional diversity and implications for ecosystem function. Journal of Plankton Research 40(6), 627-642. DOI: $\underline{10.1093 / p l a n k t / f b y 044}$ 


\subsection{Introduction}

Food webs comprise complex arrays of interactions between resources and consumers (Worm et al., 2002; Araújo et al., 2011). Despite the recognised importance of predation and competition in defining the ecological niches of different functional taxa (Hunter and Price 1992; Cloern and Dufford, 2005), the overall structure and dynamics of food webs are also greatly affected by additional factors, such as intraguild predation and omnivory (Polis et al., 1989; Williams and Martinez, 2000; Johnson et al., 2010; Granados et al., 2017). Mixotrophy is another 'twist' that can shift our understanding of ecosystem dynamics from terrestrial to aquatic environments (Tittel et al., 2003; Selosse et al., 2017).

There is a need to understand how mixotrophy, in its different forms, may change our understanding and simulations of food web dynamics and biogeochemical cycling in the oceans. For instance, CMs have been hypothesized to 'farm' bacteria in oligotrophic waters; while CMs feed on bacteria to acquire essential nutrients, they also release dissolved organic matter (DOM) which supports bacterial growth (Mitra et al., 2014b). Mixotrophs, compared to their heterotrophic competitors, can retain more nutrients from their prey as they can use them along with the organic carbon obtained through photosynthesis. An implication of this is, if mixotrophs outcompete strict heterotrophs in oligotrophic regions, then nutrient limitation of pure autotrophs (including cyanobacteria) may become more severe (Fischer et al., 2017). Furthermore, NCMs have the clear potential to achieve higher gross growth efficiencies through acquired phototrophy, potentially increasing the transfer of carbon biomass to higher trophic levels, particularly in low chlorophyll waters (Stoecker et al., 2009). Taken together these studies suggest that mixotrophy has the potential to enhance both the production of large size, fast sinking particles (e.g., faecal pellets) by mesozooplankton, which may feed on mixotrophs, and the bacterial production of recalcitrant material (Jiao et al., 2010; Polimene et al., 2017) which may be stimulated by the enhanced production of dissolved organic carbon (DOC) (Mitra et al., 2014b). Both the production of particles and recalcitrant DOC (rDOC) are key fluxes for the global carbon cycle contributing to the ocean carbon sequestration (Legendre et al., 2015).

So far, few studies have investigated the relevance of functional diversity within the mixotrophs on ecosystem functioning (Flynn and Mitra, 2009; Mitra et al., 2016; 
Ghyoot et al., 2017b). Understanding the ecological niches of mixotrophs and their strict auto- and hetero- trophic competitors helps to identify when and where different mixotrophs are major components of plankton communities and, thus, potentially affect ecosystem properties (Fischer et al., 2017; Leles et al., 2017). Mixotrophic functional diversity is a topic of particular importance in the context of climatic and anthropogenic changes on the oceans. Consider plankton communities in two contrasting marine ecosystems, oligotrophic seas and eutrophic coastal systems, characterised by nutrient and light limitation, respectively. Global warming is expected to increase ocean stratification in the former, potentially expanding the area occupied by low productive seas (Polovina et al., 2008; Behrenfeld et al., 2016). In turn, the increased runoff of nutrients and organic matter in coastal waters usually promotes unbalanced (and high) nitrogen to phosphorus ratios (Burkholder et al., 2008; Gomes et al., 2014). In both cases, mixotrophy has been shown to be a successful strategy (Burkholder et al., 2008; Zubkov and Tarran, 2008; Wilken et al., 2013; Gomes et al., 2014). Thus, acknowledging the role of mixotrophs can be key to predict the dynamics of plankton communities in a changing ocean.

While there is increasing awareness that mixotrophy is a key trait shaping biological communities, quantifying its physiological and ecological relevance is challenging (Selosse et al., 2017). This lack of knowledge is mainly due to the difficulty to accurately characterise the abundance and distributions of mixotrophs in the field (Anderson et al., 2017). Modelling studies provide a suitable platform to investigate the effects of mixotrophs on ecosystem function by using a hypothesis testing approach. Although several studies have simulated mixotrophy (Thingstad et al., 1996; Stickney et al., 2000; Flynn and Mitra, 2009, Flynn and Hansen, 2013; Våge et al., 2013; Mitra et al., 2014b; Mitra et al., 2016; Moeller et al., 2016), few have accounted for mixotrophic functional diversity and their impact on ecosystem dynamics (Ghyoot et al., 2017b). In addition, the structure of the mixotroph model is very important; mixotrophy does not simply reflect the additive interaction between phototrophy and phagotrophy and the description of metabolic switching from one strategy to the other is important to correctly simulate metabolic rates (Mitra and Flynn, 2010).

In this chapter, models of diverse types of mixotrophs across different size classes were combined with submodels of plankton as described in the European Regional Seas 
Ecosystem Model (ERSEM; Butenschön et al., 2016). The impact of mixotrophic functional diversity on key biogeochemical fluxes and plankton trophodynamics was assessed by contrasting this model with a 'non-mixotrophic' plankton food web model. Since mixotrophy is expected to dominate under resource limitation, nutrient or light limitation scenarios were simulated, akin to conditions representative of oligotrophic seas and eutrophic coastal systems, respectively. The theoretical framework presented here allows the investigation of the relative importance of constitutive and non-constitutive mixotrophs (CMs and NCMs) and of their strict autotrophic and heterotrophic competitors.

\subsection{Methods}

\subsubsection{Validating silicon dynamics for diatoms}

The implementation of silicon dynamics within the protist model represents a development in terms of model structure from the model described by Flynn and Mitra (2009) and, therefore, required validation of model assumptions (for full model description please refer to Appendix C, Eqs. 26-32). To verify if silicon dynamics were correctly implemented in the protist model, simulations were compared with the original model of diatom physiology (Flynn 2006), herein called as MDP. This means that the protist model was validated against predictions generated by another model which has been previously published and tested in the literature. In order to compare the models, parameter values in the MDP were the same as in the protist model, as defined for diatoms (Table 6.1). Simulations were performed to induce nitrate or silicate limitation since silicon dynamics differ on each scenario. Nutrient limitation was assessed through the normalized nitrogen and silicon to carbon quotas. Comparisons were made with respect to nutrient concentrations, nutrient to carbon quotas, carbon biomass, and metabolic rates. Model skill assessment was performed for specific variables through time-series plots and quantitative metrics (Chapter 5.6).

\subsubsection{Model set-up}

In the plankton food web model, protist functional types were defined through different parameterisations (Table 6.1) following the descriptions given in Chapter 5.2.3. Parameters defining the prey size spectrum $\left(S_{\min }, S_{\text {opt }}, S_{\max }\right)$ and the dilution rate of 
kleptochloroplasts $\left(\mathrm{D}_{\mathrm{Chl}}\right)$ were obtained from experimental studies performed with mixotrophic species (Tables 5.1 and 6.1). The parameters controlling photosynthesis $\left(\alpha_{\mathrm{Chl}}\right.$ and $\left.\mathrm{ChlC}_{\mathrm{abs}}\right)$ and inorganic nutrient uptake $\left(\mathrm{K}_{\mathrm{a}}, \mathrm{K}_{\mathrm{n}}\right.$, and $\left.\mathrm{K}_{\mathrm{p}}\right)$ were parameterised mainly according to cell size constraints (Table 6.1). Note that it was not possible to parameterize $\alpha_{C h l}$ separately for strict autotrophs and mixotrophs since the data compiled by Bouman et al. (2018) were obtained mostly through the analysis of pigment composition. Nutrient to carbon quotas were assumed to be the same among all phototrophic protists and phagotrophic protists were assumed to have a more constrained stoichiometry. Parameter values for the bacteria and the mesozooplankton models can be found in Tables 6.2 and 6.3 .

The plankton food webs (Fig. 5.1) were simulated through chemostat-like modelling experiments. The model assumes plankton biomass and nutrients to vary over time within a homogeneous "box" that receives a constant input of inorganic nutrients (nitrate, phosphate, silicate) through a constant dilution rate. The same dilution rate also washes out residual nutrients and other dissolved and particulate organics (including plankton) and inorganics from the system. A fixed depth of $10 \mathrm{~m}, 12: 12$ hours light-dark cycle, a constant temperature of $10^{\circ} \mathrm{C}$, and a constant dilution rate of 0.01 day $^{-1}$ were assumed. Initial numerical experiments revealed that higher dilution rates (i.e., 0.05 day $^{-1}$ and 0.1 day $^{-1}$ ) compromised the coexistence of plankton functional types. Despite low in comparison with dilution rates used in laboratory chemostat experiments, the value used here is appropriate for considerations of transfer across an ergocline (where rates are ca. $0.01-0.05$ day $^{-1}$ ). The photosynthetically active radiation (PAR) was computed from the shortwave radiation in the surface ( $\mathrm{I}_{\text {surf }}$ ), which was assumed to be constant through the period of the simulation, and an attenuation coefficient dependent on the concentration of plankton and particulate organic matter following Butenschön et al. (2016). The concentration of inorganics (e.g. dissolved inorganic nitrogen - DIN) entering the system (akin to concentrations below the mixed layer) was constant throughout a given simulation.

Two different scenarios were simulated: low light-high nutrient $\left(\mathrm{I}_{\text {surf }}=50 \mathrm{~W} \mathrm{~m}^{-2}\right.$ or $228 \mu \mathrm{mol}$ photon $\mathrm{m}^{-2} \mathrm{~s}^{-1}$; DIN $=20 \mu \mathrm{M}$ nitrate $)$ and high light-low nutrient $\left(\mathrm{I}_{\text {surf }}=250\right.$ 
$\mathrm{W} \mathrm{m} \mathrm{m}^{-2}$ or $1,140 \mu \mathrm{mol}$ photon $\mathrm{m}^{-2} \mathrm{~s}^{-1} ; \mathrm{DIN}=4 \mu \mathrm{M}$ nitrate). These irradiance and nutrient concentrations were chosen to induce light limitation or nutrient limitation among phototrophs. Light limitation was assessed through the relative rate of photosynthesis (i.e., the ratio between the actual photosynthesis rate and the maximum photosynthesis rate) while nutrient limitation was assessed through the normalised nutrient to carbon quotas. It was assumed an input 16:1 mole ratio of dissolved inorganic nitrogen (DIN; nitrate) to phosphorus, and a 1:1 mole ratio of DIN to silicon in all simulations.

Models output are presented herein through the average of the last year of simulation. The total ammonium regeneration, the trophic transfer efficiency, and the total production of labile DOC were compared between the non-mixotrophic and the mixotrophic food webs. These metrics were chosen to test the hypotheses that mixotrophy decreases the overall regeneration of inorganics, increases the transfer of biomass to higher trophic levels promoting the accumulation of biomass in larger size-classes and increases DOC production. The ratio between the total amount of food ingested by mesozooplankton and the total gross primary productivity (GPP) was used as a measure of trophic transfer efficiency. All organic carbon released by phytoplankton through primary production, egestion of unassimilated prey (mainly by protists), and natural mortality were assumed to contribute to the pool of labile DOC. The contribution of different functional groups to each of the processes was also investigated. Finally, the relative biomasses of mixotrophs and their respective autotrophic and heterotrophic competitors were evaluated in the transition from light to nutrient limitation. Thus, a third modelling experiment was conducted to simulate intermediate conditions of light and nutrient limitation $\left(\mathrm{I}_{\text {surf }}=100 \mathrm{~W} \mathrm{~m}^{-2}\right.$ or $457 \mu \mathrm{mol}$ photon $\mathrm{m}^{-2} \mathrm{~s}^{-1}, \mathrm{DIN}=16 \mu \mathrm{M}$ nitrate$)$. 
Table 6.1 Constant parameter values for the different protist functional types (for constant descriptions see Appendix C, Table C.1).

\begin{tabular}{|c|c|c|c|c|c|c|c|c|c|c|c|}
\hline Constant & PicoP & Diatoms & Nano-A & Micro-A & Nano-CMs & Micro-CMs & SNCMs & GNCMs & NanoZ & MicroZ & Ref. \\
\hline$\alpha_{\mathrm{Chl}}$ & $1.0 \times 10^{-5}$ & $6.5 \times 10^{-6}$ & $8.0 \times 10^{-6}$ & $5.0 \times 10^{-6}$ & $8.0 \times 10^{-6}$ & $5.0 \times 10^{-6}$ & $5.0 \times 10^{-6}$ & $5.0 \times 10^{-6}$ & - & - & 1,2 \\
\hline ChlC $_{\text {abs }}$ & 0.015 & 0.06 & 0.025 & 0.045 & 0.025 & 0.045 & - & - & - & - & 3,2 \\
\hline $\mathbf{D}_{\mathrm{Chl}}$ & - & - & - & - & - & - & 0.01 & 0.40 & - & - & 4,5 \\
\hline $\mathbf{k} \mathbf{P}_{\max }$ & - & - & - & - & - & - & 1.00 & 1.00 & - & - & - \\
\hline $\mathrm{K}_{\mathrm{a}}$ & 1.00 & 7.00 & 2.10 & 7.00 & 2.10 & 7.00 & 7.00 & - & - & - & 6 \\
\hline $\mathrm{K}_{\mathrm{n}}$ & 2.10 & 14.0 & 4.20 & 14.0 & 4.20 & 14.0 & 14.0 & - & - & - & 6 \\
\hline $\mathrm{K}_{\mathrm{p}}$ & 0.21 & 1.40 & 0.42 & 1.40 & 0.42 & 1.40 & 1.40 & - & - & - & 6 \\
\hline $\mathbf{K}_{\mathbf{S i}}$ & - & 15.0 & - & - & - & - & - & - & - & - & 7 \\
\hline$\mu_{\max }$ & 0.693 & 1.386 & 0.693 & 0.50 & 0.60 & 0.40 & 0.693 & 0.693 & 0.693 & 0.693 & 8,9 \\
\hline$\mu_{\text {phot }}$ & 0.693 & 1.386 & 0.693 & 0.50 & 0.60 & 0.40 & 0.35 & 0.00 & - & - & 8,9 \\
\hline $\mathrm{NC}_{\mathrm{abs}}$ & 0.25 & 0.20 & 0.20 & 0.20 & 0.20 & 0.20 & 0.20 & 0.20 & 0.20 & 0.20 & 10,6 \\
\hline $\mathrm{NC}_{\max }$ & 0.20 & 0.15 & 0.15 & 0.15 & 0.15 & 0.15 & 0.15 & 0.15 & 0.15 & 0.15 & 10,6 \\
\hline $\mathrm{NC}_{\min }$ & 0.05 & 0.05 & 0.05 & 0.05 & 0.05 & 0.05 & 0.05 & 0.05 & 0.15 & 0.15 & 10,6 \\
\hline $\mathrm{Pbal}_{\text {crit }}$ & - & - & - & - & 0.10 & 0.10 & 0.10 & 0.00 & - & - & - \\
\hline $\mathrm{PC}_{\mathrm{abs}}$ & 0.04 & 0.04 & 0.04 & 0.04 & 0.04 & 0.04 & 0.04 & 0.04 & 0.04 & 0.04 & 10,6 \\
\hline $\mathrm{PC}_{\max }$ & 0.02 & 0.02 & 0.02 & 0.02 & 0.02 & 0.02 & 0.02 & 0.02 & 0.02 & 0.02 & 10,6 \\
\hline $\mathrm{PC}_{\min }$ & 0.005 & 0.005 & 0.005 & 0.005 & 0.005 & 0.005 & 0.005 & 0.005 & 0.02 & 0.02 & 10,6 \\
\hline size & 1.00 & 20.0 & 5.00 & 20.0 & 5.00 & 20.0 & 20.0 & 20.0 & 5.00 & 20.0 & - \\
\hline$S_{\text {max }}$ & - & - & - & - & 6.00 & 30.0 & 30.0 & 30.0 & 10.0 & 40.0 & 11 \\
\hline $\mathbf{S}_{\min }$ & - & - & - & - & 0.90 & 0.20 & 0.20 & 0.20 & 0.10 & 0.20 & 11 \\
\hline $\mathrm{S}_{\text {mix }}$ & - & - & - & - & 0 & 0 & 1 & 1 & - & - & - \\
\hline $\mathbf{S}_{\text {opt }}$ & - & - & - & - & 1.00 & 5.00 & 5.00 & 5.00 & 1.00 & 5.00 & 11 \\
\hline $\mathrm{S}_{\mathrm{pd}}$ & - & - & - & - & 1 & 1 & 1 & 0 & - & - & - \\
\hline $\mathbf{S}_{\mathrm{si}}$ & 0 & 1 & 0 & 0 & 0 & 0 & 0 & 0 & 0 & 0 & - \\
\hline
\end{tabular}

${ }^{1}$ Bouman et al. 2018; ${ }^{2}$ Butenschön et al 2016; ${ }^{3}$ Sathyendranath et al. 2009; ${ }^{4}$ Myung et al. 2013; ${ }^{5}$ Schoener and McManus 2017; ${ }^{6}$ Flynn and Mitra 2009; ${ }^{7}$ Thamatrakoln and Hildebrang 2007; ${ }^{8}$ Flynn and Raven 2016; ${ }^{9}$ Laws $2013 ;{ }^{10}$ Geider and La Roche 2002; ${ }^{11}$ Table S1 
Table 6.2 List of constant parameters within the bacteria model from ERSEM (as per Butenschön et al. 2016).

\begin{tabular}{|c|c|c|c|}
\hline Constant & Description & Unit & Value \\
\hline $\operatorname{chn}$ & Michaelis-Menten constant for nitrate limitation & $\mathrm{mmol} \mathrm{N} \mathrm{m}^{-3}$ & 0.5 \\
\hline chp & $\begin{array}{l}\text { Michaelis-Menten constant for phosphate } \\
\text { limitation }\end{array}$ & $\mathrm{mmol} \mathrm{P} \mathrm{m}{ }^{-3}$ & 0.1 \\
\hline frF3 & $\begin{array}{l}\text { fraction of activity respiration converted to semi- } \\
\text { refractory DOC }\end{array}$ & - & 0.3 \\
\hline $\mathrm{NC}_{\max }$ & maximum nitrogen to carbon ratio & $\mathrm{mmol} \mathrm{N}(\mathrm{mg} \mathrm{C})^{-1}$ & 0.0167 \\
\hline $\mathrm{nRP}$ & number of substrates & - & 3 \\
\hline $\mathrm{PC}_{\max }$ & maximum phosphorus to carbon ratio & $\mathrm{mmol} \mathrm{P}(\mathrm{mg} \mathrm{C})^{-1}$ & 0.0019 \\
\hline $\mathrm{pu}_{\mathrm{B}}$ & efficiency at high oxygen levels & - & 0.6 \\
\hline $\mathrm{frDOC}_{1}$ & fraction of semi-labile DOC available to bacteria & - & 0.0075 \\
\hline $\mathrm{frDOC}_{\mathrm{r}}$ & $\begin{array}{l}\text { fraction of semi-refractory DOC available to } \\
\text { bacteria }\end{array}$ & - & 0.0025 \\
\hline $\mathrm{sd}_{\mathrm{B}}$ & specific mortality & day $^{-1}$ & 0.05 \\
\hline sDOM & $\begin{array}{l}\text { maximum turn-over rate of dissolved organic } \\
\text { matter }\end{array}$ & day $^{-1}$ & 1 \\
\hline sDOP & $\begin{array}{l}\text { mineralisation rate of labile dissolved organic } \\
\text { phosphorus }\end{array}$ & day $^{-1}$ & 0 \\
\hline sDON & $\begin{array}{l}\text { mineralisation rate of labile dissolved organic } \\
\text { nitrogen }\end{array}$ & day $^{-1}$ & 0 \\
\hline $\mathrm{rPOC}_{\mathrm{s}}$ & remineralisation of small-size $\mathrm{POM}$ & day $^{-1}$ & 0.01 \\
\hline $\mathrm{rPOC}_{\mathrm{m}}$ & remineralisation of medium-size POM & day $^{-1}$ & 0.0025 \\
\hline $\mathrm{rPOC}_{1}$ & remineralisation of large-size $\mathrm{POM}$ & day $^{-1}$ & 0.001 \\
\hline $\operatorname{srs}_{B}$ & specific rest respiration & day $^{-1}$ & 0.1 \\
\hline $\operatorname{sum}_{B}$ & maximum specific uptake & day $^{-1}$ & 2.2 \\
\hline
\end{tabular}


Table 6.3 List of constant parameters within the mesozooplankton model; parameters in bold correspond to new implementations to the model of Butenschön et al. (2016).

\begin{tabular}{|c|c|c|c|}
\hline Constant & Description & Unit & Value \\
\hline $\mathrm{Cr}_{\text {micro-CMs }}$ & slope of the encounter rate for micro-CMs & $\left(\mathrm{gC} \mathrm{gC}^{-1} \mathrm{day}^{-1}\right) \cdot\left(\mathrm{gC} \mathrm{m}^{-3}\right)^{-1}$ & 0.70 \\
\hline $\mathrm{Cr}_{\text {nano-CMs }}$ & slope of the encounter rate for nano-CMs & $\left(\mathrm{gC} \mathrm{gC}^{-1} \mathrm{day}^{-1}\right) \cdot\left(\mathrm{gC} \mathrm{m}^{-3}\right)^{-1}$ & 0.08 \\
\hline $\mathrm{Cr}_{\text {diatoms }}$ & slope of the encounter rate for diatoms & $\left(\mathrm{gC} \mathrm{gC}^{-1}\right.$ day $\left.^{-1}\right) \cdot\left(\mathrm{gC} \mathrm{m}^{-3}\right)^{-1}$ & 0.70 \\
\hline $\mathrm{Cr}_{\mathrm{GNCMs}}$ & slope of the encounter rate for GNCMs & $\left(\mathrm{gC} \mathrm{gC}^{-1}\right.$ day $\left.^{-1}\right) \cdot\left(\mathrm{gC} \mathrm{m}^{-3}\right)^{-1}$ & 0.70 \\
\hline $\mathrm{Cr}_{\text {mesozoo }}$ & $\begin{array}{l}\text { slope of the encounter rate for } \\
\text { mesozooplankton (intraguild predation) }\end{array}$ & $\left(\mathrm{gC} \mathrm{gC}^{-1} \mathrm{day}^{-1}\right) \cdot\left(\mathrm{gC} \mathrm{m}^{-3}\right)^{-1}$ & 3.00 \\
\hline $\mathrm{Cr}_{\text {microzoo }}$ & $\begin{array}{l}\text { slope of the encounter rate for } \\
\text { heterotrophic microzooplankton }\end{array}$ & $\left(\mathrm{gC} \mathrm{gC}^{-1} \mathrm{day}^{-1}\right) \cdot\left(\mathrm{gC} \mathrm{m}^{-3}\right)^{-1}$ & 0.35 \\
\hline $\mathrm{Cr}_{\text {nanozoo }}$ & $\begin{array}{l}\text { slope of the encounter rate for } \\
\text { heterotrophic nanoflagellates }\end{array}$ & $\left(\mathrm{gC} \mathrm{gC}^{-1}\right.$ day $\left.^{-1}\right) \cdot\left(\mathrm{gC} \mathrm{m}^{-3}\right)^{-1}$ & 0.04 \\
\hline $\mathrm{Cr}_{\text {POM }}$ & $\begin{array}{l}\text { slope of the encounter rate for particulate } \\
\text { organic matter }\end{array}$ & $\left(\mathrm{gC} \mathrm{gC}^{-1} \mathrm{day}^{-1}\right) \cdot\left(\mathrm{gC} \mathrm{m}^{-3}\right)^{-1}$ & 0.70 \\
\hline $\mathrm{Cr}_{\text {SNCMs }}$ & slope of the encounter rate for SNCMs & $\left(\mathrm{gC} \mathrm{gC}^{-1} \mathrm{day}^{-1}\right) \cdot\left(\mathrm{gC} \mathrm{m}^{-3}\right)^{-1}$ & 0.70 \\
\hline KI & $\begin{array}{l}\text { half-saturation constant for the ingestion } \\
\text { rate of all prey types }\end{array}$ & $\mathrm{gC} \mathrm{gC}^{-1} \mathrm{day}^{-1}$ & 0.25 \\
\hline $\mathbf{I}_{\max }$ & maximum carbon ingestion rate & $\mathrm{gC} \mathrm{gC}^{-1}$ day $^{-1}$ & 1.00 \\
\hline Minprey & food threshold for overwintering state & $\mathrm{mg} \mathrm{C} \mathrm{m}{ }^{-2}$ & 0.00 \\
\hline nprey & number of prey types & - & 9.00 \\
\hline pe_R1 & dissolved fraction of excreted matter & - & 0.50 \\
\hline $\mathrm{pu}_{\mathrm{MZ}}$ & assimilation efficiency & - & 0.60 \\
\hline pu_ea & $\begin{array}{l}\text { fraction of unassimilated prey that is } \\
\text { excreted (not respired) }\end{array}$ & - & 0.50 \\
\hline pu_eaR & $\begin{array}{l}\text { fraction of unassimilated detritus that is } \\
\text { excreted (not respired) }\end{array}$ & - & 0.90 \\
\hline qne & nitrogen to carbon ratio & $\operatorname{mmol~N}\left(\mathrm{mg} \mathrm{C}^{-1}\right.$ & 0.013 \\
\hline qpc & phosphorus to carbon ratio & $\operatorname{mmol~P}(\mathrm{mg} \mathrm{C})^{-1}$ & 0.0008 \\
\hline R1R2 & $\begin{array}{l}\text { labile fraction of produced dissolved } \\
\text { organic carbon }\end{array}$ & - & 1.00 \\
\hline $\operatorname{sd}_{\mathrm{MZ}}$ & basal mortality & day $^{-1}$ & 0.025 \\
\hline sdo & $\begin{array}{l}\text { maximum mortality due to oxygen } \\
\text { limitation }\end{array}$ & day $^{-1}$ & 0.00 \\
\hline $\operatorname{srs}_{\mathrm{MZ}}$ & specific rest respiration & day $^{-1}$ & 0.015 \\
\hline$x R 1 n$ & $\begin{array}{l}\text { transfer of nitrogen to DOM, relative to } \\
\text { POM }\end{array}$ & - & 1.00 \\
\hline $\mathrm{xR} 1 \mathrm{p}$ & $\begin{array}{l}\text { transfer of phosphorus to DOM, relative } \\
\text { to POM }\end{array}$ & - & 1.20 \\
\hline repw & specific overwintering respiration & day $^{-1}$ & 0.0025 \\
\hline mort & specific overwintering mortality & day $^{-1}$ & 0.0025 \\
\hline
\end{tabular}

* all values from Butenschön et al. (2016) except for the slopes of the encounter rates, KI, and Imax (Flynn and Mitra, 2016) 


\subsubsection{Sensitivity analyses for the mixotrophic food web}

Sensitivity analyses were performed to evaluate how the ecological processes described in the previous section (i.e., ammonium regeneration, trophic transfer efficiency, and total production of labile DOC) are affected by the choice of parameter values and nutrient concentrations in the chemostat medium for the mixotrophic food web. The sensitivity of the mixotrophic food web was evaluated for both the nutrient-limited and the light-limited scenarios. The main parameters that define functional diversity within the conceptual food web, such as those related to phototrophy, nutrient uptake, predation, respiration, and mixotrophic potential (Chapter 5.4) were selected for the analyses (Appendix D, Table D.1).

In order to rank the importance of the input parameters, an approach based on the Monte-Carlo ensemble technique was used (Chapter 5.8). A total of $m=116$ input parameters were included in the sensitivity analyses. A total of $n=2320$ realizations were performed assuming 20 realizations for each input parameter as a rule of thumb (Hair et al., 2006). Random values were generated assuming a range of $\pm 30 \%$ of the reference value of the input parameters (e.g., Sankar et al., 2018) and all parameters included in the analysis had initial values higher than zero. The analyses were performed using a Python code developed for the purpose.

In addition to these analyses, an extra sensitivity test was performed to confirm that averaged model outputs during the last year of the simulation were independent from initial conditions. Tests were performed in both limitation scenarios (i.e., light or nutrient). First, the biomass of all plankton functional types was decreased to $5 \%$ or doubled with respect to the default values used in all simulations. Then, the biomass of each functional type was doubled individually to check for any potential effect related to individual components of the model. Changes in the main ecosystem properties investigated on this study (ammonium regeneration, trophic transfer efficiency, production of labile DOC) and in plankton biomasses were evaluated for each of the tests. 


\subsection{Results}

\subsubsection{Validating silicon dynamics for diatoms}

Silicon dynamics within the protist model were validated against the MDP both under nitrate and silicate limitation (Table 6.4 and Figs. 6.1 and 6.2, respectively). Based on the time-series plots, the simulations by the protist model matched closely the simulations by the MDP (Figs. 6.1 and 6.2). In fact, all variables analysed were highly correlated, with $r>0.9$ for all variables (Table 6.4). Overall, nutrient limitation developed relatively earlier in the protist model (Figs. 6.1 and 6.2). According to the RMSE and AE metrics, differences between the protist model and the MDP were close to zero for most of the variables except for nutrient concentrations and diatom carbon biomass (Table 6.4). The RMSE and AE values were higher among these variables due to the large variability of the simulated data (Figs. 6.1 and 6.2).

Table 6.4 Model skill assessment for selected variables under nitrate or silicate limitation. $\mathrm{r}$-correlation coefficient, RMSE-root mean squared error, and AE-average error. Variables correspond to nutrients (DIN, DIP, and Silicon), normalised nutrient to carbon quotas - mNCU (nitrate), $\mathrm{mPCu}$ (phosphate), and $\mathrm{mSCu}$ (silicate); nutrient to carbon quotas $-\mathrm{N}: \mathrm{C}, \mathrm{P}: \mathrm{C}$, and $\mathrm{Si}: \mathrm{C}$, diatoms biomass, photosynthesis rate (mPS), and growth rate $(\mathrm{mCu})$.

\begin{tabular}{lcccccc}
\hline & \multicolumn{3}{c}{ Nitrate limitation } & \multicolumn{3}{c}{ Silicate limitation } \\
\cline { 2 - 7 } Model output & $\mathbf{r}$ & RMSE & AE & r & RMSE & AE \\
\hline $\mathrm{DIN}(\mu \mathrm{M})$ & 0.97 & 81.27 & -26.01 & 0.98 & 280.09 & -201.41 \\
$\mathrm{DIP}(\mu \mathrm{M})$ & 0.97 & 11.23 & -4.16 & 0.98 & 34.37 & -19.68 \\
$\mathrm{Silicon}(\mu \mathrm{M})$ & 0.98 & 482.81 & 15.34 & 0.98 & 147.25 & -48.17 \\
$\mathrm{mNCu}(\mathrm{dl})$ & 0.94 & 0.17 & -0.06 & 0.99 & 0.02 & -0.02 \\
$\mathrm{mPCu}(\mathrm{dl})$ & 0.97 & 0.04 & -0.03 & 0.99 & 0.01 & $<0.01$ \\
$\mathrm{mSCu}(\mathrm{dl})$ & 0.99 & $<0.01$ & $<0.01$ & 0.92 & 0.21 & -0.04 \\
$\mathrm{~N}: \mathrm{C}\left(\mathrm{gN} \mathrm{gC} \mathrm{gC}^{-1}\right)$ & 0.94 & 0.03 & -0.02 & 0.96 & 0.02 & -0.01 \\
$\mathrm{P}: \mathrm{C}\left(\mathrm{gP} \mathrm{gC}^{-1}\right)$ & 0.97 & $<0.01$ & $<0.01$ & 0.98 & $<0.01$ & $<0.01$ \\
$\mathrm{Si}: \mathrm{C}\left(\mathrm{gSi} \mathrm{gC}^{-1}\right)$ & 0.98 & 0.04 & -0.03 & 0.99 & 0.03 & -0.03 \\
$\mathrm{Biomass}\left(\mu \mathrm{g} \mathrm{C} \mathrm{L}^{-1}\right)$ & 0.97 & 1638.10 & 725.29 & 0.97 & 1449.23 & 925.40 \\
$\mathrm{mPS}\left(\mathrm{gC} \mathrm{gC}^{-1} \mathrm{~d}^{-1}\right)$ & 0.96 & 0.28 & $<0.01$ & 0.95 & 0.34 & 0.01 \\
$\mathrm{mCu}\left(\mathrm{gC} \mathrm{gC}^{-1} \mathrm{~d}^{-1}\right)$ & 0.94 & 0.23 & $<0.01$ & 0.95 & 0.22 & 0.01 \\
\hline
\end{tabular}



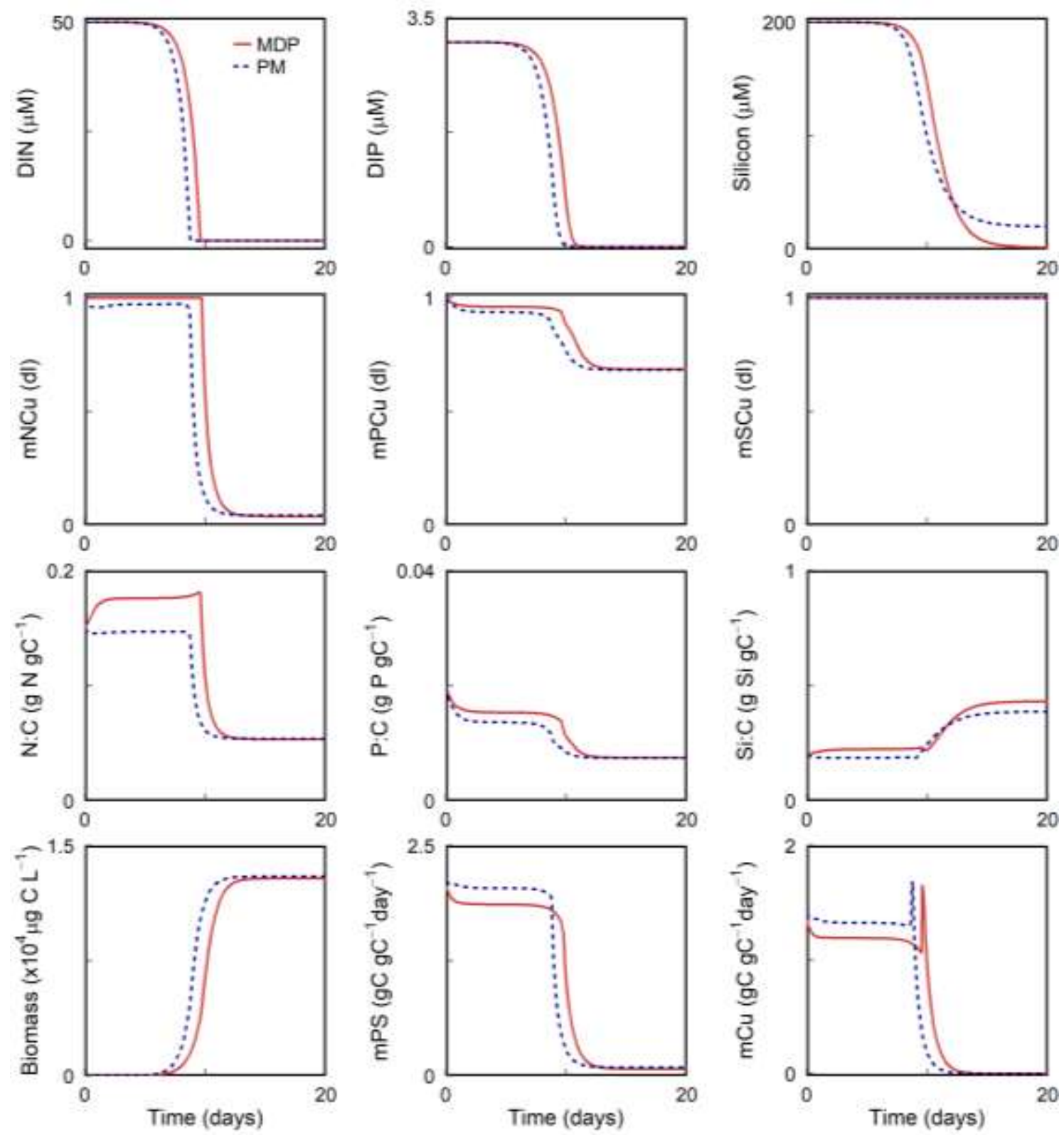

Figure 6.1 Validating silicon dynamics within the protist model (PM) under nitrate limitation. PM simulations (dotted blue lines) were compared with simulations using the original model of diatom physiology (MDP; solid red lines). Comparisons were made for nutrient concentrations (first line), normalised nutrient to carbon quotas (second line; $\mathrm{mNCu}-\mathrm{N}: \mathrm{C}, \mathrm{mPCu}-\mathrm{P}: \mathrm{C}, \mathrm{mSCu}-\mathrm{Si}: \mathrm{C}$ ), actual nutrient to carbon quotas (third line), carbon biomass and photosynthesis (mPS) and growth $(\mathrm{mCu})$ rates (fourth line). 

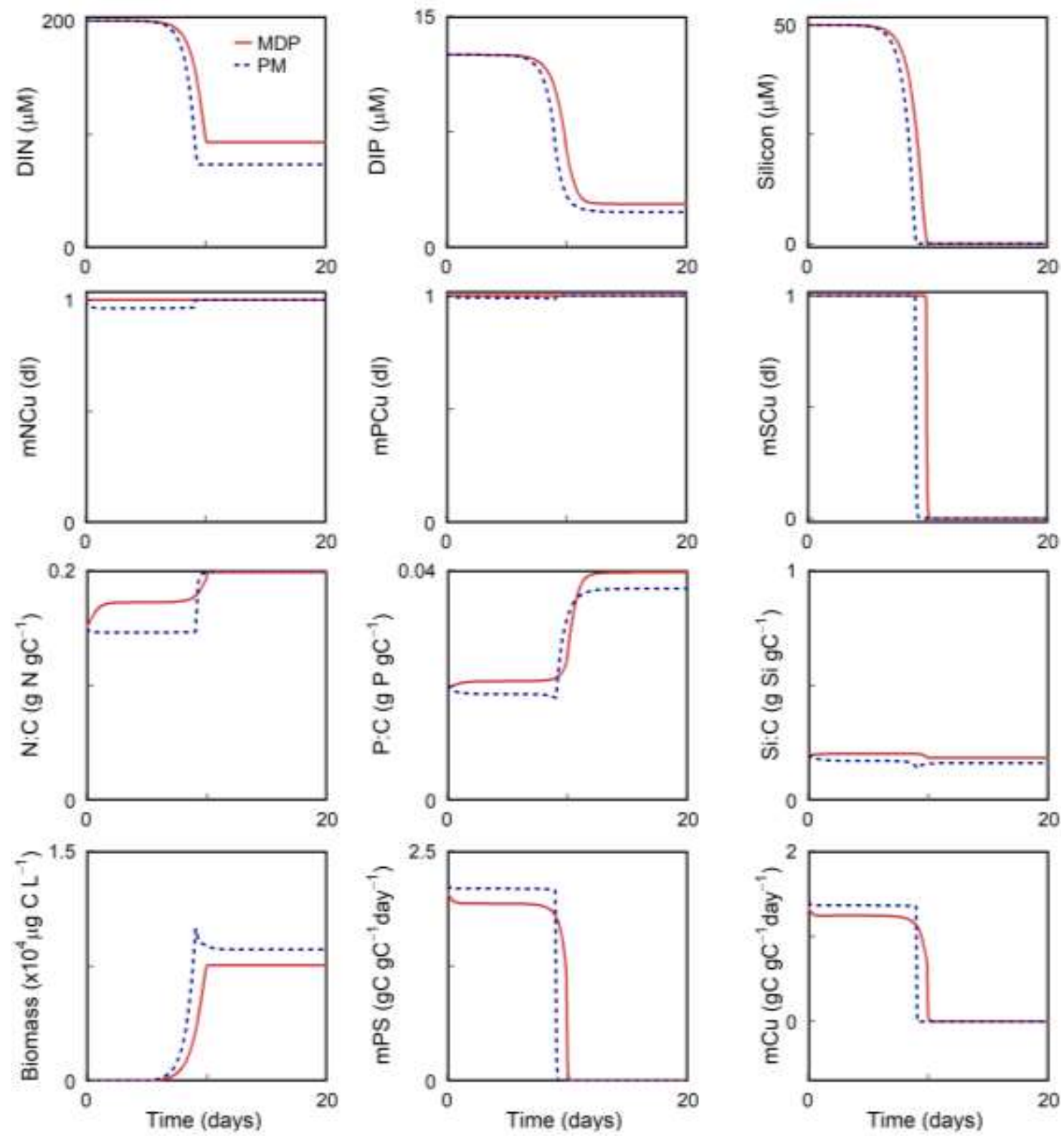

Figure 6.2 Validating silicon dynamics within the protist model (PM) under silicate limitation. PM simulations (dotted blue lines) were compared with simulations using the original model of diatom physiology (MDP; solid red lines). Comparisons were made for nutrient concentrations (first line), normalised nutrient to carbon quotas (second line; $\mathrm{mNCu}-\mathrm{N}: \mathrm{C}, \mathrm{mPCu}-\mathrm{P}: \mathrm{C}, \mathrm{mSCu}-\mathrm{Si}: \mathrm{C}$ ), actual nutrient to carbon quotas (third line), carbon biomass and photosynthesis (mPS) and growth $(\mathrm{mCu})$ rates (fourth line). 


\subsubsection{Light-limited scenario}

Ecosystem properties differed between the non-mixotrophic and the mixotrophic food webs in the light-limited scenario (Fig. 6.3). Ammonium regeneration was higher in the non-mixotrophic food web, mainly due to the activity of heterotrophic protists (Fig. 6.3a). Once mixotrophs were included, they competed with their heterotrophic counterparts and down-regulated the biomass of heterotrophic nanoflagellates (Fig. 6.4). Mixotrophs did not contribute as much to the regeneration of ammonium but supported a higher trophic transfer efficiency of carbon biomass to higher trophic levels (Fig. 6.3b). This is explained by changes in community composition, from smaller (in the nonmixotrophic food web) to larger (in the mixotrophic food web) phototrophs, since mesozooplankton exhibit a preference for larger prey items. In the absence of mixotrophs, autotrophic nanoflagellates and microflagellates were outcompeted by picophytoplankton and diatoms (Fig. 6.4), with only the latter having a cell size large enough to serve as food for mesozooplankton (Fig. 6.3b). In the mixotrophic food web, CMs thrived, with mixotrophs contributing significantly to the diet of mesozooplankton (Fig. 6.3b). In turn, the production of DOC was higher in the non-mixotrophic food web (Fig. 6.3c). This was mainly due to the higher total GPP, reflecting the high biomass levels attained by picophytoplankton and diatoms (Fig. 6.4), and due to higher mortality following the overall increase in carbon biomass. Relative to that of phototrophs, the production of DOC by phagotrophic protists was minor in the non-mixotrophic framework while being more significant in the presence of mixotrophs (Fig. 6.3c). 


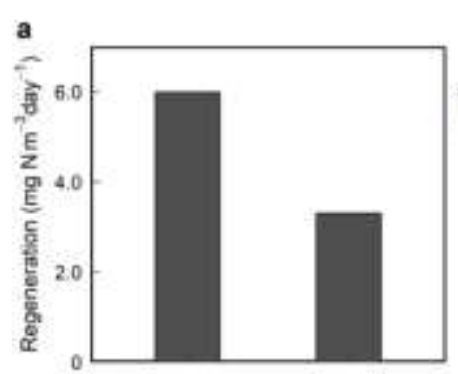

Non-mixotrophic food web
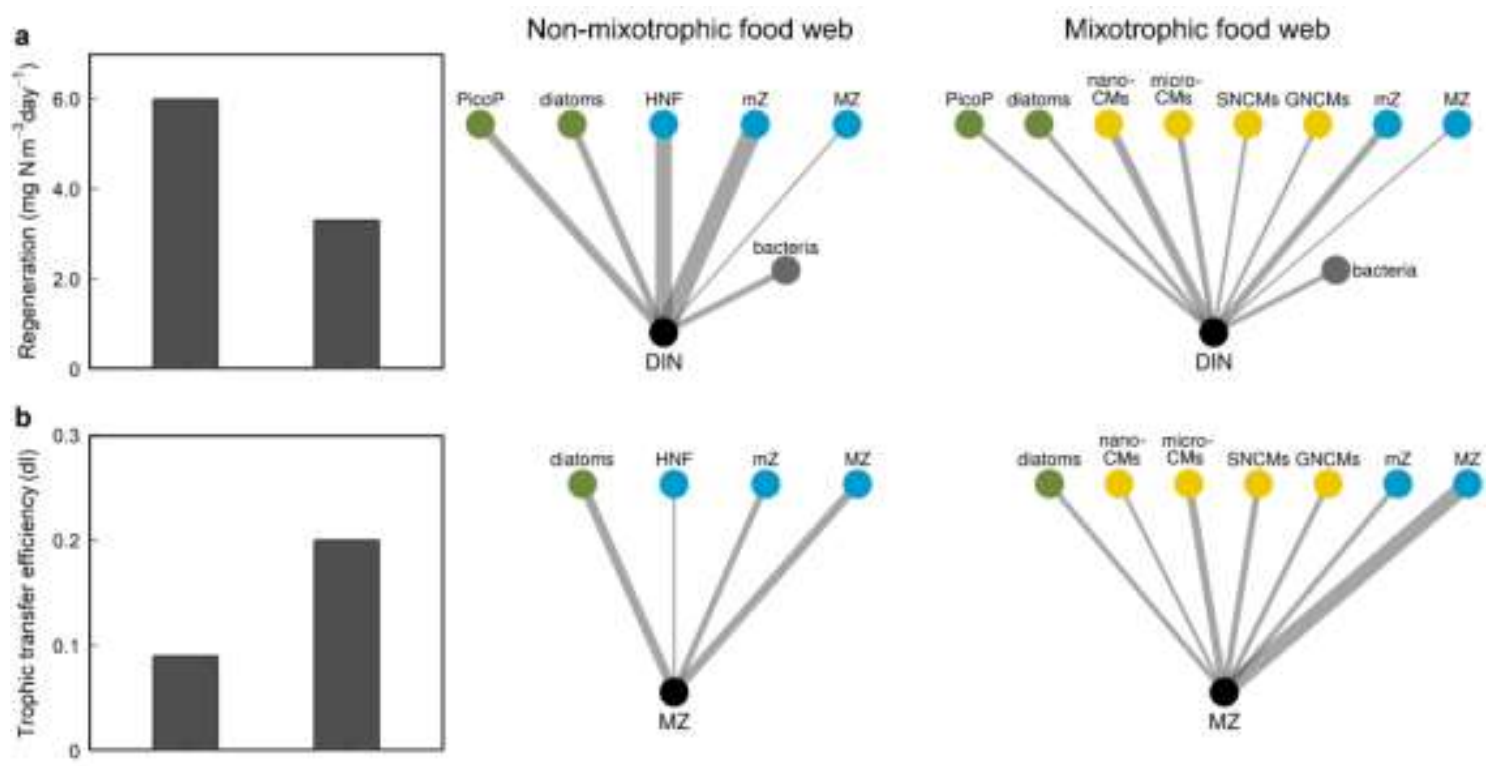

c
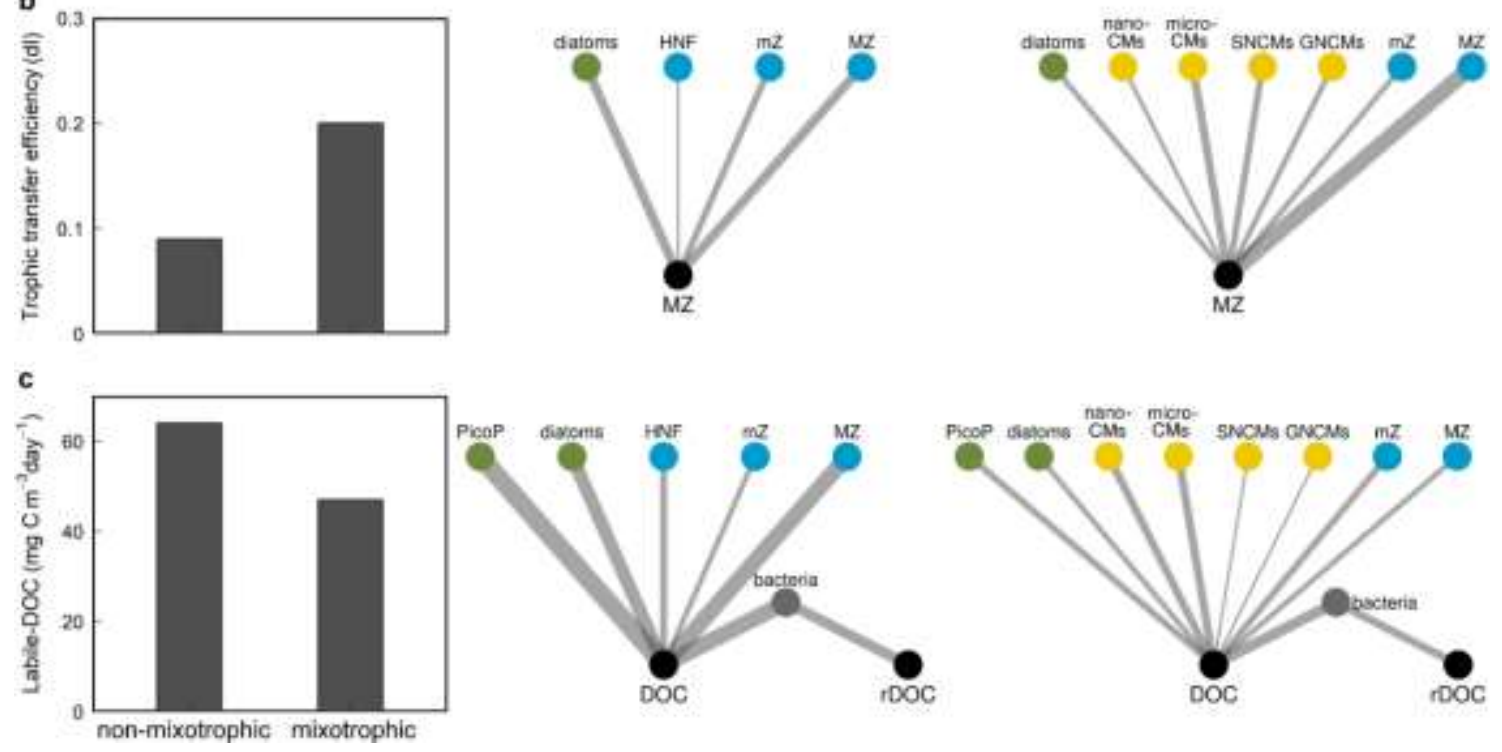

Figure 6.3 Light limited-scenario for the non-mixotrophic and the mixotrophic food webs. a) ammonium (DIN) regeneration; b) trophic transfer efficiency (measured by the ratio of the total amount of food ingested by mesozooplankton (MZ) by the total gross primary productivity); and c) total production of labile dissolved organic carbon (DOC). Data were averaged for the last year of simulation. Schematics show the relative contribution of functional groups (green-autotrophs, yellow-mixotrophs, blue-heterotrophs, greydecomposers) to each of the ecosystem properties (black nodes). In panel b, fluxes represent the amount of food ingested by mesozooplankton. dl-dimensionless; for others please refer to the Abbreviations section. 


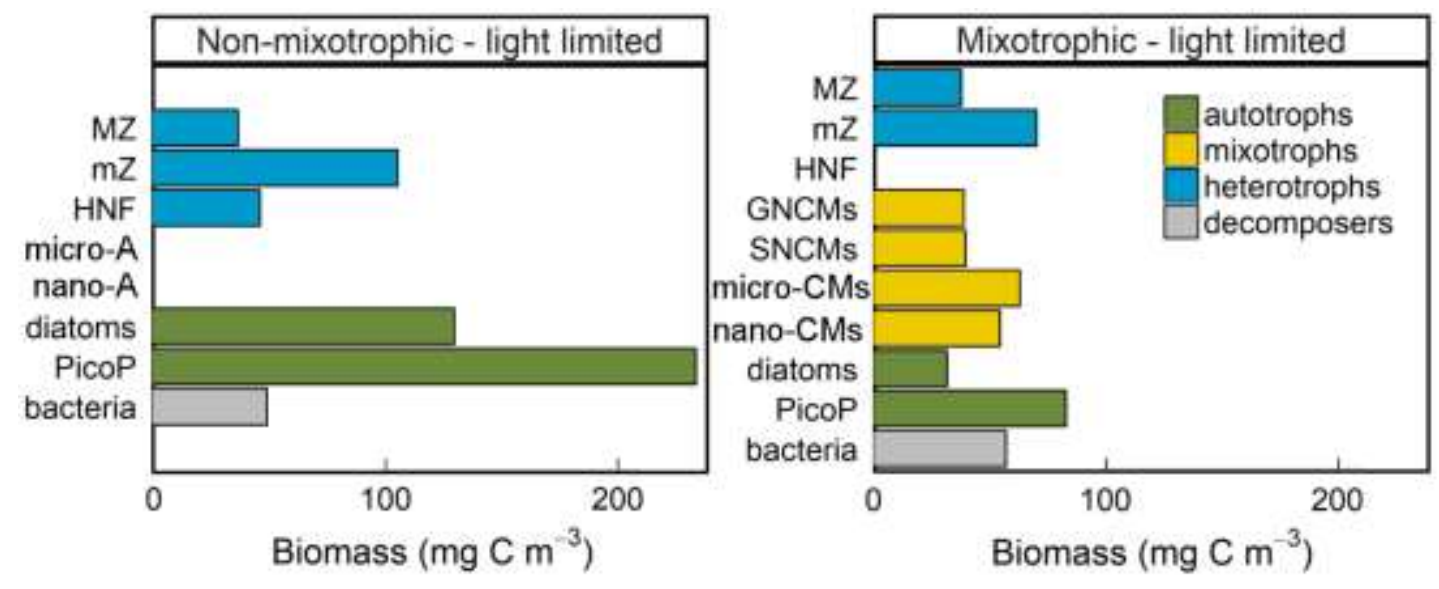

Figure 6.4 Community composition for the non-mixotrophic (left) and the mixotrophic (right) food webs in the light-limited scenario. Carbon biomass of the different functional groups are given; colours indicate different trophic strategies. Data were averaged for the last year of simulation. Please refer to the Abbreviations section for the functional types.

\subsubsection{Nutrient-limited scenario}

Mixotrophy was more successful under the nutrient-limited scenario (Figs. 6.5 and 6.6), with mixotrophs outcompeting their strict auto- and hetero- trophic counterparts, respectively. As a result, ecosystem properties differed substantially between the nonmixotrophic and the mixotrophic food webs in this scenario (Fig. 6.5). Similar to the light limited-scenario, ammonium regeneration was lower in the mixotrophic food web (Fig. 6.5a) and NCMs could outcompete their heterotrophic counterparts due to limited prey availability (Fig. 6.6). As mixotrophs did not contribute to the regeneration of ammonium (Fig. 6.5a), this in turn decreased the availability of inorganic nutrients, which favoured CMs (mainly nano-CMs) over strict autotrophs (Fig. 6.6).

The role of mixotrophy in the trophic transfer efficiency was more pronounced in the nutrient-limited scenario (Fig. 6.5b). Indeed, while in the non-mixotrophic food web mesozooplankton was limited by the paucity of suitable prey, in the mixotrophic food web, mesozooplankton could rely on NCMs which in turn were supported by the nanoCMs biomass feeding on picophytoplankton (Figs. 6.5b and 6.6). Contrary to the lightlimited scenario, mixotrophy also boosted the production of labile DOC under nutrient limitation. This was mainly related to a greater fraction of ingested prey remaining unassimilated (i.e., more inefficient predators due to lower prey quality). The main 
contributors to the production of labile DOC on this scenario were nano-CMs and GNCMs (Fig. 6.5c). The direct effect of increased availability of labile DOC is the stimulation of bacterial metabolism, which leads to enhanced production of recalcitrant DOC (Fig. 6.5c).

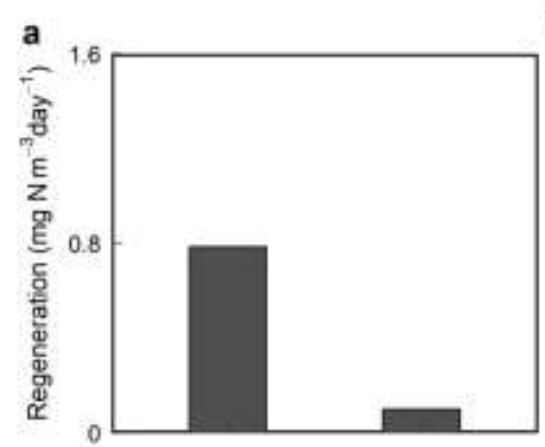

Non-mixotrophic food web

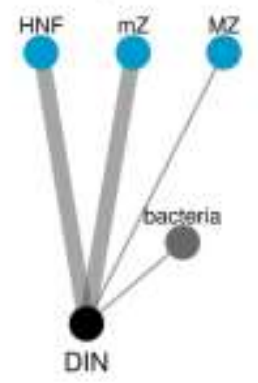

Mixotrophic food web
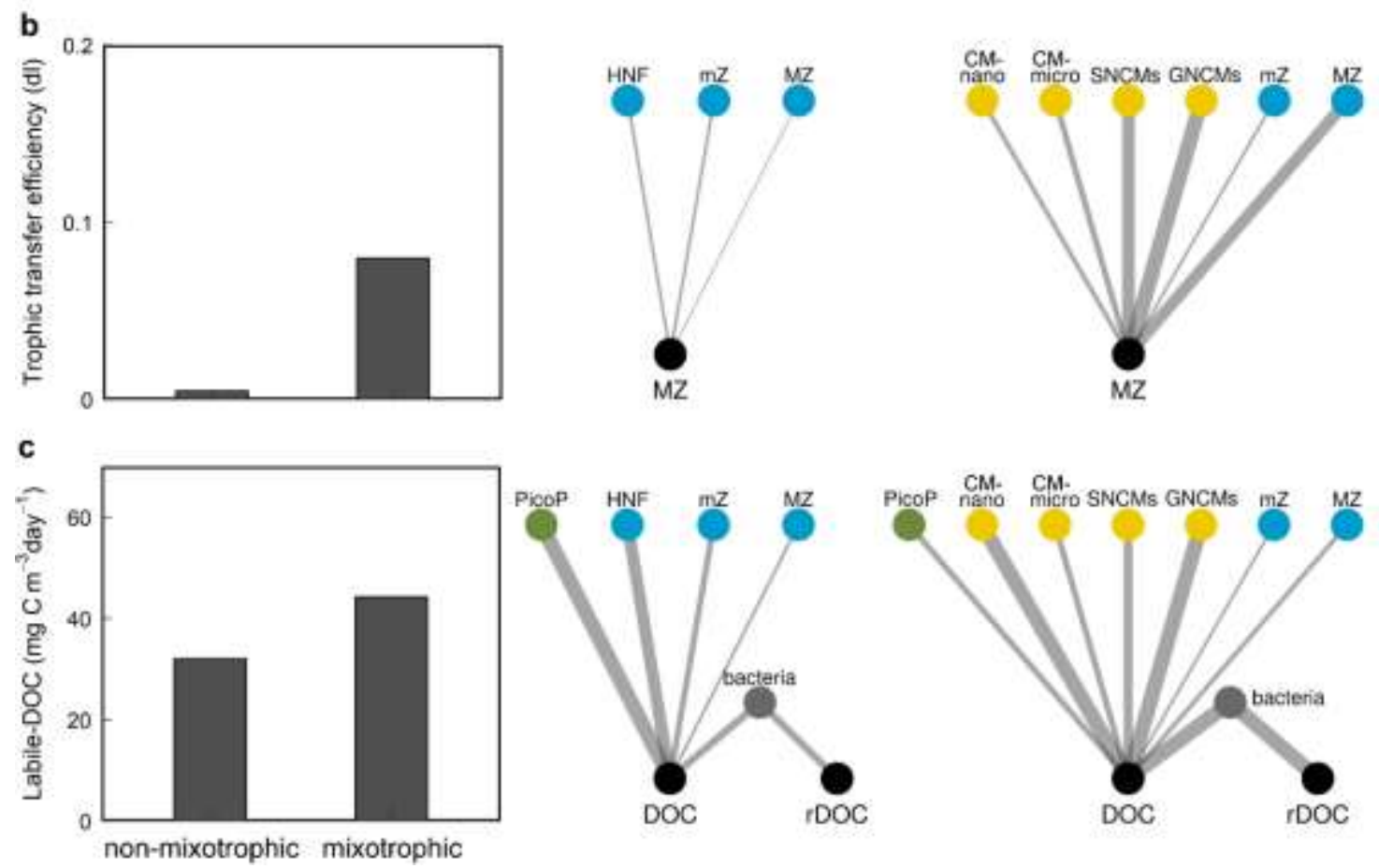

Figure 6.5 Nutrient limited-scenario for the non-mixotrophic and the mixotrophic food webs. a) ammonium (DIN) regeneration; b) trophic transfer efficiency (total amount of food ingested by mesozooplankton (MZ) to the total gross primary productivity ratio); and c) total production of labile DOC. Data were averaged for the last year of simulation. Schematics show the relative contribution of autotrophs (green), mixotrophs (yellow), heterotrophs (blue), and decomposers (grey) to each of the ecosystem properties (black nodes). In panel $b$, fluxes represent the amount of food ingested by mesozooplankton. $\mathrm{dl}$ dimensionless; for others please refer to the Abbreviations section. 


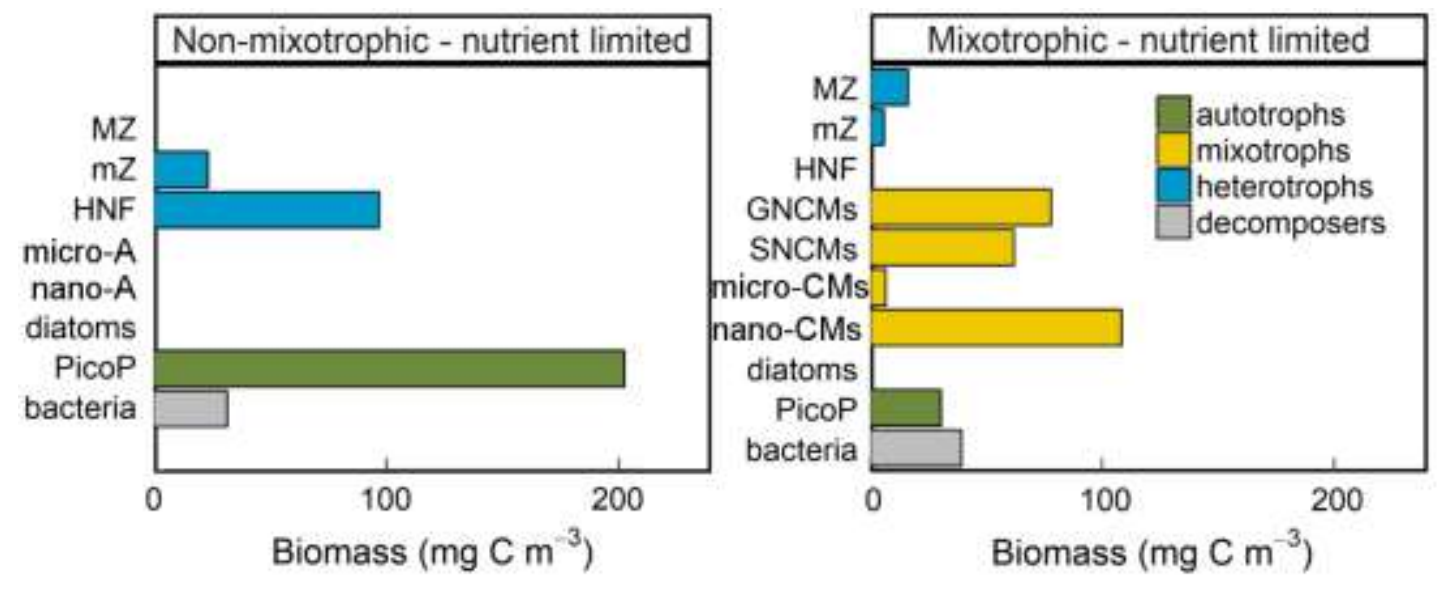

Figure 6.6 Community composition for the non-mixotrophic (left) and the mixotrophic (right) food webs in the nutrient-limited scenario. Carbon biomass of the different functional groups are given; colours indicate different trophic strategies. Data were averaged for the last year of simulation. Please refer to the Abbreviations section for the functional types.

The individual and additional effects of mixotrophic diversity were also investigated by including one or more mixotrophic types at a time (Fig. 6.7). The additional simulations were performed under nutrient limitation due to the higher importance of mixotrophy on this scenario. The changes on community structure (in terms of carbon biomass considering mixotrophs and their strict auto- and hetero- trophic competitors), ammonium regeneration, and trophic transfer efficiency were evaluated relative to the non-mixotrophic food web. When only one mixotrophic type was considered, changes on community structure were more significant for nano-CMs which, outcompeting picophytoplankton, were the only mixotrophic type to enable the growth of mesozooplankton alone (Fig. 6.7). On the other hand, micro-CMs down-regulated the biomass of strict heterotrophs, allowing higher picophytoplankton biomass and decreasing the overall regeneration of ammonium by $\sim 70 \%$ (Fig. 6.7). The individual impact of GNCMs or SNCMs was small; in fact, SNCMs did not survive because they depend on nanophytoplankton to obtain their phototrophic capacity and this group was outcompeted by picophytoplankton (Fig. 6.7). Differences were more pronounced once nano-CMs and GNCMs or SNCMs were included in the model because nano-CMs supports the biomass of NCMs which, in turn, is transferred to mesozooplankton (Fig. 6.7). Finally, including 
all mixotrophic types increased the extent of the overall niche for mixotrophy, enhancing its overall impact (Fig. 6.7).

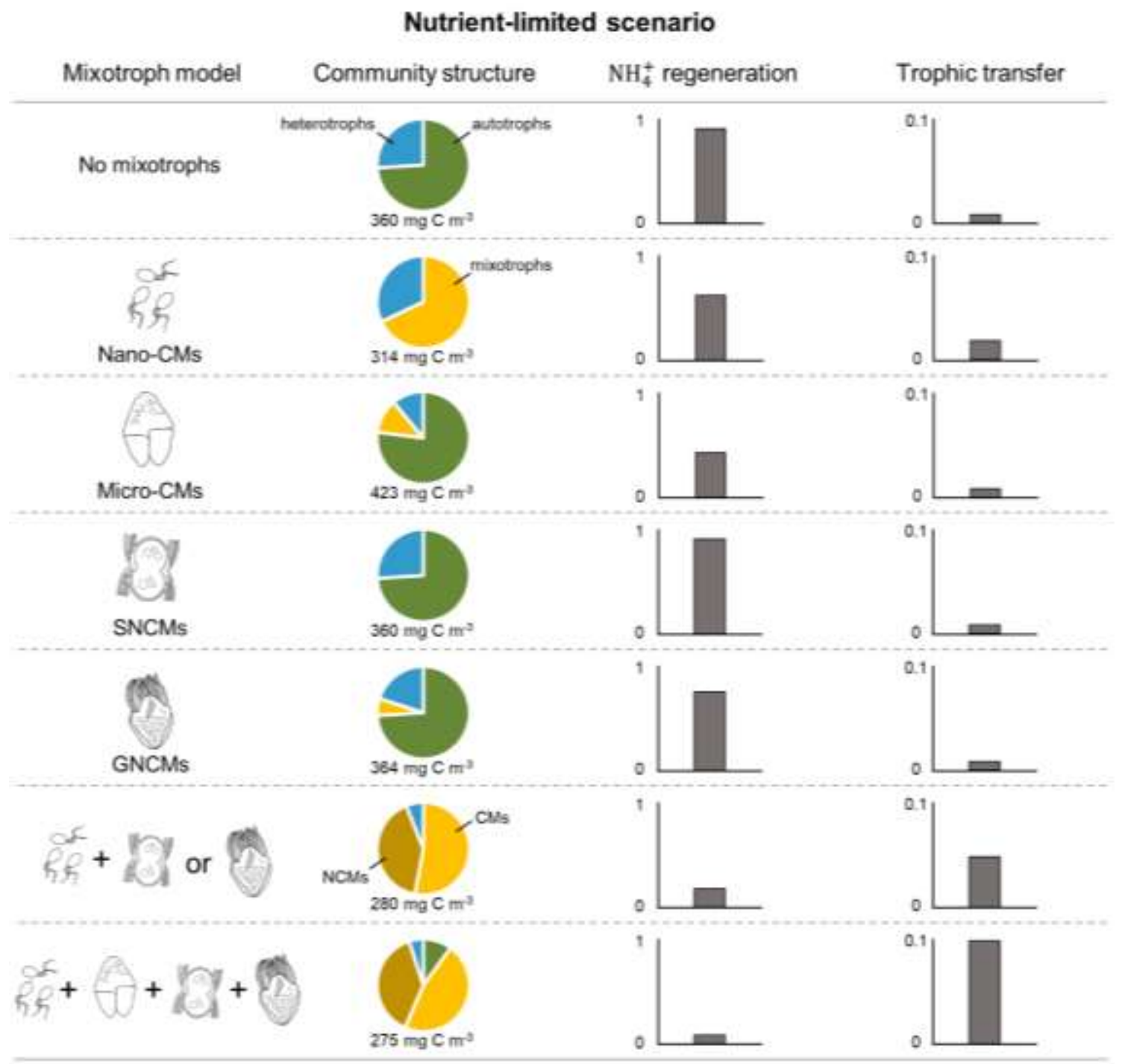

Figure 6.7 Food web models accounting for individual and additional effects of mixotrophic diversity to the community structure, ammonium regeneration $\left(\mathrm{mg} \mathrm{N} \mathrm{m} \mathrm{N}^{-3}\right.$ day $^{-1}$ ), and carbon transfer efficiency to mesozooplankton (dimensionless) within the nutrient-limited scenario. Community structure is given in terms of carbon biomass considering the mixotrophs and their strict auto- and hetero- trophic competitors. Trophic transfer is given by the ratio between mesozooplankton total ingestion and the total gross primary productivity. Please refer to the Abbreviations section for the functional types. 


\subsubsection{Sensitivity analyses for mixotrophic food web}

The overall fraction of variance explained by the multiple linear regression on the 116 selected parameters was high for all three targeted outputs in both limitation scenarios $\left(\mathrm{R}^{2}>0.9\right)$. In this chapter, the first 8 parameters ranked by highest sensitivity are presented (Tables 6.5 and 6.6); the full ranking of significant coefficients can be found in the supplementary material (Appendix D, Table D.1). The sensitivity coefficients of all the parameters reported here were statistically significant. A positive coefficient (in Tables 6.5 and 6.6) indicates that an increase in the parameter value led to an increase in the output value and vice-versa.

In the light-limited scenario, all targeted outputs were most sensitive to photosynthetic parameters (Table 6.5). Ammonium regeneration was promoted by increasing the efficiency of diatoms and picophytoplankton in harvesting light $\left(\alpha_{\mathrm{Chl}}\right.$ and ChlCabs, positive coefficients in Table 6.5) and decreased if higher maximum nitrogen to carbon quotas were considered ( $\mathrm{NC}_{\max }$, negative coefficients in Table 6.5). In turn, the trophic transfer efficiency was most sensitive to the optimal prey size $\left(\mathrm{S}_{\text {opt }}\right)$ of nano-CMs (Table 6.5). Increasing their optimal prey size increased the intraguild predation within this group, resulting in higher growth rates but lower population biomass. A cascade effect is then observed, because less prey would be available for micro-CMs, which are an important prey item for mesozooplankton in this scenario ( $\left.\mathrm{Cr}_{\text {micro-CMs }}\right)$. On the other hand, increasing $\alpha_{\mathrm{Chl}}$ and $\mathrm{ChlC}_{\mathrm{abs}}$ among CMs and diatoms supported higher trophic transfer efficiency, since these were the main prey supporting mesozooplankton biomass (positive coefficients in Table 6.5). Mesozooplankton intraguild predation ( $\mathrm{Cr}_{\text {mesozoo }}$ ) was also important and negatively impacted (i.e. decreased) the trophic transfer efficiency (ranked $\left.5^{\text {th }}\right)$. Regarding the production of labile DOC, the contribution of the major phototrophs (diatoms, picophytoplankton, and nano-CMs) was the main source of DOC in the lightlimited scenario, mainly driven by parameters controlling their phototrophic potential ( $\alpha_{\mathrm{Chl}}$ and ChlCabs; positive coefficients in Table 6.5). The optimal prey size of nano-CMs was also important, although to a lesser extent $\left(\mathrm{S}_{\mathrm{opt}}\right)$. 
In the nutrient-limited scenario, the parameterisation of bacteria and mixotrophs was more important (Table 6.6). Ammonium regeneration was negatively impacted by increasing the maximum prey size accessible by nano-CMs ( $\left.\mathrm{S}_{\max }\right)$, as well as its preferred prey size (Sopt) (negative coefficients in Table 6.6), because it favours the success of nanoCMs relative to their strict heterotrophic competitors. However, increases in the maximum phototrophic growth rate ( $\left.\mu_{\text {phot}}\right)$ of nano-CMs counterbalanced the negative effect of $S_{\text {opt. }}$ Maximum internal $\mathrm{N}$ or $\mathrm{P}$ to carbon quotas were also important (ranked $2^{\text {nd }}, 3^{\text {rd }}$, and $8^{\text {th }}$ ). Similar to the light-limited scenario, the trophic transfer efficiency was inversely related to the optimal prey size ( $\left.\mathrm{S}_{\mathrm{opt}}\right)$ of nano-CMs, resulting in less prey for GNCMs, which were an important prey for mesozooplankton under nutrient limitation (ranked $1^{\text {st }}$ and $3^{\text {rd }}$, respectively). A similar negative effect was observed when increasing $S_{\max }$ among mixotrophs (negative coefficients in Table 6.6). Parameters associated with picophytoplankton and bacteria were also important ( $\mu_{\text {phot }}$ and $\mathrm{NC}_{\max }$, respectively), but to a lesser extent than the previous ones (Table 6.6). The production of labile DOC was positively related (i.e., increased) with the maximum phototrophic growth rate of nanoCMs $\left(\mu_{\text {phot}}\right)$ and with parameters controlling the predation by NCMs ( $S_{\max }$; positive coefficients in Table 6.6). The internal stoichiometry regulation of mixotrophs and bacteria was also important, with a negative effect associated with $\mathrm{N}$ to $\mathrm{C}$ ratios (ranked $3^{\text {rd }}, 4^{\text {th }}$, and $6^{\text {th }}$ ) and a positive effect associated with $\mathrm{P}$ to $\mathrm{C}$ ratios (ranked $7^{\text {th }}$ and $8^{\text {th }}$ ).

The additional sensitivity analyses confirmed that averaged model outputs during the last year of the simulation were independent from initial conditions applied to plankton biomasses, for both light and nutrient limitation scenarios. No difference was observed for any of the ecosystem properties investigated or plankton biomasses. 
Table 6.5 Results of the Monte-Carlo sensitivity analyses for three targeted model outputs in the mixotrophic food web within the light-limited scenario (sensitivity coefficients of all parameters were statistically significant at $\mathrm{p}<0.001$ and $\mathrm{R}^{2}>0.9$ ). These are ranked with respect to their absolute value (most important first). Coefficient signs indicate a positive or negative effect on the targeted model outputs, i.e. increase or decrease of the output values, respectively.

\begin{tabular}{|c|c|c|c|}
\hline Targeted output & Functional type & Parameter & Coefficient \\
\hline \multirow[t]{8}{*}{ Ammonium regeneration } & Picophytoplankton & $\alpha_{\mathrm{Chl}}$ & 0.34 \\
\hline & Nano-CMs & $\mathrm{NC}_{\max }$ & -0.27 \\
\hline & Diatoms & $\alpha_{\text {Chl }}$ & 0.27 \\
\hline & Diatoms & $\mathrm{ChlC}_{\mathrm{abs}}$ & 0.27 \\
\hline & Picophytoplankton & $\mathrm{ChlC}_{\mathrm{abs}}$ & 0.24 \\
\hline & Micro-CMs & $\mathrm{NC}_{\max }$ & -0.21 \\
\hline & SNCMs & $\mathrm{NC}_{\max }$ & -0.18 \\
\hline & Bacteria & $\mathrm{NC}_{\max }$ & 0.17 \\
\hline \multirow[t]{8}{*}{ Trophic transfer efficiency } & Nano-CMs & $S_{\text {opt }}$ & -0.31 \\
\hline & Diatoms & $\alpha_{\mathrm{Chl}}$ & 0.27 \\
\hline & Diatoms & ChlC $_{\text {abs }}$ & 0.22 \\
\hline & Nano-CMs & ChlC $_{\text {abs }}$ & 0.20 \\
\hline & Mesozooplankton & $\mathrm{Cr}_{\text {mesozoo }}$ & -0.19 \\
\hline & Picophytoplankton & ChlC $_{\text {abs }}$ & -0.19 \\
\hline & Mesozooplankton & $\mathrm{Cr}_{\text {micro-CMs }}$ & 0.17 \\
\hline & Nano-CMs & $\alpha_{\text {Chl }}$ & 0.17 \\
\hline \multirow[t]{8}{*}{ Production of labile DOC } & Diatoms & $\alpha_{\mathrm{Chl}}$ & 0.50 \\
\hline & Diatoms & ChlC $_{\text {abs }}$ & 0.40 \\
\hline & Picophytoplankton & $\alpha_{\mathrm{Chl}}$ & 0.31 \\
\hline & Picophytoplankton & ChlC $_{\text {abs }}$ & 0.16 \\
\hline & Nano-CMs & $\alpha_{\text {Chl }}$ & 0.15 \\
\hline & Nano-CMs & ChlC $_{\text {abs }}$ & 0.12 \\
\hline & Nano-CMs & $S_{\text {opt }}$ & 0.11 \\
\hline & Diatoms & BR & -0.09 \\
\hline
\end{tabular}

$\alpha_{\mathrm{Chl}}$, initial slope of photosynthesis-irradiance curve; $\mathrm{BR}$, basal respiration rate; $\mathrm{ChlC}_{\text {abs }}$, absolute maximum $\mathrm{Chl}: \mathrm{C}$ ratio; $\mathrm{Cr}$ : slope of capture-prey abundance curve; $\mathrm{NC}_{\max }$, maximum $\mathrm{N}: \mathrm{C}$ ratio; $\mathrm{S}_{\text {opt: }}$ : optimum prey size. 
Table 6.6 Results of the Monte-Carlo sensitivity analyses for three targeted model outputs in the mixotrophic food web within the nutrient-limited scenario (sensitivity coefficients of all parameters were statistically significant at $\mathrm{p}<0.001$ and $\mathrm{R}^{2}>0.9$ ). These are ranked with respect to their absolute value (most important first). Coefficient signs indicate a positive or negative effect on the targeted model outputs, i.e., increase or decrease of the output values, respectively.

\begin{tabular}{|c|c|c|c|}
\hline Targeted output & Functional type & Parameter & Coefficient \\
\hline \multirow[t]{8}{*}{ Ammonium regeneration } & Nano-CMs & $\mathrm{S}_{\max }$ & -0.46 \\
\hline & Nano-CMs & $\mathrm{NC}_{\max }$ & -0.20 \\
\hline & Bacteria & $\mathrm{NC}_{\max }$ & 0.14 \\
\hline & GNCMs & $\mathrm{S}_{\max }$ & 0.11 \\
\hline & Nano-CMs & $\mu_{\text {phot }}$ & 0.10 \\
\hline & Nano-CMs & $\mathrm{S}_{\mathrm{opt}}$ & -0.10 \\
\hline & SNCMs & $\mathrm{S}_{\max }$ & 0.09 \\
\hline & SNCMs & $\mathrm{PC}_{\max }$ & 0.06 \\
\hline \multirow[t]{8}{*}{ Trophic transfer efficiency } & Nano-CMs & $\mathrm{S}_{\mathrm{opt}}$ & -0.42 \\
\hline & Nano-CMs & $\mathrm{S}_{\max }$ & -0.29 \\
\hline & Mesozooplankton & $\mathrm{Cr}_{\mathrm{GNCMs}}$ & 0.21 \\
\hline & GNCMs & $\mathrm{S}_{\max }$ & -0.21 \\
\hline & SNCMs & $\mathrm{S}_{\max }$ & -0.16 \\
\hline & Nano-CMs & $\mathrm{S}_{\min }$ & -0.13 \\
\hline & Picophytoplankton & $\mu_{\text {phot }}$ & -0.12 \\
\hline & Bacteria & $\mathrm{NC}_{\max }$ & 0.11 \\
\hline \multirow[t]{8}{*}{ Production of labile DOC } & Nano-CMs & $\mu_{\text {phot }}$ & 0.33 \\
\hline & SNCMs & $S_{\max }$ & 0.32 \\
\hline & Nano-CMs & $\mathrm{NC}_{\min }$ & -0.31 \\
\hline & Bacteria & $\mathrm{NC}_{\max }$ & -0.30 \\
\hline & GNCMs & $\mathrm{S}_{\max }$ & 0.25 \\
\hline & Nano-CMs & $\mathrm{NC}_{\max }$ & -0.20 \\
\hline & GNCMs & $\mathrm{PC}_{\max }$ & 0.19 \\
\hline & Bacteria & $\mathrm{PC}_{\max }$ & 0.17 \\
\hline
\end{tabular}

Cr: slope of capture-prey abundance curve; $\mathrm{NC}_{\max }$, maximum $\mathrm{N}: \mathrm{C}$ ratio; $\mathrm{NC}_{\min }$, minimum $\mathrm{N}: \mathrm{C}$ ratio; $\mu_{\text {phot }}$, maximum phototrophic growth rate; $\mathrm{PC}_{\max }$, maximum $\mathrm{P}: \mathrm{C}$ ratio; $\mathrm{S}_{\max }$, maximum prey size; $\mathrm{S}_{\min }$, minimum prey size; Sopt: optimum prey size. 


\subsection{Discussion}

This study suggests that the interpretations and predictions of the functioning of the marine planktonic ecosystem could radically change if we consider mixotrophic functional diversity in ocean models, with mixotrophy impacting nutrient availability, mass and energy transfer to higher trophic levels, and the microbial loop (Figs. 6.3-6.6). The simulations show that the relative dominance of different mixotrophic functional groups can shape the planktonic ecosystem in different ways depending on light and nutrient regimes. Size was shown to be important to determine the success of mixotrophs with an innate capacity for photosynthesis; while small cells dominated under nutrient limitation, larger cells were more important under light limitation (Fig. 6.8a). Among acquired phototrophs, the specificity of the prey from which kleptochloroplasts are obtained affected their success, with generalist forms dominating under nutrient limitation and specialist forms showing maximal contribution in intermediate conditions of light and nutrients and under nutrient limitation (Fig. 6.8b).

a

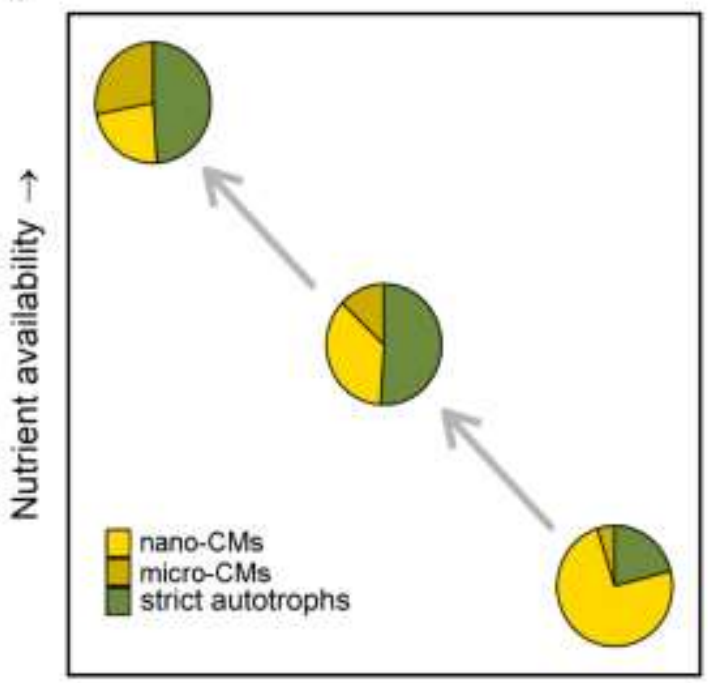

Irradiance $\rightarrow$ b

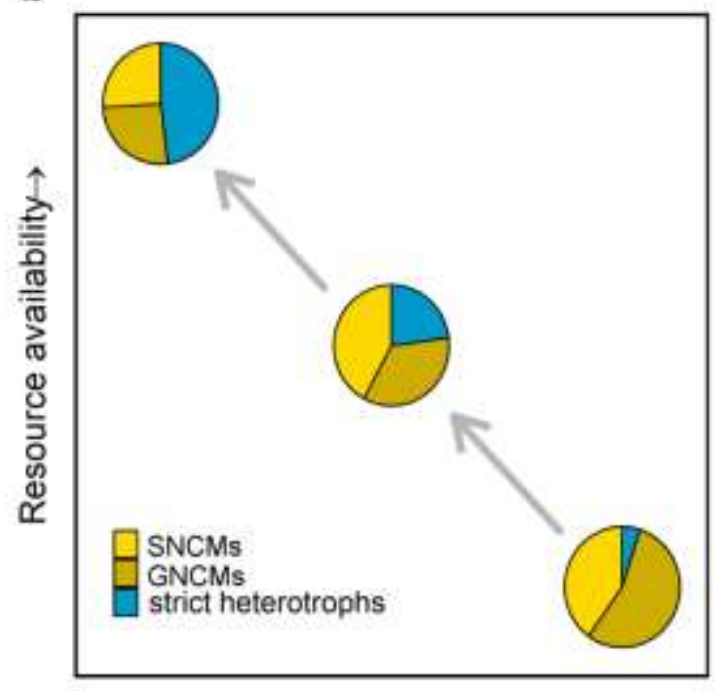

Irradiance $\rightarrow$

Figure 6.8 Relative biomass of mixotrophic, strict autotrophic, and strict heterotrophic protists in a gradient from nutrient to light limitation. a) CMs and their strict autotrophic competitors; b) NCMs and their strict heterotrophic competitors. Data were averaged for the last year of simulation. Note that area corresponding to high nutrient and high irradiance (upper right corner of the panels) are potentially mutually exclusive due to selfshading. 


\subsubsection{Model output and empirical observations}

The results presented here appear consistent with empirical observations. The nano-CMs and GNCMs (e.g. oligotrich ciliates) have been reported to be important members within oligotrophic gyres and during summer within temperate seas (Stoecker et al., 1987; Zubkov and Tarran, 2008; Hartmann et al., 2012; Unrein et al., 2014; Haraguchi et al., 2018). In contrast, micro-CMs and SNCMs (e.g., Mesodinium rubrum) can be major components of plankton assemblages in eutrophic coastal environments and during winter within temperate seas (Burkholder et al., 2008; Jeong et al., 2010c, Hansen, 2011; Johnson et al., 2013). The simulations also produced realistic estimates of the biomass ratios between NCMs and their heterotrophic competitors. In the light-limited scenario, the model predicted coexistence of NCMs and their heterotrophic counterparts, with the latter comprising half of the total assemblage (Fig. 6.8b). These results were consistent with previous observations showing that strict heterotrophs comprise on average $60 \%$ of total ciliate biomass during winter within coastal temperate seas (Chapter 3). Once limited by prey availability, strict heterotrophs survived at a very low biomass only accounting for $5 \%$ of the total assemblage (Fig. 5b). Overall, this value is lower than expected during summer (Chapter 3); minimum values were reported in the Mediterranean Sea and in the Northwest Atlantic Shelves, in which heterotrophic microzooplankton accounted for less than 15\% of total ciliate biomass (Stoecker et al. 1987; Modigh, 2001; Bernard and Rassoulzadegan, 1994). It was not possible, however, to compare the biomass ratios of nano-CMs and heterotrophic nanoflagellates (as per Chapter 4) because the latter were outcompeted in all simulations (Figs. 6.4 and 6.6).

\subsubsection{System stability and acquired phototrophy}

Acquired phototrophy has been suggested to stabilise coexistence between NCMs and the prey that provides their phototrophic potential (Moeller et al., 2016). However, the nature of this coexistence would depend on light availability, with the amplitude of repeating biomass cycles increasing with irradiance (Moeller et al., 2016). In a food web considering bottom-up (nutrients) and top-down (higher predators) controls, the amplitude of repeating cycles was considerably lower under high-light and low-nutrient conditions, approaching a constant steady-state (Fig. 6.9). In addition, when interactions between 
SNCMs, GNCMs, and strict heterotrophs were allowed, the model became more unstable with one group slowly outcompeting the others. Defining the differences between these groups is challenging. For instance, experimental evidence found similar maximum growth rates and inorganic $\mathrm{N}$ uptake between strict heterotrophs and GNCMs (Schoener and McManus, 2017). Although the assumptions of the model presented here were based on the current literature, there is little quantitative information on the costs and benefits associated to acquired phototrophy (Dolan and Pérez, 2000; Stoecker et al., 2009; McManus et al., 2012). The sensitivity experiments suggest that defining the prey size spectrum accessible and selected by each of these groups significantly impact the targeted outputs (Tables 6.5 and 6.6).

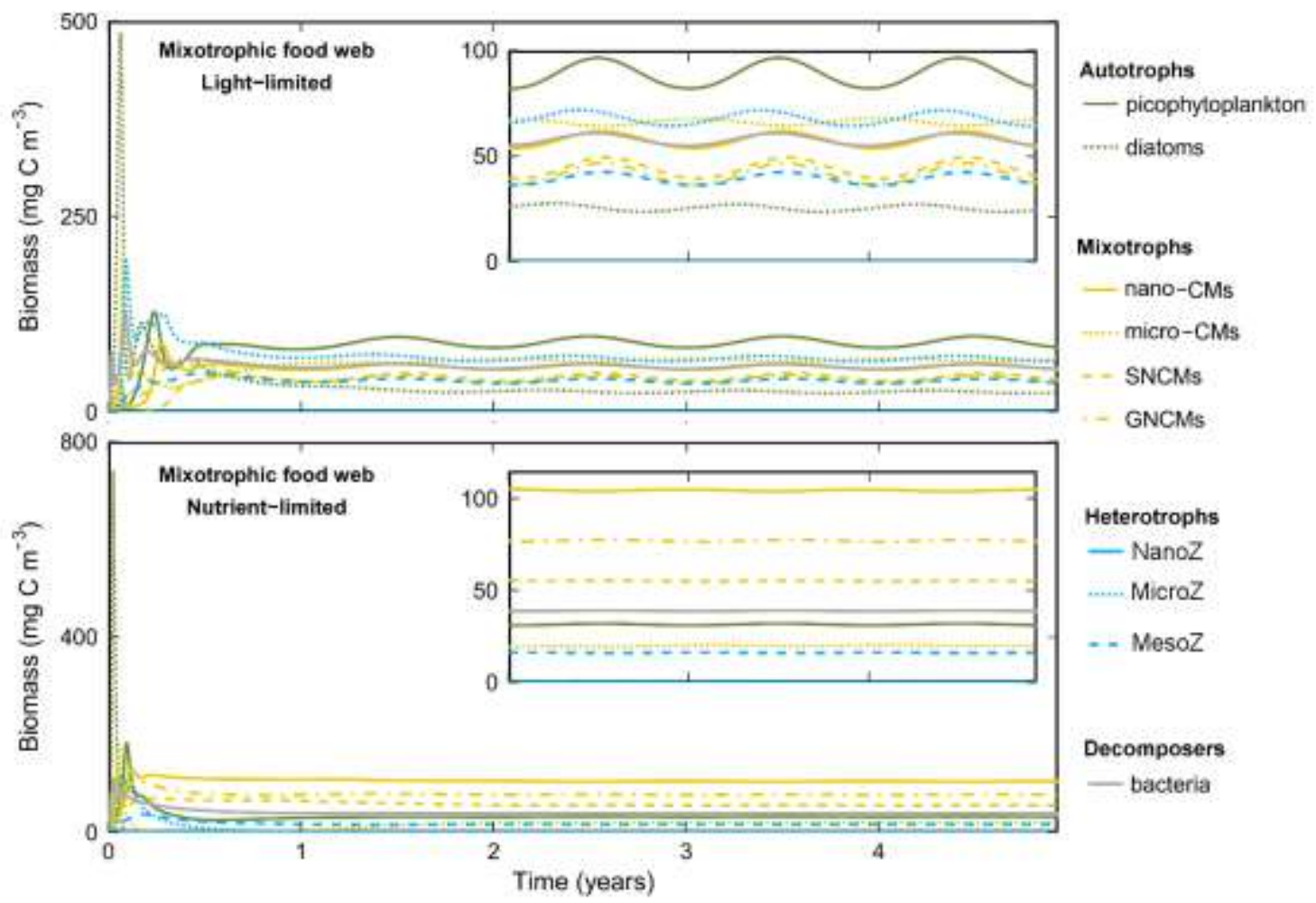

Figure 6.9 Population dynamics within the mixotrophic food web under light (upper panel) or nutrient (lower panel) limitation scenarios. Lines represent different functional types and colours indicate different trophic strategies (green - autotrophs, yellow mixotrophs, blue - heterotrophs, grey - decomposers). See also the Abbreviations section. 


\subsubsection{Nutrient cycling, trophic transfer, and DOC production}

This modelling study predicted that the dominance of mixotrophs over their strict autotrophic and heterotrophic counterparts increases in the transition from light to nutrient limitation (Fig. 6.8). Mixotrophs can outcompete strict autotrophs and strict heterotrophs by using nutrients more efficiently. Indeed, when nutrient-rich prey are ingested any surplus of $\mathrm{N}$ and $\mathrm{P}$ may be combined with newly fixed carbon instead of being excreted outside the cell (Rothhaupt, 1997; Flynn and Mitra, 2009; Fischer et al., 2017). In addition, certain species of acquired phototrophs can take up inorganic nutrients (HattenrathLehmann and Gobler, 2015; Qiu et al., 2016). These features minimise the remineralization of nutrients to the environment enhancing the bottom-up control of strict autotrophs under nutrient limitation and favouring mixotrophs over strict heterotrophs under low prey availability (Fig. 6.8). Thus, the simulations indicate that the presence of not only CMs but also NCMs can decrease nutrient regeneration. This is consistent with previous findings showing that the dinoflagellate Dinophysis acuminata and the ciliate Mesodinium rubrum take up inorganic nutrients; these two species are classifiable as SNCMs as they must acquire kleptochloroplasts from M. rubrum and red cryptophyte algal prey, respectively (Hattenrath-Lehmann and Gobler, 2015; Qiu et al., 2016). Recent evidence also shows that oligotrich ciliates (i.e. GNCMs) can take up inorganic nutrients, although it might not contribute significantly to their growth (Schoener and McManus, 2017).

Mixotrophs have been previously suggested to increase the trophic transfer efficiency to higher trophic levels (Stoecker et al., 2009; Ward and Follows, 2016; Stoecker et al., 2017). The simulations presented here support and expand this ecological concept by considering the functional diversity among mixotrophs and their strict autotrophic and heterotrophic competitors. The results suggest that $\mathrm{CMs}$ have a competitive advantage over strict autotrophic competitors, particularly under nutrient limitation, allowing the accumulation of biomass in larger prey instead of in pico-sized prey, which are too small to be consumed by higher consumers. In turn, CMs provide photosynthetic potential to NCMs, which composed the bulk of biomass that sustained higher trophic levels (Fig. 6.5b). In fact, crustacean zooplankton and fish larvae 
preferentially prey on NCMs, such as oligotrich ciliates and M. rubrum, rather than on their strict auto- and hetero- trophic competitors (Broglio et al., 2004; Figueiredo et al., 2007). This study suggests that $\sim 50 \%$ of mesozooplankton diet was composed by NCMs in the nutrient-limited scenario versus $20 \%$ under light limitation. These values are consistent with observations showing that the relative importance of the consumption of NCMs by copepods varies across environmental gradients, increasing towards less productive systems (Calbet and Saiz, 2005).

Mixed nutrition may also increase the release of labile DOC among protists (Flynn et al., 2008; Mitra et al., 2014b). The theoretical framework presented here provides a platform to explore how this might affect the production of recalcitrant DOC by bacteria. The results showed higher production of labile DOC in the mixotrophic food web only when nutrients were limiting, stimulating bacterial biomass (Mitra et al., 2014b) and, consequently, boosting the production of recalcitrant DOC (Fig. 6.3c vs Fig. 6.5c). The main source responsible for the higher production of labile DOC on this scenario was the increased release of labile DOC by protists (Fig. S8). This release, in turn, was induced by higher prey consumption combined with an overall poor prey quality, described here by internal N:C and P:C quotas (Mitra, 2006; Polimene et al., 2015). Overall, the stronger nutrient limitation in the presence of mixotrophs resulted in lower prey quality and hence less efficient microzooplankton. However, it is noteworthy that the production of labile DOC and hence of recalcitrant DOC is strongly dependent on model assumptions, particularly in the partitioning of voided material between particulate and dissolved pools.

It is also important to note that this model lacked the description of osmotrophy among CMs (Ghyoot et al., 2017b), which can decrease the net production of DOC, or even change the mixotroph from a source of DOC into a sink.

\subsubsection{Model caveats}

The results presented in this chapter are dependent on several assumptions and uncertain parameters, but the aim was to explore the emerging paradigm in marine ecology, in which the phytoplankton-zooplankton dichotomy no longer holds. Constitutive mixotrophy was particularly important to maintain phototrophy within nanoand micro- plankton size classes, which would be outcompeted by picophytoplankton 
otherwise. This result appears robust because it is mainly related to the overall predation impact, which is lower among picophytoplankton as predicted by allometric constraints. The success of different phototrophs is also dependent on their phototrophic capacities and internal stoichiometric quotas, as showed by the sensitivity tests, and these parameters are well characterised in the literature (Table 6.1). On the other hand, mixotrophs were too dominant relative to their heterotrophic competitors in the simulations. While it was possible to compile information on their total prey size spectrum (Table 5.1), it seems that we still lack information on the costs associated to mixotrophy. Further sensitivity analyses can help to parameterize the cost associated with the mixotrophic trait. Monte Carlo simulations can be used to search and identify the parameter values that minimize the error (with respect to data) to allow the survival of heterotrophic nanoflagellates. Similarly, this analysis can be used to produce more realistic scenarios in which strict heterotrophic microzooplankton coexist with NCMs whilst consuming each other. Finally, the results also suggest that NCMs may act as a sink or source of inorganic nutrients, depending on environmental conditions. Similarly, quantitative studies on the cycling of DOM by mixotrophs and consequently in the production of recalcitrant DOC by bacteria, can help to elucidate the significance of mixotrophy to the microbial carbon pump.

Despite model caveats, the importance of mixotrophy in the environmental setups used in the simulation experiments can have profound consequences in view of climatic and anthropogenic changes on the oceans, particularly in oligotrophic seas and eutrophic coastal systems. Warmer waters and stronger stratification have been previously hypothesised to favour mixotrophic plankton in oligotrophic seas (Polovina et al., 2008; Wilken et al., 2013; Behrenfeld et al., 2016). In turn, increased eutrophication in coastal waters can induce light-limitation and promote the formation of harmful algal blooms, many of which are mixotrophic species (Burkholder et al., 2008; Gomes et al., 2014). This study provides the basis for the mechanisms giving competitive advantages to different mixotrophs relative to their strict auto- and hetero- trophic competitors under such environmental conditions (i.e., nutrient or light limitation). In view of the results presented here, future studies aiming to predict the impact of environmental changes on the oceanic food webs should consider the mixotrophic potential of plankton communities. 


\subsection{Summary}

Mixotrophic functional diversity can significantly alter our understanding of ecosystem dynamics within the lower trophic levels of marine food webs, with key groups of mixotrophs controlling nutrient regulation, trophic transfer, and the microbial loop. The modelling study predicted predominance of nano-CMs and GNCMs in nutrient depleted conditions (akin to oligotrophic oceans), and a higher importance of micro-CMs and SNCMs under light limited conditions (e.g., eutrophic coastal systems). This is the first time that the roles of different mixotroph types have been explored simultaneously within plankton food webs. This work demonstrates the importance of deploying detailed descriptions of mixotroph physiology. It also shows how mixotrophy interacts in the direct and indirect control of the growth of strict autotrophic and heterotrophic populations, particularly under nutrient limitation. Moreover, it was demonstrated how mixotrophy can promote the transfer of carbon biomass to higher planktonic predators through the interplay between CMs and NCMs. Critically, a food web framework has been constructed for comprehensive quantitative exploration of the role of mixotrophic functional diversity in marine ecosystems, which can be readily implemented in a variety of settings: from chemostats to spatially structured models of the water column (1D) and the global ocean (3D). It thus provides a powerful tool to investigate the role of mixotrophy in a changing ocean. 


\section{Chapter 7}

\section{Seasonal succession of protist trophic strategies in a coastal temperate sea}




\subsection{Introduction}

It is now well recognised that mixotrophy is an important trait under conditions of resource limitation (Troost et al., 2005; Flynn et al., 2013; Stoecker et al., 2017). More attention has been given to the importance of mixotrophy in mature systems, such as within oligotrophic seas, including summer conditions in temperate seas (Zubkov and Tarran, 2008; Unrein et al., 2014; Mitra et al., 2014b; Stoecker et al., 2009). Under these conditions, mixotrophy is mainly seen as an alternative route used by constitutive mixotrophs to acquire nutrients and by non-constitutive mixotrophs to get an extra source of carbon (Mitra et al., 2016). These findings were particularly important because they change the way we understand the microbial loop and the cycling of materials in nutrient limited waters (Mitra et al., 2014b). However, mixotrophy is also common in other oceanic ecosystems (Burkholder et al., 2008; Stoecker et al., 2017) and global analyses revealed mixotrophy to be ubiquitous across different spatio-temporal scales in the oceans (Chapters 3 and 4). For instance, empirical studies have shown the importance of mixotrophy during winter within a coastal upwelling area and throughout the water column in the Baltic Sea (Vargas et al., 2012; Weber et al., 2014). The diversity of mixotrophic strategies and the synergy between phototrophy and phagotrophy allow mixotrophs to be selected under a range of environmental conditions (Mitra and Flynn, 2010; Mitra et al., 2016, Edwards, 2019). The challenge now is to better understand the mechanisms that allow mixotrophs to thrive in different ecosystems and how these relate to the succession of plankton communities (Andersen et al., 2015; Berge et al., 2017).

The succession of plankton communities is a result of physical forcing and both bottom-up and top-down controls, which can be translated into competitive outcomes and (mainly) predation losses (Sverdrup, 1953; Margalef, 1978; Reynolds, 1984; Sommer et al., 2012; Calbet, 2001; Calbet and Landry, 2004). Defining the competitive abilities between mixotrophic protists and their competitors is not a trivial task. Mixotrophic protists compete at the same time with strict autotrophs and strict heterotrophs and depending on the strategy adopted by the mixotroph different competitive outcomes may be expected (Rothhaupt, 1996; Dolan and Pérez, 2000; Flynn and Hansen, 2013; Fischer 
et al., 2017; Moeller et al., 2016). To add more complexity, most mixotrophs adopt the strategy of 'eating your competitor' and, therefore, also act as predators (Thingstad et al., 1996; Burkholder et al., 2008; Jeong et al., 2010c; Hansen, 2011). Experimental studies provide valuable information about the competitive abilities of mixotrophs and their specialised competitors; however, these are usually limited to 2-3 species kept under controlled conditions and cannot access directly top-down controls. Considering that the fitness of a population depends on the traits of organisms and the sum of ecosystem processes, one could argue that fitness is an emergent property from simulations (Flynn et al., 2015). Therefore, incorporating realistic environmental variability into models and informing these with empirical data can shed light into protist succession allowing the evaluation of bottom-up and top-down controls (e. g., Bruggeman and Kooijman, 2007).

Due to the stratification of the water column and the wide variations in light and temperature gradients, temperate seas function as model systems to study plankton seasonal succession (e.g., Bode and Fernández, 1992; Blight et al., 1995; Sommer and Lengfellner, 2008; Bunse and Pinhassi, 2017). Traditionally, it is envisaged that at the onset of thermal stratification, light is no longer limiting phototrophic growth and fastgrowing species (diatoms) tend to dominate the spring bloom (Sverdrup, 1953; Litchtman et al., 2007). Nutrient depletion and zooplankton predation are then expected to act curtailing the spring bloom. As stratification develops during summer, predators become food limited and phototrophic species with higher competitive abilities for nutrient uptake are expected to thrive (Edwards et al., 2013). As mixing increases during autumn, a second bloom might be observed if timed with still favourable light conditions (Sommer et al., 2012) and non-limiting silicate concentrations (Egge and Aksnes, 1992). These mechanisms are based mainly on physical disturbance, nutrient affinities, and predation; other important features have received far less attention in the past decades, such as zooplankton overwintering, parasitism, food quality, and the role of heterotrophic protists; therefore, the seasonal succession of plankton has been revisited (Sommer et al., 2012; Glibert et al., 2016; Bunse and Pinhassi, 2017; Atkinson et al., 2018).

Mixotrophy is another important feature to be added to this list (Bruggeman, 2009; Mitra et al., 2014b; de Vargas et al., 2015; Stoecker et al., 2017; Berge et al., 2017). Due 
to methodological limitations, few empirical studies have accounted for mixotrophy when evaluating the seasonal succession of protists and these usually consider only specific groups of mixotrophs, usually mixotrophic dinoflagellates (e.g., Barton et al., 2013a; Gran-Stadniczeñko et al., 2019). Similarly, it is also challenging to compare model predictions with observational data when evaluating mixotrophy; therefore, most modelling studies have been applied in a theoretical way (Table 1.1).

Here, the mechanisms driving the seasonal succession of protist trophic strategies (autotrophs, mixotrophs, and heterotrophs) were explored using an ecosystem model applied in a coastal stratified temperate sea, the Western English Channel at station L4 (Fig. 7.1). This study is driven mainly by three motivations: i) to investigate the relevance of mixotrophy during different periods across the seasonal cycle, ii) to evaluate how mixotrophic populations change vertically within the water column and, iii) to integrate different mixotrophic forms in a single modelling framework comparing it against observational data. The plankton food webs were based on plankton functional types and coupled to a 1D model of the water column (Chapter 5). The physical model is key to address this question since it explicitly represents depth and incorporates realistic environmental variability (i.e., temperature, light, and mixing). Based on previous modelling results that showed different mixotrophs dominating under different light and nutrient regimes (Chapter 6), different mixotrophs were included in the model to evaluate if mixotrophic diversity influences the success of mixotrophs across the seasonal cycle and throughout the water column.

\subsection{Methods}

\subsubsection{Model set-up and skill assessment}

In this Chapter, the plankton food web models described in Chapter 5 were coupled to a $1 \mathrm{D}$ physical model to investigate the seasonal succession of protists at station L4 (Chapter 7.2.2), with emphasis on different trophic strategies. Simulations by the mixotrophic and the non-mixotrophic food web models were compared to evaluate the effect of mixotrophy on the prediction of observational data. The mixotrophic model was also compared against the standard ERSEM (Butenschön et al., 2016). It is noteworthy 
that it was not possible to disentangle the effect of mixotrophy when comparing the mixotrophic model with ERSEM because these differ in structural terms, e.g., the way processes are modelled, which may involve different functional forms and parameters, and the way fluxes are connected to the different nutrient pools. However, this comparison allowed to evaluate how well the mixotrophic model can predict observational data compared to a model which is well stablished in the literature (Baretta-Bekker et al., 1995; Blackford et al., 2004; Butenschön et al., 2016). Here details are given with respect to the model set-up and skill assessment.

The biogeochemical models were implemented at L4 station through GOTM (Chapter 5.3). GOTM was set to resolve 100 vertical layers with increasing resolution towards surface waters and assuming a water column of $50 \mathrm{~m}$ depth. The biogeochemical models were run over a period of 9 years (coinciding with the period of the observational data; Chapter 7.2.2) after a 2-year spin-up period assuming a time step of 1000 seconds. Model output was obtained daily. The computational time required to run ERSEM was 20 minutes while the non-mixotrophic and the mixotrophic models required $\sim 30$ minutes.

Temperature and salinity profiles from CTD casts were used to initialise the model and, to improve the representation of the water column structure, were assimilated approximately every week into the physical model using a relaxation scheme to account for advection processes not implicitly modelled (Burchard et al., 1999; Torres et al., 2006). This method is particularly useful when simulating physical dynamics in a coastal region, in which horizontal gradients in temperature and salinity are important to determine water column structure (Verspecht et al., 2009). Meteorological data (e.g., air temperature, cloud cover, and wind speed) were used to compute fluxes of heat and momentum and were obtained from the European Centre for Medium-Range Weather Forecasts (ECMWF) using the ERA-interim products which provide data with a temporal resolution of 6 hours (Simmons et al., 2007).

Surface radiation was computed from latitude, longitude, time, fractional cloud cover and albedo. Following Butenschön et al. (2016), the photosynthetically active radiation (PAR) was computed from the shortwave radiation in the surface and an 
attenuation coefficient dependent on the concentration of plankton and particulate organic matter as well as background extinction due to clear seawater; light attenuation was modelled based on inherent optical properties. It is noteworthy that light at each vertical layer is computed as an integrated value divided by the layer depth. Tides are represented by prescribing time-varying sea surface elevation and its horizontal gradient. All models were initialised with in situ measurements of temperature, salinity, and nutrient concentrations observed during winter (e.g., DIN $=9 \mu \mathrm{M}$, DIP $=0.5 \mu \mathrm{M}$, silicate $=4.5$ $\mu \mathrm{M}$, ammonium $=0.1 \mu \mathrm{M}$; Smyth et al., 2010). All models were implemented using the ERSEM benthic coupling following Butenschön et al. (2016) and resuspension was not enabled.

Observational data used to validate the models comprise of inorganic nutrient concentrations, chlorophyll-a, and plankton biomass (Chapter 7.2.2). Model validation was initially analysed comparing simulations with observational data over the whole studied period (i.e., 9 years). However, the aim of this Chapter is to investigate the seasonal succession of protist trophic strategies; therefore, model output was averaged by month of every year and then averaged again over the years so that climatological means could be obtained. Models were then compared against observations and between themselves plotting the global monthly means and corresponding standard deviations. In addition, the correlation coefficient, the root mean squared error (RMSE), and the average error (AE) were computed and interpreted through target diagrams (Chapter 5.6). The metrics provided in the target diagrams were the normalised average error $\left(\mathrm{AE}^{*}\right)$ in the abscissa and the normalised and unbiased RMSE (RMSE*) in the ordinate (Jolliff et al., 2009; Chapter 5.6). Please refer to the Abbreviations section for other shortenings hereinafter.

\subsubsection{The $L 4$ long-term time series}

The L4 station is located $13 \mathrm{~km}$ SSW of Plymouth, in the Western English Channel, UK $\left(50^{\circ} 15^{\prime} \mathrm{N}, 4^{\circ} 13^{\prime} \mathrm{W}\right.$; Fig. 7.1), with a mean water depth of $54 \mathrm{~m}$ (Harris, 2010; Smyth et al., 2010). This monitoring station is classified as transitionally mixed, being subjected to occasional riverine inputs of the Tamar estuary as determined by rainfall, wind mixing, and tides (Smyth et al., 2010). During periods of strong southwest 
winds its hydrography is mainly influenced by oceanic water (Rees et al., 2009). Temperatures range from 9 to $16.5^{\circ} \mathrm{C}$ over the seasonal cycle and mean chlorophyll concentrations are $\sim 1 \mu \mathrm{g} \mathrm{L}^{-1}$ (Smyth et al., 2010); however, values can be notably higher if blooms are observed (Barnes et al., 2015).

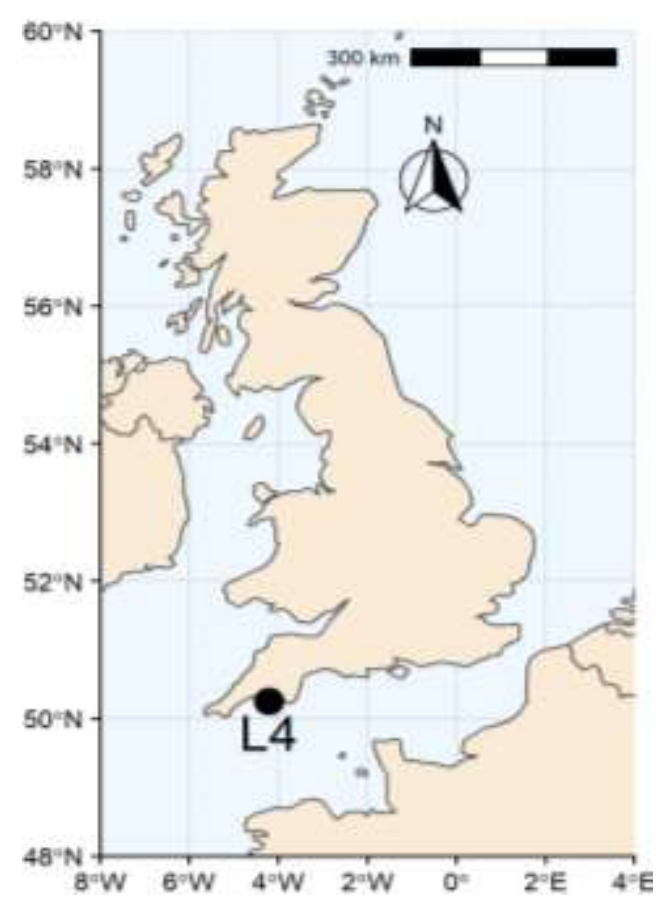

Figure 7.1 The study area (Western English Channel, UK) showing the L4 monitoring station.

The L4 long-term oceanographic and marine biodiversity time series is part of the Western Channel Observatory (WCO, https://www.westernchannelobservatory.org.uk/). The L4 observational data used here includes temperature, salinity, inorganic nutrients, chlorophyll-a, and carbon biomass of all plankton functional types included in the food web model. Data were obtained (quasi) weekly at $10 \mathrm{~m}$ depth on board of the RV Plymouth Quest. Although microplankton samples are obtained at L4 station since October 1992 (Widdicombe et al., 2010), observations from January 2006 to December 2014 were selected due to a lack of consistency regarding ciliates identification prior to this period; however, note that data from the flow cytometry and chlorophyll-a measurements (at $10 \mathrm{~m}$ depth) were available only from 2007 onwards. Data collection and analyses are described below; for full details please refer to Smyth et al. (2010) for 
physico-chemical data, Tarran and Bruun (2015) for nano- and pico- plankton data, Widdicombe et al. (2010) for microplankton data, and Eloire et al. (2010) and Atkinson et al. (2015) for mesozooplankton data.

Vertical profiles of temperature and salinity were obtained using a SeaBird CTD SBE19+ (Smyth et al., 2010). Inorganic nutrient concentrations (i.e., nitrate, nitrite, phosphate, ammonium, and silicate) were determined from live samples following recognised analytical techniques based on colorimetry (Woodward and Rees, 2001). Water samples (1-2 L) for chlorophyll-a determination were filtered through GF/F filters and stored in liquid nitrogen until analysis (Smyth et al., 2010). Then, chlorophyll-a concentrations were determined following chlorophyll-a extraction by centrifugation and measurement by a reversed-phase HPLC (Smyth et al., 2010).

Plankton samples were obtained using a 10 L Niskin bottle mounted on a rosette sampler which also housed the SeaBird CTD SBE19+. Pico- and nano- plankton subsamples were decanted in polycarbonate bottles and processed generally within $3 \mathrm{~h}$ (Tarran and Bruun, 2015). These samples were fixed with glutaraldehyde (1\% final concentration) and later stained for enumeration and sizing by flow cytometry (Tarran and Bruun, 2015). Pico- and nano- plankton cell volumes $\left(\mu \mathrm{m}^{3}\right)$ were calculated from approximations to simple geometric shapes and converted to carbon biomass ( $\mu \mathrm{g} \mathrm{C}$ cell $^{-1}$ ) using carbon to volume relationships from the literature (Fagerbakke et al., 1996; Menden-Deuer and Lessard, 2000; Romanova and Sazhin, 2010; Buitenhuis et al., 2012) and then multiplied by the numeric abundance (cells $\left.\mathrm{L}^{-1}\right)$ to derive the total carbon biomass $\left(\mu \mathrm{g} \mathrm{C} \mathrm{L}^{-1}\right)$. Microplankton samples were fixed with Lugol's iodine solution ( $2 \%$ final concentration) and kept in cool and dark conditions until identification, enumeration, and sizing were undertaken using the Utermöhl counting technique under an inverted microscope (Utermöhl, 1958); taxa were identified down to species level for most microplankton taxa usually settling $100 \mathrm{~mL}$ of the samples (Widdicombe et al., 2010). Carbon biomass (taxa specific) were determined following the same methodology as described above for picoand nano- plankton, using a general carbon to volume relationship for protists (MendenDeuer and Lessard, 2000). 
Mesozooplankton were collected with two replicate $0-50 \mathrm{~m}$ vertical hauls with a WP2 net (0.57 m diameter, $200 \mu \mathrm{m}$ mesh) and samples were fixed with $5 \%$ formalin (Eloire et al., 2010). Identification, enumeration, and sizing were conducted under a dissecting microscope to estimate numeric abundance as number of organisms per cubic meter (Eloire et al., 2010) and carbon biomass was estimated through CHN analysis (Atkinson et al., 2015).

\subsubsection{Assigning plankton taxa to functional types}

The plankton taxa within the L4 dataset were assigned to the different plankton functional types included in the food web models (Fig. 5.1). In the non-mixotrophic food web and within ERSEM, bacteria and picophytoplankton were validated by both high and low nucleic acid-containing bacteria and Synechococcus and picoeukaryotes, respectively, as enumerated by flow cytometry (Tarran and Bruun, 2015). Nanoplankton were also validated using flow cytometry data; heterotrophic nanoflagellates were enumerated as a single group and autotrophic nanoflagellates were enumerated as cryptophytes, coccolithophores, Phaeocystis (single cells) and dinoflagellates $<20 \mu \mathrm{m}$ (Tarran and Bruun, 2015). Among microplankton, the list of taxa was assigned as following: diatoms (Table E.1), autotrophic microflagellates (Table 7.1), and microzooplankton (Tables 7.1 and Table E.2). Mesozooplankton taxa can also be found in Appendix E (Table E.3).

In the mixotrophic model, changes were made in order to account for different mixotrophic functional types, which were identified according to the literature (Appendix A, Table A.1; Appendix B, Table B.1; and Table 7.1). In the case of absence of evidence for mixotrophy, protist taxa commonly perceived as pure phototrophs (with the exception of diatoms and picophytoplankton) were assumed to be CMs (Table 7.1) following the review by Flynn et al. (2013). Therefore, the datasets used to validate autotrophic nanoflagellates and microflagellates in the previous models were used to validate nanoCMs and micro-CMs instead (Table 7.1). In turn, the dataset used to validate microzooplankton was split in the mixotrophic model to account for GNCMs and SNCMs (Table 7.1). Acquired phototrophy has not been investigated among Dinophysis cf. punctata and Dinophysis nasuta; however, all species of the genus Dinophysis were assumed to be NCMs (Nishitani et al., 2012). Although taxa were classified between 
SNCMs and GNCMs, initial simulations showed that the mixotrophic model was not able to predict their coexistence, with GNCMs being outcompeted after the first years of simulation. The mixotrophic food web model was then simplified to account for a single box representing NCMs, which was then validated against the total biomass of NCMs (i.e., SNCMs plus GNCMs) observed at L4 station. NCMs were modelled assuming a specialist behaviour, i.e., phototrophic capacity could only be obtained from nano-CMs, but all other parameters were set to conform to the representation of GNCMs (Table 7.2).

Table 7.1 Assigning L4 microplankton taxa to the different mixotrophic functional types within the mixotrophic food web and their respective strict photo- or phago- trophic functional types within the non-mixotrophic food web and in ERSEM. Taxa with superscript numbers identify those from which evidence (or potential) of mixotrophy has been previously described in the literature.

\begin{tabular}{|c|c|c|c|}
\hline $\begin{array}{c}\text { Mixotrophic } \\
\text { model }\end{array}$ & $\begin{array}{c}\text { Non-mixotrophic } \\
\text { model } \\
\text { and ERSEM } \\
\end{array}$ & Taxa & $\begin{array}{c}\text { size } \\
(\mu \mathrm{m} \text { ESD) }\end{array}$ \\
\hline Micro-CMs & Micro-A & Alexandrium tamarense ${ }^{1}$ & 24 \\
\hline Micro-CMs & Micro-A & Ceratium hexacanthum $^{1}$ & 44 \\
\hline Micro-CMs & Micro-A & Ceratium longipes ${ }^{1}$ & 44 \\
\hline Micro-CMs & Micro-A & Gonyaulax digitale & 34 \\
\hline Micro-CMs & Micro-A & Gonyaulax grindleyi & 34 \\
\hline Micro-CMs & Micro-A & Gonyaulax spinifera $^{1}$ & 31 \\
\hline Micro-CMs & Micro-A & Gonyaulax verior & 27 \\
\hline Micro-CMs & Micro-A & Gyrodinium spp. (medium) $)^{1}$ & 27 \\
\hline Micro-CMs & Micro-A & Karenia mikimotoi $^{1}$ & 20 \\
\hline Micro-CMs & Micro-A & Mesoporos perforatus & 21 \\
\hline Micro-CMs & Micro-A & Nematodinium spp. & 31 \\
\hline Micro-CMs & Micro-A & Neoceratium furca ${ }^{1}$ & 39 \\
\hline Micro-CMs & Micro-A & Neoceratium fusus & 26 \\
\hline Micro-CMs & Micro-A & Neoceratium horridum & 44 \\
\hline Micro-CMs & Micro-A & Neoceratium lineatum & 27 \\
\hline Micro-CMs & Micro-A & Neoceratium macroceros & 44 \\
\hline Micro-CMs & Micro-A & Neoceratium massiliense & 46 \\
\hline Micro-CMs & Micro-A & Neoceratium tripos & 58 \\
\hline Micro-CMs & Micro-A & Polykrikos schwarzii ${ }^{1}$ & 69 \\
\hline Micro-CMs & Micro-A & Prorocentrum triestinum ${ }^{1}$ & 22 \\
\hline Micro-CMs & Micro-A & Prorocentrum micans ${ }^{1}$ & 27 \\
\hline Micro-CMs & Micro-A & Pterosperma spp. ${ }^{2}$ & 19 \\
\hline
\end{tabular}


Table 7.1 continued

\begin{tabular}{|c|c|c|c|}
\hline Micro-CMs & Micro-A & Tryblionella compressa & 22 \\
\hline NCMs (SNCMs) & Microzooplankton & Amylax triacantha ${ }^{3}$ & 29 \\
\hline NCMs (SNCMs) & Microzooplankton & Dinophysis acuminata ${ }^{3}$ & 32 \\
\hline NCMs (SNCMs) & Microzooplankton & Dinophysis acuta ${ }^{3}$ & 47 \\
\hline NCMs (SNCMs) & Microzooplankton & Dinophysis cf. punctata & 20 \\
\hline NCMs (SNCMs) & Microzooplankton & Dinophysis nasuta & 20 \\
\hline NCMs (SNCMs) & Microzooplankton & Dinophysis sacculus ${ }^{3}$ & 31 \\
\hline NCMs (SNCMs) & Microzooplankton & Dinophysis tripos ${ }^{4}$ & 47 \\
\hline NCMs (SNCMs) & Microzooplankton & Mesodinium spp. $(\text { small })^{3}$ & 15 \\
\hline NCMs (SNCMs) & Microzooplankton & Mesodinium rubrum ${ }^{3}$ & 29 \\
\hline NCMs (GNCMs) & Microzooplankton & Laboea strobila ${ }^{3}$ & 33 \\
\hline NCMs (GNCMs) & Microzooplankton & Non-identified mixotrophic ciliates & 20 \\
\hline NCMs (GNCMs) & Microzooplankton & Strombidium spp. (small) ${ }^{3}$ & 10 \\
\hline NCMs (GNCMs) & Microzooplankton & Strombidium spp. (medium) ${ }^{3}$ & 17 \\
\hline NCMs (GNCMs) & Microzooplankton & Strombidium spp. (large) $)^{3}$ & 28 \\
\hline NCMs (GNCMs) & Microzooplankton & Tontonia ovalis ${ }^{3}$ & 34 \\
\hline NCMs (GNCMs) & Microzooplankton & Tontonia spp. ${ }^{3}$ & 25 \\
\hline
\end{tabular}

\subsubsection{Model parameterization}

Model parameterization was performed comparing model simulations with observational data corresponding to the first $3 y$ of the time-series used in this analysis. Calibration experiments consisted of manipulations of (selected) single parameters at a time assuming values observed in the literature (according to references in Tables 5.1 and 7.2). The parameters were selected for the calibration experiments according to the sensitivity analyses performed in the previous Chapter (Chapter 6.3.4). The initial goal of the calibration experiments was to predict the qualitative annual evolution of nutrients and total plankton biomass. Then, predictions were calibrated to represent these variables quantitatively. To achieve these goals, the plankton food web model was calibrated by progressively adding complexity in terms of the number of functional types. The initial plankton food web consisted of diatoms, autotrophic nanoflagellates, bacteria, and 
microzooplankton; the other functional types were added one at a time to better control their impact on trophodynamics. Mixotrophy was the last feature to be added to the biogeochemical model. The final list of parameters used on this study can be found in Table 7.2 (protists), Table 6.2 (bacteria), and Table 7.3 (mesozooplankton). ERSEM parameter values have been calibrated previously and were defined according to Butenschön et al. (2016).

Even in the plankton food web with fewer functional types, phototrophic biomass was being overestimated by the model compared to observed data. This feature was associated with both phototrophic growth and with predation/losses terms. The calibration of parameters controlling phototrophic growth, i.e. the maximum growth rate $\left(\mu_{\max }\right)$, the slope of the photosynthesis-irradiance curve $\left(\alpha_{\mathrm{Chl}}\right)$, and the maximum chlorophyll to carbon ratio $\left(\mathrm{ChlC}_{\mathrm{abs}}\right)$ were key to predict nutrient concentrations and plankton biomasses quantitatively (Table 7.2). Both $\alpha_{\mathrm{Chl}}$ and $\mathrm{ChlC}_{\mathrm{abs}}$ were key to allocate biomass within the different phototrophic functional types (values within Sathyendranath et al., 2009 and Bouman et al., 2018). At this initial step, predation pressure by mesozooplankton was assumed to be constant among all prey types to facilitate the interpretation of competitive abilities between the different functional types. Following up these experiments, calibration was performed with respect to predation pressure through the manipulation of the maximum and minimum prey sizes accessible to phagotrophs $\left(S_{\min }\right.$ and $S_{\max }$, respectively) and the slope of the encounter rate between mesozooplankton and each prey type $\left(\mathrm{Cr}_{\text {prey }}\right)$ (references within Tables 7.2 and 7.3). It is noteworthy that parameters defining the size of the plankton functional types were defined based on observational data at L4 station (Table 7.2; Appendix E, Tables E.1 and E.2). In addition to these, the specific mortality was calibrated to down-regulate the total plankton (mainly phototrophic) biomass; this term is related to the internal nutrient status of the cell. Since diatoms are the only functional type represented by non-motile cells, an extra loss term (linear) was added to the diatom model to account for sinking losses (0.1-0.2 day $^{-1}$; Kiørboe et al., 1996; Flynn and Irigoien, 2009).

After a series of calibration experiments, it was not possible to achieve the coexistence of picophytoplankton and autotrophic nano- and micro- plankton, with the 
first outcompeting the other two functional types. This was the case even when calibrating the model to make picophytoplankton a weaker competitor. The reason why they could not coexist is due to the overall predation pressure, which is lower among picophytoplankton compared to the other two phototrophs (as discussed within Chapter 6). This is mainly related to mesozooplankton, which cannot access such a small prey as picophytoplankton, allowing this group to outcompete the other two due to lower predation pressure. Interestingly, once mixotrophy was considered in the model all phototrophs can coexist and nano- and micro- plankton can achieve higher biomass values compared to picophytoplankton; this is explained not only by the fact that mixotrophs can now rely on feeding to supplement their nutritional requirements but also because they can consume their picoplankton competitor. In contrast, it was not possible to allow coexistence between GNCMs and SNCMs. Competition was always strong between these two groups, as well as with micro-CMs. Therefore, NCMs were included in the model as a single functional type after the tuning; the model was parameterised applying the settings to represent GNCMs (as per Chapter 6), with the exception that NCMs could only acquire photosynthetic capacity from one specific prey type (i.e., nano-CMs).

As an attempt to increase the growth rate of heterotrophic protists, changes were made with respect to $\mu_{\max }$ and to the parameters controlling assimilation efficiency among phagotrophs ( $\mathrm{AE}_{\min }=0.4$ instead of 0.2 as in Chapter 6; Table C.1). It was also necessary to decrease the predation pressure by mesozooplankton on these groups (compared to phototrophic prey) to allow their survival in the model (Table 7.3). To improve the simulation of mesozooplankton biomass, it was necessary to calibrate the maximum ingestion rate $\left(\mathrm{I}_{\max }\right)$ and the respective half saturation constant $(\mathrm{KI})$, the assimilation efficiency ( $\mathrm{pu}_{\mathrm{Mz}}$ ), and enable the overwintering state (Minprey), in which metabolic rates are lower under a specific prey threshold (Table 7.3). The constant values mentioned above were based on the works by Mitra (2006), Mitra and Flynn (2006), Flynn and Irigoien (2009), Flynn and Mitra (2016), and Butenschön et al. (2016). The values of KI and $I_{\max }$ were set so that $\mathrm{KI}$ is equivalent to $I_{\max } / 4$ (Flynn and Mitra, 2016). Finally, it was not necessary to calibrate the bacteria model which was relatively insensitive to the several calibration experiments performed (Table 6.2). 
Table 7.2 Constant parameter values for the different protist functional types (for constant descriptions see Appendix C, Table C.1).

\begin{tabular}{|c|c|c|c|c|c|c|c|c|c|c|}
\hline Constant & PicoP & Diatoms & Nano-A & Micro-A & Nano-CMs & Micro-CMs & NCMs & NanoZ & MicroZ & Ref. \\
\hline$\alpha_{\mathrm{Chl}}$ & $1.5 \times 10^{-5}$ & $1.0 \times 10^{-5}$ & $1.2 \times 10^{-5}$ & $1.0 \times 10^{-5}$ & $1.2 \times 10^{-5}$ & $1.0 \times 10^{-5}$ & $1.2 \times 10^{-5}$ & - & - & 1,2 \\
\hline $\mathrm{ChlC}_{\mathrm{abs}}$ & 0.006 & 0.02 & 0.008 & 0.015 & 0.008 & 0.015 & - & - & - & 3,2 \\
\hline $\mathrm{D}_{\mathrm{Chl}}$ & - & - & - & - & - & - & 0.40 & - & - & 4,5 \\
\hline $\mathrm{kP}_{\max }$ & - & - & - & - & - & - & 1.00 & - & - & - \\
\hline $\mathrm{K}_{\mathrm{a}}$ & 3.50 & 7.00 & 3.50 & 7.00 & 3.50 & 7.00 & - & - & - & 6 \\
\hline $\mathrm{K}_{\mathrm{n}}$ & 7.00 & 14.0 & 7.00 & 14.0 & 7.00 & 14.0 & - & - & - & 6 \\
\hline $\mathrm{K}_{\mathrm{p}}$ & 0.70 & 1.40 & 0.70 & 1.40 & 0.70 & 1.40 & - & - & - & 6 \\
\hline $\mathrm{K}_{\mathrm{Si}}$ & - & 15.0 & - & - & - & - & - & - & - & 7 \\
\hline$\mu_{\max }$ & 0.60 & 1.00 & 0.60 & 0.60 & 0.50 & 0.50 & 0.50 & 0.60 & 0.50 & 9,10 \\
\hline$\mu_{\text {phot }}$ & 0.60 & 1.00 & 0.60 & 0.60 & 0.50 & 0.50 & 0.00 & - & - & 9,10 \\
\hline $\mathrm{NC}_{\mathrm{abs}}$ & 0.25 & 0.20 & 0.20 & 0.20 & 0.20 & 0.20 & 0.20 & 0.20 & 0.20 & 6,11 \\
\hline $\mathrm{NC}_{\max }$ & 0.20 & 0.15 & 0.15 & 0.15 & 0.15 & 0.15 & 0.15 & 0.15 & 0.15 & 6,11 \\
\hline $\mathrm{NC}_{\min }$ & 0.05 & 0.05 & 0.05 & 0.05 & 0.05 & 0.05 & 0.05 & 0.15 & 0.15 & 6,11 \\
\hline $\mathrm{Pbal}_{\text {crit }}$ & - & - & - & - & 0.10 & 0.10 & 0.00 & - & - & - \\
\hline $\mathrm{PC}_{\mathrm{abs}}$ & 0.04 & 0.04 & 0.04 & 0.04 & 0.04 & 0.04 & 0.04 & 0.04 & 0.04 & 6,11 \\
\hline $\mathrm{PC}_{\max }$ & 0.02 & 0.02 & 0.02 & 0.02 & 0.02 & 0.02 & 0.02 & 0.02 & 0.02 & 6,11 \\
\hline $\mathrm{PC}_{\min }$ & 0.005 & 0.005 & 0.005 & 0.005 & 0.005 & 0.005 & 0.005 & 0.02 & 0.02 & 6,11 \\
\hline size & 2.00 & 25.0 & 6.00 & 25.0 & 6.00 & 25.0 & 25.0 & 6.00 & 25.0 & - \\
\hline$S_{\max }$ & - & - & - & - & 10.0 & 50.0 & 40.0 & 10.0 & 50.0 & 12 \\
\hline $\mathrm{S}_{\min }$ & - & - & - & - & 0.20 & 0.20 & 0.20 & 0.20 & 0.20 & 12 \\
\hline $\mathrm{S}_{\mathrm{mix}}$ & - & - & - & - & 0 & 0 & 1 & - & - & - \\
\hline $\mathrm{S}_{\mathrm{opt}}$ & - & - & - & - & 2.00 & 6.00 & 6.00 & 2.00 & 6.00 & 12 \\
\hline $\mathrm{S}_{\mathrm{pd}}$ & - & - & - & - & . & 1 & 0 & - & - & - \\
\hline
\end{tabular}


Table 7.3 Constant parameter values for the mesozooplankton model.

\begin{tabular}{|c|c|c|c|}
\hline Constant & Description & Unit & Value \\
\hline $\mathrm{Cr}_{\text {micro-CMs }}$ & clearance rate for micro-CMs & $\mathrm{m}^{-3} \mathrm{gC}^{-1} \mathrm{day}^{-1}$ & 2.00 \\
\hline $\mathrm{Cr}_{\text {nano-CMs }}$ & clearance rate for nano-CMs & $\mathrm{m}^{-3} \mathrm{gC}^{-1} \mathrm{day}^{-1}$ & 1.50 \\
\hline $\mathrm{Cr}_{\text {diatoms }}$ & clearance rate for diatoms & $\mathrm{m}^{-3} \mathrm{gC}^{-1} \mathrm{day}^{-1}$ & 2.00 \\
\hline $\mathrm{Cr}_{\mathrm{NCMs}}$ & clearance rate for GNCMs & $\mathrm{m}^{-3} \mathrm{gC}^{-1} \mathrm{day}^{-1}$ & 1.00 \\
\hline $\mathrm{Cr}_{\text {mesozoo }}$ & $\begin{array}{l}\text { clearance rate for mesozooplankton } \\
\text { (intraguild predation) }\end{array}$ & $\mathrm{m}^{-3} \mathrm{gC}^{-1} \mathrm{day}^{-1}$ & 2.00 \\
\hline $\mathrm{Cr}_{\text {microzoo }}$ & $\begin{array}{l}\text { clearance rate for heterotrophic } \\
\text { microzooplankton }\end{array}$ & $\mathrm{m}^{-3} \mathrm{gC}^{-1}$ day $^{-1}$ & 0.50 \\
\hline $\mathrm{Cr}_{\text {nanozoo }}$ & $\begin{array}{l}\text { clearance rate for heterotrophic } \\
\text { nanoflagellates }\end{array}$ & $\mathrm{m}^{-3} \mathrm{gC}^{-1}$ day $^{-1}$ & 1.50 \\
\hline $\mathrm{Cr}_{\mathrm{POM}}$ & clearance rate for particulate organic matter & $\mathrm{m}^{-3} \mathrm{gC}^{-1} \mathrm{day}^{-1}$ & 0.00 \\
\hline KI & $\begin{array}{l}\text { half-saturation constant for the ingestion } \\
\text { rate of all prey types }\end{array}$ & $\mathrm{gC} \mathrm{gC}^{-1}$ day $^{-1}$ & 0.125 \\
\hline$I_{\max }$ & maximum carbon ingestion rate & $\mathrm{gC} \mathrm{gC}^{-1}$ day $^{-1}$ & 0.50 \\
\hline Minprey & food threshold for overwintering state & $\mathrm{mg} \mathrm{C} \mathrm{m} \mathrm{m}^{-2}$ & 3.00 \\
\hline nprey & number of prey types & - & 7.00 \\
\hline pe_R1 & dissolved fraction of excreted/dying matter & - & 0.50 \\
\hline $\mathrm{pu}_{\mathrm{MZ}}$ & assimilation efficiency & - & 0.60 \\
\hline pu_ea & $\begin{array}{l}\text { fraction of unassimilated prey that is } \\
\text { excreted (not respired) }\end{array}$ & - & 0.50 \\
\hline pu_eaR & $\begin{array}{l}\text { fraction of unassimilated detritus that is } \\
\text { excreted (not respired) }\end{array}$ & - & 0.90 \\
\hline qne & nitrogen to carbon ratio & $\operatorname{mmol~N}(\mathrm{mg} \mathrm{C})^{-1}$ & 0.013 \\
\hline qpc & phosphorus to carbon ratio & $\operatorname{mmol~P}(\mathrm{mg} \mathrm{C})^{-1}$ & 0.0008 \\
\hline R1R2 & $\begin{array}{l}\text { labile fraction of produced dissolved } \\
\text { organic carbon }\end{array}$ & - & 1.00 \\
\hline $\operatorname{sd}_{\mathrm{MZ}}$ & basal mortality & day $^{-1}$ & 0.025 \\
\hline sdo & $\begin{array}{l}\text { maximum mortality due to oxygen } \\
\text { limitation }\end{array}$ & day $^{-1}$ & 0.00 \\
\hline $\operatorname{srs}_{M Z}$ & specific rest respiration & day $^{-1}$ & 0.015 \\
\hline $\mathrm{xR} 1 \mathrm{n}$ & $\begin{array}{l}\text { transfer of nitrogen to DOM, relative to } \\
\text { POM }\end{array}$ & - & 1.00 \\
\hline$x R 1 p$ & $\begin{array}{l}\text { transfer of phosphorus to DOM, relative to } \\
\text { POM }\end{array}$ & - & 1.20 \\
\hline repw & specific overwintering respiration & day $^{-1}$ & 0.0025 \\
\hline mort & specific overwintering mortality & day $^{-1}$ & 0.0025 \\
\hline
\end{tabular}




\subsection{Results}

\subsubsection{Model skill assessment}

The mixotrophic model reproduced annual means of nutrients and chlorophyll concentrations and total plankton biomass observed at L4 by a ratio close to 1:1 (Fig. 7.2); comparisons against the full 9y time-series can be found in Appendix E (Figs. E.1-E.3). The annual means for the nutrients were similar between the different models, with ERSEM slightly overestimating nitrate and silicate concentrations (Fig. 7.2). The annual mean of total chlorophyll was similar between all models while the annual mean of total plankton biomass was overestimated by the non-mixotrophic model by $31 \%$ while underestimated by ERSEM by $12 \%$ (Fig. 7.2).
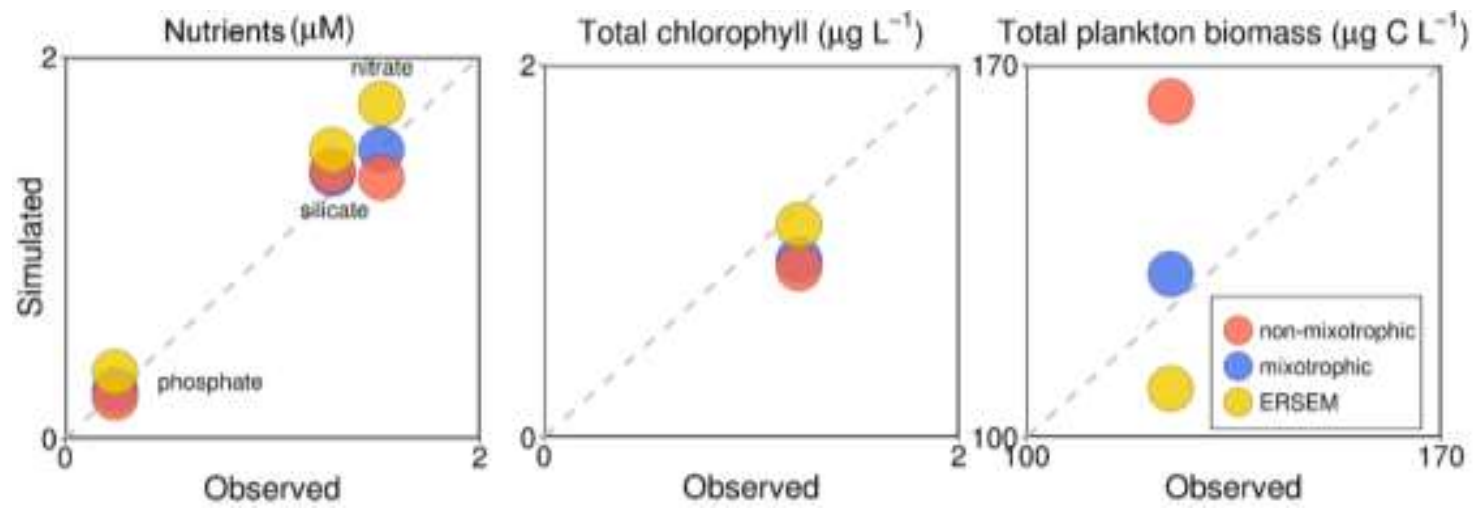

Figure 7.2 Comparisons of observed and simulated annual means of nutrients (log-scaled) and chlorophyll concentrations and total plankton biomass between the mixotrophic and the non-mixotrophic models and ERSEM; dotted lines indicate perfect fit. Mean values correspond to the period 2006-2014.

The mixotrophic model was also able to reproduce the observed seasonalities for nutrients, chlorophyll concentrations, and total plankton biomass, with simulations being similar for all models (Figs. 7.3 and E.4); however, all models overestimated the spring bloom (lower panels, Fig. 7.3). When comparing these properties, model performance was similar among all models as quantified by correlation coefficients, but the overall bias $\left(\mathrm{AE}^{*}\right)$ was lower for the mixotrophic model (except for ammonium when compared against ERSEM) (Fig. 7.4 and Table E.4). Overall, the standard deviation of the models was larger (RMSE* > 0) than the reference field's standard deviation (Fig. 7.4). 

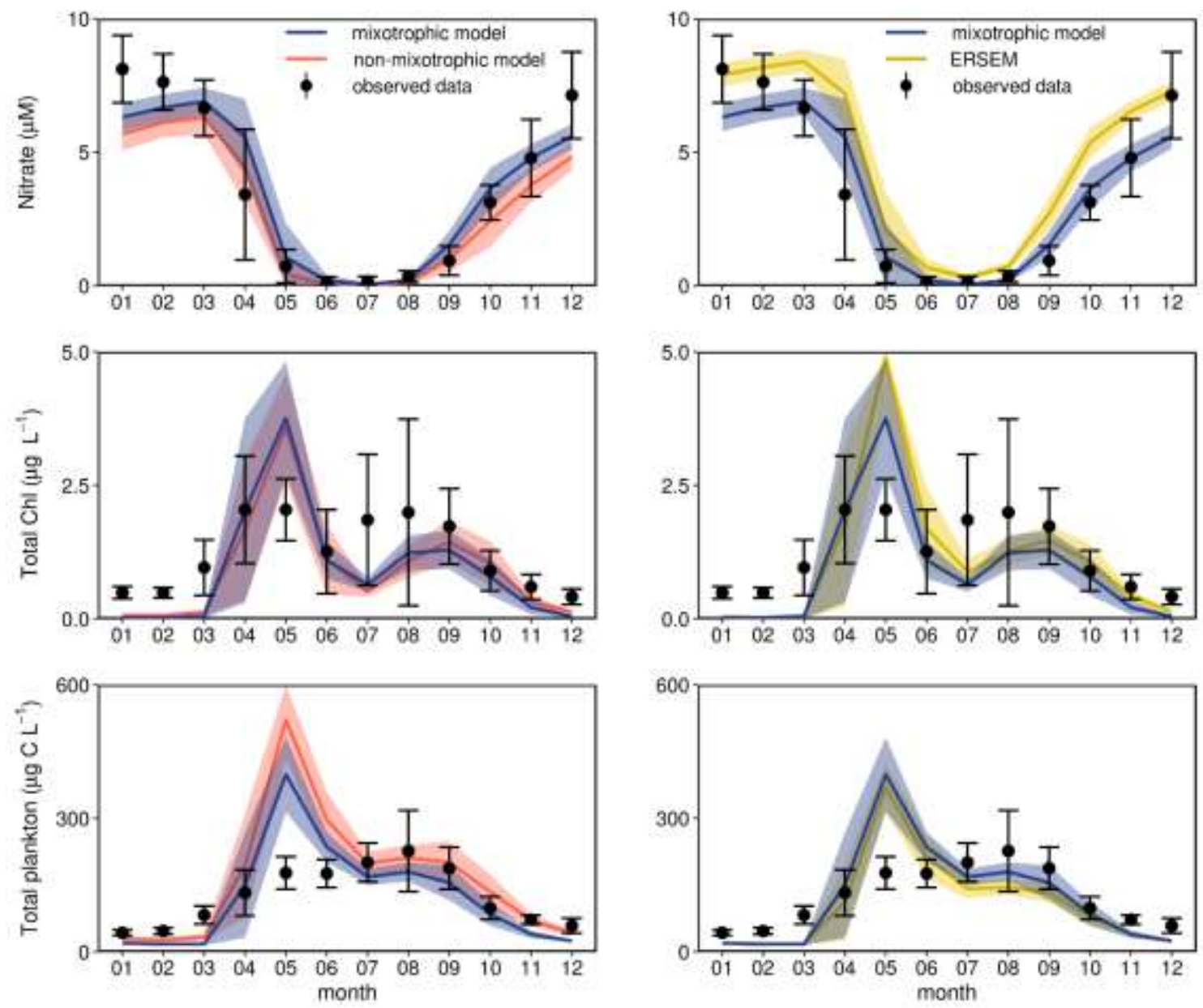

Figure 7.3 Comparisons of nitrate and chlorophyll-a (Chl) concentrations and total plankton biomass over the climatological seasonal cycle between model simulations (lines) and observations (dots) at L4 station (at $10 \mathrm{~m}$ depth). Comparisons are also made between the mixotrophic (blue) and non-mixotrophic (red) models (left panels) and between ERSEM (yellow) and the mixotrophic model (right panels). Mean $( \pm \mathrm{SD})$ values correspond to the period 2006-2014. 

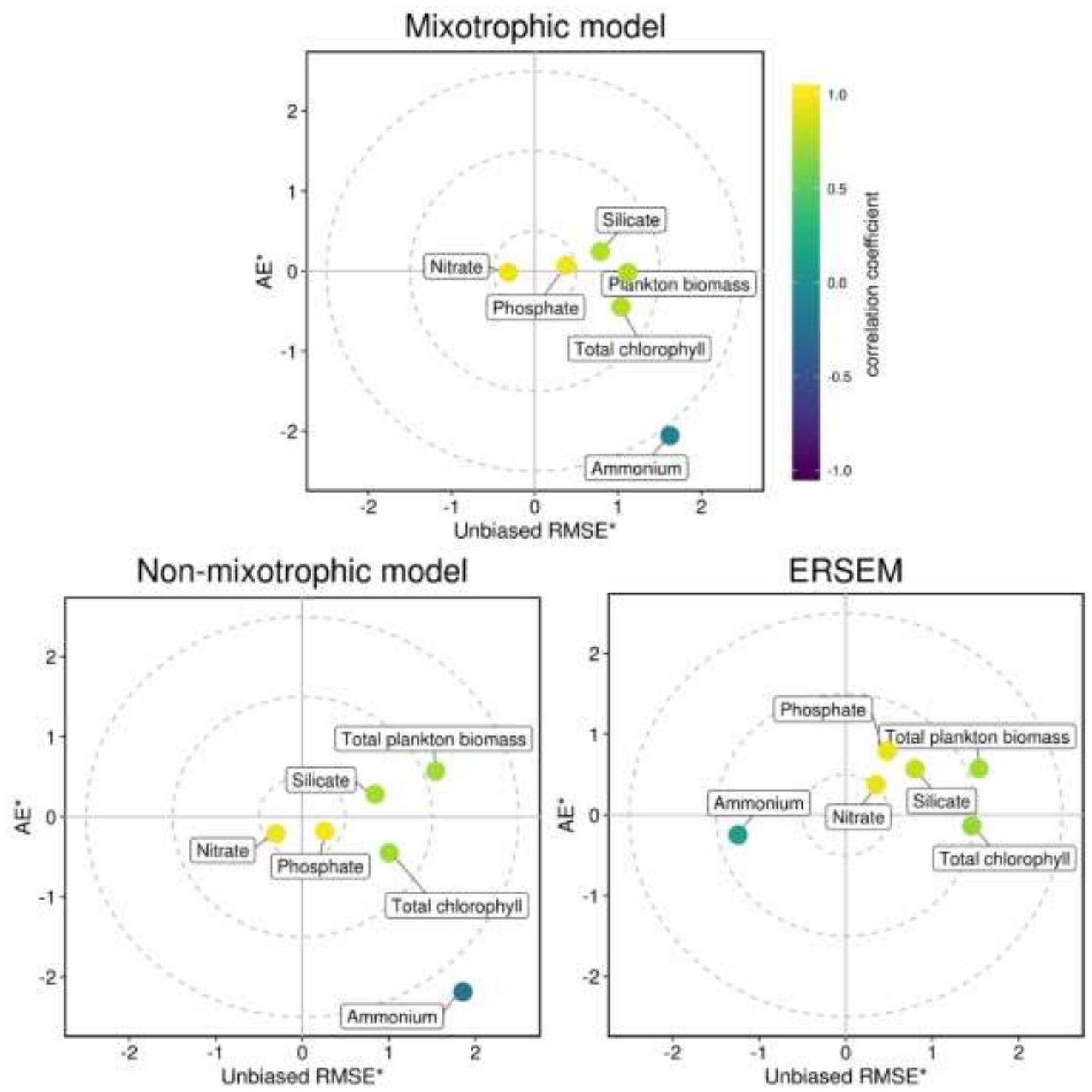

Figure 7.4 Target diagrams with bias (AE*, abscissa), RMSE* (ordinate), and correlation coefficient (colour code) for nutrients (nitrate, phosphate, silicate, ammonium), total chlorophyll, and total plankton biomass. Upper panel shows the mixotrophic model and lower panels show the non-mixotrophic model (left) and ERSEM (right). 
One feature common to all models was the inability to capture silicate depletion earlier in the production cycle (Fig. 7.5). Analyses of the 9y time-series revealed that the model does well for certain years but cannot capture the observed interannual variability at L4 (Fig. E.1). In addition, the low silicate concentrations observed during summer could only be simulated after a high diatom spring bloom (Fig. 7.5). In reality, diatoms appear only in April and reach maximum values during late summer/autumn, without ever reaching the pronounced peaks simulated by the model during spring (Fig. 7.5). This result is related to the limited capacity of the models to simulate mesozooplankton biomass earlier in the production cycle (Fig. 7.5), which could otherwise act controlling the diatom bloom. Mesozooplankton at L4 are detected earlier than their prey, which might help to explain why diatoms never reach pronounced peaks during spring (Fig. 7.5). Furthermore, the overall plankton biomass is underestimated during winter in all models (lower panel, Fig. 7.3), which might also have implications for the development of a pronounced diatom spring bloom. 

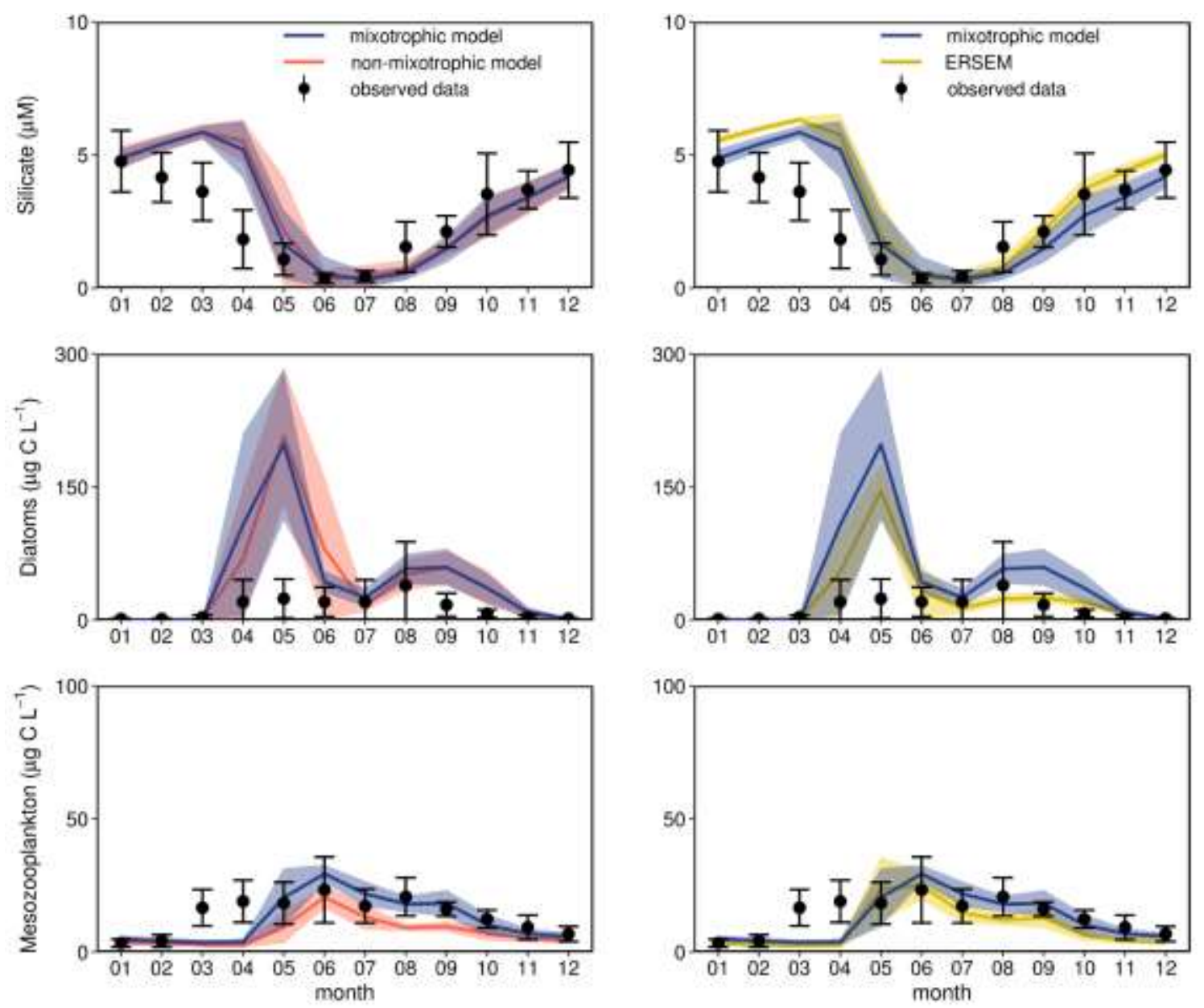

Figure 7.5 Comparisons of silicate concentrations, diatoms, and mesozooplankton biomasses over the climatological seasonal cycle between model simulations (lines) and observations (dots) at L4 station (at $10 \mathrm{~m}$ depth, except for mesozooplankton in which data were integrated over the water column). Comparisons are also made between the mixotrophic (blue) and non-mixotrophic (red) models (left panels) and between ERSEM (yellow) and the mixotrophic model (right panels). Note that y-axes scales differ among panels. Mean $( \pm$ SD) values correspond to the period 2006-2014.

The models performed differently for other plankton functional types (Figs. 7.6 and 7.7). As observed in Chapter 6, mixotrophy favours the concentration of biomass in larger size classes. The non-mixotrophic model greatly overestimates the biomass of picophytoplankton ( 15-fold higher), underestimates the biomass of autotrophic nanoflagellates, and cannot represent the observed dynamics of autotrophic microplankton (Fig. 7.6). In turn, the mixotrophic model performs equal or better for all functional types but nanozooplankton (Fig. 7.7 and Table E.4). It is noteworthy that the 
climatological data used to evaluate microzooplankton dynamics differed among the models; while both strict phagotrophs and NCMs were included for comparisons with the non-mixotrophic model (and ERSEM), only strict phagotrophs were used for comparisons with the mixotrophic model (Fig. E.4). NCMs were only explicitly modelled in the mixotrophic model, which was able to reproduce observed data (Fig. 7.8). Model comparisons were also made for three selected emergent properties to corroborate (or not) the findings from Chapter 6, including: i) ammonium regeneration, ii) trophic transfer efficiency; and iii) production of labile DOC (Fig. E.5). It was observed that the overall regeneration of ammonium was lower while the trophic transfer efficiency was higher in the presence of mixotrophs, but no difference was observed regarding the total production of labile DOC (Fig. E.5).

The mixotrophic model performed at least as well as ERSEM (Figs. 7.6 and 7.7 and Table E.4): an established ecosystem model which has been previously tested in different oceanographic regimes; however, some differences were observed. Overall, biases were higher within the mixotrophic model compared to ERSEM (Fig. 7.7), except for picophytoplankton. Picophytoplankton was overestimated during spring by ERSEM and underestimated during winter by both models (Fig. 7.6). The observed biomass peak of phototrophic nanoflagellates during summer was better represented by the mixotrophic model (Fig. 7.6). Regarding microphytoflagellates, the mixotrophic model performs well in quantitative terms, but fails to reproduce the timing of the bloom at L4, which was better represented in ERSEM (Fig. 7.6). Microzooplankton is qualitatively well represented in both models; however, biomass values are overestimated by the mixotrophic model by an averaged factor of 2.5 while underestimated in ERSEM by a factor of 0.6 (Fig. E.4). Both models performed similarly when simulating diatoms, bacteria, and mesozooplankton biomasses (Figs. 7.5, 7.7 and E.4). 

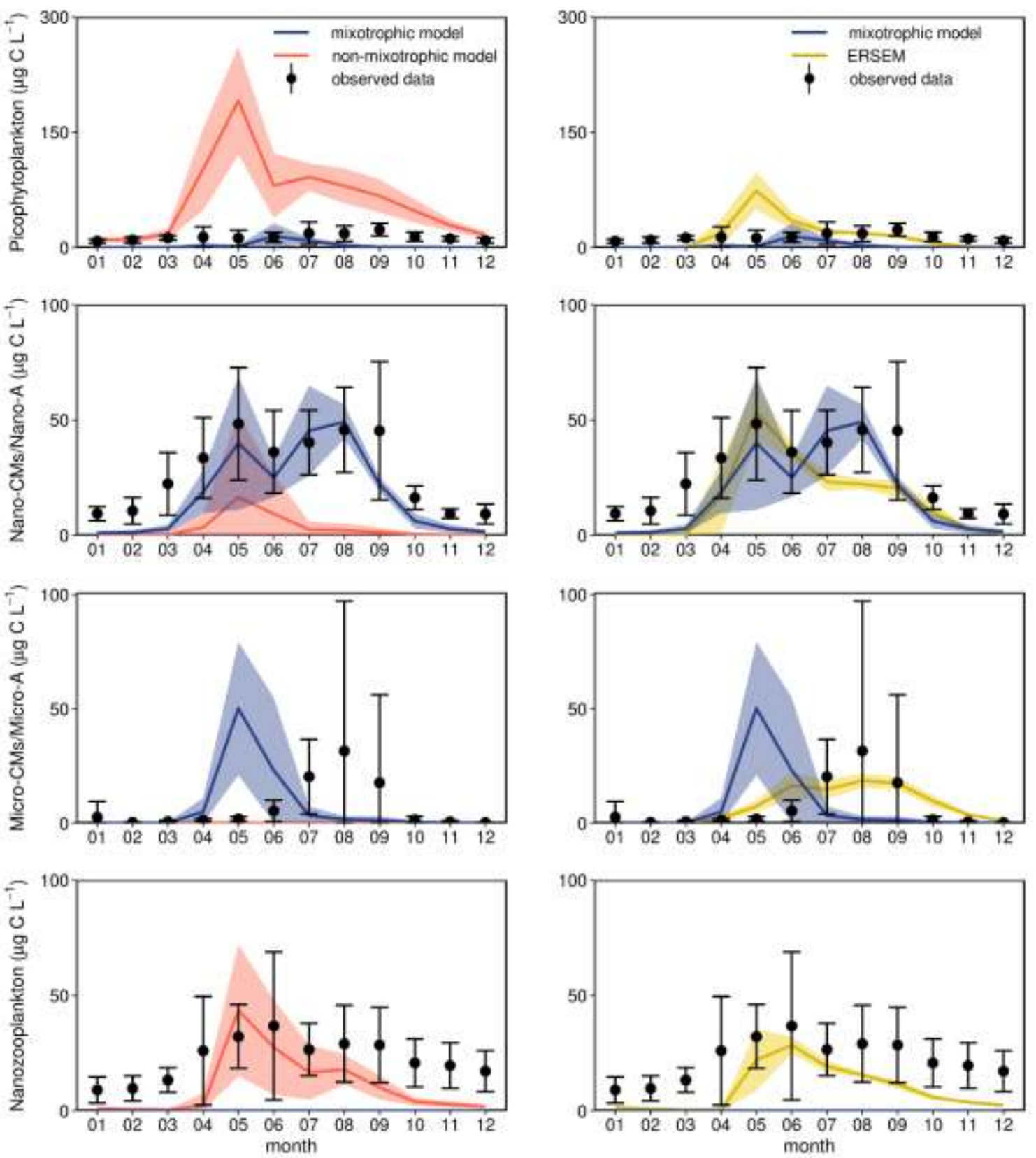

Figure 7.6 Comparisons of picophytoplankton, nanophytoflagellates (Nano-A or Nano$\mathrm{CMs}$ ), microphytoflagellates (Micro-A or Micro-CMs), and nanozooplankton biomasses over the climatological seasonal cycle between model simulations (lines) and observations (dots) at L4 station (at $10 \mathrm{~m}$ depth). Comparisons are also made between the mixotrophic (blue) and non-mixotrophic (red) models (left panels) and between ERSEM (yellow) and the mixotrophic model (right panels). Mean $( \pm \mathrm{SD})$ values correspond to the period 20062014. 

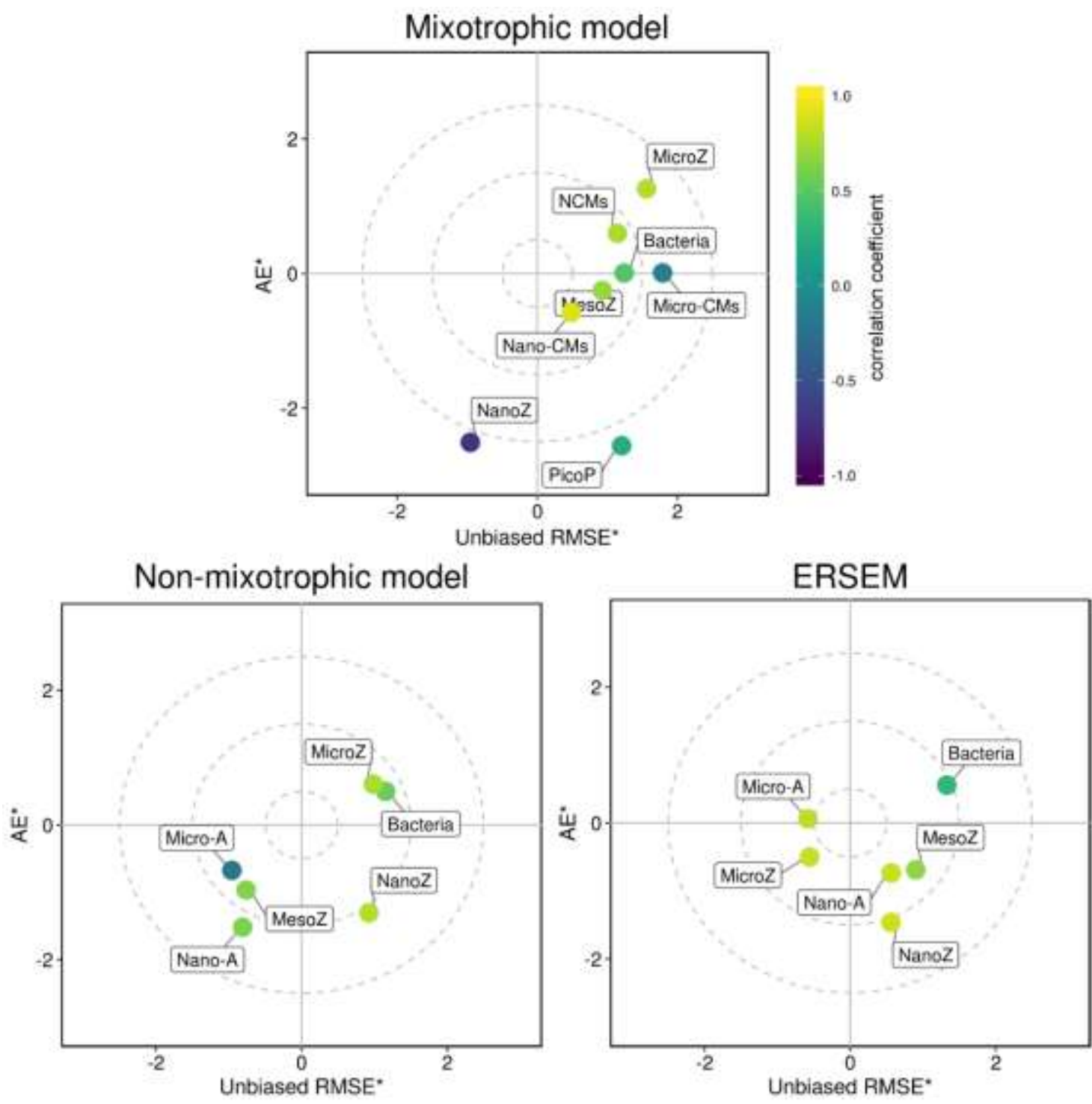

Figure 7.7 Target diagrams with bias (AE*, abscissa), RMSE* (ordinate), and correlation coefficient (colour code) for the biomass of plankton functional types. Upper panel shows the mixotrophic model and lower panels show the non-mixotrophic model (left) and ERSEM (right). Functional types that felt out of the domain (i.e., diatoms for all models and picophytoplankton for the non-mixotrophic model and ERSEM) were not represented in the plots. 


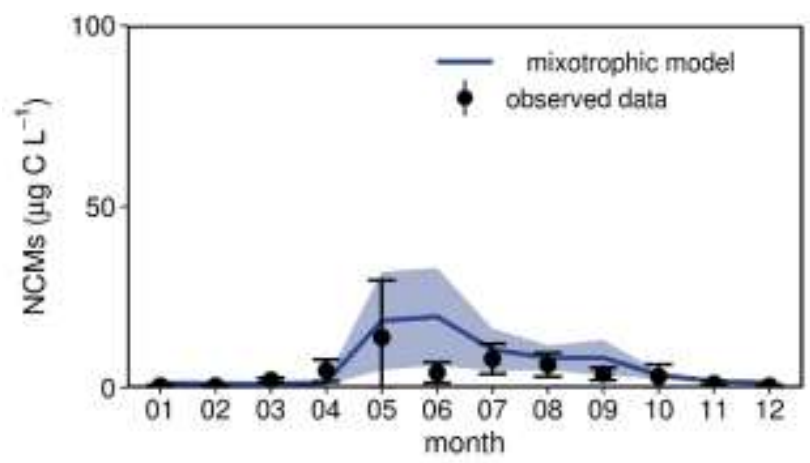

Figure 7.8 Comparison of simulated NCM biomass (line) with observations (dots) at L4 station over the climatological seasonal cycle (at $10 \mathrm{~m}$ depth). Mean $( \pm \mathrm{SD})$ values correspond to the period 2006-2014.

\subsubsection{Physico-chemical characterization of the model}

The climatological seasonal variation of PAR, temperature, diffusivity, mixed layer depth, and nitrate concentrations over the water column are shown in Fig. 7.9. Nitrate will be used herein to represent the seasonal variation of inorganic nutrients; for phosphate, silicate, and ammonium depth profiles please refer to the Appendix E (Fig. E.6). PAR can reach the bottom at L4 throughout the production cycle, but values decrease significantly with depth (Fig. 7.9). During winter, the water column is fully mixed (maximum diffusivity of $0.08 \mathrm{~m}^{2} \mathrm{~s}^{-1}$ ), with temperatures ranging from 9 to $10^{\circ} \mathrm{C}$, and nitrate concentrations reaching $6 \mu \mathrm{M}$ (Fig. 7.9). The onset of stratification develops in mid-April (MLD $\sim 20 \mathrm{~m})$ together with a decrease in nitrate concentrations $(\sim 3 \mu \mathrm{M})$ due to plankton uptake. During summer, irradiance increases, temperatures reach $16^{\circ} \mathrm{C}$ and diffusivity is minimum, resulting in the development of a shallower mixed layer (MLD $\sim 10 \mathrm{~m}$ ) and a nutricline (surface nitrate $<1 \mu \mathrm{M}$ ); therefore, the plankton community is confined to the MLD and cannot access the inorganic nutrients within deeper waters (Fig. 7.9). The increase in mixing in autumn (late September), combined with the decrease in light availability, allows the termination of the bloom and the increase in nutrient concentrations in the surface due to mixing with deeper nutrient-rich waters (Fig. 7.9). 

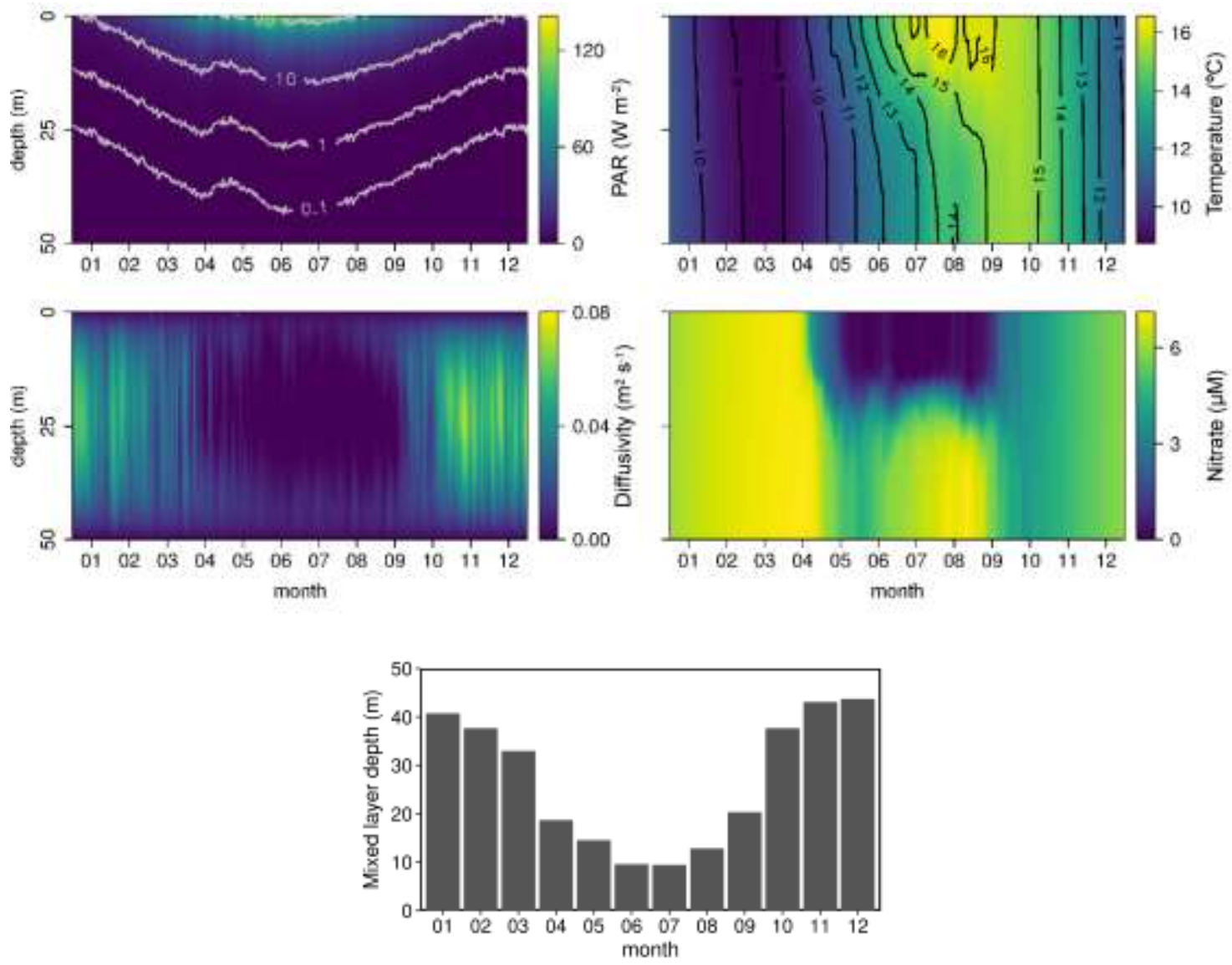

Figure 7.9 Depth profiles of PAR, temperature, diffusivity, and nitrate concentrations over the climatological seasonal cycle. The mixed layer depth is also given for each month. Mean values correspond to the period 2006-2014.

\subsubsection{Modelling the seasonal succession of protist trophic strategies}

To evaluate the succession of protist trophic strategies over the seasonal cycle and throughout the water column, the relative contribution of autotrophs (picophytoplankton and diatoms), mixotrophs (nano-CMs, micro-CMs, and NCMs), and heterotrophs (nanoand micro- zooplankton) to the total protist biomass was computed at several times and depths. To capture seasonal variation, five time periods were chosen based on water column stratification, as following: i) fully mixed, late winter (16/02 to $15 / 03)$, ii) onset stratification (16/04 to $15 / 05)$, iii) stratified (16/06 to $15 / 08)$, iv) stratification breaking down (16/08 to $15 / 09)$, and v) fully mixed, early winter (16/11 to $15 / 12)$. To account for potential differences over the water column, two depths (10 and $40 \mathrm{~m}$ ) were selected for the analysis to represent the plankton community within and below the mixed layer. To compare the relative success of the different groups, the difference between growth (i.e., 
photosynthesis minus respiration) and losses (mainly predation) rates of the different protist functional types was used as a measure of population fitness.

All trophic strategies coexisted during the analysed periods and the model showed that different trophic strategies dominate in different time periods according to water column stratification (Figs. 7.10). However, the persistence of mixotrophy across the spatio-temporal gradient analysed is clear (Fig. 7.10). Overall, the model agrees with expectations: autotrophs dominate during spring and autumn blooms, mixotrophs dominate once the water column is strongly stratified and heterotrophs maximum contribution is observed when mixing is maximal (Fig. 7.10). The main difference when compared to observational data at $10 \mathrm{~m}$ depth is the overestimation of autotrophic biomass (diatoms) and underestimation of heterotrophs during spring and autumn (Fig. 7.10 vs Fig. E.7). The model also predicts a shift from a protist community dominated by heterotrophs to mixotrophs from early to late winter, respectively (Fig. 7.10). This is supported by the data, although to a lesser extent (Fig. E.7). Below the mixed layer, the overall importance of autotrophs is lower, giving space mainly to mixotrophic forms during spring and to heterotrophic forms in the other periods of the production cycle (Fig. 7.10). It is noteworthy that the biomass growth of protist populations is negative in deeper waters or, most likely, lower than the losses terms; therefore, biomass at deeper waters might not be viable and it is more likely to have been brought by turbulent mixing.

At onset stratification, resources are plenty (Figs. E.8 and E.9) and growth rates tend to be higher than predation pressure (Fig. 7.10). Early in the spring bloom, mixing, light, and nutrients availability favours phototrophic metabolism mainly among diatoms due to their higher growth rates. Although diatoms reach growth rates twice that of microCMs (resulting in higher biomass values; Fig. E.10), silicate depletion limits diatom growth late in the spring bloom (Fig. 7.10). Micro-CMs compete with diatoms for inorganic resources and light, however, the diatom bloom is what allows the development of micro-CMs because the latter can feed on the former (Fig. 7.11). Therefore, the suppression of the diatom bloom is followed by the suppression of micro-CMs while nano-CMs, NCMs, and microzooplankton can persist late in spring (Fig. E.10). Picophytoplankton and nanozooplankton cannot grow as fast as their competitors and 
populations are down regulated by predation pressure (Fig. 7.10). In fact, nanozooplankton was not able to thrive in any of the simulated periods, with predation pressure always overcoming growth rates (Fig. 7.10).

During summer, the water column is stratified and inorganic nutrients and prey are limited; much of the spring production gets exported to deeper waters ( $\sim 45 \%$ of the total nitrogen within the first 25 meters) and does not return until autumn due to the presence of the thermocline (Fig. 7.10). Such conditions favour mixotrophy over both autotrophy and heterotrophy and a tight coupling between growth and predation rates is observed, showing an increase in the role of predation shaping the plankton community (Fig. 7.10). Population fitness was higher among smaller cells, mainly nano-CMs, which relied more on phagotrophy during this period compared to the onset of stratification (Fig. 7.11). Similarly, the contribution of phototrophy to total carbon uptake among NCMs was maximum during summer due to limited prey availability (Fig. 7.11). During this period, autotrophic protist biomass was dominated by picophytoplankton which can take advantage during low nutrient conditions (Fig. E.10). However, picophytoplankton was quickly depressed through predation (Figs. E.10 and 7.10), mainly by nano-CMs.

During the stratification breaking down, diatoms increase in relative biomass but the bloom is weaker than the one observed during spring (Fig. 7.10). In addition, the heterotrophic community observed during summer, both protists and mesozooplankton, can respond quicker to the autumn bloom and exert higher predation control compared to spring (Fig. 7.10). Despite high growth rates, micro-CMs could not thrive in the absence of a stronger bloom and were outcompeted by heterotrophic protists and NCMs (Figs. 7.10 and 7.11). In turn, nano-CMs could maintain biomass values similar to those observed during the stratified period (Fig. 7.11). After the autumn bloom, the water column is fully mixed and heterotrophs achieve their highest contribution to total protist biomass which, in turn, was considerably low in the other periods (Fig. 7.10). Intense mixing and light limitation result in slow growing populations subjected to high predation pressure (Fig. 7.10). Mixotrophs also thrived under these conditions, and phagotrophy contributed significantly to the growth of nano-CMs (Fig. 7.11). Heterotrophs are replaced by mixotrophs late in winter and predation is relaxed (Fig. 7.10). 


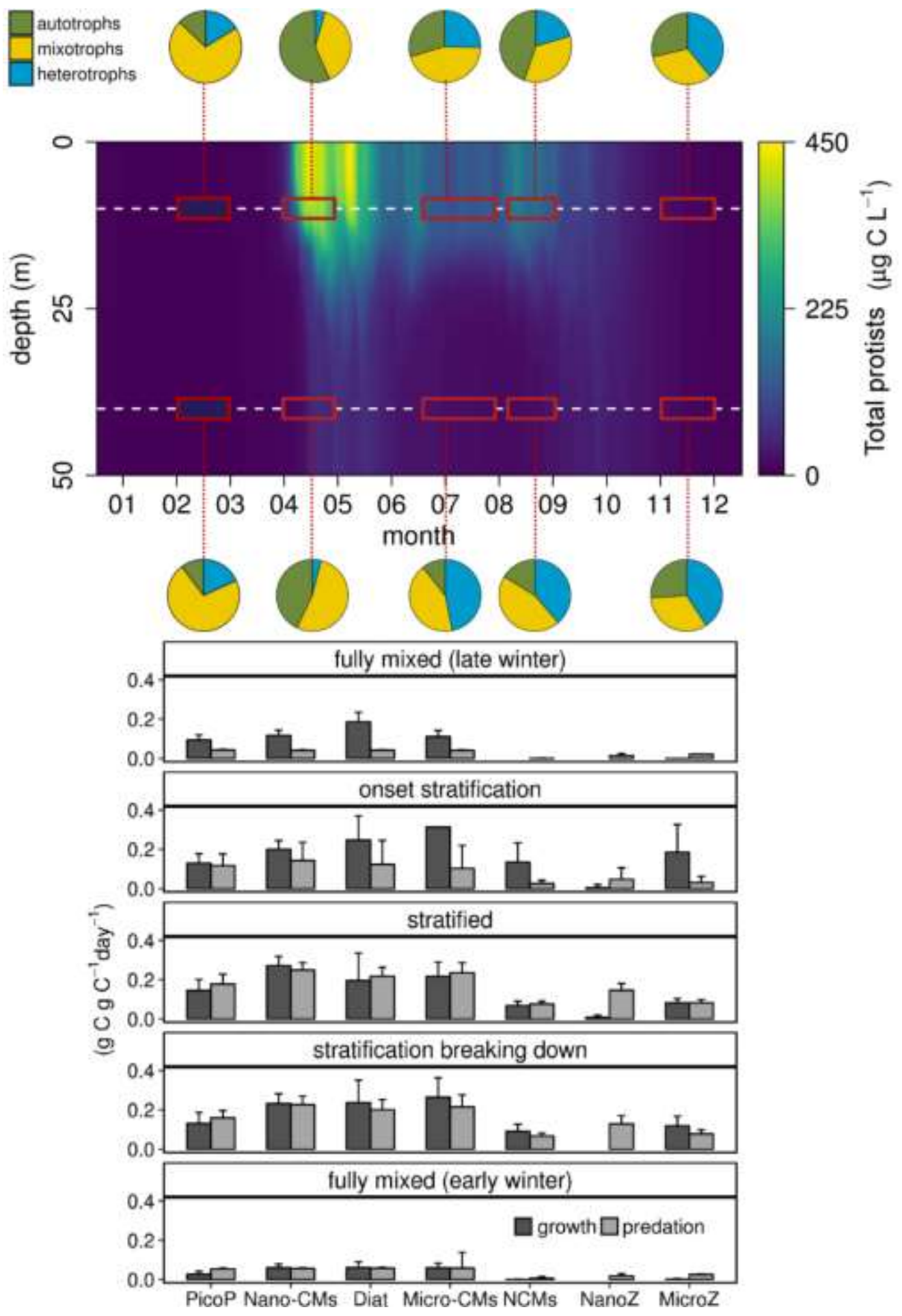

Figure 7.10 Simulated seasonal succession of plankton trophic strategies at L4 station. Depth profile shows the total protist biomass. The relative contribution of autotrophs (green), mixotrophs (yellow), and heterotrophs (blue) is given by the pie charts for selected time periods and depth. Growth (black) and mortality rates (grey) are also given at $10 \mathrm{~m}$ for each functional type as a measure of population fitness. Mean $( \pm \mathrm{SD})$ values correspond to the period 2006-2014. 
a) onset stratification

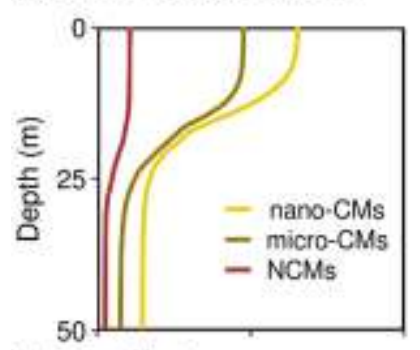

b) stratified
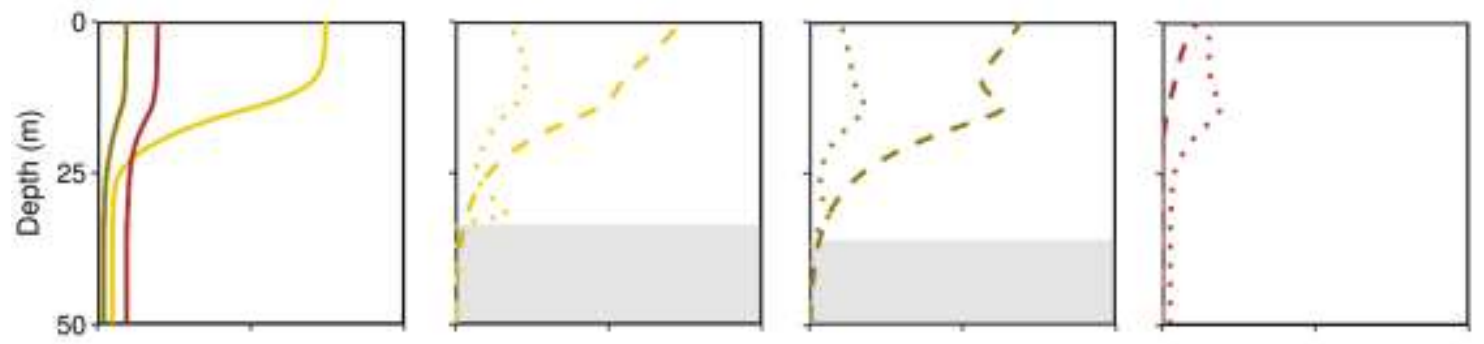

c) stratification breaking down
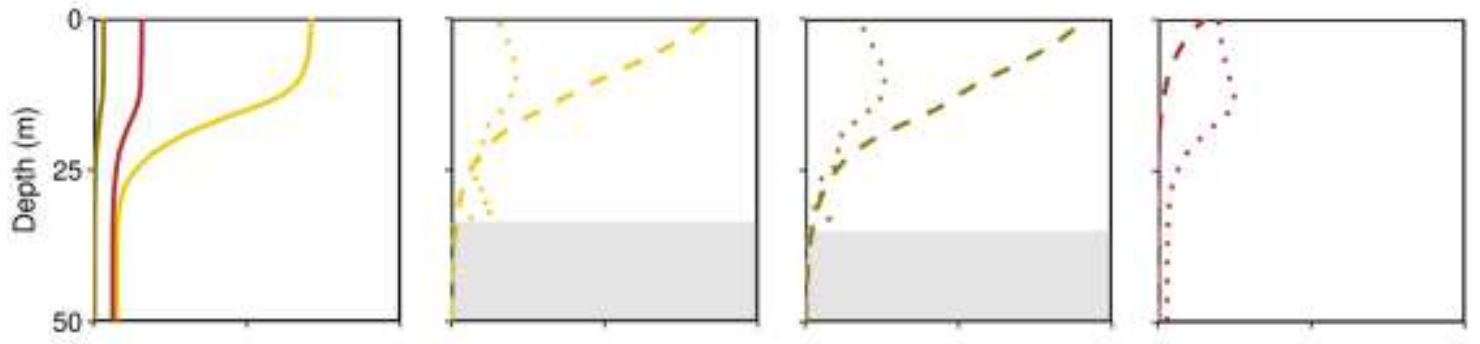

d) fully mixed
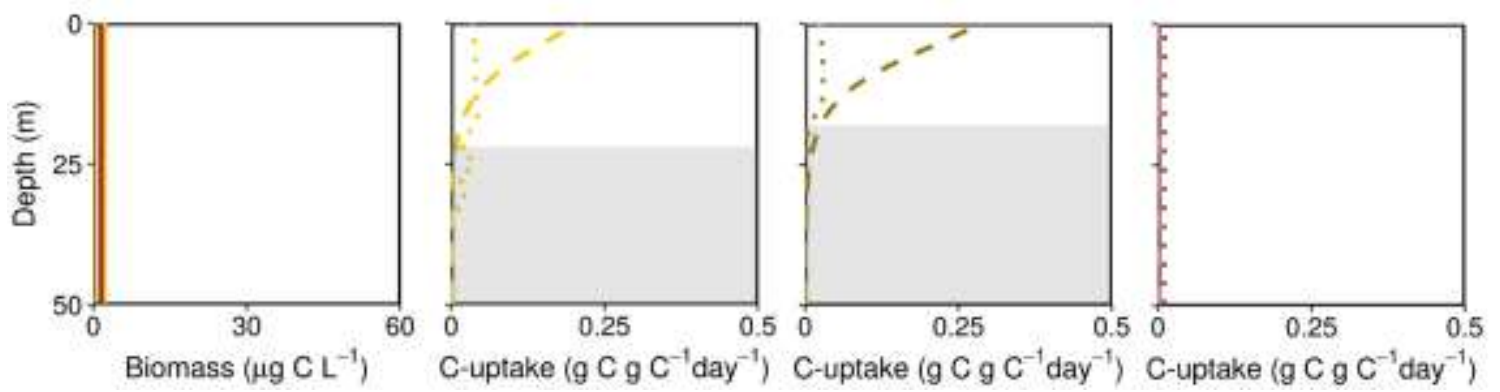

Figure 7.11 Depth profiles of simulated biomass of the different mixotrophs (nano-CMs, micro-CMs, and NCMs; $1^{\text {st }}$ column) and their respective carbon fixation (phototrophy) and carbon assimilation (phagotrophy) rates $\left(2^{\text {nd }}, 3^{\text {rd }}\right.$, and $4^{\text {th }}$ columns) within selected time periods over the seasonal cycle. Grey areas indicate negative growth. Mean values correspond to the period 2006-2014. 


\subsection{Discussion}

Model and data revealed that mixotrophy persists over the seasonal cycle in a coastal temperate sea as the succession of different protist trophic strategies takes place throughout the water column (Figs. 7.10 and E.7). The model was able to reproduce the greater importance of autotrophs at the onset of stratification and the relevant role of heterotrophs once the water column is fully mixed (Fig. 7.10). The persistence of mixotrophy is captured through the model by: i) allowing mixotrophic strategies to vary over the studied period, i.e., accounting for the synergy between phototrophy and phagotrophy, and ii) assigning different forms of mixotrophy, i.e., constitutive and nonconstitutive forms of different sizes. In addition, and perhaps more importantly, the former depends on the latter, ultimately changing the composition of mixotrophic populations and their ecological roles across the spatio-temporal gradient investigated (Fig. 7.11).

\subsubsection{The seasonal succession of protist trophic strategies}

The modelling framework used here invokes realistic environmental variability and different functional types in a plankton food web to investigate both competitive outcomes and top down controls in the seasonal succession of protist trophic strategies. Doing so, the model is successful on predicting a series of expected seasonal patterns within temperate seas and realistic growth and grazing rates which will be discussed herein. Nevertheless, results are grounded on a series of assumptions and model caveats are described throughout and in detail later in the discussion (Chapter 7.4.4 and 7.4.5).

In the beginning of the production cycle (pre-bloom conditions), simulations revealed protist populations weakly controlled by predation, particularly diatoms (Fig. 7.10). Changes in mixing conditions, affecting light and nutrient availability, may disrupt prey-predator relationships and allow populations to bloom during spring (Irigoien et al., 2005). This can be triggered by the poor nutritional status of the prey which alleviates grazing pressure (Polimene et al., 2015); in the model, however, the overall nutritional status of the prey was high during this period (Fig. E.9). During the spring bloom, predation by protistan grazers was higher than mesozooplankton predation (Fig. E.11), as previously observed in the North Sea (Löder et al., 2011). The simulated 
spring bloom was dominated by diatoms due to its ability to reach high growth rates if light and nutrient conditions favour their growth (Litchman et al., 2007). Dinoflagellates followed the diatom bloom in the model, as previously observed within the North Atlantic (Barton et al., 2013a), and mixotrophy was found to be key for their development (Fig. 7.11). Protists displaying acquired phototrophy peaked later in spring and persisted as stratification developed in the model (Fig. 7.8). Certain species of non-constitutive mixotrophs are largely dependent on phototrophy and thrive in turbulent waters, such as Mesodinium rubrum, while others (oligotrich ciliates) rely more on phagotrophy and therefore benefit from $\mathrm{C}$ gains during periods of low prey availability (Stoecker et al., 2009; Johnson et al., 2013; Stoecker et al., 2017). These features match well with the observational data at L4, with specialist forms (mainly $M$. rubrum) peaking during spring and generalist forms (i.e., oligotrich ciliates) during summer (Fig. E.12).

Under stratified conditions, predation pressure was higher (Fig. 7.10), as previously observed in the Western English Channel (Fileman et al., 2002). Model and data show that mixotrophs dominate the protist assemblage under these conditions (Figs. 7.10 and E.7) and mixotrophic activity is important to their growth (Fig. 7.11). Mixotrophic nanoflagellates (nano-CMs) can obtain nutrients feeding on bacteria which are enriched in $\mathrm{N}$ and $\mathrm{P}$ relative to $\mathrm{C}$ (Mitra et al., 2014b). These mixotrophs are now well recognised as being important bacterivores in nutrient-depleted waters (Havskum and Riemann, 1996; Zubkov and Tarran, 2008; Unrein et al., 2014). Microzooplankton also increased in relative importance from the onset of stratification to stratified conditions as showed by both model and data (Figs. 7.10 and E.7). Contrary to the data, the model could not predict the dynamics of constitutive mixotrophic dinoflagellates (micro-CMs) at L4 during stratified periods (discussed in detail in section 7.4.4).

As stratification breaks down in the model, diatoms increase again in relative importance, but not as much as during spring due to nitrate limitation (Figs. 7.10 and E.9). The overall role of predation regulating protist populations was still high, being slightly higher on prey smaller than $20 \mu \mathrm{m}$ compared to the stratified period (Fig. 7.10). Previous observations close to L4 station found that protist grazers smaller than $200 \mu \mathrm{m}$ were responsible for $100 \%$ of total primary production grazed daily during this period (Fileman 
et al., 2002; Fileman, unpublished). In the model, the higher predation pressure observed during this period is explained by both micro- and meso- zooplankton populations (70 vs $30 \%$ of total grazing, respectively; Fig. E.11) which could maintain their biomass during summer, responding quickly to prey availability (Fig. E.10). Far less attention has been given to understand autumn dynamics compared to spring bloom dynamics in the literature. Species composition in the previous autumn has been shown by a modelling study to strongly affect species composition during the following spring (Dakos et al., 2009). This is supported by observations that found protist richness to be higher during late summer/early-autumn (Gran-Stadniczeñko et al., 2019). For instance, selective grazing by ciliates and dinoflagellates can be an important factor shaping the diversity of mixotrophic nanoflagellates during autumn (Johnson et al., 2018).

Once the water column is fully mixed, simulations revealed microzooplankton as the major components of the protist assemblage, with all protist populations largely controlled by microzooplankton predation (Figs. 7.10 and E.11). However, predation control decreased throughout the winter, as supported by previous empirical studies close to L4 station (Table 7.4) and in the Mediterranean Sea (Calbet et al., 2008). Interestingly, mixotrophs found a window of opportunity during this period, increasing in biomass relative to autotrophs and heterotrophs; this feature was supported by observed data although being less pronounced than in the model (Fig. E.7). Therefore, model and data support that the protist community shifts from being dominated by heterotrophs early in winter to mixotrophs and finally to autotrophs at the onset of stratification.

The model reproduced realistic growth and grazing rates (Table 7.4). Simulated values were compared with empirical estimations obtained from dilution or gut content experiments from studies conducted in the Western English Channel and other temperate or coastal systems (Table 7.4). Overall, simulated growth $(\mu)$ and grazing rates were in the range observed in the literature, but averaged simulated values were lower (Table 7.4). The empirical studies, however, have different temporal resolutions and averaged rates are potentially overestimated due to the lack of measurements during winter. It is also noteworthy that simulated $\mu$ is given in terms of carbon, while $\mu$ from empirical studies correspond to changes in chlorophyll or cell division. The predation pressure by 
phagotrophic protists on phototrophic prey was higher than that of mesozooplankton in the model, in accordance with previous observations; however, the fraction of total primary production grazed daily in the model was lower than expected by the observations (Table 7.4). The mesozooplankton model and parameterisation followed that by Butenschön et al. (2016) and the emergent simulated growth rates ranged between -0.01 to 0.14 day $^{-1}$ (av. 0.05 day $^{-1}$ ). These values are within the range observed in the literature (e.g., Liu and Hopcroft, 2006), but as observed for protists, averaged values were lower than empirical observations (Yáñez et al., 2018). 
Table 7.4 Comparisons between simulated (this study) and observed (natural assemblages) prey growth rate $\left(\mu\right.$; day $\left.{ }^{-1}\right)$, grazing rate $(g$; day $\left.^{-1}\right)$, and proportion of total prey population grazed daily $(\mathrm{g}: \mu)$ in different temperate and/or coastal systems. Minimum, maximum, and averaged values are provided whenever possible. Data are given for phagotrophic protists smaller than $200 \mu \mathrm{m}$ and mesozooplankton grazing on phototrophic prey (A and B, respectively) and copepods grazing on phagotrophic protists (C); na - not applicable.

\begin{tabular}{|c|c|c|c|c|}
\hline \multicolumn{5}{|c|}{ A. Phagotrophic protists smaller than $200 \mu \mathrm{m}$ grazing on phototrophic prey } \\
\hline Reference & $\mu($ day-1) & $\mathrm{g}($ day-1) & $\mathrm{g}: \mu$ & Study area \\
\hline This study (model) & $0.05-0.28(0.17)$ & $0.00-0.16(0.06)$ & $0.02-0.60(0.27)$ & Western English Channel (L4 station) \\
\hline Fileman (unpublished) & $0.11-0.75(0.34)$ & $0.07-0.36(0.24)$ & $0.38-1.19(0.80)$ & Western English Channel (E1 station) \\
\hline Fileman et al. (2002) & $0.00-0.16$ & $0.94-1.03$ & 1.00 & Western English Channel \\
\hline Burkill et al. (1987) & $0.16-0.35(0.29)$ & $0.36-1.04(0.58)$ & $1.03-2.97(2.00)$ & Carmarthen Bay, South Wales \\
\hline Loebl and Van Beusekom (2008) & $0.01-1.08(0.34)$ & $0.00-1.23(0.29)$ & $0.00-1.40(0.44)$ & Wadden Sea (coastal) \\
\hline Löder et al. (2011) & $0.17-0.77(0.50)$ & $0.39-0.66(0.50)$ & $0.80-2.00(1.20)$ & Helgoland Roads, North Sea \\
\hline Schmoker et al. (2013) & $0.26-0.77(0.46)$ & $0.15-0.54(0.33)$ & $0.35-1.00(0.67)$ & Coastal Atlantic (review) \\
\hline \multicolumn{5}{|c|}{ B. Mesozooplankton grazing on phototrophic prey } \\
\hline Reference & $\mu($ day-1) & g (day-1) & $\mathrm{g}: \mu$ & Study area \\
\hline This study (model) & $0.05-0.28(0.17)$ & $0.00-0.08(0.03)$ & $0.00-0.32(0.13)$ & Western English Channel (L4 station) \\
\hline Fileman (unpublished) & $-0.44-1.14(0.46)$ & $0.00-1.10(0.25)$ & $0.00-2.00(0.36)$ & Western English Channel (E1 station) \\
\hline Löder et al. $(2011)^{1}$ & $-0.18-0.13(0.00)$ & $0.05-0.34(0.16)$ & $0.13-1.00(0.46)$ & Helgoland Roads, North Sea \\
\hline Li et al. $(2013)^{2 *}$ & na & na & $0.00-0.01$ & Southern Yellow Sea \\
\hline Lee et al. $(2012)^{3 *}$ & na & na & $0.00-0.30(0.08)$ & Asan Bay, Korean Peninsula \\
\hline \multicolumn{5}{|c|}{ C. Mesozooplankton grazing on phagotrophic protists $(20-200 \mu \mathrm{m})$} \\
\hline Reference & $\mu($ day-1) & $\mathrm{g}($ day-1) & $\mathrm{g}: \mu$ & Study area \\
\hline This study (model) & $-0.00-0.19(0.06)$ & $0.00-0.03(0.01)$ & $0.00-0.39(0.13)$ & Western English Channel (L4 station) \\
\hline Fileman et al. $(2010)^{4}$ & $-0.01-0.30(0.09)$ & $0.01-0.20(0.06)$ & $0.02-0.20(0.06)$ & Western English Channel (L4 station) \\
\hline Löder et al. $(2011)^{1}$ & $-0.04-0.27(0.13)$ & $0.40-0.78(0.50)$ & $0.00-4.00(2.00)$ & Helgoland Roads, North Sea \\
\hline
\end{tabular}


To date, few modelling studies have addressed mixotrophy when investigating the seasonal succession of plankton despite its recognised importance (Sommer et al., 2012; Mitra et al., 2014b; Stoecker et al., 2017). A class of plankton models based on trait distributions have found high investment in photosynthesis and nutrient uptake during spring and increased phagotrophy during summer (Bruggeman, 2009; Berge et al., 2017). These results are in accordance with findings from this study, with autotrophs dominating at the onset of stratification and mixotrophs during the stratified period (Fig. 7.10). Another study, however, found that the succession would develop from autotrophs to heterotrophs to mixotrophs and then back to small photoautotrophs during winter (Chakraborty et al., 2017). Here, heterotrophs were dominant only when the water column was fully mixed or at deeper waters (Fig. 7.10). In addition, the model used here could detect a succession from heterotrophs to mixotrophs within winter, highlighting the relevance of this trait under other periods that not summer in the production cycle.

The seasonal patterns of protist trophic strategies that emerges from modelling studies are largely dependent on model assumptions, particularly those associated with mixotrophy. Compared to models based on trait distributions, the modelling framework used here depends on a greater number of parameters (Flynn and Mitra, 2009); however, it allows the direct comparison of simulations against observational data through the explicit representation of different functional types (e.g., Ghyoot et al., 2017b). One could argue that the relative investments applied within the models mentioned above are difficult to measure empirically, hampering model comparison against observations.

Another important issue is how mixotrophy is related to size in models which are allometric-scaled (e.g., Baird and Suthers, 2007; Banas, 2011; Ward et al., 2012). Within these models, it is assumed that smaller cells are more likely to take up inorganic nutrients and photosynthesize while larger cells would be more likely to engage on phagotrophy (Ward and Follows, 2016; Chakraborty et al., 2017). Mixotrophs, however, range from pico- to mega- plankton in size and mixotrophic strategies, i.e., the balance between phototrophy and phagotrophy, do not follow cell size (Stoecker et al., 2017). Even closely related constitutive mixotrophs can deploy distinct mixotrophic strategies, with some strains being able to survive in darkness (Liu et al., 2016; Lie et al., 2018; Calbet et al., 
2011). On the other extreme, large unicellular plankton such as Rhizaria function primarily as phototrophs (de Vargas et al., 2015). Therefore, care must be taken when coupling mixotrophy to allometric-scaled models.

\subsubsection{Mixotrophy is a persistent trait in temperate seas}

An important finding from this study is that mixotrophs persist throughout the seasonal cycle in a coastal temperate sea (Figs. 7.10 and E.7 and Table 7.1). The model captures this feature representing different mixotrophic forms and the plasticity of mixotrophic strategies so that phototrophy and phagotrophy are adjusted according to environmental conditions to maximise growth rates. As predicted by the model, smallsized constitutive mixotrophs (nanoflagellates) are the dominant group of mixotrophs at L4 (in terms of biomass), occurring throughout the year (Fig. 7.6). Constitutive mixotrophs larger than $20 \mu \mathrm{m}$ (mainly dinoflagellates) are present at low biomass over the seasonal cycle, peaking only at late summer/early autumn, but the timing of their bloom was not captured by the model (Fig. 7.6; see also Chapter 7.4.4). Non-constitutive forms also persist over time at L4, but in lower biomass values when compared to their heterotrophic counterparts and the model was able to reproduce the dynamics of these two groups (Figs. 7.8 and E.4).

The relevance of mixotrophy (phagotrophy) among small-sized constitutive mixotrophs (nanoflagellates) was greater once inorganic nutrients or light was limiting growth; in fact, engaging on phagotrophy during winter was key to secure survival (Fig. 7.11). During summer, the importance of mixotrophy among this group is related to the benefit of acquiring nutrients from bacterial prey (Arenovski et al., 1995; Unrein et al., 2007; Mitra et al., 2014b; Stoecker et al., 2017); in turn, mixotrophy can also be used as a carbon supply under light limited conditions (Hall et al., 1993; Unrein et al., 2007; Czypionka et al., 2011; Millette et al., 2017; Edwards, 2019). Dinoflagellates in the nanoplankton spectrum, such as Prorocentrum minimum which is one of the most abundant at L4 (Widdicome et al., 2010), can use C, N, and P obtained through phagotrophy to grow, but are generally considered to be primarily phototrophic (Johnson, 2015). 
In the model, the success of larger constitutive mixotrophs (mainly dinoflagellates) was strongly dependent on the availability of prey even under conditions favourable to autotrophy, such as during the stratification breaking down (Fig. 7.11). Experimental studies have shown that the net growth of dinoflagellates can be higher when they are growing as mixotrophs as opposed to autotrophic nutrition (Jeong et al., 2005b; Adolf et al., 2008; Glibert et al., 2016); but this is highly variable among species (Jeong et al., 2010c; Hansen, 2011). In addition, they can decrease competition and thrive by adopting the strategy of 'eating your competitor' (Thingstad et al., 1996). In contrast, nonconstitutive mixotrophs (mainly ciliates) are primarily heterotrophs; therefore, mixotrophy (phototrophy) was most significant during stratified periods (Fig. 7.11) in which the availability of prey is low (Levinsen et al., 1999; Stoecker et al., 2009; Yang et al., 2014). It is also noteworthy that phototrophy among these mixotrophs is not expected to vary with prey availability (Schoener and McManus, 2017). Although the model captures well the peak of M. rubrum observed late in spring at L4, the role of phagotrophy was overestimated for this species, since it largely relies on phototrophy (Hansen et al., 2013).

So far, few studies have estimated the balance between phototrophy and phagotrophy to the carbon budgets of mixotrophs (Table 7.5). Simulated values are within the range observed in the literature; however, these studies are species-specific and are based upon experimental conditions which differ among studies (e.g., temperature, light, nutrient, and prey conditions). Furthermore, these studies also differ in providing gross or net carbon budget estimates (Table 7.5). All these aspects hinder any further comparisons between simulated values and observations from the literature (Table 7.5). Considering the diverse mixotrophic strategies adopted by protists, including within functional types (Table 7.5), further studies are necessary to estimate not only carbon but also nitrogen and phosphorus budgets among mixotrophs (Glibert et al., 2009; Carvalho and Granéli, 2010). These studies will require plankton ecologists to develop new methodologies, which will potentially involve the combination of different techniques, to quantify mixotrophy both under controlled conditions in the laboratory and within natural assemblages in the field (Stoecker and Lavrentyev, 2018; Beisner et al., 2019). 
Table 7.5 Relative contribution of phagotrophy to the carbon budget of different mixotrophs in the model (Nano-CMs, Micro-CMs, and $\mathrm{NCMs}$ ) and of specific mixotrophic species under experimental conditions. Relative estimates (\% C phagotrophy) refer to the gross or to the net budgets under a given temperature (T). Minimum, maximum, and averaged values are provided whenever possible; na - not applicable.

\begin{tabular}{lllll}
\hline $\begin{array}{l}\text { A. Nano-CMs } \\
\text { Reference }\end{array}$ & mixotrophic species & \% C phagotrophy & budget & $\mathrm{T}\left({ }^{\circ} \mathrm{C}\right)$ \\
\hline This study (model) & na & $-2.8-23.1(10.7)$ & net & $8-16$ \\
Princiotta et al. (2016) & Dinobryon sociale & $20-30$ & net & 8 \\
Princiotta et al. (2016) & Dinobryon sociale & $1.2-1.7$ & net & 20 \\
Caron et al. (1993) & Dinobryon cylindricum & $14-20(21)$ & net & 20 \\
Terrado et al. (2017) & Ochromonas sp. & $84-99$ & net & 20 \\
\hline B. Micro-CMs & & & \\
Reference & mixotrophic species & $\%$ Chagotrophy & T $\left({ }^{\circ} \mathrm{C}\right)$ \\
\hline This study (model) & na & $2.7-37.5(20.2)$ & gross & $8-16$ \\
Adolf et al. (2006) & Karlodinium micrum & $31-73$ & gross & 20 \\
Johnson (2015) & Prorocentrum minimum & $52-86(65)$ & gross & 18 \\
Skovgaard (1996) & Fragilidium subglobosum & $34-100$ & gross & 15 \\
Skovgaard (2000) & Gyrodinium resplendens & $>50 \%$ & gross & 15 \\
\hline C. NCMs & & & \\
Reference & mixotrophic species & $\%$ C phagotrophy & & T $\left({ }^{\circ} \mathrm{C}\right)$ \\
\hline This study (model) & na & $75.8-98.2(91.5)$ & net & $8-16$ \\
Stoecker and Michaels (1991) & Laboea strobila & net & 15 \\
Stoecker and Michaels (1991) & Strombidium conicum & 97 & net & 15 \\
Schoener and McManus (2017) & Strombidium rassoulzadegani & $95-100$ & net & 19 \\
Johnson and Stoecker (2005) & Mesodinium rubrum & up to 10 & net & $0-2$ \\
\hline
\end{tabular}




\subsubsection{Different mixotrophic populations across depth?}

The results described above conform mainly with the dynamics observed within the mixed layer. Seasonal differences are expected to be more pronounced in surface than in deeper waters, but the relative contribution of protist trophic strategies is also expected to change throughout the water column (Bruggeman, 2009; Gran-Stadniczeñko et al., 2019). The model showed an increase in the contribution of heterotrophs with depth and the persistence of mixotrophs (Fig. 7.10). L4 data at deeper waters were only available for pico- and nano- plankton and observations supported these results (Fig. E.13). It is not very clear, however, how mixotrophic populations vary with depth; most studies that investigated the vertical distribution of protists focused only on one specific group of mixotrophs (Arenovski et al., 1995; Havskum and Riemann, 1996; Czypionka et al., 2011; Sanders and Gast, 2012; Weber et al., 2014). What the model can tell us about it?

The model suggests that the composition of mixotrophic populations changes over the water column once stratification develops in a coastal site, shifting from being dominated by constitutive to non-constitutive forms with increasing depth (Fig. 7.11). Irrespective of seasonal changes, this is associated with the attenuation of light over the water column, which favours the heterotrophic metabolism (Figs. 7.10 and 7.11). In the model, constitutive mixotrophs cannot sustain positive growth if a critical proportion of growth is not obtained through phototrophy. In turn, non-constitutive forms do not survive without food and a source of plastids but can survive under severe light limitation. Observations support that most constitutive mixotrophs cannot grow in the dark (Andersson et al., 1989; Carvalho and Granéli, 2010; Hansen, 2011) but it is noteworthy that even closely related species may have different mixotrophic strategies (Liu et al., 2016; Lie et al., 2018; Calbet et al., 2011). In turn, non-constitutive mixotrophs can survive through the darkness of winter in polar seas (Levinsen et al., 2000; Jakobsen et al., 2000; Stoecker et al., 2009; Stoecker et al., 2017).

Data compilations presented in Chapters 3 and 4 can help to understand general patterns in the vertical distribution of different mixotrophs (Tables A.2, B.2, and B.3). Previous studies have quantified the relative contribution of constitutive mixotrophs to the total nanoflagellate biomass over the water column (Chapter 4). Their biomass 
contribution was considerably lower in the deep chlorophyll maximum (Arenovski et al., 1995; Havskum and Riemann, 1996; Czypionka et al., 2011) and they were absent in darkness (Czypionka et al., 2011). Their bacterivory impact was also considerably lower with depth (Havskum and Riemann, 1996; Czypionka et al., 2011). However, it is possible that mixotrophic nanoflagellates are present in deeper waters but were not active (Czypionka et al., 2011). Despite pointing towards a decrease in the relative contribution of mixotrophic nanoflagellates with depth, there is also evidence that certain species can thrive in deep waters, such as Dinobryon balticum (Olli et al., 2002). Among nonconstitutive mixotrophs, no study was found to quantify their contribution to total microzooplankton over depth (Table A.2). Nevertheless, many studies provided information for surface waters while others provided an integrated value throughout the water column. Disregarding temporal differences and the different study sites, the average contribution of NCMs to total microzooplankton biomass was $41 \%$ in surface waters and $46 \%$ across the water column (Appendix A, Table A.2). These numbers suggest, at least, that their relative biomass does not decrease with depth. In fact, a previous study suggest that $M$. rubrum can comprise a significant proportion of protist biomass at depth (Pérez et al., 2000; Weber et al., 2014).

Despite the evidence presented above, we still lack a clear understanding of the vertical distribution of mixotrophic populations. Although the model suggests that constitutive mixotrophs may pay a higher price than non-constitutive forms over depth, it is noteworthy that community diversity may also change vertically (Sanders and Gast, 2012). Also, the model does not account for diel vertical migration (further discussed in Section 7.4.4). Nevertheless, in terms of chlorophyll concentration, constitutive forms contributed significantly to total chlorophyll both in surface and deeper waters in the model, while NCMs contribution was minor (Fig. E.14). Due to the high dilution rate of kleptochloroplasts and due to the predominance of heterotrophic metabolism (Fig. 7.11), NCMs do not contribute significantly to total chlorophyll at any of the depth strata evaluated (Fig. E.14). However, this scenario could be different if SNCMs were explicitly included in the model because they have lower dilution rates of kleptochloroplasts and rely mostly on phototrophic metabolism to growth. Despite the challenges associated with sampling mixotrophs in the field, further studies that aim to quantity vertical changes in 
biomass, mixotrophic activity, and community diversity, both between and within the different functional types of mixotrophs, will help to elucidate this issue.

\subsubsection{The dynamics of mixotrophic dinoflagellates at $\mathrm{L} 4$}

Comparisons of simulations with observational data at $10 \mathrm{~m}$ depth at L4 showed an overall good agreement for constitutive mixotrophs smaller than $20 \mu \mathrm{m}$ and for nonconstitutive mixotrophs but not for constitutive mixotrophs larger than $20 \mu \mathrm{m}$ (Fig. 7.6). These are mainly dinoflagellates (Table 7.1) which typically peak late summer/autumn at L4, being present at low biomass values during other periods of the year (Widdicombe et al., 2010). Within this group, the most abundant species at L4 is Karenia mikimotoi (Fig. E.15). Barnes et al. (2015) conducted a 19-year time series analysis at L4 and concluded that blooms of $K$. mikimotoi were mainly associated to elevated rainfall and resultant low salinities during summertime and, to a lesser extent, to silicate concentrations (Barnes et al., 2015). However, they did not discuss the potential role of mixotrophy. This species can feed through a peduncle on heterotrophic bacteria, picophytoplankton, and nanoflagellates and it does not seem to feed on prey larger than $12 \mu \mathrm{m}$ ESD (Jeong et al., 2010a). At L4, the biomass of $K$. mikimotoi starts to develop in July and reaches its maximum in August (Fig. E.15), matching the observed increases in the biomass of its potential prey (i.e., bacteria, picophytoplankton, and nanoflagellates) as well as the biomass of diatoms (Figs. 7.5, 7.6 and E.4). Therefore, phagotrophy may be another factor that contributes to the observed blooms of $K$. mikimotoi at L4.

However, the model presented here could not predict the seasonal dynamics of $K$. mikimotoi nor that of other micro-CMs at L4 despite explicitly resolving mixotrophy (Fig. 7.6). In the model, this group of mixotrophs was assumed to feed on several prey types including diatoms based on evidence for other several species of dinoflagellates (Table 5.1). Simulations showed that dinoflagellates peak together with diatoms during spring (Figs. 7.5 and 7.6) and feeding on this group is important for their growth. Despite reaching very similar phototrophic rates during the stratification breaking down (Fig. 7.11), the model cannot reproduce a second peak of micro-CMs and this is associated to the lower contribution of phagotrophy to their growth, particularly due to the lower availability of diatoms as prey. On top of this, predation pressure is higher during this 
period (Fig. 7.11) and NCMs and microzooplankton have a competitive advantage since they can maintain their biomass values during stratified periods (Fig. E. 8). So what mechanisms is the model currently lacking that could improve the representation of the dynamics of these organisms?

During mixed or slightly stratified water columns, dinoflagellates can engage on diel vertical migration; for instance, K. mikimotoi was observed to migrate up to $15 \mathrm{~m}$ (Koizumi et al., 1996). A different behaviour appears to take place in stratified periods, with populations accumulating in fine layers in or close to the pycnocline (Bjoernsen \& Nielsen, 1991). In addition, it is known that dinoflagellates are extremely sensitive to agitation when grown in cultures and mortality can be associated with shear rate (Gentien et al., 2007). Therefore, it has been suggested that the confinement in the pycnocline is related to increased survival rate (Gentien et al., 2007). In the model presented here, such mechanisms were not explicitly described; non-predatory mortality is described as a linear function of nutrient stress (for all protists). Most biogeochemical models do not explicitly resolve mortality as a function of mixing because biological controls are expected to be more important than physical ones, particularly within slightly stratified coastal seas as L4 (Atkinson et al., 2018). Another effect not included in the model is the ability of many dinoflagellates to produce toxins (Smayda, 1997). In general terms, allelopathy is not included in ecosystem models and may be important. This adaptation provides a competitive advantage over other phototrophic species; however, it can be countered by autotoxicity, as previously observed for K. mikimotoi (Gentien et al., 2007). These mechanisms were not considered in the model and could help the correct prediction of the seasonal dynamics of mixotrophic dinoflagellates.

\subsubsection{Caveats: model vs observational data at $L 4$}

The model was able to reproduce annual averaged values of nutrients and chlorophyll concentrations and total plankton biomass at L4 (Fig. 7.2). Overall, model simulations also agreed with seasonal observational data for these variables, performing better or at least as well as ERSEM (Figs. 7.4 and 7.7). However, in all models, plankton biomass is underestimated during winter while overestimated during spring (Fig. 7.3). These limitations are not expected to be independent from each other since the plankton 
community in the beginning of the production cycle will affect the community observed during the spring bloom.

During winter, phototrophic populations were strongly limited by light availability. One could argue that, to improve their performance under light limitation, their light harvesting capacity should be increased. However, the initial slope of the photosynthesis-irradiance curve $\left(\alpha_{\mathrm{Chl}}\right)$ was parametrised based on maximum values observed within coastal temperate seas (up to $0.062 \mathrm{mg} \mathrm{C} \mathrm{mg} \mathrm{Chl}^{-1} \mathrm{~h}^{-1}$ ( $\mu$ molquanta $\mathrm{m}^{-2}$ $\left.\mathrm{s}^{-1}\right)^{-1}$ ), as revealed by a meta-analysis (Bouman et al., 2018). Previous observations at L4 found $\alpha_{\mathrm{Chl}}$ values up to $0.035 \mathrm{mg} \mathrm{C} \mathrm{mg} \mathrm{Chl}^{-1} \mathrm{~h}^{-1}$ ( $\mu$ molquanta $\left.\mathrm{m}^{-2} \mathrm{~s}^{-1}\right)^{-1}$ (Barnes et al., 2015). Nevertheless, when forcing the model with higher $\alpha_{\mathrm{Chl}}$ values, the initiation of the bloom would start relatively earlier but no improvement was observed during winter. Therefore, this limitation is associated with the physical model instead; improving how light is described in biogeochemical models is subject of future work for the ecosystem modelling community (Holt et al., 2014).

The low biomass of diatoms in the beginning of the production cycle affects silicate dynamics and the model cannot reproduce the gradual transition from replete to deplete silicate conditions observed at L4 as stratification develops (Fig. 7.5). The model can reproduce silicate concentrations observed during summer but, in turn, it requires a high diatoms peak which appears to be 'over-predicted' at L4 (Fig. 7.5). In reality, diatoms are present in lower biomass values, with similar values observed from spring to autumn. Besides the limitations associated with the light model, four mechanisms can explain the simulated dynamics of silicate and diatoms; these are related with changes in diatom community composition, the mismatch between trophic levels, advective processes, and vertical export. The diatom community changes seasonally at L4, with large centric diatoms dominating during winter while small pennate diatoms thrive during summer (Widdicombe et al., 2010), but this diversity is not represented in the model. Another interesting feature observed at L4 is that mesozooplankton timing has little relation to that of food (Atkinson et al., 2015), making it very challenging for models to correctly simulate the timing of mesozooplankton (which, as for diatoms, is considered as a single 'black' box). In addition, the 1D physical model used here does not account for advection and, as any other monitoring site, the interpretation of L4 time-series can be confounded by 
advective processes. Finally, L4 is characterised by a productive benthic community and blooms have been observed to get exported to the sea bed (Zhang et al., 2015). In fact, nitrate concentrations observed below the mixed layer are lower than the values predicted by the model (Fig. E.16). These, together with the limitations associated to the light model, suggest that the model is underestimating biological activity at depth.

Other biological mechanisms such as resting stages, parasitism, selective feeding among mesozooplankton, and temperature-related phenology were not considered in the model and may play important roles at times. The ability to form resting stages can affect plankton dynamics over the water column through export and/or import. Summer diatoms bloom, for instance, can be triggered by the germination of resting stages that sedimented after the spring bloom (Chen et al., 2009). In the lower end of plankton food webs, parasites are recognised to play significant roles in controlling population sizes, trophic transfer, biodiversity, and succession (Scholz et al., 2016). A molecular study has shown that parasites can play a role in the succession of protist communities during blooms in the Western English Channel (Genitsaris et al., 2015). Yet, most biogeochemical models do not explicitly describe this group; here, for instance, a mortality term that varies linearly with nutrient stress was configured to account for non-resolved mortality, such as viral lysis. Going up in the plankton food web, mesozooplankton can display selective feeding based on prey quality and potentially facilitate a bottom-up control with consequent higher vertical export of particulate matter (Mitra, 2006; Mitra and Flynn, 2006; Sailley et al., 2015). This mechanism was not included in the model used here; however, a previous study found that copepods display unselective feeding behaviour at L4 (Djeghri et al., 2018). Lastly, the model did not account for temperature-related phenology, which can affect both protists and mesozooplankton (Rose and Caron, 2007; Mackas et al., 2012; Atkinson et al., 2015).

Ultimately, there is no single explanation for what defines plankton succession within temperate seas and, most likely, several mechanisms take place and may differ in relative importance in different points in time and space (Atkinson et al., 2018). Model predictions are limited to the processes described and, therefore, represent simplifications of the real system being simulated. If one wants to increase the predictive power for the 
different functional types assigned in the model, automated methods of parameter estimation may provide a way forward (Schartau et al., 2017). Nevertheless, parameter tuning has its own limitations since just a few parameters can be optimised. In addition, one needs to keep in mind that the spatio-temporal scaling of the model differs greatly from that of the data and that there will always be caveats associated to any dataset. For instance, mesozooplankton at L4 is sampled with a net of $200 \mu \mathrm{m}$ mesh size, potentially underestimating the trophic role of copepodites and copepod nauplii (e.g., López et al., 2007). Even with a very complex model that is well constrained by experimental observations, data may diverge from model output (Schartau et al., 2017). The process of creating a model should be interactive and cyclic, from model to experiments then back to model and, therefore, even if the parameters are not well constrained by the data, a model can guide us on future experimental investigations.

On the other hand, one could argue that it depends on the question being posed. The main focus of this study was the seasonal succession of protist trophic strategies and, in particular, the relevance of mixotrophy over the production cycle. Based on this, the model was able to reproduce general features of the succession of protists in temperate seas. More importantly, it was able to represent the biomass of mixotrophs (except for dinoflagellates) and how different trophic strategies progress over the seasonal cycle. Despite the uncertainties associated with modelling mixotrophic nutrition, the major limitations of this study (i.e., low biomass during winter and high diatom spring bloom) were associated with other features, as discussed above, and probably require a revision of the physical model. Finally, modelling studies not only are built upon empirical data and are used for predictions but, perhaps more importantly, also generate new hypotheses and provide new directions for future research. In this sense, the present study calls attention for the persistence of mixotrophy (and its different forms) over the seasonal cycle and throughout the water column in temperate seas. 


\subsection{Summary}

Model and data confirmed that mixotrophy is a persistent trait within temperate seas. The model was able to predict general patterns on protist succession over the seasonal cycle, with autotrophs dominating during more productive periods, mixotrophs during summer, and heterotrophs once the water column is fully mixed. Interestingly, during winter, both model and data showed a succession from heterotrophic to mixotrophic protists, suggesting that the latter can find a window of opportunity to grow before autotrophs take place at the onset of stratification. Seasonal patterns were less evident in deeper waters, but the model suggests that mixotrophic populations change vertically, shifting from being dominated by constitutive forms to non-constitutive forms with increases in depth. Both seasonal and vertical patterns were observed simulating changes in mixotrophic strategies (the synergy between phototrophy and phagotrophy) and assigning different mixotrophic functional forms in the model. At L4, nano-CMs were the most abundant group of mixotrophs, while micro-CMs only peaked late summer and NCMs were found throughout the seasonal cycle but in lower biomass values. Despite model caveats, limitations were mainly associated to diatom dynamics and with the physical model (and not with the description of mixotrophic nutrition), suggesting that the next step is to integrate mixotrophy in 3D ocean models. 


\section{Chapter 8}

General Discussion 


\subsection{Introduction}

The main aims of this thesis were: i) to investigate the biogeography of mixotrophs according to their functional diversity across the global oceans and to evaluate how it relates to environmental variability; ii) to explore the competitive outcomes between mixotrophs and their auto- or hetero- trophic counterparts and the effect of mixotrophy on ecosystem functioning; and iii) to investigate the seasonal succession of protist trophic strategies and the vertical distribution of mixotrophs within a coastal temperate sea. These aims were explored through the interrogation of global databases and literature, applying theoretical modelling approaches, and utilizing long-term time-series datasets to validate model results at a specific site. Here, a summary of the results within this thesis is provided (Chapter 8.2) followed by a discussion on how these findings relate to the current literature (Chapter 8.3) and, to conclude, the challenges ahead in plankton ecology and mixotrophic research is revised (Chapter 8.4).

\subsection{Summary of thesis results}

\subsubsection{Biogeography of mixotrophs across the global oceans}

This thesis presents the first attempt to evaluate the biogeography of marine mixotrophic protists at a global scale (Chapters 2, 3, and 4). Mixotrophy among both constitutive and non-constitutive forms was found to be ubiquitous in the global oceans (Figs. 3.1 and 4.1). Among non-constitutive mixotrophs, different biogeographic patterns were observed based on mixotrophic functional diversity, with endosymbiotic forms dominating in oligotrophic waters while mixotrophs relying on acquired plastids were more important in high-biomass areas (Fig. 3.5). Interestingly, the similarities between the biogeographic patterns of the different groups suggest a continuum based on their control over acquired phototrophy, from GNCMs to pSNCMs to eSNCMs, respectively. This study also revealed that $40-60 \%$ of plankton traditionally viewed as microzooplankton are, in fact, non-constitutive mixotrophs (Chapter 3). However, their relative contribution in terms of biomass depends on the spatio-temporal scale being evaluated. Overall, the biomass of GNCMs was higher during summer in the Mediterranean Sea, the pSNCM Mesodinium was more abundant during spring within 
coastal seas, and eSNCMs were particularly abundant during autumn within equatorial upwelling zones (Fig. 3.6).

Constitutive mixotrophs vary greatly in size, taxonomy, and with respect to the level of mixotrophy that they undertake (i.e., their balance between phototrophy and phagotrophy); however, it was not possible to evaluate their biogeography at this level of detail when interrogating global databases due to sampling biases (Chapter 4). Despite confirming that sampling occurred across all biogeographic provinces but one (Fig. 4.2), most of the maps generated for the different sizes and taxonomic groups of CMs indicate that their distribution is very restricted to certain areas (Fig. 4.1) or, as verified afterwards, that there are clear gaps in the data (Figs. 4.3 and 4.4). Data are biased towards larger species, mainly dinophytes, and less data were available within oligotrophic gyres and in equatorial regions compared to the other biomes (Figs. 4.3 and 4.4). Since the results pointed to non-dinophyte species $<20 \mu \mathrm{m}$ as being the least represented group, data from global databases were compared against data from studies which particularly targeted these organisms (Fig. 4.7). While global data indicate that this group is largely limited to polar and temperate seas (and mainly in coastal areas), their true distribution encompasses open oceans including areas close to the Equator (Fig. 4.7). Moreover, the abundance of actively feeding mixotrophs is highly variable across the oceans (Chapter 4).

The abundance of mixotrophic nanoflagellates (nano-CMs) was found to increase exponentially towards more productive systems (Fig. 4.8); however, this pattern depends on prey abundance and was not observed for bacterial concentrations higher than $1 \times 10^{7}$ cells $\mathrm{mL}^{-1}$ (Fig. 4.9). In addition, this relationship was verified in surface and sub-surface waters as well as in the deep chlorophyll maximum, but it was almost absent at the thermocline and disappeared when evaluating their abundance averaged over the water column (Fig. 4.8). When comparing their biomass with that of heterotrophic nanoflagellates, nano-CMs were less abundant across all oceanic biomes (Fig. 4.10). Among the different biomes, the abundance ratio between nano-CMs and that of heterotrophic nanoflagellates was higher and also greatly variable in more productive biomes (i.e., coastal seas, equatorial upwelling, and polar seas); in turn, variability was lower within oligotrophic oceans such as the Mediterranean Sea and within the oceanic 
gyres (Fig. 4.10). Despite being less numerically abundant than heterotrophic nanoflagellates, nano-CMs contributed to $\sim$ half of the total bacterivory across the different biomes (Fig. 4.10). Their contribution was comparable among all biomes except within polar seas and in zones of coastal upwelling (Fig. 4.10).

\subsubsection{Mixotrophy, ecosystem functioning, and community composition}

A theoretical analysis of a plankton food web revealed that mixotrophy influences nutrient availability, mass and energy transfer to higher trophic levels, and the microbial loop (Chapters 5 and 6). In the light-limited scenario, ammonium regeneration was higher in the absence of mixotrophy (Fig. 6.3); once mixotrophs were included in the food web they competed with their heterotrophic counterparts and down-regulated the biomass of nano- and micro- zooplankton (Fig. 6.4). In addition, mixotrophy changed community composition, from smaller (in the non-mixotrophic food web) to larger (in the mixotrophic food web) phototrophs (Fig. 6.4). Since mesozooplankton cannot access small prey such as picophytoplankton, mixotrophy supported higher trophic transfer efficiency of carbon biomass to higher trophic levels (Fig. 6.3). The production of labile DOC was higher in the absence of mixotrophs due to the high biomass achieved by picophytoplankton and diatoms, resulting in higher GPP and consequent leakage of labile DOC (Fig. 6.3). All properties described above were most sensitive to photosynthetic parameters, particularly the slope of the PE curve and the maximum chlorophyll to carbon ratio (Table 6.5).

The effect of mixotrophy was even more pronounced in the nutrient-limited scenario (Fig. 6.5), with mixotrophs outcompeting their autotrophic and heterotrophic counterparts (Fig. 6.6). Non-constitutive forms could outcompete heterotrophic protists due to limited prey availability and the overall low regeneration of ammonium decreased the availability of inorganic nutrients, favouring constitutive mixotrophs over autotrophic protists (Figs. 6.5 and 6.6). Similarly to the light-limited scenario, the trophic transfer efficiency was higher in the presence of mixotrophs and NCMs were an important prey supporting the biomass of mesozooplankton (Fig. 6.5). In turn, the production of labile DOC was higher in the presence of mixotrophs and was mainly associated with a larger fraction of prey remaining unassimilated and being egested (Fig. 6.5). Under this scenario, the results were most sensitive to the parameterisation of bacteria and mixotrophs, 
particularly the parameters defining the prey size spectrum of nano-CMs (Table 6.7). A closer examination of the individual effects of the different mixotrophs revealed nanoCMs to have the highest impact in plankton trophodynamics, promoting higher trophic transfer efficiency by outcompeting picophytoplankton (Fig. 6.7). When only micro-CMs were considered, they had a major role decreasing the overall regeneration of ammonium and the individual impacts of GNCMs or SNCMs were minor (Fig. 6.7). The impact of mixotrophy was higher when assigning nano-CMs and GNCMs or SNCMs and maximised once all mixotrophs were considered (Fig. 6.7).

Overall, size was found to be an important trait determining the success of mixotrophs with an innate capacity for photosynthesis, with smaller cells dominating under nutrient limitation and larger cells being more important under light limitation (Fig. 6.8a). Among non-constitutive mixotrophs, results suggest that the specificity of prey from which they acquire phototrophic potential affect their success, with generalist forms dominating under nutrient limitation and specialist forms showing maximal contribution in intermediate conditions of light and nutrient limitation (Fig. 6.8b). Finally, the model predicted that the relative contribution of autotrophic and heterotrophic protists to total protist biomass increases in the transition from nutrient to light limitation (Fig. 6.8).

\subsubsection{Seasonal succession of protists in a coastal temperate sea}

In Chapter 7, the food web model developed in Chapters 5 and 6 was coupled to a physical model of the water column (GOTM). The mixotrophic model reproduced the seasonal development of nutrients and chlorophyll concentrations as well as total plankton biomass at the Western English Channel at station L4 (Chapter 7). When evaluating nutrients, chlorophyll, and total plankton biomass, model performance was similar between the mixotrophic model, the non-mixotrophic model, and ERSEM (Figs. 7.3 and 7.4). All models overestimated the spring bloom at L4 due to the high biomass of diatoms which, in turn, was necessary to simulate silicate depletion during summer (Fig. 7.5). In addition, mesozooplankton appears earlier than their prey at L4 and, therefore, this feature was not captured by the model (Fig. 7.5). However, models performed differently from each other when evaluating each plankton functional type separately (Figs. 7.6 and 7.7). 
The mixotrophic model was used to investigate the seasonal succession of protist trophic strategies at L4. Model and data support the hypothesis that mixotrophy is a persistent trait across the seasonal cycle (Figs. 7.10 and E.7). The mixotrophic model predicted the greater importance of autotrophs during more productive periods, the dominance of mixotrophs during stratified periods, and the maximum contribution of heterotrophs towards the end of the production cycle, i.e., once the water column is fully mixed (Fig. 7.10). Compared to observed data, the model overestimated autotrophic biomass while underestimating heterotrophic biomass throughout the seasonal cycle (Figs. 7.10 vs E.7). Interestingly, the contribution of heterotrophs decreased during winter, particularly just before the spring bloom, opening a window of opportunity to mixotrophs (Fig. E.7). This feature is supported by the available data but the biomass of mixotrophs was overestimated by the model during this period.

The contribution of bottom-up and top-down controls in shaping the succession of protists varied across the seasonal cycle. Fast growing species take advantage at the onset of stratification and during the breaking down of stratification, but predation pressure was higher in the latter (Fig. 7.10). The overall role of predation is important to control protist populations during stratified periods and, in particular, once the water column is fully mixed (Fig. 7.10). Mixotrophy persisted across the seasonal cycle due to the synergy between phototrophy and phagotrophy (Fig. 7.11). Even below the mixed layer, where heterotrophs increased in relative biomass, mixotrophy comprised an important fraction of the total protist biomass (Fig. 7.10). Nano-CMs were the most dominant mixotrophs at L4, micro-CMs were important at times, and NCMs were less abundant but their biomass was relatively constant throughout the seasonal cycle. Mixotrophy (phagotrophy) was key for the success of nano-CMs during summer and to secure survival during winter (Fig. 7.11). Micro-CMs were strongly dependent on phagotrophy to outnumber competitors and be able to thrive (Fig. 7.11). The importance of mixotrophy (phototrophy) among NCMs was more important during summer, but also contributed to cover respiration costs in other periods (Fig. 7.11). 


\subsubsection{On the persistence of different protist functional types in the oceans}

The persistence of the different functional types was not always consistent between the observational data (Chapters 3 and 4) and the modelling studies (Chapters 6 and 7). For instance, NCMs and their heterotrophic counterparts cooccurred in all analyses; however, in order to persist in the models, they were not allowed to feed on each other. While the meta-analysis showed heterotrophic nanoflagellates and nano-CMs persisting across different environments (Chapter 4), they could never coexist in the modelling studies (both chemostat and 1D approaches). The results above suggest that further analyses are required to better implement the cost associated with the mixotrophic trait in models. In turn, the modelling analyses presented in Chapters 6 and 7 were consistent in a series of aspects, such as: i) mixotrophy favoured the concentration of biomass in larger size classes; ii) phototrophic nanoflagellates and microflagellates could only persist in the model once constitutive mixotrophy was assigned; iii) higher trophic transfer and lower ammonium regeneration was observed in the presence of the mixotrophic trait. However, once assigning realistic environmental variability (Chapter 7), diatoms achieved higher biomass values and GNCMs and SNCMs could not persist, contrary to the chemostat experiments (Chapter 6). These results are important because evidence the need to have an holistic approach, i.e., physical and biogeochemical model applied at a long-term monitoring site (i.e., L4 station), to investigate the complexities associated to the mixotrophic trait and its potential impact in ecosystem dynamics.

\subsection{Overview of findings in the context of the current literature}

\subsubsection{Spatial distribution of mixotrophic protists}

The studies within this thesis investigated the distribution of mixotrophs across environmental gradients and the physical and biological mechanisms behind their biogeographic patterns (Chapters 3, 4, 6, and 7). As more studies seek to understand the distribution of mixotrophs, the more it becomes clear that mixotrophy in plankton is ubiquitous (Stoecker et al., 2017; Leles et al., 2017; Leles et al., 2019; Faure et al., 2019; Edwards, 2019). Defining the traits that lead to a wide colonization and diversification in microorganisms is a central question in microbial biogeography (Dolan, 2005; Foissner, 2006; Caron, 2009; Martiny et al., 2006; Barton et al., 2013b). It is possible to conclude 
from the results herein that mixotrophy is a major trait defining the biogeography of protists across the oceans (Chapters 3, 4, 6, and 7). Nevertheless, we know very little about the biogeography of this trait.

The studies presented here are the first attempts to investigate the global distribution of mixotrophs (Leles et al., 2017; Leles et al., 2019). These revealed that not all mixotrophic taxa are everywhere in significant quantities (Chapters 3 and 4). For instance, all non-constitutive mixotrophs occurred in coastal seas as showed in Chapter 3 but the importance of endosymbiotic associations was clearly low in these regions while kleptoplastidic forms were more abundant (Leles et al., 2017; Faure et al., 2019). This illustrates how microbial biogeography differs from that of multicellular organisms because the former largely depends on quantities while the latter can be defined mostly by the presence/absence of taxa (Dolan, 2005). Furthermore, it shows the importance of diverse mixotrophic strategies in shaping the biogeography of protists.

Different mixotrophic types are selected under different environmental conditions (Chapters 3, 4, 5, and 6). As exemplified above, this was clear when evaluating different functional types of non-constitutive mixotrophs (Chapter 3). However, differences may also be found within functional types; for instance, endosymbiotic forms were found to have different biogeographies based on their ability to form colonies or depending on the mode of symbiosis (Faure et al., 2019). It is also expected that different constitutive mixotrophs will have different biogeographies due to their different sizes and the observed continuum of mixotrophic strategies, with some species relying more on phagotrophy and others closer to the phototrophic end of the spectrum (Burkholder et al., 2008; Jeong et al., 2010c; Flynn et al., 2013). It was not possible, however, to test this hypothesis here when interrogating global databases due to sampling biases and due to our limited knowledge about their mixotrophic metabolism (Chapter 4). Nevertheless, the modelling experiments (Chapters 6 and 7) suggest that size differences will result in different mixotrophic metabolisms, particularly due to different prey size spectrum and selection, and, thus, in different ecological niches among constitutive mixotrophs (Leles et al., 2018). Therefore, the above calls attention to the interpretation of mixotrophic diversity in combination with other traits when evaluating the biogeography of protists. 
It is noteworthy that the spatial distribution of organisms in the oceans takes place both horizontally as well as through the water column. In this sense, mixotrophs tend to be restricted to the euphotic zone (Dolan and Pérez, 2000; Stoecker et al., 2017). Investigations of their vertical distribution can elucidate how they respond to light variability. The model study presented in Chapter 7 suggests that mixotrophic populations change across the water column once stratification develops in a coastal site, shifting from being dominated by constitutive to non-constitutive forms with increasing depth and light attenuation. However, it is not very clear if this feature is supported by empirical studies; from the data compiled in Chapters 3 and 4, most studies have focused on only one type of mixotroph (Arenovski et al., 1995; Havskum and Riemann, 1996; Czypionka et al., 2011; Sanders and Gast, 2012; Weber et al., 2014). For instance, the abundance of constitutive mixotrophs (nanoflagellates) tends to decrease with depth, being undetectable in darkness (Czypionka et al., 2011). In turn, the non-constitutive mixotroph Mesodinium rubrum can be very abundant in deeper waters (Weber et al., 2014).

\subsubsection{Temporal distribution of mixotrophic protists}

Biogeography is the study of the processes that determine both spatial and temporal distributions of organisms. Evaluating the temporal distribution of mixotrophs helps us to understand their biogeography and the succession of protist communities (Chapters 3 and 7). For instance, if the temporal distribution had been neglected, one could conclude from Chapter 3 that acquired phototrophs have different biogeographies but only when comparing endosymbiotic mixotrophs with kleptoplastidic forms (Leles et al., 2017). A study based on metabarcoding data arrived at similar conclusions without examining temporal differences (Faure et al., 2019). However, once temporal distributions were assigned in Chapter 3, it becomes clear that kleptoplastidic mixotrophs can exhibit different biogeographies based on the specificity of the prey from which kleptochloroplasts are acquired, i.e., generalist vs specialist forms (Leles et al., 2017). Within coastal seas, generalist forms are more abundant during summer while the specialist M. rubrum reaches its highest abundance during spring (Stoecker et al., 2009; Johnson et al., 2013; Stoecker et al., 2017). The mechanistic model used in Chapter 6 allowed the investigation of this mechanism, revealing that generalist non-constitutive mixotrophs dominate under high light and low nutrient conditions, while specialist forms 
are more important in intermediate conditions of light and nutrients (Leles et al., 2018). Furthermore, their different temporal distributions were corroborated validating this model at L4 station, in the Western English Channel (Chapter 7).

In Chapter 7, the seasonal succession of protist trophic strategies was evaluated, for the first time, through a modelling study validated against observational data. Model and data revealed that mixotrophy is a persistent trait in a coastal temperate sea. The seasonal succession of plankton communities has long been investigated (Sverdrup, 1953; Margalef, 1978; Reynolds, 1984; Lichtman et al., 2007; Edwards et al., 2013) but the role of mixotrophy is often overlooked (Sommer et al., 2012; Mitra et al., 2014b; Stoecker et al., 2017). It is now well accepted that mixotrophs thrive in mature systems, such as within oligotrophic oceans and during summer within temperate seas (Mitra et al., 2014b). What is intriguing is their persistence in other periods of the seasonal cycle, as verified by previous empirical investigations (Unrein et al., 2007; Czypionka et al., 2011; Vargas et al., 2012). Here, several 'windows of opportunity' that allow mixotrophs to grow over the production cycle in a coastal temperate sea were identified (Chapter 7). These are related to the different functional types of mixotrophs included in the model and due to changes in their mixotrophic strategies over the seasonal cycle. As expected, mixotrophy among both constitutive and non-constitutive forms was important during summer (Havskum and Riemann, 1996; Stoecker et al., 2009; Johnson et al., 2013). In addition, phagotrophy was key to secure survival during winter among small constitutive mixotrophs. Interestingly, model and data support that, just before the spring bloom, mixotrophs can increase in abundance relative to heterotrophs. This suggests that the succession of protist trophic strategies can change during winter, from heterotrophs to mixotrophs and then back to autotrophs during the spring bloom.

\subsubsection{Implications to ecosystem functioning}

Mixotrophy can significantly impact plankton dynamics and ecosystem functioning (Dolan and Pérez, 2000; Stoecker et al., 2009; Mitra et al., 2016; Stoecker et al., 2017; Stoecker and Lavrentyev, 2018). At the base of pelagic food webs, mixotrophic nanoflagellates are recognised as important bacterivores in oligotrophic seas (Zubkov and Tarran, 2008; Hartmann et al., 2012; Mitra et al., 2014b). Their abundance, however, 
increases towards more productive systems and despite presenting low abundance values compared to heterotrophic nanoflagellates, these mixotrophs are responsible for nearly half of total bacterivory across different ecosystems (Chapter 4). Mixotrophic bacterivory was highly variable within temperate coastal seas and polar seas, suggesting temporal variability in the relative importance of mixotrophs (Chapter 4). The model presented in Chapter 7 revealed that mixotrophic bacterivory changed over the year in a coastal temperate sea, being maximal during summer but also significant during winter. This analysis highlights the importance of quantifying mixotrophs, the abundance of their potential competitors, and their ecological impact across seasons. So far, few temporal investigations have sampled consistently over several months (Unrein et al., 2007; Tsai et al., 2011) and, therefore, further studies are required to understand changes on mixotrophic bacterivory over the year.

Modelling investigations in Chapter 6 and other studies suggest that mixotrophs can promote the accumulation of biomass in larger size classes, increasing the transfer of carbon to higher trophic levels (Ward and Follows, 2016; Leles et al., 2018). In the absence of mixotrophy, phototrophic biomass is accumulated in smaller size classes (picoplankton), particularly under nutrient limitation, which are not accessible to mesozooplankton (Leles et al., 2018). In addition, empirical data indicate that constitutive mixotrophy have a stabilizing effect on the stoichiometric content of primary producers (Moorthi et al., 2017). This is related to phagotrophic feeding on nutrient-rich prey to compensate for inorganic nutrient limitation, which can also act increasing trophic efficiencies (Moorthi et al., 2017). Non-constitutive mixotrophs, in turn, are hypothesised to increase trophic transfer efficiency by reaching higher gross growth efficiencies (GGE) than heterotrophic counterparts (Stoecker et al., 2009; Schoener and McManus, 2017); however, no previous studies have assessed this experimentally. Certain mesozooplankton, such as crustaceans and fish larvae, preferentially prey on mixotrophic ciliates (Broglio et al., 2004; Figueiredo et al., 2007). Nevertheless, other mixotrophs can negatively impact the pelagic food web, such as mixotrophic nanoflagellates and dinoflagellates (e.g., Prymnesium and Karlodinium genus) which are responsible for the formation of harmful algal blooms (Burkholder et al., 2008; Gomes et al., 2014). 
Modelling studies also suggest that mixotrophs can affect nutrient cycling (Chapters 6 and 7); however, it is not clear if non-constitutive mixotrophs act supressing or increasing the regeneration of inorganic nutrients in the system (Mitra et al., 2016; Ghyoot et al., 2017b; Leles et al., 2018). If these mixotrophs can achieve higher GGE, as discussed above, one might argue that they act to decrease inorganic nutrient regeneration since they can recycle nutrients internally (Stoecker et al., 2009). Nevertheless, more investigations are required to understand if these mixotrophs can take up inorganic nutrients (Hattenrath-Lehmann and Gobler, 2015; Qiu et al., 2016; Schoener and McManus, 2017). Specialist non-constitutive mixotrophs, such as Dinophysis acuminata and Mesodinium rubrum, can assimilate ammonium and urea, but nitrate assimilation was found to be significantly lower (Hattenrath-Lehmann and Gobler, 2015; Qiu et al., 2016). The generalist non-constitutive mixotroph Strombidium rassoulzadegani was found to take up both ammonium and nitrate but inorganic nitrogen did not contribute significantly to growth (Schoener and McManus, 2017).

The modelling results from Chapter 6 also support the hypothesis that mixotrophy can increase the production of DOC and stimulate bacterial biomass (Stoecker et al., 2009; Mitra et al., 2014b), but only under nutrient limitation. The increased production of DOC was mainly related to the overall poor prey quality, described by internal N:C and P:C quotas, resulting in inefficient protist predators which, therefore, contributed to the increased release of labile DOC (Mitra, 2006; Polimene et al., 2015). Under light limitation, total gross primary productivity (GPP) was higher in the absence of mixotrophs and the production of labile DOC was mainly associated with photosynthesis. However, results are largely dependent on how materials are partitioned between particulate and dissolved pools in the model. In addition, the model lacked the description of osmotrophy, which has the potential to change these dynamics. 


\subsection{What are the challenges ahead?}

\subsubsection{Limitations in the current literature}

If mixotrophy, on its different forms, has long been described among protists (Jones 1997; Raven, 1997; Stoecker 1998; Burkholder et al., 2008; Raven et al., 2009; Jeong et al., 2010c; Johnson et al., 2011; Flynn et al., 2013; Mitra et al., 2016), why do we know so little about the biogeography of mixotrophs and their impact on ecosystem functioning? From microscopic analyses to molecular data, ocean colour, and ecosystem models, all share something in common - these approaches traditionally neglect or, at least, underestimate mixotrophy within protist communities.

Traditional sampling protocols, based on microscopic identification and quantification, are commonly biased towards certain taxonomic groups or size classes of protists. For example, large 'zooplankton' $(>600 \mu \mathrm{m})$ are commonly characterised by copepods despite many protist taxa also occurring in this size range. The latter are underrepresented because their cells are severely damaged by plankton nets and eventually dissolve upon preservation (Biard et al., 2016). Imaging surveys revealed rhizarians, most of which are non-constitutive mixotrophs, to consist of nearly $30 \%$ of total zooplankton biomass (> $600 \mu \mathrm{m}$ ) across the oceans (Biard et al., 2016). Similarly, sampling biases also hinder the investigation of the biogeography of constitutive mixotrophs which occur mainly in the nano- and micro- plankton size spectrum (Leles et al., 2019). Their small size complicates the identification at species level through microscopy. Protist species have been traditionally defined based on morphological differences, but cryptic species exist with very similar body forms and different physiologies, particularly among nanoplankton (Lie et al., 2018). DNA sequence information arise as a powerful tool to address this shortcoming detecting protistan diversity (de Vargas et al., 2015). It is noteworthy, however, that estimates of protistan diversity from DNA sequences are strongly dependent on primer choice, amplification protocols, and sequencing and can be also biased towards certain groups (Caron and $\mathrm{Hu}$, 2019). The study by Faure et al. (2019), for instance, identified a gap of sequence data among GNCMs and pSNCMs relative to CMs and eSNCMs. 
One important fact is that neither traditional sampling protocols or highthroughput sequencing capture mixotrophic activity. Therefore, even analyses of metabarcoding data must rely on previous experimental evidence to classify the operational taxonomic units as mixotrophs (Leles et al., 2019; Faure et al., 2019). Even though not a common practice, it is relatively simple to quantify the biomass of nonconstitutive mixotrophs versus that of their heterotrophic counterparts (Leles et al., 2017). Mixotrophic ciliates, for example, are easily identifiable from heterotrophic ones through the examination of samples under epifluorescence microscopy or using the FlowCAM (e.g., Stoecker et al., 2014; Haraguchi et al., 2018). The same does not apply for constitutive mixotrophs because these are not necessarily actively feeding at all times (Chapter 4). Constitutive mixotrophs smaller than $20 \mu \mathrm{m}$ are usually distinguished from strict phototrophic counterparts through bacterivory experiments which are limited by a series of assumptions, such as constant bacterivory over short time scales and by the fact that community ingestion rates can be approximated to the average ingestion rates of all feeding individuals (Safi and Hall, 1999; McKie-Krisberg et al., 2014; Sato et al., 2017). In reality, feeding varies over the diel cycle (Tsai et al., 2009) and only a proportion of the total mixotroph assemblage is actively feeding at any time during an experiment (Christaki et al., 1999; Anderson et al., 2017). Paradoxically, most bacterivory studies do not provide information on protistan diversity (Unrein et al., 2014) and appear to not be applicable among constitutive mixotrophs larger than $20 \mu \mathrm{m}$. There is, therefore, a lack of studies that tackle both mixotrophic diversity and mixotrophic activity and, potentially, different methodologies are needed for different mixotrophs.

The problem is that, without quantifying mixotrophic activity, we cannot have a clear understanding of the impact of mixotrophs on plankton trophodynamics and on global biogeochemical cycles. In view of the limitations associated with empirical studies, numerical modelling provides a platform to explore these questions and test hypotheses. Physiological (mechanistic) models of mixotrophy with different levels of complexity have been previously formulated in the literature (Troost et al., 2005; Bruggeman, 2009; Flynn and Mitra, 2009; Ward et al., 2011; Chakraborty et al., 2017). However, the traditional split between 'phytoplankton' or 'zooplankton' still rule the way plankton is structured within ecosystem models with a few exceptions (Tables 1.1 and 1.2). There is 
a lack of modelling studies that address the diverse mixotrophic strategies adopted by protists (Flynn and Mitra, 2009; Mitra et al., 2016; Ghyoot et al., 2017a) and how these affect the competitive outcomes within protist communities (Leles et al., 2018). One could argue that mixotrophy is not described within ecosystem models due to the paucity of data. Data are needed to parameterise the models as well as to evaluate model performance and, thus, represent a challenge to the modelling community. In fact, poor parameterisation can aggravate a common feature of ecosystem models which is the difficulty to allow coexistence among the different plankton functional types (Cropp and Norbury, 2013).

Biogeochemical models, particularly 3D models, tend to compare simulations against ocean colour data due to the continuous global estimates of surface chlorophyll-a concentrations (Bracher et al., 2017). However, such data does not capture the diversity of forms and function among phototrophic taxa and does not allow to access mixotrophy. From the 1990s onwards, increasing efforts have been applied on developing algorithms that can retrieve information on the composition and size structure of phototrophic communities from ocean colour (IOCCG, 2014). The methods utilize information from phytoplankton abundance, cell size, and bio-optical properties such as pigment composition, absorption, and backscattering properties (Bracher et al., 2017). Most algorithms provide information about the dominance or the presence/absence of a particular group, or the fraction of chl-a associated with three different size classes (pico, nano-, and micro- plankton) (reviewed in Bracher et al., 2017). Such information is not, however, easily transferable to the plankton functional types within biogeochemical models. There are also the issues with the units required by biogeochemical modellers, which are usually carbon-based, and the limited application of remote sensing data within coastal areas and, of course, vertically in the water column. In turn, monitoring datasets (usually from fixed stations) provide estimates of plankton biomass in terms of carbon for different plankton functional types, including mixotrophic taxa, but have their own caveats and lack the global continuous measurements unique from ocean colour data.

A model can only be as good as the data used to parameterise it and, more importantly, on the concepts used on its construction (Flynn, 2005). Modelling 
mixotrophy is not a trivial task (Mitra and Flynn, 2010). It is important to justify model assumptions and address model caveats. Despite the refined level of mechanistic understanding compared to other mixotrophic models in the literature (Tables 1.1 and 1.2), the model used in this thesis (Flynn and Mitra, 2009) also have limitations. Overall, it is difficult to allow coexistence among mixotrophs and their auto- or hetero- trophic counterparts (Mitra et al., 2014b; Leles et al., 2018). For instance, nanozooplankton was rapidly excluded from simulations, not coexisting with constitutive mixotrophs (Mitra et al., 2014b; Leles et al., 2018). Similarly, it is not trivial to allow the coexistence between microzooplankton, GNCMs, and pSNCMs (Leles et al., 2018). These results suggest that the model can be underestimating the costs associated to mixotrophy.

Nevertheless, mixotrophy should be interpreted as a synergistic interaction, in which different mechanisms can act regulating phototrophy and/or phagotrophy (Flynn and Mitra, 2009; Stoecker et al., 2017). Therefore, models that implement mixotrophy as 'the sum of the parts', i.e., summing up phytoplankton and zooplankton descriptors, are not appropriate to represent mixotrophic populations (Mitra and Flynn, 2010). Certain modelling approaches have implemented a continuum of mixotrophic strategies through the description of trait (i.e., photosynthesis, nutrient uptake, and phagotrophy) distributions, but these are based on simpler descriptions of mixotrophy and do not account for mixotrophic functional diversity (Bruggeman, 2009; Ward et al., 2011; Berge et al., 2017; Chakraborty et al., 2017), i.e., do not differentiate between CMs and the different types of NCMs (their description is closer to that of $\mathrm{CMs}$ ). Moreover, some studies have implemented an allometric description of mixotrophy based on the assumption that phagotrophic capabilities increase with cell size whilst the opposite is true for phototrophy (Ward and Follows, 2016; Chakraborty et al., 2017); however, this approach is not correct since mixotrophic nanoflagellates can be primarily phagotrophic and large Rhizaria can function primarily as phototrophs (e.g., de Vargas et al., 2015; Lie et al., 2018).

\subsubsection{Directions for future studies}

Acknowledging the limitations associated with mixotrophic research is an important exercise to define future research questions with appropriate experimental 
design. There is increasing evidence that mixotrophic populations exhibit latitudinal gradients and seasonal distributions (Biard et al., 2016; Stoecker et al., 2017; Leles et al., 2017; Sato et al., 2017; Edwards, 2019). Nevertheless, disentangling the effects of temperature, light, and resource (nutrient/prey) availability is challenging and complicates the interpretation of competitive outcomes within protist communities. For example, oligotrophic conditions are expected to favour mixotrophy but non-constitutive mixotrophs can dominate in productive polar systems (Olson and Strom, 2002; Lavrentyev and Franzè, 2017). The response of mixotrophs to environmental changes is particularly important to understand competitive outcomes within protist communities and the impact of mixotrophs on biogeochemical cycling in the oceans. Here, the knowledge gaps associated with the different factors that influence the biogeography of mixotrophs and their impact on ecosystem functioning across the oceans are discussed. Ultimately, innovative methods are needed to obtain experimental and field data. These, combined with modelling approaches, will allow a better understanding of the distribution and role of mixotrophs in the oceans.

\section{Temperature}

The effect of temperature on mixotrophic metabolism is not well understood. Among protists, the maximal growth rates of heterotrophs equalled or exceeded that of phototrophs at temperatures above $15^{\circ} \mathrm{C}$ (Rose and Caron, 2007). On the other hand, heterotrophic protists appear to be more constrained than their phototrophic counterparts at low temperatures such as those observed at high-latitude systems (Rose and Caron, 2007). These results, however, do not address mixotrophy. Mixotrophic protists are also expected to adjust their metabolism as a function of temperature. Among constitutive mixotrophs, the mixotrophic chrysophyte Ochromonas and the haptophyte Isochrysis galbana were found to increase their heterotrophic metabolism faster than photosynthesis with increases in temperature under experimental conditions (Wilken et al., 2013; Cabrerizo et al., 2018); this might provide a competitive advantage to mixotrophs relative to strict autotrophs. Nevertheless, an opposite response was found for Dinobryon (Princiotta et al., 2016). The contrasting results might be related to the position of the different taxa along the continuum of phototrophic to phagotrophic nutritional abilities, 
with Ochromonas being primarily heterotrophic and Dinobryon being closer to the phototrophic side of the spectrum (Wilken et al., 2013; Princiotta et al., 2016; Terrado et al., 2017; Lie et al., 2018). So far, it is not clear how the mixotrophic metabolism of nonconstitutive mixotrophs shifts as a function of temperature. However, these can achieve higher growth rates than their heterotrophic counterparts at sub-zero temperatures (Franzè and Lavrentyev, 2014) while the opposite was observed for temperatures higher than 13 ${ }^{\circ} \mathrm{C}$ (Pérez et al., 1997). These observations are interesting because in order to maintain both phototrophic and heterotrophic machineries, mixotrophs have been hypothesised to have lower maximum growth rates compared to their competitors (Raven, 1997; Rothhaupt, 1996).

More studies are necessary to verify if these can be generalised for mixotrophic populations observed in the oceans since species can display very different mixotrophic strategies (Burkholder et al., 2008; Jeong et al., 2010c). So far, no studies have been conducted with natural assemblages. Incubations of natural assemblages under different temperatures combined with fluorescence methods used to detect mixotrophic nanoflagellates (Beisner et al., 2019), for instance, can provide new insights about their response to temperature.

\section{Light}

Both constitutive and non-constitutive mixotrophs are thought to depend on light to survive (Stoecker et al., 2009; Czypionka et al., 2011; Hansen, 2011). It has also been argued that high light conditions may be important for the success of mixotrophic nanoflagellates (Fischer et al., 2017; Edwards, 2019). However, this does not seem to be the rule among all mixotrophs; the non-constitutive mixotroph Mesodinium rubrum, for instance, thrive in light-limited environments (Johnson et al., 2013). So far, most experimental evidence showed that species of constitutive mixotrophs cannot fully supplement their energy requirements through feeding (Andersson et al., 1989; Carvalho and Granéli, 2010; Hansen, 2011; Anderson et al., 2018), except for certain species of the genus Ochromonas which can feed and grow heterotrophically (Liu et al., 2016; Lie et al., 2018). Non-constitutive mixotrophs have been found to grow heterotrophically (Jakobsen et al., 2000) and to persist in the dark polar winter (Levinsen et al., 2000; Stoecker et al., 
2009; Stoecker et al., 2017). The survival of M. rubrum and Laboea strobila during winter in polar systems has been explained due to lower metabolic demands under low temperatures (Levinsen et al., 2000). Nevertheless, this appears in contrast to the fact that these ciliates must acquire kleptochloroplasts from their prey (mostly constitutive mixotrophs) to survive (Stoecker et al., 2009). One potential explanation is that photodegradation of plastids is null or minimum, so that they can keep the phototrophic machinery through winter (Stoecker and Lavrentyev, 2018). In fact, M. rubrum was observed to keep functional plastids for up to 80 days in the absence of prey (Myung et al., 2013). On the other side, plastids life-span were found to be much shorter (up to two weeks) among ciliates of the genus Strombidium (Schoener and McManus, 2012).

Evaluating the vertical distribution of both constitutive and non-constitutive mixotrophs over the water column and contrasting it with their autotrophic and heterotrophic counterparts might provide valuable information on their coupling with light in the natural environment. For instance, the contribution of mixotrophs with endosymbiotic associations relative to the total biomass of Rhizaria was found to decrease with depth (Biard et al., 2016). Similarly, the biomass contribution of mixotrophic nanoflagellates was found to decrease with depth (Arenovski et al., 1995; Havskum and Riemann, 1996; Czypionka et al., 2011). Little is known about the vertical distribution of mixotrophic ciliates, but $M$. rubrum can display diel vertical migration and acquire inorganic nutrients in darker waters, near the base of the mixed layer depth (Pérez et al., 2000; Weber et al., 2014).

Diel cycles

Physiological functions of protists are known to follow diel cycles, but these are better described among phototrophs (e.g., photosynthesis and nitrate metabolism) compared to heterotrophic protists (e.g., feeding), consequently, little is known about how mixotrophs respond to day-night variations. Previous studies have found that the ingestion rates of picoplankton by constitutive mixotrophs can change over the diel cycle (Tsai et al., 2009; Anderson et al., 2017). Such changes can shift the relative importance of mixotrophic nanoflagellates to total bacterivory in short-time scales, being higher during day-time (Anderson et al., 2017). Similarly, it has been shown that feeding and growth 
rates among microzooplankton are higher during the day and lower during the night (Jakobsen and Strom, 2004; Tarangkoon and Hansen, 2011; Arias et al., 2017). One might then expect that due to the coupling with phototrophy, growth in mixotrophic ciliates is lower (or negative) in darkness compared to growth in heterotrophic ciliates of similar size. Experiments consisting on measuring feeding and growth rates in light vs dark treatments for both mixotrophs and their heterotrophic competitors of similar size could be performed to test this hypothesis.

\section{Resource availability and the diverse mixotrophic strategies}

Mixotrophy is favoured under oligotrophic conditions (Zubkov and Tarran, 2008; Stoecker et al., 2009; Hartmann et al., 2012; Mitra et al., 2016). Nevertheless, the biomass of mixotrophic nanoflagellates increases towards more productive systems, mixotrophic dinoflagellates can bloom in eutrophic coastal environments, and non-constitutive mixotrophs, for instance, can be abundant in bloom conditions in the Arctic (Burkholder et al., 2008; Stoecker and Lavrentyev, 2018; Edwards, 2019). The diversity of mixotrophic strategies across taxa can help to understand their persistence across different ecosystems. For instance, some species engage on feeding only to obtain nutrients (Carvalho and Granéli, 2010) while others can also use carbon for growth (Terrado et al., 2017). Mixotrophic nutrition among dinoflagellates is diverse; these occur in both constitutive and non-constitutive forms and their metabolic requirements vary greatly among species (Jeong et al., 2010c; Hansen, 2011; Stoecker et al., 2017). Such diversity complicates the generalization of results from experimental studies with mixotrophic species and complicates the description of mixotrophy in ecosystem models. Therefore, studies that quantify both photosynthesis and ingestion rates among different species are key to better understand mixotrophic nutrition. The ultimate goal is to identify mixotrophic populations in the natural environment and, to accomplish this task, innovative methods of feeding and photosynthesis will need to be combined with molecular tools for identification and quantification of natural assemblages (Stoecker and Lavrentyev, 2018; Beisner et al., 2019). 


\section{Acquired phototrophy: prey selectivity}

Experimental investigations have shown that non-constitutive mixotrophs can acquire plastids from diverse prey types or must rely on specific prey to engage on photosynthesis (Stoecker et al., 1989b; Stoecker et al., 2009; McManus et al., 2012; Hansen et al., 2013; Mitra et al., 2016). However, it is less clear if prey that provide kleptochloroplasts are selected in natural assemblages and if, despite being generalists, oligotrich ciliates have a primary source of plastids. Therefore, one could argue that mixotrophic ciliates have similar prey size spectrum than heterotrophic ciliates of similar size (Jonsson, 1987), but the former selects for prey that provide phototrophic potential (Dolan and Pérez, 2000; Stoecker et al., 2009). This could give a competitive advantage to heterotrophic forms in the absence of suitable prey (Dolan and Pérez, 2000; Stoecker et al., 2009). Experiments consisting on offering both autotrophic and heterotrophic prey of same size in mixed diets (assuming an abundance ratio of 1:1) to evaluate prey selectivity among a mixotrophic and a heterotrophic ciliate could shed light into their competitive abilities. Ultimately, experimental results should be verified against estimates of ingestion rates of mixotrophic vs heterotrophic ciliates on specific taxa in field assemblages.

Acquired phototrophy: plastid replacement and functionality

So far, few studies have investigated plastid replacement among non-constitutive mixotrophs (Stoecker and Silver, 1990; Schoener and McManus, 2012; Hansen et al., 2012; Peltomaa and Johnson, 2017; Kim et al., 2018). The time plastids are retained by the ciliate cell depends on three physiological processes: plastid functionality, cell division rate and plastid expulsion rate (light blue ellipses; Fig. 8.1). In turn, these physiological processes are expected to be affected by the environmental setting, i.e., food and nutrients concentration and irradiance (dark blue ellipses, Fig. 8.1). The turnover rate of plastids among mixotrophic ciliates increases with prey availability (Schoener and McManus, 2012; Hansen et al., 2012; Peltomaa and Johnson, 2017); however, the poor nutritional status of the prey (based on $\mathrm{C}, \mathrm{N}$, and $\mathrm{P}$ internal stoichiometry) can be expected to negatively affect plastid functionality and, potentially, result in higher turnover rate of plastids. In addition, plastid functionality is a function of irradiance, due to photodamage. 
Division rates increase with the increase of food concentration and light intensity until a saturation point and cell division is not expected to occur under starvation (Stoecker et al., 1988; 1989). Prey switching experiments using prey with different pigmentation (red vs green chloroplasts) can be performed to evaluate the turnover rate of plastids (e.g., Schoener and McManus, 2012) and plastid functionality under different light conditions and whilst offering poor vs high prey quality.

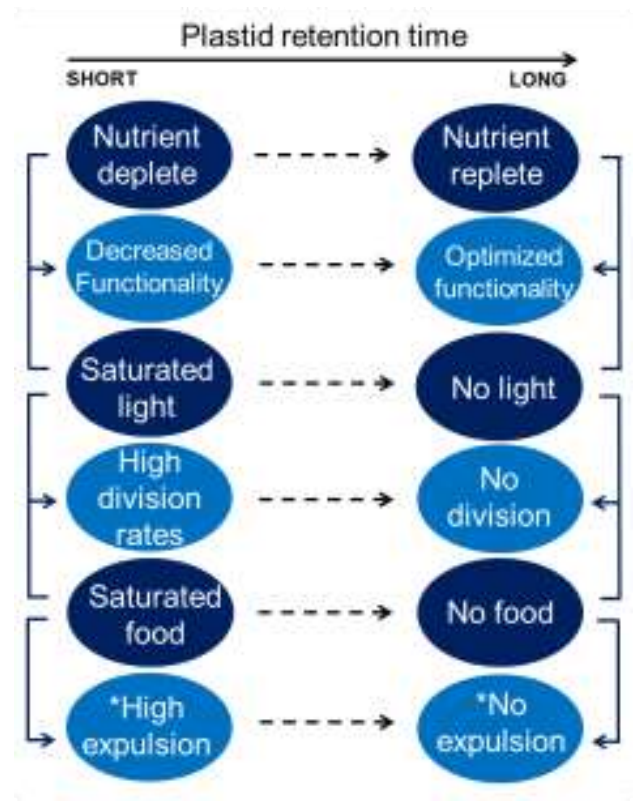

Fig. 8.1 Plastid retention time defined by the effect of the environmental setting (dark blue) in different physiological processes (light blue). Expulsion rate was highlighted (*) because it is expected to occur, but it is difficult to observe experimentally.

Protist plankton biogeography: environmental selection vs historical factors

This thesis and a previous study have evaluated the effect of environmental variation and functional diversity in the biogeography of mixotrophs (Leles et al., 2017; Faure et al., 2019). Far less is known about the role of historical factors, i.e., past selection, drift, dispersal, and/or mutation. The high dispersal ability, large population sizes, short generation times, and the low probability of local extinction can help to explain why historical factors (such as drift) are less important than biological and physical factors in determining microbial biogeography (Dolan 2005; Fenchel and Finlay, 2003). Nevertheless, these processes are not mutually exclusive, and their complex interactions 
may confound our ability to interpret their distribution (Caron, 2009). The fact is that populations defined by similar morphology may be cosmopolitan; however, this may not hold true if populations are genetically distinct (Dolan, 2005; Dolan, 2006). How can we evaluate the relative importance of environmental selection vs historical factors? Hanson et al. (2012) suggested that microbial biogeography is a result of four fundamental processes: selection, drift, dispersal, and mutation, and that these processes should not be separated into ecological and evolutionary levels (see Glossary). Drift, for instance, might act on rare taxa which correspond to most microbial taxa within a community (Hanson et al., 2012), but these are usually under sampled. In addition, many taxa may enter a dormant stage and, therefore, may not be actively reproducing, adding another complication to the analysis of historical factors (Hanson et al., 2012).

To evaluate the effect of drift, sampling methods should target rare and/or active microbial populations and should minimize environmental variation (Hanson et al., 2012). In addition, the genetic markers should target higher amount of genetic variation, particularly neutral variation, which corresponds to the fraction of genetic variation that has no effect on fitness (Hanson et al., 2012). Measurements of phenotypic traits that characterise dispersal are also valuable. Furthermore, selection and historical factors influence the biogeography of protists across spatial and temporal scales. To address the effects of time, experimental studies should be combined with temporal investigations. As previously discussed, seasonality is important on determining distribution patterns and can have implications for the interpretation of spatial patterns observed at one point in time (Chapter 7). Ultimately, ecological models should be combined with evolutionary theory and applied to explore the relative importance of the different processes on shaping protist biogeography, from latitudinal gradients to vertical distributions in the water column, and temporal (both seasonal and across years) variations (e.g., Hellweger et al., 2014).

\section{Mixotrophy in marine ecosystem models}

Models are valuable tools that allow the investigation of plankton trophodynamics. Empirical data are used to inform and/or validate models which, in turn, can provide mechanistic understanding of experimental datasets and point towards new hypotheses 
and experimental investigations. For instance, empirical evidence is needed to corroborate (or not) modelling predictions with respect to increased trophic efficiencies and production of DOC and lower nutrient regeneration in the presence of mixotrophy (Chapter 8.3.3). Models should also be applied to provide new insights into the mechanisms that allow mixotrophs to thrive in different marine ecosystems. It would be interesting, for instance, to develop modelling studies to compare the mechanisms that trigger the development of mixotrophs in tropical vs temperate vs polar seas. Understanding temporal variations both within and across years (seasonal variations and ENSO events, respectively) is important. Mixotrophy as a strategy to compensate for iron limitation (Stukel et al., 2011), particularly in HNLC (i.e., high-nutrient low-chlorophyll) regions has not been explored in modelling studies so far and is a potential interesting subject. Another interesting research area is the role of mixotrophic dinoflagellates in DMS production (Lee et al., 2012; McParland and Levine, 2019). In the presence of DMSP-rich prey cells, mixotrophic dinoflagellates can produce significantly amounts of DMS, which can be particularly important during bloom events in coastal systems (Lee et al., 2012).

All topics outlined previously in this section are key to understand the impact of mixotrophy within pelagic marine food webs. How mixotrophs respond to temperature is particularly important because it will be required by modellers to evaluate the changes in plankton communities and biogeochemical cycling in the oceans in future climate scenarios (Cabrerizo et al., 2018). It is unclear what will be the competitive outcomes at the base of pelagic food webs and consequent effects on ecosystem functioning under warmer and more stratified oceans (Polovina et al., 2008; Behrenfeld et al., 2016). Within coastal regions, eutrophication results in unbalanced (and high) ratios of nitrogen to phosphorus, increased light limitation among phototrophic populations, and hypoxia events (Burkholder et al., 2008; Gomes et al., 2014). Mixotrophy has been shown to be a successful strategy in both scenarios (Burkholder et al., 2008; Zubkov and Tarran, 2008; Wilken et al., 2013; Gomes et al., 2014; Leles et al., 2018) and, therefore, is a key component of plankton food webs that must be included in future modelling studies (Flynn et al., 2013; Mitra et al., 2016; Stoecker et al., 2017). 


\section{Models of mixotrophy}

This thesis, together with other studies in the literature, shows that including mixotrophy in ecosystem models can change the way we understand the functioning (e.g., ecological interactions, nutrient cycling, and trophic transfer) of the base of marine pelagic food webs. Therefore, in order to address questions such as 'How the plankton ecosystem will respond to climate change?' and to provide the scientific ground to assist in the management of coastal water bodies due to the recurrent events of harmful algal blooms, new (or reformulated) modelling approaches are needed. As discussed throughout Chapter 8.4.2, there are many experiments that should be prioritised to improve our understanding of the physiology of mixotrophs. These will serve to better parameterise (or revisit the structure) of ecosystem models which, in turn, will guide future experimental or field investigations. Models, experiments, and field campaigns benefit from each other and, together, they allow the advancement of the scientific knowledge. Here, for instance, we show the importance of long-term monitoring datasets coupled to ecosystem models to test hypotheses about the complex phenomena driving the persistence of mixotrophy in a costal temperate sea (Chapter 7).

The modelling studies within this thesis incorporated diverse features of plankton physiology. In addition to all the features within Table 1.2, this thesis implemented mixotrophic functional diversity in complex plankton food webs, incorporating realistic environmental variability and validating simulations against observational data (Table 8.1). Only a few modelling studies, if at all, have accomplished these tasks previously (Table 8.1). Based on these results, it is suggested that future modelling studies: i) address

mixotrophic functional diversity (i.e., CMs and the different types of NCMs), ii) explore the plasticity in the phototrophy-phagotrophy balance within each mixotrophic type, iii) investigate the costs associated to mixotrophy, and iv) channel efforts to compare simulations against field data across different spatio-temporal scales.

So far, few models described acquired phototrophy through the acquisition and retention of kleptochloroplasts (Flynn and Mitra, 2009; Mitra et al., 2016; Ghyoot et al., 2017a; Leles et al., 2018). These models can be modified in future investigations to include the mechanisms associated to the retention of kleptochloroplasts, such as the 
effects of prey quality on plastid functionality and the effect of prey quantity on plastid retention (Stoecker and Silver, 1987; Schoener and McManus 2012). In addition, model development is required to explicitly describe endosymbiotic associations among NCMs. It is also important to recognise that constitutive mixotrophs are very diverse (Jeong et al., 2010c; Leles et al., 2018). Simulating mixotrophic dinoflagellates, for instance, can be challenging and processes such as diel vertical migration, cyst formation, and allelopathy might be important and should be explored in future modelling studies (discussed in detail in Chapter 7.4.4). 
Table 8.1 Features of mixotrophic models in addition to those in Table 1.2; this thesis incorporated all features in Table 1.2.

\begin{tabular}{|c|c|c|c|c|c|}
\hline \multirow[b]{2}{*}{ Reference } & \multicolumn{5}{|c|}{ Model feature } \\
\hline & $\begin{array}{c}\text { CMs of } \\
\text { different sizes }\end{array}$ & $\begin{array}{l}\text { GNCMs and } \\
\text { pSNCMs }\end{array}$ & $\begin{array}{l}\text { allometric } \\
\text { predation }\end{array}$ & $\begin{array}{c}\text { realistic environmental } \\
\text { variability }\end{array}$ & $\begin{array}{c}\text { validation against } \\
\text { biological data }\end{array}$ \\
\hline \multicolumn{6}{|l|}{ Thingstad et al. (1996) } \\
\hline \multicolumn{6}{|l|}{ Baretta-Bekker et al. (1998) } \\
\hline \multicolumn{6}{|l|}{ Stickney et al. (2000) } \\
\hline \multicolumn{6}{|l|}{ Kooijman et al. (2002) } \\
\hline Zhang et al. (2003) & & & & & $\mathrm{x}$ \\
\hline \multicolumn{6}{|l|}{ Jost et al. (2004) } \\
\hline \multicolumn{6}{|l|}{ Hammer and Pitchford (2005) } \\
\hline \multicolumn{6}{|l|}{ Troost et al. (2005) } \\
\hline \multicolumn{6}{|l|}{ Hood et al. (2006) } \\
\hline Bruggeman (2009) & & & & $\mathrm{x}$ & \\
\hline \multicolumn{6}{|l|}{ Flynn and Mitra (2009) } \\
\hline \multicolumn{6}{|l|}{ Mitra and Flynn (2010) } \\
\hline \multicolumn{6}{|l|}{ Ward et al. (2011) } \\
\hline \multicolumn{6}{|l|}{ Flynn and Hansen (2013) } \\
\hline Våge et al. (2013) & $\mathrm{x}$ & & $\mathrm{x}$ & & \\
\hline \multicolumn{6}{|l|}{ Mitra et al. (2014) } \\
\hline \multicolumn{6}{|l|}{ Cropp and Norbury (2015) } \\
\hline Mitra et al. (2016) & & & $\mathrm{X}$ & & \\
\hline Ward and Follows (2016) & $\mathrm{X}$ & & $\mathrm{X}$ & $\mathrm{X}$ & \\
\hline \multicolumn{6}{|l|}{ Berge et al. (2017) } \\
\hline Chakraborty et al. (2017) & $\mathrm{x}$ & & $\mathrm{x}$ & & \\
\hline \multicolumn{6}{|l|}{ Ghyoot et al. (2017a) } \\
\hline Ghyoot et al. (2017b) & & & & & $\mathrm{x}$ \\
\hline Lin et al. (2018) & & & & & $\mathrm{x}$ \\
\hline \multicolumn{6}{|l|}{ Edwards (2019) } \\
\hline Chapter 6 (Leles et al., 2018) & $\mathbf{x}$ & $\mathbf{x}$ & $\mathbf{x}$ & & \\
\hline Chapter 7 & $\mathbf{x}$ & & $\mathbf{x}$ & $\mathbf{x}$ & $\mathbf{x}$ \\
\hline
\end{tabular}


In addition to the different mixotrophic functional types, it is important to address the plasticity of mixotrophic strategies within a single type (Jeong et al., 2010c; Hansen, 2011; Granéli et al., 2012). The model used in this thesis accounts for the plasticity between phototrophy and phagotrophy depending on environmental conditions. Future investigations could apply this model to explore the sensitivity of mixotrophic dynamics to changes on their dependence on phototrophy, particularly constitutive mixotrophs, which rely on phototrophy for a critical proportion of their growth (Flynn and Mitra, 2009; Mitra et al., 2016). Due to a gap in knowledge regarding the physiology of mixotrophs, models should also be used to explore the costs associated with mixotrophy. For example, simulations should be performed to explore the conditions in which mixotrophs and their autotrophic and heterotrophic counterparts coexist. Sensitivity analyses are very useful for this task in hand; such experiments provide valuable information regarding the parameters that the model is more sensitive to. The prey size spectrum and selectivity of mixotrophs, for instance, were shown here to be important to determine key ecosystem properties (Leles et al., 2018).

Ultimately, future research projects should aim to generate plankton datasets that target mixotrophic populations (Leles et al., 2019; Beisner et al., 2019) and these should be used to validate modelling studies. Addressing these shortcomings through appropriate experimental designs will shed light into the ecology of mixotrophs, their impact in the cycling of materials in the oceans and allow investigations of their role under climate change projections. 


\section{Appendix A}

Table A.1 Results from the bibliographic survey - Distribution records of non-constitutive mixotrophs (NCMs: GNCMs, pSNCMs, and eSNCMs). Geographic coordinates from each record were used to identify the corresponding Longhurst's biogeographic provinces (please refer to Chapter 2.3 Table 2.1 for province code).

\begin{tabular}{|c|c|c|c|c|c|c|}
\hline NCM & Plankton group & Taxa & $\begin{array}{l}\text { Province } \\
\text { code }\end{array}$ & Lat. & Long. & Reference \\
\hline$\overline{\text { GNCM }}$ & ciliates & $\begin{array}{l}\text { Strombidium sp, Tontonia } \\
\text { appendiculariformis, Laboea } \\
\text { strobila, Tontonia ovalis, Tontonia } \\
\text { gracilima }\end{array}$ & MEDI & 43.7 & 7.3 & Bernard and Rassoulzadegan (1994) \\
\hline GNCM & ciliates & $\begin{array}{l}\text { Laboea strobila, Tontonia } \mathrm{sp}, \\
\text { Strombidium sp, Cyrtostrombidium } \\
\mathrm{sp}\end{array}$ & MEDI & 40.7 & 14.2 & Modigh (2001) \\
\hline GNCM & ciliates & Strombidium spp, Tontonia spp & CHIN & 32.0 & 123.0 & Chiang et al. (2003) \\
\hline GNCM & ciliates & Strombidium spp, Tontonia spp & CHIN & 26.0 & 120.0 & Chiang et al. (2003) \\
\hline GNCM & ciliates & Strombidium spp, Tontonia spp & CHIN & 30.0 & 127.5 & Chiang et al. (2003) \\
\hline GNCM & ciliates & Strombidium spp, Tontonia spp & $\mathrm{CHIN}$ & 28.5 & 124.0 & Chiang et al. (2003) \\
\hline$\overline{\mathrm{GNCM}}$ & ciliates & $\begin{array}{l}\text { Strombidium capitatum, } \\
\text { Strombidium conicum, } \\
\text { Strombidium acutum, } \\
\text { Strombidium styliferum, } \\
\text { Pseudotontonia cornuta }\end{array}$ & CHIN & 36.0 & 120.0 & $\mathrm{Xu}$ et al. (2016) \\
\hline GNCM & ciliates & $\begin{array}{l}\text { Strombidium acutum, Strombidium } \\
\text { capitatum, Strombidium conicum, } \\
\text { Tontonia spp, Laboea strobila }\end{array}$ & MEDI & 39.7 & 4.9 & Christaki et al. (2011) \\
\hline
\end{tabular}




\begin{tabular}{|c|c|c|c|c|c|c|}
\hline GNCM & ciliates & $\begin{array}{l}\text { Strombidium acutum, Strombidium } \\
\text { capitatum, Strombidium conicum, } \\
\text { Tontonia spp, Laboea strobila }\end{array}$ & MEDI & 35.6 & 17.9 & Christaki et al. (2011) \\
\hline GNCM & ciliates & $\begin{array}{l}\text { Strombidium acutum, Strombidium } \\
\text { capitatum, Strombidium conicum, } \\
\text { Tontonia spp, Laboea strobila }\end{array}$ & MEDI & 34.0 & 32.2 & Christaki et al. (2011) \\
\hline GNCM & ciliates & Strombidium reticulatum & NECS & 58.4 & 8.8 & Dale and Dahl (1987) \\
\hline GNCM & ciliates & $\begin{array}{l}\text { Laboea strobila, Tontonia } \\
\text { appendiculariformis, Tontonia spp }\end{array}$ & BPLR & 69.3 & -53.6 & Levinsen et al. (1999) \\
\hline GNCM & ciliates & Laboea strobila & NWCS & 41.0 & -73.2 & McManus and Fuhrman (1986) \\
\hline GNCM & ciliates & $\begin{array}{l}\text { Lohmaniella elegans, } \\
\text { Rimostrombidium, } \\
\text { Pelagostrombidium, Laboea } \\
\text { strobila, Strombidium spp }\end{array}$ & NECS & 60.0 & 29.6 & Mironova et al. (2013) \\
\hline GNCM & ciliates & $\begin{array}{l}\text { Tontonia poopsia, Tontonia } \\
\text { simplicidens, Tontonia } \\
\text { appendiculariformis, Laboea } \\
\text { strobila }\end{array}$ & KURO & 31.0 & 123.0 & Ota and Taniguchi (2003) \\
\hline GNCM & ciliates & $\begin{array}{l}\text { Tontonia poopsia, Tontonia } \\
\text { simplicidens, Tontonia } \\
\text { appendiculariformis, Laboea } \\
\text { strobila }\end{array}$ & KURO & 30.0 & 124.0 & Ota and Taniguchi (2003) \\
\hline GNCM & ciliates & $\begin{array}{l}\text { Tontonia poopsia, Tontonia } \\
\text { simplicidens, Tontonia } \\
\text { appendiculariformis, Laboea } \\
\text { strobila }\end{array}$ & KURO & 28.5 & 126.5 & Ota and Taniguchi (2003) \\
\hline GNCM & ciliates & $\begin{array}{l}\text { Strombidium delicatussimum, } \\
\text { Strombidium conicum, } \\
\text { Strombidium sp, Strombidium } \\
\text { vestitum, Tontonia gracilima, } \\
\text { Tontonia simplicidens }\end{array}$ & MEDI & 40.3 & 25.0 & Pitta and Giannakourou (2000) \\
\hline
\end{tabular}




\begin{tabular}{|c|c|c|c|c|c|c|}
\hline GNCM & ciliates & $\begin{array}{l}\text { Strombidium delicatussimum, } \\
\text { Strombidium conicum, } \\
\text { Strombidium sp, Strombidium } \\
\text { vestitum, Tontonia gracilima, } \\
\text { Tontonia simplicidens }\end{array}$ & MEDI & 35.7 & 25.0 & Pitta and Giannakourou (2000) \\
\hline GNCM & ciliates & Strombidium conicum & KURO & 38.4 & 141.5 & Kim and Taniguchi (1995) \\
\hline GNCM & ciliates & Strombidium capitatum & KURO & 38.4 & 141.5 & Kim et al. (2008b) \\
\hline GNCM & ciliates & Strombidium capitatum & KURO & 35.2 & 128.6 & Kim et al. (2008b) \\
\hline GNCM & ciliates & no data & SARC & 75.0 & 34.0 & Putt (1990) \\
\hline GNCM & ciliates & no data & ARCT & 77.1 & -6.8 & Putt (1990) \\
\hline GNCM & ciliates & no data & SARC & 63.0 & -14.9 & Putt (1990) \\
\hline GNCM & ciliates & no data & NWCS & 30.9 & -76.5 & Dolan and Gallegos (1992) \\
\hline GNCM & ciliates & no data & CARB & 17.9 & -76.8 & Lynn et al. (1991) \\
\hline GNCM & ciliates & no data & SSTC & -39.5 & 19.5 & Froneman and Perissinotto (1996) \\
\hline GNCM & ciliates & no data & SSTC & -43.0 & 21.0 & Froneman and Perissinotto (1996) \\
\hline GNCM & ciliates & no data & SSTC & -41.5 & 22.5 & Froneman and Perissinotto (1996) \\
\hline GNCM & ciliates & no data & SSTC & -43.5 & 20.2 & Froneman and Perissinotto (1996) \\
\hline GNCM & ciliates & no data & SSTC & -41.8 & 18.7 & Froneman and Perissinotto (1996) \\
\hline GNCM & ciliates & no data & PSAG (E) & 44.2 & 157.2 & Suzuki et al. (1998) \\
\hline GNCM & ciliates & no data & PSAG (E) & 45.0 & 165.0 & Suzuki et al. (1998) \\
\hline GNCM & ciliates & no data & KURO & 39.0 & 143.0 & Suzuki et al. (1998) \\
\hline GNCM & ciliates & no data & KURO & 38.0 & 144.0 & Suzuki et al. (1998) \\
\hline GNCM & ciliates & no data & TASM & -40.0 & 155.2 & Suzuki et al. (1998) \\
\hline GNCM & ciliates & no data & TASM & -37.0 & 155.0 & Suzuki et al. (1998) \\
\hline GNCM & ciliates & no data & TASM & -34.0 & 155.0 & Suzuki et al. (1998) \\
\hline GNCM & ciliates & no data & TASM & -24.0 & 155.0 & Suzuki et al. (1998) \\
\hline GNCM & ciliates & no data & TASM & -20.0 & 155.0 & Suzuki et al. (1998) \\
\hline GNCM & ciliates & no data & CALC & 49.4 & -122.8 & Martin and Montagnes (1993) \\
\hline
\end{tabular}




\begin{tabular}{|c|c|c|c|c|c|c|}
\hline GNCM & ciliates & no data & PEQD & 0.0 & -140.0 & Stoecker et al. (1996) \\
\hline GNCM & ciliates & Strombidium spp, Tontonia spp & MEDI & 43.4 & 7.9 & Pérez et al. (2000) \\
\hline GNCM & ciliates & Laboea spp, Tontonia spp & MEDI & 41.7 & 2.8 & Vaqué et al. (1997) \\
\hline GNCM & ciliates & $\begin{array}{l}\text { Laboea strobila, Tontonia } \\
\text { gracilima, Tontonia } \\
\text { appendiculariformis }\end{array}$ & MEDI & 40.5 & 2.5 & Dolan and Marrasé (1995) \\
\hline GNCM & ciliates & Laboea spp, Tontonia spp & MEDI & 41.0 & 6.2 & Dolan et al. (1999) \\
\hline GNCM & ciliates & Laboea spp, Tontonia spp & MEDI & 39.0 & 6.2 & Dolan et al. (1999) \\
\hline GNCM & ciliates & Laboea spp, Tontonia spp & MEDI & 39.0 & 14.0 & Dolan et al. (1999) \\
\hline GNCM & ciliates & Laboea spp, Tontonia spp & MEDI & 38.0 & 6.2 & Dolan et al. (1999) \\
\hline GNCM & ciliates & Laboea spp, Tontonia spp & MEDI & 37.0 & 9.0 & Dolan et al. (1999) \\
\hline GNCM & ciliates & Laboea spp, Tontonia spp & MEDI & 38.0 & 11.3 & Dolan et al. (1999) \\
\hline GNCM & ciliates & Laboea spp, Tontonia spp & MEDI & 36.5 & 13.2 & Dolan et al. (1999) \\
\hline GNCM & ciliates & Laboea spp, Tontonia spp & MEDI & 36.0 & 15.7 & Dolan et al. (1999) \\
\hline GNCM & ciliates & Laboea spp, Tontonia spp & MEDI & 34.0 & 30.0 & Dolan et al. (1999) \\
\hline GNCM & ciliates & Laboea spp, Tontonia spp & MEDI & 41.3 & 10.6 & Dolan et al. (1999) \\
\hline GNCM & ciliates & Laboea spp, Tontonia spp & NWCS & 43.9 & -69.6 & Sanders (1995) \\
\hline GNCM & ciliates & Laboea strobila, Strombidium spp & NWCS & 41.5 & -70.7 & Stoecker et al. (1987) \\
\hline GNCM & ciliates & $\begin{array}{l}\text { Laboea strobila, Tontonia spp, } \\
\text { Strombidium } \mathrm{spp}\end{array}$ & NWCS & 42.2 & -67.7 & Stoecker et al. (1989a) \\
\hline GNCM & ciliates & $\begin{array}{l}\text { Laboea strobila, Tontonia } \mathrm{spp}, \\
\text { Strombidium } \mathrm{spp}\end{array}$ & NWCS & 42.1 & -67.5 & Stoecker et al. (1989a) \\
\hline GNCM & ciliates & $\begin{array}{l}\text { Laboea strobila, Tontonia spp, } \\
\text { Strombidium spp }\end{array}$ & NWCS & 41.8 & -67.0 & Stoecker et al. (1989a) \\
\hline GNCM & ciliates & $\begin{array}{l}\text { Laboea strobila, Tontonia } \mathrm{spp} \text {, } \\
\text { Strombidium } \mathrm{spp}\end{array}$ & NWCS & 41.6 & -66.6 & Stoecker et al. (1989a) \\
\hline GNCM & ciliates & $\begin{array}{l}\text { Laboea strobila, Tontonia } \mathrm{spp} \text {, } \\
\text { Strombidium } \mathrm{spp}\end{array}$ & GFST & 40.5 & -65.8 & Stoecker et al. (1989a) \\
\hline
\end{tabular}




\begin{tabular}{|c|c|c|c|c|c|c|}
\hline GNCM & ciliates & $\begin{array}{l}\text { Laboea strobila, Tontonia spp, } \\
\text { Strombidium } \mathrm{spp}\end{array}$ & NWCS & 40.4 & -67.3 & Stoecker et al. (1989a) \\
\hline GNCM & ciliates & $\begin{array}{l}\text { Laboea strobila, Tontonia } \mathrm{spp}, \\
\text { Strombidium } \mathrm{spp}\end{array}$ & NWCS & 41.1 & -68.7 & Stoecker et al. (1989a) \\
\hline GNCM & ciliates & $\begin{array}{l}\text { Laboea strobila, Tontonia } \mathrm{spp}, \\
\text { Strombidium } \mathrm{spp}\end{array}$ & NADR & 47.0 & -18.0 & Stoecker et al. (1994b) \\
\hline GNCM & ciliates & $\begin{array}{l}\text { Laboea strobila, Tontonia spp, } \\
\text { Strombidium } \mathrm{spp}\end{array}$ & NADR & 45.0 & -16.0 & Stoecker et al. (1994b) \\
\hline GNCM & ciliates & $\begin{array}{l}\text { Laboea strobila, Tontonia } \mathrm{spp}, \\
\text { Strombidium } \mathrm{spp}\end{array}$ & NADR & 50.0 & -19.0 & Stoecker et al. (1994b) \\
\hline GNCM & ciliates & no data & APLR & -77.3 & -166.0 & Stocker et al. (1995) \\
\hline GNCM & ciliates & Strombidium spp, Laboea strobila & BERS & 61.5 & -179.0 & Stoecker et al. (2014) \\
\hline GNCM & ciliates & Strombidium spp, Laboea strobila & BERS & 61.5 & -173.0 & Stoecker et al. (2014) \\
\hline GNCM & ciliates & Strombidium spp, Laboea strobila & BERS & 61.5 & -167.0 & Stoecker et al. (2014) \\
\hline GNCM & ciliates & Strombidium spp, Laboea strobila & BERS & 58.5 & -179.0 & Stoecker et al. (2014) \\
\hline GNCM & ciliates & Strombidium spp, Laboea strobila & BERS & 58.5 & -173.0 & Stoecker et al. (2014) \\
\hline GNCM & ciliates & Strombidium spp, Laboea strobila & BERS & 58.5 & -169.0 & Stoecker et al. (2014) \\
\hline GNCM & ciliates & Strombidium spp, Laboea strobila & BERS & 58.5 & -166.0 & Stoecker et al. (2014) \\
\hline GNCM & ciliates & $\begin{array}{l}\text { Laboea spp, Tontonia } \\
\text { appendiculariformis }\end{array}$ & PSAG (E) & 50.0 & -138.0 & Sime-Ngando et al. (1992) \\
\hline GNCM & ciliates & \begin{tabular}{|l|} 
Laboea spp, Tontonia \\
appendiculariformis
\end{tabular} & PSAG $(W)$ & 46.8 & -130.8 & Sime-Ngando et al. (1992) \\
\hline GNCM & ciliates & Laboea strobila & NECS & 60.8 & 5.2 & Dale (1987) \\
\hline GNCM & ciliates & $\begin{array}{l}\text { Laboea strobila, Strombidium } \mathrm{sp}, \\
\text { Strombidium reticulatum, } \\
\text { Strombidium conicum, } \\
\text { Strombidium vestitum } \\
\end{array}$ & NECS & 58.9 & 11.1 & Jonsson (1987) \\
\hline GNCM & ciliates & Laboea strobila, Strombidium spp & BPLR & 69.3 & -53.6 & Levinsen and Nielsen (2002) \\
\hline GNCM & ciliates & Laboea strobila, Strombidium spp & NECS & 56.2 & 12.1 & Levinsen and Nielsen (2002) \\
\hline GNCM & ciliates & Laboea strobila, Strombidium spp & NECS & 56.2 & 12.1 & Levinsen and Nielsen (2002) \\
\hline
\end{tabular}




\begin{tabular}{|c|c|c|c|c|c|c|}
\hline GNCM & ciliates & $\begin{array}{l}\text { Strombidium capitatum, } \\
\text { Strombidium conicum, } \\
\text { Strombidium acutum, Strombidium } \\
\text { chlorophilum }\end{array}$ & NWCS & 41.5 & -70.7 & Stoecker et al. (1989b) \\
\hline GNCM & ciliates & $\begin{array}{l}\text { Strombidium, Laboea spp, } \\
\text { Tontonia spp }\end{array}$ & NWCS & 43.0 & -70.6 & Montagnes et al. (1988) \\
\hline GNCM & ciliates & $\begin{array}{l}\text { Strombidium, Laboea spp, } \\
\text { Tontonia spp }\end{array}$ & NWCS & 43.0 & -70.6 & Montagnes et al. (1988) \\
\hline GNCM & ciliates & $\begin{array}{l}\text { Strombidium, Laboea spp, } \\
\text { Tontonia spp }\end{array}$ & NWCS & 43.0 & -70.6 & Montagnes et al. (1988) \\
\hline GNCM & ciliates & Strombidium crassulum & NECS & 50.2 & -4.3 & Reid (1997) \\
\hline GNCM & ciliates & $\begin{array}{l}\text { Strombidium elegans, Strombidium } \\
\text { delicatissimum, Strombidium } \\
\text { crassulum, Strombidium } \\
\text { capitatum, Strombidium acutum, } \\
\text { Laboea strobila, Strombidium } \\
\text { typicum, Strombidium vestitum, } \\
\text { Tontonia gracilima, Tontonia } \\
\text { ovalis }\end{array}$ & MEDI & 43.7 & 7.3 & $\begin{array}{l}\text { Laval-Peuto and Rassoulzadegan } \\
\text { (1988) }\end{array}$ \\
\hline GNCM & ciliates & Strombidium stylifer & NECS & 54.2 & -4.4 & McManus et al. (2004) \\
\hline GNCM & ciliates & Strombidium stylifer & NECS & 53.3 & -6.1 & McManus et al. (2004) \\
\hline GNCM & ciliates & Strombidium stylifer & NECS & 53.2 & -9.3 & McManus et al. (2004) \\
\hline GNCM & ciliates & Strombidium stylifer & NWCS & 41.1 & -72.9 & McManus et al. (2004) \\
\hline GNCM & ciliates & Strombidium stylifer & NECS & 48.9 & -2.3 & Jonsson (1994) \\
\hline GNCM & ciliates & Pseudotontonia cornuta & NECS & 56.2 & 6.6 & Skovgaard and Legrand (2005) \\
\hline pSNCM & ciliates & Mesodinium rubrum & NECS & 55.0 & 18.3 & Witek (1998) \\
\hline pSNCM & ciliates & Mesodinium rubrum & NECS & 54.5 & 18.7 & Witek (1998) \\
\hline pSNCM & ciliates & Mesodinium rubrum & NECS & 54.8 & 19.4 & Witek (1998) \\
\hline GNCM & ciliates & no data & KURO & 34.3 & 132.4 & Kamiyama et al. (2003) \\
\hline GNCM & ciliates & no data & KURO & 34.3 & 132.4 & Kamiyama et al. (2003) \\
\hline
\end{tabular}


Appendices. Appendix A, Table A.I

\begin{tabular}{|c|c|c|c|c|c|c|}
\hline GNCM & ciliates & no data & KURO & 34.2 & 132.3 & Kamiyama et al. (2003) \\
\hline GNCM & ciliates & no data & KURO & 34.1 & 132.4 & Kamiyama et al. (2003) \\
\hline GNCM & ciliates & no data & KURO & 34.0 & 132.4 & Kamiyama et al. (2003) \\
\hline GNCM & ciliates & $\begin{array}{l}\text { Tontonia ovalis, Tontonia } \\
\text { simplecidens, Strombidium } \\
\text { vestitum, Strombidium } \\
\text { delicatissimum }\end{array}$ & MEDI & 33.0 & 22.0 & Pitta et al. (2001) \\
\hline GNCM & ciliates & $\begin{array}{l}\text { Tontonia ovalis, Tontonia } \\
\text { simplecidens, Strombidium } \\
\text { vestitum, Strombidium } \\
\text { delicatissimum }\end{array}$ & MEDI & 34.0 & 30.0 & Pitta et al. (2001) \\
\hline GNCM & ciliates & $\begin{array}{l}\text { Tontonia ovalis, Tontonia } \\
\text { simplecidens, Strombidium } \\
\text { vestitum, Strombidium } \\
\text { delicatissimum }\end{array}$ & MEDI & 33.0 & 27.0 & Pitta et al. (2001) \\
\hline GNCM & ciliates & Strombidium rassoulzadegani & NWCS & 42.0 & -74.0 & Schoener and McManus (2012) \\
\hline GNCM & ciliates & $\begin{array}{l}\text { Laboea strobila, Spirotontonia cf. } \\
\text { grandia, Strombidium acutum, } \\
\text { Strombidium capitatum, Tontonia } \\
\text { gracilima, Tontonia } \mathrm{sp}\end{array}$ & BPLR & 78.0 & -170.0 & Jiang et al. (2015) \\
\hline GNCM & ciliates & $\begin{array}{l}\text { Laboea strobila, Spirotontonia cf. } \\
\text { grandia, Strombidium acutum, } \\
\text { Strombidium capitatum, Tontonia } \\
\text { gracilima, Tontonia } \mathrm{sp}\end{array}$ & BPLR & 76.0 & -170.0 & Jiang et al. (2015) \\
\hline GNCM & ciliates & $\begin{array}{l}\text { Laboea strobila, Spirotontonia cf. } \\
\text { grandia, Strombidium acutum, } \\
\text { Strombidium capitatum, Tontonia } \\
\text { gracilima, Tontonia } \mathrm{sp}\end{array}$ & BPLR & 82.0 & 170.0 & Jiang et al. (2015) \\
\hline GNCM & ciliates & $\begin{array}{l}\text { Laboea strobila, Spirotontonia cf. } \\
\text { grandia, Strombidium acutum, }\end{array}$ & BPLR & 81.0 & 170.0 & Jiang et al. (2015) \\
\hline
\end{tabular}




\begin{tabular}{|c|c|c|c|c|c|c|}
\hline & & $\begin{array}{l}\text { Strombidium capitatum, Tontonia } \\
\text { gracilima, Tontonia } \mathrm{sp}\end{array}$ & & & & \\
\hline GNCM & ciliates & $\begin{array}{l}\text { Laboea strobila, Spirotontonia cf. } \\
\text { grandia, Strombidium acutum, } \\
\text { Strombidium capitatum, Tontonia } \\
\text { gracilima, Tontonia } \mathrm{sp}\end{array}$ & BPLR & 79.0 & 172.0 & Jiang et al. (2015) \\
\hline GNCM & ciliates & $\begin{array}{l}\text { Laboea strobila, Spirotontonia cf. } \\
\text { grandia, Strombidium acutum, } \\
\text { Strombidium capitatum, Tontonia } \\
\text { gracilima, Tontonia } \mathrm{sp}\end{array}$ & BPLR & 78.0 & 173.0 & Jiang et al. (2015) \\
\hline GNCM & ciliates & $\begin{array}{l}\text { Laboea strobila, Spirotontonia cf. } \\
\text { grandia, Strombidium acutum, } \\
\text { Strombidium capitatum, Tontonia } \\
\text { gracilima, Tontonia } \mathrm{sp}\end{array}$ & BPLR & 76.0 & 173.0 & Jiang et al. (2015) \\
\hline GNCM & ciliates & $\begin{array}{l}\text { Laboea strobila, Spirotontonia cf. } \\
\text { grandia, Strombidium acutum, } \\
\text { Strombidium capitatum, Tontonia } \\
\text { gracilima, Tontonia } \mathrm{sp}\end{array}$ & BPLR & 77.0 & -175.0 & Jiang et al. (2015) \\
\hline GNCM & ciliates & $\begin{array}{l}\text { Laboea strobila, Spirotontonia cf. } \\
\text { grandia, Strombidium acutum, } \\
\text { Strombidium capitatum, Tontonia } \\
\text { gracilima, Tontonia } \mathrm{sp}\end{array}$ & BPLR & 76.0 & -175.0 & Jiang et al. (2015) \\
\hline GNCM & ciliates & $\begin{array}{l}\text { Laboea strobila, Spirotontonia cf. } \\
\text { grandia, Strombidium acutum, } \\
\text { Strombidium capitatum, Tontonia } \\
\text { gracilima, Tontonia } \mathrm{sp}\end{array}$ & BPLR & 75.0 & -175.0 & Jiang et al. (2015) \\
\hline GNCM & ciliates & $\begin{array}{l}\text { Laboea strobila, Spirotontonia cf. } \\
\text { grandia, Strombidium acutum, } \\
\text { Strombidium capitatum, Tontonia } \\
\text { gracilima, Tontonia } \mathrm{sp}\end{array}$ & BPLR & 78.0 & -165.0 & Jiang et al. (2015) \\
\hline GNCM & ciliates & $\begin{array}{l}\text { Laboea strobila, Spirotontonia cf. } \\
\text { grandia, Strombidium acutum, }\end{array}$ & BPLR & 76.0 & -165.0 & Jiang et al. (2015) \\
\hline
\end{tabular}




\begin{tabular}{|c|c|c|c|c|c|c|}
\hline & & $\begin{array}{l}\text { Strombidium capitatum, Tontonia } \\
\text { gracilima, Tontonia } \mathrm{sp}\end{array}$ & & & & \\
\hline GNCM & ciliates & $\begin{array}{l}\text { Laboea strobila, Spirotontonia cf. } \\
\text { grandia, Strombidium acutum, } \\
\text { Strombidium capitatum, Tontonia } \\
\text { gracilima, Tontonia } \mathrm{sp}\end{array}$ & BPLR & 75.0 & -165.0 & Jiang et al. (2015) \\
\hline GNCM & ciliates & $\begin{array}{l}\text { Laboea strobila, Spirotontonia cf. } \\
\text { grandia, Strombidium acutum, } \\
\text { Strombidium capitatum, Tontonia } \\
\text { gracilima, Tontonia } \mathrm{sp}\end{array}$ & BPLR & 74.0 & -165.0 & Jiang et al. (2015) \\
\hline GNCM & ciliates & $\begin{array}{l}\text { Laboea strobila, Spirotontonia cf. } \\
\text { grandia, Strombidium acutum, } \\
\text { Strombidium capitatum, Tontonia } \\
\text { gracilima, Tontonia } \mathrm{sp}\end{array}$ & BPLR & 73.0 & -165.0 & Jiang et al. (2015) \\
\hline GNCM & ciliates & $\begin{array}{l}\text { Laboea strobila, Spirotontonia cf. } \\
\text { grandia, Strombidium acutum, } \\
\text { Strombidium capitatum, Tontonia } \\
\text { gracilima, Tontonia } \mathrm{sp}\end{array}$ & BPLR & 77.0 & -155.0 & Jiang et al. (2015) \\
\hline GNCM & ciliates & $\begin{array}{l}\text { Laboea strobila, Spirotontonia cf. } \\
\text { grandia, Strombidium acutum, } \\
\text { Strombidium capitatum, Tontonia } \\
\text { gracilima, Tontonia } \mathrm{sp}\end{array}$ & BPLR & 76.0 & -155.0 & Jiang et al. (2015) \\
\hline GNCM & ciliates & Strombidium conicum & CHIN & 21.8 & 108.6 & Liu et al. (2015) \\
\hline GNCM & ciliates & $\begin{array}{l}\text { Strombidium acutum, Strombidium } \\
\text { capitatum, Strombidium conicum, } \\
\text { Laboea strobila, Strombidium } \\
\text { sulcatum, Strombidium stylifer }\end{array}$ & CHIN & 21.2 & 108.4 & Wang et al. (2014) \\
\hline GNCM & ciliates & $\begin{array}{l}\text { Strombidium acutum, Strombidium } \\
\text { capitatum, Strombidium conicum, } \\
\text { Laboea strobila, Strombidium } \\
\text { sulcatum, Strombidium stylifer }\end{array}$ & CHIN & 20.3 & 107.0 & Wang et al. (2014) \\
\hline
\end{tabular}




\begin{tabular}{|c|c|c|c|c|c|c|}
\hline GNCM & ciliates & $\begin{array}{l}\text { Strombidium acutum, Strombidium } \\
\text { capitatum, Strombidium conicum, } \\
\text { Laboea strobila, Strombidium } \\
\text { sulcatum, Strombidium stylifer }\end{array}$ & CHIN & 20.3 & 109.2 & Wang et al. (2014) \\
\hline GNCM & ciliates & $\begin{array}{l}\text { Strombidium acutum, Strombidium } \\
\text { capitatum, Strombidium conicum, } \\
\text { Laboea strobila, Strombidium } \\
\text { sulcatum, Strombidium stylifer }\end{array}$ & CHIN & 19.4 & 108.2 & Wang et al. (2014) \\
\hline GNCM & ciliates & $\begin{array}{l}\text { Strombidium acutum, Strombidium } \\
\text { capitatum, Strombidium conicum, } \\
\text { Laboea strobila, Strombidium } \\
\text { sulcatum, Strombidium stylifer }\end{array}$ & CHIN & 36.1 & 120.3 & Jiang et al. (2011) \\
\hline GNCM & ciliates & no data & KURO & 37.7 & 137.5 & Suzuki and Taniguchi (1998) \\
\hline GNCM & ciliates & Strombidium spp, Laboea spp & INDW & 15.4 & 73.9 & Gauns et al. (2015) \\
\hline GNCM & ciliates & $\begin{array}{l}\text { Strombidium conicum, } \\
\text { Lohmaniella spp }\end{array}$ & INDE & 20.4 & 87.7 & Jyothibabu et al. (2008) \\
\hline GNCM & ciliates & $\begin{array}{l}\text { Strombidium conicum, } \\
\text { Lohmaniella } \mathrm{spp}\end{array}$ & INDE & 19.0 & 85.8 & Jyothibabu et al. (2008) \\
\hline GNCM & ciliates & $\begin{array}{l}\text { Strombidium conicum, } \\
\text { Lohmaniella } \mathrm{spp}\end{array}$ & INDE & 17.0 & 83.5 & Jyothibabu et al. (2008) \\
\hline GNCM & ciliates & $\begin{array}{l}\text { Strombidium conicum, } \\
\text { Lohmaniella } \mathrm{spp}\end{array}$ & INDE & 15.4 & 80.6 & Jyothibabu et al. (2008) \\
\hline GNCM & ciliates & $\begin{array}{l}\text { Strombidium conicum, } \\
\text { Lohmaniella } \mathrm{spp}\end{array}$ & INDE & 13.9 & 80.7 & Jyothibabu et al. (2008) \\
\hline GNCM & ciliates & $\begin{array}{l}\text { Strombidium conicum, } \\
\text { Lohmaniella } \mathrm{spp}\end{array}$ & INDE & 12.1 & 80.5 & Jyothibabu et al. (2008) \\
\hline GNCM & ciliates & $\begin{array}{l}\text { Strombidium conicum, } \\
\text { Lohmaniella spp }\end{array}$ & INDE & 20.4 & 90.7 & Jyothibabu et al. (2008) \\
\hline GNCM & ciliates & $\begin{array}{l}\text { Strombidium conicum, } \\
\text { Lohmaniella } \mathrm{spp}\end{array}$ & INDE & 19.0 & 87.8 & Jyothibabu et al. (2008) \\
\hline GNCM & ciliates & $\begin{array}{l}\text { Strombidium conicum, } \\
\text { Lohmaniella spp }\end{array}$ & INDE & 17.0 & 86.5 & Jyothibabu et al. (2008) \\
\hline
\end{tabular}




\begin{tabular}{|c|c|c|c|c|c|c|}
\hline GNCM & ciliates & $\begin{array}{l}\text { Strombidium conicum, } \\
\text { Lohmaniella } \mathrm{spp}\end{array}$ & INDE & 15.4 & 83.6 & Jyothibabu et al. (2008) \\
\hline GNCM & ciliates & $\begin{array}{l}\text { Strombidium conicum, } \\
\text { Lohmaniella spp }\end{array}$ & INDE & 13.9 & 83.7 & Jyothibabu et al. (2008) \\
\hline GNCM & ciliates & $\begin{array}{l}\text { Strombidium conicum, } \\
\text { Lohmaniella } \mathrm{spp}\end{array}$ & INDE & 12.1 & 83.5 & Jyothibabu et al. (2008) \\
\hline GNCM & ciliates & no data & EAFR & -33.7 & 26.9 & Froneman and McQuaid (1997) \\
\hline GNCM & ciliates & Strombididum spp, Laboea strobila & BENG & -30.6 & 13.0 & Matthews (1991) \\
\hline GNCM & ciliates & Strombididum spp, Laboea strobila & BENG & -31.8 & 14.0 & Matthews (1991) \\
\hline GNCM & ciliates & Strombididum spp, Laboea strobila & BENG & -33.7 & 15.2 & Matthews (1991) \\
\hline GNCM & ciliates & $\begin{array}{l}\text { Strombidium spp, Laboea strobila, } \\
\text { Tontonia spp }\end{array}$ & NATR & 22.0 & -21.0 & Quevedo et al. (2003) \\
\hline GNCM & ciliates & $\begin{array}{l}\text { Strombidium spp, Laboea strobila, } \\
\text { Tontonia spp }\end{array}$ & NAST (E) & 28.0 & -24.0 & Quevedo et al. (2003) \\
\hline GNCM & ciliates & $\begin{array}{l}\text { Strombidium spp, Laboea strobila, } \\
\text { Tontonia spp }\end{array}$ & NAST (E) & 28.0 & -20.0 & Quevedo et al. (2003) \\
\hline GNCM & ciliates & $\begin{array}{l}\text { Strombidium spp, Laboea strobila, } \\
\text { Tontonia spp }\end{array}$ & NAST (E) & 30.0 & -30.0 & Quevedo et al. (2003) \\
\hline GNCM & ciliates & $\begin{array}{l}\text { Strombidium spp, Laboea strobila, } \\
\text { Tontonia spp }\end{array}$ & NAST (E) & 30.0 & -27.0 & Quevedo et al. (2003) \\
\hline GNCM & ciliates & $\begin{array}{l}\text { Strombidium spp, Laboea strobila, } \\
\text { Tontonia spp }\end{array}$ & NAST (E) & 30.0 & -22.0 & Quevedo et al. (2003) \\
\hline GNCM & ciliates & $\begin{array}{l}\text { Strombidium spp, Laboea strobila, } \\
\text { Tontonia spp }\end{array}$ & NAST (W) & 35.0 & -35.0 & Quevedo et al. (2003) \\
\hline GNCM & ciliates & $\begin{array}{l}\text { Strombidium spp, Laboea strobila, } \\
\text { Tontonia spp }\end{array}$ & NAST (W) & 35.0 & -30.0 & Quevedo et al. (2003) \\
\hline GNCM & ciliates & $\begin{array}{l}\text { Strombidium spp, Laboea strobila, } \\
\text { Tontonia spp }\end{array}$ & NAST (E) & 35.0 & -20.0 & Quevedo et al. (2003) \\
\hline GNCM & ciliates & $\begin{array}{l}\text { Strombidium spp, Laboea strobila, } \\
\text { Tontonia spp }\end{array}$ & NAST (E) & 40.0 & -22.0 & Quevedo et al. (2003) \\
\hline
\end{tabular}




\begin{tabular}{|c|c|c|c|c|c|c|}
\hline GNCM & ciliates & $\begin{array}{l}\text { Strombidium spp, Laboea strobila, } \\
\text { Tontonia spp }\end{array}$ & NAST (E) & 42.5 & -20.0 & Quevedo et al. (2003) \\
\hline GNCM & ciliates & $\begin{array}{l}\text { Strombidium spp, Laboea strobila, } \\
\text { Tontonia spp }\end{array}$ & NADR & 48.0 & -18.0 & Quevedo et al. (2003) \\
\hline GNCM & ciliates & $\begin{array}{l}\text { Strombidium spp, Strombidium } \\
\text { conicum, Tontonia spp, Laboea } \\
\text { spp }\end{array}$ & APLR & -69.4 & -77.8 & Garzio and Steinberg (2013) \\
\hline GNCM & ciliates & $\begin{array}{l}\text { Strombidium spp, Strombidium } \\
\text { conicum, Tontonia spp, Laboea } \\
\text { spp }\end{array}$ & APLR & -68.7 & -80.0 & Garzio and Steinberg (2013) \\
\hline GNCM & ciliates & $\begin{array}{l}\text { Strombidium spp, Strombidium } \\
\text { conicum, Tontonia spp, Laboea } \\
\text { spp }\end{array}$ & APLR & -68.0 & -83.0 & Garzio and Steinberg (2013) \\
\hline GNCM & ciliates & $\begin{array}{l}\text { Strombidium spp, Strombidium } \\
\text { conicum, Tontonia spp, Laboea } \\
\text { spp }\end{array}$ & APLR & -68.0 & -80.0 & Garzio and Steinberg (2013) \\
\hline GNCM & ciliates & $\begin{array}{l}\text { Strombidium spp, Strombidium } \\
\text { conicum, Tontonia spp, Laboea } \\
\text { spp }\end{array}$ & APLR & -64.0 & 72.0 & Garzio and Steinberg (2013) \\
\hline GNCM & ciliates & $\begin{array}{l}\text { Strombidium spp, Strombidium } \\
\text { conicum, Tontonia spp, Laboea } \\
\text { spp }\end{array}$ & APLR & -65.5 & -75.0 & Garzio and Steinberg (2013) \\
\hline GNCM & ciliates & $\begin{array}{l}\text { Strombidium spp, Strombidium } \\
\text { conicum, Tontonia spp, Laboea } \\
\text { spp }\end{array}$ & APLR & -67.5 & 77.5 & Garzio and Steinberg (2013) \\
\hline GNCM & ciliates & $\begin{array}{l}\text { Strombidium spp, Strombidium } \\
\text { conicum, Tontonia spp, Laboea } \\
\text { spp }\end{array}$ & APLR & -64.0 & -68.0 & Garzio and Steinberg (2013) \\
\hline GNCM & ciliates & $\begin{array}{l}\text { Strombidium spp, Strombidium } \\
\text { conicum, Tontonia spp, Laboea } \\
\text { spp }\end{array}$ & APLR & -64.8 & -68.0 & Garzio and Steinberg (2013) \\
\hline
\end{tabular}




\begin{tabular}{|c|c|c|c|c|c|c|}
\hline GNCM & ciliates & $\begin{array}{l}\text { Strombidium spp, Strombidium } \\
\text { conicum, Tontonia spp, Laboea } \\
\text { spp }\end{array}$ & APLR & -63.0 & -61.0 & Garzio and Steinberg (2013) \\
\hline GNCM & ciliates & $\begin{array}{l}\text { Strombidium spp, Strombidium } \\
\text { conicum, Tontonia spp, Laboea sp }\end{array}$ & APLR & -63.0 & -68.0 & Garzio and Steinberg (2013) \\
\hline GNCM & ciliates & $\begin{array}{l}\text { Tontonia antarctica, Strombidium } \\
\text { antarcticum, Strombidium } \\
\text { conicum, Laboea strobila }\end{array}$ & SANT & -51.5 & 78.0 & Christaki et al. (2008) \\
\hline GNCM & ciliates & $\begin{array}{l}\text { Tontonia antarctica, Strombidium } \\
\text { antarcticum, Strombidium } \\
\text { conicum, Laboea strobila }\end{array}$ & SANT & -51.0 & 75.0 & Christaki et al. (2008) \\
\hline GNCM & ciliates & $\begin{array}{l}\text { Tontonia antarctica, Strombidium } \\
\text { antarcticum, Strombidium } \\
\text { conicum, Laboea strobila }\end{array}$ & SANT & -49.0 & 74.0 & Christaki et al. (2008) \\
\hline GNCM & ciliates & $\begin{array}{l}\text { Laboea strobila, Strombidium } \\
\text { conicum }\end{array}$ & SANT & -43.0 & 167.0 & Chang (1990) \\
\hline GNCM & ciliates & Strombidium conicum & ARAB & 20.0 & 59.0 & Jyothibabu et al. (2008) \\
\hline pSNCM & ciliates & Mesodinium rubrum & SANT & -43.0 & 167.0 & Chang (1990) \\
\hline $\mathrm{pSNCM}$ & ciliates & Mesodinium rubrum & MEDI & 43.7 & 7.3 & Bernard and Rassoulzadegan (1994) \\
\hline pSNCM & ciliates & Mesodinium rubrum & MEDI & 40.7 & 14.2 & Modigh (2001) \\
\hline pSNCM & ciliates & Mesodinium rubrum & MEDI & 39.7 & 4.9 & Christaki et al. (2011) \\
\hline pSNCM & ciliates & Mesodinium rubrum & MEDI & 35.6 & 17.9 & Christaki et al. (2011) \\
\hline pSNCM & ciliates & Mesodinium rubrum & MEDI & 34.0 & 32.2 & Christaki et al. (2011) \\
\hline pSNCM & ciliates & Mesodinium rubrum & NECS & 58.4 & 8.8 & Dale and Dahl (1987) \\
\hline pSNCM & ciliates & Mesodinium rubrum & CHIN & 35.2 & 128.5 & Jeong et al. (2013) \\
\hline $\mathrm{pSNCM}$ & ciliates & Mesodinium rubrum & NWCS & 37.5 & -76.1 & Johnson et al. (2013) \\
\hline pSNCM & ciliates & Mesodinium rubrum & BPLR & 69.3 & -53.6 & Levinsen et al. (1999) \\
\hline pSNCM & ciliates & Mesodinium rubrum & NECS & 60.0 & 29.6 & Mironova et al. (2013) \\
\hline pSNCM & ciliates & Mesodinium rubrum & KURO & 31.0 & 123.0 & Ota and Taniguchi (2003) \\
\hline pSNCM & ciliates & Mesodinium rubrum & KURO & 30.0 & 124.0 & Ota and Taniguchi (2003) \\
\hline
\end{tabular}




\begin{tabular}{|c|c|c|c|c|c|c|}
\hline $\mathrm{pSNCM}$ & ciliates & Mesodinium rubrum & KURO & 28.5 & 126.5 & Ota and Taniguchi (2003) \\
\hline pSNCM & ciliates & Mesodinium rubrum & MEDI & 43.4 & 7.9 & Pérez et al. (2000) \\
\hline pSNCM & ciliates & Mesodinium rubrum & MEDI & 41.7 & 2.8 & Vaqué et al. (1997) \\
\hline pSNCM & ciliates & Mesodinium rubrum & MEDI & 40.5 & 2.5 & Dolan and Marrasé (1995) \\
\hline $\mathrm{pSNCM}$ & ciliates & Mesodinium rubrum & NWCS & 43.9 & -69.6 & Sanders (1995) \\
\hline pSNCM & ciliates & Mesodinium rubrum & NWCS & 42.2 & -67.7 & Stoecker et al. (1989a) \\
\hline $\mathrm{pSNCM}$ & ciliates & Mesodinium rubrum & NWCS & 42.1 & -67.5 & Stoecker et al. (1989a) \\
\hline pSNCM & ciliates & Mesodinium rubrum & NWCS & 41.8 & -67.0 & Stoecker et al. (1989a) \\
\hline $\mathrm{pSNCM}$ & ciliates & Mesodinium rubrum & NWCS & 41.6 & -66.6 & Stoecker et al. (1989a) \\
\hline pSNCM & ciliates & Mesodinium rubrum & GFST & 40.5 & -65.8 & Stoecker et al. (1989a) \\
\hline pSNCM & ciliates & Mesodinium rubrum & NWCS & 40.4 & -67.3 & Stoecker et al. (1989a) \\
\hline $\mathrm{pSNCM}$ & ciliates & Mesodinium rubrum & NWCS & 41.1 & -68.7 & Stoecker et al. (1989a) \\
\hline $\mathrm{pSNCM}$ & ciliates & Mesodinium rubrum & NWCS & 41.6 & -70.7 & Stoecker et al. (1991) \\
\hline $\mathrm{pSNCM}$ & ciliates & Mesodinium rubrum & NWCS & 41.5 & -70.6 & Stoecker et al. (1991) \\
\hline pSNCM & ciliates & Mesodinium rubrum & BPLR & 69.3 & -53.6 & Levinsen and Nielsen (2002) \\
\hline pSNCM & ciliates & Mesodinium rubrum & NECS & 56.2 & 12.1 & Levinsen and Nielsen (2002) \\
\hline $\mathrm{pSNCM}$ & ciliates & Mesodinium rubrum & NECS & 56.2 & 12.1 & Levinsen and Nielsen (2002) \\
\hline $\mathrm{pSNCM}$ & ciliates & Mesodinium rubrum & APLR & -79.0 & -168.0 & Gustafson et al. (2000) \\
\hline pSNCM & ciliates & Mesodinium rubrum & CHIN & 35.5 & 126.4 & Yih et al. (2004) \\
\hline $\mathrm{pSNCM}$ & ciliates & Mesodinium rubrum & NECS & 55.8 & 11.8 & Hansen and Fenchel (2006) \\
\hline pSNCM & ciliates & Mesodinium rubrum & NECS & 55.0 & 18.3 & Witek (1998) \\
\hline $\mathrm{pSNCM}$ & ciliates & Mesodinium rubrum & NECS & 54.5 & 18.7 & Witek (1998) \\
\hline $\mathrm{pSNCM}$ & ciliates & Mesodinium rubrum & NECS & 54.8 & 19.4 & Witek (1998) \\
\hline pSNCM & ciliates & Mesodinium rubrum & NECS & 57.3 & 19.6 & Setälä and Kivi (2003) \\
\hline $\mathrm{pSNCM}$ & ciliates & Mesodinium rubrum & NECS & 59.6 & 23.4 & Setälä and Kivi (2003) \\
\hline $\mathrm{pSNCM}$ & ciliates & Mesodinium rubrum & NECS & 55.3 & 16.1 & Setälä and Kivi (2003) \\
\hline pSNCM & ciliates & Mesodinium rubrum & NECS & 55.0 & 13.8 & Setälä and Kivi (2003) \\
\hline
\end{tabular}




\begin{tabular}{|c|c|c|c|c|c|c|}
\hline $\mathrm{pSNCM}$ & ciliates & Mesodinium rubrum & NECS & 60.0 & 20.4 & Crawford et al. (1997) \\
\hline pSNCM & ciliates & Mesodinium rubrum & HUMB & -15.0 & -75.5 & Smith and Barber (1979) \\
\hline pSNCM & ciliates & Mesodinium rubrum & HUMB & -15.1 & -75.5 & Smith and Barber (1979) \\
\hline pSNCM & ciliates & Mesodinium rubrum & HUMB & -15.0 & -75.5 & Smith and Barber (1979) \\
\hline pSNCM & ciliates & Mesodinium rubrum & HUMB & -15.1 & -75.6 & Smith and Barber (1979) \\
\hline pSNCM & ciliates & Mesodinium rubrum & HUMB & -15.2 & -75.7 & Smith and Barber (1979) \\
\hline $\mathrm{pSNCM}$ & ciliates & Mesodinium rubrum & HUMB & -15.2 & -75.7 & Smith and Barber (1979) \\
\hline pSNCM & ciliates & Mesodinium rubrum & BRAZ & -22.8 & -43.2 & Gomes et al. (2007) \\
\hline $\mathrm{pSNCM}$ & ciliates & Mesodinium rubrum & BRAZ & -22.9 & -43.2 & Gomes et al. (2007) \\
\hline pSNCM & ciliates & Mesodinium rubrum & BRAZ & -23.6 & -45.1 & Owen et al. (1992) \\
\hline pSNCM & ciliates & Mesodinium rubrum & BPLR & 78.0 & -170.0 & Jiang et al. (2015) \\
\hline $\mathrm{pSNCM}$ & ciliates & Mesodinium rubrum & BPLR & 76.0 & -170.0 & Jiang et al. (2015) \\
\hline pSNCM & ciliates & Mesodinium rubrum & BPLR & 82.0 & 170.0 & Jiang et al. (2015) \\
\hline pSNCM & ciliates & Mesodinium rubrum & BPLR & 81.0 & 170.0 & Jiang et al. (2015) \\
\hline $\mathrm{pSNCM}$ & ciliates & Mesodinium rubrum & BPLR & 79.0 & 172.0 & Jiang et al. (2015) \\
\hline $\mathrm{pSNCM}$ & ciliates & Mesodinium rubrum & BPLR & 78.0 & 173.0 & Jiang et al. (2015) \\
\hline pSNCM & ciliates & Mesodinium rubrum & BPLR & 76.0 & 173.0 & Jiang et al. (2015) \\
\hline $\mathrm{pSNCM}$ & ciliates & Mesodinium rubrum & BPLR & 77.0 & -175.0 & Jiang et al. (2015) \\
\hline pSNCM & ciliates & Mesodinium rubrum & BPLR & 76.0 & -175.0 & Jiang et al. (2015) \\
\hline pSNCM & ciliates & Mesodinium rubrum & BPLR & 75.0 & -175.0 & Jiang et al. (2015) \\
\hline pSNCM & ciliates & Mesodinium rubrum & BPLR & 78.0 & -165.0 & Jiang et al. (2015) \\
\hline pSNCM & ciliates & Mesodinium rubrum & BPLR & 76.0 & -165.0 & Jiang et al. (2015) \\
\hline $\mathrm{pSNCM}$ & ciliates & Mesodinium rubrum & BPLR & 75.0 & -165.0 & Jiang et al. (2015) \\
\hline pSNCM & ciliates & Mesodinium rubrum & BPLR & 74.0 & -165.0 & Jiang et al. (2015) \\
\hline pSNCM & ciliates & Mesodinium rubrum & BPLR & 73.0 & -165.0 & Jiang et al. (2015) \\
\hline $\mathrm{pSNCM}$ & ciliates & Mesodinium rubrum & BPLR & 77.0 & -155.0 & Jiang et al. (2015) \\
\hline pSNCM & ciliates & Mesodinium rubrum & BPLR & 76.0 & -155.0 & Jiang et al. (2015) \\
\hline
\end{tabular}




\begin{tabular}{|c|c|c|c|c|c|c|}
\hline pSNCM & ciliates & Mesodinium rubrum & APLR & -68.5 & 77.7 & Van den Hoff and Bell (2015) \\
\hline pSNCM & ciliates & Mesodinium rubrum & APLR & -68.5 & 78.8 & Van den Hoff and Bell (2015) \\
\hline pSNCM & ciliates & Mesodinium rubrum & HUMB & -41.5 & -72.0 & Santoferrara et al. (2014) \\
\hline pSNCM & dinoflagellates & Amylax triacantha & CHIN & 35.0 & 128.7 & Park et al. (2013) \\
\hline $\mathrm{pSNCM}$ & dinoflagellates & Dinophysis mitra & KURO & 41.0 & 140.8 & Nishitani et al. (2012) \\
\hline pSNCM & dinoflagellates & Dinophysis mitra & KURO & 33.8 & 132.2 & Nishitani et al. (2012) \\
\hline pSNCM & dinoflagellates & Dinophysis mitra & KURO & 32.5 & 130.5 & Nishitani et al. (2012) \\
\hline $\mathrm{pSNCM}$ & ciliates & Mesodinium rubrum & NECS & 56.9 & 9.0 & Andersen and Sørensen (1986) \\
\hline pSNCM & ciliates & Mesodinium rubrum & CHIN & 37.6 & 126.0 & Yih et al. (2013) \\
\hline pSNCM & ciliates & Mesodinium rubrum & CHIN & 37.4 & 126.3 & Yih et al. (2013) \\
\hline pSNCM & ciliates & Mesodinium rubrum & CHIN & 37.1 & 126.6 & Yih et al. (2013) \\
\hline pSNCM & ciliates & Mesodinium rubrum & CHIN & 36.5 & 126.4 & Yih et al. (2013) \\
\hline $\mathrm{pSNCM}$ & ciliates & Mesodinium rubrum & CHIN & 36.2 & 126.3 & Yih et al. (2013) \\
\hline pSNCM & ciliates & Mesodinium rubrum & CHIN & 35.9 & 126.3 & Yih et al. (2013) \\
\hline pSNCM & ciliates & Mesodinium rubrum & CHIN & 34.3 & 126.4 & Yih et al. (2013) \\
\hline $\mathrm{pSNCM}$ & ciliates & Mesodinium rubrum & CHIN & 34.4 & 127.5 & Yih et al. (2013) \\
\hline pSNCM & ciliates & Mesodinium rubrum & CHIN & 34.8 & 127.9 & Yih et al. (2013) \\
\hline pSNCM & ciliates & Mesodinium rubrum & CHIN & 34.8 & 128.3 & Yih et al. (2013) \\
\hline pSNCM & ciliates & Mesodinium rubrum & CHIN & 34.8 & 128.6 & Yih et al. (2013) \\
\hline pSNCM & ciliates & Mesodinium rubrum & CHIN & 34.9 & 128.7 & Yih et al. (2013) \\
\hline pSNCM & ciliates & Mesodinium rubrum & CHIN & 35.0 & 129.2 & Yih et al. (2013) \\
\hline pSNCM & ciliates & Mesodinium rubrum & CHIN & 35.5 & 129.5 & Yih et al. (2013) \\
\hline pSNCM & ciliates & Mesodinium rubrum & CHIN & 36.2 & 129.5 & Yih et al. (2013) \\
\hline pSNCM & dinoflagellates & Dinophysis acuminata & CHIN & 37.1 & 126.6 & Yih et al. (2013) \\
\hline pSNCM & ciliates & Mesodinium rubrum & NECS & 56.0 & 11.8 & Fenchel and Hansen (2006) \\
\hline pSNCM & dinoflagellates & Dinophysis acuminata & NECS & 55.9 & 12.0 & Garcia-Cuetos et al. (2010) \\
\hline pSNCM & dinoflagellates & Dinophysis acuminata & NECS & 56.0 & 7.6 & Hansen et al. (2016) \\
\hline
\end{tabular}




\begin{tabular}{|c|c|c|c|c|c|c|}
\hline pSNCM & ciliates & Mesodinium rubrum & NECS & 56.0 & 12.6 & Hansen et al. (2016) \\
\hline pSNCM & dinoflagellates & Dinophysis acuminata & CHIN & 34.8 & 128.1 & Kim et al. (2012) \\
\hline pSNCM & dinoflagellates & Dinophysis acuminata & CHIN & 36.7 & 126.7 & Kim et al. (2012) \\
\hline pSNCM & dinoflagellates & Dinophysis acuminata & CHIN & 36.0 & 128.0 & Kim et al. (2012) \\
\hline pSNCM & dinoflagellates & Dinophysis acuminata & CHIN & 35.0 & 130.0 & Kim et al. (2012) \\
\hline pSNCM & dinoflagellates & Dinophysis acuminata & CHIN & 36.0 & 130.0 & Kim et al. (2012) \\
\hline pSNCM & dinoflagellates & Dinophysis acuminata & CHIN & 35.4 & 130.0 & Park et al. (2006) \\
\hline pSNCM & dinoflagellates & $\begin{array}{l}\text { Gymnodinium graciletum, } \\
\text { Amphidinium poecilochroum }\end{array}$ & NECS & 55.6 & 12.7 & Jakobsen et al. (2000) \\
\hline pSNCM & ciliates & Mesodinium rubrum & HUMB & -15.0 & -75.5 & Wilkerson and Grunseich (1990) \\
\hline pSNCM & ciliates & Mesodinium rubrum & HUMB & -15.0 & -75.4 & Wilkerson and Grunseich (1990) \\
\hline $\mathrm{pSNCM}$ & ciliates & Mesodinium rubrum & HUMB & -15.1 & -75.6 & Wilkerson and Grunseich (1990) \\
\hline pSNCM & ciliates & Mesodinium rubrum & HUMB & -15.2 & -75.6 & Wilkerson and Grunseich (1990) \\
\hline pSNCM & ciliates & Mesodinium rubrum & HUMB & -15.0 & -75.6 & Wilkerson and Grunseich (1990) \\
\hline pSNCM & ciliates & Mesodinium rubrum & HUMB & -15.4 & -77.7 & Wilkerson and Grunseich (1990) \\
\hline pSNCM & ciliates & Mesodinium rubrum & HUMB & -15.4 & -75.9 & Wilkerson and Grunseich (1990) \\
\hline pSNCM & ciliates & Mesodinium rubrum & HUMB & -15.3 & -75.8 & Wilkerson and Grunseich (1990) \\
\hline pSNCM & dinoflagellates & Dinophysis acuminata & NECS & 41.5 & -70.7 & Wisecaver and Hackett (2010) \\
\hline $\mathrm{pSNCM}$ & ciliates & Mesodinium rubrum & NWCS & 40.9 & -73.6 & Dierssen et al. (2015) \\
\hline pSNCM & ciliates & Mesodinium rubrum & CHIN & 21.8 & 108.6 & Liu et al. (2015) \\
\hline pSNCM & ciliates & Mesodinium rubrum & CNRY & 38.8 & -9.0 & Brito et al. (2015) \\
\hline pSNCM & dinoflagellates & Dinophysis acuminata & CNRY & 38.8 & -9.0 & Brito et al. (2015) \\
\hline pSNCM & ciliates & $\begin{array}{l}\text { Mesodinium rubrum, Mesodinium } \\
\text { major }\end{array}$ & NECS & 54.2 & 7.9 & Yang et al. (2014) \\
\hline pSNCM & ciliates & Mesodinium rubrum & NECS & 50.0 & -6.2 & Rychert et al. (2014) \\
\hline $\mathrm{pSNCM}$ & ciliates & Mesodinium rubrum & NADR & 44.0 & -10.6 & Rychert et al. (2014) \\
\hline $\mathrm{pSNCM}$ & ciliates & Mesodinium rubrum & NAST (E) & 35.9 & -21.8 & Rychert et al. (2014) \\
\hline pSNCM & ciliates & Mesodinium rubrum & NATR & 16.1 & -25.9 & Rychert et al. (2014) \\
\hline
\end{tabular}




\begin{tabular}{|c|c|c|c|c|c|c|}
\hline $\mathrm{pSNCM}$ & ciliates & Mesodinium rubrum & WTRA & 0.5 & -28.9 & Rychert et al. (2014) \\
\hline pSNCM & ciliates & Mesodinium rubrum & SATL & -9.1 & -31.7 & Rychert et al. (2014) \\
\hline pSNCM & ciliates & Mesodinium rubrum & BRAZ & -22.8 & -38.5 & Rychert et al. (2014) \\
\hline pSNCM & ciliates & Mesodinium rubrum & FKLD & -36.8 & -54.7 & Rychert et al. (2014) \\
\hline $\mathrm{pSNCM}$ & ciliates & Mesodinium rubrum & FKLD & -47.3 & -58.4 & Rychert et al. (2014) \\
\hline pSNCM & ciliates & Mesodinium rubrum & FKLD & -51.0 & -60.3 & Rychert et al. (2014) \\
\hline pSNCM & dinoflagellates & Dinophysis acuminata & BRAZ & -25.5 & -48.4 & Mafra et al. (2014) \\
\hline pSNCM & dinoflagellates & Dinophysis acuminata & BRAZ & -25.9 & -48.6 & Mafra et al. (2014) \\
\hline $\mathrm{pSNCM}$ & dinoflagellates & Dinophysis acuminata & BRAZ & -26.2 & -48.6 & Mafra et al. (2014) \\
\hline pSNCM & ciliates & Mesodinium rubrum & BRAZ & -25.9 & -48.6 & Mafra et al. (2014) \\
\hline pSNCM & dinoflagellates & Dinophysis acuminata & CARB & 27.8 & -97.0 & Campbell et al. (2010) \\
\hline pSNCM & ciliates & Mesodinium rubrum & CARB & 28.2 & -96.0 & Harred and Campbell (2014) \\
\hline $\mathrm{pSNCM}$ & ciliates & Mesodinium rubrum & CHIN & 22.4 & 114.2 & Liu et al. (2014) \\
\hline $\mathrm{pSNCM}$ & ciliates & Mesodinium rubrum & CHIN & 22.5 & 114.3 & Liu et al. (2014) \\
\hline $\mathrm{pSNCM}$ & dinoflagellates & Dinophysis acuminata & CNRY & 42.4 & -8.9 & Velo-Suárez et al. (2014) \\
\hline $\mathrm{pSNCM}$ & ciliates & Mesodinium rubrum & CNRY & 43.0 & -9.0 & Velo-Suárez et al. (2014) \\
\hline pSNCM & ciliates & Mesodinium rubrum & CALC & 46.2 & -123.8 & Herfort et al. (2012) \\
\hline pSNCM & ciliates & Mesodinium rubrum & CHIN & 35.9 & 121.4 & Sun et al. (2013) \\
\hline pSNCM & ciliates & Mesodinium rubrum & CHIN & 36.5 & 125.3 & Sun et al. (2013) \\
\hline pSNCM & ciliates & Mesodinium rubrum & CHIN & 32.7 & 122.1 & Sun et al. (2013) \\
\hline pSNCM & ciliates & Mesodinium rubrum & CHIN & 34.2 & 125.3 & Sun et al. (2013) \\
\hline $\mathrm{pSNCM}$ & ciliates & Mesodinium rubrum & MEDI & 43.1 & 36.8 & Selifonova (2011) \\
\hline $\mathrm{pSNCM}$ & dinoflagellates & Dinophysis acuminata & CHIN & 35.4 & 130.0 & Park et al. (2006) \\
\hline pSNCM & ciliates & Mesodinium rubrum & AUSE & -28.5 & 153.6 & Ajani et al. (2011) \\
\hline pSNCM & ciliates & Mesodinium rubrum & AUSE & -30.5 & 153.5 & Ajani et al. (2011) \\
\hline $\mathrm{pSNCM}$ & ciliates & Mesodinium rubrum & AUSE & -32.5 & 153.4 & Ajani et al. (2011) \\
\hline pSNCM & ciliates & Mesodinium rubrum & AUSE & -34.5 & 153.0 & Ajani et al. (2011) \\
\hline
\end{tabular}




\begin{tabular}{|c|c|c|c|c|c|c|}
\hline pSNCM & ciliates & Mesodinium rubrum & AUSE & -36.0 & 152.0 & Ajani et al. (2011) \\
\hline pSNCM & ciliates & Mesodinium rubrum & AUSE & -37.1 & 150.0 & Ajani et al. (2011) \\
\hline pSNCM & ciliates & Mesodinium rubrum & TASM & -30.5 & 157.0 & Ajani et al. (2011) \\
\hline pSNCM & ciliates & Mesodinium rubrum & TASM & -34.5 & 157.5 & Ajani et al. (2011) \\
\hline $\mathrm{pSNCM}$ & ciliates & Mesodinium rubrum & TASM & -37.1 & 157.0 & Ajani et al. (2011) \\
\hline pSNCM & ciliates & Mesodinium rubrum & KURO & 37.7 & 137.5 & Suzuki et al. (1998) \\
\hline pSNCM & ciliates & Mesodinium rubrum & PSAG (E) & 44.2 & 157.2 & Suzuki et al. (1998) \\
\hline pSNCM & ciliates & Mesodinium rubrum & PSAG (E) & 45.0 & 165.0 & Suzuki et al. (1998) \\
\hline pSNCM & ciliates & Mesodinium rubrum & KURO & 39.0 & 143.0 & Suzuki et al. (1998) \\
\hline pSNCM & ciliates & Mesodinium rubrum & KURO & 38.0 & 144.0 & Suzuki et al. (1998) \\
\hline pSNCM & ciliates & Mesodinium rubrum & TASM & -40.0 & 155.2 & Suzuki et al. (1998) \\
\hline pSNCM & ciliates & Mesodinium rubrum & TASM & -37.0 & 155.0 & Suzuki et al. (1998) \\
\hline pSNCM & ciliates & Mesodinium rubrum & AUSE & -34.0 & 155.0 & Suzuki et al. (1998) \\
\hline pSNCM & ciliates & Mesodinium rubrum & AUSE & -24.0 & 155.0 & Suzuki et al. (1998) \\
\hline pSNCM & ciliates & Mesodinium rubrum & AUSE & -20.0 & 155.0 & Suzuki et al. (1998) \\
\hline $\mathrm{pSNCM}$ & dinoflagellates & Dinophysis acuminata & INDE & 20.4 & 87.7 & Jyothibabu et al. (2008) \\
\hline pSNCM & dinoflagellates & Dinophysis acuminata & INDE & 19.0 & 85.8 & Jyothibabu et al. (2008) \\
\hline pSNCM & dinoflagellates & Dinophysis acuminata & INDE & 17.0 & 83.5 & Jyothibabu et al. (2008) \\
\hline pSNCM & dinoflagellates & Dinophysis acuminata & INDE & 15.4 & 80.6 & Jyothibabu et al. (2008) \\
\hline $\mathrm{pSNCM}$ & dinoflagellates & Dinophysis acuminata & INDE & 13.9 & 80.7 & Jyothibabu et al. (2008) \\
\hline pSNCM & dinoflagellates & Dinophysis acuminata & INDE & 12.1 & 80.5 & Jyothibabu et al. (2008) \\
\hline pSNCM & dinoflagellates & Dinophysis acuminata & INDE & 20.4 & 90.7 & Jyothibabu et al. (2008) \\
\hline pSNCM & dinoflagellates & Dinophysis acuminata & INDE & 19.0 & 87.8 & Jyothibabu et al. (2008) \\
\hline pSNCM & dinoflagellates & Dinophysis acuminata & INDE & 17.0 & 86.5 & Jyothibabu et al. (2008) \\
\hline pSNCM & dinoflagellates & Dinophysis acuminata & INDE & 15.4 & 83.6 & Jyothibabu et al. (2008) \\
\hline pSNCM & dinoflagellates & Dinophysis acuminata & INDE & 13.9 & 83.7 & Jyothibabu et al. (2008) \\
\hline pSNCM & dinoflagellates & Dinophysis acuminata & INDE & 12.1 & 83.5 & Jyothibabu et al. (2008) \\
\hline
\end{tabular}




\begin{tabular}{|c|c|c|c|c|c|c|}
\hline $\mathrm{pSNCM}$ & ciliates & Mesodinium rubrum & BENG & -30.6 & 13.0 & Matthews (1991) \\
\hline $\mathrm{pSNCM}$ & ciliates & Mesodinium rubrum & BENG & -31.8 & 14.0 & Matthews (1991) \\
\hline $\mathrm{pSNCM}$ & ciliates & Mesodinium rubrum & BENG & -33.7 & 15.2 & Matthews (1991) \\
\hline $\mathrm{pSNCM}$ & ciliates & Mesodinium rubrum & BPLR & 65.0 & -35.0 & Montagnes et al. (1988) \\
\hline pSNCM & ciliates & Mesodinium rubrum & ARCT & 65.0 & -31.3 & Montagnes et al. (1988) \\
\hline pSNCM & ciliates & Mesodinium rubrum & ARCT & 65.0 & -27.4 & Montagnes et al. (1988) \\
\hline $\mathrm{pSNCM}$ & ciliates & Mesodinium rubrum & ARCT & 62.0 & -35.0 & Montagnes et al. (1988) \\
\hline $\mathrm{pSNCM}$ & ciliates & Mesodinium rubrum & ARCT & 62.0 & -31.3 & Montagnes et al. (1988) \\
\hline pSNCM & ciliates & Mesodinium rubrum & ARCT & 62.0 & -27.4 & Montagnes et al. (1988) \\
\hline $\mathrm{pSNCM}$ & ciliates & Mesodinium rubrum & NADR & 55.0 & -35.0 & Montagnes et al. (1988) \\
\hline $\mathrm{pSNCM}$ & ciliates & Mesodinium rubrum & NADR & 55.0 & -31.3 & Montagnes et al. (1988) \\
\hline pSNCM & ciliates & Mesodinium rubrum & NADR & 55.0 & -27.4 & Montagnes et al. (1988) \\
\hline pSNCM & ciliates & Mesodinium rubrum & APLR & -69.4 & -77.8 & Garzio et al. (2013) \\
\hline pSNCM & ciliates & Mesodinium rubrum & APLR & -68.7 & -80.0 & Garzio et al. (2013) \\
\hline pSNCM & ciliates & Mesodinium rubrum & APLR & -68.0 & -83.0 & Garzio et al. (2013) \\
\hline pSNCM & ciliates & Mesodinium rubrum & APLR & -68.0 & -80.0 & Garzio et al. (2013) \\
\hline pSNCM & ciliates & Mesodinium rubrum & APLR & -64.0 & 72.0 & Garzio et al. (2013) \\
\hline $\mathrm{pSNCM}$ & ciliates & Mesodinium rubrum & APLR & -65.5 & -75.0 & Garzio et al. (2013) \\
\hline pSNCM & ciliates & Mesodinium rubrum & APLR & -67.5 & 77.5 & Garzio et al. (2013) \\
\hline pSNCM & ciliates & Mesodinium rubrum & APLR & -64.0 & -68.0 & Garzio et al. (2013) \\
\hline pSNCM & ciliates & Mesodinium rubrum & APLR & -64.8 & -68.0 & Garzio et al. (2013) \\
\hline pSNCM & ciliates & Mesodinium rubrum & APLR & -63.0 & -61.0 & Garzio et al. (2013) \\
\hline pSNCM & ciliates & Mesodinium rubrum & APLR & -63.0 & -68.0 & Garzio et al. (2013) \\
\hline pSNCM & ciliates & Mesodinium rubrum & BRAZ & -23.1 & -42.8 & McManus et al. (2007) \\
\hline $\mathrm{pSNCM}$ & ciliates & Mesodinium rubrum & BRAZ & -24.0 & -42.5 & McManus et al. (2007) \\
\hline pSNCM & ciliates & Mesodinium rubrum & BRAZ & -24.0 & -42.0 & McManus et al. (2007) \\
\hline pSNCM & ciliates & Mesodinium rubrum & BRAZ & -23.0 & -42.0 & McManus et al. (2007) \\
\hline
\end{tabular}




\begin{tabular}{|c|c|c|c|c|c|c|}
\hline $\mathrm{pSNCM}$ & ciliates & Mesodinium rubrum & BRAZ & -23.1 & -42.1 & McManus et al. (2007) \\
\hline pSNCM & ciliates & Mesodinium rubrum & BRAZ & -23.4 & -42.6 & McManus et al. (2007) \\
\hline pSNCM & ciliates & Mesodinium rubrum & BRAZ & -23.3 & -42.8 & McManus et al. (2007) \\
\hline pSNCM & ciliates & Mesodinium rubrum & BRAZ & -23.0 & -42.2 & McManus et al. (2007) \\
\hline pSNCM & ciliates & Mesodinium rubrum & BRAZ & -23.0 & -42.3 & McManus et al. (2007) \\
\hline pSNCM & dinoflagellates & Dinophysis acuminata & CALC & 26.5 & -115.0 & Reguera et al. (2012) \\
\hline $\mathrm{pSNCM}$ & dinoflagellates & Dinophysis acuminata & CAMR & 18.0 & -103.6 & Reguera et al. (2012) \\
\hline pSNCM & dinoflagellates & Dinophysis acuminata & CAMR & 15.5 & -94.4 & Reguera et al. (2012) \\
\hline $\mathrm{pSNCM}$ & dinoflagellates & Dinophysis acuminata & HUMB & -29.0 & -71.7 & Reguera et al. (2012) \\
\hline pSNCM & dinoflagellates & Dinophysis acuminata & GUIA & 6.4 & -56.7 & Reguera et al. (2012) \\
\hline pSNCM & dinoflagellates & Dinophysis acuminata & NECS & 62.9 & 5.8 & Reguera et al. (2012) \\
\hline $\mathrm{pSNCM}$ & dinoflagellates & Dinophysis acuminata & NECS & 55.7 & -8.5 & Reguera et al. (2012) \\
\hline $\mathrm{pSNCM}$ & dinoflagellates & Dinophysis acuminata & NECS & 55.7 & -1.3 & Reguera et al. (2012) \\
\hline $\mathrm{pSNCM}$ & dinoflagellates & Dinophysis acuminata & NECS & 51.7 & -8.2 & Reguera et al. (2012) \\
\hline $\mathrm{pSNCM}$ & dinoflagellates & Dinophysis acuminata & NECS & 54.4 & -10.3 & Reguera et al. (2012) \\
\hline $\mathrm{pSNCM}$ & dinoflagellates & Dinophysis acuminata & INDW & 20.4 & 70.3 & Reguera et al. (2012) \\
\hline pSNCM & dinoflagellates & Dinophysis acuminata & INDW & 11.1 & 75.1 & Reguera et al. (2012) \\
\hline pSNCM & dinoflagellates & Dinophysis acuminata & ARCH & 9.5 & 98.0 & Reguera et al. (2012) \\
\hline pSNCM & dinoflagellates & Dinophysis acuminata & ARCH & 12.7 & 97.4 & Reguera et al. (2012) \\
\hline $\mathrm{pSNCM}$ & dinoflagellates & Dinophysis acuminata & AUSW & -33.9 & 114.0 & Reguera et al. (2012) \\
\hline pSNCM & dinoflagellates & Dinophysis acuminata & AUSW & -33.0 & 127.4 & Reguera et al. (2012) \\
\hline pSNCM & dinoflagellates & Dinophysis acuminata & SANT & -44.3 & 167.2 & Reguera et al. (2012) \\
\hline $\mathrm{pSNCM}$ & dinoflagellates & Dinophysis acuminata & TASM & -38.2 & 173.9 & Reguera et al. (2012) \\
\hline pSNCM & dinoflagellates & Dinophysis acuminata & NEWZ & -44.4 & 172.1 & Reguera et al. (2012) \\
\hline $\mathrm{pSNCM}$ & dinoflagellates & Dinophysis acuminata & GUIN & 6.3 & 3.6 & Zendong et al. (2016) \\
\hline $\mathrm{pSNCM}$ & dinoflagellates & Dinophysis acuminata & GUIN & 4.2 & 6.9 & Zendong et al. (2016) \\
\hline pSNCM & dinoflagellates & Dinophysis acuminata & GUIN & 4.4 & 7.9 & Zendong et al. (2016) \\
\hline
\end{tabular}




\begin{tabular}{|c|c|c|c|c|c|c|}
\hline $\mathrm{pSNCM}$ & dinoflagellates & Dinophysis acuminata & REDS & 16.4 & 42.0 & Kürten et al. (2015) \\
\hline pSNCM & dinoflagellates & Dinophysis acuminata & $\mathrm{ARCH}$ & 12.7 & 98.4 & Su-Myat et al. (2012) \\
\hline pSNCM & dinoflagellates & Dinophysis acuminata & SUND & 11.5 & 125.7 & Marasigan et al. (2001) \\
\hline pSNCM & dinoflagellates & Dinophysis acuminata & SUND & -5.7 & 107.0 & Praseno (1981) \\
\hline $\mathrm{pSNCM}$ & dinoflagellates & Dinophysis acuminata & ARAB & 22.9 & 62.0 & Gul and Saifullah (2010) \\
\hline pSNCM & ciliates & Mesodinium spp & CNRY & 36.6 & -6.5 & Gimmler et al. (2016) \\
\hline $\mathrm{pSNCM}$ & ciliates & Mesodinium spp & MEDI & 37.0 & 1.9 & Gimmler et al. (2016) \\
\hline pSNCM & ciliates & Mesodinium spp & MEDI & 39.1 & 5.8 & Gimmler et al. (2016) \\
\hline $\mathrm{pSNCM}$ & ciliates & Mesodinium spp & MEDI & 41.6 & 2.8 & Gimmler et al. (2016) \\
\hline pSNCM & ciliates & Mesodinium spp & MEDI & 37.4 & 15.4 & Gimmler et al. (2016) \\
\hline pSNCM & ciliates & Mesodinium spp & MEDI & 35.7 & 14.2 & Gimmler et al. (2016) \\
\hline $\mathrm{pSNCM}$ & ciliates & Mesodinium spp & MEDI & 34.4 & 14.9 & Gimmler et al. (2016) \\
\hline $\mathrm{pSNCM}$ & ciliates & Mesodinium spp & MEDI & 39.7 & 17.4 & Gimmler et al. (2016) \\
\hline $\mathrm{pSNCM}$ & ciliates & Mesodinium spp & MEDI & 42.2 & 17.7 & Gimmler et al. (2016) \\
\hline pSNCM & ciliates & Mesodinium spp & MEDI & 42.2 & 17.7 & Gimmler et al. (2016) \\
\hline $\mathrm{pSNCM}$ & ciliates & Mesodinium spp & MEDI & 42.4 & 18.0 & Gimmler et al. (2016) \\
\hline pSNCM & ciliates & Mesodinium spp & MEDI & 39.3 & 19.4 & Gimmler et al. (2016) \\
\hline pSNCM & ciliates & Mesodinium spp & MEDI & 38.4 & 20.1 & Gimmler et al. (2016) \\
\hline pSNCM & ciliates & Mesodinium spp & MEDI & 33.9 & 32.3 & Gimmler et al. (2016) \\
\hline $\mathrm{pSNCM}$ & ciliates & Mesodinium spp & REDS & 27.1 & 34.8 & Gimmler et al. (2016) \\
\hline pSNCM & ciliates & Mesodinium spp & REDS & 23.4 & 37.2 & Gimmler et al. (2016) \\
\hline $\mathrm{pSNCM}$ & ciliates & Mesodinium spp & REDS & 23.4 & 37.2 & Gimmler et al. (2016) \\
\hline pSNCM & ciliates & Mesodinium spp & REDS & 22.1 & 38.2 & Gimmler et al. (2016) \\
\hline pSNCM & ciliates & Mesodinium spp & REDS & 18.4 & 39.9 & Gimmler et al. (2016) \\
\hline pSNCM & ciliates & Mesodinium spp & ARAB & 20.8 & 63.5 & Gimmler et al. (2016) \\
\hline $\mathrm{pSNCM}$ & ciliates & Mesodinium spp & ARAB & 19.0 & 64.6 & Gimmler et al. (2016) \\
\hline pSNCM & ciliates & Mesodinium spp & ARAB & 18.7 & 66.7 & Gimmler et al. (2016) \\
\hline
\end{tabular}




\begin{tabular}{|c|c|c|c|c|c|c|}
\hline $\mathrm{pSNCM}$ & ciliates & Mesodinium spp & ARAB & 14.6 & 70.0 & Gimmler et al. (2016) \\
\hline $\mathrm{pSNCM}$ & ciliates & Mesodinium spp & INDE & 6.0 & 73.9 & Gimmler et al. (2016) \\
\hline pSNCM & ciliates & Mesodinium spp & MONS & 2.8 & 71.5 & Gimmler et al. (2016) \\
\hline pSNCM & ciliates & Mesodinium spp & MONS & -9.4 & 66.3 & Gimmler et al. (2016) \\
\hline pSNCM & ciliates & Mesodinium spp & EAFR & -17.0 & 53.5 & Gimmler et al. (2016) \\
\hline pSNCM & ciliates & Mesodinium spp & EAFR & -29.0 & 37.9 & Gimmler et al. (2016) \\
\hline pSNCM & ciliates & Mesodinium spp & EAFR & -35.2 & 26.3 & Gimmler et al. (2016) \\
\hline pSNCM & ciliates & Mesodinium spp & EAFR & -34.0 & 18.0 & Gimmler et al. (2016) \\
\hline pSNCM & ciliates & Mesodinium spp & BENG & -32.3 & 17.0 & Gimmler et al. (2016) \\
\hline $\mathrm{pSNCM}$ & ciliates & Mesodinium spp & SATL & -31.0 & 4.6 & Gimmler et al. (2016) \\
\hline pSNCM & ciliates & Mesodinium spp & SATL & -20.0 & -3.4 & Gimmler et al. (2016) \\
\hline pSNCM & ciliates & Mesodinium spp & SATL & -21.0 & -35.0 & Gimmler et al. (2016) \\
\hline pSNCM & ciliates & Mesodinium spp & SATL & -30.0 & -43.0 & Gimmler et al. (2016) \\
\hline $\mathrm{pSNCM}$ & ciliates & Mesodinium spp & FKLD & -47.0 & -58.0 & Gimmler et al. (2016) \\
\hline $\mathrm{pSNCM}$ & ciliates & Mesodinium spp & APLR & -60.3 & -60.5 & Gimmler et al. (2016) \\
\hline $\mathrm{pSNCM}$ & ciliates & Mesodinium spp & APLR & -62.0 & -49.5 & Gimmler et al. (2016) \\
\hline pSNCM & ciliates & Mesodinium spp & SPSG & -26.0 & -110.0 & Gimmler et al. (2016) \\
\hline $\mathrm{pSNCM}$ & ciliates & Mesodinium spp & SPSG & -13.0 & -96.0 & Gimmler et al. (2016) \\
\hline $\mathrm{pSNCM}$ & ciliates & Mesodinium spp & HUMB & -5.2 & -85.0 & Gimmler et al. (2016) \\
\hline $\mathrm{pSNCM}$ & ciliates & Mesodinium spp & PNEC & 1.8 & -84.5 & Gimmler et al. (2016) \\
\hline pSNCM & ciliates & Mesodinium spp & SPSG & -17.0 & -100.7 & Gimmler et al. (2016) \\
\hline pSNCM & ciliates & Mesodinium spp & SPSG & -9.0 & -139.4 & Gimmler et al. (2016) \\
\hline $\mathrm{pSNCM}$ & ciliates & Mesodinium spp & SPSG & -8.9 & -140.3 & Gimmler et al. (2016) \\
\hline $\mathrm{pSNCM}$ & ciliates & Mesodinium spp & SPSG & -9.0 & -140.6 & Gimmler et al. (2016) \\
\hline $\mathrm{pSNCM}$ & ciliates & Mesodinium spp & SUND & -9.0 & 142.0 & Gimmler et al. (2016) \\
\hline pSNCM & dinoflagellates & Dinophysis acuminata & GUIN & 6.3 & 3.6 & Reguera et al. (2012) \\
\hline pSNCM & dinoflagellates & Dinophysis acuminata & GUIN & 4.2 & 6.9 & Reguera et al. (2012) \\
\hline
\end{tabular}




\begin{tabular}{|c|c|c|c|c|c|c|}
\hline pSNCM & dinoflagellates & Dinophysis acuminata & GUIN & 4.4 & 7.9 & Reguera et al. (2012) \\
\hline eSNCM & dinoflagellates & Noctiluca scintillans & NECS & 56.9 & 9.0 & Andersen and Sørensen (1986) \\
\hline pSNCM & dinoflagellates & $\begin{array}{l}\text { Gymnodinium graciletum, } \\
\text { Amphidinium poecilochroum }\end{array}$ & NECS & 55.6 & 12.7 & Jakobsen et al. (2000) \\
\hline $\mathrm{pSNCM}$ & dinoflagellates & Pfiesteria piscicida & NWCS & 35.4 & -76.7 & Lewitus et al. (1999) \\
\hline pSNCM & dinoflagellates & Fragilidium duplocampanaeforme & CHIN & 36.0 & 127.0 & Park et al. (2015) \\
\hline eSNCM & dinoflagellates & Noctiluca scintillans & CHIN & 22.4 & 114.2 & Liu et al. (2014) \\
\hline eSNCM & dinoflagellates & Noctiluca scintillans & INDW & 23.0 & 64.0 & Gomes et al. (2014) \\
\hline eSNCM & dinoflagellates & Noctiluca scintillans & INDW & 23.0 & 69.0 & Gomes et al. (2014) \\
\hline eSNCM & dinoflagellates & Noctiluca scintillans & INDW & 19.0 & 64.0 & Gomes et al. (2014) \\
\hline eSNCM & dinoflagellates & Noctiluca scintillans & INDW & 19.0 & 69.0 & Gomes et al. (2014) \\
\hline eSNCM & dinoflagellates & Noctiluca scintillans & AUSE & -28.5 & 153.6 & Ajani et al. (2011) \\
\hline eSNCM & dinoflagellates & Noctiluca scintillans & AUSE & -30.5 & 153.5 & Ajani et al. (2011) \\
\hline eSNCM & dinoflagellates & Noctiluca scintillans & AUSE & -32.5 & 153.4 & Ajani et al. (2011) \\
\hline eSNCM & dinoflagellates & Noctiluca scintillans & AUSE & -34.5 & 153.0 & Ajani et al. (2011) \\
\hline eSNCM & dinoflagellates & Noctiluca scintillans & AUSE & -36.0 & 152.0 & Ajani et al. (2011) \\
\hline eSNCM & dinoflagellates & Noctiluca scintillans & TASM & -37.1 & 150.0 & Ajani et al. (2011) \\
\hline eSNCM & dinoflagellates & Noctiluca scintillans & TASM & -30.5 & 157.0 & Ajani et al. (2011) \\
\hline eSNCM & dinoflagellates & Noctiluca scintillans & TASM & -34.5 & 157.5 & Ajani et al. (2011) \\
\hline eSNCM & dinoflagellates & Noctiluca scintillans & TASM & -37.1 & 157.0 & Ajani et al. (2011) \\
\hline eSNCM & dinoflagellates & Noctiluca scintillans & INDE & 20.4 & 87.7 & Jyothibabu et al. (2008) \\
\hline eSNCM & dinoflagellates & Noctiluca scintillans & INDE & 19.0 & 85.8 & Jyothibabu et al. (2008) \\
\hline eSNCM & dinoflagellates & Noctiluca scintillans & INDE & 17.0 & 83.5 & Jyothibabu et al. (2008) \\
\hline eSNCM & dinoflagellates & Noctiluca scintillans & INDE & 15.4 & 80.6 & Jyothibabu et al. (2008) \\
\hline eSNCM & dinoflagellates & Noctiluca scintillans & INDE & 13.9 & 80.7 & Jyothibabu et al. (2008) \\
\hline eSNCM & dinoflagellates & Noctiluca scintillans & INDE & 12.1 & 80.5 & Jyothibabu et al. (2008) \\
\hline eSNCM & dinoflagellates & Noctiluca scintillans & INDE & 20.4 & 90.7 & Jyothibabu et al. (2008) \\
\hline eSNCM & dinoflagellates & Noctiluca scintillans & INDE & 19.0 & 87.8 & Jyothibabu et al. (2008) \\
\hline
\end{tabular}




\begin{tabular}{|c|c|c|c|c|c|c|}
\hline eSNCM & dinoflagellates & Noctiluca scintillans & INDE & 17.0 & 86.5 & Jyothibabu et al. (2008) \\
\hline eSNCM & dinoflagellates & Noctiluca scintillans & INDE & 15.4 & 83.6 & Jyothibabu et al. (2008) \\
\hline eSNCM & dinoflagellates & Noctiluca scintillans & INDE & 13.9 & 83.7 & Jyothibabu et al. (2008) \\
\hline eSNCM & dinoflagellates & Noctiluca scintillans & INDE & 12.1 & 83.5 & Jyothibabu et al. (2008) \\
\hline eSNCM & dinoflagellates & Noctiluca scintillans & CNRY & 43.5 & -6.0 & Harrison et al. (2011) \\
\hline eSNCM & dinoflagellates & Noctiluca scintillans & CNRY & 43.5 & -2.0 & Harrison et al. (2011) \\
\hline eSNCM & dinoflagellates & Noctiluca scintillans & MEDI & 42.0 & 28.0 & Harrison et al. (2011) \\
\hline eSNCM & dinoflagellates & Noctiluca scintillans & MEDI & 45.0 & 30.0 & Harrison et al. (2011) \\
\hline eSNCM & dinoflagellates & Noctiluca scintillans & BPLR & 67.7 & 45.9 & Harrison et al. (2011) \\
\hline eSNCM & dinoflagellates & Noctiluca scintillans & CHIN & 22.5 & 114.3 & Harrison et al. (2011) \\
\hline eSNCM & dinoflagellates & Noctiluca scintillans & $\mathrm{ARCH}$ & 5.0 & 99.0 & Harrison et al. (2011) \\
\hline eSNCM & dinoflagellates & Noctiluca scintillans & SUND & 10.0 & 102.0 & Harrison et al. (2011) \\
\hline eSNCM & dinoflagellates & Noctiluca scintillans & SUND & 13.0 & 106.0 & Harrison et al. (2011) \\
\hline eSNCM & dinoflagellates & Noctiluca scintillans & SUND & 12.0 & 104.0 & Harrison et al. (2011) \\
\hline eSNCM & dinoflagellates & Noctiluca scintillans & SUND & 21.0 & 105.0 & Harrison et al. (2011) \\
\hline eSNCM & dinoflagellates & Noctiluca scintillans & INDE & 9.0 & 78.6 & Harrison et al. (2011) \\
\hline eSNCM & dinoflagellates & Noctiluca scintillans & INDE & 8.3 & 76.5 & Harrison et al. (2011) \\
\hline eSNCM & dinoflagellates & Noctiluca scintillans & INDW & 12.3 & 85.0 & Harrison et al. (2011) \\
\hline eSNCM & dinoflagellates & Noctiluca scintillans & SUND & 6.4 & 106.5 & Harrison et al. (2011) \\
\hline eSNCM & dinoflagellates & Noctiluca scintillans & CARB & 15.0 & -88.0 & Harrison et al. (2011) \\
\hline eSNCM & dinoflagellates & Noctiluca scintillans & CARB & 25.0 & -88.0 & Harrison et al. (2011) \\
\hline eSNCM & dinoflagellates & Noctiluca scintillans & CARB & 33.0 & -88.0 & Harrison et al. (2011) \\
\hline eSNCM & dinoflagellates & Noctiluca scintillans & CALC & 33.0 & -117.3 & Harrison et al. (2011) \\
\hline eSNCM & dinoflagellates & Noctiluca scintillans & CALC & 23.2 & -106.0 & Harrison et al. (2011) \\
\hline eSNCM & dinoflagellates & Noctiluca scintillans & SPSG & -36.0 & 175.0 & Harrison et al. (2011) \\
\hline eSNCM & dinoflagellates & Noctiluca scintillans & REDS & 19.9 & 42.3 & Harrison et al. (2011) \\
\hline eSNCM & dinoflagellates & Noctiluca scintillans & GUIN & 3.0 & 4.0 & Harrison et al. (2011) \\
\hline
\end{tabular}




\begin{tabular}{|c|c|c|c|c|c|c|}
\hline eSNCM & dinoflagellates & Noctiluca scintillans & $\mathrm{ARAB}$ & 18.0 & 52.0 & Harrison et al. (2011) \\
\hline eSNCM & dinoflagellates & Noctiluca scintillans & ARAB & 26.0 & 60.0 & Harrison et al. (2011) \\
\hline eSNCM & dinoflagellates & Noctiluca scintillans & SUND & 2.8 & 125.9 & Harrison et al. (2011) \\
\hline eSNCM & dinoflagellates & Noctiluca scintillans & SUND & 14.7 & 125.5 & Harrison et al. (2011) \\
\hline eSNCM & dinoflagellates & Noctiluca scintillans & AUSW & -6.4 & 104.6 & Harrison et al. (2011) \\
\hline eSNCM & foraminiferans & Orbulina universa & NAST (W) & 32.5 & -64.9 & Gast and Caron (1996) \\
\hline eSNCM & $\begin{array}{l}\text { foraminiferans, } \\
\text { radiolarians }\end{array}$ & no data & ANTA & -70.6 & -42.2 & Stoecker et al. (2009) \\
\hline eSNCM & $\begin{array}{l}\text { foraminiferans, } \\
\text { radiolarians }\end{array}$ & no data & APLR & -57.5 & -40.0 & Stoecker et al. (2009) \\
\hline eSNCM & foraminiferans & Orbulina universa & CALC & 33.4 & -118.4 & Stoecker et al. (2009) \\
\hline eSNCM & $\begin{array}{l}\text { foraminiferans, } \\
\text { radiaolarians }\end{array}$ & no data & NATR & 13.3 & -56.3 & Spero and Angel (1991) \\
\hline eSNCM & $\begin{array}{l}\text { foraminiferans, } \\
\text { radiaolarians }\end{array}$ & no data & NAST (W) & 30.2 & -39.6 & Spero and Angel (1991) \\
\hline eSNCM & $\begin{array}{l}\text { foraminiferans, } \\
\text { radiaolarians }\end{array}$ & no data & NWCS & 44.8 & -58.0 & Spero and Angel (1991) \\
\hline eSNCM & $\begin{array}{l}\text { foraminiferans, } \\
\text { radiaolarians }\end{array}$ & no data & NWCS & 41.0 & -63.0 & Spero and Angel (1991) \\
\hline eSNCM & radiolarians & $\begin{array}{l}\text { Diploconus fasces, Amphilonche } \\
\text { elongata, Acanthometra pellucida }\end{array}$ & REDS & 28.7 & 34.7 & Kimor et al. (1992) \\
\hline eSNCM & radiolarians & $\begin{array}{l}\text { Diploconus fasces, Amphilonche } \\
\text { elongata, Acanthometra pellucida, } \\
\text { Sphaerozoum spp. }\end{array}$ & MEDI & 32.4 & 33.0 & Kimor et al. (1992) \\
\hline eSNCM & radiolarians & $\begin{array}{l}\text { Diploconus fasces, Amphilonche } \\
\text { elongata, Acanthometra pellucida }\end{array}$ & CALC & 32.7 & -117.9 & Kimor et al. (1992) \\
\hline eSNCM & foraminiferans & Nonionella stella & CALC & 34.3 & -120.0 & Grzymski et al. (2002) \\
\hline eSNCM & radiolarians & $\begin{array}{l}\text { Spongastaurus, Dictyocoryne } \\
\text { truncatum }\end{array}$ & GUIA & 5.5 & -51.4 & Foster et al. (2006) \\
\hline eSNCM & radiolarians & $\begin{array}{l}\text { Spongastaurus, Dictyocoryne } \\
\text { truncatum }\end{array}$ & GUIA & 7.0 & -52.0 & Foster et al. (2006) \\
\hline
\end{tabular}


Appendices. Appendix A, Table A.I

\begin{tabular}{|c|c|c|c|c|c|c|}
\hline eSNCM & radiolarians & $\begin{array}{l}\text { Spongastaurus, Dictyocoryne } \\
\text { truncatum }\end{array}$ & GUIA & 15.0 & -33.0 & Foster et al. (2006) \\
\hline eSNCM & radiolarians & no data & NATR & 22.5 & -65.8 & Taylor (1982) \\
\hline eSNCM & foraminiferans & $\begin{array}{l}\text { Amphisorus hemprichii, Sorites } \\
\text { spp, Marginopora vertebralis, } \\
\text { Elphidium }\end{array}$ & $\mathrm{ARCH}$ & -5.1 & 145.8 & Langer and Lipps (2003) \\
\hline eSNCM & foraminiferans & $\begin{array}{l}\text { Amphisorus hemprichii, Sorites } \\
\text { spp, Marginopora vertebralis, } \\
\text { Elphidium }\end{array}$ & CARB & 24.8 & -82.4 & Pawlowski et al. (2001) \\
\hline eSNCM & foraminiferans & $\begin{array}{l}\text { Amphisorus hemprichii, Sorites } \\
\text { spp, Marginopora vertebralis, } \\
\text { Elphidium }\end{array}$ & NPTG (W) & 13.6 & 144.0 & Pawlowski et al. (2001) \\
\hline eSNCM & foraminiferans & $\begin{array}{l}\text { Amphisorus hemprichii, Sorites } \\
\text { spp, Marginopora vertebralis, } \\
\text { Elphidium }\end{array}$ & AUSE & -14.4 & 145.7 & Pawlowski et al. (2001) \\
\hline eSNCM & foraminiferans & $\begin{array}{l}\text { Amphisorus hemprichii, } \\
\text { Marginopora vertebralis, Sorites } \\
\text { spp }\end{array}$ & REDS & 29.4 & 34.8 & Pawlowski et al. (2001) \\
\hline eSNCM & foraminiferans & $\begin{array}{l}\text { Amphisorus hemprichii, } \\
\text { Marginopora vertebralis, Sorites } \\
\text { spp }\end{array}$ & MONS & 2.0 & 73.4 & Pawlowski et al. (2001) \\
\hline eSNCM & foraminiferans & $\begin{array}{l}\text { Amphisorus hemprichii, } \\
\text { Marginopora vertebralis, Sorites } \\
\text { spp }\end{array}$ & REDS & 26.7 & 34.8 & Pawlowski et al. (2001) \\
\hline eSNCM & foraminiferans & $\begin{array}{l}\text { Neogloboquadrina duterrei, } \\
\text { Pulleniatina obliquiloculata, } \\
\text { Globorotalia tumida, Globorotalia } \\
\text { Menardii, Globoquadrina } \\
\text { conglomerata, Globigerinita } \\
\text { glutinata, Globigerinoides } \\
\text { sacculifer, Globigerinoides ruber, } \\
\text { Globigerinoides conglobatus, }\end{array}$ & PEQD & 9.0 & -140.0 & Watkins et al. (1996) \\
\hline
\end{tabular}




\begin{tabular}{|c|c|c|c|c|c|c|}
\hline & & $\begin{array}{l}\text { Globigerinella aequilateralis, } \\
\text { Globigerinella calida }\end{array}$ & & & & \\
\hline eSNCM & foraminiferans & $\begin{array}{l}\text { Neogloboquadrina duterrei, } \\
\text { Pulleniatina obliquiloculata, } \\
\text { Globorotalia tumida, Globorotalia } \\
\text { Menardii, Globoquadrina } \\
\text { conglomerata, Globigerinita } \\
\text { glutinata, Globigerinoides } \\
\text { sacculifer, Globigerinoides ruber, } \\
\text { Globigerinoides conglobatus, } \\
\text { Globigerinella aequilateralis, } \\
\text { Globigerinella calida }\end{array}$ & PEQD & 7.0 & -140.0 & Watkins et al. (1996) \\
\hline eSNCM & foraminiferans & $\begin{array}{l}\text { Neogloboquadrina duterrei, } \\
\text { Pulleniatina obliquiloculata, } \\
\text { Globorotalia tumida, Globorotalia } \\
\text { Menardii, Globoquadrina } \\
\text { conglomerata, Globigerinita } \\
\text { glutinata, Globigerinoides } \\
\text { sacculifer, Globigerinoides ruber, } \\
\text { Globigerinoides conglobatus, } \\
\text { Globigerinella aequilateralis, } \\
\text { Globigerinella calida }\end{array}$ & $\begin{array}{l}\text { PEQD } \\
\end{array}$ & 5.0 & -140.0 & Watkins et al. (1996) \\
\hline eSNCM & foraminiferans & $\begin{array}{l}\text { Neogloboquadrina duterrei, } \\
\text { Pulleniatina obliquiloculata, } \\
\text { Globorotalia tumida, Globorotalia } \\
\text { Menardii, Globoquadrina } \\
\text { conglomerata, Globigerinita } \\
\text { glutinata, Globigerinoides } \\
\text { sacculifer, Globigerinoides ruber, } \\
\text { Globigerinoides conglobatus, } \\
\text { Globigerinella aequilateralis, } \\
\text { Globigerinella calida }\end{array}$ & PEQD & 3.0 & -140.0 & Watkins et al. (1996) \\
\hline
\end{tabular}




\begin{tabular}{|c|c|c|c|c|c|c|}
\hline eSNCM & foraminiferans & $\begin{array}{l}\text { Neogloboquadrina duterrei, } \\
\text { Pulleniatina obliquiloculata, } \\
\text { Globorotalia tumida, Globorotalia } \\
\text { Menardii, Globoquadrina } \\
\text { conglomerata, Globigerinita } \\
\text { glutinata, Globigerinoides } \\
\text { sacculifer, Globigerinoides ruber, } \\
\text { Globigerinoides conglobatus, } \\
\text { Globigerinella aequilateralis, } \\
\text { Globigerinella calida }\end{array}$ & PEQD & 2.0 & -140.0 & Watkins et al. (1996) \\
\hline eSNCM & foraminiferans & $\begin{array}{l}\text { Neogloboquadrina duterrei, } \\
\text { Pulleniatina obliquiloculata, } \\
\text { Globorotalia tumida, Globorotalia } \\
\text { Menardii, Globoquadrina } \\
\text { conglomerata, Globigerinita } \\
\text { glutinata, Globigerinoides } \\
\text { sacculifer, Globigerinoides ruber, } \\
\text { Globigerinoides conglobatus, } \\
\text { Globigerinella aequilateralis, } \\
\text { Globigerinella calida }\end{array}$ & PEQD & 1.0 & -140.0 & Watkins et al. (1996) \\
\hline eSNCM & foraminiferans & $\begin{array}{l}\text { Neogloboquadrina duterrei, } \\
\text { Pulleniatina obliquiloculata, } \\
\text { Globorotalia tumida, Globorotalia } \\
\text { Menardii, Globoquadrina } \\
\text { conglomerata, Globigerinita } \\
\text { glutinata, Globigerinoides } \\
\text { sacculifer, Globigerinoides ruber, } \\
\text { Globigerinoides conglobatus, } \\
\text { Globigerinella aequilateralis, } \\
\text { Globigerinella calida }\end{array}$ & PEQD & 0.0 & -140.0 & Watkins et al. (1996) \\
\hline eSNCM & foraminiferans & $\begin{array}{l}\text { Neogloboquadrina duterrei, } \\
\text { Pulleniatina obliquiloculata, }\end{array}$ & PEQD & -1.0 & -140.0 & Watkins et al. (1996) \\
\hline
\end{tabular}




\begin{tabular}{|c|c|c|c|c|c|c|}
\hline & & $\begin{array}{l}\text { Globorotalia tumida, Globorotalia } \\
\text { Menardii, Globoquadrina } \\
\text { conglomerata, Globigerinita } \\
\text { glutinata, Globigerinoides } \\
\text { sacculifer, Globigerinoides ruber, } \\
\text { Globigerinoides conglobatus, } \\
\text { Globigerinella aequilateralis, } \\
\text { Globigerinella calida }\end{array}$ & & & & \\
\hline eSNCM & foraminiferans & $\begin{array}{l}\text { Neogloboquadrina duterrei, } \\
\text { Pulleniatina obliquiloculata, } \\
\text { Globorotalia tumida, Globorotalia } \\
\text { Menardii, Globoquadrina } \\
\text { conglomerata, Globigerinita } \\
\text { glutinata, Globigerinoides } \\
\text { sacculifer, Globigerinoides ruber, } \\
\text { Globigerinoides conglobatus, } \\
\text { Globigerinella aequilateralis, } \\
\text { Globigerinella calida }\end{array}$ & PEQD & -2.0 & -140.0 & Watkins et al. (1996) \\
\hline eSNCM & foraminiferans & $\begin{array}{l}\text { Neogloboquadrina duterrei, } \\
\text { Pulleniatina obliquiloculata, } \\
\text { Globorotalia tumida, Globorotalia } \\
\text { Menardii, Globoquadrina } \\
\text { conglomerata, Globigerinita } \\
\text { glutinata, Globigerinoides } \\
\text { sacculifer, Globigerinoides ruber, } \\
\text { Globigerinoides conglobatus, } \\
\text { Globigerinella aequilateralis, } \\
\text { Globigerinella calida }\end{array}$ & PEQD & $x^{2}$ & -140.0 & Watkins et al. (1996) \\
\hline eSNCM & foraminiferans & $\begin{array}{l}\text { Neogloboquadrina duterrei, } \\
\text { Pulleniatina obliquiloculata, } \\
\text { Globorotalia tumida, Globorotalia } \\
\text { Menardii, Globoquadrina }\end{array}$ & PEQD & -12.0 & -140.0 & Watkins et al. (1996) \\
\hline
\end{tabular}




\begin{tabular}{|c|c|c|c|c|c|c|}
\hline & & $\begin{array}{l}\text { conglomerata, Globigerinita } \\
\text { glutinata, Globigerinoides } \\
\text { sacculifer, Globigerinoides ruber, } \\
\text { Globigerinoides conglobatus, } \\
\text { Globigerinella aequilateralis, } \\
\text { Globigerinella calida }\end{array}$ & & & & \\
\hline eSNCM & radiolarians & no data & PEQD & 0.0 & -140.0 & Stoecker et al. (1996) \\
\hline eSNCM & foraminiferans & Globigerinella siphonifera & GUIA & 12.4 & -68.6 & Huber et al. (1997) \\
\hline eSNCM & radiolarians & Actinomma spp & BERS & 54.0 & 146.0 & Abelmann and Nimmergut (2005) \\
\hline eSNCM & radiolarians & Actinomma spp & BERS & 50.0 & 146.0 & Abelmann and Nimmergut (2005) \\
\hline eSNCM & radiolarians & Actinomma spp & BERS & 46.0 & 146.0 & Abelmann and Nimmergut (2005) \\
\hline eSNCM & radiolarians & Actinomma spp & BERS & 54.0 & 150.0 & Abelmann and Nimmergut (2005) \\
\hline eSNCM & radiolarians & Actinomma spp & BERS & 50.0 & 150.0 & Abelmann and Nimmergut (2005) \\
\hline eSNCM & radiolarians & Actinomma spp & BERS & 46.0 & 150.0 & Abelmann and Nimmergut (2005) \\
\hline eSNCM & radiolarians & Actinomma spp & BERS & 50.0 & 154.0 & Abelmann and Nimmergut (2005) \\
\hline eSNCM & $\begin{array}{l}\text { foraminiferans, } \\
\text { radiaolarians }\end{array}$ & no data & CALC & 30.0 & -122.0 & Dennett et al. (2002) \\
\hline eSNCM & $\begin{array}{l}\text { foraminiferans, } \\
\text { radiaolarians }\end{array}$ & no data & NPTG (E) & 30.0 & -126.0 & Dennett et al. (2002) \\
\hline eSNCM & $\begin{array}{l}\text { foraminiferans, } \\
\text { radiaolarians }\end{array}$ & no data & NPTG (E) & 30.0 & -130.0 & Dennett et al. (2002) \\
\hline eSNCM & $\begin{array}{l}\text { foraminiferans, } \\
\text { radiaolarians }\end{array}$ & no data & NPTG (E) & 30.0 & -135.0 & Dennett et al. (2002) \\
\hline eSNCM & $\begin{array}{l}\text { foraminiferans, } \\
\text { radiaolarians }\end{array}$ & no data & NPTG (E) & 30.0 & -140.0 & Dennett et al. (2002) \\
\hline eSNCM & $\begin{array}{l}\text { foraminiferans, } \\
\text { radiaolarians }\end{array}$ & no data & NPTG (E) & 30.0 & -145.0 & Dennett et al. (2002) \\
\hline eSNCM & $\begin{array}{l}\text { foraminiferans, } \\
\text { radiaolarians }\end{array}$ & no data & NPTG (E) & 30.0 & -150.0 & Dennett et al. (2002) \\
\hline
\end{tabular}




\begin{tabular}{|c|c|c|c|c|c|c|}
\hline eSNCM & $\begin{array}{l}\text { foraminiferans, } \\
\text { radiaolarians }\end{array}$ & no data & NPTG (E) & 30.0 & -153.0 & Dennett et al. (2002) \\
\hline eSNCM & $\begin{array}{l}\text { foraminiferans, } \\
\text { radiaolarians }\end{array}$ & no data & NPTG (E) & 30.0 & -157.0 & Dennett et al. (2002) \\
\hline eSNCM & foraminiferans & Globigerina spp & ARCT & 77.0 & 3.4 & Bé and Tolderlind (1971) \\
\hline eSNCM & foraminiferans & Globigerina spp & ARCT & 74.0 & -2.0 & Bé and Tolderlind (1971) \\
\hline eSNCM & foraminiferans & Globigerina spp & SARC & 68.9 & 12.4 & Bé and Tolderlind (1971) \\
\hline eSNCM & foraminiferans & Globigerina spp & BPLR & 64.7 & -52.0 & Bé and Tolderlind (1971) \\
\hline eSNCM & foraminiferans & $\begin{array}{l}\text { Globigerina spp, Globigerinoides } \\
\text { spp, Globoratalia menardii }\end{array}$ & NADR & 52.2 & -41.4 & Bé and Tolderlind (1971) \\
\hline eSNCM & foraminiferans & $\begin{array}{l}\text { Globigerina spp, Globigerinoides } \\
\text { spp, Globoratalia menardii }\end{array}$ & NAST (E) & 42.4 & -23.0 & Bé and Tolderlind (1971) \\
\hline eSNCM & foraminiferans & \multicolumn{2}{|l|}{$\begin{array}{l}\text { Globigerina spp, Globigerinoides spp, } \\
\text { Globoratalia menardii }\end{array}$} & 29.2 & 29.2 & -48.0 \\
\hline eSNCM & foraminiferans & $\begin{array}{l}\text { Globigerina spp, Globigerinoides } \\
\text { spp, Globoratalia menardii }\end{array}$ & SATL & -22.0 & -34.0 & Bé and Tolderlind (1971) \\
\hline eSNCM & foraminiferans & Globigerina $\mathrm{spp}$ & SANT & -57.0 & -20.0 & Bé and Tolderlind (1971) \\
\hline eSNCM & foraminiferans & Globigerina spp & SSTC & -45.0 & -15.0 & Bé and Tolderlind (1971) \\
\hline eSNCM & foraminiferans & $\begin{array}{l}\text { Globigerinoides spp, Globoratalia } \\
\text { menardii }\end{array}$ & GUIN & 5.0 & -5.0 & Bé and Tolderlind (1971) \\
\hline eSNCM & foraminiferans & $\begin{array}{l}\text { Globigerinoides spp, Globoratalia } \\
\text { menardii }\end{array}$ & ETRA & 5.0 & -20.0 & Bé and Tolderlind (1971) \\
\hline eSNCM & foraminiferans & $\begin{array}{l}\text { Globigerinoides spp, Globoratalia } \\
\text { menardii }\end{array}$ & WTRA & 5.0 & -30.0 & Bé and Tolderlind (1971) \\
\hline eSNCM & foraminiferans & $\begin{array}{l}\text { Globigerinoides spp, Globoratalia } \\
\text { menardii }\end{array}$ & ARAB & 5.0 & 53.0 & Bé and Tolderlind (1971) \\
\hline eSNCM & foraminiferans & $\begin{array}{l}\text { Globigerinoides spp, Globoratalia } \\
\text { menardii }\end{array}$ & EAFR & -35.0 & 23.0 & Bé and Tolderlind (1971) \\
\hline eSNCM & foraminiferans & $\begin{array}{l}\text { Globigerinoides spp, Globoratalia } \\
\text { menardii }\end{array}$ & ISSG & -30.0 & 75.0 & Bé and Tolderlind (1971) \\
\hline
\end{tabular}




\begin{tabular}{|c|c|c|c|c|c|c|}
\hline eSNCM & foraminiferans & $\begin{array}{l}\text { Globigerinoides spp, Globoratalia } \\
\text { menardii }\end{array}$ & MONS & -5.0 & 65.0 & Bé and Tolderlind (1971) \\
\hline eSNCM & foraminiferans & $\begin{array}{l}\text { Globigerina spp, Globigerinoides } \\
\text { spp, Globoratalia menardii }\end{array}$ & AUSW & -37.0 & 120.0 & Bé and Tolderlind (1971) \\
\hline eSNCM & foraminiferans & Globigerina spp & SANT & -57.0 & -20.0 & Bé and Tolderlind (1971) \\
\hline eSNCM & foraminiferans & $\begin{array}{l}\text { Globigerina spp, Globigerinoides } \\
\text { spp, Globoratalia menardii }\end{array}$ & SATL & -16.0 & -20.0 & Bé and Tolderlind (1971) \\
\hline eSNCM & foraminiferans & $\begin{array}{l}\text { Globigerina spp, Globigerinoides } \\
\text { spp, Globoratalia menardii }\end{array}$ & SATL & -20.0 & -6.0 & Bé and Tolderlind (1971) \\
\hline eSNCM & foraminiferans & $\begin{array}{l}\text { Globigerina spp, Globigerinoides } \\
\text { spp, Globoratalia menardii, } \\
\text { Orbulina universa, Pulleniatina } \\
\text { obliqueloculata }\end{array}$ & SATL & -16.0 & -20.0 & Bé and Tolderlind (1971) \\
\hline eSNCM & radiolarians & $\begin{array}{l}\text { Acanthosphaera spp, Actinomma } \\
\text { spp, Collosphaera spp, Euchitonia } \\
\text { elegans, Hexacontium spp, } \\
\text { Plegmosphaera spp }\end{array}$ & CALC & 26.0 & -113.0 & Boltovskoy and Riedel (1977) \\
\hline eSNCM & radiolarians & $\begin{array}{l}\text { Acanthosphaera spp, Actinomma } \\
\text { spp, Collosphaera spp, Euchitonia } \\
\text { elegans, Hexacontium spp, } \\
\text { Plegmosphaera spp }\end{array}$ & CALC & 25.0 & -115.0 & Boltovskoy and Riedel (1977) \\
\hline eSNCM & radiolarians & $\begin{array}{l}\text { Acanthosphaera spp, Actinomma } \\
\text { spp, Collosphaera spp, Euchitonia } \\
\text { elegans, Hexacontium spp, } \\
\text { Plegmosphaera spp }\end{array}$ & CALC & 34.0 & -122.0 & Boltovskoy and Riedel (1977) \\
\hline eSNCM & radiolarians & $\begin{array}{l}\text { Pterocanium praetextum, } \\
\text { Spongaster tetras }\end{array}$ & NPPF & 39.5 & -158.0 & Casey (1971) \\
\hline eSNCM & radiolarians & $\begin{array}{l}\text { Pterocanium praetextum, } \\
\text { Spongaster tetras }\end{array}$ & NPTG (E) & 28.5 & -135.9 & Casey (1971) \\
\hline eSNCM & radiolarians & $\begin{array}{l}\text { Pterocanium praetextum, } \\
\text { Spongaster tetras }\end{array}$ & NPTG (E) & 20.0 & -154.9 & Casey (1971) \\
\hline
\end{tabular}




\begin{tabular}{|c|c|c|c|c|c|c|}
\hline eSNCM & radiolarians & $\begin{array}{l}\text { Pterocanium praetextum, } \\
\text { Spongaster tetras }\end{array}$ & PNEC & 5.4 & -160.5 & Casey (1971) \\
\hline eSNCM & radiolarians & $\begin{array}{l}\text { Pterocanium praetextum, } \\
\text { Spongaster tetras }\end{array}$ & PNEC & 10.8 & -105.0 & Casey (1971) \\
\hline eSNCM & radiolarians & Spongaster tetras & SPSG & -154.8 & -24.7 & Casey (1971) \\
\hline eSNCM & radiolarians & Spongaster tetras & SANT & -178.0 & -52.7 & Casey (1971) \\
\hline eSNCM & radiolarians & Acantharia & MEDI & 40.7 & 14.3 & Decelle et al. (2012) \\
\hline eSNCM & radiolarians & Acantharia & MEDI & 43.6 & 7.4 & Decelle et al. (2012) \\
\hline eSNCM & radiolarians & Acantharia & MEDI & 29.5 & 35.0 & Decelle et al. (2012) \\
\hline eSNCM & radiolarians & Acantharia & NPTG $(\mathrm{W})$ & 26.2 & 127.2 & Decelle et al. (2012) \\
\hline eSNCM & radiolarians & Acantharia & INDW & 18.8 & 66.9 & Decelle et al. (2012) \\
\hline eSNCM & radiolarians & Acantharia & INDW & 14.6 & 69.9 & Decelle et al. (2012) \\
\hline eSNCM & radiolarians & Acantharia & NECS & 48.8 & -4.0 & Decelle et al. (2012) \\
\hline eSNCM & radiolarians & Acantharia & APLR & -60.2 & -60.5 & Decelle et al. (2012) \\
\hline eSNCM & radiolarians & Acantharia & APLR & -62.0 & -49.2 & Decelle et al. (2012) \\
\hline eSNCM & radiolarians & Acantharia & APLR & -64.4 & -53.0 & Decelle et al. (2012) \\
\hline eSNCM & radiolarians & Acantharia & APLR & -63.4 & -56.9 & Decelle et al. (2012) \\
\hline eSNCM & radiolarians & Acantharia & FKLD & -40.7 & -52.2 & Decelle et al. (2012) \\
\hline eSNCM & foraminiferans & $\begin{array}{l}\text { Globigerinoides ruber, } \\
\text { Globigerinella spinosa, Orbulina } \\
\text { universa }\end{array}$ & MEDI & 29.5 & 35.0 & Lombard et al. (2009) \\
\hline eSNCM & radiolarians & Collodaria & SPSG & -21.3 & -105.0 & Probert et al. (2014) \\
\hline eSNCM & radiolarians & Collodaria & SPSG & -23.8 & -107.3 & Probert et al. (2014) \\
\hline eSNCM & radiolarians & Collodaria & SPSG & -24.8 & -110.0 & Probert et al. (2014) \\
\hline eSNCM & radiolarians & Collodaria & SPSG & -24.3 & -113.0 & Probert et al. (2014) \\
\hline eSNCM & radiolarians & Collodaria & SPSG & -23.3 & -131.0 & Probert et al. (2014) \\
\hline eSNCM & radiolarians & $\begin{array}{l}\text { Collodaria (Collozoum colony), } \\
\text { Spumellaria, Nassellaria }\end{array}$ & KURO & 26.7 & 127.9 & Probert et al. (2014) \\
\hline
\end{tabular}


Appendices. Appendix A, Table A.I

\begin{tabular}{|c|c|c|c|c|c|c|}
\hline eSNCM & radiolarians & $\begin{array}{l}\text { Collodaria (Collozoum colony), } \\
\text { Spumellaria }\end{array}$ & MEDI & 43.8 & 7.2 & Probert et al. (2014) \\
\hline eSNCM & foraminiferans & $\begin{array}{l}\text { Globigerinella siphonifera, } \\
\text { Globigerinoides ruber, } \\
\text { Globorotalia menardii, Orbulina } \\
\text { universa, Neogloboquadrina } \\
\text { dutertrei }\end{array}$ & BRAZ & -24.5 & -43.7 & Sousa et al. (2014) \\
\hline eSNCM & foraminiferans & $\begin{array}{l}\text { Globigerinella siphonifera, } \\
\text { Globigerinoides ruber, } \\
\text { Globorotalia menardii, Orbulina } \\
\text { universa, Neogloboquadrina } \\
\text { dutertrei }\end{array}$ & BRAZ & -24.2 & -43.5 & Sousa et al. (2014) \\
\hline eSNCM & foraminiferans & $\begin{array}{l}\text { Globigerinella siphonifera, } \\
\text { Globigerinoides ruber, } \\
\text { Globorotalia menardii, Orbulina } \\
\text { universa, Neogloboquadrina } \\
\text { dutertrei }\end{array}$ & BRAZ & -24.4 & -43.0 & Sousa et al. (2014) \\
\hline eSNCM & foraminiferans & $\begin{array}{l}\text { Globigerinella siphonifera, } \\
\text { Globigerinoides ruber, } \\
\text { Globorotalia menardii, Orbulina } \\
\text { universa, Neogloboquadrina } \\
\text { dutertrei }\end{array}$ & BRAZ & -24.0 & -43.2 & Sousa et al. (2014) \\
\hline eSNCM & foraminiferans & $\begin{array}{l}\text { Globigerinella siphonifera, } \\
\text { Globigerinoides ruber, } \\
\text { Globorotalia menardii, Orbulina } \\
\text { universa, Neogloboquadrina } \\
\text { dutertrei }\end{array}$ & BRAZ & -23.6 & -43.8 & Sousa et al. (2014) \\
\hline eSNCM & foraminiferans & $\begin{array}{l}\text { Globigerinella siphonifera, } \\
\text { Globigerinoides ruber, } \\
\text { Globorotalia menardii, Orbulina } \\
\text { universa, Neogloboquadrina } \\
\text { dutertrei }\end{array}$ & BRAZ & -24.2 & -43.5 & Sousa et al. (2014) \\
\hline
\end{tabular}




\begin{tabular}{|c|c|c|c|c|c|c|}
\hline eSNCM & foraminiferans & $\begin{array}{l}\text { Globigerinella siphonifera, } \\
\text { Globigerinoides ruber, } \\
\text { Globorotalia menardii, Orbulina } \\
\text { universa, Neogloboquadrina } \\
\text { dutertrei }\end{array}$ & BRAZ & -23.5 & -41.0 & Sousa et al. (2014) \\
\hline eSNCM & foraminiferans & $\begin{array}{l}\text { Globigerinella siphonifera, } \\
\text { Globigerinoides ruber, } \\
\text { Globorotalia menardii, Orbulina } \\
\text { universa, Neogloboquadrina } \\
\text { dutertrei }\end{array}$ & BRAZ & -23.4 & -40.4 & Sousa et al. (2014) \\
\hline eSNCM & foraminiferans & Globigerinella siphonifera & ETRA & -7.6 & -14.5 & de Vargas et al. (2002) \\
\hline eSNCM & foraminiferans & Globigerinella siphonifera & ETRA & -4.0 & -15.5 & de Vargas et al. (2002) \\
\hline eSNCM & foraminiferans & Globigerinella siphonifera & ETRA & 0.0 & -16.7 & de Vargas et al. (2002) \\
\hline eSNCM & foraminiferans & Globigerinella siphonifera & ETRA & 4.0 & -17.9 & de Vargas et al. (2002) \\
\hline eSNCM & foraminiferans & Globigerinella siphonifera & ETRA & 7.9 & -19.0 & de Vargas et al. (2002) \\
\hline eSNCM & foraminiferans & Globigerinella siphonifera & ETRA & 11.5 & -20.1 & de Vargas et al. (2002) \\
\hline eSNCM & foraminiferans & Globigerinella siphonifera & NATR & 15.1 & -21.0 & de Vargas et al. (2002) \\
\hline eSNCM & foraminiferans & Globigerinella siphonifera & NATR & 18.9 & -21.0 & de Vargas et al. (2002) \\
\hline eSNCM & foraminiferans & Globigerinella siphonifera & NATR & 22.9 & -21.0 & de Vargas et al. (2002) \\
\hline eSNCM & foraminiferans & Globigerinella siphonifera & NAST (E) & 34.4 & -20.9 & de Vargas et al. (2002) \\
\hline eSNCM & foraminiferans & Globigerinella siphonifera & $\operatorname{NAST}(\mathrm{E})$ & 34.4 & -20.8 & de Vargas et al. (2002) \\
\hline eSNCM & foraminiferans & Globigerinella siphonifera & $\operatorname{NAST}(\mathrm{E})$ & 38.0 & -20.0 & de Vargas et al. (2002) \\
\hline eSNCM & foraminiferans & Globigerinella siphonifera & NAST (E) & 41.9 & -20.0 & de Vargas et al. (2002) \\
\hline eSNCM & foraminiferans & Globigerinella siphonifera & NADR & 47.0 & -20.0 & de Vargas et al. (2002) \\
\hline eSNCM & foraminiferans & Globigerinella siphonifera & NECS & 48.0 & -9.7 & de Vargas et al. (2002) \\
\hline eSNCM & radiolarians & Dictyocoryne profunda & KURO & 26.8 & 127.9 & Yuasa et al. (2012) \\
\hline eSNCM & radiolarians & Collozoum spp, Thalassicolla spp & NAST $(\mathrm{W})$ & 27.0 & -66.0 & Gast and Caron (2001) \\
\hline eSNCM & radiolarians & Dictyocoryne truncatum & CARB & & & Anderson and Matusuoka (1992) \\
\hline
\end{tabular}




\begin{tabular}{|c|c|c|c|c|c|c|}
\hline eSNCM & radiolarians & $\begin{array}{l}\text { Sphaerozoum spp, Pterocanium } \\
\text { praetextum, Phorticium pylonium }\end{array}$ & KURO & 26.7 & 127.8 & Takahashi et al. (2003) \\
\hline eSNCM & radiolarians & $\begin{array}{l}\text { Acanthosphaera spp, Actiomma } \\
\text { spp, Plegmosphaera spp, } \\
\text { Hexacontium spp, Spongotrochus } \\
\text { spp }\end{array}$ & NAST (W) & 27.0 & -66.0 & Stoecker et al. (2009) \\
\hline eSNCM & radiolarians & $\begin{array}{l}\text { Collozoum caudatum, Acrosphaera } \\
\text { spinosa }\end{array}$ & GFST & nd & nd & Stoecker et al. (2009) \\
\hline eSNCM & radiolarians & Collosphaera spp & NAST (W) & nd & nd & Stoecker et al. (2009) \\
\hline eSNCM & radiolarians & $\begin{array}{l}\text { Spongaster tetras, Dictyocoryne } \\
\text { truncatum }\end{array}$ & CARB & & & Stoecker et al. (2009) \\
\hline eSNCM & radiolarians & Physematium muelleri & CARB & & & Stoecker et al. (2009) \\
\hline eSNCM & radiolarians & $\begin{array}{l}\text { Phorticum pylonium, Ceratospyris } \\
\text { hyperborea, Androcyclas } \\
\text { gamphonyca }\end{array}$ & NECS & & & Stoecker et al. (2009) \\
\hline eSNCM & radiolarians & Astrosphaeridae & NAST $(W)$ & 27.0 & -66.0 & Stoecker et al. (2009) \\
\hline eSNCM & foraminiferans & Globigerinella siphonifera & NAST (W) & 27.0 & -66.0 & Gast and Caron (2001) \\
\hline eSNCM & radiolarians & Collodaria and Acantharia & SANT & -55.6 & -63.1 & Biard et al. (2016) \\
\hline eSNCM & radiolarians & Collodaria and Acantharia & FKLD & -48.0 & -60.0 & Biard et al. (2016) \\
\hline eSNCM & radiolarians & Collodaria and Acantharia & SSTC & -47.0 & -52.0 & Biard et al. (2016) \\
\hline eSNCM & radiolarians & Collodaria and Acantharia & BRAZ & -40.0 & -54.0 & Biard et al. (2016) \\
\hline eSNCM & radiolarians & Collodaria and Acantharia & PEQD & -35.6 & -49.1 & Biard et al. (2016) \\
\hline eSNCM & radiolarians & Collodaria and Acantharia & PEQD & -27.7 & -40.0 & Biard et al. (2016) \\
\hline eSNCM & radiolarians & Collodaria and Acantharia & PEQD & -20.0 & -35.0 & Biard et al. (2016) \\
\hline eSNCM & radiolarians & Collodaria and Acantharia & PEQD & -13.0 & -27.0 & Biard et al. (2016) \\
\hline eSNCM & radiolarians & Collodaria and Acantharia & PEQD & -8.0 & -19.0 & Biard et al. (2016) \\
\hline eSNCM & radiolarians & Collodaria and Acantharia & ETRA & -8.0 & -7.0 & Biard et al. (2016) \\
\hline eSNCM & radiolarians & Collodaria and Acantharia & PEQD & -18.0 & 3.0 & Biard et al. (2016) \\
\hline eSNCM & radiolarians & Collodaria and Acantharia & PEQD & -29.0 & 5.0 & Biard et al. (2016) \\
\hline
\end{tabular}




\begin{tabular}{|c|c|c|c|c|c|c|}
\hline eSNCM & radiolarians & Collodaria and Acantharia & BENG & -33.0 & 17.6 & Biard et al. (2016) \\
\hline eSNCM & radiolarians & Collodaria and Acantharia & EAFR & -35.0 & 26.0 & Biard et al. (2016) \\
\hline eSNCM & radiolarians & Collodaria and Acantharia & EAFR & -32.0 & 34.4 & Biard et al. (2016) \\
\hline eSNCM & radiolarians & Collodaria and Acantharia & EAFR & -28.0 & 40.6 & Biard et al. (2016) \\
\hline eSNCM & radiolarians & Collodaria and Acantharia & EAFR & -16.0 & 42.0 & Biard et al. (2016) \\
\hline eSNCM & radiolarians & Collodaria and Acantharia & MONS & -18.0 & 53.0 & Biard et al. (2016) \\
\hline eSNCM & radiolarians & Collodaria and Acantharia & MONS & -8.0 & 59.0 & Biard et al. (2016) \\
\hline eSNCM & radiolarians & Collodaria and Acantharia & ARAB & 15.0 & 69.0 & Biard et al. (2016) \\
\hline eSNCM & radiolarians & Collodaria and Acantharia & ARAB & 21.7 & 61.2 & Biard et al. (2016) \\
\hline eSNCM & radiolarians & Collodaria and Acantharia & MONS & 1.0 & 76.0 & Biard et al. (2016) \\
\hline eSNCM & radiolarians & Collodaria and Acantharia & SANT & -51.0 & 72.0 & Biard et al. (2016) \\
\hline eSNCM & radiolarians & Collodaria and Acantharia & BPLR & 76.0 & 75.0 & Biard et al. (2016) \\
\hline eSNCM & radiolarians & Collodaria and Acantharia & BPLR & 75.0 & 52.0 & Biard et al. (2016) \\
\hline eSNCM & radiolarians & Collodaria and Acantharia & SARC & 70.0 & 43.0 & Biard et al. (2016) \\
\hline eSNCM & radiolarians & Collodaria and Acantharia & BPLR & 75.0 & 120.0 & Biard et al. (2016) \\
\hline eSNCM & radiolarians & Collodaria and Acantharia & SARC & 69.0 & 10.0 & Biard et al. (2016) \\
\hline eSNCM & radiolarians & Collodaria and Acantharia & SARC & 63.7 & 1.7 & Biard et al. (2016) \\
\hline eSNCM & radiolarians & Collodaria and Acantharia & ARCT & 75.0 & -0.3 & Biard et al. (2016) \\
\hline eSNCM & radiolarians & Collodaria and Acantharia & NECS & 59.5 & -7.0 & Biard et al. (2016) \\
\hline eSNCM & radiolarians & Collodaria and Acantharia & NADR & 51.9 & -21.7 & Biard et al. (2016) \\
\hline eSNCM & radiolarians & Collodaria and Acantharia & BPLR & 68.0 & -59.0 & Biard et al. (2016) \\
\hline eSNCM & radiolarians & Collodaria and Acantharia & $\mathrm{ARCT}$ & 62.0 & -52.8 & Biard et al. (2016) \\
\hline eSNCM & radiolarians & Collodaria and Acantharia & BPLR & 74.0 & -89.0 & Biard et al. (2016) \\
\hline eSNCM & radiolarians & Collodaria and Acantharia & NAST (E) & 43.4 & -12.7 & Biard et al. (2016) \\
\hline eSNCM & radiolarians & Collodaria and Acantharia & NAST (E) & 43.0 & -21.0 & Biard et al. (2016) \\
\hline eSNCM & radiolarians & Collodaria and Acantharia & NAST (W) & 32.0 & -35.0 & Biard et al. (2016) \\
\hline eSNCM & radiolarians & Collodaria and Acantharia & NAST (W) & 30.0 & -48.0 & Biard et al. (2016) \\
\hline
\end{tabular}




\begin{tabular}{|c|c|c|c|c|c|c|}
\hline eSNCM & radiolarians & Collodaria and Acantharia & NAST (W) & 28.0 & -61.0 & Biard et al. (2016) \\
\hline eSNCM & radiolarians & Collodaria and Acantharia & GFST & 35.0 & -73.0 & Biard et al. (2016) \\
\hline eSNCM & radiolarians & Collodaria and Acantharia & BPLR & 71.0 & -132.0 & Biard et al. (2016) \\
\hline eSNCM & radiolarians & Collodaria and Acantharia & PNEC & 3.0 & -85.0 & Biard et al. (2016) \\
\hline eSNCM & radiolarians & Collodaria and Acantharia & HUMB & -3.0 & -85.0 & Biard et al. (2016) \\
\hline eSNCM & radiolarians & Collodaria and Acantharia & PEQD & -9.0 & -90.0 & Biard et al. (2016) \\
\hline eSNCM & radiolarians & Collodaria and Acantharia & PEQD & -17.0 & -98.0 & Biard et al. (2016) \\
\hline eSNCM & radiolarians & Collodaria and Acantharia & PEQD & -25.0 & -112.0 & Biard et al. (2016) \\
\hline eSNCM & radiolarians & Collodaria and Acantharia & PEQD & -27.0 & -140.0 & Biard et al. (2016) \\
\hline eSNCM & radiolarians & Collodaria and Acantharia & PEQD & -28.0 & -100.0 & Biard et al. (2016) \\
\hline eSNCM & radiolarians & Collodaria and Acantharia & HUMB & -30.0 & -74.0 & Biard et al. (2016) \\
\hline eSNCM & radiolarians & Collodaria and Acantharia & PEQD & -42.0 & -90.0 & Biard et al. (2016) \\
\hline eSNCM & radiolarians & Collodaria and Acantharia & HUMB & -44.0 & -80.0 & Biard et al. (2016) \\
\hline eSNCM & radiolarians & Collodaria and Acantharia & NPTG (E) & 34.0 & -162.0 & Biard et al. (2016) \\
\hline eSNCM & radiolarians & Collodaria and Acantharia & NATR & 14.0 & -33.0 & Biard et al. (2016) \\
\hline eSNCM & radiolarians & Collodaria and Acantharia & NATR & 14.0 & -24.0 & Biard et al. (2016) \\
\hline eSNCM & radiolarians & Collodaria and Acantharia & WTRA & 7.0 & -24.0 & Biard et al. (2016) \\
\hline eSNCM & radiolarians & Collodaria and Acantharia & WTRA & 0.0 & -24.0 & Biard et al. (2016) \\
\hline eSNCM & radiolarians & Collodaria and Acantharia & NATR & 14.0 & -43.0 & Biard et al. (2016) \\
\hline eSNCM & radiolarians & Collodaria and Acantharia & ISSG & -25.0 & 59.0 & Biard et al. (2016) \\
\hline eSNCM & radiolarians & Collodaria and Acantharia & APLR & -65.0 & -56.0 & Biard et al. (2016) \\
\hline eSNCM & dinoflagellates & $\begin{array}{l}\text { Histioneis spp, Parahistioneis spp, } \\
\text { Ornithocercus spp, Amphisolenia } \\
\text { spp, Tricosolenia sp, Citharistes sp }\end{array}$ & MEDI & 41.0 & 12.0 & Tarangkoon et al. (2010) \\
\hline eSNCM & dinoflagellates & $\begin{array}{l}\text { Histioneis spp, Parahistioneis spp, } \\
\text { Ornithocercus spp, Amphisolenia } \\
\text { spp, Tricosolenia sp, Citharistes sp }\end{array}$ & MEDI & 46.0 & 18.0 & Tarangkoon et al. (2010) \\
\hline
\end{tabular}




\begin{tabular}{|c|c|c|c|c|c|c|}
\hline eSNCM & dinoflagellates & $\begin{array}{l}\text { Histioneis spp, Parahistioneis spp, } \\
\text { Ornithocercus spp, Amphisolenia } \\
\text { spp, Tricosolenia sp, Citharistes sp }\end{array}$ & MEDI & 36.7 & 35.7 & Tarangkoon et al. (2010) \\
\hline eSNCM & dinoflagellates & $\begin{array}{l}\text { Histioneis spp, Parahistioneis spp, } \\
\text { Ornithocercus spp, Amphisolenia } \\
\text { spp, Tricosolenia sp, Citharistes sp }\end{array}$ & MEDI & 36.0 & 33.5 & Tarangkoon et al. (2010) \\
\hline eSNCM & dinoflagellates & $\begin{array}{l}\text { Histioneis spp, Parahistioneis spp, } \\
\text { Ornithocercus spp, Amphisolenia } \\
\text { spp, Tricosolenia sp, Citharistes sp }\end{array}$ & KURO & 35.0 & 139.0 & Tarangkoon et al. (2010) \\
\hline eSNCM & dinoflagellates & $\begin{array}{l}\text { Histioneis spp, Parahistioneis spp, } \\
\text { Ornithocercus spp, Amphisolenia } \\
\text { spp, Tricosolenia sp, Citharistes sp }\end{array}$ & KURO & 36.0 & 140.0 & Tarangkoon et al. (2010) \\
\hline eSNCM & dinoflagellates & $\begin{array}{l}\text { Histioneis spp, Parahistioneis spp, } \\
\text { Ornithocercus spp, Amphisolenia } \\
\text { spp, Tricosolenia sp, Citharistes sp }\end{array}$ & REDS & 29.5 & 35.0 & Tarangkoon et al. (2010) \\
\hline eSNCM & dinoflagellates & $\begin{array}{l}\text { Histioneis spp, Parahistioneis spp, } \\
\text { Ornithocercus spp, Amphisolenia } \\
\text { spp, Tricosolenia sp, Citharistes sp }\end{array}$ & NWCS & 33.0 & -79.0 & Tarangkoon et al. (2010) \\
\hline eSNCM & dinoflagellates & $\begin{array}{l}\text { Histioneis spp, Parahistioneis spp, } \\
\text { Ornithocercus spp, Amphisolenia } \\
\text { spp, Tricosolenia sp, Citharistes sp }\end{array}$ & CALC & 32.0 & -120.0 & Tarangkoon et al. (2010) \\
\hline eSNCM & dinoflagellates & $\begin{array}{l}\text { Histioneis spp, Parahistioneis spp, } \\
\text { Ornithocercus spp, Amphisolenia } \\
\text { spp, Tricosolenia sp, Citharistes sp }\end{array}$ & NPTG (E) & 33.0 & -133.0 & Tarangkoon et al. (2010) \\
\hline eSNCM & dinoflagellates & $\begin{array}{l}\text { Histioneis spp, Parahistioneis spp, } \\
\text { Ornithocercus spp, Amphisolenia } \\
\text { spp, Tricosolenia sp, Citharistes sp }\end{array}$ & NPTG (E) & 23.7 & -159.0 & Tarangkoon et al. (2010) \\
\hline eSNCM & dinoflagellates & $\begin{array}{l}\text { Histioneis spp, Parahistioneis spp, } \\
\text { Ornithocercus spp, Amphisolenia } \\
\text { spp, Tricosolenia sp, Citharistes sp }\end{array}$ & NPTG (E) & 27.0 & -158.0 & Tarangkoon et al. (2010) \\
\hline
\end{tabular}




\begin{tabular}{|c|c|c|c|c|c|c|}
\hline eSNCM & dinoflagellates & $\begin{array}{l}\text { Histioneis spp, Parahistioneis spp, } \\
\text { Ornithocercus spp, Amphisolenia } \\
\text { spp, Tricosolenia sp, Citharistes sp }\end{array}$ & CARB & 20.9 & -73.0 & Tarangkoon et al. (2010) \\
\hline eSNCM & dinoflagellates & $\begin{array}{l}\text { Histioneis spp, Parahistioneis spp, } \\
\text { Ornithocercus spp, Amphisolenia } \\
\text { spp, Tricosolenia sp, Citharistes sp }\end{array}$ & CARB & 22.0 & -74.0 & Tarangkoon et al. (2010) \\
\hline eSNCM & dinoflagellates & $\begin{array}{l}\text { Histioneis spp, Parahistioneis spp, } \\
\text { Ornithocercus spp, Amphisolenia } \\
\text { spp, Tricosolenia sp, Citharistes sp }\end{array}$ & INDE & 12.0 & 93.0 & Tarangkoon et al. (2010) \\
\hline eSNCM & dinoflagellates & $\begin{array}{l}\text { Histioneis spp, Parahistioneis spp, } \\
\text { Ornithocercus spp, Amphisolenia } \\
\text { spp, Tricosolenia sp, Citharistes sp }\end{array}$ & GUIA & 10.0 & -60.0 & Tarangkoon et al. (2010) \\
\hline eSNCM & dinoflagellates & $\begin{array}{l}\text { Histioneis spp, Parahistioneis spp, } \\
\text { Ornithocercus spp, Amphisolenia } \\
\text { spp, Tricosolenia sp, Citharistes sp }\end{array}$ & GUIA & 12.0 & -70.0 & Tarangkoon et al. (2010) \\
\hline eSNCM & dinoflagellates & $\begin{array}{l}\text { Histioneis spp, Parahistioneis spp, } \\
\text { Ornithocercus spp, Amphisolenia } \\
\text { spp, Tricosolenia sp, Citharistes sp }\end{array}$ & GUIA & 15.0 & -75.0 & Tarangkoon et al. (2010) \\
\hline eSNCM & dinoflagellates & $\begin{array}{l}\text { Histioneis spp, Parahistioneis spp, } \\
\text { Ornithocercus spp, Amphisolenia } \\
\text { spp, Tricosolenia sp, Citharistes sp }\end{array}$ & CARB & 20.0 & -80.0 & Tarangkoon et al. (2010) \\
\hline eSNCM & dinoflagellates & $\begin{array}{l}\text { Histioneis spp, Parahistioneis spp, } \\
\text { Ornithocercus spp, Amphisolenia } \\
\text { spp, Tricosolenia sp, Citharistes sp }\end{array}$ & CARB & 25.0 & -90.0 & Tarangkoon et al. (2010) \\
\hline eSNCM & dinoflagellates & $\begin{array}{l}\text { Histioneis spp, Parahistioneis spp, } \\
\text { Ornithocercus spp, Amphisolenia } \\
\text { spp, Tricosolenia sp, Citharistes sp }\end{array}$ & MONS & 7.0 & 80.0 & Tarangkoon et al. (2010) \\
\hline eSNCM & dinoflagellates & $\begin{array}{l}\text { Histioneis spp, Parahistioneis spp, } \\
\text { Ornithocercus spp, Amphisolenia } \\
\text { spp, Tricosolenia sp, Citharistes sp }\end{array}$ & MONS & 12.0 & 84.0 & Tarangkoon et al. (2010) \\
\hline
\end{tabular}




\begin{tabular}{|c|c|c|c|c|c|c|}
\hline eSNCM & dinoflagellates & $\begin{array}{l}\text { Histioneis spp, Parahistioneis spp, } \\
\text { Ornithocercus spp, Amphisolenia } \\
\text { spp, Tricosolenia sp, Citharistes sp }\end{array}$ & INDE & 20.0 & 89.0 & Tarangkoon et al. (2010) \\
\hline eSNCM & dinoflagellates & $\begin{array}{l}\text { Histioneis spp, Parahistioneis spp, } \\
\text { Ornithocercus spp, Amphisolenia } \\
\text { spp, Tricosolenia sp, Citharistes sp }\end{array}$ & ISSG & -25.0 & 70.0 & Tarangkoon et al. (2010) \\
\hline eSNCM & dinoflagellates & $\begin{array}{l}\text { Histioneis spp, Parahistioneis spp, } \\
\text { Ornithocercus spp, Amphisolenia } \\
\text { spp, Tricosolenia sp, Citharistes sp }\end{array}$ & ISSG & 21.0 & 63.0 & Tarangkoon et al. (2010) \\
\hline eSNCM & dinoflagellates & $\begin{array}{l}\text { Histioneis spp, Parahistioneis spp, } \\
\text { Ornithocercus spp, Amphisolenia } \\
\text { spp, Tricosolenia sp, Citharistes sp }\end{array}$ & ARAB & -7.0 & 43.0 & Tarangkoon et al. (2010) \\
\hline eSNCM & dinoflagellates & $\begin{array}{l}\text { Histioneis spp, Parahistioneis spp, } \\
\text { Ornithocercus spp, Amphisolenia } \\
\text { spp, Tricosolenia sp, Citharistes sp }\end{array}$ & ISSG & -26.0 & 105.0 & Tarangkoon et al. (2010) \\
\hline eSNCM & dinoflagellates & $\begin{array}{l}\text { Histioneis spp, Parahistioneis spp, } \\
\text { Ornithocercus spp, Amphisolenia } \\
\text { spp, Tricosolenia sp, Citharistes sp }\end{array}$ & ISSG & -30.0 & 95.0 & Tarangkoon et al. (2010) \\
\hline eSNCM & dinoflagellates & $\begin{array}{l}\text { Histioneis spp, Parahistioneis spp, } \\
\text { Ornithocercus spp, Amphisolenia } \\
\text { spp, Tricosolenia sp, Citharistes sp }\end{array}$ & ISSG & -32.0 & 90.0 & Tarangkoon et al. (2010) \\
\hline eSNCM & dinoflagellates & $\begin{array}{l}\text { Histioneis spp, Parahistioneis spp, } \\
\text { Ornithocercus spp, Amphisolenia } \\
\text { spp, Tricosolenia sp, Citharistes sp }\end{array}$ & ISSG & -27.0 & 79.0 & Tarangkoon et al. (2010) \\
\hline eSNCM & dinoflagellates & $\begin{array}{l}\text { Histioneis spp, Parahistioneis spp, } \\
\text { Ornithocercus spp, Amphisolenia } \\
\text { spp, Tricosolenia sp, Citharistes sp }\end{array}$ & ISSG & -19.0 & 80.0 & Tarangkoon et al. (2010) \\
\hline eSNCM & dinoflagellates & $\begin{array}{l}\text { Histioneis spp, Parahistioneis spp, } \\
\text { Ornithocercus spp, Amphisolenia } \\
\text { spp, Tricosolenia sp, Citharistes sp }\end{array}$ & ISSG & -10.0 & 79.0 & Tarangkoon et al. (2010) \\
\hline
\end{tabular}




\begin{tabular}{|c|c|c|c|c|c|c|}
\hline eSNCM & dinoflagellates & $\begin{array}{l}\text { Histioneis spp, Parahistioneis spp, } \\
\text { Ornithocercus spp, Amphisolenia } \\
\text { spp, Tricosolenia sp, Citharistes sp }\end{array}$ & ISSG & -2.0 & 80.0 & Tarangkoon et al. (2010) \\
\hline eSNCM & dinoflagellates & $\begin{array}{l}\text { Histioneis spp, Parahistioneis spp, } \\
\text { Ornithocercus spp, Amphisolenia } \\
\text { spp, Tricosolenia sp, Citharistes sp }\end{array}$ & ISSG & 2.0 & 80.0 & Tarangkoon et al. (2010) \\
\hline eSNCM & dinoflagellates & $\begin{array}{l}\text { Histioneis spp, Parahistioneis spp, } \\
\text { Ornithocercus spp, Amphisolenia } \\
\text { spp, Tricosolenia sp, Citharistes sp }\end{array}$ & ISSG & 5.0 & 80.0 & Tarangkoon et al. (2010) \\
\hline eSNCM & dinoflagellates & $\begin{array}{l}\text { Histioneis spp, Parahistioneis spp, } \\
\text { Ornithocercus spp, Amphisolenia } \\
\text { spp, Tricosolenia sp, Citharistes sp }\end{array}$ & ISSG & 16.0 & 70.0 & Tarangkoon et al. (2010) \\
\hline eSNCM & dinoflagellates & $\begin{array}{l}\text { Histioneis spp, Parahistioneis spp, } \\
\text { Ornithocercus spp, Amphisolenia } \\
\text { spp, Tricosolenia sp, Citharistes sp }\end{array}$ & ISSG & 13.0 & 70.0 & Tarangkoon et al. (2010) \\
\hline eSNCM & dinoflagellates & $\begin{array}{l}\text { Histioneis spp, Parahistioneis spp, } \\
\text { Ornithocercus spp, Amphisolenia } \\
\text { spp, Tricosolenia sp, Citharistes sp }\end{array}$ & ISSG & 11.0 & 70.0 & Tarangkoon et al. (2010) \\
\hline eSNCM & dinoflagellates & $\begin{array}{l}\text { Histioneis spp, Parahistioneis spp, } \\
\text { Ornithocercus spp, Amphisolenia } \\
\text { spp, Tricosolenia sp, Citharistes sp }\end{array}$ & ISSG & 9.0 & 70.0 & Tarangkoon et al. (2010) \\
\hline eSNCM & dinoflagellates & $\begin{array}{l}\text { Histioneis spp, Parahistioneis spp, } \\
\text { Ornithocercus spp, Amphisolenia } \\
\text { spp, Tricosolenia sp, Citharistes sp }\end{array}$ & ISSG & 7.0 & 70.0 & Tarangkoon et al. (2010) \\
\hline eSNCM & dinoflagellates & $\begin{array}{l}\text { Histioneis spp, Parahistioneis spp, } \\
\text { Ornithocercus spp, Amphisolenia } \\
\text { spp, Tricosolenia sp, Citharistes sp }\end{array}$ & ISSG & 6.0 & 65.0 & Tarangkoon et al. (2010) \\
\hline eSNCM & dinoflagellates & $\begin{array}{l}\text { Histioneis spp, Parahistioneis spp, } \\
\text { Ornithocercus spp, Amphisolenia } \\
\text { spp, Tricosolenia sp, Citharistes sp }\end{array}$ & ISSG & 2.5 & 55.0 & Tarangkoon et al. (2010) \\
\hline
\end{tabular}




\begin{tabular}{|c|c|c|c|c|c|c|}
\hline eSNCM & dinoflagellates & $\begin{array}{l}\text { Histioneis spp, Parahistioneis spp, } \\
\text { Ornithocercus spp, Amphisolenia } \\
\text { spp, Tricosolenia sp, Citharistes sp }\end{array}$ & ISSG & -1.0 & 59.0 & Tarangkoon et al. (2010) \\
\hline eSNCM & dinoflagellates & $\begin{array}{l}\text { Histioneis spp, Parahistioneis spp, } \\
\text { Ornithocercus spp, Amphisolenia } \\
\text { spp, Tricosolenia sp, Citharistes sp }\end{array}$ & ISSG & -22.0 & 55.0 & Tarangkoon et al. (2010) \\
\hline eSNCM & dinoflagellates & $\begin{array}{l}\text { Histioneis spp, Parahistioneis spp, } \\
\text { Ornithocercus spp, Amphisolenia } \\
\text { spp, Tricosolenia sp, Citharistes sp }\end{array}$ & ISSG & -14.0 & 70.0 & Tarangkoon et al. (2010) \\
\hline eSNCM & dinoflagellates & $\begin{array}{l}\text { Histioneis spp, Parahistioneis spp, } \\
\text { Ornithocercus spp, Amphisolenia } \\
\text { spp, Tricosolenia sp, Citharistes sp }\end{array}$ & ISSG & -10.0 & 70.0 & Tarangkoon et al. (2010) \\
\hline eSNCM & dinoflagellates & $\begin{array}{l}\text { Histioneis spp, Parahistioneis spp, } \\
\text { Ornithocercus spp, Amphisolenia } \\
\text { spp, Tricosolenia sp, Citharistes sp }\end{array}$ & ISSG & -22.0 & 55.0 & Tarangkoon et al. (2010) \\
\hline eSNCM & dinoflagellates & $\begin{array}{l}\text { Histioneis spp, Parahistioneis spp, } \\
\text { Ornithocercus spp, Amphisolenia } \\
\text { spp, Tricosolenia sp, Citharistes sp }\end{array}$ & ISSG & -25.0 & 60.0 & Tarangkoon et al. (2010) \\
\hline eSNCM & dinoflagellates & $\begin{array}{l}\text { Histioneis spp, Parahistioneis spp, } \\
\text { Ornithocercus spp, Amphisolenia } \\
\text { spp, Tricosolenia sp, Citharistes sp }\end{array}$ & WARM & 0.0 & 160.0 & Tarangkoon et al. (2010) \\
\hline eSNCM & dinoflagellates & $\begin{array}{l}\text { Histioneis spp, Parahistioneis spp, } \\
\text { Ornithocercus spp, Amphisolenia } \\
\text { spp, Tricosolenia sp, Citharistes sp }\end{array}$ & WARM & 0.0 & 170.0 & Tarangkoon et al. (2010) \\
\hline eSNCM & dinoflagellates & $\begin{array}{l}\text { Histioneis spp, Parahistioneis spp, } \\
\text { Ornithocercus spp, Amphisolenia } \\
\text { spp, Tricosolenia sp, Citharistes sp }\end{array}$ & WARM & 0.0 & 175.0 & Tarangkoon et al. (2010) \\
\hline eSNCM & dinoflagellates & $\begin{array}{l}\text { Histioneis spp, Parahistioneis spp, } \\
\text { Ornithocercus spp, Amphisolenia } \\
\text { spp, Tricosolenia sp, Citharistes sp }\end{array}$ & FKLD & 29.0 & -159.0 & Tarangkoon et al. (2010) \\
\hline
\end{tabular}




\begin{tabular}{|c|c|c|c|c|c|c|}
\hline eSNCM & dinoflagellates & $\begin{array}{l}\text { Histioneis spp, Parahistioneis spp, } \\
\text { Ornithocercus spp, Amphisolenia } \\
\text { spp, Tricosolenia sp, Citharistes sp }\end{array}$ & FKLD & -52.0 & -59.0 & Tarangkoon et al. (2010) \\
\hline eSNCM & dinoflagellates & $\begin{array}{l}\text { Histioneis spp, Parahistioneis spp, } \\
\text { Ornithocercus spp, Amphisolenia } \\
\text { spp, Tricosolenia sp, Citharistes sp }\end{array}$ & SPSG & -20.0 & -135.0 & Tarangkoon et al. (2010) \\
\hline eSNCM & dinoflagellates & $\begin{array}{l}\text { Histioneis spp, Parahistioneis spp, } \\
\text { Ornithocercus spp, Amphisolenia } \\
\text { spp, Tricosolenia sp, Citharistes sp }\end{array}$ & SPSG & -19.0 & -132.0 & Tarangkoon et al. (2010) \\
\hline eSNCM & dinoflagellates & $\begin{array}{l}\text { Histioneis spp, Parahistioneis spp, } \\
\text { Ornithocercus spp, Amphisolenia } \\
\text { spp, Tricosolenia sp, Citharistes sp }\end{array}$ & SPSG & -18.0 & -129.0 & Tarangkoon et al. (2010) \\
\hline eSNCM & dinoflagellates & $\begin{array}{l}\text { Histioneis spp, Parahistioneis spp, } \\
\text { Ornithocercus spp, Amphisolenia } \\
\text { spp, Tricosolenia sp, Citharistes sp }\end{array}$ & SPSG & -17.0 & -126.0 & Tarangkoon et al. (2010) \\
\hline eSNCM & dinoflagellates & $\begin{array}{l}\text { Histioneis spp, Parahistioneis spp, } \\
\text { Ornithocercus spp, Amphisolenia } \\
\text { spp, Tricosolenia sp, Citharistes sp }\end{array}$ & SPSG & -16.0 & -123.0 & Tarangkoon et al. (2010) \\
\hline eSNCM & dinoflagellates & $\begin{array}{l}\text { Histioneis spp, Parahistioneis spp, } \\
\text { Ornithocercus spp, Amphisolenia } \\
\text { spp, Tricosolenia sp, Citharistes sp }\end{array}$ & SPSG & -15.0 & -120.0 & Tarangkoon et al. (2010) \\
\hline eSNCM & dinoflagellates & $\begin{array}{l}\text { Histioneis spp, Parahistioneis spp, } \\
\text { Ornithocercus spp, Amphisolenia } \\
\text { spp, Tricosolenia sp, Citharistes sp }\end{array}$ & SPSG & -14.0 & -117.0 & Tarangkoon et al. (2010) \\
\hline eSNCM & dinoflagellates & $\begin{array}{l}\text { Histioneis spp, Parahistioneis spp, } \\
\text { Ornithocercus spp, Amphisolenia } \\
\text { spp, Tricosolenia sp, Citharistes sp }\end{array}$ & SPSG & -13.0 & -114.0 & Tarangkoon et al. (2010) \\
\hline eSNCM & dinoflagellates & $\begin{array}{l}\text { Histioneis spp, Parahistioneis spp, } \\
\text { Ornithocercus spp, Amphisolenia } \\
\text { spp, Tricosolenia sp, Citharistes sp }\end{array}$ & SPSG & -12.0 & -110.0 & Tarangkoon et al. (2010) \\
\hline
\end{tabular}




\begin{tabular}{|c|c|c|c|c|c|c|}
\hline eSNCM & dinoflagellates & $\begin{array}{l}\text { Histioneis spp, Parahistioneis spp, } \\
\text { Ornithocercus spp, Amphisolenia } \\
\text { spp, Tricosolenia sp, Citharistes sp }\end{array}$ & SPSG & -11.0 & -107.0 & Tarangkoon et al. (2010) \\
\hline eSNCM & dinoflagellates & $\begin{array}{l}\text { Histioneis spp, Parahistioneis spp, } \\
\text { Ornithocercus spp, Amphisolenia } \\
\text { spp, Tricosolenia sp, Citharistes sp }\end{array}$ & PEQD & -10.0 & -104.0 & Tarangkoon et al. (2010) \\
\hline eSNCM & dinoflagellates & $\begin{array}{l}\text { Histioneis spp, Parahistioneis spp, } \\
\text { Ornithocercus spp, Amphisolenia } \\
\text { spp, Tricosolenia sp, Citharistes sp }\end{array}$ & SPSG & -30.0 & -101.0 & Tarangkoon et al. (2010) \\
\hline eSNCM & dinoflagellates & $\begin{array}{l}\text { Histioneis spp, Parahistioneis spp, } \\
\text { Ornithocercus spp, Amphisolenia } \\
\text { spp, Tricosolenia sp, Citharistes sp }\end{array}$ & SPSG & -28.0 & -98.0 & Tarangkoon et al. (2010) \\
\hline eSNCM & dinoflagellates & $\begin{array}{l}\text { Histioneis spp, Parahistioneis spp, } \\
\text { Ornithocercus spp, Amphisolenia } \\
\text { spp, Tricosolenia sp, Citharistes sp }\end{array}$ & SPSG & -26.0 & -95.0 & Tarangkoon et al. (2010) \\
\hline eSNCM & dinoflagellates & $\begin{array}{l}\text { Histioneis spp, Parahistioneis spp, } \\
\text { Ornithocercus spp, Amphisolenia } \\
\text { spp, Tricosolenia sp, Citharistes sp }\end{array}$ & SPSG & -24.0 & -92.0 & Tarangkoon et al. (2010) \\
\hline eSNCM & dinoflagellates & $\begin{array}{l}\text { Histioneis spp, Parahistioneis spp, } \\
\text { Ornithocercus spp, Amphisolenia } \\
\text { spp, Tricosolenia sp, Citharistes sp }\end{array}$ & SPSG & -22.0 & -95.0 & Tarangkoon et al. (2010) \\
\hline eSNCM & dinoflagellates & $\begin{array}{l}\text { Histioneis spp, Parahistioneis spp, } \\
\text { Ornithocercus spp, Amphisolenia } \\
\text { spp, Tricosolenia sp, Citharistes sp }\end{array}$ & PEQD & -9.0 & -98.0 & Tarangkoon et al. (2010) \\
\hline eSNCM & dinoflagellates & $\begin{array}{l}\text { Histioneis spp, Parahistioneis spp, } \\
\text { Ornithocercus spp, Amphisolenia } \\
\text { spp, Tricosolenia sp, Citharistes sp }\end{array}$ & PEQD & -8.0 & -96.0 & Tarangkoon et al. (2010) \\
\hline eSNCM & dinoflagellates & $\begin{array}{l}\text { Histioneis spp, Parahistioneis spp, } \\
\text { Ornithocercus spp, Amphisolenia } \\
\text { spp, Tricosolenia sp, Citharistes sp }\end{array}$ & PEQD & -7.0 & -94.0 & Tarangkoon et al. (2010) \\
\hline
\end{tabular}




\begin{tabular}{|c|c|c|c|c|c|c|}
\hline eSNCM & dinoflagellates & $\begin{array}{l}\text { Histioneis spp, Parahistioneis spp, } \\
\text { Ornithocercus spp, Amphisolenia } \\
\text { spp, Tricosolenia sp, Citharistes sp }\end{array}$ & PEQD & -6.0 & -92.0 & Tarangkoon et al. (2010) \\
\hline eSNCM & dinoflagellates & $\begin{array}{l}\text { Histioneis spp, Parahistioneis spp, } \\
\text { Ornithocercus spp, Amphisolenia } \\
\text { spp, Tricosolenia sp, Citharistes sp }\end{array}$ & PEQD & -4.0 & -90.0 & Tarangkoon et al. (2010) \\
\hline eSNCM & dinoflagellates & $\begin{array}{l}\text { Histioneis spp, Parahistioneis spp, } \\
\text { Ornithocercus spp, Amphisolenia } \\
\text { spp, Tricosolenia sp, Citharistes sp }\end{array}$ & HUMB & -2.0 & -88.0 & Tarangkoon et al. (2010) \\
\hline eSNCM & dinoflagellates & $\begin{array}{l}\text { Histioneis spp, Parahistioneis spp, } \\
\text { Ornithocercus spp, Amphisolenia } \\
\text { spp, Tricosolenia sp, Citharistes sp }\end{array}$ & CAMR & -1.0 & -86.0 & Tarangkoon et al. (2010) \\
\hline eSNCM & dinoflagellates & $\begin{array}{l}\text { Histioneis spp, Parahistioneis spp, } \\
\text { Ornithocercus spp, Amphisolenia } \\
\text { spp, Tricosolenia sp, Citharistes sp }\end{array}$ & CAMR & 0.0 & -84.0 & Tarangkoon et al. (2010) \\
\hline eSNCM & dinoflagellates & $\begin{array}{l}\text { Histioneis spp, Parahistioneis spp, } \\
\text { Ornithocercus spp, Amphisolenia } \\
\text { spp, Tricosolenia sp, Citharistes sp }\end{array}$ & PNEC & 15.0 & -100.0 & Tarangkoon et al. (2010) \\
\hline eSNCM & dinoflagellates & $\begin{array}{l}\text { Histioneis spp, Parahistioneis spp, } \\
\text { Ornithocercus spp, Amphisolenia } \\
\text { spp, Tricosolenia sp, Citharistes sp }\end{array}$ & PNEC & 13.5 & -102.0 & Tarangkoon et al. (2010) \\
\hline eSNCM & dinoflagellates & $\begin{array}{l}\text { Histioneis spp, Parahistioneis spp, } \\
\text { Ornithocercus spp, Amphisolenia } \\
\text { spp, Tricosolenia sp, Citharistes sp }\end{array}$ & PNEC & 12.0 & -104.0 & Tarangkoon et al. (2010) \\
\hline eSNCM & dinoflagellates & $\begin{array}{l}\text { Histioneis spp, Parahistioneis spp, } \\
\text { Ornithocercus spp, Amphisolenia } \\
\text { spp, Tricosolenia sp, Citharistes sp }\end{array}$ & PNEC & 10.5 & -106.0 & Tarangkoon et al. (2010) \\
\hline eSNCM & dinoflagellates & $\begin{array}{l}\text { Histioneis spp, Parahistioneis spp, } \\
\text { Ornithocercus spp, Amphisolenia } \\
\text { spp, Tricosolenia sp, Citharistes sp }\end{array}$ & PNEC & 9.0 & -108.0 & Tarangkoon et al. (2010) \\
\hline
\end{tabular}




\begin{tabular}{|c|c|c|c|c|c|c|}
\hline eSNCM & dinoflagellates & $\begin{array}{l}\text { Histioneis spp, Parahistioneis spp, } \\
\text { Ornithocercus spp, Amphisolenia } \\
\text { spp, Tricosolenia sp, Citharistes sp }\end{array}$ & PNEC & 7.5 & -110.0 & Tarangkoon et al. (2010) \\
\hline eSNCM & dinoflagellates & $\begin{array}{l}\text { Histioneis spp, Parahistioneis spp, } \\
\text { Ornithocercus spp, Amphisolenia } \\
\text { spp, Tricosolenia sp, Citharistes sp }\end{array}$ & PNEC & 6.0 & -112.0 & Tarangkoon et al. (2010) \\
\hline eSNCM & dinoflagellates & $\begin{array}{l}\text { Histioneis spp, Parahistioneis spp, } \\
\text { Ornithocercus spp, Amphisolenia } \\
\text { spp, Tricosolenia sp, Citharistes sp }\end{array}$ & PEQD & 4.5 & -114.0 & Tarangkoon et al. (2010) \\
\hline eSNCM & dinoflagellates & $\begin{array}{l}\text { Histioneis spp, Parahistioneis spp, } \\
\text { Ornithocercus spp, Amphisolenia } \\
\text { spp. Tricosolenia sp. Citharistes sp }\end{array}$ & PEQD & 3.0 & -116.0 & Tarangkoon et al. (2010) \\
\hline eSNCM & dinoflagellates & $\begin{array}{l}\text { Histioneis spp, Parahistioneis spp, } \\
\text { Ornithocercus spp, Amphisolenia } \\
\text { spp, Tricosolenia sp, Citharistes sp }\end{array}$ & PEQD & 1.5 & -118.0 & Tarangkoon et al. (2010) \\
\hline eSNCM & dinoflagellates & $\begin{array}{l}\text { Histioneis spp, Parahistioneis spp, } \\
\text { Ornithocercus spp, Amphisolenia } \\
\text { spp, Tricosolenia sp, Citharistes sp }\end{array}$ & PEQD & 0.0 & -119.0 & Tarangkoon et al. (2010) \\
\hline pSNCM & dinoflagellates & Dinophysis sacculus & MEDI & 43.0 & -8.0 & Riobó et al. (2013) \\
\hline $\mathrm{pSNCM}$ & dinoflagellates & Dinophysis acuta & MEDI & 43.0 & -8.0 & Hernández-Urcera et al. (2018) \\
\hline
\end{tabular}


Table A.2 Results from the bibliographic survey - Abundance and biomass data of the total ciliate assemblage and the relative contribution (\%) of heterotrophs (HC) and mixotrophs (GNCMs and red-pigmented Mesodinium (MES) spp.). Period of the year (season) and sampling depth (punctual or range) are also given. Geographic coordinates from each record (Table A.1) were used to identify the corresponding Longhurst's biogeographic province and biome (please refer to Chapter 2.3, Table 2.1 for province code); na - not applicable, nd - not determined.

\begin{tabular}{lllllllll}
\hline $\begin{array}{l}\text { Province } \\
\text { code }\end{array}$ & Season & $\begin{array}{l}\text { Sampling } \\
\text { depth }(\mathbf{m})\end{array}$ & $\begin{array}{l}\text { Total ciliate } \\
\text { abundance } \\
\left(\mathbf{c e l l} \mathbf{~ m L}^{-\mathbf{1}}\right)\end{array}$ & $\begin{array}{l}\text { Total ciliate } \\
\text { biomass } \\
\left(\mathbf{n g C} \mathbf{~ m L} \mathbf{~}^{-\mathbf{1}}\right)\end{array}$ & $\begin{array}{l}\text { HC } \\
(\boldsymbol{\%})\end{array}$ & $\begin{array}{l}\text { MES } \\
(\boldsymbol{\%})\end{array}$ & $\begin{array}{l}\text { GNCMs } \\
(\boldsymbol{\%})\end{array}$ & Reference \\
\hline MEDI & spring & surface & na & 15.9 & 23 & 14 & 64 & Bernard and Rassoulzadegan (1994) \\
MEDI & summer & surface & na & 10.6 & 13 & 25 & 62 & Bernard and Rassoulzadegan (1994) \\
MEDI & autumn & surface & na & 21.3 & 51 & 11 & 38 & Bernard and Rassoulzadegan (1994) \\
MEDI & winter & surface & na & 2.7 & 63 & 0 & 37 & Bernard and Rassoulzadegan (1994) \\
MEDI & summer & $0-200$ & 0.43 & na & 60 & nd & nd & Christaki et al. (2011) \\
NECS & spring & $0-50$ & 2083.33 & na & 39 & 1 & 60 & Dale and Dahl (1987) \\
CHIN & spring & $0-100$ & 0.00 & na & 60 & 0 & 40 & Chiang et al. (2003) \\
CHIN & summer & $0-100$ & 1.50 & na & 50 & 0 & 50 & Chiang et al. (2003) \\
CHIN & autumn & $0-100$ & 0.00 & na & 55 & 0 & 45 & Chiang et al. (2003) \\
CHIN & winter & $0-100$ & 0.00 & na & 90 & 0 & 10 & Chiang et al. (2003) \\
BPLR & summer & $0-35$ & na & 23.6 & 47 & 3 & 50 & Levinsen et al. (1999) \\
BPLR & spring & $0-35$ & na & 9.0 & 65 & 5 & 30 & Levinsen et al. (1999) \\
NECS & summer & $0-22$ & na & 74.6 & 33 & nd & nd & Mironova et al. (2013) \\
MEDI & summer & surface & na & 48.0 & 7 & 4 & 89 & Modigh (2001) \\
MEDI & spring & surface & na & 60.0 & 50 & nd & nd & Modigh (2001) \\
MEDI & autumn & surface & na & 14.0 & 50 & nd & nd & Modigh (2001) \\
MEDI & winter & surface & na & 12.0 & 53 & 40 & 7 & Modigh (2001) \\
CHIN & summer & $0-200$ & na & 61 mg C m ${ }^{-2}$ & 54 & 1 & 45 & Ota and Taniguchi (2003)
\end{tabular}




\begin{tabular}{|c|c|c|c|c|c|c|c|c|}
\hline CHIN & autumn & $0-200$ & na & $29 \mathrm{mg} \mathrm{C} \mathrm{m}^{-2}$ & 54 & 2 & 44 & Ota and Taniguchi (2003) \\
\hline CHIN & winter & $0-200$ & na & $42 \mathrm{mg} \mathrm{C} \mathrm{m}^{-2}$ & 77 & 4.5 & 19 & Ota and Taniguchi (2003) \\
\hline MEDI & spring & $0-100$ & na & 55.2 & 73 & 0 & 27 & Pitta and Giannakourou (2000) \\
\hline NWCS & spring & surface & 17.37 & na & 81 & 0 & 19 & Dolan and Gallegos (1992) \\
\hline SSTC & winter & surface & 0.10 & na & 90 & 0 & 10 & Froneman and Perissinotto (1996) \\
\hline PEQD & autumn & $0-120$ & na & 0.2 & 90 & 0 & 10 & Stoecker et al. (1996) \\
\hline PEQD & spring & $0-120$ & na & 0.1 & 75 & 0 & 25 & Stoecker et al. (1996) \\
\hline MEDI & spring & $0-50$ & 3.30 & na & 53 & 7 & 40 & Pérez et al. (2000) \\
\hline MEDI & spring & surface & na & 6.8 & 47 & 14 & 39 & Vaqué et al. (1997) \\
\hline MEDI & summer & surface & na & 6.2 & 65 & 10 & 25 & Vaqué et al. (1997) \\
\hline MEDI & autumn & surface & na & 11.7 & 83 & 11 & 6 & Vaqué et al. (1997) \\
\hline MEDI & winter & surface & na & 8.6 & 86 & 9 & 5 & Vaqué et al. (1997) \\
\hline MEDI & summer & surface & 0.63 & na & 30 & 7 & 63 & Dolan and Marrasé (1995) \\
\hline MEDI & spring & $0-80$ & na & 0.7 & 71 & nd & nd & Dolan et al. (1999) \\
\hline MEDI & spring & $0-80$ & na & 1.0 & 50 & nd & nd & Dolan et al. (1999) \\
\hline MEDI & spring & $0-80$ & na & 0.4 & 80 & nd & nd & Dolan et al. (1999) \\
\hline NWCS & spring & surface & 2.10 & na & 70 & 27 & 3 & Sanders (1995) \\
\hline NWCS & autumn & surface & 1.60 & na & 84 & 11 & 5 & Sanders (1995) \\
\hline NWCS & winter & surface & 1.00 & na & 53 & 45 & 2 & Sanders (1995) \\
\hline NWCS & spring & surface & 5.79 & na & 58 & 0 & 42 & Stoecker et al. (1987) \\
\hline NWCS & summer & surface & 4.00 & na & 10 & 0 & 90 & Stoecker et al. (1987) \\
\hline NWCS & autumn & surface & 15.00 & na & 90 & 0 & 10 & Stoecker et al. (1987) \\
\hline NWCS & winter & surface & 2.00 & na & 90 & 0 & 10 & Stoecker et al. (1987) \\
\hline NWCS & summer & $0-40$ & 5.38 & na & 65 & 0 & 35 & Stoecker et al. (1989a) \\
\hline NWCS & summer & $0-40$ & 1.90 & na & 52 & 2 & 46 & Stoecker et al. (1989a) \\
\hline NWCS & summer & $0-40$ & 12.97 & na & 61 & 16 & 23 & Stoecker et al. (1989a) \\
\hline NADR & spring & surface & 2.86 & na & 36 & 1 & 63 & Stoecker et al. (1994) \\
\hline APLR & spring & $0-150$ & na & no data & 74 & 1 & 25 & Stoecker et al. (1995) \\
\hline
\end{tabular}




\begin{tabular}{|c|c|c|c|c|c|c|c|c|}
\hline APLR & summer & $0-150$ & na & no data & 52 & 1 & 47 & Stoecker et al. (1995) \\
\hline APLR & spring & $0-150$ & 0.24 & na & 43 & 27 & 30 & Stoecker et al. (1995) \\
\hline APLR & summer & $0-150$ & 2.03 & na & 53 & 4 & 43 & Stoecker et al. (1995) \\
\hline BPLR & summer & $0-150$ & na & 9.1 & 42 & 34 & 24 & Jiang et al. (2015) \\
\hline $\mathrm{CHIN}$ & summer & $0-30$ & na & 4.5 & 60 & 20 & 20 & Wang et al. (2014) \\
\hline $\mathrm{CHIN}$ & spring & surface & na & 32.2 & 8 & 90 & 2 & Sun et al. (2013) \\
\hline MEDI & spring & $0-100$ & na & 29.8 & 38 & 0 & 62 & Pitta and Giannakourou (2000) \\
\hline SARC & summer & surface & 0.69 & na & 38 & nd & nd & Putt (1990) \\
\hline BERS & spring & surface & 6.00 & na & 40 & 30 & 30 & Sorokin et al. (1996) \\
\hline PSAG(E) & summer & $10-80$ & na & 3.6 & 60 & nd & 40 & Sime-Ngando et al. (1992) \\
\hline PSAG(W) & spring & no data & na & no data & 60 & nd & nd & Pitta and Giannakourou (2000) \\
\hline $\operatorname{PSAG}(\mathrm{W})$ & spring & $0-200$ & na & no data & 52 & nd & 48 & Suzuki and Taniguchi (1998) \\
\hline $\operatorname{PSAG}(\mathrm{W})$ & spring & $0-200$ & 0.99 & na & 67 & 5 & 28 & Suzuki and Taniguchi (1998) \\
\hline AUSE & spring & $0-200$ & 0.30 & na & 46 & 20 & 34 & Suzuki and Taniguchi (1998) \\
\hline KURO & summer & $0-200$ & 0.60 & na & 37 & 5 & 58 & Suzuki and Taniguchi (1998) \\
\hline KURO & spring & $0-200$ & 0.40 & na & 25 & 50 & 25 & Suzuki and Taniguchi (1998) \\
\hline KURO & autumn & $0-200$ & 1.00 & na & 60 & 30 & 10 & Suzuki and Taniguchi (1998) \\
\hline SANT & summer & $5-100$ & na & $30 \mathrm{mg} \mathrm{C} \mathrm{m}^{-2}$ & 40 & 0 & 60 & Christaki et al. (2008) \\
\hline SANT & summer & $5-100$ & na & $224 \mathrm{mg} \mathrm{C} \mathrm{m}^{-2}$ & 80 & 0 & 20 & Christaki et al. (2008) \\
\hline SANT & summer & $5-100$ & na & $90 \mathrm{mg} \mathrm{C} \mathrm{m}^{-2}$ & 60 & 0 & 40 & Christaki et al. (2008) \\
\hline NECS & spring & $0-130$ & 30.00 & na & 26 & 67 & 7 & Witek (1998) \\
\hline NECS & summer & $0-130$ & 8.00 & na & 35 & 15 & 50 & Witek (1998) \\
\hline NECS & winter & $0-130$ & 3.00 & na & 40 & 30 & 30 & Witek (1998) \\
\hline NECS & autumn & $0-130$ & 15.00 & na & 25 & 50 & 25 & Witek (1998) \\
\hline BERS & summer & surface & na & 14.0 & 34 & nd & 66 & Stoecker et al. (2014) \\
\hline MEDI & autumn & surface & no data & na & 60 & nd & 40 & Laval-Peuto and Rassoulzadegan (1988) \\
\hline MEDI & winter & surface & no data & na & 60 & nd & 40 & Laval-Peuto and Rassoulzadegan (1988) \\
\hline CHIN & summer & $0-40$ & na & 14.4 & 25 & nd & 75 & Kamiyama et al. (2003) \\
\hline
\end{tabular}




\begin{tabular}{|c|c|c|c|c|c|c|c|c|}
\hline TASM & spring & $0-200$ & na & 1.1 & 43 & nd & 57 & Suzuki et al. (1998) \\
\hline NAST(E) & spring & $0-150$ & na & 2.2 & 50 & 0 & 50 & Quevedo et al. (2003) \\
\hline NAST(W) & spring & $0-150$ & na & 0.9 & 50 & 0 & 50 & Quevedo et al. (2003) \\
\hline NATR & spring & surface & na & 0.1 & 96 & 4 & nd & Rychert et al. (2014) \\
\hline NATR & spring & surface & 0.33 & na & 94 & 6 & nd & Rychert et al. (2014) \\
\hline SATL & autumn & surface & na & 0.1 & 95 & 5 & nd & Rychert et al. (2014) \\
\hline SATL & autumn & surface & 0.30 & na & 93 & 7 & nd & Rychert et al. (2014) \\
\hline NECS & spring & surface & na & 1.3 & 15 & 85 & nd & Rychert et al. (2014) \\
\hline NECS & spring & surface & 1.50 & na & 27 & 73 & nd & Rychert et al. (2014) \\
\hline NAST(E) & spring & surface & na & 0.3 & 94 & 6 & nd & Rychert et al. (2014) \\
\hline NAST(E) & spring & surface & 0.06 & na & 92 & 8 & nd & Rychert et al. (2014) \\
\hline NECS & summer & $0-100$ & na & 8.0 & 70 & 30 & nd & Setälä and Kivi (2003) \\
\hline BRAZ & summer & surface & na & 2.4 & 96 & 4 & nd & McManus et al. (2007) \\
\hline NECS & summer & surface & na & 11.0 & 65 & 35 & nd & Yang et al. (2014) \\
\hline MEDI & summer & 10 & na & 0.6 & 75 & 25 & nd & Selifonova (2011) \\
\hline BPLR & winter & $2-15$ & 0.10 & na & 81 & 1 & 18 & Levinsen et al. (2000) \\
\hline BPLR & spring & $2-15$ & 4.00 & na & 61 & 2 & 37 & Levinsen et al. (2000) \\
\hline BPLR & summer & $2-15$ & 6.00 & na & 46 & 4 & 50 & Levinsen et al. (2000) \\
\hline BPLR & autumn & $2-15$ & 1.00 & na & 74 & 1 & 25 & Levinsen et al. (2000) \\
\hline BPLR & winter & $2-15$ & na & $26 \mathrm{mgC} \mathrm{m}^{-2}$ & 99 & 1 & nd & Levinsen et al. (2000) \\
\hline BPLR & spring & $2-15$ & na & $320 \mathrm{mgC} \mathrm{m}^{-2}$ & 93.8 & 6.2 & nd & Levinsen et al. (2000) \\
\hline BPLR & summer & $2-15$ & na & $550 \mathrm{mgC} \mathrm{m}^{-2}$ & 90 & 10 & nd & Levinsen et al. (2000) \\
\hline BPLR & autumn & $2-15$ & na & $34 \mathrm{mgC} \mathrm{m}^{-2}$ & 94 & 6 & nd & Levinsen et al. (2000) \\
\hline NECS & winter & 28 & na & 7.1 & 47 & 42 & 11 & Nielsen and Kiørboe (1994) \\
\hline NECS & spring & 28 & na & 37.5 & 15 & 80 & 5 & Nielsen and Kiørboe (1994) \\
\hline NECS & summer & 28 & na & 10.8 & 44 & 46 & 9 & Nielsen and Kiørboe (1994) \\
\hline NECS & autumn & 28 & na & 3.9 & 62 & 26 & 13 & Nielsen and Kiørboe (1994) \\
\hline
\end{tabular}




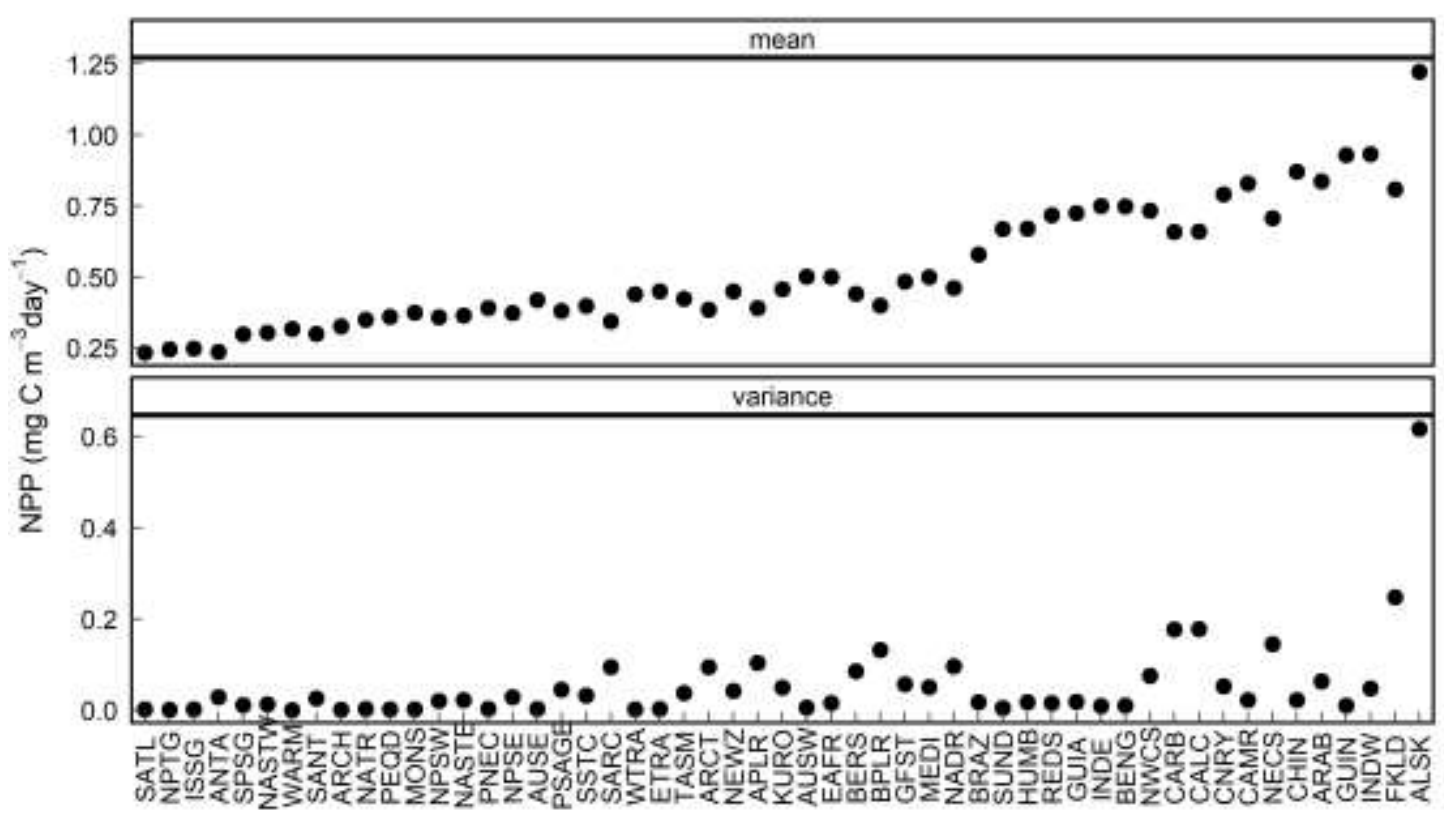

Figure A.1 Net primary productivity (NPP) data among the different biogeographic provinces compiled from Longhurst (2007). Mean and variance correspond to monthly values from 1997-2002. 


\section{Appendix B}

Table B.1 List of CM species including species name, species synonyms/basionyms, species name in the original publication, type of evidence for mixotrophy, and reference to original source(s).

\begin{tabular}{|c|c|c|c|c|c|}
\hline Group & $\begin{array}{l}\text { Current species } \\
\text { name }\end{array}$ & $\begin{array}{l}\text { Synonyms / } \\
\text { Basyonyms }\end{array}$ & $\begin{array}{l}\text { Species name in the } \\
\text { original publication }\end{array}$ & $\begin{array}{l}\text { type of evidence of } \\
\text { mixotrophy }\end{array}$ & Reference \\
\hline Dinophyceae & Akashiwo sanguinea & $\begin{array}{l}\text { Gymnodinium } \\
\text { sanguineum }\end{array}$ & $\begin{array}{l}\text { Gymnodinium } \\
\text { sanguineum }\end{array}$ & ingestion of ciliates & $\begin{array}{l}\text { Bockstahler and Coasts } \\
\text { (1993) }\end{array}$ \\
\hline Dinophyceae & Alexandrium affine & $\begin{array}{l}\text { Protogonyaulax } \\
\text { affinis }\end{array}$ & Alexandrium affine & not informed & Jeong et al. (2010c) \\
\hline Dinophyceae & $\begin{array}{l}\text { Alexandrium } \\
\text { andersonii }\end{array}$ & na & $\begin{array}{l}\text { Alexandrium } \\
\text { andersonii }\end{array}$ & $\begin{array}{l}\text { ingestion of } \\
\text { Pyramimonas sp., } \\
\text { Teleaulax sp., } \\
\text { Heterocapsa } \\
\text { rotundata, }\end{array}$ & Lee et al. (2016) \\
\hline Dinophyceae & $\begin{array}{l}\text { Alexandrium } \\
\text { catenella }\end{array}$ & $\begin{array}{l}\text { Gonyaulax } \\
\text { catenella, } \\
\text { Protogonyaulax } \\
\text { catenella, } \\
\text { Gessnerium } \\
\text { catenella, } \\
\text { Gonyaulax } \\
\text { washingtonensis, } \\
\text { Alexandrium } \\
\text { fundyense }\end{array}$ & $\begin{array}{l}\text { Alexandrium } \\
\text { tamarense }\end{array}$ & $\begin{array}{l}\text { ingestion of } \\
\text { Skeletonema } \\
\text { costatum }\end{array}$ & Yoo et al. (2009) \\
\hline Dinophyceae & $\begin{array}{l}\text { Alexandrium } \\
\text { minutum }\end{array}$ & $\begin{array}{l}\text { Pyrodinium } \\
\text { minutum, } \\
\text { Alexandrium }\end{array}$ & $\begin{array}{l}\text { Alexandrium } \\
\text { minutum }\end{array}$ & $\begin{array}{l}\text { ingestion of } \\
\text { Synechococcus }\end{array}$ & Jeong et al. (2005a) \\
\hline
\end{tabular}




\begin{tabular}{|c|c|c|c|c|c|}
\hline & & $\begin{array}{l}\text { ibericum, } \\
\text { Alexandrium } \\
\text { lusitanicum, } \\
\text { Alexandrium } \\
\text { angustitabulatum }\end{array}$ & & & \\
\hline Dinophyceae & $\begin{array}{l}\text { Alexandrium } \\
\text { ostenfeldii }\end{array}$ & $\begin{array}{l}\text { Goniodoma } \\
\text { ostenfeldii, } \\
\text { Gonyaulax } \\
\text { ostenfeldii, } \\
\text { Heteraulacus } \\
\text { ostenfeldii, } \\
\text { Gessnerium } \\
\text { ostenfeldii, } \\
\text { Triadinium } \\
\text { ostenfeldii, } \\
\text { Protogonyaulax } \\
\text { ostenfeldii }\end{array}$ & $\begin{array}{l}\text { Alexandrium } \\
\text { ostenfeldii }\end{array}$ & ingestion of ciliates & $\begin{array}{l}\text { Jacobson and Anderson } \\
\text { (1996) }\end{array}$ \\
\hline Dinophyceae & $\begin{array}{l}\text { Alexandrium } \\
\text { pohangense }\end{array}$ & na & $\begin{array}{l}\text { Alexandrium } \\
\text { pohangense }\end{array}$ & $\begin{array}{l}\text { ingestion of } \\
\text { Cochlodinium } \\
\text { polykrikoides }\end{array}$ & Lim et al. (2015) \\
\hline Dinophyceae & $\begin{array}{l}\text { Alexandrium } \\
\text { pseudogonyaulax }\end{array}$ & na & $\begin{array}{l}\text { Alexandrium } \\
\text { pseudogonyaulax }\end{array}$ & $\begin{array}{l}\text { ingestion of Teleaulax } \\
\text { acuta }\end{array}$ & Blossom et al. (2017) \\
\hline Dinophyceae & $\begin{array}{l}\text { Amphidinium } \\
\text { carterae }\end{array}$ & $\begin{array}{l}\text { Amphidinium } \\
\text { microvephalum, } \\
\text { Amphidinium klebsii }\end{array}$ & $\begin{array}{l}\text { Amphidinium } \\
\text { carterae }\end{array}$ & $\begin{array}{l}\text { ingestion of } \\
\text { Skeletonema } \\
\text { costatum }\end{array}$ & Yoo et al. (2009) \\
\hline Raphidophyceae & $\begin{array}{l}\text { Chattonella marina } \\
\text { var. ovata }\end{array}$ & Chattonella ovata & Chattonella ovata & uptake of eubacteria & Seong et al. (2006) \\
\hline Raphidophyceae & Chattonella subsalsa & na & Chattonella subsalsa & $\begin{array}{l}\text { uptake of } \\
\text { Synechococcus }\end{array}$ & Jeong et al. (2010b) \\
\hline Haptophyte & $\begin{array}{l}\text { Chrysochromulina } \\
\text { brevifilum }\end{array}$ & na & $\begin{array}{l}\text { Chrysochromulina } \\
\text { brevifilum }\end{array}$ & $\begin{array}{l}\text { ingestion of } \\
\text { Marsupiomonas } \\
\text { pelliculata }\end{array}$ & Jones et al. (1993) \\
\hline
\end{tabular}


Appendices. Appendix B, Table B.I

\begin{tabular}{|c|c|c|c|c|c|}
\hline Haptophyte & Haptolina ericina & $\begin{array}{l}\text { Chrysochromulina } \\
\text { ericina }\end{array}$ & $\begin{array}{l}\text { Chrysochromulina } \\
\text { ericina }\end{array}$ & $\begin{array}{l}\text { ingestion of } \\
\text { Marsupiomonas } \\
\text { pelliculata }\end{array}$ & $\begin{array}{l}\text { Hansen and Hjorth } \\
(2002)\end{array}$ \\
\hline Haptophyte & Haptolina hirta & $\begin{array}{l}\text { Chrysochromulina } \\
\text { hirta }\end{array}$ & $\begin{array}{l}\text { Chrysochromulina } \\
\text { hirta }\end{array}$ & $\begin{array}{l}\text { ingestion of bacteria- } \\
\text { sized microspheres, } \\
\text { Nannochlropsis } \\
\text { oculata, Micromonas } \\
\text { Pusilla, and small- } \\
\text { sized Coscinodiscus } \\
\text { sp. }\end{array}$ & Kawachi et al. (1991) \\
\hline Haptophyte & $\begin{array}{l}\text { Chrysochromulina } \\
\text { leadbeateri }\end{array}$ & na & $\begin{array}{l}\text { Chrysochromulina } \\
\text { leadbeateri }\end{array}$ & $\begin{array}{l}\text { enhanced growth with } \\
\text { high concentrations of } \\
\text { putracine simulating } \\
\text { conditions in fish } \\
\text { kills; and observation } \\
\text { of uptake of } \\
\text { chloroplast fragments }\end{array}$ & Burkholder et al. (2008) \\
\hline Haptophyte & $\begin{array}{l}\text { Prymnesium } \\
\text { polylepis }\end{array}$ & $\begin{array}{l}\text { Chrysochromulina } \\
\text { polylepis }\end{array}$ & $\begin{array}{l}\text { Chrysochromulina } \\
\text { polylepis }\end{array}$ & $\begin{array}{l}\text { ingestion of } \\
\text { eubacteria }\end{array}$ & $\begin{array}{l}\text { Nygaard and Tobiesen } \\
\text { (1993) }\end{array}$ \\
\hline Dinophyceae & $\begin{array}{l}\text { Margalefidinium } \\
\text { polikrikoides }\end{array}$ & $\begin{array}{l}\text { Cochlodinium } \\
\text { polykrikoides }\end{array}$ & $\begin{array}{l}\text { Cochlodinium } \\
\text { polykrikoides }\end{array}$ & $\begin{array}{l}\text { ingestion of } \\
\text { criptophytes, } \\
\text { dinoflagellates, } \\
\text { Synechococcus, } \\
\text { eubacteria }\end{array}$ & Jeong et al. (2004a) \\
\hline Chrysophyte & $\begin{array}{l}\text { Dinobryon } \\
\text { faculiferum }\end{array}$ & $\begin{array}{l}\text { Dinobryon } \\
\text { petiolatum }\end{array}$ & $\begin{array}{l}\text { Dinobryon } \\
\text { faculiferum }\end{array}$ & ingestion of bacteria & Unrein et al. (2010) \\
\hline Dinophyceae & $\begin{array}{l}\text { Fragilidium } \\
\text { duplocampanaeforme }\end{array}$ & na & $\begin{array}{l}\text { Fragilidium } \\
\text { duplocampanaeforme }\end{array}$ & $\begin{array}{l}\text { ingestion of } \\
\text { Dinophysis sp. }\end{array}$ & $\begin{array}{l}\text { Nézan and Chomérat } \\
(2009)\end{array}$ \\
\hline Dinophyceae & $\begin{array}{l}\text { Fragilidium } \\
\text { mexicanum }\end{array}$ & na & $\begin{array}{l}\text { Fragilidium } \\
\text { mexicanum }\end{array}$ & $\begin{array}{l}\text { ingestion of } \\
\text { Protoperidinium cf. } \\
\text { divergens }\end{array}$ & Jeong et al. (1997) \\
\hline
\end{tabular}


Appendices. Appendix B, Table B.I

\begin{tabular}{|c|c|c|c|c|c|}
\hline Dinophyceae & $\begin{array}{l}\text { Fragilidium } \\
\text { subglobosum }\end{array}$ & $\begin{array}{l}\text { Helgolandinium } \\
\text { subglobosum }\end{array}$ & $\begin{array}{l}\text { Fragilidium } \\
\text { subglobosum }\end{array}$ & $\begin{array}{l}\text { ingestion of Ceratium } \\
\text { spp. }\end{array}$ & Skovgaard (1996) \\
\hline Dinophyceae & $\begin{array}{l}\text { Gambierdiscus } \\
\text { toxicus }\end{array}$ & na & $\begin{array}{l}\text { Gambierdiscus } \\
\text { toxicus }\end{array}$ & $\begin{array}{l}\text { presence of feeding } \\
\text { vacuoles, unknown } \\
\text { prey }\end{array}$ & Burkholder et al. (2008) \\
\hline Cryptophyte & $\begin{array}{l}\text { Geminigera } \\
\text { cryophila }\end{array}$ & $\begin{array}{l}\text { Cryptomonas } \\
\text { cryophila }\end{array}$ & $\begin{array}{l}\text { Geminigera } \\
\text { cryophila }\end{array}$ & $\begin{array}{l}\text { ingestion of } \\
\text { Paraphysomonas } \\
\text { antarctica }\end{array}$ & $\begin{array}{l}\text { McKie-Krisberg et al. } \\
(2015)\end{array}$ \\
\hline Dinophyceae & Gonyaulax diegensis & na & Gonyaulax diegensis & ingestion of ciliates & $\begin{array}{l}\text { Jacobson and Anderson } \\
(1996)\end{array}$ \\
\hline Dinophyceae & $\begin{array}{l}\text { Gonyaulax } \\
\text { polygramma }\end{array}$ & Gonyaulax schuettii & $\begin{array}{l}\text { Gonyaulax } \\
\text { polygramma }\end{array}$ & $\begin{array}{l}\text { ingestion of } \\
\text { cryptophyte species, } \\
\text { Amphidinium } \\
\text { carterae, Heterosigma } \\
\text { akashiwo, } \\
\text { Heterocapsa triquetra, } \\
\text { Isochrysis galbana, } \\
\text { Prorocentrum } \\
\text { minimum, } \\
\text { Scrippsiella sp. }\end{array}$ & Jeong et al. (2005c) \\
\hline Dinophyceae & Gonyaulax spinifera & $\begin{array}{l}\text { Peridinium } \\
\text { spiniferum, } \\
\text { Peridinium } \\
\text { levanderi, } \\
\text { Spiniferites } \\
\text { ramosus, Gonyaulax } \\
\text { levanderi, } \\
\text { Tectatodinium } \\
\text { pellitum }\end{array}$ & Gonyaulax spinifera & $\begin{array}{l}\text { ingestion of } \\
\text { Synechococcus }\end{array}$ & Jeong et al. (2005a) \\
\hline Dinophyceae & $\begin{array}{l}\text { Gymnodinium } \\
\text { aureolum }\end{array}$ & $\begin{array}{l}\text { Gyrodinium } \\
\text { aureolum, Karenia } \\
\text { aureola }\end{array}$ & $\begin{array}{l}\text { Gymnodinium } \\
\text { aureolum }\end{array}$ & $\begin{array}{l}\text { ingestion of } \\
\text { heterotrophic } \\
\text { bacteria, }\end{array}$ & Jeong et al. (2010a) \\
\hline
\end{tabular}


Appendices. Appendix B, Table B.I

\begin{tabular}{|c|c|c|c|c|c|}
\hline & & & & $\begin{array}{l}\text { Synechococcus sp., } \\
\text { nanophytoflagellates }\end{array}$ & \\
\hline Dinophyceae & $\begin{array}{l}\text { Gymnodinium } \\
\text { catenatum }\end{array}$ & na & $\begin{array}{l}\text { Gymnodinium } \\
\text { catenatum }\end{array}$ & $\begin{array}{l}\text { ingestion of } \\
\text { Synechococcus/food } \\
\text { vacuoles containing } \\
\text { Amphidinium } \\
\text { carterae, unidentified } \\
\text { cryptophytes, } \\
\text { Heterosigma } \\
\text { akashiwo, Isochrysis } \\
\text { galbana, Rhodomonas } \\
\text { salina, Prorocentrum } \\
\text { minimum }\end{array}$ & Jeong et al. (2005b) \\
\hline Dinophyceae & $\begin{array}{l}\text { Gymnodinium } \\
\text { impudicum }\end{array}$ & $\begin{array}{l}\text { Gyrodinium } \\
\text { impudicum }\end{array}$ & $\begin{array}{l}\text { Gymnodinium } \\
\text { impudicum }\end{array}$ & $\begin{array}{l}\text { ingestion of } \\
\text { Synechococcus/Amph } \\
\text { idinium carterae, } \\
\text { unidentified } \\
\text { cryptophytes, } \\
\text { Hetersigma akashiwo, } \\
\text { Prorocentrum } \\
\text { minimum, } \\
\text { Rhodmonas salina }\end{array}$ & Jeong et al. (2005b) \\
\hline Dinophyceae & Levanderina fissa & $\begin{array}{l}\text { Gymnodinium } \\
\text { uncatenum, } \\
\text { Gyrodinium } \\
\text { uncatenum, } \\
\text { Gyrodinium } \\
\text { instriatum, } \\
\text { Gyrodinium } \\
\text { pavillardi } \\
\end{array}$ & $\begin{array}{l}\text { Gymnodinium } \\
\text { uncatenum }\end{array}$ & ingestion of ciliates & $\begin{array}{l}\text { Bockstahler and Coasts } \\
\text { (1993) }\end{array}$ \\
\hline
\end{tabular}


Appendices. Appendix B, Table B.I

\begin{tabular}{|c|c|c|c|c|c|}
\hline Dinophyceae & $\begin{array}{l}\text { Karlodinium } \\
\text { veneficum }\end{array}$ & $\begin{array}{l}\text { Gymnodinium } \\
\text { veneficum, } \\
\text { Gymnodinium } \\
\text { galatheanum, } \\
\text { Gymnodinium } \\
\text { micrum, } \\
\text { Gyrodinium } \\
\text { galatheanum, } \\
\text { Woloszynskia micra, } \\
\text { Karlodinium } \\
\text { micrum, } \\
\text { Gymnodinium } \\
\text { eusturiale }\end{array}$ & $\begin{array}{l}\text { Gyrodinium } \\
\text { galatheanum }\end{array}$ & $\begin{array}{l}\text { ingestion of various } \\
\text { cryptophytes/ingestio } \\
n \text { of eubacteria }\end{array}$ & Li et al. (1996) \\
\hline Dinophyceae & $\begin{array}{l}\text { Barrufeta } \\
\text { resplendens }\end{array}$ & $\begin{array}{l}\text { Gyrodinium } \\
\text { resplendens }\end{array}$ & $\begin{array}{l}\text { Gyrodinium } \\
\text { resplendens }\end{array}$ & $\begin{array}{l}\text { ingestion of } \\
\text { Prorocentrum } \\
\text { minimum }\end{array}$ & Skovgaard (2000) \\
\hline Dinophyceae & $\begin{array}{l}\text { Heterocapsa } \\
\text { circularisquama }\end{array}$ & na & $\begin{array}{l}\text { Heterocapsa } \\
\text { circularisquama }\end{array}$ & not informed & Yamaguchi et al. (1997) \\
\hline Dinophyceae & $\begin{array}{l}\text { Heterocapsa } \\
\text { rotundata }\end{array}$ & $\begin{array}{l}\text { Amphidinium } \\
\text { rotundatum }\end{array}$ & $\begin{array}{l}\text { Heterocapsa } \\
\text { rotundata }\end{array}$ & $\begin{array}{l}\text { ingestion of } \\
\text { Skeletonema } \\
\text { costatum }\end{array}$ & Yoo et al. (2009) \\
\hline Dinophyceae & $\begin{array}{l}\text { Heterocapsa } \\
\text { triquetra }\end{array}$ & $\begin{array}{l}\text { Glenodinium } \\
\text { triquetrum, } \\
\text { Peridinium } \\
\text { triquetra, } \\
\text { Properidinium } \\
\text { heterocapsa, } \\
\text { Peridinium } \\
\text { triquetrum }\end{array}$ & $\begin{array}{l}\text { Heterocapsa } \\
\text { triquetra }\end{array}$ & $\begin{array}{l}\text { ingestion of } \\
\text { Thalassiosira } \\
\text { pseudonana }\end{array}$ & Legrand et al. (1998) \\
\hline Dinophyceae & $\begin{array}{l}\text { Heterosigma } \\
\text { akashiwo }\end{array}$ & $\begin{array}{l}\text { Entomosigma } \\
\text { akashiwo, } \\
\text { Chattonella }\end{array}$ & $\begin{array}{l}\text { Heterosigma } \\
\text { akashiwo }\end{array}$ & uptake of eubacteria & $\begin{array}{l}\text { Nygaard and Tobiesen } \\
\text { (1993) }\end{array}$ \\
\hline
\end{tabular}




\begin{tabular}{|c|c|c|c|c|c|}
\hline & & $\begin{array}{l}\text { akashiwo, } \\
\text { Olisthodiscus } \\
\text { carterae, } \\
\text { Heterosigma } \\
\text { inlandica, } \\
\text { Heterosigma } \\
\text { carterae }\end{array}$ & & & \\
\hline Dinophyceae & Karenia brevis & $\begin{array}{l}\text { Gymnodinium } \\
\text { breve, Ptychodiscus } \\
\text { brevis }\end{array}$ & Karenia brevis & $\begin{array}{l}\text { ingestion of } \\
\text { Synechococcus }\end{array}$ & Glibert et al. (2009) \\
\hline Dinophyceae & Karenia mikimotoi & $\begin{array}{l}\text { Gymnodinium } \\
\text { mikimotoi }\end{array}$ & Karenia mikimotoi & $\begin{array}{l}\text { ingestion of } \\
\text { fluorescent } \\
\text { microspheres, } \\
\text { heterotrophic } \\
\text { bacteria, Isochysis } \\
\text { galbana }\end{array}$ & Zhang et al. (2011) \\
\hline Dinophyceae & Karlodinium armiger & na & Karlodinium armiger & $\begin{array}{l}\text { ingestion of } \\
\text { Heterocapsa triquetra } \\
\text { and Rhodomonas } \\
\text { salina }\end{array}$ & Berge et al. (2008) \\
\hline Dinophyceae & $\begin{array}{l}\text { Lingulodinium } \\
\text { polyedra }\end{array}$ & $\begin{array}{l}\text { Gonyaulax } \\
\text { polyedra, } \\
\text { Lingulodinium } \\
\text { machaerophorum }\end{array}$ & $\begin{array}{l}\text { Lingulodinium } \\
\text { polyedrum }\end{array}$ & $\begin{array}{l}\text { ingestion of } \\
\text { Skeletonema } \\
\text { costatum }\end{array}$ & Yoo et al. (2009) \\
\hline Prasinophyte & $\begin{array}{l}\text { Mantoniella } \\
\text { antarctica }\end{array}$ & na & $\begin{array}{l}\text { Mantoniella } \\
\text { antarctica }\end{array}$ & $\begin{array}{l}\text { ingestion of bacteria- } \\
\text { sized fluorescent } \\
\text { microspheres }\end{array}$ & $\begin{array}{l}\text { McKie-Krisberg et al. } \\
\text { (2015) }\end{array}$ \\
\hline Prasinophyte & Micromonas spp & na & Micromonas spp & $\begin{array}{l}\text { ingestion of bacteria- } \\
\text { sized fluorescent } \\
\text { microspheres }\end{array}$ & $\begin{array}{l}\text { McKie-Krisberg and } \\
\text { Sanders (2014) }\end{array}$ \\
\hline Dinophyceae & Tripos furca & $\begin{array}{l}\text { Neoceratium furca, } \\
\text { Peridinium furca, }\end{array}$ & Ceratium furca & $\begin{array}{l}\text { ingestion of oligotrich } \\
\text { ciliates }\end{array}$ & $\begin{array}{l}\text { Smalley and Coats } \\
(2002)\end{array}$ \\
\hline
\end{tabular}


Appendices. Appendix B, Table B.I

\begin{tabular}{|c|c|c|c|c|c|}
\hline & & $\begin{array}{l}\text { Ceratophorus furca, } \\
\text { Biceratium furca, } \\
\text { Ceratium furca }\end{array}$ & & & \\
\hline Dinophyceae & Tripos longipes & $\begin{array}{l}\text { Ceratium arcticum, } \\
\text { Peridinium } \\
\text { longipes, Ceratium } \\
\text { longipes, } \\
\text { Neoceratium } \\
\text { longipes }\end{array}$ & Ceratium longipes & $\begin{array}{l}\text { presence of feeding } \\
\text { vacuoles, unknown } \\
\text { prey }\end{array}$ & $\begin{array}{l}\text { Jacobson and Anderson } \\
\text { (1996) }\end{array}$ \\
\hline Chrysophyte & Ochromonas sp & na & Ochromonas sp & $\begin{array}{l}\text { presence of feeding } \\
\text { vacuoles, unknown } \\
\text { prey }\end{array}$ & Andersson et al. (1989) \\
\hline Dinophyceae & $\begin{array}{l}\text { Ostreopsis } \\
\text { lenticularis }\end{array}$ & na & $\begin{array}{l}\text { Ostreopsis } \\
\text { lenticularis }\end{array}$ & $\begin{array}{l}\text { presence of feeding } \\
\text { vacuoles, unknown } \\
\text { prey }\end{array}$ & Burkholder et al. (2008) \\
\hline Dinophyceae & Ostreopsis ovata & na & Ostreopsis ovata & $\begin{array}{l}\text { presence of feeding } \\
\text { vacuoles, unknown } \\
\text { prey }\end{array}$ & Burkholder et al. (2008) \\
\hline Dinophyceae & Ostreopsis siamensis & na & Ostreopsis siamensis & $\begin{array}{l}\text { presence of feeding } \\
\text { vacuoles, unknown } \\
\text { prey }\end{array}$ & Burkholder et al. (2008) \\
\hline Dinophyceae & $\begin{array}{l}\text { Paragymnodinium } \\
\text { shiwhaense }\end{array}$ & na & $\begin{array}{l}\text { Paragymnodinium } \\
\text { shiwhaense }\end{array}$ & not informed & Kang et al. (2010) \\
\hline Dinophyceae & $\begin{array}{l}\text { Polykrikos } \\
\text { hartmannii }\end{array}$ & $\begin{array}{l}\text { Pheopolykrikos } \\
\text { hartmannii }\end{array}$ & $\begin{array}{l}\text { Polykrikos } \\
\text { hartmannii }\end{array}$ & $\begin{array}{l}\text { ingestion of } \\
\text { Cochlodinium } \\
\text { polykrokoides and } \\
\text { Gymnodinium } \\
\text { catenatum }\end{array}$ & Lee et al. (2015) \\
\hline Dinophyceae & $\begin{array}{l}\text { Prorocentrum } \\
\text { belizeanum }\end{array}$ & na & $\begin{array}{l}\text { Prorocentrum } \\
\text { belizeanum }\end{array}$ & $\begin{array}{l}\text { presence of feeding } \\
\text { vacuoles, unknown } \\
\text { prey }\end{array}$ & Burkholder et al. (2008) \\
\hline
\end{tabular}


Appendices. Appendix B, Table B.I

\begin{tabular}{|c|c|c|c|c|c|}
\hline Dinophyceae & $\begin{array}{l}\text { Prorocentrum } \\
\text { donghaiense }\end{array}$ & na & $\begin{array}{l}\text { Prorocentrum } \\
\text { donghaiense }\end{array}$ & $\begin{array}{l}\text { ingestion of } \\
\text { Amphidinium } \\
\text { carterae, unidentified } \\
\text { cryptophytes, } \\
\text { Heterosigma } \\
\text { akashiwo, Isochrysis } \\
\text { galbana, } \\
\text { Prorocentrum } \\
\text { minimum, } \\
\text { Prorocentrum } \\
\text { triestinum, } \\
\text { Rhodomonas salina }\end{array}$ & Jeong et al. (2005b) \\
\hline Dinophyceae & Prorocentrum lima & $\begin{array}{l}\text { Prorocentrum } \\
\text { arenarium }\end{array}$ & $\begin{array}{l}\text { Prorocentrum } \\
\text { arenarium }\end{array}$ & $\begin{array}{l}\text { presence of feeding } \\
\text { vacuoles, unknown } \\
\text { prey }\end{array}$ & Burkholder et al. (2008) \\
\hline Dinophyceae & $\begin{array}{l}\text { Prorocentrum } \\
\text { micans }\end{array}$ & $\begin{array}{l}\text { Prorocentrum } \\
\text { schilleri, } \\
\text { Prorocentrum } \\
\text { levantinoides, } \\
\text { Prorocentrum } \\
\text { pacificum }\end{array}$ & $\begin{array}{l}\text { Prorocentrum } \\
\text { micans }\end{array}$ & $\begin{array}{l}\text { consumed Isochrysis } \\
\text { galbana, Heterosigma } \\
\text { akashiwo, } \\
\text { Rhodomonas salina } \\
\text { and a second } \\
\text { unidenitifed } \\
\text { cryptophyte species, } \\
\text { Amphidinium } \\
\text { carterae, Heterocapsa } \\
\text { triquestra, } \\
\text { Prorocentrum } \\
\text { minimum }\end{array}$ & Jeong et al. (2005b) \\
\hline Dinophyceae & $\begin{array}{l}\text { Prorocentrum } \\
\text { cordatum }\end{array}$ & $\begin{array}{l}\text { Exuviaella minima, } \\
\text { Exuviaella } \\
\text { pyriformis, } \\
\text { Prorocentrum } \\
\text { minimum, }\end{array}$ & $\begin{array}{l}\text { Prorocentrum } \\
\text { minimum }\end{array}$ & $\begin{array}{l}\text { ingestion of } \\
\text { Amphidinium } \\
\text { carterae, unidentified } \\
\text { cryptophytes, } \\
\text { Heterosigma }\end{array}$ & Jeong et al. (2005b) \\
\hline
\end{tabular}




\begin{tabular}{|c|c|c|c|c|c|}
\hline & & $\begin{array}{l}\text { Exuviaella peisonis, } \\
\text { Exuviaella pacifica }\end{array}$ & & $\begin{array}{l}\text { akashiwo, Isochrysis } \\
\text { galbana, Rhodomonas } \\
\text { salina }\end{array}$ & \\
\hline Dinophyceae & $\begin{array}{l}\text { Prorocentrum } \\
\text { triestinum }\end{array}$ & $\begin{array}{l}\text { Prorocentrum } \\
\text { redfieldii, } \\
\text { Prorocentrum } \\
\text { pyrenoideum, } \\
\text { Prorocentrum } \\
\text { setoutii }\end{array}$ & $\begin{array}{l}\text { Prorocentrum } \\
\text { triestinum }\end{array}$ & $\begin{array}{l}\text { ingestion of } \\
\text { eubacteria / ingestion } \\
\text { of Amphidinum } \\
\text { carterae, unidentified } \\
\text { cryptophytes, } \\
\text { Heterosigma } \\
\text { akashiwo, Isochrysis } \\
\text { galbana, } \\
\text { Prorocentrum } \\
\text { minimum, } \\
\text { Rhodomonas salina }\end{array}$ & Jeong et al. (2005b) \\
\hline Haptophyte & Prymnesium parvum & na & Prymnesium parvum & $\begin{array}{l}\text { ingestion of Oxyrrhis } \\
\text { marina, unknown } \\
\text { chlorococcales, } \\
\text { Dunaliella sp., } \\
\text { Gyrodinium sp., } \\
\text { unknown amoeba }\end{array}$ & Tillmann (1998) \\
\hline Prasinophyte & $\begin{array}{l}\text { Pyramimonas } \\
\text { gelidicola }\end{array}$ & na & $\begin{array}{l}\text { Pyramimonas } \\
\text { gelidicola }\end{array}$ & $\begin{array}{l}\text { ingestion of } \\
\text { fluorescent labelled } \\
\text { bacteria }\end{array}$ & $\begin{array}{l}\text { Bell and Laybourn- } \\
\text { Parry (2003) }\end{array}$ \\
\hline Prasinophyte & $\begin{array}{l}\text { Pyramimonas } \\
\text { tychotreta }\end{array}$ & na & $\begin{array}{l}\text { Pyramimonas } \\
\text { tychotreta }\end{array}$ & $\begin{array}{l}\text { ingestion of bacteria- } \\
\text { sized fluorescent } \\
\text { microspheres }\end{array}$ & $\begin{array}{l}\text { McKie-Krisberg et al. } \\
(2015)\end{array}$ \\
\hline Dinophyceae & $\begin{array}{l}\text { Scrippsiella } \\
\text { acuminata }\end{array}$ & $\begin{array}{l}\text { Peridinium } \\
\text { acuminatum, } \\
\text { Heteraulacus } \\
\text { acuminatus, } \\
\text { Goniodoma } \\
\text { acuminatum, }\end{array}$ & $\begin{array}{l}\text { Scrippsiella } \\
\text { trochoidea }\end{array}$ & $\begin{array}{l}\text { ingestion of } \\
\text { Lingulodinium } \\
\text { polyedrum }\end{array}$ & Jeong et al. (2005b) \\
\hline
\end{tabular}




\begin{tabular}{|c|c|c|c|c|c|}
\hline & & $\begin{array}{l}\text { Glenodinium } \\
\text { acuminatum, } \\
\text { Glenodinium } \\
\text { trochoideum, } \\
\text { Peridinium } \\
\text { trochoideum, } \\
\text { Scrippsiella } \\
\text { trochoidea }\end{array}$ & & & \\
\hline Dinophyceae & Symbiodinium sp. & na & Symbiodinium sp. & $\begin{array}{l}\text { ingestion of } \\
\text { heterotrophic } \\
\text { bacteria, } \\
\text { Synechococcus sp., } \\
\text { nanophytoflagellates }\end{array}$ & Jeong et al. (2012) \\
\hline Dinophyceae & Takayama helix & na & Takayama helix & $\begin{array}{l}\text { ingestion of several } \\
\text { dinoflagellate species } \\
\text { from } 15-60 \mu \mathrm{m}\end{array}$ & Jeong et al. (2016) \\
\hline Cryptophyte & $\begin{array}{l}\text { Teleaulax } \\
\text { amphioxeia }\end{array}$ & $\begin{array}{l}\text { Rhodomonas } \\
\text { amphioxeia, } \\
\text { Chroomonas } \\
\text { amphioxieia } \\
\end{array}$ & $\begin{array}{l}\text { Teleaulax } \\
\text { amphioxeia }\end{array}$ & $\begin{array}{l}\text { ingestion of } \\
\text { heterotrophic bacteria } \\
\text { and Synechococcus } \\
\text { sp. }\end{array}$ & Yoo et al. (2017) \\
\hline Dinophyceae & Yihiella yeosuensis & na & Yihiella yeosuensis & $\begin{array}{l}\text { ingestion of } \\
\text { Pyramimonas sp. and } \\
\text { Teleaulax sp. }\end{array}$ & Jang et al. (2017) \\
\hline Dinophyceae & Tripos hexacanthus & $\begin{array}{l}\text { Ceratium } \\
\text { hexacanthum, } \\
\text { Neoceratium } \\
\text { hexacanthum }\end{array}$ & $\begin{array}{l}\text { Neoceratium } \\
\text { hexacanthum }\end{array}$ & not informed & Aldridge et al. (2014) \\
\hline Dinophyceae & Tripos candelabrum & $\begin{array}{l}\text { Peridinium } \\
\text { candelabrum, } \\
\text { Ceratium } \\
\text { candelabrum, }\end{array}$ & $\begin{array}{l}\text { Neoceratium } \\
\text { candelabrum }\end{array}$ & not informed & Aldridge et al. (2014) \\
\hline
\end{tabular}




\begin{tabular}{|c|c|c|c|c|c|}
\hline & & $\begin{array}{l}\text { Neoceratium } \\
\text { candelabrum }\end{array}$ & & & \\
\hline Dinophyceae & Polykrikos lebourae & Polykrikos schwarzii & Polykrikos lebourae & $\begin{array}{l}\text { ingestion of } \\
\text { Chroomonas spp., } \\
\text { Rhodomonas spp., } \\
\text { Amphidinium sp., } \\
\text { Heterocapsa sp., } \\
\text { Thecadinium kofoidii, } \\
\text { and Prorocentrum } \\
\text { fukuyoi }\end{array}$ & Kim et al. (2015) \\
\hline Raphidophyceae & Fibrocapsa japonica & na & Chattonella japonica & $\begin{array}{l}\text { ingestion of } \\
\text { Synechoccocus }\end{array}$ & Jeong et al. (2005a) \\
\hline Chrysophyte & Ochromonas monicis & na & Ochromonas monicis & ingestion of bacteria & $\begin{array}{l}\text { Doddema and Van der } \\
\text { Veer (1983) }\end{array}$ \\
\hline Chrysophyte & Dinobryon balticum & $\begin{array}{l}\text { Dinodendron } \\
\text { balticum }\end{array}$ & Dinobryon balticum & $\begin{array}{l}\text { ingestion of } \\
\text { fluorescent latex- } \\
\text { beads }\end{array}$ & McKenrie et al. (1995) \\
\hline Dinophyceae & Biecheleria cincta & Woloszynskia cincta & Woloszynskia cincta & $\begin{array}{l}\text { ingestion of Isochysis } \\
\text { galbana, Teleaulax } \\
\text { sp., Rhodomonas } \\
\text { salina, Heterosigma } \\
\text { akashiwo, Eutreptiella } \\
\text { gymnastica, } \\
\text { Heterocapsa } \\
\text { rotundata, and } \\
\text { Amphidinium } \\
\text { carterae }\end{array}$ & Kang et al. (2011) \\
\hline Prasinophyte & $\begin{array}{l}\text { Mantoniella } \\
\text { squamata }\end{array}$ & $\begin{array}{l}\text { Micromonas } \\
\text { squamata }\end{array}$ & $\begin{array}{l}\text { Mantoniella } \\
\text { squamata }\end{array}$ & $\begin{array}{l}\text { ingestion of bacteria- } \\
\text { sized fluorescent } \\
\text { microspheres }\end{array}$ & $\begin{array}{l}\text { McKie-Krisberg et al. } \\
(2015)\end{array}$ \\
\hline Prasinophyte & $\begin{array}{l}\text { Cymbomonas } \\
\text { tetramitiformis }\end{array}$ & na & $\begin{array}{l}\text { Cymbomonas } \\
\text { tetramitiformis }\end{array}$ & $\begin{array}{l}\text { ingestion of } \\
\text { heterotrophic bacteria }\end{array}$ & Maruyama et al. (2013) \\
\hline
\end{tabular}


Appendices. Appendix B, Table B.I

\begin{tabular}{|c|c|c|c|c|c|}
\hline Chrysophyte & $\begin{array}{l}\text { Sulcochrysis } \\
\text { biplastida }\end{array}$ & na & $\begin{array}{l}\text { Sulcochrysis } \\
\text { biplastida }\end{array}$ & $\begin{array}{l}\text { uptake of fluorescent } \\
\text { non-carboxylated } \\
\text { latex microspheres }\end{array}$ & Honda et al. (1995) \\
\hline Chlorarachniophyte & $\begin{array}{l}\text { Cryptochlora } \\
\text { perforans }\end{array}$ & na & $\begin{array}{l}\text { Cryptochlora } \\
\text { perforans }\end{array}$ & $\begin{array}{l}\text { uptake by } \\
\text { pseudopodia of the } \\
\text { diatom Amphora sp. }\end{array}$ & $\begin{array}{l}\text { Calderon-Saenz and } \\
\text { Schnetter (1989) }\end{array}$ \\
\hline Chlorarachniophyte & Lotharella oceanica & na & Lotharella oceanica & not informed & Ota et al. (2009) \\
\hline Synchromophyte & Synchroma grande & na & Synchroma grande & $\begin{array}{l}\text { ingestion of } \\
\text { Phaeodactylum } \\
\text { tricornutum. }\end{array}$ & Koch et al. (2011) \\
\hline
\end{tabular}


Table B.2 Characterization of the studies which specifically targeted CMs smaller than $20 \mu \mathrm{m}$ including Longhurst's biogeographic province (please refer to Chapter 2.3 Table 2.1 for province code), oceanic biome, latitude, longitude, scale of the study, season, sampling depth code, and reference.

\begin{tabular}{|c|c|c|c|c|c|c|c|}
\hline Province & Oceanic biome & Lat. & Long. & Scale & Season & Depth code & Reference \\
\hline NASTW & Oligotrophic Gyres & 30 & -70 & spatio-temporal & summer & surface & Arenovski et al. (1995) \\
\hline NASTW & Oligotrophic Gyres & 30 & -70 & spatio-temporal & summer & surface & Arenovski et al. (1995) \\
\hline NASTW & Oligotrophic Gyres & 30 & -70 & spatio-temporal & summer & surface & Arenovski et al. (1995) \\
\hline NASTW & Oligotrophic Gyres & 30 & -70 & spatio-temporal & summer & surface & Arenovski et al. (1995) \\
\hline NASTW & Oligotrophic Gyres & 30 & -70 & spatio-temporal & summer & surface & Arenovski et al. (1995) \\
\hline NASTW & Oligotrophic Gyres & 30 & -70 & spatio-temporal & summer & surface & Arenovski et al. (1995) \\
\hline NASTW & Oligotrophic Gyres & 30 & -70 & spatio-temporal & summer & surface & Arenovski et al. (1995) \\
\hline NASTW & Oligotrophic Gyres & 30 & -70 & spatio-temporal & summer & $\mathrm{DCM}$ & Arenovski et al. (1995) \\
\hline NASTW & Oligotrophic Gyres & 30 & -70 & spatio-temporal & summer & DCM & Arenovski et al. (1995) \\
\hline NASTW & Oligotrophic Gyres & 30 & -70 & spatio-temporal & summer & DCM & Arenovski et al. (1995) \\
\hline NASTW & Oligotrophic Gyres & 30 & -70 & spatio-temporal & summer & DCM & Arenovski et al. (1995) \\
\hline NASTW & Oligotrophic Gyres & 30 & -70 & spatio-temporal & summer & DCM & Arenovski et al. (1995) \\
\hline NASTW & Oligotrophic Gyres & 30 & -70 & spatio-temporal & summer & DCM & Arenovski et al. (1995) \\
\hline NASTW & Oligotrophic Gyres & 30 & -70 & spatio-temporal & spring & surface & Arenovski et al. (1995) \\
\hline NASTW & Oligotrophic Gyres & 30 & -70 & spatio-temporal & spring & sub-surface & Arenovski et al. (1995) \\
\hline NASTW & Oligotrophic Gyres & 30 & -70 & spatio-temporal & spring & sub-surface & Arenovski et al. (1995) \\
\hline NASTW & Oligotrophic Gyres & 30 & -70 & spatio-temporal & spring & $\mathrm{DCM}$ & Arenovski et al. (1995) \\
\hline NASTW & Oligotrophic Gyres & 30 & -70 & spatio-temporal & spring & thermocline & Arenovski et al. (1995) \\
\hline NASTW & Oligotrophic Gyres & 30 & -70 & spatio-temporal & spring & sub-surface & Arenovski et al. (1995) \\
\hline NASTW & Oligotrophic Gyres & 30 & -70 & spatio-temporal & spring & sub-surface & Arenovski et al. (1995) \\
\hline NASTW & Oligotrophic Gyres & 30 & -70 & spatio-temporal & spring & DCM & Arenovski et al. (1995) \\
\hline NASTW & Oligotrophic Gyres & 30 & -70 & spatio-temporal & spring & thermocline & Arenovski et al. (1995) \\
\hline NASTW & Oligotrophic Gyres & 30 & -70 & spatio-temporal & spring & thermocline & Arenovski et al. (1995) \\
\hline NWCS & Coastal Seas & 42 & -68 & spatio-temporal & autumn & sub-surface & Sanders et al. (2000) \\
\hline
\end{tabular}




\begin{tabular}{|c|c|c|c|c|c|c|c|}
\hline NWCS & Coastal Seas & 42 & -68 & spatio-temporal & autumn & sub-surface & Sanders et al. (2000) \\
\hline NWCS & Coastal Seas & 42 & -68 & spatio-temporal & autumn & surface & Sanders et al. (2000) \\
\hline NWCS & Coastal Seas & 42 & -68 & spatio-temporal & autumn & surface & Sanders et al. (2000) \\
\hline NWCS & Coastal Seas & 42 & -68 & spatio-temporal & summer & surface & Sanders et al. (2000) \\
\hline NWCS & Coastal Seas & 42 & -68 & spatio-temporal & summer & surface & Sanders et al. (2000) \\
\hline NWCS & Coastal Seas & 42 & -68 & spatio-temporal & summer & surface & Sanders et al. (2000) \\
\hline NWCS & Coastal Seas & 42 & -68 & spatio-temporal & summer & surface & Sanders et al. (2000) \\
\hline NASTW & Oligotrophic Gyres & 35 & -65 & spatio-temporal & autumn & sub-surface & Sanders et al. (2000) \\
\hline NASTW & Oligotrophic Gyres & 35 & -65 & spatio-temporal & autumn & sub-surface & Sanders et al. (2000) \\
\hline NASTW & Oligotrophic Gyres & 35 & -65 & spatio-temporal & autumn & sub-surface & Sanders et al. (2000) \\
\hline NASTW & Oligotrophic Gyres & 35 & -65 & spatio-temporal & autumn & sub-surface & Sanders et al. (2000) \\
\hline NASTW & Oligotrophic Gyres & 35 & -65 & spatio-temporal & summer & sub-surface & Sanders et al. (2000) \\
\hline NASTW & Oligotrophic Gyres & 35 & -65 & spatio-temporal & summer & sub-surface & Sanders et al. (2000) \\
\hline NASTW & Oligotrophic Gyres & 35 & -65 & spatio-temporal & summer & sub-surface & Sanders et al. (2000) \\
\hline NASTW & Oligotrophic Gyres & 35 & -65 & spatio-temporal & summer & sub-surface & Sanders et al. (2000) \\
\hline APLR & Polar Seas & -70 & 180 & spatio-temporal & spring & surface & Moorthi et al. (2009) \\
\hline APLR & Polar Seas & -70 & 180 & spatio-temporal & spring & surface & Moorthi et al. (2009) \\
\hline APLR & Polar Seas & -70 & 180 & spatio-temporal & spring & surface & Moorthi et al. (2009) \\
\hline APLR & Polar Seas & -70 & 180 & spatio-temporal & spring & surface & Moorthi et al. (2009) \\
\hline APLR & Polar Seas & -70 & 180 & spatio-temporal & spring & surface & Moorthi et al. (2009) \\
\hline APLR & Polar Seas & -70 & 180 & spatio-temporal & spring & surface & Moorthi et al. (2009) \\
\hline APLR & Polar Seas & -70 & 180 & spatio-temporal & spring & surface & Moorthi et al. (2009) \\
\hline APLR & Polar Seas & -70 & 180 & spatio-temporal & spring & surface & Moorthi et al. (2009) \\
\hline APLR & Polar Seas & -70 & 180 & spatio-temporal & spring & surface & Moorthi et al. (2009) \\
\hline APLR & Polar Seas & -70 & 180 & spatio-temporal & spring & surface & Moorthi et al. (2009) \\
\hline APLR & Polar Seas & -70 & 180 & spatio-temporal & spring & surface & Moorthi et al. (2009) \\
\hline APLR & Polar Seas & -70 & 180 & spatio-temporal & spring & surface & Moorthi et al. (2009) \\
\hline APLR & Polar Seas & -70 & 180 & spatio-temporal & spring & surface & Moorthi et al. (2009) \\
\hline
\end{tabular}




\begin{tabular}{|c|c|c|c|c|c|c|c|}
\hline CHIN & Coastal Seas & 25 & 121 & temporal & spring & surface & Tsai et al. (2011) \\
\hline CHIN & Coastal Seas & 25 & 121 & temporal & spring & surface & Tsai et al. (2011) \\
\hline CHIN & Coastal Seas & 25 & 121 & temporal & spring & surface & Tsai et al. (2011) \\
\hline $\mathrm{CHIN}$ & Coastal Seas & 25 & 121 & temporal & spring & surface & Tsai et al. (2011) \\
\hline $\mathrm{CHIN}$ & Coastal Seas & 25 & 121 & temporal & spring & surface & Tsai et al. (2011) \\
\hline $\mathrm{CHIN}$ & Coastal Seas & 25 & 121 & temporal & summer & surface & Tsai et al. (2011) \\
\hline CHIN & Coastal Seas & 25 & 121 & temporal & summer & surface & Tsai et al. (2011) \\
\hline CHIN & Coastal Seas & 25 & 121 & temporal & summer & surface & Tsai et al. (2011) \\
\hline $\mathrm{CHIN}$ & Coastal Seas & 25 & 121 & temporal & summer & surface & Tsai et al. (2011) \\
\hline $\mathrm{CHIN}$ & Coastal Seas & 25 & 121 & temporal & summer & surface & Tsai et al. (2011) \\
\hline CHIN & Coastal Seas & 25 & 121 & temporal & summer & surface & Tsai et al. (2011) \\
\hline $\mathrm{CHIN}$ & Coastal Seas & 25 & 121 & temporal & summer & surface & Tsai et al. (2011) \\
\hline $\mathrm{CHIN}$ & Coastal Seas & 25 & 121 & temporal & summer & surface & Tsai et al. (2011) \\
\hline CHIN & Coastal Seas & 25 & 121 & temporal & summer & surface & Tsai et al. (2011) \\
\hline $\mathrm{CHIN}$ & Coastal Seas & 25 & 121 & temporal & summer & surface & Tsai et al. (2011) \\
\hline CHIN & Coastal Seas & 25 & 121 & temporal & summer & surface & Tsai et al. (2011) \\
\hline CHIN & Coastal Seas & 25 & 121 & temporal & summer & surface & Tsai et al. (2011) \\
\hline $\mathrm{CHIN}$ & Coastal Seas & 25 & 121 & temporal & autumn & surface & Tsai et al. (2011) \\
\hline $\mathrm{CHIN}$ & Coastal Seas & 25 & 121 & temporal & autumn & surface & Tsai et al. (2011) \\
\hline CHIN & Coastal Seas & 25 & 121 & temporal & autumn & surface & Tsai et al. (2011) \\
\hline $\mathrm{CHIN}$ & Coastal Seas & 25 & 121 & temporal & autumn & surface & Tsai et al. (2011) \\
\hline MEDI & Mediterranean Sea & 37 & 25 & spatio-temporal & spring & integrated & Christaki et al. (1999) \\
\hline MEDI & Mediterranean Sea & 37 & 25 & spatio-temporal & spring & integrated & Christaki et al. (1999) \\
\hline MEDI & Mediterranean Sea & 37 & 25 & spatio-temporal & spring & integrated & Christaki et al. (1999) \\
\hline MEDI & Mediterranean Sea & 37 & 25 & spatio-temporal & spring & integrated & Christaki et al. (1999) \\
\hline MEDI & Mediterranean Sea & 37 & 25 & spatio-temporal & spring & integrated & Christaki et al. (1999) \\
\hline MEDI & Mediterranean Sea & 37 & 25 & spatio-temporal & spring & integrated & Christaki et al. (1999) \\
\hline MEDI & Mediterranean Sea & 37 & 25 & spatio-temporal & spring & integrated & Christaki et al. (1999) \\
\hline
\end{tabular}




\begin{tabular}{|c|c|c|c|c|c|c|c|}
\hline MEDI & Mediterranean Sea & 37 & 25 & spatio-temporal & spring & integrated & Christaki et al. (1999) \\
\hline MEDI & Mediterranean Sea & 37 & 25 & spatio-temporal & spring & integrated & Christaki et al. (1999) \\
\hline MEDI & Mediterranean Sea & 37 & 25 & spatio-temporal & spring & integrated & Christaki et al. (1999) \\
\hline MEDI & Mediterranean Sea & 37 & 25 & spatio-temporal & autumn & integrated & Christaki et al. (1999) \\
\hline MEDI & Mediterranean Sea & 37 & 25 & spatio-temporal & autumn & integrated & Christaki et al. (1999) \\
\hline MEDI & Mediterranean Sea & 37 & 25 & spatio-temporal & autumn & integrated & Christaki et al. (1999) \\
\hline MEDI & Mediterranean Sea & 37 & 25 & spatio-temporal & autumn & integrated & Christaki et al. (1999) \\
\hline MEDI & Mediterranean Sea & 37 & 25 & spatio-temporal & autumn & integrated & Christaki et al. (1999) \\
\hline MEDI & Mediterranean Sea & 37 & 25 & spatio-temporal & autumn & integrated & Christaki et al. (1999) \\
\hline MEDI & Mediterranean Sea & 37 & 25 & spatio-temporal & autumn & integrated & Christaki et al. (1999) \\
\hline HUMB & Coastal Upwelling & -45 & -73 & temporal & winter & surface & Czypionka et al. (2011) \\
\hline HUMB & Coastal Upwelling & -45 & -73 & temporal & winter & surface & Czypionka et al. (2011) \\
\hline HUMB & Coastal Upwelling & -45 & -73 & temporal & winter & surface & Czypionka et al. (2011) \\
\hline HUMB & Coastal Upwelling & -45 & -73 & temporal & winter & $\mathrm{DCM}$ & Czypionka et al. (2011) \\
\hline HUMB & Coastal Upwelling & -45 & -73 & temporal & winter & DCM & Czypionka et al. (2011) \\
\hline HUMB & Coastal Upwelling & -45 & -73 & temporal & winter & DCM & Czypionka et al. (2011) \\
\hline HUMB & Coastal Upwelling & -45 & -73 & temporal & spring & surface & Czypionka et al. (2011) \\
\hline HUMB & Coastal Upwelling & -45 & -73 & temporal & spring & surface & Czypionka et al. (2011) \\
\hline HUMB & Coastal Upwelling & -45 & -73 & temporal & spring & surface & Czypionka et al. (2011) \\
\hline HUMB & Coastal Upwelling & -45 & -73 & temporal & spring & $\mathrm{DCM}$ & Czypionka et al. (2011) \\
\hline HUMB & Coastal Upwelling & -45 & -73 & temporal & spring & DCM & Czypionka et al. (2011) \\
\hline HUMB & Coastal Upwelling & -45 & -73 & temporal & spring & DCM & Czypionka et al. (2011) \\
\hline SARC & Temperate Seas & 60 & -20 & spatio-temporal & autumn & sub-surface & Hartmann et al. (2012) \\
\hline NATR & Oligotrophic Gyres & 20 & -40 & spatio-temporal & autumn & sub-surface & Hartmann et al. (2012) \\
\hline WTRA & Equatorial Upwelling & 0 & -30 & spatio-temporal & spring & sub-surface & Hartmann et al. (2012) \\
\hline SATL & Oligotrophic Gyres & -20 & -25 & spatio-temporal & spring & sub-surface & Hartmann et al. (2012) \\
\hline SATL & Oligotrophic Gyres & -20 & -25 & spatio-temporal & spring & sub-surface & Hartmann et al. (2012) \\
\hline SATL & Oligotrophic Gyres & -20 & -25 & spatio-temporal & spring & sub-surface & Hartmann et al. (2012) \\
\hline
\end{tabular}




\begin{tabular}{|c|c|c|c|c|c|c|c|}
\hline SSTC & Temperate Seas & -40 & -35 & spatio-temporal & spring & sub-surface & Hartmann et al. (2012) \\
\hline NECS & Coastal Seas & 56 & 10 & temporal & summer & surface & Havskum and Riemann (1996) \\
\hline NECS & Coastal Seas & 56 & 10 & temporal & summer & DCM & Havskum and Riemann (1996) \\
\hline NECS & Coastal Seas & 59 & 6 & temporal & summer & surface & Havskum and Hansen (1997) \\
\hline NECS & Coastal Seas & 59 & 6 & temporal & summer & surface & Havskum and Hansen (1997) \\
\hline NEWZ & Coastal Seas & -41 & 179 & spatio-temporal & summer & sub-surface & Safi and Hall (1999) \\
\hline NEWZ & Coastal Seas & -44 & 177 & spatio-temporal & summer & sub-surface & Safi and Hall (1999) \\
\hline NEWZ & Coastal Seas & -47 & 177 & spatio-temporal & summer & sub-surface & Safi and Hall (1999) \\
\hline NECS & Coastal Seas & 56 & 12 & spatio-temporal & winter & surface & Anderson et al. (2017) \\
\hline NECS & Coastal Seas & 56 & 12 & spatio-temporal & winter & surface & Anderson et al. (2017) \\
\hline NECS & Coastal Seas & 56 & 12 & spatio-temporal & winter & surface & Anderson et al. (2017) \\
\hline NECS & Coastal Seas & 56 & 12 & spatio-temporal & summer & surface & Anderson et al. (2017) \\
\hline NECS & Coastal Seas & 56 & 12 & spatio-temporal & summer & surface & Anderson et al. (2017) \\
\hline NECS & Coastal Seas & 56 & 12 & spatio-temporal & summer & surface & Anderson et al. (2017) \\
\hline CHIN & Coastal Seas & 25 & 122 & temporal & summer & surface & Tsai et al. (2009) \\
\hline NEWZ & Coastal Seas & 43 & 172 & spatio-temporal & winter & sub-surface & Hall et al. (1993) \\
\hline NEWZ & Coastal Seas & 43 & 172 & spatio-temporal & winter & sub-surface & Hall et al. (1993) \\
\hline NEWZ & Coastal Seas & 43 & 172 & spatio-temporal & winter & sub-surface & Hall et al. (1993) \\
\hline NEWZ & Coastal Seas & 43 & 172 & spatio-temporal & winter & sub-surface & Hall et al. (1993) \\
\hline NEWZ & Coastal Seas & 43 & 172 & spatio-temporal & winter & sub-surface & Hall et al. (1993) \\
\hline NEWZ & Coastal Seas & 43 & 172 & spatio-temporal & winter & sub-surface & Hall et al. (1993) \\
\hline NEWZ & Coastal Seas & 43 & 172 & spatio-temporal & winter & sub-surface & Hall et al. (1993) \\
\hline NEWZ & Coastal Seas & 43 & 172 & spatio-temporal & winter & sub-surface & Hall et al. (1993) \\
\hline MEDI & Mediterranean Sea & 41 & 3 & temporal & winter & surface & Unrein et al. (2007) \\
\hline MEDI & Mediterranean Sea & 41 & 3 & temporal & autumn & surface & Unrein et al. (2007) \\
\hline MEDI & Mediterranean Sea & 41 & 3 & temporal & autumn & surface & Unrein et al. (2007) \\
\hline MEDI & Mediterranean Sea & 41 & 3 & temporal & autumn & surface & Unrein et al. (2007) \\
\hline MEDI & Mediterranean Sea & 41 & 3 & temporal & summer & surface & Unrein et al. (2007) \\
\hline
\end{tabular}




\begin{tabular}{|c|c|c|c|c|c|c|c|}
\hline MEDI & Mediterranean Sea & 41 & 3 & temporal & summer & surface & Unrein et al. (2007) \\
\hline MEDI & Mediterranean Sea & 41 & 3 & temporal & summer & surface & Unrein et al. (2007) \\
\hline MEDI & Mediterranean Sea & 41 & 3 & temporal & spring & surface & Unrein et al. (2007) \\
\hline MEDI & Mediterranean Sea & 41 & 3 & temporal & spring & surface & Unrein et al. (2007) \\
\hline MEDI & Mediterranean Sea & 41 & 3 & temporal & spring & surface & Unrein et al. (2007) \\
\hline MEDI & Mediterranean Sea & 41 & 3 & temporal & winter & surface & Unrein et al. (2007) \\
\hline MEDI & Mediterranean Sea & 41 & 3 & temporal & winter & surface & Unrein et al. (2007) \\
\hline SARC & Temperate Seas & 59 & -20 & spatio-temporal & summer & surface & Zubkov and Tarran (2008) \\
\hline SARC & Temperate Seas & 59 & -20 & spatio-temporal & summer & sub-surface & Zubkov and Tarran (2008) \\
\hline NATR & Oligotrophic Gyres & 22 & -30 & spatio-temporal & summer & sub-surface & Zubkov and Tarran (2008) \\
\hline NATR & Oligotrophic Gyres & 17 & -30 & spatio-temporal & summer & sub-surface & Zubkov and Tarran (2008) \\
\hline NATR & Oligotrophic Gyres & 13 & -30 & spatio-temporal & summer & sub-surface & Zubkov and Tarran (2008) \\
\hline BPLR & Polar Seas & 77 & -151 & spatio-temporal & spring & surface & Sanders and Gast (2012) \\
\hline BPLR & Polar Seas & 77 & 170 & spatio-temporal & spring & DCM & Sanders and Gast (2012) \\
\hline BPLR & Polar Seas & 77 & 170 & spatio-temporal & spring & surface & Sanders and Gast (2012) \\
\hline BPLR & Polar Seas & 77 & 170 & spatio-temporal & spring & DCM & Sanders and Gast (2012) \\
\hline BPLR & Polar Seas & 77 & 170 & spatio-temporal & spring & surface & Sanders and Gast (2012) \\
\hline BPLR & Polar Seas & 77 & 170 & spatio-temporal & spring & DCM & Sanders and Gast (2012) \\
\hline BPLR & Polar Seas & 77 & 170 & spatio-temporal & spring & surface & Sanders and Gast (2012) \\
\hline BPLR & Polar Seas & 77 & 170 & spatio-temporal & spring & DCM & Sanders and Gast (2012) \\
\hline BPLR & Polar Seas & 77 & 170 & spatio-temporal & spring & surface & Sanders and Gast (2012) \\
\hline BPLR & Polar Seas & 77 & 170 & spatio-temporal & spring & DCM & Sanders and Gast (2012) \\
\hline BPLR & Polar Seas & 77 & 170 & spatio-temporal & spring & surface & Sanders and Gast (2012) \\
\hline BPLR & Polar Seas & 77 & 170 & spatio-temporal & spring & DCM & Sanders and Gast (2012) \\
\hline BPLR & Polar Seas & 77 & 170 & spatio-temporal & spring & surface & Sanders and Gast (2012) \\
\hline BPLR & Polar Seas & 77 & 170 & spatio-temporal & spring & DCM & Sanders and Gast (2012) \\
\hline BPLR & Polar Seas & 77 & 170 & spatio-temporal & spring & DCM & Sanders and Gast (2012) \\
\hline BPLR & Polar Seas & 77 & 170 & spatio-temporal & spring & surface & Sanders and Gast (2012) \\
\hline
\end{tabular}




\begin{tabular}{|c|c|c|c|c|c|c|c|}
\hline BPLR & Polar Seas & 77 & 170 & spatio-temporal & spring & DCM & Sanders and Gast (2012) \\
\hline BPLR & Polar Seas & 77 & 170 & spatio-temporal & spring & surface & Sanders and Gast (2012) \\
\hline BPLR & Polar Seas & 77 & 170 & spatio-temporal & spring & DCM & Sanders and Gast (2012) \\
\hline BPLR & Polar Seas & 77 & 170 & spatio-temporal & spring & surface & Sanders and Gast (2012) \\
\hline BPLR & Polar Seas & 77 & 170 & spatio-temporal & spring & DCM & Sanders and Gast (2012) \\
\hline PEQD & Equatorial Upwelling & 1 & -125 & spatio-temporal & spring & sub-surface & Stukel et al. (2011) \\
\hline PEQD & Equatorial Upwelling & 2 & -125 & spatio-temporal & spring & sub-surface & Stukel et al. (2011) \\
\hline PEQD & Equatorial Upwelling & 1 & -125 & spatio-temporal & spring & sub-surface & Stukel et al. (2011) \\
\hline PEQD & Equatorial Upwelling & 1 & -125 & spatio-temporal & spring & sub-surface & Stukel et al. (2011) \\
\hline APLR & Polar Seas & -70 & 170 & spatio-temporal & summer & surface & Gast et al. (2014) \\
\hline APLR & Polar Seas & -70 & 170 & spatio-temporal & summer & surface & Gast et al. (2014) \\
\hline APLR & Polar Seas & -70 & 170 & spatio-temporal & summer & surface & Gast et al. (2014) \\
\hline APLR & Polar Seas & -70 & 170 & spatio-temporal & summer & surface & Gast et al. (2014) \\
\hline APLR & Polar Seas & -70 & 170 & spatio-temporal & summer & surface & Gast et al. (2014) \\
\hline APLR & Polar Seas & -70 & 170 & spatio-temporal & summer & surface & Gast et al. (2014) \\
\hline APLR & Polar Seas & -70 & 170 & spatio-temporal & summer & surface & Gast et al. (2014) \\
\hline APLR & Polar Seas & -70 & 170 & spatio-temporal & summer & surface & Gast et al. (2014) \\
\hline APLR & Polar Seas & -70 & 170 & spatio-temporal & summer & surface & Gast et al. (2014) \\
\hline APLR & Polar Seas & -70 & 170 & spatio-temporal & summer & surface & Gast et al. (2014) \\
\hline APLR & Polar Seas & -70 & 170 & spatio-temporal & summer & surface & Gast et al. (2014) \\
\hline APLR & Polar Seas & -70 & 170 & spatio-temporal & summer & surface & Gast et al. (2014) \\
\hline APLR & Polar Seas & -70 & 170 & spatio-temporal & summer & surface & Gast et al. (2014) \\
\hline APLR & Polar Seas & -70 & 170 & spatio-temporal & summer & DCM & Gast et al. (2014) \\
\hline APLR & Polar Seas & -70 & 170 & spatio-temporal & summer & DCM & Gast et al. (2014) \\
\hline APLR & Polar Seas & -70 & 170 & spatio-temporal & summer & DCM & Gast et al. (2014) \\
\hline APLR & Polar Seas & -70 & 170 & spatio-temporal & summer & DCM & Gast et al. (2014) \\
\hline APLR & Polar Seas & -70 & 170 & spatio-temporal & summer & DCM & Gast et al. (2014) \\
\hline APLR & Polar Seas & -70 & 170 & spatio-temporal & summer & DCM & Gast et al. (2014) \\
\hline
\end{tabular}




\begin{tabular}{|c|c|c|c|c|c|c|c|}
\hline APLR & Polar Seas & -70 & 170 & spatio-temporal & summer & DCM & Gast et al. (2014) \\
\hline APLR & Polar Seas & -70 & 170 & spatio-temporal & summer & DCM & Gast et al. (2014) \\
\hline APLR & Polar Seas & -70 & 170 & spatio-temporal & summer & DCM & Gast et al. (2014) \\
\hline APLR & Polar Seas & -70 & 170 & spatio-temporal & summer & DCM & Gast et al. (2014) \\
\hline APLR & Polar Seas & -70 & 170 & spatio-temporal & summer & DCM & Gast et al. (2014) \\
\hline APLR & Polar Seas & -70 & 170 & spatio-temporal & summer & DCM & Gast et al. (2014) \\
\hline APLR & Polar Seas & -70 & 170 & spatio-temporal & summer & DCM & Gast et al. (2014) \\
\hline NECS & Coastal Seas & 59 & 10 & temporal & nd & surface & Nygaard and Tobiesen (1993) \\
\hline NECS & Coastal Seas & 59 & 10 & temporal & nd & surface & Nygaard and Tobiesen (1993) \\
\hline PEQD & Equatorial Upwelling & 0 & -170 & spatio-temporal & summer & sub-surface & Sato et al. (2017) \\
\hline PNEC & Equatorial Upwelling & 5 & -170 & spatio-temporal & summer & sub-surface & Sato et al. (2017) \\
\hline PNEC & Equatorial Upwelling & 10 & -170 & spatio-temporal & summer & sub-surface & Sato et al. (2017) \\
\hline NPTGE & Oligotrophic Gyres & 15 & -170 & spatio-temporal & summer & sub-surface & Sato et al. (2017) \\
\hline NPTGE & Oligotrophic Gyres & 20 & -170 & spatio-temporal & summer & sub-surface & Sato et al. (2017) \\
\hline NPTGE & Oligotrophic Gyres & 24 & -170 & spatio-temporal & summer & sub-surface & Sato et al. (2017) \\
\hline NPTGE & Oligotrophic Gyres & 30 & -170 & spatio-temporal & summer & sub-surface & Sato et al. (2017) \\
\hline NPPF & Temperate Seas & 35 & -170 & spatio-temporal & summer & sub-surface & Sato et al. (2017) \\
\hline NPPF & Temperate Seas & 40 & -170 & spatio-temporal & summer & sub-surface & Sato et al. (2017) \\
\hline PSAGE & Temperate Seas & 45 & -170 & spatio-temporal & summer & sub-surface & Sato et al. (2017) \\
\hline PSAGE & Temperate Seas & 50 & -170 & spatio-temporal & summer & sub-surface & Sato et al. (2017) \\
\hline BPLR & Polar Seas & 55 & -170 & spatio-temporal & summer & sub-surface & Sato et al. (2017) \\
\hline BPLR & Polar Seas & 60 & -170 & spatio-temporal & summer & sub-surface & Sato et al. (2017) \\
\hline BPLR & Polar Seas & 65 & -170 & spatio-temporal & summer & sub-surface & Sato et al. (2017) \\
\hline HUMB & Coastal Upwelling & -36 & -73 & spatio-temporal & summer & surface & Vargas et al. (2012) \\
\hline HUMB & Coastal Upwelling & -36 & -73 & spatio-temporal & summer & $\mathrm{DCM}$ & Vargas et al. (2012) \\
\hline HUMB & Coastal Upwelling & -36 & -73 & spatio-temporal & summer & thermocline & Vargas et al. (2012) \\
\hline HUMB & Coastal Upwelling & -36 & -73 & spatio-temporal & summer & surface & Vargas et al. (2012) \\
\hline HUMB & Coastal Upwelling & -36 & -73 & spatio-temporal & summer & DCM & Vargas et al. (2012) \\
\hline
\end{tabular}


Appendices. Appendix B, Table B.2

\begin{tabular}{llllllll} 
HUMB & Coastal Upwelling & -36 & -73 & spatio-temporal & summer & thermocline & Vargas et al. (2012) \\
HUMB & Coastal Upwelling & -36 & -73 & spatio-temporal & winter & surface & Vargas et al. (2012) \\
HUMB & Coastal Upwelling & -36 & -73 & spatio-temporal & winter & DCM & Vargas et al. (2012) \\
HUMB & Coastal Upwelling & -36 & -73 & spatio-temporal & winter & thermocline & Vargas et al. (2012) \\
HUMB & Coastal Upwelling & -36 & -73 & spatio-temporal & winter & surface & Vargas et al. (2012) \\
HUMB & Coastal Upwelling & -36 & -73 & spatio-temporal & winter & DCM & Vargas et al. (2012) \\
HUMB & Coastal Upwelling & -36 & -73 & spatio-temporal & winter & thermocline & Vargas et al. (2012) \\
\hline
\end{tabular}


Table B.3 Studies which specifically targeted CMs smaller than $20 \mu \mathrm{m}$ including nitrate and phosphate concentrations, method (FLB fluorescent labelled bacteria; FLC - fluorescent labelled cyanobacteria; FLA - fluorescent labelled algae; or beads), bacterial abundance, abundance of autotrophic nanoflagellates (ANF), abundance of heterotrophic nanoflagellates (HNF), abundance of mixotrophic nanoflagellates actively feeding (MNF), percentage of total bacterivory attributed to MNF, and references. Data are also given for net primary productivity (NPP) corresponding to each of Longhurst's provinces (Chapter 2.3); nd - not determined.

\begin{tabular}{|c|c|c|c|c|c|c|c|c|c|}
\hline NPP & $\mathrm{NO}_{3}^{-}$ & $\mathrm{PO}_{4}^{3-}$ & Method & 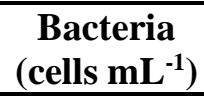 & $\begin{array}{c}\mathrm{ANF} \\
\left(\text { cells } \mathbf{m L}^{-1}\right)\end{array}$ & $\begin{array}{c}\text { HNF } \\
\left(\text { cells } \mathbf{~ m L}^{-1}\right) \\
\end{array}$ & $\begin{array}{c}\text { MNF } \\
\left(\text { cells mL } \mathbf{L}^{-1}\right)\end{array}$ & $\begin{array}{c}\text { Bacterivory } \\
(\%)\end{array}$ & Reference \\
\hline 0.3 & nd & nd & FLB/FLC & 216000 & 503.1 & 619.0 & 12.9 & nd & Arenovski et al. (1995) \\
\hline 0.3 & nd & nd & FLB/FLC & 239000 & 368.6 & 516.0 & 9.5 & nd & Arenovski et al. (1995) \\
\hline 0.3 & nd & nd & FLB/FLC & 221000 & 114.1 & 293.0 & 2.9 & nd & Arenovski et al. (1995) \\
\hline 0.3 & nd & nd & FLB/FLC & 171000 & 108.2 & 496.0 & 2.8 & nd & Arenovski et al. (1995) \\
\hline 0.3 & nd & nd & FLB/FLC & 166000 & 62.4 & 517.0 & 1.6 & nd & Arenovski et al. (1995) \\
\hline 0.3 & nd & nd & FLB/FLC & 205000 & 127.8 & 535.0 & 144.2 & nd & Arenovski et al. (1995) \\
\hline 0.3 & nd & nd & FLB/FLC & 187000 & 69.5 & 305.0 & 69.5 & nd & Arenovski et al. (1995) \\
\hline 0.3 & nd & nd & FLB/FLC & 155000 & 2544.8 & 460.0 & 65.3 & nd & Arenovski et al. (1995) \\
\hline 0.3 & nd & nd & FLB/FLC & 139000 & 921.4 & 372.0 & 23.6 & nd & Arenovski et al. (1995) \\
\hline 0.3 & nd & nd & FLB/FLC & 148000 & 163.8 & 304.0 & 4.2 & nd & Arenovski et al. (1995) \\
\hline 0.3 & nd & nd & FLB/FLC & 106000 & 175.5 & 233.0 & 4.5 & nd & Arenovski et al. (1995) \\
\hline 0.3 & nd & nd & FLB/FLC & 104000 & 144.3 & 171.0 & 3.7 & nd & Arenovski et al. (1995) \\
\hline 0.3 & nd & nd & FLB/FLC & 109000 & 288.6 & 108.0 & 7.4 & nd & Arenovski et al. (1995) \\
\hline 0.5 & nd & nd & FLB/FLC & 390000 & 174.7 & 974.3 & 21.5 & nd & Arenovski et al. (1995) \\
\hline 0.5 & nd & nd & FLB/FLC & 370000 & 185.1 & 930.4 & 25.1 & nd & Arenovski et al. (1995) \\
\hline 0.5 & nd & nd & FLB/FLC & 390000 & 367.8 & 410.3 & 15.9 & nd & Arenovski et al. (1995) \\
\hline 0.5 & nd & nd & FLB/FLC & 315000 & 1203.5 & 659.3 & 0.3 & nd & Arenovski et al. (1995) \\
\hline 0.5 & nd & nd & FLB/FLC & 171000 & 102.5 & 644.7 & 0.7 & nd & Arenovski et al. (1995) \\
\hline 0.5 & nd & nd & FLB/FLC & 186000 & 323.5 & 539.3 & 21.5 & nd & Arenovski et al. (1995) \\
\hline 0.5 & nd & nd & FLB/FLC & 248000 & 782.1 & 635.1 & 37.6 & nd & Arenovski et al. (1995) \\
\hline
\end{tabular}




\begin{tabular}{|c|c|c|c|c|c|c|c|c|c|}
\hline 0.5 & nd & nd & FLB/FLC & 252000 & 1140.0 & 959.1 & 3.3 & nd & Arenovski et al. (1995) \\
\hline 0.5 & nd & nd & FLB/FLC & 159000 & 885.2 & 889.2 & 8.5 & nd & Arenovski et al. (1995) \\
\hline 0.5 & nd & nd & FLB/FLC & 138000 & 568.4 & 818.2 & 0.8 & nd & Arenovski et al. (1995) \\
\hline 0.9 & nd & nd & FLB/FLC & 1210000 & 3550.0 & 3530.0 & 2210.0 & nd & Sanders et al. (2000) \\
\hline 0.9 & nd & nd & FLB/FLC & 1550000 & 1250.0 & 1690.0 & 420.0 & nd & Sanders et al. (2000) \\
\hline 0.9 & nd & nd & FLB/FLC & 2080000 & 5050.0 & 1240.0 & 100.0 & nd & Sanders et al. (2000) \\
\hline 0.9 & nd & nd & FLB/FLC & 2370000 & 3970.0 & 1370.0 & 170.0 & nd & Sanders et al. (2000) \\
\hline 1.0 & nd & nd & FLB/FLC & 1300000 & 4910.0 & 3230.0 & 370.0 & nd & Sanders et al. (2000) \\
\hline 1.0 & nd & nd & FLB/FLC & 1220000 & 4940.0 & 2840.0 & 380.0 & nd & Sanders et al. (2000) \\
\hline 1.0 & nd & nd & FLB/FLC & 1070000 & 5410.0 & 3650.0 & 540.0 & nd & Sanders et al. (2000) \\
\hline 1.0 & nd & nd & FLB/FLC & 1000000 & 1360.0 & 1940.0 & 580.0 & nd & Sanders et al. (2000) \\
\hline 0.2 & nd & nd & FLB/FLC & 412000 & 425.0 & 245.0 & 95.0 & nd & Sanders et al. (2000) \\
\hline 0.2 & nd & nd & FLB/FLC & 638000 & 350.0 & 220.0 & 60.0 & nd & Sanders et al. (2000) \\
\hline 0.2 & nd & nd & FLB/FLC & 416000 & 195.0 & 230.0 & 30.0 & nd & Sanders et al. (2000) \\
\hline 0.2 & nd & nd & FLB/FLC & 483000 & 415.0 & 330.0 & 55.0 & nd & Sanders et al. (2000) \\
\hline 0.3 & nd & nd & FLB/FLC & 197000 & 400.0 & 390.0 & 70.0 & nd & Sanders et al. (2000) \\
\hline 0.3 & nd & nd & FLB/FLC & 302000 & 435.0 & 680.0 & 25.0 & nd & Sanders et al. (2000) \\
\hline 0.3 & nd & nd & FLB/FLC & 261000 & 265.0 & 450.0 & 20.0 & nd & Sanders et al. (2000) \\
\hline 0.3 & nd & nd & FLB/FLC & 519000 & 140.0 & 330.0 & 30.0 & nd & Sanders et al. (2000) \\
\hline 0.3 & nd & nd & FLB/FLC & 6180000 & 1000.0 & 552.0 & 90.0 & nd & Moorthi et al. (2009) \\
\hline 0.3 & nd & nd & FLB/FLC & 5820000 & 600.0 & 653.7 & 95.0 & nd & Moorthi et al. (2009) \\
\hline 0.5 & nd & nd & FLB/FLC & 5530000 & 900.0 & 790.5 & 96.0 & nd & Moorthi et al. (2009) \\
\hline 0.5 & nd & nd & FLB/FLC & 5240000 & 900.0 & 425.1 & 130.0 & nd & Moorthi et al. (2009) \\
\hline 0.5 & nd & nd & FLB/FLC & 4480000 & 1000.0 & 561.0 & 150.0 & nd & Moorthi et al. (2009) \\
\hline 0.5 & nd & nd & FLB/FLC & 5470000 & 1300.0 & 803.4 & 160.0 & nd & Moorthi et al. (2009) \\
\hline 0.5 & nd & nd & FLB/FLC & 5540000 & 1250.0 & 320.3 & 65.0 & nd & Moorthi et al. (2009) \\
\hline 0.5 & nd & nd & FLB/FLC & 4750000 & 1100.0 & 349.0 & 60.0 & nd & Moorthi et al. (2009) \\
\hline 0.5 & nd & nd & FLB/FLC & 6200000 & 10000.0 & 579.0 & 70.0 & nd & Moorthi et al. (2009) \\
\hline
\end{tabular}


Appendices. Appendix B, Table B.3

\begin{tabular}{|c|c|c|c|c|c|c|c|c|c|}
\hline 0.5 & nd & nd & FLB/FLC & 6130000 & 12000.0 & 551.6 & 65.0 & nd & Moorthi et al. (2009) \\
\hline 0.5 & nd & nd & FLB/FLC & 5400000 & 750.0 & 595.2 & 70.0 & nd & Moorthi et al. (2009) \\
\hline 1.0 & nd & nd & FLB/FLC & 5190000 & 1100.0 & 930.5 & 65.0 & nd & Moorthi et al. (2009) \\
\hline 1.0 & nd & nd & FLB/FLC & 5740000 & 1000.0 & 100.0 & 100.0 & nd & Moorthi et al. (2009) \\
\hline na & nd & nd & FLB & 760000 & 416.0 & 354.3 & nd & 54.0 & Tsai et al. (2011) \\
\hline na & 1.3 & 0.2 & FLB & 800000 & 405.0 & 544.6 & nd & 54.0 & Tsai et al. (2011) \\
\hline na & 0.6 & 0.1 & FLB & 880000 & 518.0 & 355.5 & nd & 54.0 & Tsai et al. (2011) \\
\hline na & 0.4 & 0.1 & FLB & 890000 & 330.0 & 996.9 & nd & 54.0 & Tsai et al. (2011) \\
\hline na & 3.2 & 0.2 & FLB & 1220000 & 463.0 & 412.9 & nd & 54.0 & Tsai et al. (2011) \\
\hline na & 0.4 & 0.1 & FLB & 1310000 & 408.0 & 592.7 & nd & 54.0 & Tsai et al. (2011) \\
\hline na & 0.6 & 0.1 & FLB & 1040000 & 602.0 & 572.8 & nd & 54.0 & Tsai et al. (2011) \\
\hline na & 0.4 & 0.1 & FLB & 1290000 & 211.0 & 444.7 & nd & 54.0 & Tsai et al. (2011) \\
\hline na & 0.2 & 0.1 & FLB & 1320000 & 416.0 & 435.7 & nd & 54.0 & Tsai et al. (2011) \\
\hline na & 0.2 & 0.1 & FLB & 960000 & 223.0 & 323.3 & nd & 54.0 & Tsai et al. (2011) \\
\hline na & 0.4 & 0.2 & FLB & 850000 & 225.0 & 292.9 & nd & 54.0 & Tsai et al. (2011) \\
\hline na & nd & nd & FLB & 1100000 & 340.0 & 426.3 & nd & 54.0 & Tsai et al. (2011) \\
\hline na & 3.1 & 0.4 & FLB & 890000 & 369.0 & 267.7 & nd & 54.0 & Tsai et al. (2011) \\
\hline na & 1.8 & 0.2 & FLB & 1440000 & 235.0 & 273.4 & nd & 54.0 & Tsai et al. (2011) \\
\hline na & 1.7 & 0.1 & FLB & 1520000 & 245.0 & 325.2 & nd & 54.0 & Tsai et al. (2011) \\
\hline na & 1.4 & 0.1 & FLB & 860000 & 245.0 & 325.7 & nd & 54.0 & Tsai et al. (2011) \\
\hline na & 6.6 & 0.3 & FLB & 1440000 & 310.0 & 387.5 & nd & 54.0 & Tsai et al. (2011) \\
\hline na & 2.0 & 0.4 & FLB & 850000 & 620.0 & 388.0 & nd & 54.0 & Tsai et al. (2011) \\
\hline na & nd & nd & FLB & 780000 & 195.0 & 527.0 & nd & 54.0 & Tsai et al. (2011) \\
\hline na & nd & nd & FLB & 700000 & 389.0 & 224.8 & nd & 54.0 & Tsai et al. (2011) \\
\hline na & nd & nd & FLB & 610000 & 345.0 & 510.0 & nd & 54.0 & Tsai et al. (2011) \\
\hline 0.8 & nd & nd & beads & 719000 & 600.0 & 300.0 & 90.0 & 12.5 & Christaki et al. (1999) \\
\hline 0.8 & nd & nd & beads & 448000 & 460.0 & 250.0 & 80.0 & 12.5 & Christaki et al. (1999) \\
\hline 0.8 & nd & nd & beads & 1071000 & 590.0 & 380.0 & 50.0 & 12.5 & Christaki et al. (1999) \\
\hline
\end{tabular}




\begin{tabular}{|c|c|c|c|c|c|c|c|c|c|}
\hline 0.8 & nd & nd & beads & 745000 & 640.0 & 430.0 & 60.0 & 12.5 & Christaki et al. (1999) \\
\hline 0.3 & nd & nd & beads & 351000 & 360.0 & 860.0 & 30.0 & 12.5 & Christaki et al. (1999) \\
\hline 0.3 & nd & nd & beads & 550000 & 530.0 & 1010.0 & 80.0 & 12.5 & Christaki et al. (1999) \\
\hline 0.3 & nd & nd & beads & 562000 & 400.0 & 870.0 & 130.0 & 12.5 & Christaki et al. (1999) \\
\hline 0.3 & nd & nd & beads & 746000 & 710.0 & 1510.0 & 100.0 & 12.5 & Christaki et al. (1999) \\
\hline 0.3 & nd & nd & beads & 1078000 & 660.0 & 1460.0 & 120.0 & 12.5 & Christaki et al. (1999) \\
\hline 0.3 & nd & nd & beads & 839000 & 800.0 & 1150.0 & 70.0 & 12.5 & Christaki et al. (1999) \\
\hline 0.3 & nd & nd & beads & 728000 & 740.0 & 600.0 & 50.0 & 12.5 & Christaki et al. (1999) \\
\hline 0.3 & nd & nd & beads & 711000 & 1200.0 & 1610.0 & 80.0 & 12.5 & Christaki et al. (1999) \\
\hline 0.3 & nd & nd & beads & 709000 & 830.0 & 1080.0 & 120.0 & 12.5 & Christaki et al. (1999) \\
\hline 0.3 & nd & nd & beads & 738000 & 860.0 & 1060.0 & 90.0 & 12.5 & Christaki et al. (1999) \\
\hline 0.3 & nd & nd & beads & 689000 & 910.0 & 990.0 & 120.0 & 12.5 & Christaki et al. (1999) \\
\hline 0.3 & nd & nd & beads & 704000 & 840.0 & 1070.0 & 80.0 & 12.5 & Christaki et al. (1999) \\
\hline 0.3 & nd & nd & beads & 623000 & 880.0 & 1260.0 & 70.0 & 12.5 & Christaki et al. (1999) \\
\hline 0.6 & 10.0 & 0.5 & FLB & 4900000 & 226.4 & 2332.3 & 103.1 & nd & Czypionka et al. (2011) \\
\hline 0.6 & 10.0 & 0.5 & FLB & 5900000 & 665.9 & 1635.1 & 848.0 & nd & Czypionka et al. (2011) \\
\hline 0.6 & 10.0 & 0.5 & FLB & 3500000 & 1182.6 & 1318.6 & 33.0 & nd & Czypionka et al. (2011) \\
\hline 0.6 & 20.0 & 1.6 & FLB & 3600000 & 666.9 & 1047.7 & 100.0 & nd & Czypionka et al. (2011) \\
\hline 0.6 & 20.0 & 1.6 & FLB & 3000000 & 1045.2 & 64.2 & 80.0 & nd & Czypionka et al. (2011) \\
\hline 0.6 & 20.0 & 1.6 & FLB & 1300000 & 811.2 & 840.2 & 45.2 & nd & Czypionka et al. (2011) \\
\hline 0.7 & 0.0 & 0.0 & FLB & 9700000 & 963.3 & 3973.1 & 113.9 & nd & Czypionka et al. (2011) \\
\hline 0.7 & 0.0 & 0.0 & FLB & 5000000 & 3266.9 & 4434.2 & 102.6 & nd & Czypionka et al. (2011) \\
\hline 0.7 & 0.0 & 0.0 & FLB & 8800000 & 4467.9 & 5871.4 & 169.8 & nd & Czypionka et al. (2011) \\
\hline 0.7 & 10.0 & 1.4 & FLB & 3900000 & 195.7 & 2890.5 & 70.6 & nd & Czypionka et al. (2011) \\
\hline 0.7 & 10.0 & 1.4 & FLB & 2800000 & 436.2 & 4060.2 & 75.3 & nd & Czypionka et al. (2011) \\
\hline 0.7 & 10.0 & 1.4 & FLB & 3500000 & 1562.9 & 2332.1 & 10.0 & nd & Czypionka et al. (2011) \\
\hline na & nd & nd & FLB & nd & 9322.6 & 641.3 & nd & 23.2 & Hartmann et al. (2012) \\
\hline na & nd & nd & FLB & nd & 1146.0 & 517.6 & nd & 52.6 & Hartmann et al. (2012) \\
\hline
\end{tabular}




\begin{tabular}{|c|c|c|c|c|c|c|c|c|c|}
\hline na & nd & nd & FLB & nd & 3196.5 & 589.5 & nd & 47.4 & Hartmann et al. (2012) \\
\hline na & nd & nd & FLB & nd & 1641.2 & 624.5 & nd & 67.5 & Hartmann et al. (2012) \\
\hline na & nd & nd & FLB & nd & 1822.4 & 405.9 & nd & 52.6 & Hartmann et al. (2012) \\
\hline na & nd & nd & FLB & nd & 2377.8 & 780.6 & nd & 44.9 & Hartmann et al. (2012) \\
\hline na & nd & nd & FLB & nd & 10627.1 & 1784.5 & nd & 47.2 & Hartmann et al. (2012) \\
\hline na & nd & nd & FLB & 800000 & nd & nd & nd & 86.0 & Havskum \& Riemann (1996) \\
\hline na & nd & nd & FLB & 1200000 & nd & nd & nd & 19.0 & Havskum \& Riemann (1996) \\
\hline na & nd & nd & FLA & 1900000 & nd & nd & nd & 34.0 & Havskum \& Hansen (1997) \\
\hline na & nd & nd & FLA & 3800000 & nd & nd & nd & 12.0 & Havskum \& Hansen (1997) \\
\hline 0.7 & 0.2 & 0.0 & beads & 1500000 & nd & 295.0 & 528.0 & 48.6 & Safi \& Hall (1999) \\
\hline 0.7 & 0.5 & 0.1 & beads & 3000000 & nd & 486.0 & 680.0 & 53.7 & Safi \& Hall (1999) \\
\hline 0.7 & 7.5 & 0.6 & beads & 2800000 & nd & 689.0 & 1232.0 & 55.0 & Safi \& Hall (1999) \\
\hline 0.2 & nd & nd & $\mathrm{AP}$ & 1800000 & 1696.7 & 1400.0 & 203.3 & 56.3 & Anderson et al. (2017) \\
\hline 0.2 & nd & nd & $\mathrm{AP}$ & 1400000 & 2071.2 & 2200.0 & 328.8 & 48.1 & Anderson et al. (2017) \\
\hline 0.2 & nd & nd & $\mathrm{AP}$ & 1900000 & 1984.8 & 1800.0 & 415.2 & 66.7 & Anderson et al. (2017) \\
\hline 1.1 & nd & nd & $\mathrm{AP}$ & 1700000 & 12809.5 & 5300.0 & 890.5 & 27.1 & Anderson et al. (2017) \\
\hline 1.1 & nd & nd & $\mathrm{AP}$ & 2100000 & 26658.6 & 6500.0 & 1641.4 & 45.7 & Anderson et al. (2017) \\
\hline 1.1 & nd & nd & $\mathrm{AP}$ & 2500000 & 42705.0 & 9400.0 & 2295.0 & 50.8 & Anderson et al. (2017) \\
\hline na & nd & nd & beads & NA & 2700.0 & nd & nd & nd & Tsai et al. (2009) \\
\hline na & nd & nd & beads & 410000000 & 286.0 & 191.0 & nd & 36.0 & Hall et al. (1993) \\
\hline na & nd & nd & beads & 540000000 & 285.0 & 199.0 & nd & 36.0 & Hall et al. (1993) \\
\hline na & nd & nd & beads & 435000000 & 363.0 & 201.0 & nd & 36.0 & Hall et al. (1993) \\
\hline na & nd & nd & beads & 484000000 & 331.0 & 198.0 & nd & 36.0 & Hall et al. (1993) \\
\hline na & nd & nd & beads & 335000000 & 347.0 & 149.0 & nd & 36.0 & Hall et al. (1993) \\
\hline na & nd & nd & beads & 318000000 & 381.0 & 214.0 & nd & 36.0 & Hall et al. (1993) \\
\hline na & nd & nd & beads & 346000000 & 408.0 & 272.0 & nd & 36.0 & Hall et al. (1993) \\
\hline na & nd & nd & beads & 347000000 & 377.0 & 288.0 & nd & 36.0 & Hall et al. (1993) \\
\hline na & nd & nd & FLB & 1100000 & 2404.0 & 2566.0 & nd & 58.4 & Unrein et al. (2007) \\
\hline
\end{tabular}




\begin{tabular}{|c|c|c|c|c|c|c|c|c|c|}
\hline na & nd & nd & FLB & 1200000 & 4448.0 & 6504.0 & nd & 51.1 & Unrein et al. (2007) \\
\hline na & nd & nd & FLB & 793000 & 1818.0 & 3151.0 & nd & nd & Unrein et al. (2007) \\
\hline na & nd & nd & FLB & 565000 & 1556.0 & 2280.0 & nd & 36.4 & Unrein et al. (2007) \\
\hline na & nd & nd & FLB & 830000 & 2043.0 & 2354.0 & nd & 44.1 & Unrein et al. (2007) \\
\hline na & nd & nd & FLB & 903000 & 2342.0 & 3400.0 & nd & 38.4 & Unrein et al. (2007) \\
\hline na & nd & nd & FLB & 706000 & 1756.0 & 2928.0 & nd & 57.5 & Unrein et al. (2007) \\
\hline na & nd & nd & FLB & 770000 & 883.0 & 2778.0 & nd & 38.6 & Unrein et al. (2007) \\
\hline na & nd & nd & FLB & 1110000 & 1457.0 & 2304.0 & nd & 51.6 & Unrein et al. (2007) \\
\hline na & nd & nd & FLB & 1400000 & 6230.0 & 4896.0 & nd & 60.3 & Unrein et al. (2007) \\
\hline na & nd & nd & FLB & 779000 & 1769.0 & 1868.0 & nd & 34.5 & Unrein et al. (2007) \\
\hline na & nd & nd & FLB & 673000 & 1681.0 & 2504.0 & nd & 64.5 & Unrein et al. (2007) \\
\hline na & nd & nd & FLB & nd & 10600.0 & 650.0 & nd & 55.1 & Zubkov and Tarran (2008) \\
\hline na & nd & nd & FLB & nd & 3200.0 & 160.0 & nd & 62.8 & Zubkov and Tarran (2008) \\
\hline na & nd & nd & FLB & nd & 1600.0 & 400.0 & nd & 58.3 & Zubkov and Tarran (2008) \\
\hline na & nd & nd & FLB & nd & 12000.0 & 1000.0 & nd & 71.4 & Zubkov and Tarran (2008) \\
\hline na & nd & nd & FLB & nd & 2600.0 & 500.0 & nd & 37.5 & Zubkov and Tarran (2008) \\
\hline 0.2 & nd & nd & FLB & 550000 & 18.0 & 86.0 & 7.0 & 41.3 & Sanders and Gast (2012) \\
\hline 0.2 & nd & nd & FLB & nd & nd & nd & nd & nd & Sanders and Gast (2012) \\
\hline 0.2 & nd & nd & FLB & 220000 & 13.0 & 43.0 & 2.0 & 14.6 & Sanders and Gast (2012) \\
\hline 0.2 & nd & nd & FLB & 181000 & 79.0 & 20.0 & 3.0 & 24.6 & Sanders and Gast (2012) \\
\hline 0.2 & nd & nd & FLB & 180000 & 60.0 & 55.0 & 11.0 & 31.6 & Sanders and Gast (2012) \\
\hline 0.2 & nd & nd & FLB & 220000 & 43.0 & 47.0 & 5.0 & 50.0 & Sanders and Gast (2012) \\
\hline 0.2 & nd & nd & FLB & 249000 & 51.0 & 87.0 & 3.0 & 10.5 & Sanders and Gast (2012) \\
\hline 0.2 & nd & nd & FLB & 193000 & 18.0 & 41.0 & 16.0 & 84.7 & Sanders and Gast (2012) \\
\hline 0.2 & nd & nd & FLB & 170000 & 27.0 & 102.0 & 19.0 & 36.5 & Sanders and Gast (2012) \\
\hline 0.2 & nd & nd & FLB & 198000 & 61.0 & 127.0 & 16.0 & 57.1 & Sanders and Gast (2012) \\
\hline 0.2 & nd & nd & FLB & 184000 & 61.0 & 93.0 & 36.0 & 28.0 & Sanders and Gast (2012) \\
\hline 0.2 & nd & nd & FLB & 218000 & 121.0 & 104.0 & 22.0 & 34.5 & Sanders and Gast (2012) \\
\hline
\end{tabular}




\begin{tabular}{|c|c|c|c|c|c|c|c|c|c|}
\hline 0.2 & nd & nd & FLB & 133000 & 37.0 & 122.0 & 8.0 & nd & Sanders and Gast (2012) \\
\hline 0.2 & nd & nd & FLB & 117000 & 116.0 & 92.0 & 10.0 & 21.1 & Sanders and Gast (2012) \\
\hline 0.2 & nd & nd & FLB & 160000 & 86.0 & 103.0 & 5.0 & 20.0 & Sanders and Gast (2012) \\
\hline 0.2 & nd & nd & FLB & 165000 & 104.0 & 204.0 & 2.0 & 8.0 & Sanders and Gast (2012) \\
\hline 0.2 & nd & nd & FLB & 137000 & 81.0 & 159.0 & 19.0 & 37.7 & Sanders and Gast (2012) \\
\hline 0.2 & nd & nd & FLB & 131000 & 43.0 & 89.0 & 8.0 & 6.1 & Sanders and Gast (2012) \\
\hline 0.2 & nd & nd & FLB & 251000 & 63.0 & 112.0 & 2.0 & 10.6 & Sanders and Gast (2012) \\
\hline 0.2 & nd & nd & FLB & 165000 & 32.0 & 180.0 & 13.0 & 30.0 & Sanders and Gast (2012) \\
\hline 0.2 & nd & nd & FLB & 124000 & 18.0 & 59.0 & 7.0 & 44.8 & Sanders and Gast (2012) \\
\hline 0.4 & 4.3 & 0.6 & FLB & 896000 & 2380.0 & 42.6 & 50.0 & 49.0 & Stukel et al. (2011) \\
\hline 0.4 & 4.8 & 0.5 & FLB & 955000 & 1790.0 & nd & 60.0 & 49.0 & Stukel et al. (2011) \\
\hline 0.4 & 6.4 & 0.6 & FLB & 832000 & 2392.0 & nd & 58.0 & 49.0 & Stukel et al. (2011) \\
\hline 0.4 & 7.8 & 0.6 & FLB & 932000 & 2300.0 & nd & 60.0 & 49.0 & Stukel et al. (2011) \\
\hline 0.9 & 18.0 & 1.3 & beads & 575000 & 1311.0 & 165.0 & 75.0 & nd & Gast et al. (2014) \\
\hline 0.9 & 18.5 & 1.0 & beads & 602000 & 1289.0 & 231.0 & 157.0 & nd & Gast et al. (2014) \\
\hline 0.9 & 25.5 & 1.6 & beads & 316000 & 1686.0 & 925.0 & 301.0 & nd & Gast et al. (2014) \\
\hline 0.9 & 25.9 & 1.7 & beads & 341000 & 1256.0 & 529.0 & 338.0 & nd & Gast et al. (2014) \\
\hline 0.9 & 28.6 & 1.7 & beads & 322000 & 1840.0 & 264.0 & 630.0 & nd & Gast et al. (2014) \\
\hline 0.9 & 16.4 & 0.8 & beads & 862000 & 1377.0 & 925.0 & 133.0 & nd & Gast et al. (2014) \\
\hline 0.5 & 26.1 & 1.6 & beads & 229000 & 463.0 & 848.0 & 198.0 & nd & Gast et al. (2014) \\
\hline 0.5 & 15.0 & 1.0 & beads & 254000 & 3261.0 & 123.0 & 123.0 & nd & Gast et al. (2014) \\
\hline 0.5 & 18.0 & 1.5 & beads & 314000 & 2071.0 & 192.0 & 192.0 & nd & Gast et al. (2014) \\
\hline 0.5 & 18.0 & 1.5 & beads & 233000 & 948.0 & 183.0 & 183.0 & nd & Gast et al. (2014) \\
\hline 0.5 & 21.8 & 1.4 & beads & 238000 & 793.0 & 55.0 & 55.0 & nd & Gast et al. (2014) \\
\hline 0.5 & 18.0 & 1.5 & beads & 261000 & 793.0 & 55.0 & 55.0 & nd & Gast et al. (2014) \\
\hline 0.5 & 18.0 & 1.5 & beads & 247000 & 1179.0 & 99.0 & 44.0 & nd & Gast et al. (2014) \\
\hline 0.9 & 21.2 & 1.5 & beads & 963000 & 1609.0 & 253.0 & 287.0 & nd & Gast et al. (2014) \\
\hline 0.9 & 21.1 & 1.2 & beads & 559000 & 1917.0 & 826.0 & 413.0 & nd & Gast et al. (2014) \\
\hline
\end{tabular}




\begin{tabular}{|c|c|c|c|c|c|c|c|c|c|}
\hline 0.9 & 29.3 & 1.9 & beads & 242000 & 1454.0 & 286.0 & 267.0 & nd & Gast et al. (2014) \\
\hline 0.9 & 27.7 & 1.9 & beads & 214000 & 1355.0 & 705.0 & 252.0 & nd & Gast et al. (2014) \\
\hline 0.9 & 27.3 & 1.7 & beads & 440000 & 1190.0 & 286.0 & 149.0 & nd & Gast et al. (2014) \\
\hline 0.9 & 18.9 & 1.1 & beads & 871000 & 1146.0 & 738.0 & 186.0 & nd & Gast et al. (2014) \\
\hline 0.5 & 26.0 & 1.5 & beads & 630000 & 650.0 & 331.0 & 315.0 & nd & Gast et al. (2014) \\
\hline 0.5 & 26.8 & 1.8 & beads & 314000 & 2843.0 & 474.0 & 267.0 & nd & Gast et al. (2014) \\
\hline 0.5 & 22.3 & 1.6 & beads & 376000 & 1289.0 & 33.0 & 82.0 & nd & Gast et al. (2014) \\
\hline 0.5 & 19.6 & 1.6 & beads & 349000 & 1245.0 & 253.0 & 208.0 & nd & Gast et al. (2014) \\
\hline 0.5 & 22.3 & 1.5 & beads & 432000 & 452.0 & 88.0 & 30.0 & nd & Gast et al. (2014) \\
\hline 0.5 & 31.4 & 1.9 & beads & 298000 & 452.0 & 88.0 & 30.0 & nd & Gast et al. (2014) \\
\hline 0.5 & 31.3 & 2.0 & beads & 39000 & 165.0 & 88.0 & 11.0 & nd & Gast et al. (2014) \\
\hline na & nd & nd & FLB & nd & nd & nd & nd & 60.0 & Nygaard and Tobiesen (1993) \\
\hline na & nd & nd & FLB & nd & nd & nd & nd & 4.0 & Nygaard and Tobiesen (1993) \\
\hline 0.4 & 4.6 & 0.6 & FLB & 263974 & 1474.4 & 293.5 & 27.3 & 49.7 & Sato et al. (2017) \\
\hline 0.4 & 0.0 & 0.1 & FLB & 322411 & 122.9 & 75.0 & 20.5 & 41.3 & Sato et al. (2017) \\
\hline 0.4 & 0.0 & 0.2 & FLB & 317345 & 238.9 & 211.6 & 27.3 & 39.9 & Sato et al. (2017) \\
\hline 0.3 & 0.0 & 0.1 & FLB & 306716 & 122.9 & 184.3 & 20.5 & 32.4 & Sato et al. (2017) \\
\hline 0.3 & 0.0 & 0.1 & FLB & 266325 & 116.0 & 225.2 & 20.5 & 31.8 & Sato et al. (2017) \\
\hline 0.3 & 0.0 & 0.0 & FLB & 391113 & 102.4 & 197.9 & 13.6 & 46.8 & Sato et al. (2017) \\
\hline 0.3 & 0.0 & 0.0 & FLB & 228374 & 61.4 & 102.4 & 6.8 & 31.9 & Sato et al. (2017) \\
\hline na & 0.0 & 0.0 & FLB & 400110 & 75.1 & 102.4 & 8.0 & 39.1 & Sato et al. (2017) \\
\hline na & 0.3 & 0.2 & FLB & 463816 & 245.7 & 122.8 & 13.6 & 41.2 & Sato et al. (2017) \\
\hline 0.4 & 10.1 & 0.9 & FLB & 397344 & 812.3 & 266.2 & 27.3 & 31.9 & Sato et al. (2017) \\
\hline 0.4 & 7.7 & 0.9 & FLB & 830238 & 1549.5 & 395.9 & 75.0 & 63.7 & Sato et al. (2017) \\
\hline 0.7 & 0.5 & 0.5 & FLB & 588376 & 1924.9 & 1071.7 & 68.2 & 52.9 & Sato et al. (2017) \\
\hline 0.7 & 0.5 & 0.5 & FLB & 437753 & 757.7 & 511.9 & 40.9 & 36.2 & Sato et al. (2017) \\
\hline 0.7 & 0.4 & 1.5 & FLB & 377612 & 334.5 & 348.1 & 0.1 & 1.0 & Sato et al. (2017) \\
\hline 0.9 & 1.5 & 0.5 & FLB & 1055426 & 8829.2 & 2297.8 & 306.2 & 16.8 & Vargas et al. (2012) \\
\hline
\end{tabular}


Appendices. Appendix B, Table B.3

\begin{tabular}{cccccccccc}
0.9 & 18.0 & 2.2 & FLB & 1517907 & 5553.8 & 2705.7 & 153.9 & 19.0 & Vargas et al. (2012) \\
0.9 & 27.0 & 1.5 & FLB & 1382265 & 4524.8 & 969.7 & 103.3 & 43.0 & Vargas et al. (2012) \\
0.9 & 2.5 & 1.0 & FLB & 2110603 & 837.5 & 3471.1 & 102.4 & 13.1 & Vargas et al. (2012) \\
0.9 & 10.0 & 1.0 & FLB & 918334 & 728.4 & 3063.7 & 102.0 & 7.7 & Vargas et al. (2012) \\
0.9 & 25.0 & 3.0 & FLB & 403917 & 363.6 & 816.3 & 51.8 & 12.2 & Vargas et al. (2012) \\
0.5 & 12.0 & 1.8 & FLB & 1220815 & 1081.8 & 7589.3 & 205.5 & 8.0 & Vargas et al. (2012) \\
0.5 & 10.0 & 2.0 & FLB & 1137270 & 934.6 & 7642.4 & 204.5 & 3.8 & Vargas et al. (2012) \\
0.5 & 15.0 & 2.0 & FLB & 708320 & 530.6 & 3437.0 & 102.7 & 19.2 & Vargas et al. (2012) \\
0.5 & 19.0 & 2.0 & FLB & 1001449 & 1004.8 & 4052.6 & 101.9 & 4.7 & Vargas et al. (2012) \\
0.5 & 17.0 & 2.0 & FLB & 991165 & 858.6 & 4770.2 & 50.7 & 1.9 & Vargas et al. (2012) \\
0.5 & 18.0 & 2.0 & FLB & 762141 & 763.0 & 3180.1 & 153.7 & 16.8 & Vargas et al. (2012) \\
\hline
\end{tabular}




\section{Appendix C}

$\underline{\text { Model equations }}$

General protist model

The general protist model is a development from previous modelling works (Flynn and Mitra, 2009, Mitra et al., 2016). The equations are given in full herein, in which the developments/changes from the original modelling works correspond to the: i) representation of diatoms (Eqs. 6 and 26-32); ii) description of acquired phototrophy (Eqs. 38-41); iii) allometric description of predation (Eqs. 44-51); and iv) non-predatory mortality term (Eq. 115). In addition to these, state variables described by quotas relative to the core mixotroph carbon biomass in Flynn and Mitra (2009) were modified to agree with model units in ERSEM (i.e., nutrient concentration per water volume).

The protist model has eight state variables accounting for carbon $(\mathrm{C})$, nitrogen $(\mathrm{N})$, phosphorus (P), and chlorophyll (Chl) associated to the mixotroph core biomass (Eqs. 14 , respectively) and to the material within feeding vacuoles (Eq. 5). In equation $5, X$ represents either $\mathrm{C}, \mathrm{N}, \mathrm{P}$, or $\mathrm{Chl}$ within the feeding vacuoles $(\mathrm{F})$. In order to simulate diatoms, an additional state variable is incorporated to represent silicon ( $\mathrm{Si}$ ) associated to the mixotroph core biomass (Eq. 6). All state variables are expressed in units of element quantity per water volume (e.g., $\mathrm{mg} \mathrm{C} \mathrm{m}^{-3}$ ) and will be referred between '[ ]' throughout the text. State variables are driven by inorganic carbon fixation (fixation ${ }_{C}$; Eq. 40), inorganic nutrient uptake (uptake , uptake $_{\mathrm{P}}$, uptake $\mathrm{Si}_{\mathrm{Si}}$; Eqs. 24, 25, and 32, respectively), ingestion (ingestion ${ }_{\mathrm{FX}}$; Eq. 57), digestion (digestion $\mathrm{FX}$; Eqs. 73-77), and prey assimilation

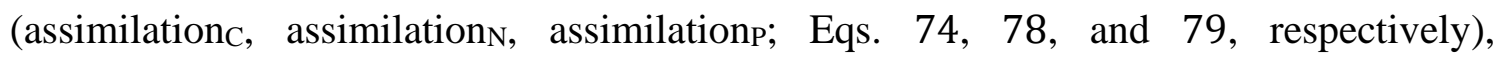
chlorophyll synthesis and degradation (balance $\mathrm{Chl}$; Eq. 83), respiration (respirationc; Eq. 96), regeneration of nutrients (regeneration ${ }_{N}$ and regeneration ${ }_{P}$; Eqs. 99, and 100), and non-predatory mortality (mortalityx; Eq. 115). Different protist functional types were included in the food web model enabling (disabling) one or more of these processes. Details on how each of these processes are modelled are given below. Please see Table C.1 for the definitions and units of constant parameters mentioned hereinafter. 


$$
\begin{gathered}
\frac{\mathrm{d}[\mathrm{C}]}{\mathrm{dt}}=\text { fixation }_{\mathrm{C}}+\operatorname{assimilation}_{\mathrm{C}}-\text { respiration }_{\mathrm{C}}-\text { mortality }_{\mathrm{C}} \\
\frac{\mathrm{d}[\mathrm{N}]}{\mathrm{dt}}=\text { uptake }_{\mathrm{N}}+\operatorname{assimilation}_{\mathrm{N}}-\text { regeneration }_{\mathrm{N}}-\text { mortality }_{\mathrm{N}} \\
\frac{\mathrm{d}[\mathrm{P}]}{\mathrm{dt}}=\text { uptake }_{\mathrm{P}}+\text { assimilation }_{\mathrm{P}}-\text { regeneration }_{\mathrm{P}}-\text { mortality }_{\mathrm{P}} \\
\frac{\mathrm{d}[\mathrm{FX}]}{\mathrm{dt}}=\frac{\text { ingestion }}{\mathrm{dt}}=\text { balance }_{\mathrm{Chl}}-\text { mortality }_{\mathrm{Chl}} \\
\frac{\mathrm{d}[\mathrm{Si}]}{\mathrm{dt}}=\text { uptake }_{\mathrm{Si}}-\text { mortality }_{\mathrm{Fi}}-\text { mortality }_{\mathrm{FX}}
\end{gathered}
$$

Feedback response curves

A common feature of the plankton model is the usage of normalised sigmoidal functions to describe feedback response curves (Fig. C.1). The general form of this function $(\mathrm{fb})$ is described in Eq. 7, in which $\mathrm{S}$ is a quotient describing the stimulus of the process, $\mathrm{K}$ is a half-saturation constant, and $\mathrm{H}$ is the Hill number (controlling the shape of the sigmoidal curve):

$$
\mathrm{fb}=\frac{\left(1+\mathrm{K}^{\mathrm{H}}\right) \cdot \mathrm{S}^{\mathrm{H}}}{\mathrm{S}^{\mathrm{H}}+\mathrm{K}^{\mathrm{H}}}
$$

\section{Normalised nutrient status}

A key characteristic of the model is the definition of the nutrient status of plankton through normalised nutrient (e.g., nitrogen or phosphorus) to carbon quotas. The normalised response curves are defined by a function of the simulated $\mathrm{NC}$ or PC quotas (Eqs. 8 and 9, respectively) and the constant parameters delimiting the minimum $\left(\mathrm{NC}_{\min }\right.$ and $\left.\mathrm{PC}_{\min }\right)$ and maximum $\left(\mathrm{NC}_{\max }\right.$ and $\left.\mathrm{PC}_{\max }\right)$ values of the internal quotas (Eqs. 10-11); the form of the response curve is given in Fig. C.1a. A minimum function of the normalised response curves (NPCu) ultimately defines the nutrient status of plankton (Eq. 12). To simulate diatoms, NPCu also depends on the normalised silicate response curve (Eq. 27).

$$
\mathrm{NC}=\frac{[\mathrm{N}]}{[\mathrm{C}]}
$$




$$
\begin{aligned}
& \mathrm{PC}=\frac{[\mathrm{P}]}{[\mathrm{C}]} \\
& \mathrm{NCu}=\left\{\begin{array}{lr}
0, & \mathrm{NC}<\mathrm{NC}_{\text {min }} \\
\left(1+\mathrm{K}_{\mathrm{qn}}\right) \cdot \frac{\left(\mathrm{NC}-\mathrm{NC}_{\min }\right)}{\left(\mathrm{NC}-\mathrm{NC}_{\min }\right)+\mathrm{K}_{\mathrm{qn}} \cdot\left(\mathrm{NC}_{\max }-\mathrm{NC}_{\min }\right)}, & \mathrm{NC}_{\text {max }} \geq \mathrm{NC} \geq \mathrm{NC}_{\text {min }} \\
1, & \mathrm{NC}>\mathrm{NC}_{\text {max }}
\end{array}\right. \\
& \mathrm{PCu}=\left\{\begin{array}{lr}
0, & \mathrm{PC}<\mathrm{PC}_{\min } \\
\left(1+\mathrm{K}_{\mathrm{qp}}\right) \cdot \frac{\left(\mathrm{PC}-\mathrm{PC}_{\min }\right)}{\left(\mathrm{PC}-\mathrm{PC}_{\min }\right)+\mathrm{K}_{\mathrm{qp}} \cdot\left(\mathrm{PC}_{\max }-\mathrm{PC}_{\min }\right)}, & \mathrm{PC}_{\text {max }} \geq \mathrm{PC} \geq \mathrm{PC}_{\min } \\
1, & \mathrm{PC}>\mathrm{PC}_{\max }
\end{array}\right. \\
& \mathrm{NPCu}=\min (\mathrm{NCu}, \mathrm{PCu})
\end{aligned}
$$

\section{Nitrogen and phosphorus uptake}

Nutrient uptake is based on previous modelling studies (Flynn 2001, Flynn 2002, Flynn 2003). Nitrogen uptake is the sum of ammonium and nitrate uptake, simulated through reference to their potential transport rates $\left(\mathrm{A}_{\mathrm{pt}}\right.$ and $\mathrm{N}_{\mathrm{pt}}$, respectively). In equations 13 and $14, \mathrm{~A}_{\mathrm{pt}}$ and $\mathrm{N}_{\mathrm{pt}}\left(\mathrm{gN} \mathrm{gC}^{-1} \mathrm{day}^{-1}\right)$ are defined by the external nutrient concentration $\left(\left[\mathrm{NH}_{4}\right]\right.$ and $\left.\left[\mathrm{NO}_{3}\right]\right)$, the half-saturation constants for nutrient uptake $\left(\mathrm{K}_{\mathrm{a}}\right.$ and $\left.\mathrm{K}_{\mathrm{n}}\right)$, the relative preferences for each nutrient $\left(A_{\text {pref }}\right.$ and $\left.N_{\text {pref }}\right)$, and the maximum potential nutrient uptake which, in turn, is defined by the maximum phototrophic growth rate $\left(\mu_{\text {phot }}\right)$ and the maximum $\mathrm{NC}$ quota $\left(\mathrm{NC}_{\max }\right)$. The actual transport rates $\left(\mathrm{A}_{\mathrm{t}}\right.$ and $\mathrm{N}_{\mathrm{t}} ; \mathrm{gN} \mathrm{gC}^{-1}$ day $\left.^{-1}\right)$ are defined to meet the demands and assume ammonium as the most energetically efficient form of nitrogen; thus, nitrate uptake only occurs if ammonium transport is not sufficient to meet demands (Eqs. 15 and 16).

$$
\begin{aligned}
& A_{p t}=\left(\mu_{\text {phot }} \cdot \mathrm{NC}_{\text {max }}\right) \cdot A_{\text {pref }} \cdot \frac{\left[\mathrm{NH}_{4}\right]}{\left[\mathrm{NH}_{4}\right]+\mathrm{K}_{\mathrm{a}}} \\
& \mathrm{N}_{\mathrm{pt}}=\left(\mu_{\mathrm{phot}} \cdot \mathrm{NC}_{\text {max }}\right) \cdot \mathrm{N}_{\mathrm{pref}} \cdot \frac{\left[\mathrm{NO}_{3}\right]}{\left[\mathrm{NO}_{3}\right]+\mathrm{K}_{\mathrm{n}}} \\
& A_{t}= \begin{cases}A_{p t}, & A_{p t}<\mu_{p h o t} \cdot N_{\text {max }} \\
\mu_{p h o t} \cdot N_{\text {max }}, & A_{p t}>\mu_{\text {phot }} \cdot \mathrm{NC}_{\text {max }}\end{cases} \\
& \mathrm{N}_{\mathrm{t}}=\left\{\begin{array}{lr}
\mathrm{N}_{\mathrm{pt}}, & \mu_{\mathrm{phot}} \cdot \mathrm{NC}_{\max }>\mathrm{A}_{\mathrm{pt}}+\mathrm{N}_{\mathrm{pt}} \\
\mu_{\mathrm{phot}} \cdot \mathrm{NC}_{\text {max }}-\mathrm{A}_{\mathrm{pt}}, & \mathrm{A}_{\mathrm{pt}}<\mu_{\mathrm{phot}} \cdot \mathrm{NC}_{\max }<\mathrm{A}_{\mathrm{pt}}+\mathrm{N}_{\mathrm{pt}} \\
0, & \mu_{\mathrm{phot}} \cdot \mathrm{NC}_{\max }<\mathrm{A}_{\mathrm{pt}}
\end{array}\right.
\end{aligned}
$$




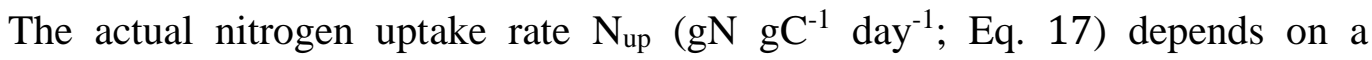
normalised feedback response curve $\left(\mathrm{NC}_{\mathrm{fb}}\right)$ which blocks the uptake as $\mathrm{NC}$ approaches the absolute $\mathrm{NC}$ quota $\left(\mathrm{NC}_{\mathrm{abs}}\right)$ (Eq. 18).

$$
\begin{gathered}
\mathrm{N}_{\mathrm{up}}= \begin{cases}\left(\mathrm{A}_{\mathrm{t}}+\mathrm{N}_{\mathrm{t}}\right) \cdot \mathrm{NC}_{\mathrm{fb}}, & \mathrm{NCu}=\mathrm{NPCu} \\
\left(\mathrm{A}_{\mathrm{t}}+\mathrm{N}_{\mathrm{t}}\right) \cdot \mathrm{NC}_{\mathrm{fb}} \cdot \mathrm{NPCu}^{\beta}, & \mathrm{NCu}>\mathrm{NPCu}\end{cases} \\
\mathrm{NC}_{\mathrm{fb}}=\left(1+\mathrm{K}_{\mathrm{q}}^{\mathrm{H}_{\mathrm{q}}}\right) \cdot \frac{\left(1-\frac{\mathrm{NC}}{\mathrm{NC}_{\mathrm{abs}}}\right)^{\mathrm{H}_{\mathrm{q}}}}{\left(1-\frac{N C}{\mathrm{NC}_{\mathrm{abs}}}\right)^{\mathrm{H}_{\mathrm{q}}}+\mathrm{K}_{\mathrm{q}}^{\mathrm{H}_{\mathrm{q}}}}
\end{gathered}
$$

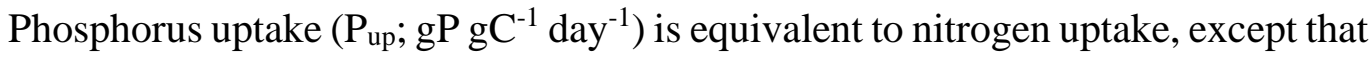
there is only one source of $\mathrm{P}$ (Eqs. 19-21).

$$
\begin{gathered}
\mathrm{P}_{\mathrm{t}}=\left(\mu_{\mathrm{phot}} \cdot \mathrm{PC}_{\text {max }}\right) \cdot \frac{\left[\mathrm{PO}_{4}\right]}{\left[\mathrm{PO}_{4}\right]+\mathrm{K}_{\mathrm{p}}} \\
\mathrm{P}_{\mathrm{up}}=\left\{\begin{array}{l}
\mathrm{P}_{\mathrm{t}} \cdot \mathrm{PC}_{\mathrm{fb}}, \\
\mathrm{P}_{\mathrm{t}} \cdot \mathrm{NPCu}^{\beta} \cdot \mathrm{PC}_{\mathrm{fb}}, \quad \mathrm{PCu}=\mathrm{NPCu}
\end{array}\right. \\
\mathrm{PC}_{\mathrm{fb}}=\left(1+\mathrm{K}_{\mathrm{q}}^{\mathrm{H}_{\mathrm{q}}}\right) \cdot \frac{\left(1-\frac{\mathrm{PC}}{\mathrm{PC}_{\mathrm{abs}}}\right)^{\mathrm{H}_{\mathrm{q}}}}{\left(1-\frac{\mathrm{PC}}{\mathrm{PC}_{\mathrm{abs}}}\right)^{\mathrm{H}_{\mathrm{q}}}+\mathrm{K}_{\mathrm{q}}^{\mathrm{H}_{\mathrm{q}}}}
\end{gathered}
$$

The population uptake of ammonium, nitrate, total $\mathrm{N}\left(\mathrm{mg} \mathrm{N} \mathrm{m}^{-3}\right.$ day $\left.^{-1}\right)$, and $\mathrm{P}(\mathrm{mg}$ $\mathrm{P} \mathrm{m}^{-3}$ day $\left.^{-1}\right)$, respectively, is then given by:

$$
\begin{gathered}
\text { uptake }_{\mathrm{NH} 4}=\mathrm{N}_{\mathrm{up}} \cdot\left(1-\frac{\mathrm{N}_{\mathrm{t}}}{\mathrm{N}_{\mathrm{t}}+\mathrm{A}_{\mathrm{t}}}\right) \cdot[\mathrm{C}] \\
\text { uptake }_{\mathrm{NO3}}=\mathrm{N}_{\mathrm{up}} \cdot \frac{\mathrm{N}_{\mathrm{t}}}{\mathrm{N}_{\mathrm{t}}+\mathrm{A}_{\mathrm{t}}} \cdot[\mathrm{C}] \\
\text { uptake }_{\mathrm{N}}=\mathrm{N}_{\mathrm{up}} \cdot[\mathrm{C}] \\
\text { uptake }_{\mathrm{P}}=\mathrm{P}_{\mathrm{up}} \cdot[\mathrm{C}]
\end{gathered}
$$

\section{Silicon dynamics for diatoms}

Diatoms representation differs from other phototrophic protists due to their physiological requirement for silicon to build their frustules. In turn, silicon uptake differs fundamentally from that of nitrogen and phosphorus because the external silicon 
concentration, instead of the internal silicon concentration, ultimately affects growth, consequently, quota models do not apply for silicon dynamics (Martin-Jézéquel et al., 2000; Flynn and Martin-Jézéquel, 2000). In order to represent diatoms, the uptake of silicon was implemented following Flynn (2005). The potential uptake rate of silicon $\left(\mathrm{S}_{\mathrm{t}}\right.$, $\mathrm{gSi} \mathrm{gC}^{-1}$ day $^{-1}$; Eq. 26) is a function of $\mu_{\mathrm{phot}}$, the maximum internal silicon to carbon ratio $\left(\mathrm{SC}_{\max }\right)$, the external silicon availability ([Si]), and a half-saturation constant for silicon uptake $\left(\mathrm{K}_{\mathrm{Si}}\right)$. In turn, the normalised quota for silicon $\left(\mathrm{SCu}\right.$; Eq. 27) is a function of $\mathrm{S}_{\mathrm{t}}$, $\mu_{\text {phot }}$, the minimum internal silicon to carbon ratio $\left(\mathrm{SC}_{\mathrm{min}}\right)$, and the internal silicon to carbon ratio (SC; Eq. 28). Thus, contrary to the other phototrophic groups, the normalised nutrient status $(\mathrm{NPCu})$ among diatoms is a minimum function of the normalised nitrogen, phosphorus, and silicon status (Eq. 29).

$$
\begin{gathered}
\mathrm{S}_{\mathrm{t}}=\left(\mu_{\mathrm{phot}} \cdot \mathrm{SC}_{\mathrm{max}}\right) \cdot \frac{[\mathrm{Si}]}{[\mathrm{Si}]+\mathrm{K}_{\mathrm{Si}}} \\
\mathrm{SCu}= \begin{cases}0, & \mathrm{SC} \leq \mathrm{SC}_{\min } \\
\frac{\mathrm{S}_{\mathrm{t}}}{\left(\mu_{\mathrm{phot}} \cdot \mathrm{SC}_{\mathrm{min}}\right)}, & \mathrm{S}_{\mathrm{t}}<\mu_{\mathrm{phot}} \cdot \mathrm{SC}_{\text {min }} \\
1, & \mathrm{~S}_{\mathrm{t}} \geq \mu_{\mathrm{phot}} \cdot \mathrm{SC}_{\mathrm{min}}\end{cases} \\
\mathrm{SC}=\frac{[\mathrm{S}]}{[\mathrm{C}]} \\
\mathrm{NPCu}=\min (\mathrm{NCu}, \mathrm{PCu}, \mathrm{SCu})
\end{gathered}
$$

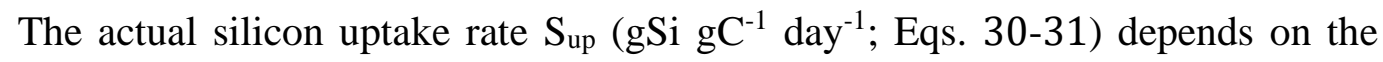
current growth rate and not on NPCu as previously described for N and P uptake.

$$
\begin{gathered}
S_{\text {up }}= \begin{cases}S_{\mathrm{t}} \cdot \mathrm{SC}_{\mathrm{fb}}, & \mathrm{SCu}=\mathrm{NPCu} \\
\mathrm{S}_{\mathrm{t}} \cdot\left(\frac{\mu}{\mu_{\mathrm{phot}}}\right)^{\beta} \cdot \mathrm{SC}_{\mathrm{fb}}, \quad \mathrm{SCu}>\mathrm{NPCu}\end{cases} \\
\mathrm{SC}_{\mathrm{fb}}=\frac{\left(1-\frac{\mathrm{SC}}{\mathrm{SC}_{\mathrm{abs}}}\right)^{\mathrm{H}_{\mathrm{q}}}}{\left(1-\frac{\mathrm{SC}}{\mathrm{SC}_{\mathrm{abs}}}\right)^{\mathrm{H}_{\mathrm{q}}}+\mathrm{K}_{\mathrm{q}}}
\end{gathered}
$$

The diatom population uptake of silicon is obtained by multiplying $S_{\text {up }}$ by diatom carbon biomass [C]: 


$$
\text { uptake }_{S i}=S_{\text {up }} \cdot[\mathrm{C}]
$$

\section{Photosynthesis}

Photosynthesis is described assuming constitutive (inherent; $\mathrm{P}_{\mathrm{CM}}$ ) or nonconstitutive (acquired; $\mathrm{P}_{\mathrm{NCM}}$ ) capacities. $\mathrm{P}_{\mathrm{CM}}$ and $\mathrm{P}_{\mathrm{NCM}}$ are calculated through the Smith negative exponential equation, in which the photosystem size is associated to Chl in the core biomass in the former and to Chl within feeding vacuoles in the later (Eqs. 33 and 34 , respectively).

$$
\begin{gathered}
\text { ChlC }=\frac{[\mathrm{Chl}]}{[\mathrm{C}]} \\
\text { FChlC }=\frac{[\mathrm{FChl}]}{[\mathrm{FC}]}
\end{gathered}
$$

Following Flynn (2001), the maximum rate of carbon fixation among plankton

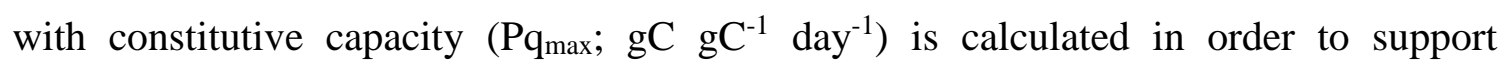
phototrophy at the current nutrient status, covering the costs associated to respiration, reduction of nitrate to ammonium, and amino acids synthesis (Eq. 35). Respiration (BR $\mathrm{B}_{\mathrm{op}}$;

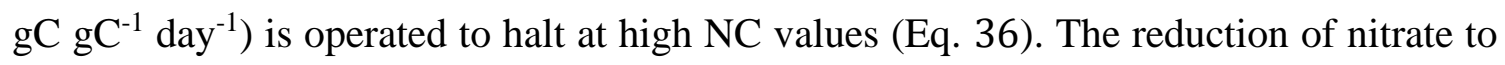
ammonium and amino acids synthesis are controlled by constant parameters (redo and $\mathrm{AA}_{\mathrm{syn}}$, respectively). Met $\mathrm{M}$ is a constant parameter equal to 1 for all functional types except for dinoflagellates, in which there is an extra cost to achieve the required gross

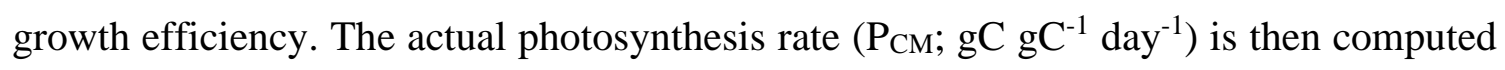
through equation 37 , in which $\alpha_{\mathrm{Chl}}$ is the initial slope of the photosynthesis-irradiance curve and PAR is the integrated photosynthetic active radiation, multiplied by 4.27 to convert units of $\mathrm{W} \mathrm{m}^{-2}$ to PFD (Photon Flux Density; $\mu$ mol photon $\mathrm{m}^{-1} \mathrm{~s}^{-1}$ ) and by $24 \times$ $60 \times 60$ to convert PFD into units of per day.

$$
\begin{gathered}
\mathrm{Pq}_{\text {max }}=\left(\mu_{\mathrm{phot}}+\mathrm{BR}_{\mathrm{op}}+\left(\mu_{\mathrm{phot}} \cdot \mathrm{NC}_{\text {max }}\right) \cdot\left(\text { redco }+\mathrm{AA}_{\text {syn }} \cdot \mathrm{Met}_{\mathrm{M}}\right)\right) \cdot \mathrm{NPCu} \\
\mathrm{BR}_{\mathrm{op}}=\mu_{\max } \cdot \mathrm{BR} \cdot \frac{\frac{\mathrm{NC}_{\mathrm{abs}}-\mathrm{NC}}{\mathrm{NC}_{\mathrm{abs}}-\mathrm{NC}_{\min }}}{\frac{\mathrm{NC}_{\mathrm{abs}}-\mathrm{NC}}{\mathrm{NC}_{\mathrm{abs}}-\mathrm{NC}_{\min }}+0.01} \\
\mathrm{P}_{\mathrm{CM}}=\mathrm{Pq}_{\max } \cdot\left(1-\exp \left(\frac{-\alpha_{\mathrm{Chl}} \cdot \mathrm{ChlC} \cdot \mathrm{PAR} \cdot 4.57 \cdot 24 \cdot 60 \cdot 60}{\mathrm{Pq}_{\max }}\right)\right)
\end{gathered}
$$


The maximum rate of carbon fixation among plankton with non-constitutive

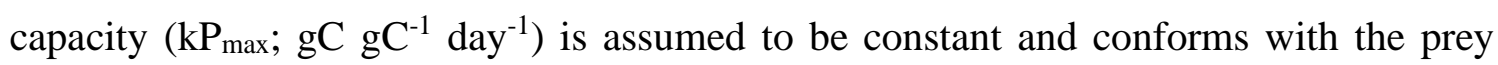
under ideal nutrient status $(\mathrm{NPCu}=1)$. Thus, $\mathrm{P}_{\mathrm{NCM}}\left(\mathrm{gC} \mathrm{gC}^{-1}\right.$ day $\left.^{-1}\right)$ is computed through equation 38.

$$
\mathrm{P}_{\mathrm{NCM}}=\mathrm{kP}_{\max } \cdot \mathrm{NPCu} \cdot\left(1-\exp \left(\frac{-\alpha_{\mathrm{Chl}} \cdot \mathrm{FChlC} \cdot \mathrm{PAR} \cdot 4.57 \cdot 24 \cdot 60 \cdot 60}{\mathrm{kP}_{\max } \cdot \mathrm{NPCu}}\right)\right)
$$

Total carbon fixation is then computed by the sum of $\mathrm{P}_{\mathrm{CM}}$ and $\mathrm{P}_{\mathrm{NCM}}$ (Eq. 39; $\mathrm{gC}$ $\mathrm{gC}^{-1}$ day $^{-1}$ ) which, in turn, is multiplied by the plankton biomass to obtain the total carbon fixation by the plankton population (Eq. $40 ; \mathrm{mg} \mathrm{C} \mathrm{m}^{-3}$ day $^{-1}$ ).

$$
\begin{gathered}
\mathrm{P}_{\text {tot }}=\mathrm{P}_{\mathrm{CM}}+\mathrm{P}_{\mathrm{NCM}} \\
\text { fixation }_{\mathrm{C}}=\mathrm{P}_{\text {tot }} \cdot[\mathrm{C}]
\end{gathered}
$$

To simulate non-constitutive mixotrophs, i.e. acquired phototrophy, chlorophyll within the feeding vacuoles was assumed to be diluted over time at a constant rate instead of digested and, thus, Eq. 5 was replaced by Eq. 41:

$$
\begin{gathered}
\frac{\mathrm{d}[\mathrm{FChl}]}{\mathrm{dt}}=\text { ingestion }_{\mathrm{Chl}}-\text { dilution }_{\mathrm{Chl}}-\text { mortality }_{\mathrm{Chl}} \\
\text { in which dilution } \\
\mathrm{Chl}
\end{gathered}
$$

\section{Ingestion}

The ingestion of prey is controlled by the demand for heterotrophic nutrition relative to the maximum growth rate $\left(\mu_{\max }\right)$. There will always be a demand for heterotrophic nutrition if $\mu_{\max }$ is higher than $\mu_{\mathrm{phot}}$ (and neglecting any satiation feedback). The demand for any/all nutrients is expressed in the model by a quotient $\left(\mu_{\text {rel }}\right)$ that relates the $24 \mathrm{~h}$ averaged growth rate and $\mu_{\max }$ (Eq. 42). The constant parameter $S_{\text {mix }}$ enable/disable the control of phototrophy over heterotrophy assuming values of 0 or 1 . If phototrophy down-regulates heterotrophy (i.e., $S_{\text {mix }}=0$ ), $\mu_{\text {rel }}$ controls the maximum possible size of the feeding vacuoles $\left(\mathrm{FC}_{\max }\right)$ through a normalised response curve, with $\mathrm{FC}_{\max }$ varying between minimum ( $\left.\mathrm{FC}_{\mathrm{min}}\right)$ and absolute ( $\left.\mathrm{FC}_{\mathrm{abs}}\right)$ maximum values (Eq. 43). Overall, if enough $\mathrm{C}$ is provided through photosynthesis there is less need to obtain $\mathrm{C}$ through feeding. On the other hand, if phototrophy has no control over heterotrophy (i.e., $\left.\mathrm{S}_{\text {mix }}=1\right), \mu_{\text {rel }}$ is not of importance and $\mathrm{FC}_{\max }$ is constant and equals to $\mathrm{FC}_{\mathrm{abs}}(\mathrm{Eq}$. 43).

$$
\mu_{\mathrm{rel}}=\min \left(1, \frac{\mu_{\mathrm{avg}}}{\mu_{\max }}\right)
$$




$$
\mathrm{FC}_{\text {max }}= \begin{cases}\mathrm{FC}_{\text {min }}+\left(1-\frac{\left(1+\mathrm{K}_{\mathrm{het}}^{\mathrm{H}_{\text {het }}}\right) \cdot \mu_{\mathrm{rel}}^{\mathrm{H}_{\text {het }}}}{\left(\mu_{\mathrm{rel}}^{\mathrm{H}_{\text {het }}}+\mathrm{K}_{\mathrm{het}}^{\mathrm{H}_{\text {het }}}\right)}\right) \cdot\left(\mathrm{FC}_{\mathrm{abs}}-\mathrm{FC}_{\text {min }}\right), & \mathrm{S}_{\text {mix }}=0 \\ \mathrm{FC}_{\mathrm{abs}}, & \mathrm{S}_{\text {mix }}=1\end{cases}
$$

Ingestion was implemented following the allometric description of predation by Flynn and Mitra (2016). This formulation simulates the kinetics of prey capture and ingestion relating prey abundance and encounter rates to a prey-selection function controlled by satiation. Since the model is biomass-based, prey numeric abundance was estimated diving total prey biomass by the carbon content per prey cell (cellC $\mathrm{p}_{\mathrm{p}}$, $\mathrm{ng} \mathrm{cell}^{-}$ ${ }^{1}$ ). Allometric regressions from the literature controlled by the values of $a$ and $b$ in Eq. 44 were used to obtain protist cellC $\mathrm{C}_{\mathrm{i}}$, in which subscript $i$ refers to the prey or to the predator (Menden-Deuer and Lessard 2000). In equation 44, size ( size $_{\mathrm{i}}$ ) is expressed in $\mu \mathrm{m}$ ESD:

$$
\text { cellC }_{i}=\frac{a}{1000} \cdot\left(\left(\frac{4}{3} \cdot \pi \cdot \frac{\text { size }_{i}}{2}\right)^{3}\right)^{b}
$$

Potential encounter rates between prey and predator are computed based on predator and prey radius $\left(\mathrm{r}_{\mathrm{i}} ; \mathrm{m}\right)$, their respective speeds of motion $\left(\mathrm{SM}_{\mathrm{i}} ; \mathrm{m} \mathrm{s}^{-1}\right)$, prey abundance (NA; cells $\mathrm{m}^{-3}$ ), and the root-mean-squared turbulence $\left(\mathrm{w} ; \mathrm{m} \mathrm{s}^{-1}\right)$. The equations describing $\mathrm{r}_{\mathrm{i}}, \mathrm{SM}_{\mathrm{i}}$, and NA are as following:

$$
\begin{gathered}
\mathrm{r}_{\mathrm{i}}=\frac{\text { size }_{\mathrm{i}}}{2 \cdot 10^{6}} \\
\mathrm{SM}_{\mathrm{i}}=10^{-6} \cdot\left(38.542 \cdot \operatorname{size}_{\mathrm{i}}{ }^{0.5424}\right) \\
\mathrm{NA}=\frac{\left[\text { prey }_{\mathrm{C}}\right] \cdot 10^{6}}{\text { cellC }_{\mathrm{i}}}
\end{gathered}
$$

$\mathrm{SM}_{\mathrm{i}}$ was assumed to follow ballistic paths for protists that are motile to maximize encounters. In equation 47, [prey $\mathrm{C}$ ] corresponds to the biomass of prey population (mg C $\mathrm{m}^{-3}$ ). Finally, Enc $\mathrm{s}$ (prey predator ${ }^{-1} \mathrm{~s}^{-1}$ ) calculates the encounter rates between prey (subscript p) and predator (subscript P) following Rothschild and Osborn (1988):

$$
\mathrm{Enc}_{\mathrm{s}}=\pi \cdot\left(\mathrm{r}_{\mathrm{p}}+\mathrm{r}_{\mathrm{P}}\right) \cdot \mathrm{NA} \cdot\left(\mathrm{SM}_{\mathrm{p}}^{2}+3 \cdot \mathrm{SM}_{\mathrm{P}}^{2}+4 \cdot \mathrm{w}^{2}\right) \cdot\left(\mathrm{SM}_{\mathrm{P}}^{2}+\mathrm{w}^{2}\right)^{-0.5} \cdot 3^{-1}
$$

Equation 48 is then converted from units of $\mathrm{s}^{-1}$ to day ${ }^{-1}$ to conform to model units (Enc $\mathrm{d}_{\mathrm{d}}$; prey predator ${ }^{-1}$ day $\left.^{-1}\right)$ :

$$
\text { Enc }_{\mathrm{d}}=\mathrm{Enc}_{\mathrm{s}} \cdot 24 \cdot 60 \cdot 60
$$


Assuming that all encountered prey are consumed, and ignoring other controls, the maximum potential ingestion rate $\left(\mathrm{C}_{\mathrm{p}} ; \mathrm{gC} \mathrm{gC}^{-1} \mathrm{day}^{-1}\right)$ is computed referring to Enc $\mathrm{d}_{\mathrm{d}}$ and to the carbon contents per cell for the prey $\left(\right.$ cellC $\left._{\mathrm{p}}\right)$ and for the predator $\left(\right.$ cellC $\left._{\mathrm{P}}\right)$ :

$$
\mathrm{C}_{\mathrm{p}}=\text { Enc }_{\mathrm{d}} \cdot \frac{\text { cellC }_{\mathrm{p}}}{\text { cellC }_{\mathrm{P}}}
$$

The actual ingestion rate is controlled by size selectivity and by satiation (Flynn, 2018). In the model, size selectivity $\left(\mathrm{C}_{\mathrm{con}}\right)$ refers to the total prey size spectrum accessible by the predator, defined by the minimum, maximum, and optimal prey sizes $\left(S_{\min }, S_{\max }\right.$, and $\mathrm{S}_{\mathrm{opt}}$, respectively); thus, capture is minimum on both extremes of the prey size spectrum and increases linearly towards $S_{\text {opt }}$ (Eq. 51; Fig. C.1b). If prey size is not within the defined prey size spectrum, capture does not take place.

$$
C_{\text {con }}= \begin{cases}\frac{\operatorname{size}_{p}-S_{\text {min }}}{S_{\text {opt }}-S_{\text {min }}}, & \text { size }_{p}<S_{\text {opt }} \\ \frac{S_{\max }-\operatorname{size}_{p}}{S_{\text {max }}-S_{\text {opt }}}, & \text { size }_{p}>S_{\text {opt }}\end{cases}
$$

The satiation control $\left(\mathrm{S}_{\mathrm{con}}\right)$ is defined by a normalised sigmoidal function of the relative capacity of the feeding vacuoles $\left(\mathrm{FC}_{\text {relv }}\right)$, decreasing as $\mathrm{FC}_{\text {relv }}$ approaches $\mathrm{FC}_{\max }$ (Eqs. 52-54; Fig. C.1c).

$$
\begin{gathered}
\mathrm{FC}=\frac{[\mathrm{FC}]}{[\mathrm{C}]} \\
\mathrm{FC}_{\text {relV }}=\min \left(1, \frac{\mathrm{FC}}{\mathrm{FC}_{\text {max }}}\right) \\
\mathrm{S}_{\text {con }}=\left(1+\mathrm{K}_{\text {ing }}^{\mathrm{H}_{\text {ing }}}\right) \cdot \frac{\left(1-\mathrm{FC}_{\text {relV }}\right)^{\mathrm{H}_{\text {ing }}}}{\left(1-\mathrm{FC}_{\text {relV }}\right)^{\mathrm{H}_{\text {ing }}}+\mathrm{K}_{\text {ing }}^{\mathrm{H}_{\text {ing }}}}
\end{gathered}
$$

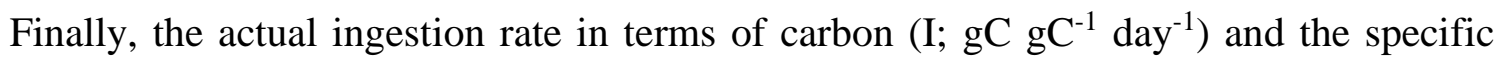
ingestion rate $\left(\mathrm{sI} ; \mathrm{day}^{-1}\right)$ are computed by:

$$
\begin{gathered}
\mathrm{I}=\mathrm{C}_{\mathrm{p}} \cdot \mathrm{C}_{\text {con }} \cdot \mathrm{S}_{\text {con }} \\
\mathrm{sI}=\frac{\mathrm{I} \cdot[\mathrm{C}]}{\left[\text { prey }_{\mathrm{C}}\right]}
\end{gathered}
$$


The total ingestion of prey by the protist population is obtained by Eq. 57, in which $\mathrm{X}$

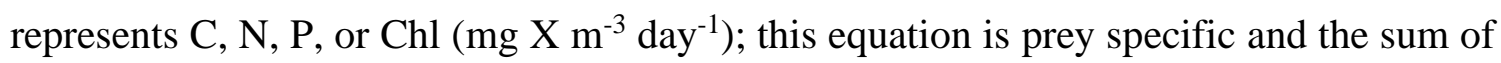
the ingestion of $n$ prey types ultimately defines the total ingestion.

$$
\text { ingestion }_{\mathrm{X}}=\mathrm{sI} \cdot\left[\mathrm{prey}_{\mathrm{X}}\right]
$$

\section{Digestion and assimilation}

Digestion of ingested prey is down regulated by phototrophy if $S_{\mathrm{pd}}=1$ and/or $\mathrm{Pbal}_{\text {crit }}>0$. First, if enough carbon is flowing from photosynthesis, there is less need to digest food (i.e. $\left.S_{p d}=1\right)$. This control $\left(D_{c o n}\right)$ is regulated by the relative rate of photosynthesis ( $\left.\mathrm{PS}_{\text {rel }}\right)$ through a normalised sigmoidal function (Eqs. 58-59; Fig. C.1d).

$$
\begin{gathered}
\mathrm{D}_{\mathrm{con}}=1-\mathrm{S}_{\mathrm{pd}} \cdot \frac{\left(1+\mathrm{K}_{\mathrm{pd}}^{\mathrm{H}_{\mathrm{pd}}}\right) \cdot \mathrm{PS}_{\mathrm{rel}}^{\mathrm{H}_{\mathrm{pd}}}}{\mathrm{PS}_{\mathrm{rel}}^{\mathrm{H}_{\mathrm{pd}}}+\mathrm{K}_{\mathrm{pd}}^{\mathrm{H}_{\mathrm{pd}}}} \\
\mathrm{PS}_{\mathrm{rel}}=\min \left(1, \frac{\mathrm{P}_{\text {tot }}}{\mathrm{Pq}_{\text {max }}}\right)
\end{gathered}
$$

The second control is associated to the need to obtain a critical proportion of growth through photosynthesis (i.e., $\mathrm{Pbal}_{\text {crit }}>0$ ). The relative contribution of photosynthesis to growth rate $\left(\mathrm{P}_{\text {bal }}\right)$ is related to $\mathrm{Pbal}_{\text {crit }}$ to define the normalised response

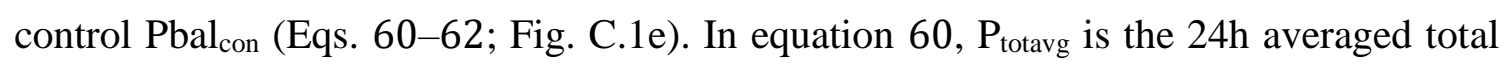
photosynthesis rate.

$$
\begin{gathered}
\mathrm{P}_{\text {bal }}=\min \left(1, \frac{\mathrm{P}_{\text {totavg }}}{\mu_{\text {avg }}}\right) \\
\mathrm{Bal}_{\text {res }}= \begin{cases}0, & \mathrm{P}_{\text {bal }}-\mathrm{Pbal}_{\text {crit }} \\
1-\mathrm{Pbal}_{\text {crit }} & \mathrm{P}_{\text {bal }}>\mathrm{Pbal}_{\text {crit }}\end{cases} \\
\text { Pbal }_{\text {con }}= \begin{cases}1, & \mathrm{Pbal}_{\text {crit }}=0 \\
\frac{\left(1+\mathrm{K}_{\mathrm{pbal}}^{\mathrm{H}_{\text {pbal }}}\right) \cdot \mathrm{Bal}_{\text {res }}^{\mathrm{H}_{\text {pbal }}}}{\mathrm{Bal}_{\text {res }}^{\mathrm{H}_{\text {pbal }}}+\mathrm{K}_{\mathrm{pbal}}^{\mathrm{H}_{\text {pbal }}},} & \mathrm{Pbal}_{\text {crit }}>0\end{cases}
\end{gathered}
$$

Setting $\mathrm{S}_{\mathrm{pd}}=0$ and $\mathrm{Pbal}_{\text {crit }}=0$ turns $\mathrm{D}_{\mathrm{con}}$ and Pbal $\mathrm{P}_{\text {con }}$ off, disabling any control of phototrophy on the digestion of prey carbon.

The digestion of prey carbon depends also on the assimilation efficiency (AE), which varies as a function of prey quality or quantity (Mitra 2006). AE decreases as prey 
quality (i.e. NC or PC) decreases. The stoichiometric value of the prey is referred through the description of food quality within feeding vacuoles (FNC and FPC; Eqs. 63-64) relative to the optimal core mixotroph values ( $\operatorname{Rel}_{\mathrm{NC}}$ and Rel${ }_{\mathrm{PC}}$; Eqs. 65-66). There is also evidence that assimilation efficiency is down-regulated in the presence of toxins and other secondary metabolites, which are implemented in the model through a function of the constant parameter $\mathrm{K}_{\mathrm{ec}}$. Thus, the actual control associated with prey quality ( $\left.\mathrm{AE}_{\text {qual }}\right)$ is a function of the minimal stoichiometric control (i.e., $\operatorname{Rel}_{\mathrm{NC}}$ or $\operatorname{Rel}_{\mathrm{PC}}-\mathrm{MIN}_{\mathrm{up}}$ ) and $\mathrm{K}_{\mathrm{ec}}$, assuming that $\mathrm{AE}$ is constrained between a minimal $\left(\mathrm{AE}_{\min }\right)$ and a maximal $\left(\mathrm{AE}_{\max }\right)$ values (Eq. 67; Fig. C.1f).

$$
\begin{gathered}
\mathrm{FNC}=\frac{[\mathrm{FN}]}{[\mathrm{C}]} \\
\mathrm{FPC}=\frac{[\mathrm{FP}]}{[\mathrm{C}]} \\
\operatorname{Rel}_{\mathrm{NC}}=\frac{\mathrm{FNC}}{[\mathrm{FC}] \cdot \mathrm{NC}_{\max }} \\
\operatorname{Rel}_{\mathrm{PC}}=\frac{\mathrm{FPC}}{[\mathrm{FC}] \cdot \mathrm{PC} \text { max }} \\
\mathrm{AE}_{\text {qual }}=\mathrm{AE}_{\text {min }}+\left(\mathrm{AE}_{\mathrm{max}}-\mathrm{AE}_{\mathrm{min}}\right) \cdot \frac{\left(1+\mathrm{K}_{\mathrm{ec}}\right) \cdot \mathrm{MIN}_{\mathrm{up}}}{\mathrm{MIN}_{\mathrm{up}}+\mathrm{K}_{\mathrm{ec}}} \\
\text { in which } \mathrm{MIN}_{\mathrm{up}}=\min \left(1, \operatorname{Rel}_{\mathrm{NC}}, \operatorname{Rel}_{\mathrm{NC}}\right)
\end{gathered}
$$

The control associated with prey quantity $\left(\mathrm{AE}_{\text {quan }}\right)$ is linked in the model to $\mu_{\text {rel }}$ (Eq. 42), decreasing AE with growth approaching maximum (Eq. 68; Fig. C.1g). Thus, the actual value of $\mathrm{AE}$ is set by equation 5.6.

$$
\begin{gathered}
\mathrm{AE}_{\text {quan }}=\mathrm{AE}_{\text {min }}+\left(\mathrm{AE}_{\text {max }}-\mathrm{AE}_{\text {min }}\right) \cdot \frac{\left(1+\mathrm{K}_{\mathrm{eq}}^{\mathrm{H}_{\mathrm{eq}}}\right) \cdot\left(1-\mu_{\mathrm{rel}}\right)^{\mathrm{H}_{\mathrm{eq}}}}{\left(1-\mu_{\mathrm{rel}}\right)^{\mathrm{H}_{\mathrm{eq}}}+\mathrm{K}_{\mathrm{eq}}^{\mathrm{H}_{\mathrm{eq}}}} \\
\mathrm{AE}=\mathrm{MIN}_{\mathrm{up}} \cdot \min \left(\mathrm{AE}_{\text {qual }}, \mathrm{AE}_{\text {quan }}\right)
\end{gathered}
$$

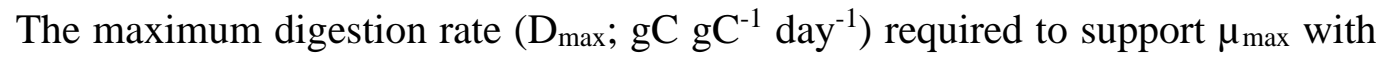
the current food quality is a function of $\mathrm{BR}_{\mathrm{op}}, \mathrm{AE}$, and the relative cost of metabolic respiration $\mathrm{MR}$ (Eq. 70). Finally, the actual digestion rate $\left(\mathrm{D}_{\mathrm{C}} ; \mathrm{gC} \mathrm{gC}^{-1} \mathrm{day}^{-1}\right)$ is a function of $\mathrm{D}_{\max }, \mathrm{D}_{\mathrm{con}}, \mathrm{Pbal}_{\mathrm{con}}$, and a normalised response curve of the relative content within the 


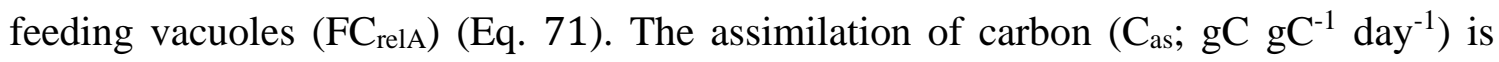
defined by equation 72 .

$$
\begin{gathered}
\mathrm{D}_{\text {max }}=\frac{\mu_{\text {max }}+\mathrm{BR}_{\mathrm{op}}}{\mathrm{AE} \cdot(1-\mathrm{MR})} \\
\mathrm{D}_{\mathrm{C}}=\mathrm{D}_{\text {max }} \cdot \mathrm{D}_{\text {con }} \cdot \mathrm{Pbal}_{\text {con }} \cdot \frac{\left(1+\mathrm{K}_{\mathrm{as}}^{\mathrm{H}_{\mathrm{as}}}\right) \cdot \mathrm{FC}_{\mathrm{relA}}^{\mathrm{H}_{\mathrm{as}}}}{\mathrm{FC}_{\mathrm{ralA}}^{\mathrm{H}_{\mathrm{as}}}+\mathrm{K}_{\mathrm{as}}^{\mathrm{H}_{\mathrm{as}}}} \\
\text { in which } \mathrm{FC}_{\mathrm{relA}}=\frac{\mathrm{FC}}{\mathrm{FC}_{\mathrm{abs}}} \\
\mathrm{C}_{\mathrm{as}}=\mathrm{AE} \cdot \mathrm{D}_{\mathrm{C}}
\end{gathered}
$$

The total digestion (digestion $\mathrm{C} ; \mathrm{mg} \mathrm{C} \mathrm{m}^{-3}$ day $^{-1}$ ) and assimilation (assimilation $\mathrm{C}$; $\mathrm{mg} \mathrm{C} \mathrm{m} \mathrm{may}^{-1}$ ) of carbon by the population is obtained through equations 73-74:

$$
\begin{gathered}
\operatorname{digestion~}_{\mathrm{C}}=\mathrm{D}_{\mathrm{C}} \cdot[\mathrm{C}] \\
\text { assimilation }_{\mathrm{C}}=\mathrm{C}_{\mathrm{as}} \cdot[\mathrm{C}]
\end{gathered}
$$

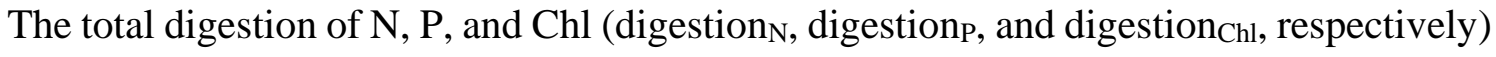
are calculated based on the materials within the feeding vacuoles (Eqs. 75-77) while the

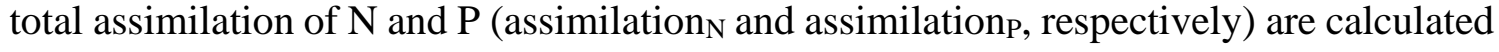
based on the maximum nutrient to carbon quotas (Eqs. 78-79):

$$
\begin{gathered}
\operatorname{digestion}_{\mathrm{N}}=\frac{\mathrm{D}_{\mathrm{C}}}{\mathrm{FC}} \cdot[\mathrm{FN}] \\
\operatorname{digestion}_{\mathrm{P}}=\frac{\mathrm{D}_{\mathrm{C}}}{\mathrm{FC}} \cdot[\mathrm{FP}] \\
\operatorname{digestion}_{\mathrm{Chl}}=\frac{\mathrm{D}_{\mathrm{C}}}{\mathrm{FC}} \cdot[\mathrm{FChl}] \\
\operatorname{assimilation}_{\mathrm{N}}=\mathrm{C}_{\mathrm{as}} \cdot \mathrm{NC} \text { max } \\
\operatorname{assimilation}_{\mathrm{P}}=\mathrm{C}_{\mathrm{as}} \cdot \mathrm{PC}_{\mathrm{max}} \cdot[\mathrm{C}]
\end{gathered}
$$

\section{Photoacclimation}

Photoacclimation is described in the model through the synthesis and degradation of chlorophyll (Flynn 2001); only protists with constitutive ability to photosynthesize were assumed to be able to photoacclimate. The total input of carbon $\left(\mathrm{TC}_{\mathrm{in}}\right)$ controls the demand for chlorophyll; if $S_{\text {mix }}=0$, both carbon obtained through photosynthesis and prey assimilation are considered; if $S_{\text {mix }}=1$, only carbon obtained through photosynthesis is 
considered (Eq. 80). The synthesis and degradation of chlorophyll ( $\left.\mathrm{gChl} \mathrm{gC}^{-1} \mathrm{day}^{-1}\right)$ is then defined by equations 81 and 82 , respectively. If the internal chlorophyll to carbon ratio approaches its absolute maximum value, the balance between synthesis and degradation is equal to zero (Eq. 83).

$$
\begin{aligned}
& \mathrm{TC}_{\text {in }}=\left(1-\min \left(1, \frac{\mathrm{C}_{\text {in }}}{\mathrm{Pq}_{\max }}\right)\right)^{0.5} \\
& \text { in which } \mathrm{C}_{\text {in }}= \begin{cases}\mathrm{P}_{\text {tot }}+\mathrm{C}_{\text {as }}, & \mathrm{S}_{\text {mix }}=0 \\
\mathrm{P}_{\text {tot }}, & \mathrm{S}_{\text {mix }}=1\end{cases} \\
& \text { synthesis }=\mathrm{ChlC}_{\mathrm{abs}} \cdot \mathrm{NPC}_{\mathrm{u}} \cdot 3 \cdot \mu_{\mathrm{phot}} \cdot \mathrm{TC}_{\mathrm{in}} \cdot \mathrm{Chl}_{\mathrm{fb}} \\
& \text { in which } \operatorname{Chl}_{\mathrm{fb}}=\frac{(1+0.05) \cdot\left(1-\frac{\text { ChlC }}{\text { ChlC }_{\mathrm{abs}}}\right)}{\left(1-\frac{\text { ChlC }}{\text { ChlC }_{\mathrm{abs}}}\right)+0.05} \\
& \text { degradation }=\text { ChlC } \cdot\left(1-\mathrm{NC}_{\mathrm{u}}\right) \cdot \mu_{\mathrm{phot}} \\
& \text { balance }_{\mathrm{Chl}}= \begin{cases}\text { synthesis }- \text { degradation, } & \mathrm{ChlC}<\mathrm{ChlC}_{\mathrm{abs}} \\
0, & \mathrm{ChlC} \geq \mathrm{ChlC}_{\mathrm{abs}}\end{cases}
\end{aligned}
$$

Respiration and internal re-assimilation

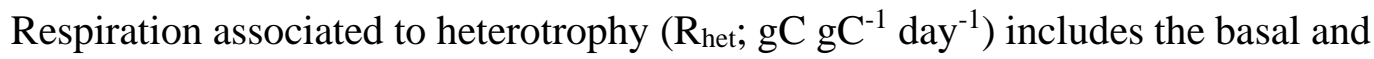

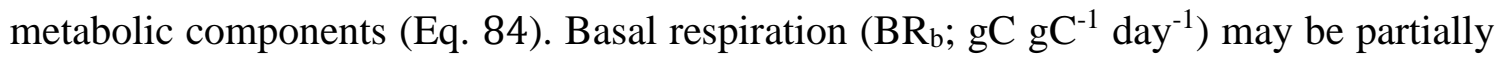
or totally met by the digestion of prey carbon according to the protist stoichiometric status and its prey (Eqs. 85-87). Metabolic respiration is defined by a constant proportion (MR) of the assimilated prey carbon (Eq. 84).

$$
\begin{gathered}
\mathrm{R}_{\text {het }}=\mathrm{BR}_{\mathrm{b}}+\mathrm{C}_{\mathrm{as}} \cdot \mathrm{MR} \\
\mathrm{XSC}=\mathrm{AE}_{\text {qual }} \cdot \mathrm{D}_{\mathrm{C}} \cdot\left(1-\mathrm{MIN}_{\mathrm{up}}\right) \\
\mathrm{BR}_{\mathrm{i}}= \begin{cases}\mathrm{BR}_{\mathrm{op}}, & \mathrm{BR}_{\mathrm{op}} \leq \mathrm{XSC} \\
\mathrm{XSC}, & \mathrm{BR}_{\mathrm{op}}>\mathrm{XSC}\end{cases} \\
\mathrm{BR}_{\mathrm{b}}=\mathrm{BR}_{\mathrm{op}}-\mathrm{BR}_{\mathrm{i}}
\end{gathered}
$$

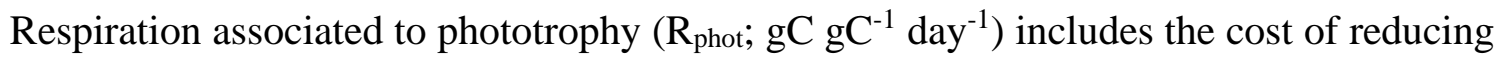
nitrate to ammonium (redco) and, once mixotrophs were modelled, the cost of reassimilating ammonium ( $\mathrm{NH} 4_{\text {reas }} ; \mathrm{gN} \mathrm{gC}^{-1} \mathrm{day}^{-1}$ ) which would otherwise be regenerated through heterotrophic processing (Eq. 88). Internal re-assimilation of ammonium is 
blocked as $\mathrm{NC}$ approaches $\mathrm{NC}_{\mathrm{abs}}\left(\mathrm{N}_{\text {rep }}\right.$; Eq. 89); thus, carbon respiration is just associated to the regeneration of $\mathrm{N}$ if $\mathrm{NC}$ approaches maximum values. A normalised response curve of $\mathrm{N}_{\text {rep }}$ ultimately controls the re-assimilation of ammonium (Eq. 90-91; Fig. C.1h).

$$
\begin{gathered}
\mathrm{R}_{\text {phot }}=\operatorname{redco} \cdot \mathrm{N}_{\text {up }} \cdot\left(\frac{\mathrm{N}_{\mathrm{t}}}{\mathrm{N}_{\mathrm{t}}+\mathrm{A}_{\mathrm{t}}}\right)+\left(\mathrm{N}_{\mathrm{up}}+\mathrm{NH} 4_{\text {reas }}\right) \cdot \mathrm{AA}_{\mathrm{syn}} \cdot \mathrm{Met}_{\mathrm{M}} \\
\mathrm{N}_{\text {rep }}= \begin{cases}0, & \mathrm{NC} \leq \mathrm{NC}_{\text {max }} \\
1-\frac{\mathrm{NC}_{\mathrm{abs}}-\mathrm{NC}}{\mathrm{NC}_{\mathrm{abs}}-\mathrm{NC}_{\max }}, & \mathrm{NC}>\mathrm{NC}_{\text {max }}\end{cases} \\
\operatorname{RegN}_{\mathrm{con}}=\frac{\left(1+\mathrm{K}_{\mathrm{rc}}^{\mathrm{H}_{\mathrm{rc}}}\right) \cdot \mathrm{N}_{\mathrm{rep}}^{\mathrm{H}_{\mathrm{rc}}}}{\mathrm{N}_{\mathrm{rep}}^{\mathrm{H}_{\mathrm{rc}}}+\mathrm{K}_{\mathrm{rc}}^{\mathrm{H}_{\mathrm{rc}}}} \\
\mathrm{NH}_{\text {reas }}=\mathrm{R}_{\text {het }} \cdot \mathrm{NC}_{\text {max }} \cdot\left(1-\mathrm{RegN}_{\text {con }}\right)
\end{gathered}
$$

Similarly, the internal re-assimilation of $\mathrm{P}$ among mixotrophs is described through the following equations (Eqs. 92-94):

$$
\begin{gathered}
\mathrm{P}_{\text {rep }}= \begin{cases}0, & \mathrm{PC} \leq \mathrm{PC}_{\max } \\
1-\frac{\mathrm{PC}_{\mathrm{abs}}-\mathrm{PC}}{\mathrm{PC}_{\mathrm{abs}}-\mathrm{PC}_{\max }}, & \mathrm{PC}>\mathrm{PC}_{\max }\end{cases} \\
\operatorname{RegP}_{\mathrm{con}}=\frac{\left(1+\mathrm{K}_{\mathrm{rc}}^{\mathrm{H}_{\mathrm{rc}}}\right) \cdot \mathrm{P}_{\mathrm{rep}}^{\mathrm{H}_{\mathrm{rc}}}}{\mathrm{P}_{\mathrm{rep}}^{\mathrm{H}_{\mathrm{rc}}}+\mathrm{K}_{\mathrm{rc}}^{\mathrm{H}_{\mathrm{rc}}}} \\
\mathrm{P}_{\text {reas }}=\mathrm{R}_{\text {het }} \cdot \mathrm{PC}_{\max } \cdot\left(1-\operatorname{RegP}_{\mathrm{con}}\right)
\end{gathered}
$$

Finally, total respiration loss from protist population (respiration $\mathrm{C} ; \mathrm{mg} \mathrm{C} \mathrm{m}^{-3} \mathrm{day}^{-}$ ${ }^{1}$ ) is defined by:

$$
\begin{gathered}
\mathrm{R}_{\text {tot }}=\mathrm{R}_{\text {het }}+\mathrm{R}_{\text {phot }} \\
\text { respiration }_{\mathrm{C}}=\mathrm{R}_{\text {tot }} \cdot[\mathrm{C}]
\end{gathered}
$$

\section{Regeneration and voiding}

Nutrient regeneration linked to heterotrophic respiration are defined as following for $\mathrm{N}\left(\mathrm{N}_{\text {reg }} ; \mathrm{gN} \mathrm{gC}^{-1} \mathrm{day}^{-1}\right)$ and $\mathrm{P}\left(\mathrm{P}_{\text {reg }} ; \mathrm{gP} \mathrm{gC}^{-1}\right.$ day $\left.^{-1}\right)$ assuming that the base $\mathrm{XC}$ of core biological material accords with maximum $\mathrm{XC}$ quotas:

$$
\begin{gathered}
\mathrm{N}_{\text {reg }}=\mathrm{R}_{\text {het }} \cdot \mathrm{NC}_{\text {max }} \cdot \operatorname{RegN}_{\text {con }} \\
\mathrm{P}_{\text {reg }}=\mathrm{R}_{\text {het }} \cdot \mathrm{PC}_{\text {max }} \cdot \mathrm{RegP}_{\text {con }}
\end{gathered}
$$


Regeneration of $\mathrm{N}$ (regeneration ${ }_{\mathrm{N}} ; \mathrm{mg} \mathrm{N} \mathrm{m}^{-3}$ day $^{-1}$ ) and $\mathrm{P}$ (regeneration $\mathrm{P} ; \mathrm{mg} \mathrm{P} \mathrm{m}^{-}$

${ }^{3}$ day $^{-1}$ ) associated to carbon respiration by the protist population is then defined by:

$$
\begin{aligned}
\text { regeneration }_{N} & =\mathrm{N}_{\text {reg }} \cdot[\mathrm{C}] \\
\text { regeneration }_{\mathrm{P}} & =\mathrm{P}_{\mathrm{reg}} \cdot[\mathrm{C}]
\end{aligned}
$$

All prey carbon that was digested but not assimilated nor respired is voided $\left(\mathrm{C}_{\mathrm{void}}\right.$; $\mathrm{gC} \mathrm{gC}^{-1}$ day $^{-1}$ ) (Eq. 101). $\mathrm{N}$ and $\mathrm{P}$ associated with carbon material must be voided as well.

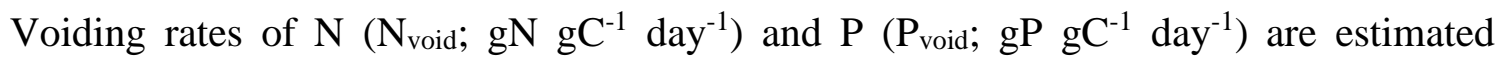
considering the assimilation rates required to maintain protist maximum nutrient to carbon quotas (Eqs. 102-103). Since $\mathrm{BR}_{\mathrm{i}}$ uses XSC (Eq. 85) there is no associated term for the voiding of $\mathrm{N}$ and $\mathrm{P}$ in equations 102 and 103.

$$
\begin{gathered}
\mathrm{C}_{\text {void }}=\mathrm{D}_{\mathrm{C}}-\mathrm{C}_{\mathrm{as}}-\mathrm{BR}_{\mathrm{i}} \\
\mathrm{N}_{\text {void }}=\mathrm{D}_{\mathrm{C}} \cdot \frac{\mathrm{FNC}}{\mathrm{FC}}-\mathrm{C}_{\mathrm{as}} \cdot \mathrm{NC}_{\max } \\
\mathrm{P}_{\text {void }}=\mathrm{D}_{\mathrm{C}} \cdot \frac{\mathrm{FPC}}{\mathrm{FC}}-\mathrm{C}_{\mathrm{as}} \cdot \mathrm{PC}_{\max }
\end{gathered}
$$

Due to chemical constraints, there is a limitation on the nutrient to carbon quotas that can be voided as organic material; these are controlled in the model by the constant parameters $\mathrm{NC}_{\mathrm{m}}$ and $\mathrm{PC}_{\mathrm{m}}$ (Eqs. 104-105). The surplus is then voided as inorganic material (Eqs. 106-107).

$$
\begin{aligned}
& \text { DON }_{\text {void }}= \begin{cases}\mathrm{N}_{\text {void }}, & \frac{\mathrm{N}_{\text {void }}}{\mathrm{C}_{\text {void }}} \leq \mathrm{NC}_{\mathrm{m}} \\
\mathrm{C}_{\text {void }} \cdot \mathrm{NC}_{\mathrm{m}}, & \frac{\mathrm{N}_{\text {void }}}{\mathrm{C}_{\text {void }}}>\mathrm{NC}_{\mathrm{m}}\end{cases} \\
& \text { DOP }_{\text {void }}= \begin{cases}\mathrm{P}_{\text {void }}, & \frac{\mathrm{P}_{\text {void }}}{\mathrm{C}_{\text {void }}} \leq \mathrm{PC}_{\mathrm{m}} \\
\mathrm{C}_{\text {void }} \cdot \mathrm{PC}_{\mathrm{m}}, & \frac{\mathrm{P}_{\text {void }}}{\mathrm{C}_{\text {void }}}>\mathrm{PC}_{\mathrm{m}}\end{cases} \\
& \text { DIN }_{\text {void }}=\mathrm{N}_{\text {void }}-\text { DON }_{\text {void }} \\
& \mathrm{DIP}_{\text {void }}=\mathrm{P}_{\text {void }}-\text { DOP }_{\text {void }}
\end{aligned}
$$

Finally, regeneration of inorganic nutrients $\left(\right.$ Tregeneration $_{N}$ and Tregeneration ${ }_{P}$ ) and voiding of organic material (voiding , $_{\text {, voiding }}$, and voiding ${ }_{\mathrm{P}}$ ) by the plankton population $\left(\mathrm{mg} \mathrm{X} \mathrm{m} \mathrm{Xay}^{-1}\right.$ ) are defined as: 


$$
\begin{gathered}
\text { voiding }_{\mathrm{C}}=\mathrm{C}_{\text {void }} \cdot[\mathrm{C}] \\
\text { voiding }_{\mathrm{N}}=\mathrm{DON}_{\mathrm{void}} \cdot[\mathrm{C}] \\
\text { voiding }_{\mathrm{P}}=\mathrm{DOP}_{\text {void }} \cdot[\mathrm{C}] \\
\text { Tregeneration }_{\mathrm{N}}=\left(\mathrm{N}_{\mathrm{reg}}+\mathrm{DIN}_{\mathrm{void}}\right) \cdot[\mathrm{C}] \\
\text { Tregeneration }_{\mathrm{P}}=\left(\mathrm{P}_{\mathrm{reg}}+\mathrm{DIP}_{\mathrm{void}}\right) \cdot[\mathrm{C}]
\end{gathered}
$$

\section{Non-predatory mortality}

Non-predatory mortality is computed through a constant parameter defining the maximum specific non-predatory mortality $\left(\mathrm{sd} ; \mathrm{day}^{-1}\right)$ and a normalised level of stress which is defined by the quotient between the actual growth rate $\mu$ and $\mu_{\max }$ (Eqs. 113115). In Eq. 115, $X$ applies to all state variables of the protist model.

$$
\begin{array}{r}
\mu=C_{a s}+P_{\text {tot }}-R_{\text {tot }} \\
\text { stress }=\left\{\begin{array}{lr}
1, & \mu \leq 0 \\
1-\frac{\mu}{\mu_{\max }}, & \mu_{\max }>\mu>0 \\
0, & \mu \geq \mu_{\max }
\end{array}\right. \\
\text { mortality }_{X}=\text { sd } \cdot \text { stress } \cdot[X]
\end{array}
$$

\section{Bacteria model}

The bacteria model corresponds to the one in ERSEM (i.e. dynamic decomposition version) and accounts for variable stoichiometry (Butenschön et al., 2016). The state variables $[\mathrm{BC}],[\mathrm{BN}]$, and $[\mathrm{BP}]$ vary according to uptake, respiration, release of nutrients, and non-predatory mortality and are resolved for carbon, nitrogen, and phosphorus, respectively (Eqs. 116-118). These equations have the same units as the state variables in the protist model (e.g., $\mathrm{mg} \mathrm{C} \mathrm{m}^{-3}$ ).

$$
\begin{aligned}
\frac{d[B C]}{d t}= & \text { uptake }_{B C}-\text { respiration }_{B C}-\text { release }_{B C}-\text { mortality }_{\mathrm{BC}} \\
& \frac{d[B N]}{d t}=\text { uptake }_{\mathrm{BN}}-\text { release }_{\mathrm{BN}}-\text { mortality }_{\mathrm{BN}} \\
& \frac{\mathrm{d}[\mathrm{BP}]}{\mathrm{dt}}=\text { uptake }_{\mathrm{BP}}-\text { release }_{\mathrm{BP}}-\text { mortality }_{\mathrm{BP}}
\end{aligned}
$$

Bacteria feed on both dissolved and particulate organic matter. The maximum potential uptake of carbon for the bacterial population is given by Eq. 119 (rumbC; mg C 
$\mathrm{m}^{-3}$ day $^{-1}$ ) assuming a constant maximum specific uptake $\operatorname{sum}_{\mathrm{B}}\left(\right.$ day $\left.^{-1}\right)$. Equation 120 describes the total carbon substrate available (total $\mathrm{sub}_{\mathrm{s}}, \mathrm{mg} \mathrm{C}^{-3}$ ) in which the subscript $\mathrm{i}$ refers to the different forms of DOC (i.e., labile or recalcitrant) and the subscript $\mathrm{j}$ refers to the different size-classes of POM (i.e. small, medium, or large). Only a fraction of the respective DOC pool is available to bacterial consumption, as determined by the non-

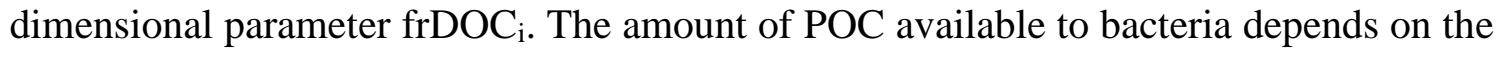
ratio between the respective remineralization rates $\left(\mathrm{rPOC}_{\mathrm{j}} ; \mathrm{day}^{-1}\right)$ and the maximum turnover rate of DOM (sDOM; $\left.\mathrm{day}^{-1}\right)$. Finally, the actual carbon uptake is given in units per day in Eq. 121 ( $\operatorname{sug}_{\mathrm{BC}}$ ) and in units of $\mathrm{mg} \mathrm{C} \mathrm{m}^{-3}$ day $^{-1}$ in Eq. 122 (uptake $\mathrm{BC}_{\mathrm{C}}$ ).

$$
\begin{aligned}
& \operatorname{rum}_{\mathrm{BC}}=\operatorname{sum}_{\mathrm{B}} \cdot[\mathrm{BC}] \\
& \operatorname{total}_{\text {sub }}=\sum\left[\mathrm{DOC}_{\mathrm{i}}\right] \cdot \mathrm{frDOC}_{\mathrm{i}}+\sum \frac{\left[\mathrm{POC}_{\mathrm{j}}\right] \cdot \mathrm{rPOC}_{\mathrm{j}}}{\mathrm{sDOM}} \\
& \operatorname{sug}_{\mathrm{BC}}= \begin{cases}0, & \text { total }_{\text {sub }} \leq 0 \\
\frac{\operatorname{rum}_{\mathrm{BC}}}{\max \left(\frac{\text { rum }_{\mathrm{BC}}}{\mathrm{sDOM}}, \text { total }_{\text {sub }}\right)}, & \text { total }_{\text {sub }}>0\end{cases} \\
& \text { uptake }_{\mathrm{BC}}=\operatorname{sug}_{\mathrm{BC}} \cdot \text { total }_{\text {sub }}
\end{aligned}
$$

The uptake of dissolved organic phosphorus and nitrogen by the bacterial population (uptake $\mathrm{BN}_{\mathrm{BN}}$ and uptake $\mathrm{BP}_{\mathrm{BP}}$, respectively) are thus obtained through the following equations:

$$
\begin{aligned}
& \text { uptake }_{\mathrm{BN}}=\operatorname{sug}_{\mathrm{BC}} \cdot\left[\mathrm{DOP}_{\mathrm{lab}}\right] \\
& \text { uptake }_{\mathrm{BP}}=\operatorname{sug}_{\mathrm{BC}} \cdot\left[\mathrm{DON}_{\mathrm{lab}}\right]
\end{aligned}
$$

The uptake or release of inorganic nitrogen (release ${ }_{\mathrm{BN}} ; \mathrm{mg} \mathrm{N} \mathrm{m}^{-3} \mathrm{day}^{-1}$ ) and phosphorus (release $\mathrm{BP}_{\mathrm{BP}} ; \mathrm{mg} \mathrm{P} \mathrm{m}^{-3} \mathrm{day}^{-1}$ ) by bacteria depends on the current stoichiometric status of bacteria in terms of nitrogen and phosphorus (Eqs. 125-126, respectively) and on the quality (i.e., $\mathrm{N}$ and $\mathrm{P}$ relative content) of the organic matter, as described in equations 127-128.

$$
\begin{aligned}
& \mathrm{BNC}=\frac{[\mathrm{BN}]}{[\mathrm{BC}]} \\
& \mathrm{BPC}=\frac{[\mathrm{BP}]}{[\mathrm{BC}]}
\end{aligned}
$$




$$
\begin{aligned}
& \text { release }_{\mathrm{BN}}= \begin{cases}\left(\mathrm{BNC}-\mathrm{NC}_{\text {max }}^{\mathrm{B}}\right) \cdot[\mathrm{BC}], & \mathrm{BNC}-\mathrm{NC}_{\text {max }}^{\mathrm{B}} \geq 0 \\
\left(\mathrm{BNC}-\mathrm{NC}_{\max }^{\mathrm{B}}\right) \cdot[\mathrm{BC}] \cdot \frac{\left[\mathrm{NH}_{4}\right]}{\left(\left[\mathrm{NH}_{4}\right]+\mathrm{chn}\right)}, & \mathrm{BNC}-\mathrm{NC}_{\text {max }}^{\mathrm{B}}<0\end{cases} \\
& \text { release }_{\mathrm{BP}}= \begin{cases}\left(\mathrm{BPC}-\mathrm{PC}_{\max }^{\mathrm{B}}\right) \cdot[\mathrm{BC}], & \mathrm{BPC}-\mathrm{PC}_{\max }^{\mathrm{B}} \geq 0 \\
\left(\mathrm{BPC}-\mathrm{PC}_{\max }^{\mathrm{B}}\right) \cdot[\mathrm{BC}] \cdot \frac{\left[\mathrm{PO}_{4}\right]}{\left(\left[\mathrm{PO}_{4}\right]+\mathrm{chp}\right)}, & \mathrm{BPC}-\mathrm{PC}_{\max }^{\mathrm{B}}<0\end{cases}
\end{aligned}
$$

Bacteria respiration (respiration ${ }_{\mathrm{BC}} ; \mathrm{mg} \mathrm{C} \mathrm{m}^{-3} \mathrm{day}^{-1}$ ) is defined by the sum of the metabolic and basal respiration costs assuming no oxygen limitation (Eqs. 129-131). Metabolic respiration (met_respiration ${ }_{\mathrm{BC}} ; \mathrm{mg} \mathrm{C} \mathrm{m}^{-3} \mathrm{day}^{-1}$ ) is defined by a constant fraction $\mathrm{pu}_{\mathrm{B}}$ of the uptake activity while basal respiration (bas_respiration ${ }_{\mathrm{BC}} ; \mathrm{mg} \mathrm{C} \mathrm{m}^{-3}$ day $^{-1}$ ) is defined by a constant maximum respiration rate $\operatorname{srs}_{\mathrm{B}}\left(\right.$ day $\left.^{-1}\right)$.

$$
\begin{gathered}
\text { met_respiration }_{\mathrm{BC}}=\operatorname{uptake}_{\mathrm{BC}} \cdot \mathrm{pu}_{\mathrm{B}} \\
\text { bas_respiration }_{\mathrm{BC}}=\mathrm{srs}_{\mathrm{B}} \cdot[\mathrm{BC}] \\
\text { respiration }_{\mathrm{BC}}=\text { met_respiration }_{\mathrm{BC}}+\text { bas_respiration }_{\mathrm{BC}}
\end{gathered}
$$

Carbon uptake is not nutrient limited as the internal stoichiometric quota of bacteria is balanced directly through the release of carbon into semi-labile organic matter. The proportion of semi-labile carbon released (release $\mathrm{BC}_{\mathrm{BC}}$ ) depends on the current stoichiometric status of bacteria in terms of nitrogen and phosphorus (Eqs. 125-126, respectively) and their maximum nutrient quotas $\left(\mathrm{NC}_{\max }^{\mathrm{B}}\right.$ and $\mathrm{PC}_{\max }^{\mathrm{B}}$, respectively), as defined by Eq. $132\left(\mathrm{rel}_{\mathrm{BC}} ;\right.$ day $\left.^{-1}\right)$. Bacteria also produces recalcitrant carbon (met_release $\mathrm{BC}_{\mathrm{BC}}$ ) as a fixed fraction (frF3) of the metabolic respiration (Eq. 133). Thus, the bacterial population release of carbon is defined by equation 134 .

$$
\begin{gathered}
\text { rel }_{\mathrm{BC}}=\max \left(0, \max \left(1-\frac{\mathrm{BNC}}{\mathrm{NC}_{\max }^{\mathrm{B}}}, 1-\frac{\mathrm{BPC}}{\mathrm{PC}_{\max }^{\mathrm{B}}}\right)\right) \cdot 1 \\
\text { met_release }_{\mathrm{BC}}=\text { met_respiration }_{\mathrm{BC}} \cdot \mathrm{frF3} \\
\text { release }_{\mathrm{BC}}=\mathrm{rel}_{\mathrm{BC}} \cdot[\mathrm{BC}]+\text { met_release }_{\mathrm{BC}}
\end{gathered}
$$

The mineralisation of dissolved organic nitrogen and phosphorus by the bacterial population are described through a linear function between the respective pools of DOM and the constant specific mineralisation rates for each nutrient (sDON and sDOP, respectively, in units of per day), following the equations: 


$$
\begin{aligned}
\operatorname{miner}_{\mathrm{BN}} & =\mathrm{sDON} \cdot\left[\mathrm{DON}_{\mathrm{lab}}\right] \\
\operatorname{miner}_{\mathrm{BP}} & =\mathrm{sDOP} \cdot\left[\mathrm{DOP}_{\mathrm{lab}}\right]
\end{aligned}
$$

In equation 137, non-predatory mortality is described through a constant parameter $\operatorname{sd}_{\mathrm{B}}\left(\right.$ day $\left.^{-1}\right)$, in which $\mathrm{X}$ represents $\mathrm{C}, \mathrm{N}$ or $\mathrm{P}$.

$$
\text { mortality }_{\mathrm{BX}}=\mathrm{sd}_{\mathrm{B}} \cdot[\mathrm{BX}]
$$

\section{Mesozooplankton model}

The mesozooplankton model was taken from ERSEM (Butenschön et al., 2016) and assumes constant stoichiometry, thus, it is composed only by one state variable measured in terms of carbon biomass ([MZ]; $\mathrm{mg} \mathrm{C} \mathrm{m}^{-3}$ ), describing the ingestion of prey, excretion, respiration, and non-predatory mortality, as following:

$$
\frac{\mathrm{d}[\mathrm{MZ}]}{\mathrm{dt}}=\text { ingestion }_{\mathrm{MZ}}-\text { excretion }_{\mathrm{MZ}}-\text { respiration }_{\mathrm{MZ}}-\text { mortality }_{\mathrm{MZ}}
$$

In ERSEM, predator-prey interactions are described through a rectangular hyperbolic function. Here, this was modified by a simpler function to agree with the description used in the common protist model following Flynn and Mitra (2016). It is also noteworthy that the overwintering state was disabled in the model (i.e., Minprey $=0$; Table $\mathrm{S} 5)$. In equation $139, \mathrm{Cp}_{\mathrm{i}}$ is the clearance rate $\left(\mathrm{gC} \mathrm{gC}^{-1}\right.$ day $\left.^{-1}\right)$ of prey type $\mathrm{i}$, [preyc] is prey $\mathrm{i}$ biomass $\left(\mathrm{mg} \mathrm{C} \mathrm{m}^{-3}\right)$ and $\mathrm{Cr}_{\mathrm{i}}$ is the slope of the relationship between prey $\mathrm{i}$ biomass

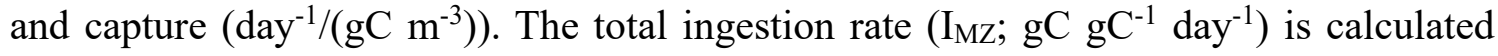
through a minimum function of the total possible ingestion rate and a curvilinear function that empirically describes a satiation feedback through the constant parameter KI and the

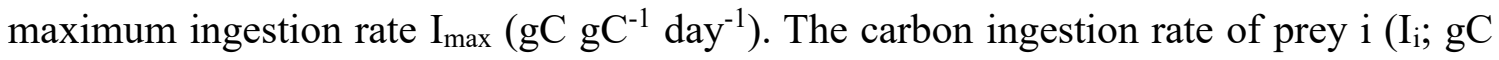
$\mathrm{gC}^{-1}$ day $\left.^{-1}\right)$ and the specific ingestion rate of prey $\mathrm{i}\left(\mathrm{sI}_{\mathrm{MZ}}\right.$; day $\left.{ }^{-1}\right)$ are then computed through Eqs. 141-142. The total ingestion rate by the mesozooplankton population $\left(\mathrm{mg} \mathrm{C} \mathrm{m}^{-3} \mathrm{day}^{-}\right.$ ${ }^{1}$ ) is then obtained through Eq. 143.

$$
\begin{gathered}
\mathrm{Cp}_{\mathrm{i}}=\mathrm{C}_{\mathrm{ri}} \cdot\left[\text { prey }_{\mathrm{C}}\right] \\
\mathrm{I}_{\mathrm{MZ}}=\left(\mathrm{I}_{\mathrm{max}} \cdot \frac{\sum \mathrm{Cpi}}{\left(\sum \mathrm{Cpi}+\mathrm{KI}\right)}, \sum \mathrm{Cpi}\right) \\
\mathrm{I}_{\mathrm{i}}=\mathrm{I}_{\mathrm{MZ}} \cdot \frac{\mathrm{Cp}_{\mathrm{i}}}{\sum \mathrm{Cpi}}
\end{gathered}
$$




$$
\begin{gathered}
\operatorname{si}_{\mathrm{MZ}}=\mathrm{I}_{\mathrm{i}} \cdot \frac{[\mathrm{MZ}]}{\left[\text { prey }_{\mathrm{C}}\right]} \\
\text { ingestion }_{\mathrm{MZ}}=\mathrm{I}_{\mathrm{MZ}} \cdot[\mathrm{MZ}]
\end{gathered}
$$

The assimilation efficiency is described through a constant and non-dimensional parameter $\mathrm{pu}_{\mathrm{MZ}}$; all prey carbon that was not assimilated is excreted $\left(\right.$ excretion $_{\mathrm{MZ}}$ ) or respired $\left(\right.$ respiration $_{\mathrm{MZ}}$ ). The constant parameter $\mathrm{pu}$-ea determines the fraction of the unassimilated food that is excreted (Eq. 144; $\mathrm{mg} \mathrm{C} \mathrm{m}^{-3}$ day $^{-1}$ ), part as dissolved organic carbon (DOC) and part as particulate organic carbon (POC) (Eqs. 145-146). The value of pu_ea is equal for all prey types except for mesozooplankton scavenging on particulate organic matter (pu_eaR; Table S5).

$$
\begin{gathered}
\text { excretion }_{\mathrm{MZ}}=\text { ingestion }_{\mathrm{MZ}} \cdot\left(1-\mathrm{pu}_{\mathrm{MZ}}\right) \cdot \mathrm{pu} \_ \text {ea } \\
\text { DoCexcretion }_{\mathrm{MZ}}=\text { excretion }_{\mathrm{MZ}} \cdot \mathrm{pe} \_\mathrm{R} 1 \\
\text { POCexcretion }_{\mathrm{MZ}}=\text { excretion }_{\mathrm{MZ}} \cdot(1-\text { pe_R} 1)
\end{gathered}
$$

The total respiration rate by the mesozooplankton population (respiration ${ }_{\mathrm{MZ}}$; $\mathrm{mg}$ $\mathrm{C} \mathrm{m}^{-3}$ day $^{-1}$ ) is described by the sum of metabolic (met_respiration $\mathrm{MZ}_{\text {) }}$ ) and basal $\left(\right.$ bas_respiration $_{\mathrm{MZ}}$ ) respiration terms, as described below (Eqs. 147-149). The metabolic respiration is obtained considering all non-assimilated prey and the carbon excreted (Eq. 147). The basal respiration component is described through a linear function of mesozooplankton biomass and a constant parameters srsmz (Eq. 148).

$$
\begin{gathered}
\text { met_respiration }_{\mathrm{MZ}}=\text { ingestion }_{\mathrm{MZ}} \cdot\left(1-\mathrm{pu}_{\mathrm{MZ}}\right)-\text { excretion }_{\mathrm{MZ}} \\
\text { bas_respiration }_{\mathrm{MZ}}=\operatorname{srs}_{\mathrm{MZ}} \cdot[\mathrm{MZ}] \\
\text { respiration }_{\mathrm{MZ}}=\text { met_respiration }_{\mathrm{MZ}}+\text { bas_respiration }_{\mathrm{MZ}}
\end{gathered}
$$

Similarly, non-predatory mortality ( mortality $_{\mathrm{MZ}}$ ) is described through a constant parameter $\operatorname{sd}_{\mathrm{MZ}}\left(\mathrm{day}^{-1}\right)$ assuming no oxygen limitation ( $\mathrm{sdo}=0$; Table S5) and contributes to both DOC and POC pools (Eqs. 150-152). The constant parameter pe_R1 defines the fraction that goes to the DOC pool.

$$
\begin{gathered}
\text { mortality }_{\mathrm{MZ}}=\operatorname{sd}_{\mathrm{MZ}} \cdot[\mathrm{MZ}] \\
\text { DOCmortality }_{\mathrm{MZ}}=\text { mortality }_{\mathrm{MZ}} \cdot \text { pe_R1 }_{-} \\
\text {POCmortality }_{\mathrm{MZ}}=\text { mortality }_{\mathrm{MZ}}-\text { DOCmortality }_{\mathrm{MZ}}
\end{gathered}
$$


Total DOC and POC losses $\left(\mathrm{mg} \mathrm{C} \mathrm{m}^{-3}\right.$ day $\left.^{-1}\right)$ are then described as following:

$$
\begin{aligned}
& \text { DOC }_{\mathrm{MZ}}=\text { DOCexcretion }_{\mathrm{MZ}}+\text { DOCmortality }_{\mathrm{MZ}} \\
& \text { POC }_{\mathrm{MZ}}=\text { POCexcretion }_{\mathrm{MZ}}+\text { POCmortality }_{\mathrm{MZ}}
\end{aligned}
$$

To determine the losses to the dissolved and particulate organic nitrogen ([DON $\left.\mathrm{Dab}_{\mathrm{l}}\right]$ and $\left[\mathrm{PON}_{1}\right]$, respectively) and phosphorus ([DOP $\left.\mathrm{Dab}_{1}\right]$ and $\left[\mathrm{PON}_{1}\right]$, respectively) the model assumes that mesozooplankton have constant nutrient to carbon ratios (qne and qpc, respectively); then, constant parameters ( $x R 1 n$ and $x R 1 p)$ control the fraction that is transferred to the dissolved and particulate pools (Eqs. 155-158).

$$
\begin{gathered}
\mathrm{DON}_{\mathrm{MZ}}=\min \left(\left(\mathrm{DOC}_{\mathrm{MZ}}+\mathrm{POC}_{\mathrm{MZ}}\right) \cdot \mathrm{qnc}, \mathrm{DOC}_{\mathrm{MZ}} \cdot \mathrm{qnc} \cdot \mathrm{xR} 1 \mathrm{n}\right) \\
\mathrm{PON}_{\mathrm{MZ}}=\left(\mathrm{DOC}_{\mathrm{MZ}}+\mathrm{POC}_{\mathrm{MZ}}\right) \cdot \mathrm{qnc}-\mathrm{DON}_{\mathrm{MZ}} \\
\mathrm{DOP}_{\mathrm{MZ}}=\min \left(\left(\mathrm{DOC}_{\mathrm{MZ}}+\mathrm{POC}_{\mathrm{MZ}}\right) \cdot \mathrm{qpc}, \mathrm{DOC}_{\mathrm{MZ}} \cdot \mathrm{qpc} \cdot \mathrm{xR} 1 \mathrm{p}\right) \\
\mathrm{POP}_{\mathrm{MZ}}=\left(\mathrm{DOC}_{\mathrm{MZ}}+\mathrm{POC}_{\mathrm{MZ}}\right) \cdot \mathrm{qpc}-\mathrm{DOP}_{\mathrm{MZ}}
\end{gathered}
$$

Nutrients, dissolved, and particulate organic matter

Equations 159-169 describe how the different processes defined in the protist, bacteria, and mesozooplankton models relate to the inorganics, dissolved, and particulate pools.

The nitrate pool varies according to the uptake by protist populations and to a constant external supply of nitrate, described through a constant dilution rate Dil $\left(\right.$ day $\left.^{-1}\right)$ and a constant external bulk of nitrate extDIN ( $\mathrm{mg} \mathrm{N} \mathrm{m}^{-3}$ ) (Eq. 159). The ammonium and phosphate pools vary according to the uptake by protist populations and bacteria, regeneration by protists, and mineralization of DON and DOP by bacteria (Eqs. 160-161, respectively). The system is also subjected to a constant external supply of phosphate and silicate, in which extDIP and extSi correspond to the constant external bulks of phosphate and silicate, in $\mathrm{mg} \mathrm{P} \mathrm{m}^{-3}$ and $\mathrm{mg} \mathrm{Si} \mathrm{m}^{-3}$, respectively (Eqs. 161-6). Silicate also varies according to the uptake by diatoms (Eq. 6).

$$
\frac{\mathrm{d}\left[\mathrm{NO}_{3}\right]}{\mathrm{dt}}=- \text { uptake }_{\mathrm{NO} 3}+\text { Dil } \cdot \mathrm{eDIN}
$$




$$
\begin{gathered}
\frac{\mathrm{d}\left[\mathrm{NH}_{4}\right]}{\mathrm{dt}}=- \text { uptake }_{\mathrm{NH} 4}+\text { Tregeneration }_{\mathrm{N}} \\
+ \text { release }_{\mathrm{BN}}+\text { miner }_{\mathrm{BN}} \\
\frac{\mathrm{d}\left[\mathrm{PO}_{4}\right]}{\mathrm{dt}}=- \text { uptake }_{\mathrm{P}}+\text { Tregeneration }_{\mathrm{P}} \\
+ \text { release }_{\mathrm{BP}}+\text { miner }_{\mathrm{BP}}+\mathrm{Dil} \cdot \mathrm{eDIP} \\
\frac{\mathrm{d}[\mathrm{Si}]}{\mathrm{dt}}=- \text { uptake }_{\mathrm{Si}}+\text { Dil } \cdot \mathrm{eSi}
\end{gathered}
$$

The respiration by all plankton groups contribute to the dissolved inorganic carbon (DIC) pool (Eq. 163). In addition, a constant proportion of the DIC fixed through primary production $\left(\mathrm{DOC}_{\mathrm{pc}}\right)$ is released as labile dissolved organic carbon (DOClab) (Eqs. 163 and 164). All voided material and the terms associated to non-predatory mortality of the protist and bacteria models contribute to the dissolved pools (Eqs. 164-166). Both dissolved and particulate pools are subjected to the uptake by bacteria (Eqs. 164-169); in equation 168, X refers to $\mathrm{N}, \mathrm{P}$, or $\mathrm{C}$ and the subscript $\mathrm{j}$ to the size class of POM, i.e., small or medium. Bacteria also release carbon in recalcitrant forms (Eq. 167). In addition, the non-assimilated material and the non-predatory mortality of mesozooplankton contributes to both dissolved and particulate (only the large size class) pools (Eqs. 164-166 and 169); in equation $169, \mathrm{X}$ refers to $\mathrm{N}, \mathrm{P}$, or $\mathrm{C}$.

$$
\begin{aligned}
& \frac{\mathrm{d}[\mathrm{DIC}]}{\mathrm{dt}}=- \text { fixation }_{\mathrm{C}}-\left(\text { fixation }_{\mathrm{C}} \cdot \text { DOC }_{\mathrm{pc}}\right)+\left(\mathrm{R}_{\text {tot }}+\mathrm{BR}_{\mathrm{i}}\right) \cdot[\mathrm{C}]+ \\
& + \text { respiration }_{\mathrm{BC}} \\
& + \text { respiration }_{\mathrm{MZ}} \\
& \frac{\mathrm{d}\left[\mathrm{DOC}_{\mathrm{lab}}\right]}{\mathrm{dt}}=\left(\text { fixation }_{\mathrm{C}} \cdot \text { DOC }_{\mathrm{pc}}\right)+\text { voiding }_{\mathrm{C}}+\text { mortality }_{\mathrm{C}} \\
& -\operatorname{sug}_{\mathrm{BC}} \cdot\left[\mathrm{DOC}_{\mathrm{lab}}\right] \cdot \text { frDOC }_{\mathrm{l}}+\text { mortality }_{\mathrm{BC}} \\
& +\mathrm{DOC}_{\mathrm{MZ}} \\
& \frac{\mathrm{d}\left[\mathrm{DON}_{\text {lab }}\right]}{\mathrm{dt}}=\text { voiding }_{\mathrm{N}}+\text { mortality }_{\mathrm{N}} \\
& - \text { uptake }_{\mathrm{BN}}+\text { mortality }_{\mathrm{BN}}-\text { miner }_{\mathrm{BN}} \\
& +\mathrm{DON}_{\mathrm{MZ}} \\
& \frac{\mathrm{d}\left[\mathrm{DOP}_{\mathrm{lab}}\right]}{\mathrm{dt}}=\text { voiding }_{\mathrm{P}}+\text { mortality }_{\mathrm{P}} \\
& - \text { uptake }_{\mathrm{BP}}+\text { mortality }_{\mathrm{BP}}-\text { miner }_{\mathrm{BP}} \\
& +\mathrm{DOP}_{\mathrm{MZ}}
\end{aligned}
$$




$$
\begin{gathered}
\frac{d\left[\mathrm{DOC}_{\mathrm{r}}\right]}{\mathrm{dt}}=-\operatorname{sug}_{\mathrm{BC}} \cdot\left[\mathrm{DOC}_{\mathrm{r}}\right] \cdot \text { frDOC }_{\mathrm{r}}+\text { release }_{\mathrm{BC}} \\
\frac{\mathrm{d}\left[\mathrm{POX}_{\mathrm{j}}\right]}{\mathrm{dt}}=-\operatorname{sug}_{\mathrm{BC}} \cdot\left[\mathrm{POX}_{\mathrm{j}}\right] \cdot \mathrm{rPOC}_{\mathrm{j}} \\
\frac{\mathrm{d}\left[\mathrm{POX}_{\mathrm{l}}\right]}{\mathrm{dt}}=-\operatorname{sug}_{\mathrm{BC}} \cdot\left[\mathrm{POX}_{\mathrm{j}}\right] \cdot \mathrm{rPOC}_{\mathrm{j}}+\mathrm{POX}_{\mathrm{MZ}}
\end{gathered}
$$


Table C.1 List of constant parameters within the protist model; parameters in bold correspond to new implementations to the model of Flynn and Mitra (2009).

\begin{tabular}{|c|c|c|c|}
\hline Parameter & Description & Units & Value \\
\hline $\mathbf{a}$ & $\begin{array}{l}\text { constant a for the allometric relationship of } \\
\text { ESD and carbon content }\end{array}$ & - & 0.216 \\
\hline $\mathrm{AA}_{\mathrm{syn}}$ & cost for amino acid synthesis & $\mathrm{gC}(\mathrm{gN})^{-1}$ & 1.50 \\
\hline$\alpha_{\mathrm{Chl}}$ & initial slope of PE curve & 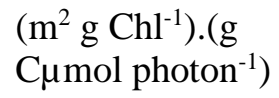 & Tables $6.1 \& 7.2$ \\
\hline $\mathrm{AE}_{\max }$ & maximum assimilation efficiency & - & 0.80 \\
\hline $\mathrm{AE}_{\min }$ & minimum assimilation efficiency & - & 0.20 \\
\hline$A_{\text {pref }}$ & relative preference for ammonium & - & 2.0 \\
\hline b & $\begin{array}{l}\text { constant } b \text { for the allometric relationship of } \\
\text { ESD and carbon content }\end{array}$ & - & 0.939 \\
\hline$\beta$ & control for nutrient uptake & - & 0.05 \\
\hline $\mathrm{BR}$ & basal respiration rate & $\mathrm{gC}(\mathrm{gC})^{-1}$ day $^{-1}$ & 0.05 \\
\hline $\mathrm{ChlC}_{\mathrm{abs}}$ & absolute maximum Chl:C & $\mathrm{gChl}(\mathrm{gC})^{-1}$ & Tables $6.1 \& 7.2$ \\
\hline $\mathbf{D}_{\text {Chl }}$ & decay rate of kleptochloroplasts & day $^{-1}$ & Tables $6.1 \& 7.2$ \\
\hline $\mathrm{DOC}_{\mathrm{pc}}$ & $\begin{array}{l}\text { proportion of inorganic carbon fixation } \\
\text { released }\end{array}$ & - & 0.20 \\
\hline $\mathrm{FC}_{\mathrm{abs}}$ & maximum feeding vacuole size & $\mathrm{gC}(\mathrm{gC})^{-1}$ & 0.20 \\
\hline $\mathrm{FC}_{\min }$ & minimum feeding vacuole size & $\mathrm{gC}(\mathrm{gC})^{-1}$ & 0.00 \\
\hline $\mathrm{H}_{\mathrm{as}}$ & Hill number for digestion rate & - & 2.00 \\
\hline $\mathrm{H}_{\mathrm{eq}}$ & Hill number for quantity-linked $\mathrm{AE}$ & - & 4.00 \\
\hline $\mathrm{H}_{\text {het }}$ & Hill number for control of $\mathrm{FC}_{\max }$ & - & 20.0 \\
\hline $\mathrm{H}_{\text {ing }}$ & Hill number for ingestion control & - & 4.00 \\
\hline $\mathrm{H}_{\text {pbal }}$ & $\begin{array}{l}\text { Hill number for digestion link to critical C- } \\
\text { fixation }\end{array}$ & - & 4.00 \\
\hline $\mathrm{H}_{\mathrm{pd}}$ & Hill number for digestion suppression & - & 10.0 \\
\hline $\mathrm{H}_{\mathrm{q}}$ & Hill number for uptake control & - & 4.00 \\
\hline $\mathrm{H}_{\mathrm{rc}}$ & Hill number for regeneration & - & 4.00 \\
\hline iop $_{A B S}$ & specific shortwave absorption & $\mathrm{m}^{2} \mathrm{mg} \mathrm{Chl}^{-1}$ & Tables $6.1 \& 7.2$ \\
\hline $\mathrm{K}_{\mathrm{a}}$ & half-saturation for ammonium uptake & $\mathrm{mg} \mathrm{N} \mathrm{m}^{-3}$ & Tables $6.1 \& 7.2$ \\
\hline $\mathrm{K}_{\mathrm{as}}$ & half-saturation for digestion rate & - & 0.50 \\
\hline $\mathrm{K}_{\mathrm{ec}}$ & response control to prey quality & - & 10.0 \\
\hline $\mathrm{K}_{\mathrm{eq}}$ & response control to ingestion quantity & - & $1 \times 10^{-6}$ \\
\hline $\mathrm{K}_{\text {het }}$ & half-saturation for $\mathrm{FC}_{\max }$ & - & 1.00 \\
\hline $\mathrm{K}_{\text {ing }}$ & half-saturation for ingestion control & - & 0.20 \\
\hline $\mathrm{K}_{\mathrm{n}}$ & half-saturation for nitrate uptake & $\mathrm{mg} \mathrm{N} \mathrm{m}^{-3}$ & Tables $6.1 \& 7.2$ \\
\hline $\mathrm{K}_{\mathrm{p}}$ & half-saturation for phosphate uptake & $\mathrm{mg} \mathrm{P} \mathrm{m}{ }^{-3}$ & Tables $6.1 \& 7.2$ \\
\hline $\mathrm{K}_{\mathrm{pbal}}$ & $\begin{array}{l}\text { half-saturation for digestion link to critical } \\
\text { C-fixation }\end{array}$ & - & 0.10 \\
\hline
\end{tabular}




\begin{tabular}{|c|c|c|c|}
\hline $\mathrm{K}_{\mathrm{pd}}$ & half-saturation for digestion supression & - & 1.00 \\
\hline $\mathbf{k} \mathbf{P}_{\max }$ & $\begin{array}{l}\text { kleptochloroplasts maximum possible } \\
\text { phototrophic growth rate }\end{array}$ & $\mathrm{gC}(\mathrm{gC})^{-1}$ day $^{-1}$ & Tables $6.1 \& 7.2$ \\
\hline $\mathrm{K}_{\mathrm{q}}$ & $\begin{array}{l}\text { half-saturation for control of nutrient } \\
\text { uptake }\end{array}$ & - & 0.10 \\
\hline $\mathrm{K}_{\mathrm{qn}}$ & half-saturation for nitrogen quota curve & - & 10.0 \\
\hline $\mathrm{K}_{\mathrm{qp}}$ & half-saturation for phosphate quota curve & - & 0.10 \\
\hline $\mathbf{K}_{\mathbf{q s}}$ & half-saturation for silicate uptake & $\mathrm{mg} \mathrm{Si} \mathrm{m}{ }^{-3}$ & Tables $6.1 \& 7.2$ \\
\hline $\mathrm{K}_{\mathrm{rc}}$ & $\begin{array}{l}\text { half-saturation for control of nutrient } \\
\text { regeneration vs re-assimilation }\end{array}$ & - & 1.00 \\
\hline $\operatorname{Met}_{M}$ & $\begin{array}{l}\text { Metabolic cost multiplier to achieve } \\
\text { required gross growth efficiency for } \\
\text { dinoflagellates }\end{array}$ & - & Tables $6.1 \& 7.2$ \\
\hline$\mu_{\max }$ & maximum possible growth rate & $\mathrm{gC}(\mathrm{gC})^{-1}$ day $^{-1}$ & Tables $6.1 \& 7.2$ \\
\hline$\mu_{\text {phot }}$ & $\begin{array}{l}\text { maximum possible phototrophic growth } \\
\text { rate }\end{array}$ & $\mathrm{gC}(\mathrm{gC})^{-1}$ day $^{-1}$ & Tables $6.1 \& 7.2$ \\
\hline MR & metabolic respiration & $\mathrm{gC}(\mathrm{gC})^{-1}$ & 0.20 \\
\hline $\mathrm{NC}_{\mathrm{abs}}$ & absolute maximum $\mathrm{N}: \mathrm{C}$ & $\mathrm{gN}(\mathrm{gC})^{-1}$ & Tables $6.1 \& 7.2$ \\
\hline $\mathrm{NC}_{\mathrm{m}}$ & $\begin{array}{l}\text { maximum } \mathrm{N}: \mathrm{C} \text { which could be attained in } \\
\text { the organic form }\end{array}$ & $\mathrm{gN}(\mathrm{gC})^{-1}$ & 0.30 \\
\hline $\mathrm{NC}_{\max }$ & maximum $\mathrm{N}: \mathrm{C}$ affecting growth rate & $\mathrm{gN}(\mathrm{gC})^{-1}$ & Tables $6.1 \& 7.2$ \\
\hline $\mathrm{NC}_{\min }$ & minimum $\mathrm{N}: \mathrm{C}$ & $\mathrm{gN}(\mathrm{gC})^{-1}$ & Tables $6.1 \& 7.2$ \\
\hline $\mathrm{N}_{\text {pref }}$ & relative preference for nitrate & - & 1.00 \\
\hline $\mathrm{Pbal}_{\text {crit }}$ & $\begin{array}{l}\text { minimum critical proportion of growth } \\
\text { supported by photosynthesis }\end{array}$ & - & Tables $6.1 \& 7.2$ \\
\hline $\mathrm{PC}_{\mathrm{abs}}$ & absolute maximum P:C & $\mathrm{gP}(\mathrm{gC})^{-1}$ & Tables $6.1 \& 7.2$ \\
\hline $\mathrm{PC}_{\mathrm{m}}$ & $\begin{array}{l}\text { maximum } \mathrm{P}: \mathrm{C} \text { which could be obatined in } \\
\text { the organic form }\end{array}$ & $\mathrm{gP}(\mathrm{gC})^{-1}$ & 0.03 \\
\hline $\mathrm{PC}_{\max }$ & maximum $\mathrm{P}: \mathrm{C}$ affecting growth rate & $\mathrm{gP}(\mathrm{gC})^{-1}$ & Tables $6.1 \& 7.2$ \\
\hline $\mathrm{PC}_{\min }$ & minimum $\mathrm{P}: \mathrm{C}$ & $\mathrm{gP}(\mathrm{gC})^{-1}$ & Tables $6.1 \& 7.2$ \\
\hline$P_{\text {pref }}$ & preference for DIP & - & 1.00 \\
\hline redco & cost of nitrate reduction to ammonium & $\mathrm{gC}(\mathrm{gN})^{-1}$ & 1.71 \\
\hline sd & specific mortality & day $^{-1}$ & 0.02 \\
\hline size & cell size & $\mu \mathrm{m}$ ESD & Tables $6.1 \& 7.2$ \\
\hline $\mathbf{S}_{\max }$ & maximum prey size & $\mu \mathrm{m}$ ESD & Tables $6.1 \& 7.2$ \\
\hline$S_{\min }$ & minimum prey size & $\mu \mathrm{m}$ ESD & Tables $6.1 \& 7.2$ \\
\hline$S_{\text {mix }}$ & $\begin{array}{l}\text { switch to enable phototrophy vs } \\
\text { heterotrophy control }\end{array}$ & $(0$ or 1$)$ & Tables $6.1 \& 7.2$ \\
\hline $\mathbf{S}_{\text {opt }}$ & optimum prey size & $\mu \mathrm{m}$ ESD & Tables $6.1 \& 7.2$ \\
\hline $\mathrm{S}_{\mathrm{pd}}$ & $\begin{array}{l}\text { switch to enable phototrophy down } \\
\text { regulating heterotrophy }\end{array}$ & $(0$ or 1$)$ & Tables $6.1 \& 7.2$ \\
\hline $\mathbf{S}_{\mathrm{si}}$ & switch to enable silicate uptake & $(0$ or 1$)$ & Tables $6.1 \& 7.2$ \\
\hline $\mathbf{w}$ & root-mean-squared turbulence & $\mathrm{m} \mathrm{s}^{-1}$ & 0.00 \\
\hline
\end{tabular}



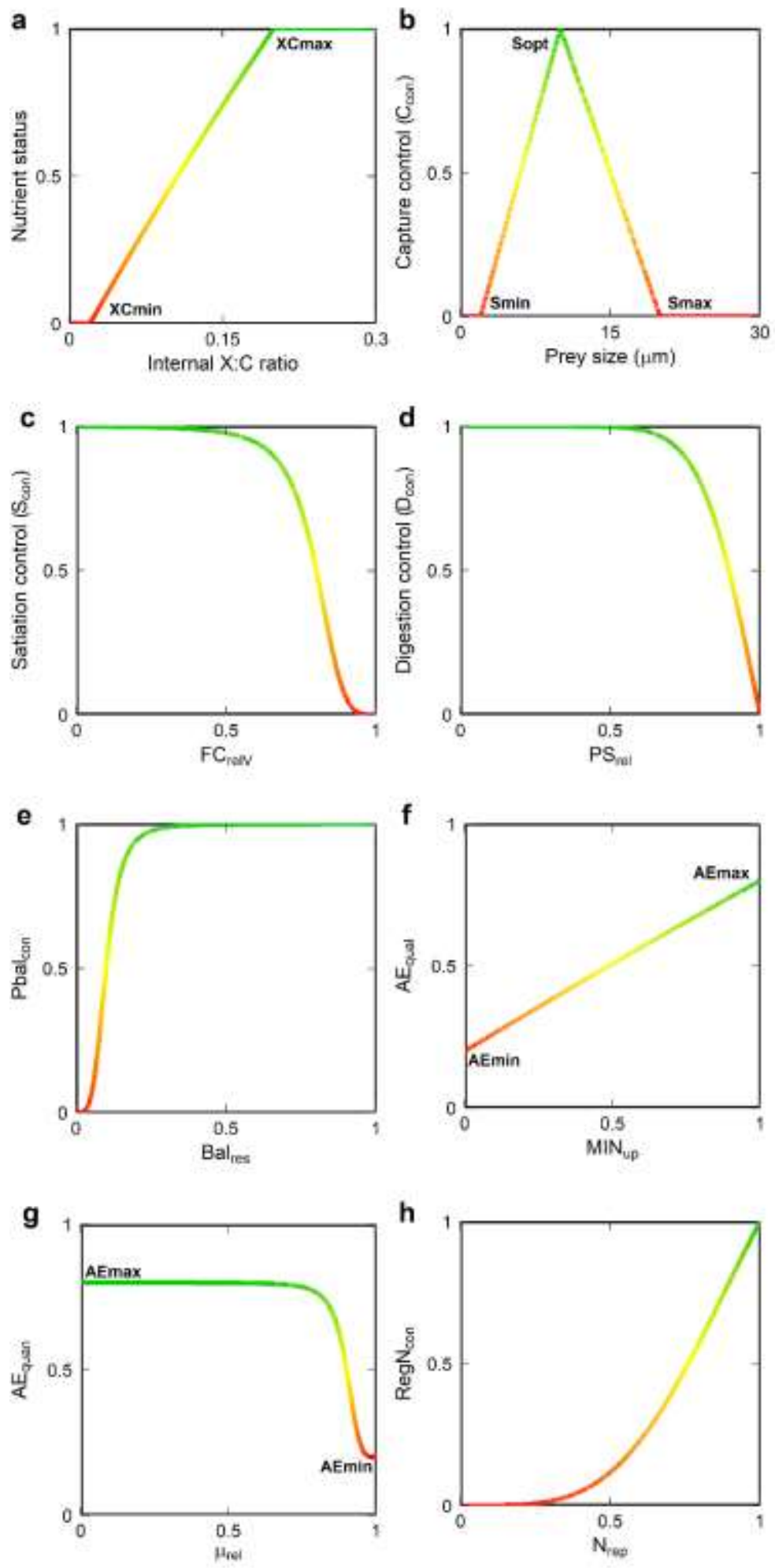

Figure C.1 Response curves for diverse normalised feedbacks used in the protist model (Eqs. 10-11, 51, 52-54, 58-59, 60-62, 67, 68, and 89-90, respectively). The colour gradient indicates the magnitude of the feedback, increasing from the green to the red. 


\section{Appendix D}

Table D.1 List of results of the Monte-Carlo sensitivity analyses for three targeted model outputs in the mixotrophic food web within the light-limited and the nutrient-limited scenarios (overall $\mathrm{R}^{2}>0.9$ ). Results are given for sensitivity coefficients of all parameters statistically significant at $p<0.05$. These are ranked with respect to their absolute value (most important first). Coefficient signs indicate a positive or negative effect on the targeted model outputs, i.e., increase or decrease of the output values, respectively.

\begin{tabular}{|c|c|c|c|c|}
\hline Scenario & Targeted output & Functional type & Parameter & Coefficient \\
\hline Light-limited & Production of labile DOC & Diatoms & $\alpha_{\mathrm{Chl}}$ & 0.50 \\
\hline Light-limited & Production of labile DOC & Diatoms & $\mathrm{ChlC}_{\mathrm{abs}}$ & 0.41 \\
\hline Light-limited & Production of labile DOC & PicoP & $\alpha_{\mathrm{Chl}}$ & 0.31 \\
\hline Light-limited & Production of labile DOC & PicoP & $\mathrm{ChlC}_{\mathrm{abs}}$ & 0.16 \\
\hline Light-limited & Production of labile DOC & Nano-CMs & $\alpha_{\mathrm{Chl}}$ & 0.16 \\
\hline Light-limited & Production of labile DOC & Nano-CMs & $\mathrm{ChlC}_{\mathrm{abs}}$ & 0.13 \\
\hline Light-limited & Production of labile DOC & Nano-CMs & $S_{\text {opt }}$ & 0.11 \\
\hline Light-limited & Production of labile DOC & Diatoms & $\mathrm{BR}$ & -0.09 \\
\hline Light-limited & Production of labile DOC & SNCMs & $\mathrm{S}_{\max }$ & -0.09 \\
\hline Light-limited & Production of labile DOC & Micro-CMs & $S_{\max }$ & -0.09 \\
\hline Light-limited & Production of labile DOC & Nano-CMs & $\mu_{\text {phot }}$ & 0.08 \\
\hline Light-limited & Production of labile DOC & Diatoms & $\mu_{\text {phot }}$ & 0.08 \\
\hline Light-limited & Production of labile DOC & Diatoms & $\mu_{\max }$ & -0.08 \\
\hline Light-limited & Production of labile DOC & MicroZ & $S_{\max }$ & -0.08 \\
\hline Light-limited & Production of labile DOC & Bacteria & $\operatorname{srs}_{B}$ & -0.07 \\
\hline Light-limited & Production of labile DOC & PicoP & $\mu_{\max }$ & -0.07 \\
\hline Light-limited & Production of labile DOC & PicoP & $\mathrm{BR}$ & -0.06 \\
\hline Light-limited & Production of labile DOC & PicoP & $\mu_{\text {phot }}$ & 0.05 \\
\hline Light-limited & Production of labile DOC & Nano-CMs & $\mathrm{PC}_{\max }$ & -0.04 \\
\hline Light-limited & Production of labile DOC & Diatoms & $\mathrm{K}_{\mathrm{a}}$ & -0.04 \\
\hline Light-limited & Production of labile DOC & GNCMs & $\mathrm{S}_{\max }$ & -0.04 \\
\hline Light-limited & Production of labile DOC & MicroZ & $\mathrm{NC}_{\max }$ & -0.03 \\
\hline Light-limited & Production of labile DOC & Diatoms & $\mathrm{NC}_{\max }$ & 0.03 \\
\hline Light-limited & Production of labile DOC & NanoZ & $\mathrm{NC}_{\max }$ & -0.03 \\
\hline Light-limited & Production of labile DOC & MesoZ & $\mathrm{Cr}_{\text {nanoZ }}$ & 0.03 \\
\hline Light-limited & Production of labile DOC & Diatoms & $\mathrm{PC}_{\min }$ & -0.03 \\
\hline Light-limited & Production of labile DOC & Bacteria & $\operatorname{sum}_{B}$ & 0.03 \\
\hline Light-limited & Production of labile DOC & Micro-CMs & $\alpha_{\mathrm{Chl}}$ & 0.03 \\
\hline
\end{tabular}




\begin{tabular}{|c|c|c|c|c|}
\hline Light-limited & Production of labile DOC & SNCMs & $\alpha_{\mathrm{Chl}}$ & 0.03 \\
\hline Light-limited & Production of labile DOC & MesoZ & $\mathrm{Cr}_{\text {diatoms }}$ & -0.03 \\
\hline Light-limited & Production of labile DOC & MesoZ & $\mathrm{Cr}_{\mathrm{GNCMs}}$ & 0.03 \\
\hline Light-limited & Ammonium regeneration & PicoP & $\alpha_{\text {Chl }}$ & 0.34 \\
\hline Light-limited & Ammonium regeneration & Nano-CMs & $\mathrm{NC}_{\max }$ & -0.27 \\
\hline Light-limited & Ammonium regeneration & Diatoms & $\alpha_{\mathrm{Chl}}$ & 0.27 \\
\hline Light-limited & Ammonium regeneration & Diatoms & $\mathrm{ChlC}_{\mathrm{abs}}$ & 0.27 \\
\hline Light-limited & Ammonium regeneration & PicoP & $\mathrm{ChlC}_{\mathrm{abs}}$ & 0.24 \\
\hline Light-limited & Ammonium regeneration & Micro-CMs & $\mathrm{NC}_{\max }$ & -0.21 \\
\hline Light-limited & Ammonium regeneration & SNCMs & $\mathrm{NC}_{\max }$ & -0.18 \\
\hline Light-limited & Ammonium regeneration & Bacteria & $\mathrm{NC}_{\max }$ & 0.17 \\
\hline Light-limited & Ammonium regeneration & Nano-CMs & $S_{\text {opt }}$ & 0.16 \\
\hline Light-limited & Ammonium regeneration & MicroZ & $\mathrm{S}_{\max }$ & 0.15 \\
\hline Light-limited & Ammonium regeneration & Nano-CMs & $\mathrm{ChlC}_{\mathrm{abs}}$ & -0.12 \\
\hline Light-limited & Ammonium regeneration & PicoP & $\mathrm{NC}_{\max }$ & -0.10 \\
\hline Light-limited & Ammonium regeneration & Diatoms & $\mu_{\mathrm{phot}}$ & 0.08 \\
\hline Light-limited & Ammonium regeneration & PicoP & $\mu_{\text {phot }}$ & 0.07 \\
\hline Light-limited & Ammonium regeneration & Nano-CMs & $\mathrm{S}_{\max }$ & 0.07 \\
\hline Light-limited & Ammonium regeneration & PicoP & $\mu_{\max }$ & -0.07 \\
\hline Light-limited & Ammonium regeneration & PicoP & $\mathrm{BR}$ & -0.06 \\
\hline Light-limited & Ammonium regeneration & Micro-CMs & $\mathrm{ChlC}_{\mathrm{abs}}$ & -0.06 \\
\hline Light-limited & Ammonium regeneration & Micro-CMs & $\mathrm{S}_{\max }$ & -0.06 \\
\hline Light-limited & Ammonium regeneration & MesoZ & $\mathrm{Cr}_{\text {diatoms }}$ & -0.05 \\
\hline Light-limited & Ammonium regeneration & Micro-CMs & $\alpha_{\text {Chl }}$ & -0.05 \\
\hline Light-limited & Ammonium regeneration & Nano-CMs & $\alpha_{\text {Chl }}$ & -0.05 \\
\hline Light-limited & Ammonium regeneration & MesoZ & $\mathrm{Cr}_{\text {mesoZ }}$ & 0.05 \\
\hline Light-limited & Ammonium regeneration & GNCMs & $\mathrm{S}_{\max }$ & -0.05 \\
\hline Light-limited & Ammonium regeneration & SNCMs & $\mathrm{BR}$ & 0.04 \\
\hline Light-limited & Ammonium regeneration & Diatoms & $\mathrm{NC}_{\max }$ & -0.04 \\
\hline Light-limited & Ammonium regeneration & SNCMs & $\mathrm{S}_{\max }$ & -0.03 \\
\hline Light-limited & Ammonium regeneration & Nano-CMs & $\mathrm{S}_{\min }$ & 0.03 \\
\hline Light-limited & Ammonium regeneration & SNCMs & $\mathrm{D}_{\mathrm{Chl}}$ & -0.03 \\
\hline Light-limited & Ammonium regeneration & Bacteria & $\operatorname{srs}_{B}$ & -0.03 \\
\hline Light-limited & Ammonium regeneration & Diatoms & $\mathrm{K}_{\mathrm{a}}$ & -0.03 \\
\hline Light-limited & Ammonium regeneration & NanoZ & $\mathrm{NC}_{\max }$ & -0.03 \\
\hline Light-limited & Ammonium regeneration & Micro-CMs & $\mathrm{S}_{\mathrm{opt}}$ & 0.03 \\
\hline Light-limited & Ammonium regeneration & MicroZ & $\mu_{\max }$ & 0.03 \\
\hline Light-limited & Ammonium regeneration & GNCMs & $\alpha_{\mathrm{Chl}}$ & -0.03 \\
\hline Light-limited & Ammonium regeneration & Nano-CMs & $\mathrm{PC}_{\max }$ & -0.03 \\
\hline Light-limited & Ammonium regeneration & Micro-CMs & $\mu_{\max }$ & 0.03 \\
\hline
\end{tabular}




\begin{tabular}{|c|c|c|c|c|}
\hline Light-limited & Ammonium regeneration & MicroZ & $\mathrm{S}_{\min }$ & 0.03 \\
\hline Light-limited & Ammonium regeneration & PicoP & $\mathrm{K}_{\mathrm{a}}$ & -0.03 \\
\hline Light-limited & Trophic transfer efficiency & Nano-CMs & $\mathrm{S}_{\mathrm{opt}}$ & -0.31 \\
\hline Light-limited & Trophic transfer efficiency & Diatoms & $\alpha_{\text {Chl }}$ & 0.27 \\
\hline Light-limited & Trophic transfer efficiency & Diatoms & $\mathrm{ChlC}_{\mathrm{abs}}$ & 0.22 \\
\hline Light-limited & Trophic transfer efficiency & Nano-CMs & $\mathrm{ChlC}_{\mathrm{abs}}$ & 0.21 \\
\hline Light-limited & Trophic transfer efficiency & MesoZ & $\mathrm{Cr}_{\text {mesoZ }}$ & -0.19 \\
\hline Light-limited & Trophic transfer efficiency & PicoP & ChlC $_{a b s}$ & -0.19 \\
\hline Light-limited & Trophic transfer efficiency & MesoZ & $\mathrm{Cr}_{\text {micro-CMs }}$ & 0.17 \\
\hline Light-limited & Trophic transfer efficiency & Nano-CMs & $\alpha_{\text {Chl }}$ & 0.17 \\
\hline Light-limited & Trophic transfer efficiency & PicoP & $\alpha_{\text {Chl }}$ & -0.17 \\
\hline Light-limited & Trophic transfer efficiency & Micro-CMs & $\mathrm{S}_{\max }$ & -0.15 \\
\hline Light-limited & Trophic transfer efficiency & MesoZ & $\mathrm{Cr}_{\text {diatoms }}$ & 0.15 \\
\hline Light-limited & Trophic transfer efficiency & Nano-CMs & $S_{\max }$ & -0.14 \\
\hline Light-limited & Trophic transfer efficiency & MicroZ & $\mathrm{S}_{\max }$ & -0.13 \\
\hline Light-limited & Trophic transfer efficiency & SNCMs & $\mathrm{S}_{\max }$ & -0.11 \\
\hline Light-limited & Trophic transfer efficiency & Diatoms & $\mathrm{BR}$ & -0.11 \\
\hline Light-limited & Trophic transfer efficiency & Nano-CMs & $\mathrm{S}_{\min }$ & -0.10 \\
\hline Light-limited & Trophic transfer efficiency & MesoZ & $\mathrm{Cr}_{\text {microz }}$ & 0.09 \\
\hline Light-limited & Trophic transfer efficiency & MesoZ & $\operatorname{srs}_{B}$ & -0.08 \\
\hline Light-limited & Trophic transfer efficiency & MesoZ & $\mathrm{Cr}_{\mathrm{GNCMs}}$ & 0.08 \\
\hline Light-limited & Trophic transfer efficiency & Diatoms & $\mu_{\max }$ & -0.08 \\
\hline Light-limited & Trophic transfer efficiency & Nano-CMs & $\mu_{\text {phot }}$ & 0.07 \\
\hline Light-limited & Trophic transfer efficiency & Diatoms & $\mu_{\text {phot }}$ & 0.05 \\
\hline Light-limited & Trophic transfer efficiency & MicroZ & $\mathrm{NC}_{\max }$ & -0.05 \\
\hline Light-limited & Trophic transfer efficiency & Nano-CMs & $\mu_{\max }$ & -0.05 \\
\hline Light-limited & Trophic transfer efficiency & PicoP & $\mathrm{BR}$ & 0.05 \\
\hline Light-limited & Trophic transfer efficiency & PicoP & $\mu_{\max }$ & 0.05 \\
\hline Light-limited & Trophic transfer efficiency & Nano-CMs & $\mathrm{NC}_{\max }$ & 0.05 \\
\hline Light-limited & Trophic transfer efficiency & Nano-CMs & $\mathrm{BR}$ & -0.05 \\
\hline Light-limited & Trophic transfer efficiency & Micro-CMs & $\mathrm{ChlC}_{\mathrm{abs}}$ & -0.05 \\
\hline Light-limited & Trophic transfer efficiency & MicroZ & $\mathrm{BR}$ & 0.04 \\
\hline Light-limited & Trophic transfer efficiency & Bacteria & $\operatorname{srs}_{B}$ & -0.04 \\
\hline Light-limited & Trophic transfer efficiency & SNCMs & $\mu_{\max }$ & -0.04 \\
\hline Light-limited & Trophic transfer efficiency & PicoP & $\mu_{\text {phot }}$ & -0.04 \\
\hline Light-limited & Trophic transfer efficiency & NanoZ & $\mathrm{S}_{\min }$ & 0.04 \\
\hline Light-limited & Trophic transfer efficiency & Bacteria & $\mathrm{PC}_{\max }$ & 0.03 \\
\hline Light-limited & Trophic transfer efficiency & Micro-CMs & $S_{\mathrm{opt}}$ & 0.03 \\
\hline Light-limited & Trophic transfer efficiency & PicoP & $\mathrm{K}_{\mathrm{a}}$ & 0.03 \\
\hline Light-limited & Trophic transfer efficiency & SNCMs & $S_{\mathrm{opt}}$ & -0.03 \\
\hline
\end{tabular}




\begin{tabular}{|c|c|c|c|c|}
\hline Light-limited & Trophic transfer efficiency & PicoP & $\mathrm{NC}_{\max }$ & 0.03 \\
\hline Light-limited & Trophic transfer efficiency & Micro-CMs & $\mathrm{NC}_{\max }$ & -0.03 \\
\hline Light-limited & Trophic transfer efficiency & SNCMs & $\mathrm{K}_{\mathrm{a}}$ & -0.03 \\
\hline Nutrient-limited & Production of labile DOC & Nano-CMs & $\mu_{\text {phot }}$ & 0.33 \\
\hline Nutrient-limited & Production of labile DOC & SNCMs & $\mathrm{S}_{\max }$ & 0.32 \\
\hline Nutrient-limited & Production of labile DOC & Nano-CMs & $\mathrm{NC}_{\min }$ & -0.31 \\
\hline Nutrient-limited & Production of labile DOC & Bacteria & $\mathrm{NC}_{\max }$ & -0.30 \\
\hline Nutrient-limited & Production of labile DOC & GNCMs & $\mathrm{S}_{\max }$ & 0.25 \\
\hline Nutrient-limited & Production of labile DOC & Nano-CMs & $\mathrm{NC}_{\max }$ & -0.20 \\
\hline Nutrient-limited & Production of labile DOC & GNCMs & $\mathrm{PC}_{\max }$ & 0.19 \\
\hline Nutrient-limited & Production of labile DOC & Bacteria & $\mathrm{PC}_{\max }$ & 0.17 \\
\hline Nutrient-limited & Production of labile DOC & SNCMs & $\mathrm{PC}_{\max }$ & 0.13 \\
\hline Nutrient-limited & Production of labile DOC & Nano-CMs & $\mathrm{PC}_{\max }$ & -0.12 \\
\hline Nutrient-limited & Production of labile DOC & Nano-CMs & $S_{\mathrm{opt}}$ & -0.10 \\
\hline Nutrient-limited & Production of labile DOC & GNCMs & $S_{\mathrm{opt}}$ & 0.09 \\
\hline Nutrient-limited & Production of labile DOC & Nano-CMs & $\mathrm{S}_{\min }$ & -0.07 \\
\hline Nutrient-limited & Production of labile DOC & MicroZ & $S_{\max }$ & 0.06 \\
\hline Nutrient-limited & Production of labile DOC & SNCMs & $\mathrm{S}_{\mathrm{opt}}$ & 0.06 \\
\hline Nutrient-limited & Production of labile DOC & SNCMs & $\mathrm{NC}_{\min }$ & -0.05 \\
\hline Nutrient-limited & Production of labile DOC & SNCMs & $\mu_{\max }$ & -0.05 \\
\hline Nutrient-limited & Production of labile DOC & PicoP & $\mathrm{NC}_{\max }$ & -0.05 \\
\hline Nutrient-limited & Production of labile DOC & SNCMs & $\mathrm{BR}$ & -0.05 \\
\hline Nutrient-limited & Production of labile DOC & MesoZ & $\mathrm{Cr}_{\mathrm{GNCMs}}$ & -0.05 \\
\hline Nutrient-limited & Production of labile DOC & MicroZ & $\mathrm{BR}$ & 0.04 \\
\hline Nutrient-limited & Production of labile DOC & MicroZ & $\mathrm{PC}_{\max }$ & 0.04 \\
\hline Nutrient-limited & Production of labile DOC & Micro-CMs & $\mathrm{S}_{\max }$ & 0.04 \\
\hline Nutrient-limited & Production of labile DOC & Micro-CMs & $\mathrm{NC}_{\max }$ & 0.04 \\
\hline Nutrient-limited & Production of labile DOC & PicoP & $\mu_{\text {phot }}$ & 0.04 \\
\hline Nutrient-limited & Production of labile DOC & NanoZ & $\mathrm{NC}_{\max }$ & 0.04 \\
\hline Nutrient-limited & Production of labile DOC & Diatoms & $\mathrm{BR}$ & -0.04 \\
\hline Nutrient-limited & Production of labile DOC & PicoP & $\alpha_{\mathrm{Chl}}$ & 0.04 \\
\hline Nutrient-limited & Production of labile DOC & MesoZ & $\mathrm{Cr}_{\mathrm{POM}}$ & -0.04 \\
\hline Nutrient-limited & Production of labile DOC & MicroZ & $\mu_{\max }$ & 0.04 \\
\hline Nutrient-limited & Production of labile DOC & SNCMs & $\alpha_{\mathrm{Chl}}$ & 0.03 \\
\hline Nutrient-limited & Production of labile DOC & Micro-CMs & $\mathrm{NC}_{\min }$ & 0.03 \\
\hline Nutrient-limited & Production of labile DOC & Nano-CMs & $\mathrm{BR}$ & -0.03 \\
\hline Nutrient-limited & Production of labile DOC & GNCMs & $\mu_{\max }$ & -0.03 \\
\hline Nutrient-limited & Production of labile DOC & PicoP & $\mathrm{K}_{\mathrm{a}}$ & 0.03 \\
\hline Nutrient-limited & Production of labile DOC & SNCMs & $\mathrm{NC}_{\max }$ & -0.03 \\
\hline Nutrient-limited & Production of labile DOC & NanoZ & $\mathrm{S}_{\max }$ & -0.03 \\
\hline
\end{tabular}




\begin{tabular}{|c|c|c|c|c|}
\hline Nutrient-limited & Production of labile DOC & PicoP & $\mathrm{PC}_{\max }$ & 0.03 \\
\hline Nutrient-limited & Production of labile DOC & SNCMs & $\mathrm{K}_{\mathrm{p}}$ & -0.03 \\
\hline Nutrient-limited & Ammonium regeneration & Nano-CMs & $\mathrm{S}_{\max }$ & -0.46 \\
\hline Nutrient-limited & Ammonium regeneration & Nano-CMs & $\mathrm{NC}_{\max }$ & -0.20 \\
\hline Nutrient-limited & Ammonium regeneration & Bacteria & $\mathrm{NC}_{\max }$ & 0.14 \\
\hline Nutrient-limited & Ammonium regeneration & GNCMs & $S_{\max }$ & 0.11 \\
\hline Nutrient-limited & Ammonium regeneration & Nano-CMs & $\mu_{\text {phot }}$ & 0.10 \\
\hline Nutrient-limited & Ammonium regeneration & Nano-CMs & $\mathrm{S}_{\mathrm{opt}}$ & -0.09 \\
\hline Nutrient-limited & Ammonium regeneration & SNCMs & $\mathrm{S}_{\max }$ & 0.09 \\
\hline Nutrient-limited & Ammonium regeneration & SNCMs & $\mathrm{PC}_{\max }$ & 0.06 \\
\hline Nutrient-limited & Ammonium regeneration & GNCMs & $\mathrm{PC}_{\max }$ & 0.06 \\
\hline Nutrient-limited & Ammonium regeneration & Nano-CMs & $\mathrm{NC}_{\min }$ & -0.05 \\
\hline Nutrient-limited & Ammonium regeneration & PicoP & $\mu_{\text {phot }}$ & -0.05 \\
\hline Nutrient-limited & Ammonium regeneration & GNCMs & $\mathrm{S}_{\mathrm{opt}}$ & 0.05 \\
\hline Nutrient-limited & Ammonium regeneration & SNCMs & $\mathrm{S}_{\min }$ & -0.04 \\
\hline Nutrient-limited & Ammonium regeneration & PicoP & $\mathrm{K}_{\mathrm{p}}$ & 0.04 \\
\hline Nutrient-limited & Ammonium regeneration & SNCMs & $S_{\mathrm{opt}}$ & 0.04 \\
\hline Nutrient-limited & Ammonium regeneration & Micro-CMs & $\mathrm{K}_{\mathrm{p}}$ & -0.04 \\
\hline Nutrient-limited & Ammonium regeneration & PicoP & $\mathrm{NC}_{\max }$ & 0.03 \\
\hline Nutrient-limited & Trophic transfer efficiency & Nano-CMs & $\mathrm{S}_{\mathrm{opt}}$ & -0.42 \\
\hline Nutrient-limited & Trophic transfer efficiency & Nano-CMs & $\mathrm{S}_{\max }$ & -0.30 \\
\hline Nutrient-limited & Trophic transfer efficiency & MesoZ & $\mathrm{Cr}_{\mathrm{GNCMs}}$ & 0.21 \\
\hline Nutrient-limited & Trophic transfer efficiency & GNCMs & $\mathrm{S}_{\max }$ & -0.21 \\
\hline Nutrient-limited & Trophic transfer efficiency & SNCMs & $\mathrm{S}_{\max }$ & -0.16 \\
\hline Nutrient-limited & Trophic transfer efficiency & Nano-CMs & $\mathrm{S}_{\min }$ & -0.14 \\
\hline Nutrient-limited & Trophic transfer efficiency & PicoP & $\mu_{\text {phot }}$ & -0.12 \\
\hline Nutrient-limited & Trophic transfer efficiency & Bacteria & $\mathrm{NC}_{\max }$ & 0.11 \\
\hline Nutrient-limited & Trophic transfer efficiency & GNCMs & $\mathrm{PC}_{\max }$ & -0.08 \\
\hline Nutrient-limited & Trophic transfer efficiency & Nano-CMs & $\mathrm{NC}_{\min }$ & 0.07 \\
\hline Nutrient-limited & Trophic transfer efficiency & Nano-CMs & $\mathrm{PC}_{\max }$ & 0.07 \\
\hline Nutrient-limited & Trophic transfer efficiency & GNCMs & $\mathrm{NC}_{\max }$ & -0.07 \\
\hline Nutrient-limited & Trophic transfer efficiency & MesoZ & $\mathrm{Cr}_{\text {micro-CMs }}$ & 0.07 \\
\hline Nutrient-limited & Trophic transfer efficiency & GNCMs & $\mathrm{S}_{\mathrm{opt}}$ & -0.07 \\
\hline Nutrient-limited & Trophic transfer efficiency & Bacteria & $\operatorname{srs}_{B}$ & -0.06 \\
\hline Nutrient-limited & Trophic transfer efficiency & MicroZ & $\mathrm{S}_{\max }$ & -0.06 \\
\hline Nutrient-limited & Trophic transfer efficiency & MesoZ & $\mathrm{Cr}_{\text {mesoZ }}$ & -0.06 \\
\hline Nutrient-limited & Trophic transfer efficiency & PicoP & $\mathrm{PC}_{\max }$ & -0.06 \\
\hline Nutrient-limited & Trophic transfer efficiency & SNCMs & $\mathrm{NC}_{\min }$ & 0.05 \\
\hline Nutrient-limited & Trophic transfer efficiency & Nano-CMs & $\mu_{\max }$ & -0.05 \\
\hline Nutrient-limited & Trophic transfer efficiency & Micro-CMs & $\mathrm{NC}_{\max }$ & -0.05 \\
\hline
\end{tabular}




\begin{tabular}{llllr} 
Nutrient-limited & Trophic transfer efficiency & PicoP & $\mathrm{PC}_{\min }$ & 0.05 \\
Nutrient-limited & Trophic transfer efficiency & PicoP & $\mathrm{ChlC}_{\mathrm{abs}}$ & -0.04 \\
Nutrient-limited & Trophic transfer efficiency & PicoP & $\mathrm{NC}_{\max }$ & -0.04 \\
Nutrient-limited & Trophic transfer efficiency & Nano-CMs & $\mathrm{K}_{\mathrm{n}}$ & -0.04 \\
Nutrient-limited & Trophic transfer efficiency & SNCMs & $\alpha_{\text {Chl }}$ & -0.04 \\
Nutrient-limited & Trophic transfer efficiency & MesoZ & $\mathrm{Cr}_{\text {nano-CMs }}$ & 0.04 \\
Nutrient-limited & Trophic transfer efficiency & Nano-CMs & $\mathrm{NC}_{\text {max }}$ & 0.03 \\
Nutrient-limited & Trophic transfer efficiency & MesoZ & $\mathrm{Cr}_{\text {microZ }}$ & 0.03 \\
Nutrient-limited & Trophic transfer efficiency & PicoP & $\mathrm{K}_{\mathrm{a}}$ & 0.03 \\
Nutrient-limited & Trophic transfer efficiency & Micro-CMs & $\mathrm{S}_{\text {opt }}$ & -0.03 \\
Nutrient-limited & Trophic transfer efficiency & MesoZ & $\mathrm{Cr}_{\text {diatoms }}$ & -0.03 \\
\hline
\end{tabular}




\section{Appendix E}

Table E.1 L4 list of taxa assigned as diatoms in all models. Size is given in equivalent spherical diameter (ESD) and is specific for L4.

\begin{tabular}{|c|c|c|c|}
\hline Taxa & $\begin{array}{l}\text { Size } \\
(\mu \mathrm{m})\end{array}$ & Taxa & $\begin{array}{c}\text { Size } \\
(\mu \mathrm{m})\end{array}$ \\
\hline Achnanthes longipes & 22 & Chaetoceros teres & 26 \\
\hline Actinocyclus spp. & 30 & Chaetoceros tortissimus & 6 \\
\hline Actinoptychus senarius & 28 & Chaetoceros wighamii & 6 \\
\hline Asterionellopsis glacialis & 10 & Chaetoceros willei & 8 \\
\hline Attheya septentrionalis & 3 & Corethron pennatum & 33 \\
\hline Bacillaria paxillifera & 20 & Coscinodiscus asteromphalus & 58 \\
\hline Bacteriastrum furcatum & 20 & Coscinodiscus centralis & 84 \\
\hline Brockmanniella brockmannii & 15 & Coscinodiscus concinnus & 160 \\
\hline Cerataulina pelagica & 26 & Coscinodiscus granii & 84 \\
\hline Chaetoceros affinis & 8 & Coscinodiscus radiatus & 42 \\
\hline Chaetoceros anastomosans & 8 & Coscinodiscus wailesii & 260 \\
\hline Chaetoceros brevis & 8 & Cylindrotheca closterium & 6 \\
\hline Chaetoceros compressus & 6 & Dactyliosolen blavyanus & 60 \\
\hline Chaetoceros concavicornis & 18 & Dactyliosolen fragilissimus & 22 \\
\hline Chaetoceros costatus & 8 & Delphineis & 10 \\
\hline Chaetoceros curvisetus & 11 & Detonula pumila & 26 \\
\hline Chaetoceros danicus & 18 & Diploneis crabro & 16 \\
\hline Chaetoceros debilis & 12 & Ditylum brightwellii & 36 \\
\hline Chaetoceros decipiens & 15 & Ephemera planamembranacea & 38 \\
\hline Chaetoceros densus & 20 & Eucampia zodiacus & 20 \\
\hline Chaetoceros didymus & 11 & Fragilaria spp. & 4 \\
\hline Chaetoceros eibenii & 20 & Fragilariopsis spp. & 6 \\
\hline Chaetoceros externus & 8 & Grammatophora spp. & 24 \\
\hline Chaetoceros $f$. borealis & 8 & Guinardia delicatula & 22 \\
\hline Chaetoceros filiformis & 5 & Guinardia flaccida & 79 \\
\hline Chaetoceros fragilis & 5 & Guinardia striata & 15 \\
\hline Chaetoceros laciniosus & 8 & Guinardia striata (large) & 41 \\
\hline Chaetoceros lauderi & 18 & Haslea wawrikae & 9 \\
\hline Chaetoceros peruvianus & 12 & Helicotheca tamesis & 22 \\
\hline Chaetoceros protuberans & 11 & Lauderia annulata & 29 \\
\hline Chaetoceros radicans & 6 & Leptocylindrus danicus & 7 \\
\hline Chaetoceros similis & 3 & Leptocylindrus mediterraneus & 6 \\
\hline Chaetoceros simplex & 5 & Leptocylindrus minimus & 3 \\
\hline Chaetoceros socialis & 6 & Licmophora spp. & 26 \\
\hline Chaetoceros spp. & 4 & Lioloma delicatulum & 9 \\
\hline
\end{tabular}


Table E.1 continued

\begin{tabular}{|c|c|c|c|}
\hline Lithodesmium undulatum & 20 & Rhizosolenia setigera small & 16 \\
\hline Medium Pennate & 8 & Rhizosolenia setigera large & 55 \\
\hline Melosira spp. & 16 & Rhizosolenia styliformis & 71 \\
\hline Meuniera membranacea & 29 & Roperia tesselata & 28 \\
\hline Nanoneis hasleae & 2 & Skeletonema costatum & 4 \\
\hline Navicula distans & 16 & Small non-identified pennate & 5 \\
\hline Navicula spp. & 6 & Stephanopyxis palmeriana & 56 \\
\hline Neocalyptrella robusta & 156 & Thalassionema nitzschioides & 7 \\
\hline Nitzschia sigmoidea & 7 & Thalassiosira sp1 & 2 \\
\hline Odontella mobiliensis & 57 & Thalassiosira sp2 & 3 \\
\hline Odontella sinensis & 82 & Thalassiosira $\mathrm{sp} 3$ & 5 \\
\hline Paralia sulcata & 18 & Thalassiosira sp4 & 9 \\
\hline Planktoniella sol & 13 & Thalassiosira sp5 & 18 \\
\hline Pleurosigma planctonicum & 52 & Thalassiosira sp6 & 26 \\
\hline Pleurosigma spp. & 26 & Thalassiosira sp7 & 28 \\
\hline Podosira stelligera & 48 & Thalassiosira $\mathrm{sp} 8$ & 42 \\
\hline Proboscia alata large & 39 & Thalassiosira anguste-lineata & 26 \\
\hline Proboscia alata syn. f. gracillima & 14 & Thalassiosira cf. angulata & 30 \\
\hline Proboscia alata small & 22 & Thalassiosira eccentrica & 42 \\
\hline Proboscia truncata & 71 & Thalassiosira gravida small & 15 \\
\hline Psammodictyon panduriforme & 16 & Thalassiosira gravida large & 26 \\
\hline Pseudo-nitzschia delicatissima & 5 & Thalassiosira punctigera & 42 \\
\hline Pseudo-nitzschiaf. pungens & 10 & Thalassiosira rotula & 26 \\
\hline Pseudo-nitzschia f. seriata & 10 & Thalassiosira subtilis & 17 \\
\hline Rhizosolenia chunii & 24 & Thalassiothrix spp. & 14 \\
\hline Rhizosolenia hebetata f. semispina & 34 & Trigonium alternans & 21 \\
\hline Rhizosolenia imbricata small & 22 & Tropidoneis spp. & 14 \\
\hline Rhizosolenia imbricata medium & 34 & Undetermined Diatom & 4 \\
\hline Rhizosolenia imbricata large & 43 & Undetermined Pennate Diatom & 11 \\
\hline
\end{tabular}


Table E.2 L4 list of taxa assigned as microzooplankton in all models. Size is given in equivalent spherical diameter (ESD) and is specific for L4.

\begin{tabular}{|c|c|c|c|}
\hline Taxa & $\begin{array}{c}\text { Size } \\
(\mu \mathrm{m} \text { ESD })\end{array}$ & Taxa & $\begin{array}{c}\text { Size } \\
(\mu \mathrm{m} \\
\text { ESD })\end{array}$ \\
\hline Ascampbelliella spp. & 32 & Protoperidinium brevipes & 20 \\
\hline Askenasia stellaris & 53 & Protoperidinium curtipes & 52 \\
\hline Didinium spp. & 45 & Protoperidinium depressum & 96 \\
\hline Diplopsalis & 37 & Protoperidinium divergens & 38 \\
\hline Epiplocylis undella & 23 & Protoperidinium obtusum & 91 \\
\hline Eutintinnus spp. & 26 & Protoperidinium oceanicum & 96 \\
\hline Favella helgolandica & 26 & Protoperidinium ovatum & 91 \\
\hline Gyrodinium spirale & 63 & Protoperidinium pyriforme & 33 \\
\hline Gyrodinium spp. (large) & 32 & Protoperidinium spp. & 42 \\
\hline Helicostomella spp. & 26 & Protoperidinium steinii & 33 \\
\hline Kofoidinium lebourae & 66 & Pyrophacus horologicum & 37 \\
\hline Leegaardiella spp. & 26 & Salpingella spp. & 19 \\
\hline Lohmanniella spp. & 28 & Strobilidium spp. & 24 \\
\hline Parafavella spp. & 26 & Strombidinopsis spp. & 33 \\
\hline Phalacroma rotundatum & 35 & Tiarina spp. & 31 \\
\hline Preperidinium spp. & 68 & Tintinnopsis spp. & 18 \\
\hline Pronoctiluca pelagica & 20 & Torodinium teredo & 32 \\
\hline Proplectella spp. & 35 & Unidentified Peridiniales (large) & 18 \\
\hline Protoperidinium bipes & 26 & Unidentified tintinnid/ciliate & 41 \\
\hline
\end{tabular}


Table E.3 L4 list of taxa assigned as mesozooplankton in all models. Size is equivalent to the maximum longest axis of the taxa and was obtained through direct measurements at L4 or consulting Conway (2012).

\begin{tabular}{lclc}
\hline Taxa & $\begin{array}{c}\text { Size } \\
(\mathbf{m m})\end{array}$ & Taxa & Size \\
(mm)
\end{tabular}


Table E.4 Misfits given as the correlation coefficient (r), the root mean squared error (RMSE), and the average error (AE) for the mixotrophic, non-mixotrophic, and ERSEM models; na - not applicable.

\begin{tabular}{|c|c|c|c|c|}
\hline Statistic Metric & Variable & Mixotrophic & Non-mixotrophic & ERSEM \\
\hline \multirow[t]{14}{*}{$\mathrm{r}$} & Nitrate & 0.95 & 0.96 & 0.94 \\
\hline & Phosphate & 0.95 & 0.97 & 0.95 \\
\hline & Silicate & 0.78 & 0.76 & 0.83 \\
\hline & Ammonium & -0.12 & -0.22 & 0.11 \\
\hline & Total Chlorophyll & 0.78 & 0.71 & 0.69 \\
\hline & Picophytoplankton & 0.22 & 0.36 & 0.20 \\
\hline & Nano-CMs/Nano-A & 0.90 & 0.59 & 0.84 \\
\hline & Diatoms & 0.62 & 0.56 & 0.51 \\
\hline & Micro-CMs/Micro-A & -0.14 & -0.21 & 0.80 \\
\hline & $\mathrm{NCMs}$ & 0.75 & na & na \\
\hline & Microzooplankton & 0.78 & 0.77 & 0.84 \\
\hline & Bacteria & 0.47 & 0.54 & 0.31 \\
\hline & Nanozooplankton & -0.71 & 0.79 & 0.85 \\
\hline & Mesozooplankton & 0.69 & 0.63 & 0.67 \\
\hline \multirow[t]{14}{*}{ RMSE } & Nitrate & 1.00 & 1.15 & 1.62 \\
\hline & Phosphate & 0.07 & 0.06 & 0.17 \\
\hline & Silicate & 1.31 & 1.37 & 1.55 \\
\hline & Ammonium & 0.26 & 0.28 & 0.13 \\
\hline & Total Chlorophyll & 0.75 & 0.82 & 0.97 \\
\hline & Picophytoplankton & 12.32 & 73.99 & 20.29 \\
\hline & Nano-CMs/Nano-A & 12.05 & 31.10 & 14.75 \\
\hline & Diatoms & 58.43 & 70.77 & 37.09 \\
\hline & Micro-CMs/Micro-A & 18.67 & 12.14 & 6.03 \\
\hline & $\mathrm{NCMs}$ & 4.98 & na & na \\
\hline & Microzooplankton & 15.86 & 15.10 & 8.01 \\
\hline & Bacteria & 6.41 & 6.99 & 7.44 \\
\hline & Nanozooplankton & 23.99 & 13.98 & 14.00 \\
\hline & Mesozooplankton & 6.41 & 8.50 & 7.55 \\
\hline \multirow[t]{14}{*}{$\mathrm{AE}$} & Nitrate & -0.05 & -0.59 & 1.18 \\
\hline & Phosphate & 0.01 & -0.04 & 0.14 \\
\hline & Silicate & 0.37 & 0.39 & 0.90 \\
\hline & Ammonium & -0.20 & -0.21 & -0.03 \\
\hline & Total Chlorophyll & -0.30 & -0.24 & -0.09 \\
\hline & Picophytoplankton & -11.21 & 52.07 & 2.06 \\
\hline & Nano-CMs/Nano-A & -9.42 & -27.16 & -11.71 \\
\hline & Diatoms & 31.75 & 34.84 & 13.95 \\
\hline & Micro-CMs/Micro-A & 0.24 & -7.00 & 0.63 \\
\hline & NCMs & 2.27 & na & na \\
\hline & Microzooplankton & 10.02 & 8.85 & -5.36 \\
\hline & Bacteria & 0.11 & 3.13 & 2.88 \\
\hline & Nanozooplankton & -22.41 & -11.85 & -13.08 \\
\hline & Mesozooplankton & -1.61 & -6.90 & -4.59 \\
\hline
\end{tabular}



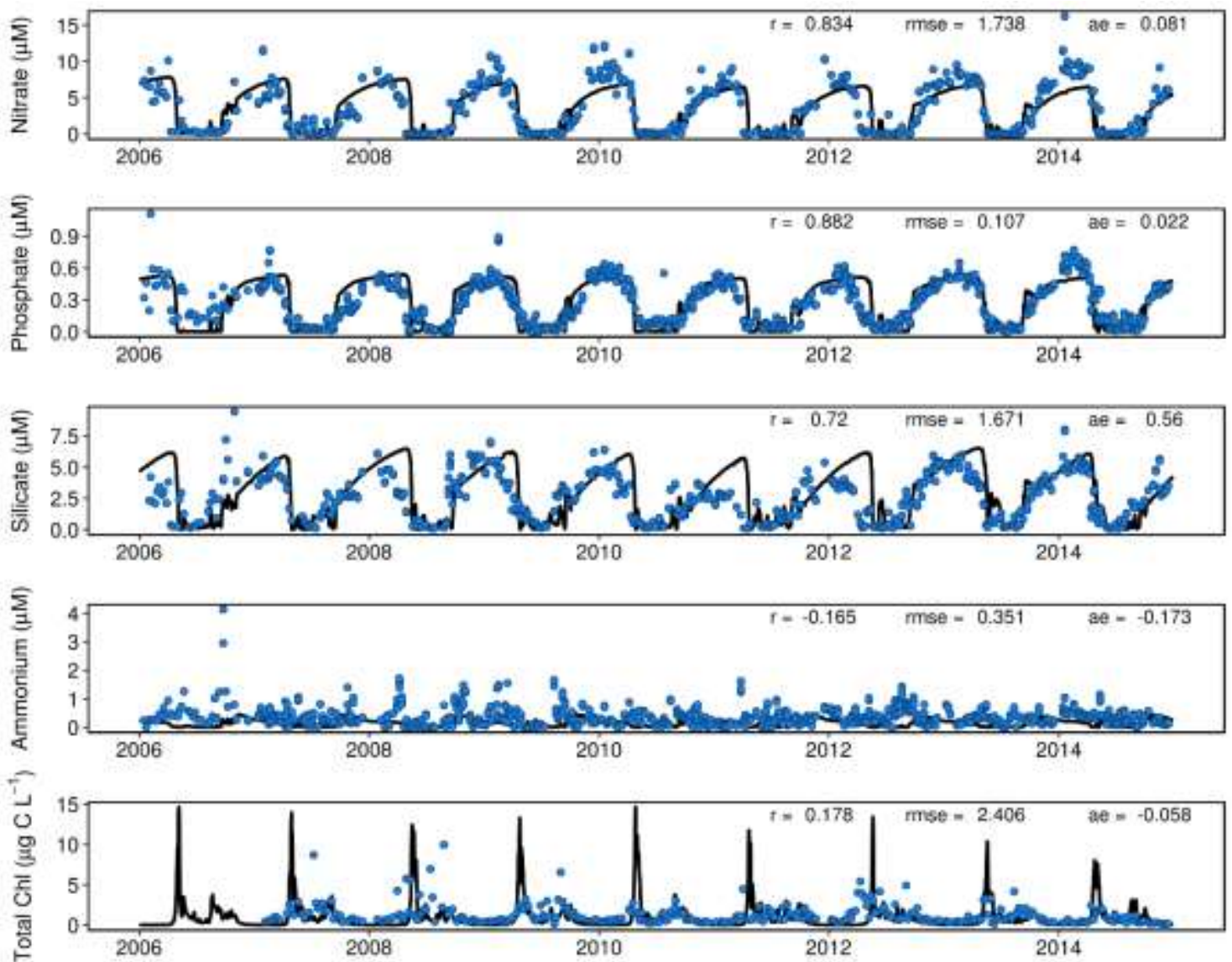

Figure E.1 Comparisons of nutrients and chlorophyll concentrations between model simulations (lines) and observations (dots) from 2006 to 2014 at L4 station (at $10 \mathrm{~m}$ depth). Model misfits are given for each variable in the upper right corner ( $\mathrm{r}=$ correlation coefficient; rmse = root mean squared error; ae = average error). Note that y-axes scales differ among panels. 

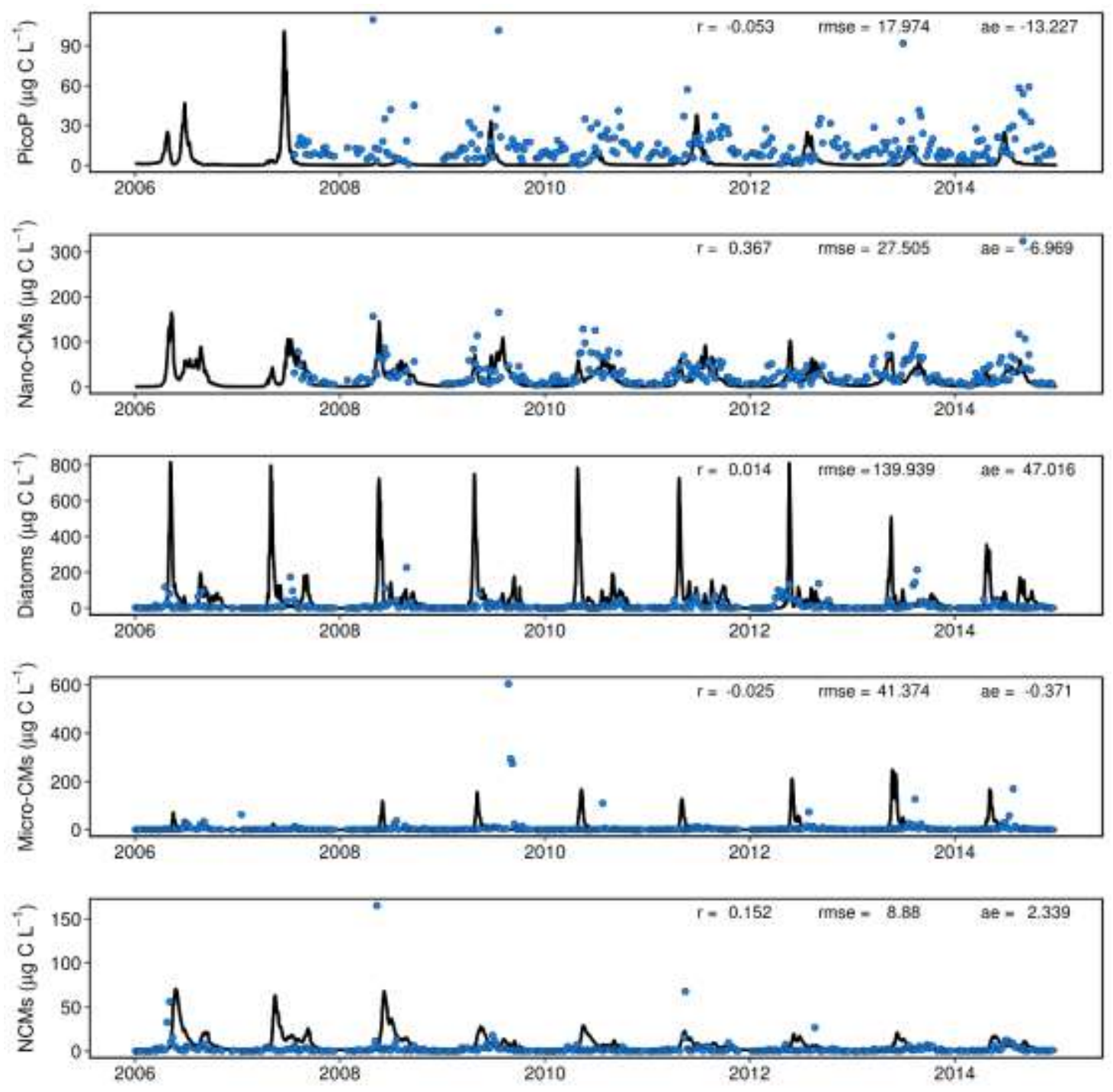

Figure E.2 Comparisons of phototrophic plankton biomasses from 2006 to 2014 between model simulations (lines) and observations (dots) from 2006 to 2014 at L4 station (at 10 $\mathrm{m}$ depth). Model misfits are given for each variable in the upper right corner $(\mathrm{r}=$ correlation coefficient; rmse = root mean squared error; ae = average error). Note that $y$ axes scales differ among panels; picophytoplankton (PicoP) and Nano-CMs data were available only from 2007 onwards. Please refer to the Abbreviations section. 

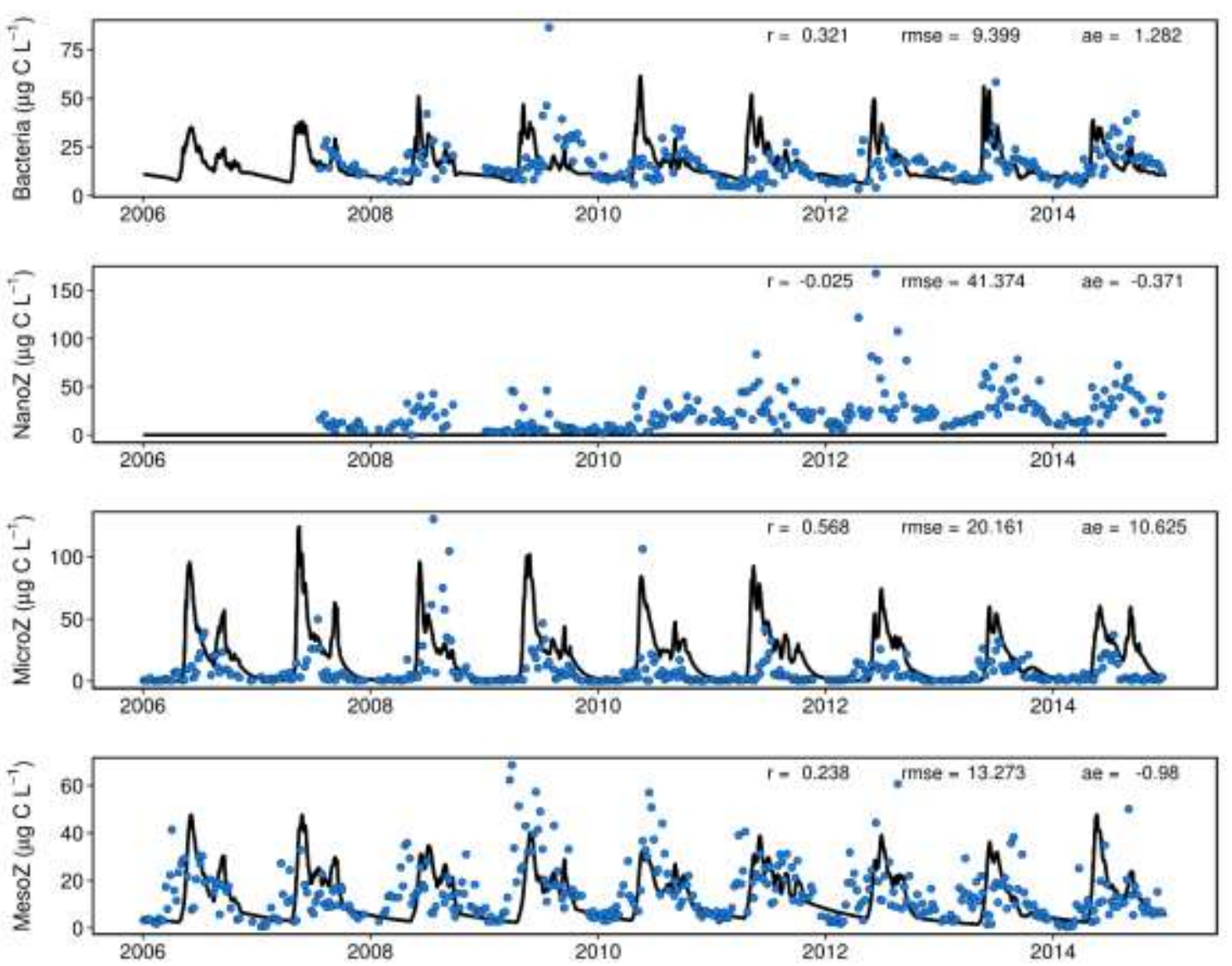

Figure E.3 Comparisons of decomposer and strict heterotrophic plankton biomasses from 2006 to 2014 between model simulations (lines) and observations (dots) from 2006 to 2014 at L4 station (at $10 \mathrm{~m}$ depth). Model misfits are given for each variable in the upper right corner $(\mathrm{r}=$ correlation coefficient; $\mathrm{rmse}=$ root mean squared error; ae = average error). Note that $\mathrm{y}$-axes scales differ among panels; bacteria and NanoZ data were available only from 2007 onwards. Please refer to the Abbreviations section. 

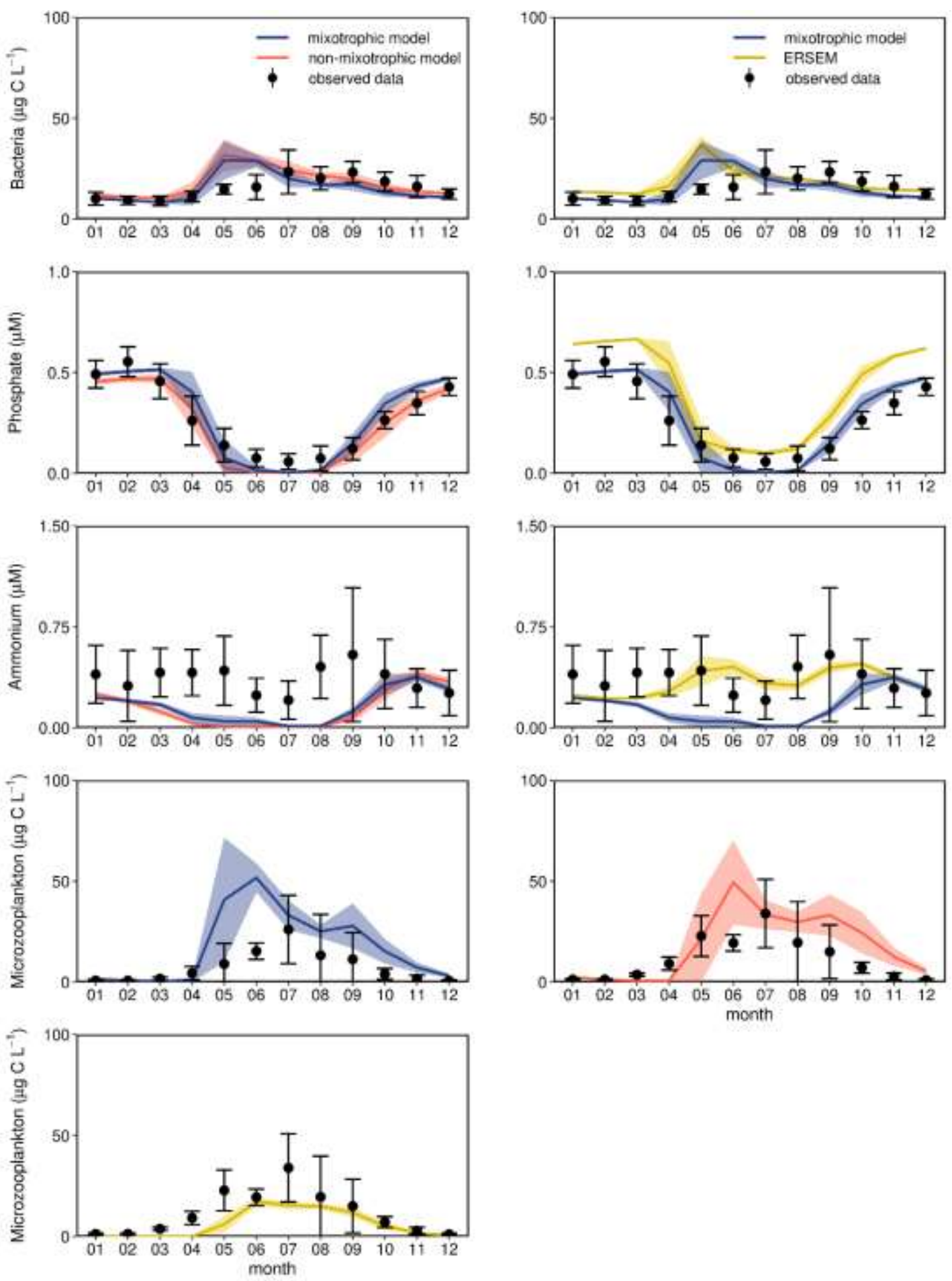

Figure E.4 Comparisons of bacteria, phosphate, ammonium, and microzooplankton values over the climatological seasonal cycle between model simulations (lines) and observations (dots) at L4 station (at $10 \mathrm{~m}$ depth). Simulations are given for the mixotrophic (blue), non-mixotrophic (red), and ERSEM (yellow) models. Note that yaxes scales and units differ among panels. Mean $( \pm \mathrm{SD})$ values correspond to the period $2006-2014$. 

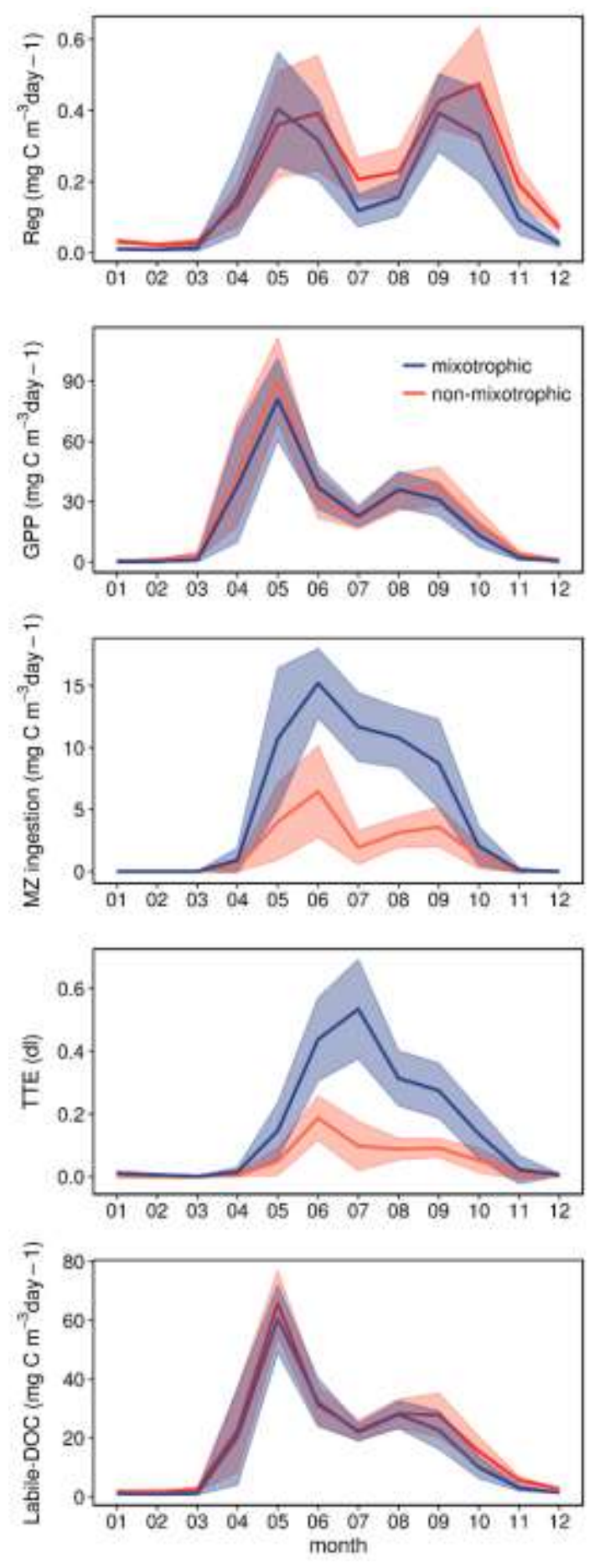

Figure E.5 Comparisons of targeted ecosystem properties over the climatological seasonal cycle between the mixotrophic (blue) and the non-mixotrophic (red) models. Monthly means and standard deviations correspond to the period 2006-2014. Results are given for the total ammonium regeneration (Reg), gross primary production (GPP), total amount of food ingested by mesozooplankton (MZ ingestion), trophic transfer efficiency (TTE), and total production of labile DOC (Labile-DOC). Trophic transfer efficiency was estimated by the ratio between the total amount of food ingested by mesozooplankton and the total gross primary productivity. 


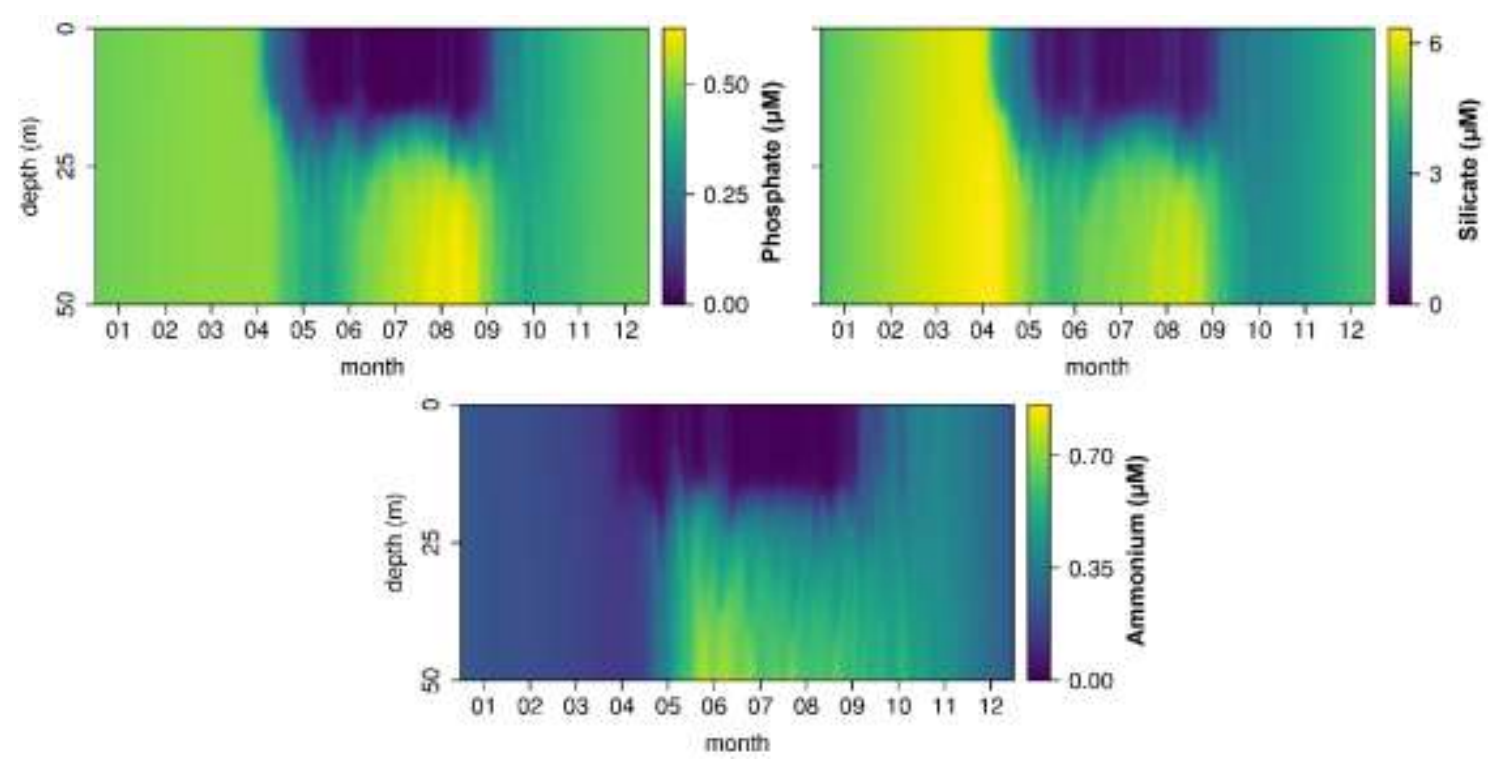

Figure E.6 Depth profiles of nutrient concentrations (phosphate, silicate, and ammonium) over the climatological seasonal cycle. Mean values correspond to the period 2006-2014.

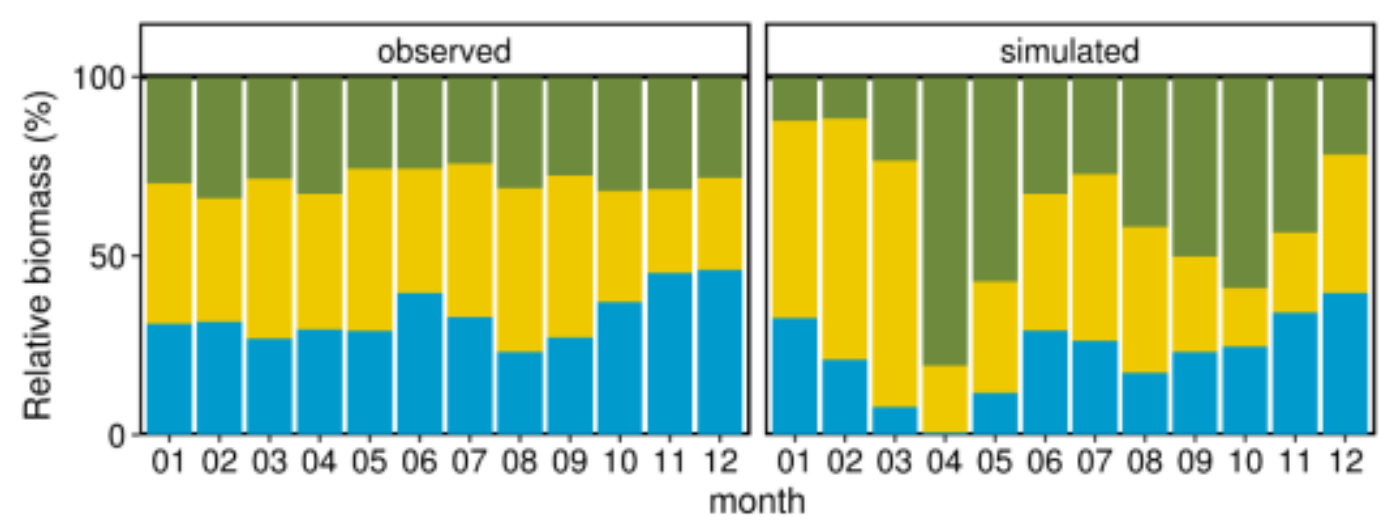

Figure E.7 Observed and simulated seasonal succession of protist trophic strategies at L4 (10 $\mathrm{m}$ depth) given by the relative biomass of autotrophs (green), mixotrophs (yellow), and heterotrophs (blue). Mean values correspond to the period 2006-2014. 


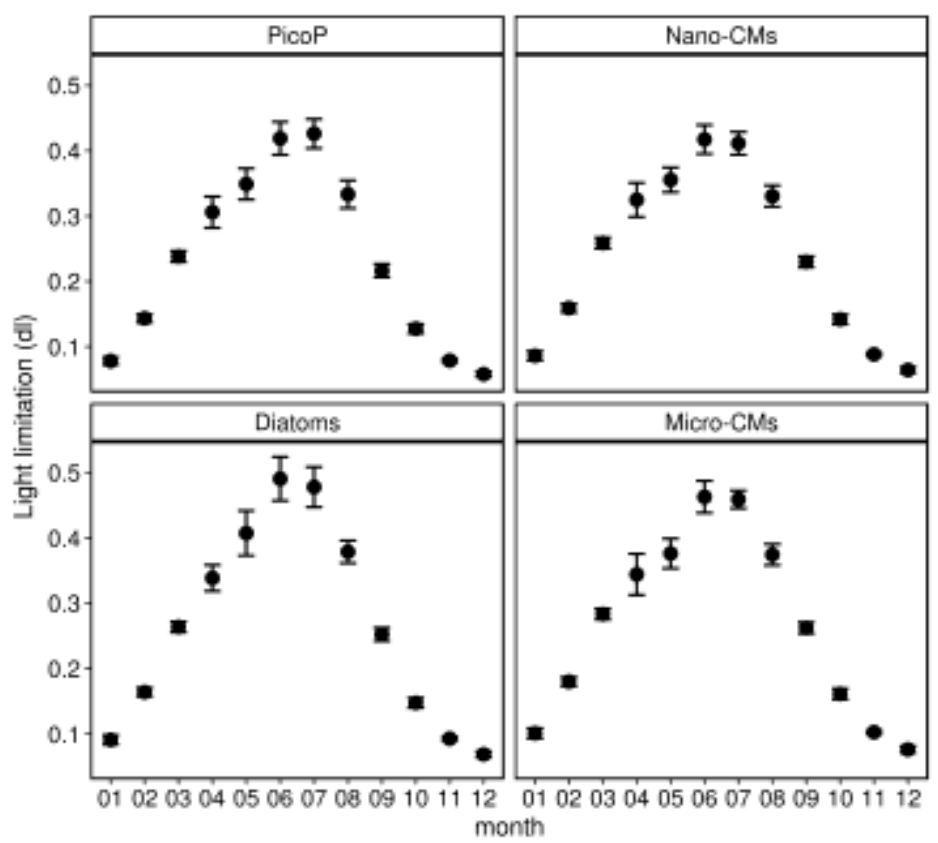

Figure E.8 Simulated seasonal development of light limitation among picophytoplankton (PicoP), nano-CMs, diatoms, and micro-CMs given by the ratio between the actual photosynthesis rate and the maximum photosynthesis rate; values closer to 0.5 indicate no light limitation. Mean $( \pm \mathrm{SD})$ values correspond to the period $2006-2014$.

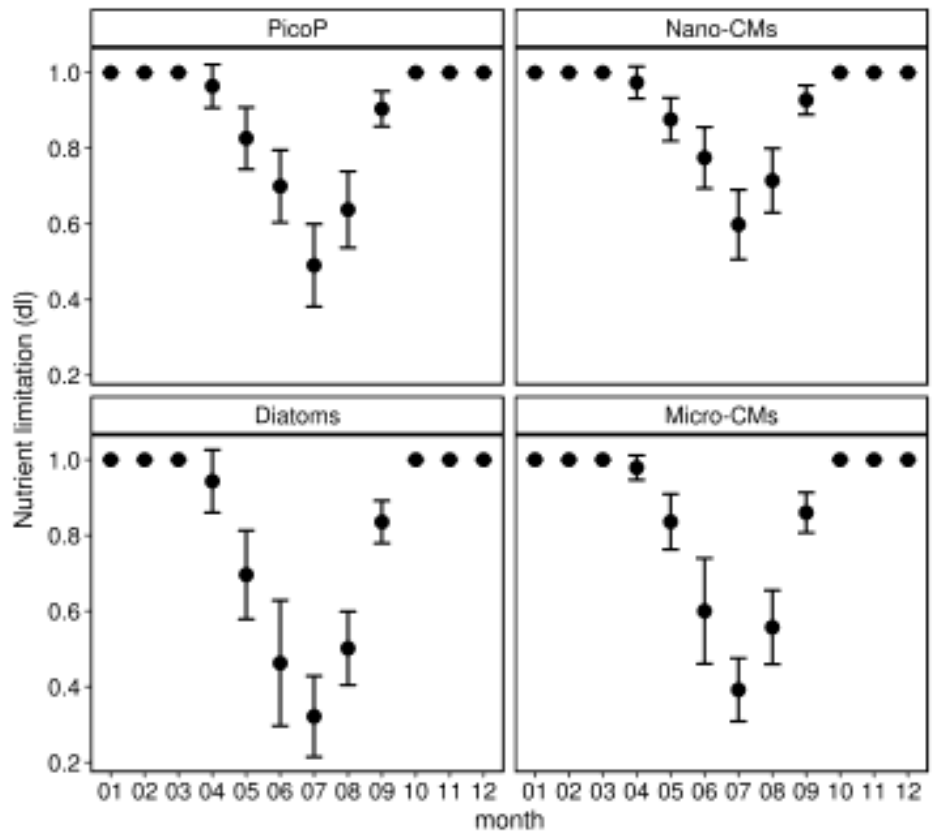

Figure E.9 Simulated seasonal development of nutrient limitation among picophytoplankton (PicoP), nano-CMs, diatoms, and micro-CMs given by the normalised internal nutrient status; values closer to 1 indicate no nutrient limitation. Mean $( \pm \mathrm{SD})$ values correspond to the period $2006-2014$. 

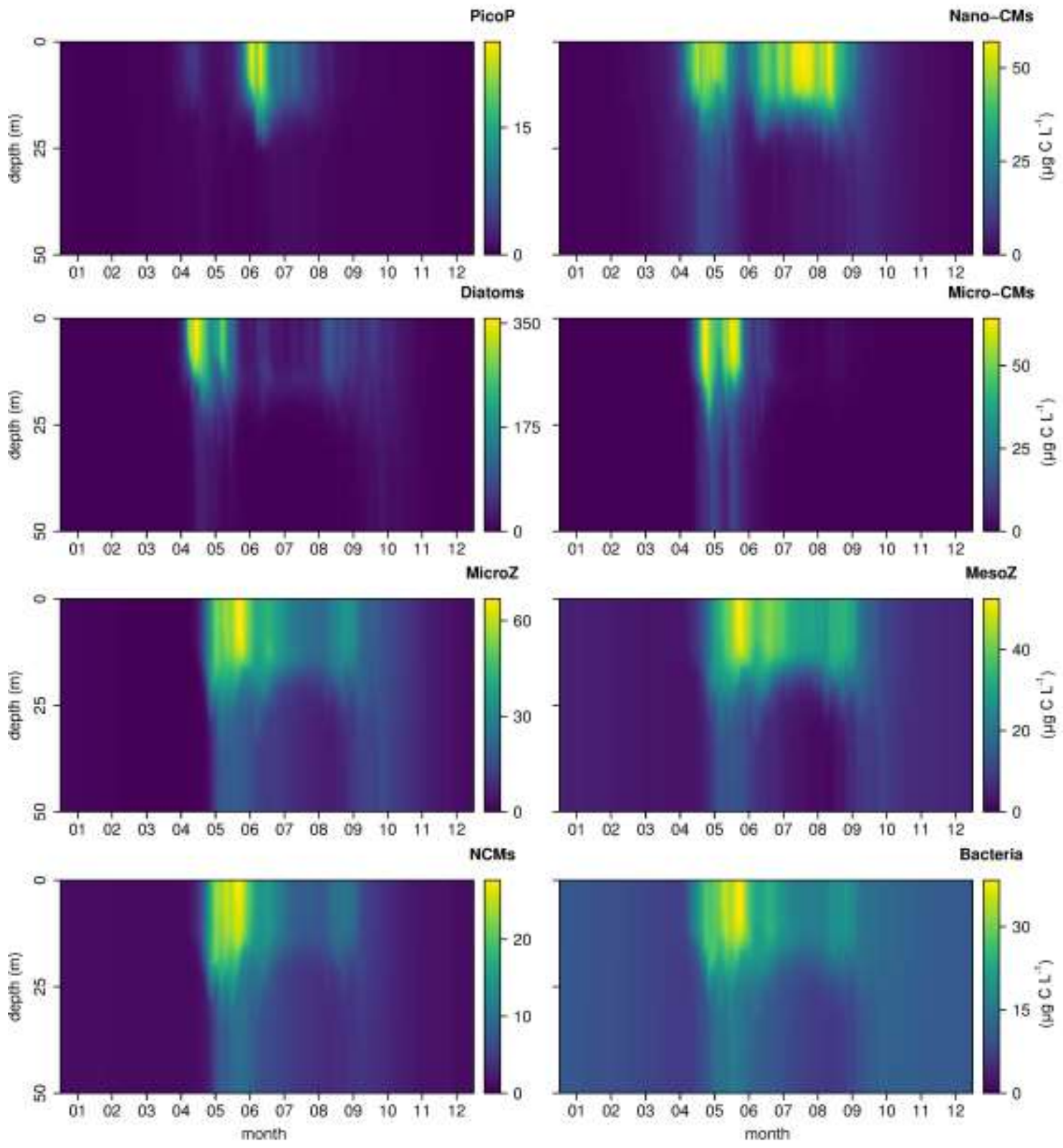

Figure E.10 Depth profiles of plankton biomasses $\left(\mu \mathrm{g} \mathrm{C} \mathrm{L}^{-1}\right)$ over the climatological seasonal cycle. Mean values correspond to the period 2006-2014. Please refer to the Abbreviations section for shortenings. 


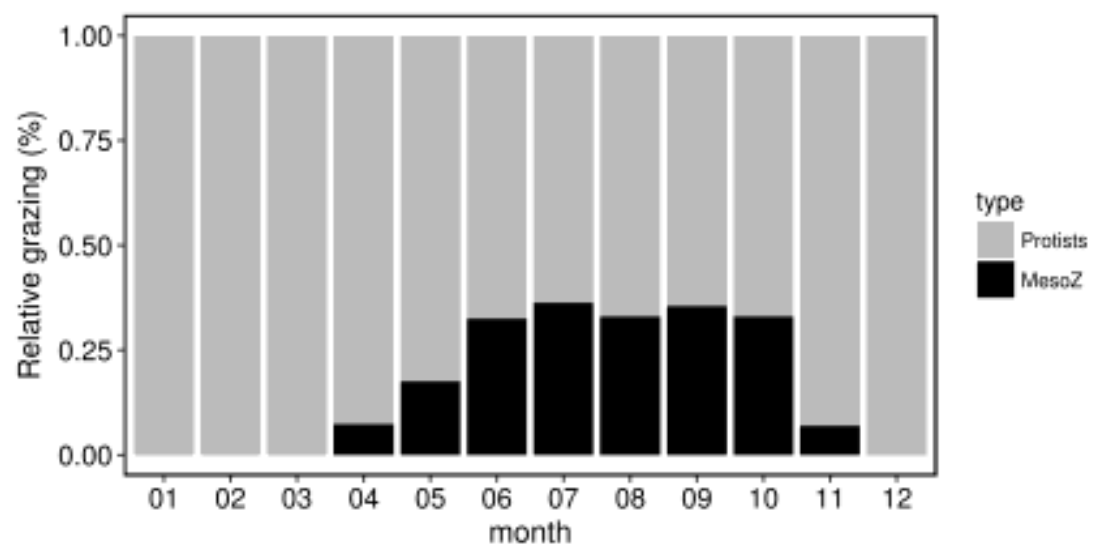

Figure E.11 Relative contribution of phagotrophic protists (grey) and mesozooplankton (black; MesoZ) to the total grazing of phototrophic prey in the model. Mean values correspond to the period 2006-2014.

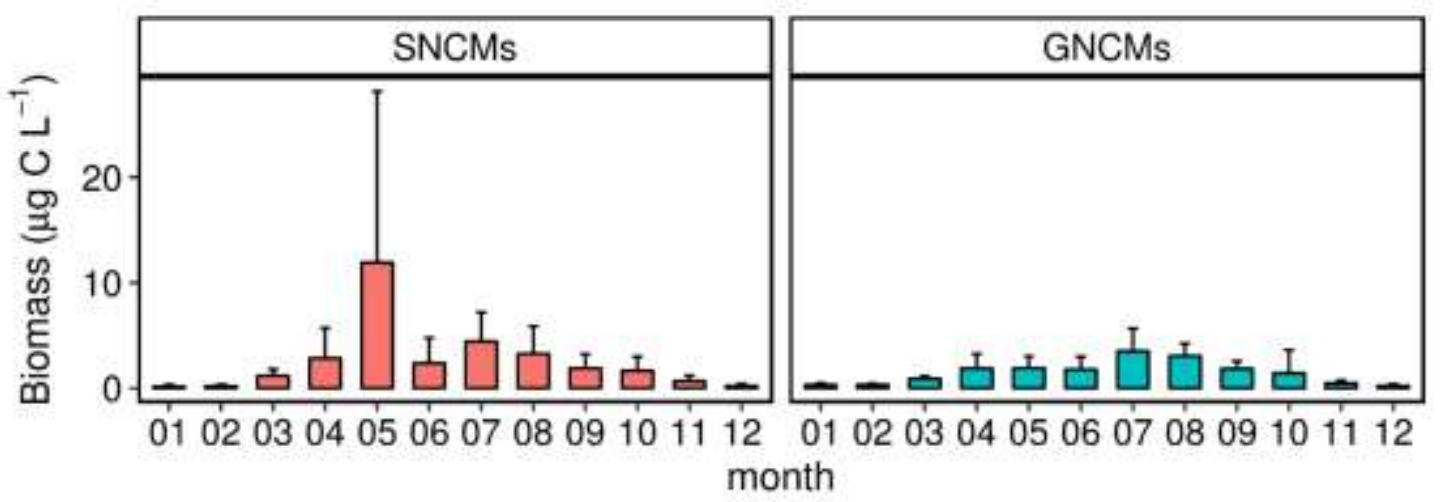

Figure E.12 Observed seasonal succession of SNCMs and GNCMs at L4 station. Mean ( \pm SD) values correspond to the period 2006-2014. 


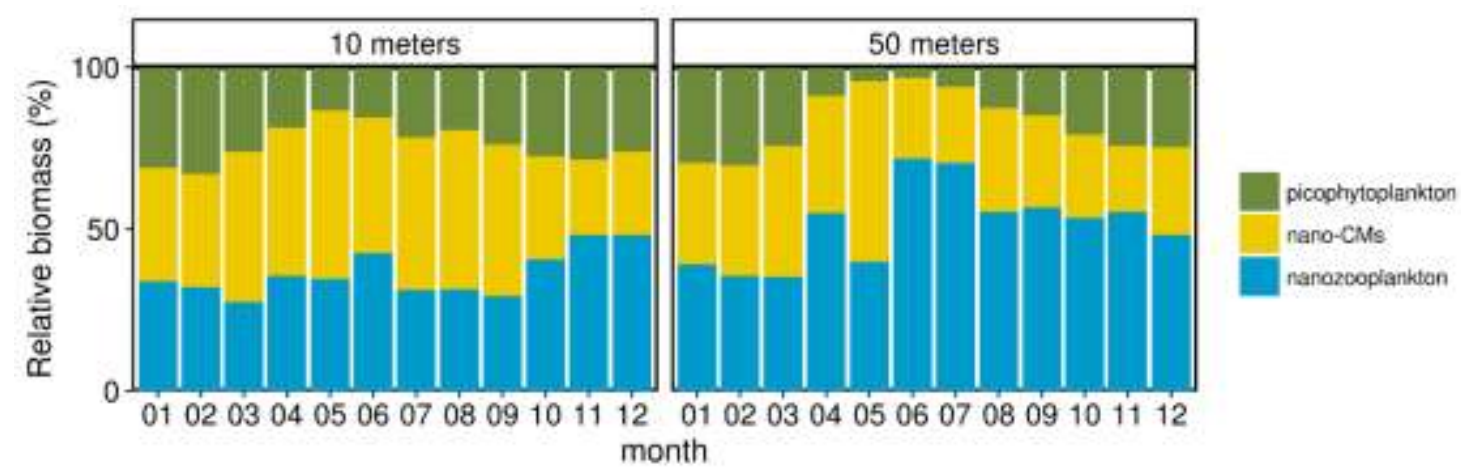

Figure E.13 Observed seasonal succession of picophytoplankton (green), nano-CMs (yellow), and nanozooplankton (blue) at L4 station given by their relative biomass. Mean values correspond to the period 2006-2014.

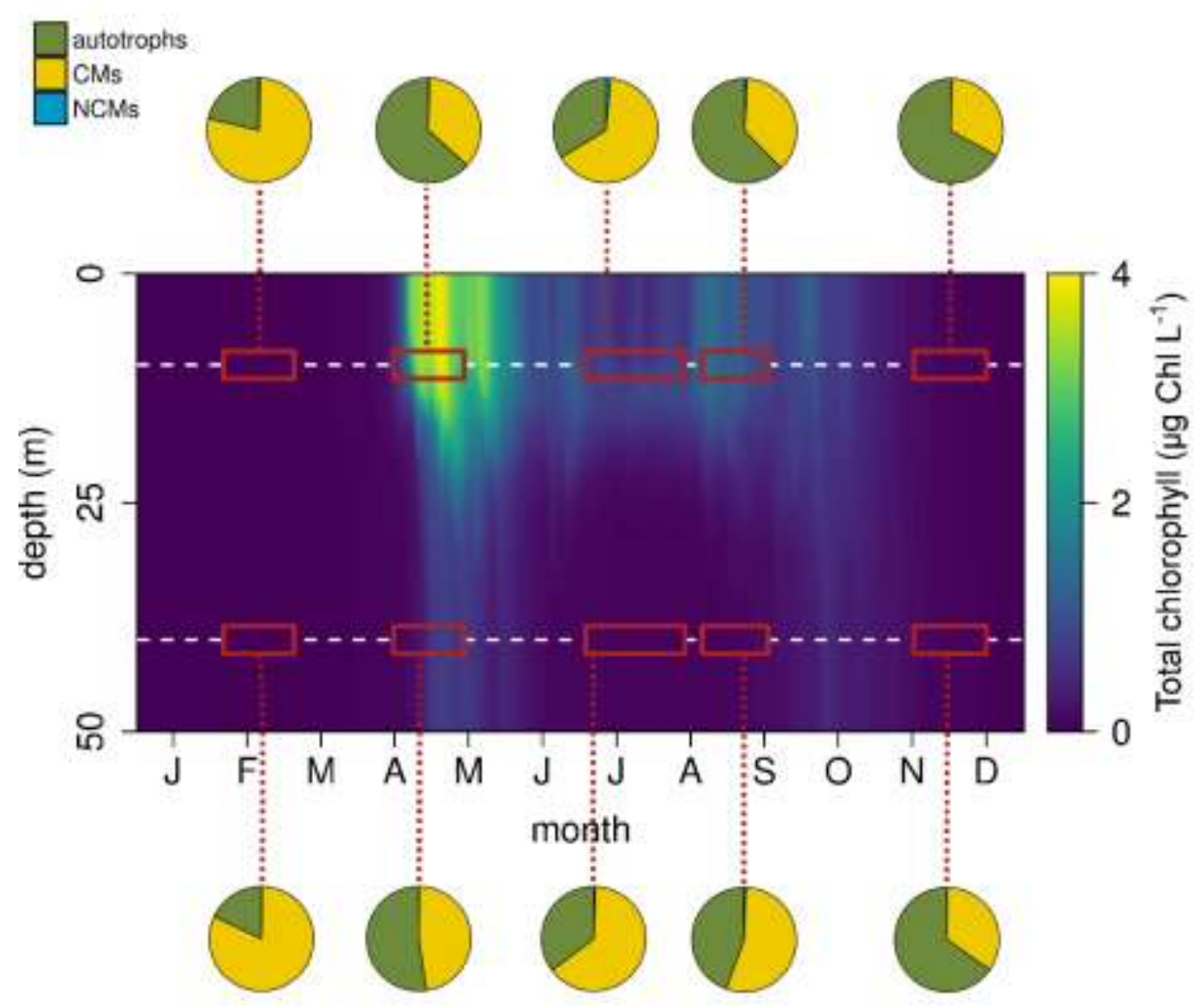

Figure E.14 Simulated seasonal succession of total chlorophyll at L4 station. Depth profile shows the total chlorophyll. The relative contribution of autotrophs (green), constitutive mixotrophs (CMs; yellow), and non-constitutive mixotrophs (NCMs; blue) is given by the pie charts for selected time periods and depth. Mean $( \pm$ SD) values correspond to the period 20062014. 


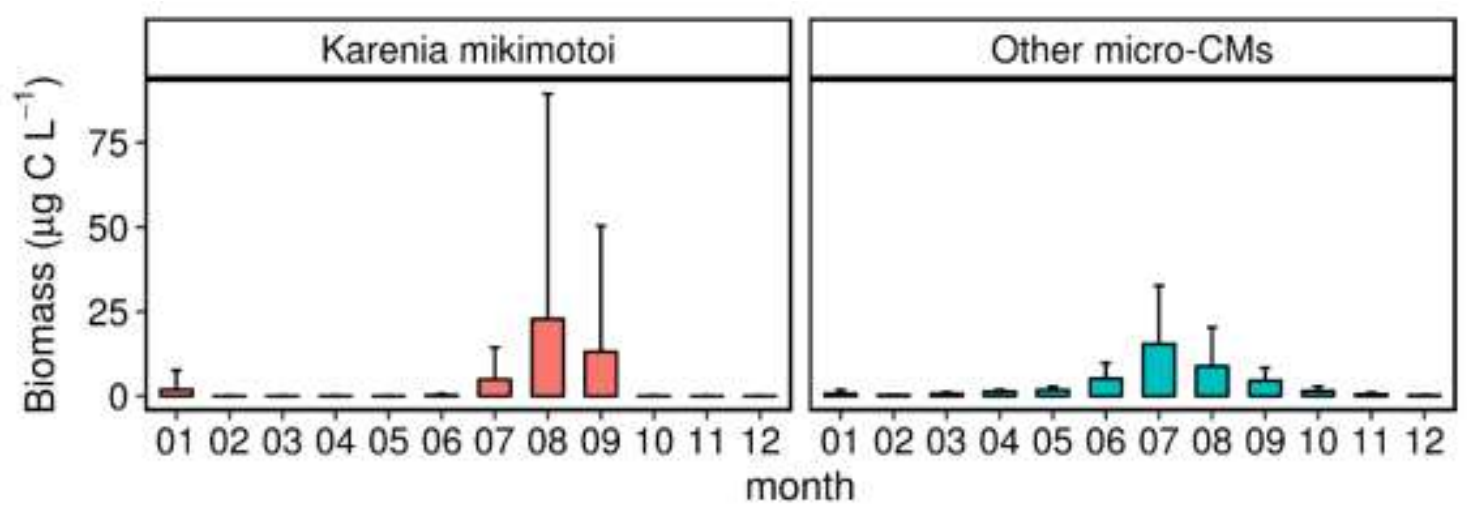

Figure E.15 Observed seasonal succession of Karenia mikimotoi and other micro-CMs at L4 station. Mean ( \pm SD) values correspond to the period 2006-2014.

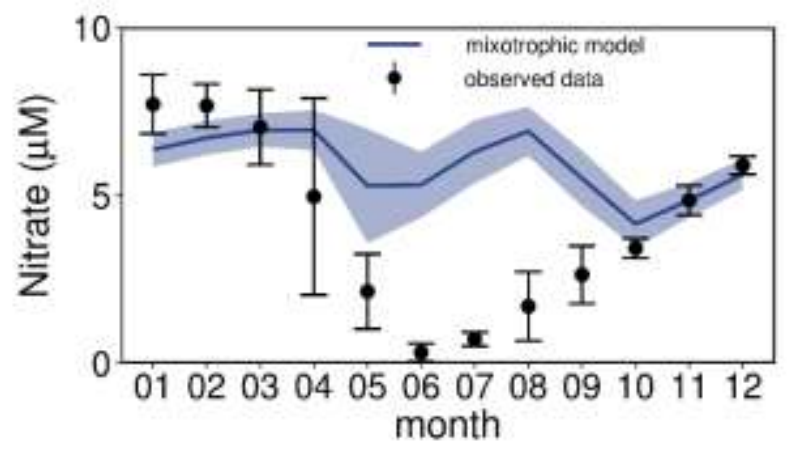

Figure E.16 Comparison of simulated nitrate (line) with observations (dots) at L4 station over the climatological seasonal cycle (at $50 \mathrm{~m}$ depth). Means $( \pm \mathrm{SD})$ values correspond to the period 2006-2014. 


\section{Glossary}

Conceptual model: akin to flow diagrams or food web schematics; help the formulation of ideas and the identification of strengths and weakness in knowledge (Flynn and McGillicuddy, 2018).

Constitutive mixotrophs: phagotrophic protists with an inherent capacity for phototrophy (Mitra et al., 2016).

Correlative models: used to describe nature and find functional relationships among observed phenomena, but no causal story is built into the model (Coelho et al., 2018).

Dispersal: the movement and successful establishment of genotypes and taxa at a new location (Hanson et al., 2012).

Drift: changes in the frequencies of genotypes and species owing to chance demographic fluctuations (Hanson et al., 2012).

Fitness: ability to survive and reproduce; it depends on the traits of the organism and the ecosystem (Flynn et al. 2015).

Functional type: group of species that show similar responses to the environment and effects on ecosystem functioning, sharing functional traits, features, or properties (Flynn et al., 2015).

Mechanistic model: used to explain nature and propose causal explanations of a biological phenomenon; contrary to correlative models, parameters within mechanistic models are designed with biological meaning (Coelho et al., 2018).

Mixotrophy: the combination of photoautotrophic and phagotrophic modes of nutrition (Mitra et al., 2016).

Model skill assessment: the use of quantitative metrics and graphical approaches to assess how well the model fit the observations (Stow et al., 2009).

Mutation: non-directed change in nucleotide sequences responsible for diversification among taxa, including speciation, together with selection, drift, and dispersal (Hanson et al., 2012).

Non-constitutive mixotrophs: phagotrophic protists which acquire their phototrophic capacity from their prey (Mitra et al., 2016).

Response curves: mathematical formulations of relationships between driver and consequence used to convert a conceptual model into a numerical model (Flynn and McGillicuddy, 2018).

Selection: alters the relative abundance of genes, genotypes, cells, and species on the basis of their ability to survive and reproduce (Hanson et al., 2012). 
State variable: property of a numerical model used to describe quantities such as nutrients and biomass (Flynn, 2018).

Trait: defining characteristic that influences organismal performance (Flynn et al., 2015). 


\section{References}

ABELMANN, A. \& NimMERGUT, A. (2005) Radiolarians in the Sea of Okhotsk and their ecological implication for paleoenvironmental reconstructions. Deep-Sea Research Part II: Topical Studies in Oceanography 52, 2302-2331.

Acha, E.M., Mianzan, H.W., Guerrero, R.A., FAVEro, M. \& BAVA, J. (2004) Marine fronts at the continental shelves of austral South America: Physical and ecological processes. Journal of Marine Systems 44, 83-105.

Adolf, J.E., BachVaroff, T. \& Place, A.R. (2008) Can cryptophyte abundance trigger toxic Karlodinium veneficum blooms in eutrophic estuaries? Harmful Algae 8, 119-128.

Adolf, J.E., Stoecker, D.K. \& Harding, L.W. (2006) The balance of autotrophy and heterotrophy during mixotrophic growth of Karlodinium micrum (Dinophyceae). Journal of Plankton Research 28, 737-751.

Ajani, P., Ingleton, T., Pritchard, T. \& Armand, L. (2011) Microalgal blooms in the coastal waters of New South Wales, Australia. Proceedings of the Linnean Society of New South Wales 133, 15-32.

Aldridge, D., Purdie, D.A. \& ZubKov, M. V. (2014) Growth and survival of Neoceratium hexacanthum and Neoceratium candelabrum under simulated nutrient-depleted conditions. Journal of Plankton Research 36, 439-449.

Allen, J.I., Holt, J.T., BlACKFORD, J. \& PROCTOR, R. (2007) Error quantification of a highresolution coupled hydrodynamic-ecosystem coastal-ocean model: Part 2. Chlorophyll-a, nutrients and SPM. Journal of Marine Systems 68, 381-404.

ALlEn, J.I. \& POLIMENE, L. (2011) Linking physiology to ecology: Towards a new generation of plankton models. Journal of Plankton Research 33, 989-997.

ALLEN, J.I., SidDORN, J.R., BLACKFORD, J.C. \& GILBERT, F.J. (2004) Turbulence as a control on the microbial loop in a temperate seasonally stratified marine systems model. Journal of Sea Research 52, 1-20.

Andersen, K.H., AKSNES, D.L., Berge, T., FIKSEN, Ø. \& VisSER, A. (2015) Modelling emergent trophic strategies in plankton. Journal of Plankton Research 37, 862-868.

Andersen, K.H., Berge, T., Gonçalves, R.J., Hartvig, M., Heuschele, J., Hylander, S., Jacobsen, N.S., Lindemann, C., Martens, E.A., Neuheimer, A.B., Olsson, K., PAlacz, A., Prowe, F., Sainmont, J., Traving, S.J., ET AL. (2016) Characteristic Sizes of Life in the Oceans, from Bacteria to Whales. Annual Review of Marine Science 8, 1-25.

Andersen, P. \& SøRENSEN, H.M. (1986) Population dynamics and trophic coupling in pelagic microorganisms in eutrophic coastal waters. Mar. Ecol. Prog. Ser 33, 99-109.

ANDERSON, O.R. \& MATUSUOKA, A. (1992) Endocytoplasmic microalgae and bacteroids within the central capsule of the radiolarian Dictyocoryne truncatum. Symbiosis 12, 237-247.

Anderson, R., Charvet, S. \& Hansen, P.J. (2018) Mixotrophy in Chlorophytes and Haptophytes - Effect of Irradiance, Macronutrient, Micronutrient and Vitamin Limitation. Frontiers in Microbiology 9, 1-13. 
ANDERSON, R., JÜRGENS, K. \& HANSEN, P.J. (2017) Mixotrophic phytoflagellate bacterivory field measurements strongly biased by standard approaches: a case study. Frontiers in Microbiology 8, 1-12.

ANDERSON, T.R. (2005) Plankton functional type modelling: Running before we can walk? Journal of Plankton Research 27, 1073-1081.

Andersson, A., FAlK, S., SAMUElsson, G. \& HAGSTRÖM, Å. (1989) Nutritional characteristics of a mixotrophic nanoflagellate, Ochromonas sp. Microbial Ecology 17, 251-262.

ARAÚJo, M.S., BolniCK, D.I. \& LAYMAN, C.A. (2011) The ecological causes of individual specialisation. Ecology Letters 14, 948-958.

ARENOVSKI, A.L., LIM, E.L. \& CARON, D.A. (1995) Mixotrophic nanoplankton in oligotrophic surface waters of the Sargasso Sea may employ phagotrophy to obtain major nutrients. Journal of Plankton Research 17, 801-820.

ARIAS, A., SAIZ, E. \& CALBET, A. (2017) Diel feeding rhythms in marine microzooplankton: effects of prey concentration, prey condition, and grazer nutritional history. Marine Biology 164:205.

Atkinson, A., Harmer, R.A., Widdicombe, C.E., McEvoy, A.J., SMYTh, T.J., Cummings, D.G., SOMERfIEld, P.J., MAUd, J.L. \& MCCONVILlE, K. (2015) Questioning the role of phenology shifts and trophic mismatching in a planktonic food web. Progress in Oceanography 137, 498-512.

Atkinson, A., Polimene, L., Fileman, E.S., Widdicombe, C.E., McEvoy, A.J., SMYTh, T.J., DJEGHRI, N., SAILlEY, S.F. \& CORNWEll, L.E. (2018) Comment. What drives plankton seasonality in a stratifying shelf sea? Some competing and complementary theories. Limnology and Oceanography 63, 2877-2884.

AZAM, F., Fenchel, T., Field, J.G., GRAy, J.S., Meyer-ReIl, L.A. \& Thingstad, F. (1983) The ecological role of water-column microbes in the sea *. Marine Ecology Progress Series 10, 257-263.

BAIRD, M.E. \& SUTHERS, I.M. (2007) A size-resolved pelagic ecosystem model. Ecological Modelling 203, 185-203.

BANAS, N.S. (2011) Adding complex trophic interactions to a size-spectral plankton model: Emergent diversity patterns and limits on predictability. Ecological Modelling 222, 26632675.

BarettA-BeKKer, J.G., Baretta, J.W., HANSEN, A.S. \& RiemanN, B. (1998) An improved model of carbon and nutrient dynamics in the microbial food web in marine enclosures. Aquatic Microbial Ecology 14, 91-108.

BARETTA-BEKKER, J.G., B ARETTA, J.W. \& Koch RASMUSSEn, E. (1995) The microbial food web in the European Regional Seas Ecosystem Model. Netherlands Journal of Sea Research 33, 363-379.

BARETTA, J.W., EBEnHÖH, W. \& RUARDIJ, P. (1995) The European regional seas ecosystem model, a complex marine ecosystem model. Netherlands Journal of Sea Research 33, 233246.

Barnes, M.K., Tilstone, G.H., Smyth, T.J., Widdicombe, C.E., GloËL, J., Robinson, C., KAISER, J. \& SUGGETT, D.J. (2015) Drivers and effects of Karenia mikimotoi blooms in the 
western English Channel. Progress in Oceanography 137, 456-469.

BARnetT, A.J., Finlay, K. \& BeISNER, B.E. (2007) Functional diversity of crustacean zooplankton communities: Towards a trait-based classification. Freshwater Biology 52, 796-813.

BARTON, A.D., Finkel, Z. V, WARD, B.A., Johns, D.G. \& Follows, M.J. (2013a) On the roles of cell size and trophic strategy in North Atlantic diatom and dinoflagellate communities. Limnology and Oceanography 58, 254-266.

Barton, A.D., Pershing, A.J., Litchman, E., Record, N.R., EdWARds, K.F., FinKel, Z. V., KIØRBOE, T. \& WARD, B.A. (2013b) The biogeography of marine plankton traits. Ecology Letters 16, 522-534.

BATES, D., MÄCHLER, M., BOLKER, B. \& WALKER, S. (2015) Fitting linear mixed-effects models using lme4. Journal of Statistical Software 67, 1-48.

BÉ, A. \& TOLDERLUnD, D. (1971) The micropalaeontology of oceans. In Micropalaeontology of marine bottom sediments (eds B. FUNNELL \& W. RIEDEL), pp. 105-149. Cambridge University Press.

Behrenfeld, M.J., O’Malley, R.T., Boss, E.S., Westberry, T.K., GrafF, J.R., Halsey, K.H., Milligan, A.J., SiEgEl, D.A. \& BRown, M.B. (2016) Revaluating ocean warming impacts on global phytoplankton. Nature Climate Change 6, 323-330.

BEIJERINCK, M.W. (1913) De infusies en de ontdekking der backteriën. Jaarboek van de Koninklijke Akademie voor Wetenschappen, Amsterdam, the Netherlands: Müller.

BeISNER, B.E., GROSSART, H.-P. \& GASOL, J.M. (2019) A guide to methods for estimating phagomixotrophy in nanophytoplankton. Journal of Plankton Research 41, 77-89.

Bell, E.M. \& LAYBOURN-PARRY, J. (2003) Mixotrophy in the antarctic phytoflagellate, Pyramimonas gelidicola (Chlorophyta: Prasinophyceae). Journal of Phycology 39, 644-649.

Berge, T., Chakraborty, S., Hansen, P.J. \& Andersen, K.H. (2017) Modeling succession of key resource-harvesting traits of mixotrophic plankton. ISME Journal 11, 212-223.

Berge, T., Hansen, P.J. \& Moestrup, Ø. (2008a) Prey size spectrum and bioenergetics of the mixotrophic dinoflagellate Karlodinium armiger. Aquatic Microbial Ecology 50, 289-299.

Berge, T., Hansen, P.J. \& Moestrup, Ø. (2008b) Feeding mechanism, prey specificity and growth in light and dark of the plastidic dinoflagellate Karlodinium armiger. Aquatic Microbial Ecology 50, 279-288.

BERnARD, C. \& RASSOUlzadegan, F. (1994) Seasonal variations of mixotrophic ciliates in the northwest Mediterranean Sea. Marine Ecology Progress Series 108, 295-302.

Biard, T., Stemmann, L., Picheral, M., Mayot, N., Vandromme, P., Hauss, H., Gorsky, G., GUIDI, L., KIKO, R. \& NOT, F. (2016) In situ imaging reveals the biomass of giant protists in the global ocean. Nature 532, 504-507.

BIRD, D.F. \& KALFF, J. (1986) Bacterial Grazing by Planktonic Algae. Science 231, 493-495.

BJORNSEN, P.K. \& NIELSEN, T.G. (1991) Decimeter scale heterogeneity in the plankton during a pycnocline bloom of Gyrodinium aureolum. Marine Ecology Progress Series 73, 263-267.

Blackford, J., Allen, J.I., Anderson, T.R. \& Rose, K.A. (2010) Challenges for a new 
generation of marine ecosystem models: Overview of the Advances in Marine Ecosystem Modelling Research (AMEMR) Symposium, 23-26 June 2008, Plymouth UK. Journal of Marine Systems 81, 1-3.

BlackFord, J.C., Allen, J.I. \& Gilbert, F.J. (2004) Ecosystem dynamics at six contrasting sites: a generic modelling study. Journal of Marine Systems 52, 191-215.

Blight, S.P., Bentley, T.L., LefeVre, D., Robinson, C., Rodrigues, R., Rowlands, J., Le, P.J. \& WiLliams, P.J. LEB. (1995) Phasing of autotrophic and heterotrophic plankton metabolism in a temperate coastal ecosystem. Marine Ecology Progress Series 128, 61-75.

Blossom, H.E., BæDKel, T.D., Tillmann, U. \& Hansen, P.J. (2017) A search for mixotrophy and mucus trap production in Alexandrium spp. and the dynamics of mucus trap formation in Alexandrium pseudogonyaulax. Harmful Algae 64, 51-62.

BockstAhleR, K.R. \& COATS, D.W. (1993) Grazing of the mixotrophic dinoflagellate Gymnodinium sanguineum on ciliate populations of Chesapeake Bay. Marine Biology 116, 477-487.

BODE, A. \& FERNÁNDEZ, E. (1992) Influence of water-column stability on phytoplankton size and biomass succession patterns in the central Cantabrian Sea (Bay of Biscay). Journal of Plankton Research 14, 885-902.

BolKer, B.M. (2007) Ecological Models and Data in R. Princeton University Press.

Bolker, B.M., Brooks, M.E., Clark, C.J., Geange, S.W., Poulsen, J.R., Stevens, M.H.H. \& WHITE, J.S.S. (2009) Generalized linear mixed models: a practical guide for ecology and evolution. Trends in Ecology and Evolution 24, 127-135.

Boltovskoy, D. \& CorReA, N. (2017) Planktonic equatorial diversity troughs: Fact or artifact? Latitudinal diversity gradients in Radiolaria. Ecology 98, 112-124.

Boltovskoy, D. \& RIEDEL, W. (1977) Polycystine radiolaria of the California Current region: seasonal and geographic patterns. In Marine Micropaleontology p. 1977.

BONACHELA, J.A., RAGHIB, M. \& LEVIN, S.A. (2011) Dynamic model of flexible phytoplankton nutrient uptake. Proceedings of the National Academy of Sciences 108, 20633-20638.

Bosch, S., Tyberghein, L., Deneudt, K., Hernandez, F. \& De Clerck, O. (2017) In search of relevant predictors for marine species distribution modelling using the MarineSPEED benchmark dataset. Diversity and Distributions, 1-14.

Bouman, H.A., Platt, T., Doblin, M., Figueiras, F.G., Gudmundsson, K., GudFinnsson, H.G., Huang, B., Hickman, A., Hiscock, M., Jackson, T., Lutz, V.A., MÉlin, F., Rey, F., PePin, P., SEgurA, V., ET AL. (2018) Photosynthesis-irradiance parameters of marine phytoplankton: synthesis of a global data set. Earth System Science Data 10, 251-266.

Bracher, A., Bouman, H.A., Brewin, R.J.W., Bricaud, A., Brotas, V., Ciotti, A.M., Clementson, L., Devred, E., Di CicCo, A., DutKiewicz, S., Hardman-MounTFord, N.J., HickMAN, A.E., HiERONYMI, M., HiRATA, T., LOSA, S.N., ET AL. (2017) Obtaining Phytoplankton Diversity from Ocean Color: A Scientific Roadmap for Future Development. Frontiers in Marine Science 4, 1-15.

Brito, A.C., Moita, T., Gameiro, C., Silva, T., Anselmo, T. \& Brotas, V. (2015) Changes in the Phytoplankton Composition in a Temperate Estuarine System (1960 to 2010). Estuaries and Coasts 38, 1678-1691. 
Broglio, E., Saiz, E., Calbet, A., Trepat, I. \& Alcaraz, M. (2004) Trophic impact and prey selection by crustacean zooplankton on the microbial communities of an oligotrophic coastal area (NW Mediterranean Sea). Aquatic Microbial Ecology 35, 65-78.

BRUGGEMAN, J. (2009) Succession in plankton communities: a trait-based perspective. Gildeprint Drukkerijen, Amsterdam.

BRUGGEMAN, J. \& BolDiNG, K. (2014) A general framework for aquatic biogeochemical models. Environmental Modelling and Software 61, 249-265.

Bruggeman, J. \& KoolJman, S.A.L.M. (2007) A biodiversity-inspired approach to aquatic ecosystem modeling. Limnology and Oceanography 52, 1533-1544.

Bucklin, A., Lindeque, P.K., Rodriguez-EzPeleta, N., Albaina, A. \& Lehtiniemi, M. (2016) Metabarcoding of marine zooplankton: prospects, progress and pitfalls. Journal of Plankton Research 38, 393-400.

Buitenhuis, E.T., LI, W.K.W., VAulot, D., LOMAS, M.W., LANDRY, M.R., PARTENSKY, F., Karl, D.M., Ulloa, O., CAMPBell, L., JaCQuet, S., LANTOINe, F., Chavez, F., MaCias, D., Gosselin, M. \& MCMANUS, G.B. (2012) Picophytoplankton biomass distribution in the global ocean. Earth System Science Data, Copernicus Publications 4, 201-242.

Bunse, C. \& PINHASSI, J. (2017) Marine Bacterioplankton Seasonal Succession Dynamics. Trends in Microbiology 25, 494-505. Elsevier Ltd.

Burchard, H., BOLDing, K. \& VillarReAl, M. (1999) GOTM, a general ocean turbulence model. Theory, implementation and test cases. Technical Report EUR 18745 EN, European Comission.

Burkholder, J.M., Glibert, P.M. \& Skelton, H.M. (2008) Mixotrophy , a major mode of nutrition for harmful algal species in eutrophic waters. Harmful Algae 8, 77-93.

Burridge, A.K., Goetze, E., Wall-Palmer, D., Le Double, S.L., Huisman, J. \& PEIJNENBURG, K.T.C.A. (2017) Diversity and abundance of pteropods and heteropods along a latitudinal gradient across the Atlantic Ocean. Progress in Oceanography 158, 213-223.

ButenschÖN, M., Clark, J., Aldridge, J.N., ICARUS Allen, J., ARTiOl, Y., BlaCKFord, J., Bruggeman, J., Cazenave, P., Ciavatta, S., Kay, S., Lessin, G., Van Leeuwen, S., VAn Der Molen, J., De Mora, L., POlimene, L., ET AL. (2016) ERSEM 15.06: A generic model for marine biogeochemistry and the ecosystem dynamics of the lower trophic levels. Geoscientific Model Development 9, 1293-1339.

CABrerizo, M.J., GonzÁlez-Olalla, J.M., Hinojosa-LóPez, V.J., Peralta-Cornejo, F.J. \& CARRILLO, P. (2018) A shifting balance: responses of mixotrophic marine algae to cooling and warming under UVR. New Phytologist 221, 1317-1327.

Cáceres, C., Rivera, A., González, S. \& Anadón, R. (2017) Phytoplankton community structure and dynamics in the North Atlantic subtropical gyre. Progress in Oceanography 151, 177-188.

CALBET, A. (2001) Mesozooplankton grazing effect on primary production: A global comparative analysis in marine ecosystems. Limnology and Oceanography 46, 1824-1830.

Calbet, A., Bertos, M., Fuentes-Grünewald, C., Alacid, E., Figueroa, R., Renom, B. \& GARCÉS, E. (2011) Intraspecific variability in Karlodinium veneficum: Growth rates, mixotrophy, and lipid composition. Harmful Algae 10, 654-667. 
CALBET, A. \& LANDRY, M.R. (2004) Phytoplankton growth, microzooplankton grazing, and carbon cycling in marine systems. Limnology and Oceanography 49, 51-57.

CAlBet, A. \& SAIZ, E. (2005) The ciliate-copepod link in marine ecosystems. Aquatic Microbial Ecology 38, 157-167.

Calbet, A., Trepat, I., Almeda, R., Saló, V., Saiz, E., Movilla, J.I., Alcaraz, M., Yebra, L. \& SIMÓ, R. (2008) Impact of micro- And nanograzers on phytoplankton assessed by standard and size-fractionated dilution grazing experiments. Aquatic Microbial Ecology 50, $145-156$.

Calderon-Saenz, E. \& Schnetter, R. (1989) Morphology, biology, and systematics of Cryptochlora perforans (Chlorarachniophyta), a phagotrophic marine alga. Plant Systematics and Evolution 163, 165-176.

CAllahan, B.J., McMurdie, P.J. \& HolmeS, S.P. (2017) Exact sequence variants should replace operational taxonomic units in marker-gene data analysis. ISME Journal 11, 2639-2643.

Campbell, L., Olson, R.J., Sosik, H.M., Abraham, A., Henrichs, D.W., Hyatt, C.J. \& BusKeY, E.J. (2010) First harmful Dinophysis (Dinophyceae, Dinophysiales) bloom in the U.S. is revealed by automated imaging flow cytometry. Journal of Phycology 46, 66-75.

CARON, D.A. (2009) Past presidents address: Protistan biogeography: Why all the fuss? Journal of Eukaryotic Microbiology 56, 105-112.

CARON, D.A. \& Hu, S.K. (2019) Are We Overestimating Protistan Diversity in Nature? Trends in Microbiology 27, 197-205.

CAron, D.A., Michaels, A.F., Swanberg, N.R. \& Howse, F.A. (1995) Primary productivity by symbiont-bearing planktonic sarcodines (Acantharia, Radiolaria, Foraminifera) in surface waters near Bermuda. Journal of Plankton Research 17, 103-129.

Caron, D.A., Sanders, R.W., Lim, E.L., Marrasé, C., Amaral, L.A., Whitney, S., Aoki, R.B. \& PORTERS, K.G. (1993) Light-dependent phagotrophy in the freshwater mixotrophic chrysophyte Dinobryon cylindricum. Microbial Ecology 25, 93-111.

CARVAlHo, W.F. \& GRANÉLI, E. (2010) Contribution of phagotrophy versus autotrophy to Prymnesium parvum growth under nitrogen and phosphorus sufficiency and deficiency. Harmful Algae 9, 105-115.

CASEY, R. (1971) Radiolarians as indicators of past and present water-masses. In The Micropaleontology of Oceans (ed W. FUNNEL, BM; RIEDEL), pp. 331-341. Cambridge at the University Press.

CERMEÑo, P. \& FALKOWSKI, P. (2009) Controls on Diatom Biogeography in the Ocean. Science 325, 1539-1542.

Chakraborty, S., NiElsen, L.T. \& Andersen, K.H. (2017) Trophic Strategies of Unicellular Plankton. The American Naturalist 189, E77-E90.

CHANG, F.H. (1990) Quantitative distribution of microzooplankton off Westland, New Zealand. New Zealand Journal of Marine and Freshwater Research 24, 187-195.

Chen, B., Liu, H., LAndry, M., Chen, M., Sun, J., SheK, L., Chen, X. \& Harrison, P. (2009) Estuarine nutrient loading affects phytoplankton growth and microzooplankton grazing at two contrasting sites in Hong Kong coastal waters. Marine Ecology Progress Series 379, 
$77-90$.

Chiang, K.P., Lin, C.Y., LeE, C.H., ShiAH, F.K. \& Chang, J. (2003) The coupling of oligotrich ciliate populations and hydrography in the East China Sea: Spatial and temporal variations. Deep-Sea Research Part II: Topical Studies in Oceanography 50, 1279-1293.

Christaki, U., Obernosterer, I., VAn Wambeke, F., Veldhuis, M., Garcia, N. \& Catala, P. (2008) Microbial food web structure in a naturally iron-fertilized area in the Southern Ocean (Kerguelen Plateau). Deep-Sea Research Part II: Topical Studies in Oceanography 55, 706-719.

Christaki, U., VAn WambeKe, F. \& Dolan, J.R. (1999) Nanoflagellates (mixotrophs, heterotrophs and autotrophs) in the oligotrophic eastern Mediterranean: Standing stocks, bacterivory and relationships with bacterial production. Marine Ecology Progress Series 181, 297-307.

Christaki, U., Van Wambeke, F., Lefevre, D., Lagaria, A., Prieur, L., Pujo-Pay, M., Grattepanche, J.-D., Colombet, J., Psarra, S., Dolan, J.R., Sime-Ngando, T., Conan, P., Weinbauer, M.G. \& Moutin, T. (2011) Microbial food webs and metabolic state across oligotrophic waters of the Mediterranean Sea during summer. Biogeosciences $\mathbf{8}$, $1839-1852$.

Cleveland, W.S. (1979) Robust Locally Weighted Regression and Smoothing Scatterplots. Journal of the American Statistical Association 74, 829-836.

Cloern, J. \& Dufford, R. (2005) Phytoplankton community ecology: Principles applied in San Francisco Bay. Marine Ecology Progress Series 285, 11-28.

Coelho, M.T.P., Diniz-Filho, J.A. \& RANGel, T.F. (2018) A parsimonious view of the parsimony principle in ecology and evolution. Ecography, ecog.04228.

Conway, D. (2012) Marine zooplankton of southern Britain. Part 2: Arachnida, Pycnogonida, Cladocera, Facetotecta, Cirripedia and Copepoda. Occasional Publications, Marine Biological Association, Plymouth, UK.

Corbineau, A., Rouyer, T., Cazelles, B., Fromentin, J.-M., Fonteneau, A. \& Ménard, F. (2008) Time series analysis of tuna and swordfish catches and climate variability in the Indian Ocean (1968-2003). Aquatic Living Resources 21, 277-285.

Costello, M.J., Tsai, P., Wong, P.S., Cheung, A.K.L., Basher, Z. \& Chaudhary, C. (2017) Marine biogeographic realms and species endemicity. Nature Communications 8, 1-9.

CRAWFORD, D. (1989) Mesodinium rubrum: the phytoplankter that wasn't . Marine Ecology Progress Series 58, 161-174.

Crawford, D.W., Purdie, D. A, Lockwood, A P.M. \& Weissman, P. (1997) Recurrent Redtides in the Southampton Water Estuary Caused by the Phototrophic Ciliate Mesodinium rubrum. Estuarine, Coastal and Shelf Science 45, 799-812.

Croft, M.T., WArren, M.J. \& SMith, A.G. (2006) Algae Need Their Vitamins. Eukaryotic Cell 5, 1175-1183.

CROPP, R. \& NORBURY, J. (2013) Modelling plankton ecosystems and the Library of Lotka. Journal of Marine Systems 125, 3-13. Elsevier B.V.

CropP, R. \& NORBURY, J. (2015) Mixotrophy: the missing link in consumer-resource-based 
ecologies. Theoretical Ecology 8, 245-260.

Czypionka, T., VArgas, C.A., Silva, N., DANERI, G., GonZÁlez, H.E. \& IRIARTE, J.L. (2011) Importance of mixotrophic nanoplankton in Aysén Fjord (Southern Chile) during austral winter. Continental Shelf Research 31, 216-224.

Dakos, V., Benincà, E., van Nes, E.H., Philippart, C.J.M., SchefFer, M. \& Huisman, J. (2009) Interannual variability in species composition explained as seasonally entrained chaos. Proceedings of the Royal Society B: Biological Sciences 276, 2871-2880.

DALE, T. (1987) Diel vertical distribution of planktonic ciliates in Lindåspollene, Western Norway. Marine Microbial Food Webs 2, 15-28.

DALE, T. \& DAHL, E. (1987) Mass occurrence of planktonic oligotrichous ciliates in a bay in southern Norway. Journal of Plankton Research 9, 871-879.

DAVIDSON, A.T., SCOTT, F.J., NAsh, G. V., Wright, S.W. \& RAYMOND, B. (2010) Physical and biological control of protistan community composition, distribution and abundance in the seasonal ice zone of the Southern Ocean between 30 and $80^{\circ}$ E. Deep Sea Research Part II: Topical Studies in Oceanography 57, 828-848. Elsevier.

Decelle, J., Probert, I., Bittner, L., Desdevises, Y., Colin, S., Vargas, C. De, Galí, M., SiMÓ, R. \& FABRICE, N. (2012) An original mode of symbiosis in open ocean plankton Johan. Proceedings of the National Academy of Sciences 109, 18000-18005.

DemarcQ, H., Reygondeau, G., Alvain, S. \& Vantrepotte, V. (2012) Monitoring marine phytoplankton seasonality from space. Remote Sensing of Environment 117, 211-222.

Dennett, M.R., Caron, D.A., Michaels, A.F., Gallager, S.M. \& DaVis, C.S. (2002) Video plankton recorder reveals high abundances of colonial Radiolaria in surface waters of the central North Pacific. Journal of Plankton Research 24, 797.

Dierssen, H., McManus, G.B., Chlus, A., QIU, D., GaO, B.-C. \& Lin, S. (2015) Space station image captures a red tide ciliate bloom at high spectral and spatial resolution. Proceedings of the National Academy of Sciences 112, 14783-14787.

Dittami, S., Arboleda, E., Auguet, J.-C., Bigalke, A., Briand, E., Cardini, U., Decelle, J., Engelen, A., EVEILlard, DAmien, Griffiths, S., HARder, T., KAYAl, E., KAZAMiA, E., MarZinelli, E., Morganti, T., ET AL. (2019) A community perspective on the concept of marine holobionts: state-of-the-art, challenges, and future directions. PeerJ Preprints.

DJEGHRI, N., AtKinson, A., Fileman, E.S., HARMER, R.A., WidDicombe, C.E., McEvoy, A.J., CORNWELL, L. \& MAYOR, D.J. (2018) High prey-predator size ratios and unselective feeding in copepods: A seasonal comparison of five species with contrasting feeding modes. Progress in Oceanography 165, 63-74.

DODDEMA, H. \& VAN DER VEeR, J. (1983) Ochromonas monicis sp. nov., a particle feeder with bacterial symbionts. Cryptogamie: Algologie 4 (1/2), 89-97, III pl.

DolAn, J.R. (2005) An introduction to the biogeography of aquatic microbes. Aquatic Microbial Ecology 41, 39-48.

DOLAN, J.R. (2006) Microbial biogeography? Journal of Biogeography 33, 199-200.

Dolan, J.R. \& GALlEGOS, C.C. (1992) Trophic role of planktonic rotifers in the Rhode River 
Estuary, spring-summer 1991. Marine Ecology Progress Series 85, 187-199.

DOLAN, J.R. \& MARRASÉ, C. (1995) Planktonic ciliate distribution relative to a deep chlorophyll maximum: Catalan Sea, N.W. Mediterranean, June 1993. Deep-Sea Research Part I 42, $1965-1987$.

DOLAN, J.R. \& PÉREZ, M.T. (2000) Costs, benefits and characteristics of mixotrophy in marine oligotrichs. Freshwater Biology 45, 227-238.

Dolan, J.R., Vidussi, F. \& Claustre, H. (1999) Planktonic ciliates in the Mediterranean Sea: Longitudinal trends. Deep-Sea Research Part I: Oceanographic Research Papers 46, 20252039.

Doron, M., Brasseur, P., Brankart, J.-M., Losa, S.N. \& Melet, A. (2013) Stochastic estimation of biogeochemical parameters from Globcolour ocean colour satellite data in a North Atlantic 3D ocean coupled physical-biogeochemical model. Journal of Marine Systems 117-118, 81-95.

DroOP, M.R. (1968) Vitamin B-12 and marine biology. IV. The kinetics of uptake growth and inhibition in Monochrysis lutheri. J.Mar.Biol. 48, 689-733. Library and Information Centre Swansea University.

DRUON, J.-N. \& FEVRE, J. LE (1999) Sensitivity of a pelagic ecosystem model to variations of process parameters within a realistic range. Journal of Marine Systems 19, 1-26.

DrushKa, K., ASher, W., WARD, B. \& WALESBy, K. (2016) Understanding the formation and evolution of rain-formed fresh lenses at the ocean surface. Journal of Geophysical Research:Oceans 121, 2673-2689.

DunN, O. J. (1961) Multiple comparisons among means. Journal of the American Statistical Association 56, 52-64.

EDWARDS, K.F. (2019) Mixotrophy in nanoflagellates across environmental gradients in the ocean. Proceedings of the National Academy of Sciences 116, 6211-6220.

Edwards, K.F., Litchman, E. \& KlausmeieR, C.A. (2013) Functional traits explain phytoplankton community structure and seasonal dynamics in a marine ecosystem. Ecology Letters 16, 56-63.

Edwards, M., Johns, D., Leterme, S.C., SVEndsen, E. \& Richardson, A.J. (2006) Regional climate change and harmful algal blooms in the northeast Atlantic. Limnology and Oceanography 51, 820-829.

EGGE, J.K. \& AKSNES, D.L. (1992) Silicate as regulating nutrient in phytoplankton competition. Marine Ecology Progress Series 83, 281-289.

Eloire, D., SOMERfield, P.J., Conway, D.V.P., HAlsband-Lenk, C., HARRis, R. \& BonNet, D. (2010) Temporal variability and community composition of zooplankton at station L4 in the Western Channel: 20 years of sampling. Journal of Plankton Research 32, 657-679.

Everett, J.D., Baird, M.E., Buchanan, P., Bulman, C., DAvies, C., Downie, R., GrifFiths, C., Heneghan, R., Kloser, R.J., Laiolo, L., Lara-Lopez, A., Lozano-Montes, H., MATEAR, R.J., MCEnNUlty, F., Robson, B., ET AL. (2017) Modeling What We Sample and Sampling What We Model: Challenges for Zooplankton Model Assessment. Frontiers in Marine Science 4, 1-19. 
FAgerbakKe, K.M., Heldal, M. \& Norland, S. (1996) Content of carbon, nitrogen, oxygen, sulfur and phosphorus in native aquatic and cultured bacteria. Aquatic Microbial Ecology 10, 15-27.

FAShaM, M.J.R., DuCKLOW, H.W. \& McKelvie, S.M. (1990) A nitrogen-based model of plankton dynamics in the oceanic mixed layer. Journal of Marine Research 48, 591-639.

Faure, E., Not, F., Benoiston, A.S., Labadie, K., Bittner, L. \& Ayata, S.D. (2019) Mixotrophic protists display contrasted biogeographies in the global ocean. ISME Journal 13, 1072-1083.

FENCHEL, T. \& Finlay, B.J. (2003) Is microbial diversity fundamentally different from biodiversity of larger animals and plants? European Journal of Protistology 39, 486-490.

FENCHEL, T. \& HANSEN, P.J. (2006) Motile behaviour of the bloom-forming ciliate Mesodinium rubrum. Marine Biology Research 2, 169-177.

Field, C.B., BEHRENFELD, M.J., RANDERSON, J.T. \& FALKOWSKI, P. (1998) Primary production of the biosphere: integrating terrestrial and oceanic components. Science 281, 237-240.

Figueiredo, G.M., NASH, R.D.M. \& MontagneS, D.J.S. (2007) Do protozoa contribute significantly to the diet of larval fish in the Irish Sea? Journal of the Marine Biological Association of the United Kingdom 87, 843-850.

Fileman, E., Petropavlovsky, A. \& Harris, R. (2010) Grazing by the copepods Calanus helgolandicus and Acartia clausi on the protozooplankton community at station L4 in the Western English Channel. Journal of Plankton Research 32, 709-724.

Fileman, E.S., Cummings, D.G. \& Llewellyn, C.A. (2002) Microplankton community structure and the impact of microzooplankton grazing during an Emiliania huxleyi bloom, off the Devon coast. Journal of the Marine Biological Association of the United Kingdom 82, 359-368.

Finkel, Z. V., Beardall, J., Flynn, K.J., QuigG, A., Rees, T.A. V \& RAVen, J.A. (2010) Phytoplankton in a changing world: Cell size and elemental stoichiometry. Journal of Plankton Research 32, 119-137.

FinLAY, B.J. \& CLARKE, K.J. (1999) Ubiquitous dispersal of microbial species. Nature 400, 828.

Fischer, H., Schmitt, J., LÜthi, D., Stocker, T.F., Tschumi, T., Parekh, P., Joos, F., KÖHLER, P., VÖlKer, C., Gersonde, R., BARbante, C., Le Floch, M., RAYNAUd, D. \& WOLFF, E. (2010) The role of Southern Ocean processes in orbital and millennial CO2 variations - A synthesis. Quaternary Science Reviews 29, 193-205.

Fischer, R., Giebel, H.A., Hillebrand, H. \& PTACNIK, R. (2017) Importance of mixotrophic bacterivory can be predicted by light and loss rates. Oikos 126, 713-722.

FLYNN, K.J. (1998) Estimation of kinetic parameters for the transport of nitrate and ammonium into marine phytoplankton. Marine Ecology Progress Series 169, 13-28.

FLYNN, K.J. (2001) A mechanistic model for describing dynamic multi-nutrient, light, temperature interactions in phytoplankton. Journal of Plankton Research 23, 977-997.

FLYNN, K.J. (2005) Castles built on sand: Dysfunctionality in plankton models and the inadequacy of dialogue between biologists and modellers. Journal of Plankton Research 27, 1205-1210.

FLYNN, K.J. (2006) Mechanistic models of algal physiology. In Algal Cultures, analogues of 
blooms and applications, ed. DV Subba Rao, Science Publishers, Enfield USA, 501-532.

FLYNN, K.J. (2010) Ecological modelling in a sea of variable stoichiometry: Dysfunctionality and the legacy of Redfield and Monod. Progress in Oceanography 84, 52-65. Elsevier Ltd.

FLYNN, K.J. (2018) Dynamic Ecology - an introduction to the art of simulating trophic dynamics. Swansea University, Swansea.

FLYNN, K.J., ClARK, D.R. \& XUE, Y. (2008) Modeling the release of dissolved organic matter by phytoplankton. Journal of Phycology 44, 1171-1187.

FlynN, K.J. \& HANSEN, P.J. (2013) Cutting the canopy to defeat the 'selfish gene'; conflicting selection pressures for the integration of phototrophy in mixotrophic protists. Protist 164, 811-823.

FLYNN, K.J. \& IRIGOIEN, X. (2009) Aldehyde-induced insidious effects cannot be considered as a diatom defence mechanism against copepods. Marine Ecology Progress Series 377, 79-89.

FLYNN, K.J. \& MARTIN-JÉZÉQuel, V. (2000) Modelling Si-N-limited growth of diatoms. Journal of Plankton Research 22, 447-472.

FlynN, K.J. \& MCGiLlicudDy, D.J. (2018) Modeling Marine Harmful Algal Blooms: Current Status and Future Prospects. In Harmful Algal Blooms: A Compendium Desk Reference (eds S. SE, B. J-AM \& M. S), pp. 115-134. Wiley Science Publishers.

FLYNN, K.J. \& MiTRA, A. (2009) Building the 'perfect beast': modelling mixotrophic plankton. Journal of Plankton Research 31, 965-992.

FlynN, K.J. \& MitRA, A. (2016) Why Plankton Modelers Should Reconsider Using Rectangular Hyperbolic (Michaelis-Menten, Monod) Descriptions of Predator-Prey Interactions. Frontiers in Marine Science 3.

FLYNN, K.J. \& RAVEN, J.A. (2016) What is the limit for photoautotrophic plankton growth rates? Journal of Plankton Research 00, 1-10.

FLYNN, K.J., SKIBINSKI, D.O.F. \& LINDEMANN, C. (2018) Effects of growth rate, cell size, motion, and elemental stoichiometry on nutrient transport kinetics. PLoS Computational Biology 14, $1-30$.

Flynn, K.J., St John, M., RAVEn, J. A., Skibinski, D.O.F., Allen, J.I., Mitra, A. \& Hofmann, E.E. (2015) Acclimation, adaptation, traits and trade-offs in plankton functional type models: reconciling terminology for biology and modelling. Journal of Plankton Research 37, 683691.

Flynn, K.J., Stoecker, D.K., Mitra, A., RAVEn, J.A., Glibert, P.M., HANSEN, P.J., GRANÉLI, E. \& BURKHOLDER, J.M. (2013) Misuse of the phytoplankton-zooplankton dichotomy: The need to assign organisms as mixotrophs within plankton functional types. Journal of Plankton Research 35, 3-11.

FOISSNER, W. (2006) Biogeography and dispersal of micro-organisms: A review emphasizing protists. Acta Protozoologica 45, 111-136.

FoISSNER, W., ChaO, A. \& KATZ, L.A. (2008) Diversity and geographic distribution of ciliates (Protista: Ciliophora). Biodiversity and Conservation 17, 345-363.

Follows, M.J. \& DuTKIEWICZ, S. (2011) Modeling diverse communities of marine microbes. Annual Review of Marine Science 3, 427-451. 
Foster, R.A., CARPENTER, E.J. \& BERGMAN, B. (2006) Unicellular cyanobionts in open ocean dinoflagellates, radiolarians, and tintinnids: Ultrastructural characterization and immunolocalization of phycoerythrin and nitrogenase. Journal of Phycology 42, 453-463.

FRANKS, P.J.S. (2002) NPZ models of plankton dynamics: their construction, coupling to physics, and application. Journal of Oceanography 58, 379-387.

FRANKS, P.J.S. (2009) Planktonic ecosystem models: Perplexing parameterizations and a failure to fail. Journal of Plankton Research 31, 1299-1306.

FranZE, G. \& LAVRENTYeV, P.J. (2014) Microzooplankton growth rates examined across a temperature gradient in the Barents Sea. PloS One 9, e86429.

FriedrichS, M.A.M., CARR, M.E., BARbER, R.T., SCARDI, M., ANTOINE, D., ARMSTRONG, R.A., Asanuma, I., Behrenfeld, M.J., Buitenhuis, E.T., Chai, F., Christian, J.R., CiotTi, A.M., DONEY, S.C., Dowell, M., DunNe, J., ET AL. (2009) Assessing the uncertainties of model estimates of primary productivity in the tropical Pacific Ocean. Journal of Marine Systems 76, 113-133.

Froneman, P.W. \& MCQUAID, C.D. (1997) Preliminary investigation of the ecological role of microzooplankton in the Kariega Estuary, South Africa. Estuarine, Coastal and Shelf Science 45, 689-695.

Froneman, P.W. \& PerissinotTo, R. (1996) Structure and grazing of the microzooplankton communities of the Subtropical Convergence and a warm-core eddy in the Atlantic sector of the Southern Ocean. Marine Ecology Progress Series 135, 237-245.

Fuhrman, J.A., Steele, J.A., Hewson, I., Schwalbach, M.S., Brown, M. V., Green, J.L. \& BROWN, J.H. (2008) A latitudinal diversity gradient in planktonic marine bacteria. Proceedings of the National Academy of Sciences 105, 7774-7778.

Fulton, E. A. (2010) Approaches to end-to-end ecosystem models. Journal of Marine Systems 81, 171-183.

Garcia-Cuetos, L., Moestrup, Ø., Hansen, P.J. \& Daugbjerg, N. (2010) The toxic dinoflagellate Dinophysis acuminata harbors permanent chloroplasts of cryptomonad origin, not kleptochloroplasts. Harmful Algae 9, 25-38.

GARZIO, L.M. \& STEINBERG, D.K. (2013) Microzooplankton community composition along the Western Antarctic Peninsula. Deep-Sea Research Part I: Oceanographic Research Papers 77, 36-49.

GAST, R.J. \& CARON, D.A. (1996) Molecular phylogeny of symbiotic dinoflagellates from planktonic foraminifera and radiolaria. Molecular Biology and Evolution 13, 1192-1197.

GAST, R.J. \& CARON, D.A. (2001) Photosymbiotic associations in planktonic foraminifera and radiolaria. Hydrobiologia 461, 1-7.

Gast, R.J., McKie-Krisberg, Z.M., FAY, S.A., Rose, J.M. \& SANDERS, R.W. (2014) Antarctic mixotrophic protist abundances by microscopy and molecular methods. FEMS Microbiology Ecology 89, 388-401.

Gauns, M., Mochemadkar, S., Patil, S., Pratihary, A., Naqvi, S.W.A. \& Madhupratap, M. (2015) Seasonal variations in abundance, biomass and grazing rates of microzooplankton in a tropical monsoonal estuary. Journal of Oceanography 71, 345-359. 
GEIDER, R. \& LA RoCHE, J. (2002) Redfield revisited: variability of C:N:P in marine microalgae and its biochemical basis. European Journal of Protistology 37, 1-17.

Gelman, A. \& HILl, J. (2007) Data Analysis Using Regression and Multilevel/Hierarchical Models. Cambridge University Press.

Genitsaris, S., Monchy, S., Viscogliosi, E., Sime-Ngando, T., Ferreira, S. \& Christaki, U. (2015) Seasonal variations of marine protist community structure based on taxon-specific traits using the eastern English Channel as a model coastal system. FEMS Microbiology Ecology 91, fiv034.

Gentien, P., Lunven, M., Lazure, P., Youenou, A. \& Crassous, M.. (2007) Motility and autotoxicity in Karenia mikimotoi (Dinophyceae). Philosophical Transactions of the Royal Society B: Biological Sciences 362, 1937-1946.

Gentleman, W., Leising, A., Frost, B., Strom, S. \& Murray, J. (2003) Functional responses for zooplankton feeding on multiple resources: a review of assumptions and biological dynamics. Deep Sea Research Part II: Topical Studies in Oceanography 50, 2847-2875.

Ghyoot, C., Flynn, K.J., Mitra, A., LAnCelot, C. \& Gypens, N. (2017a) Modeling Plankton Mixotrophy: A Mechanistic Model Consistent with the Shuter-Type Biochemical Approach. Frontiers in Ecology and Evolution 5, 1-16.

Ghyoot, C., Lancelot, C., Flynn, K.J., Mitra, A. \& Gypens, N. (2017b) Introducing mixotrophy into a biogeochemical model describing an eutrophied coastal ecosystem: The Southern North Sea. Progress in Oceanography 157, 1-11.

GIFFORD, D.J. \& CARON, D.A. (2000) Sampling, preservation, enumeration and biomass of marine protozooplankton. In ICES Zooplankton Methodology Manual Book (eds R. HARRIS, P. WieBE, J. LENZ, H.R. SKJOLDAL \& M. HunTLEY), pp. 193-221.

Gimmler, A., Korn, R., De Vargas, C., Audic, S. \& Stoeck, T. (2016) The Tara Oceans voyage reveals global diversity and distribution patterns of marine planktonic ciliates. Scientific Reports 6, 33555.

Glibert, P. \& Burford, M. (2017) Globally Changing Nutrient Loads and Harmful Algal Blooms. Oceanography 30, 58-69.

Glibert, P.M., Burkholder, J.M., Kana, T.M., AleXander, J., Skelton, H. \& Shilling, C. (2009) Grazing by Karenia brevis on Synechococcus enhances its growth rate and may help to sustain blooms. Aquatic Microbial Ecology 55, 17-30.

Glibert, P.M., Wilkerson, F.P., Dugdale, R.C., RAVEn, J.A., DuPONT, C.L., LEAVITT, P.R., PARKER, A.E., BURKHOLDER, J.M. \& KANA, T.M. (2016) Pluses and minuses of ammonium and nitrate uptake and assimilation by phytoplankton and implications for productivity and community composition, with emphasis on nitrogen-enriched conditions. Limnology and Oceanography 61, 165-197.

Gomes, E.A.., Santos, V.S., Tenenbaum, D.R. \& Villac, M.C. (2007) Protozooplankton characterization of two contrasting sites in a tropical coastal ecosystem (Guanabara Bay, RJ). Brazilian Journal of Oceanography 55, 29-38.

Gomes, H. Do R., Goes, J.I., Matondkar, S.G.P., Buskey, E.J., Basu, S., PARAB, S. \& THOPPIL, P. (2014) Massive outbreaks of Noctiluca scintillans blooms in the Arabian Sea due to spread of hypoxia. Nature Communications $\mathbf{5}, 4862$. 
Gomes, H. do R., McKee, K., Mile, A., Thandapu, S., Al-Hashmi, K., Jiang, X. \& Goes, J.I. (2018) Influence of light availability and prey type on the growth and photophysiological rates of the mixotroph Noctiluca scintillans. Frontiers in Marine Science $\mathbf{5}$, $1-12$.

Gotelli, N. \& Ellison, A. (2012) A primer of ecological statistics(2nd). Oxford University Press, Sinauer Associates.

Gotelli, N.J., Anderson, M.J., Arita, H.T., Chao, A., Colwell, R.K., Connolly, S.R., Currie, D.J., Dunn, R.R., Graves, G.R., Green, J.L., GrYtnes, J.A., JiAng, Y.H., JeTZ, W., KATHLEen LyOnS, S., MCCAIN, C.M., ET AL. (2009) Patterns and causes of species richness: A general simulation model for macroecology. Ecology Letters 12, 873-886.

Gran-StadniczeñKo, S., Egge, E., Hostyeva, V., Logares, R., Eikrem, W. \& Edvardsen, B. (2018) Protist Diversity and Seasonal Dynamics in Skagerrak Plankton Communities as Revealed by Metabarcoding and Microscopy. Journal of Eukaryotic Microbiology 66, 494513.

Granados, M., Duffy, S., McKindsey, C.W. \& Fussmann, G.F. (2017) Stabilizing mechanisms in a food web with an introduced omnivore. Ecology and Evolution 7, 50165025 .

GranÉli, E., Edvardsen, B., Roelke, D.L. \& Hagström, J. A. (2012) The ecophysiology and bloom dynamics of Prymnesium spp. Harmful Algae 14, 260-270.

GRZYMSKI, J., SCHOFIELD, O.M., FALKOWSKI, P.G. \& BERNHARD, J.M. (2002) The function of plastids in the deep-sea benthic foraminifer, Nonionella stella. Limnology and Oceanography 47, 1569-1580.

GUL, S. \& SAIfUllaH, S.M. (2010) Taxonomic and ecological studies on three marine genera of Dinophysiales from Arabian Sea shelf of Pakistan. Pakistan Journal of Botany 42, 26472660.

Gustafson, D.E., Stoecker, D.K., Johnson, M.D., Van Heukelem, W.F. \& Sneider, K. (2000) Cryptophyte algae are robbed of their organelles by the marine ciliate Mesodinium rubrum. Nature 405, 1049-1052.

HALl, J.A., BARRETT, D.P. \& JAMES, M.R. (1993) The importance of phytoflagellate, heterotrophic flagellate and ciliate grazing on bacteria and picophytoplankton sized prey in a coastal marine environment. Journal of Plankton Research 15, 1075-1086.

HAMMER, A.C. \& PITCHFORD, J.W. (2005) The role of mixotrophy in plankton bloom dynamics, and the consequences for productivity. ICES Journal of Marine Science 62, 833-840.

HANSELl, D.A. (2013) Recalcitrant Dissolved Organic Carbon Fractions. Annual Review of Marine Science 5, 421-445.

Hansen, P.J. (2011) The Role of Photosynthesis and Food Uptake for the Growth of Marine Mixotrophic Dinoflagellates. Journal of Eukaryotic Microbiology 58, 203-214.

HANSEN, P.J. \& FENCHEL, T. (2006) The bloom-forming ciliate Mesodinium rubrum harbours a single permanent endosymbiont. Marine Biology Research 2, 169-177.

HANSEN, P.J. \& HJORTH, M. (2002) Growth and grazing responses of Chrysochromulina ericina (Prymnesiophyceae): The role of irradiance, prey concentration and pH. Marine Biology 141, 975-983. 
Hansen, P.J., Moldrup, M., TarangKoon, W., Garcia-Cuetos, L. \& Moestrup (2012) Direct evidence for symbiont sequestration in the marine red tide ciliate Mesodinium rubrum. Aquatic Microbial Ecology 66, 63-75.

Hansen, P.J., Nielsen, L.T., Johnson, M., Berge, T. \& Flynn, K.J. (2013) Acquired phototrophy in Mesodinium and Dinophysis - A review of cellular organization, prey selectivity, nutrient uptake and bioenergetics. Harmful Algae 28, 126-139. Elsevier B.V.

HANSEN, P.J. \& NIELSEN, T.G. (1997) Mixotrophic feeding of Fragilidium subglobosum (dinophyceae) on three species of Ceratium: Effects of prey concentration, prey species and light intensity. Marine Ecology Progress Series 147, 187-196.

Hansen, P.J., OjamÄE, K., Berge, T., Trampe, E.C.L., Nielsen, L.T., LiPS, I. \& KÜHL, M. (2016) Photoregulation in a kleptochloroplastidic dinoflagellate, Dinophysis acuta. Frontiers in Microbiology 7:785.

Hanson, C.A., Fuhrman, J.A., Horner-Devine, M.C. \& Martiny, J.B.H. (2012) Beyond biogeographic patterns: Processes shaping the microbial landscape. Nature Reviews Microbiology 10, 497-506.

Haraguchi, L., Jakobsen, H.H., Lundholm, N. \& CARSTensen, J. (2018) Phytoplankton Community Dynamic: A Driver for Ciliate Trophic Strategies. Frontiers in Marine Science $5,1-16$.

HARRED, L.B. \& CAMPBell, L. (2014) Predicting harmful algal blooms: A case study with Dinophysis ovum in the Gulf of Mexico. Journal of Plankton Research 36, 1434-1445.

HARRIS, R. (2010) The L4 time-series: The first 20 years. Journal of Plankton Research 32, 577583.

HARrison, P.J., FuruYa, K., GLibert, P.M., Xu, J., LiU, H.B., Yin, K., LEe, J.H.W., ANDERSON, D.M., GowEN, R., AL-AZRI, A.R. \& Ho, A.Y.T. (2011) Geographical distribution of red and green Noctiluca scintillans. Chinese Journal of Oceanology and Limnology 29, 807-831.

HARRISON, X.A. (2014) Using observation-level random effects to model overdispersion in count data in ecology and evolution. PeerJ 2, e616.

Hartmann, M., Grob, C., TARRAn, G. A, Martin, A.P., Burkill, P.H. \& SCANLAN, D.J. (2012) Mixotrophic basis of Atlantic oligotrophic ecosystems. Proceedings of the National Academy of Sciences 109, 5756-5760.

Hartmann, M., Zubkov, M. V., Scanlan, D.J. \& Lepère, C. (2013) In situ interactions between photosynthetic picoeukaryotes and bacterioplankton in the Atlantic Ocean: Evidence for mixotrophy. Environmental Microbiology Reports 5, 835-840.

HATTEnRATH-LehMANn, T. \& GoBleR, C.J. (2015) The contribution of inorganic and organic nutrients to the growth of a North American isolate of the mixotrophic dinoflagellate, Dinophysis acuminata. Limnology and Oceanography 60, 1588-1603.

HAVSKUM, H. \& HANSEN, A S. (1997) Importance of pigmented and colourless nano-sized protists as grazers on nanoplankton in a phosphate-depleted Norwegian fjord and in enclosures. Aquatic Microbial Ecology 12, 139-151.

HAVSKUM, H. \& RIEMANN, B. (1996) Ecological importance of bacterivorous, pigmented flagellates (mixotrophs) in the Bay of Aarhus, Denmark. Marine Ecology Progress Series 137, 251-263. 
HEARn, C.J. (2008) The dynamics of coastal models. Cambridge University Press, Cambridge.

Hellweger, F.L., VAN SEbiLle, E. \& FredRICK, N.D. (2014) Biogeographic patterns in ocean microbes emerge in a neutral agent-based model. Science 345, 1346-1349.

Herfort, L., Peterson, T.D., Prahl, F.G., McCue, L.A., Needoba, J.A., Crump, B.C., ROEGNER, G.C., CAMPBElL, V. \& ZUBER, P. (2012) Red Waters of Myrionecta rubra are Biogeochemical Hotspots for the Columbia River Estuary with Impacts on Primary/Secondary Productions and Nutrient Cycles. Estuaries and Coasts 35, 878-891.

Hernández-Urcera, J., Rial, P., García-Portela, M., Lourés, P., Kilcoyne, J., Rodríguez, F., FERnández-VILlamarín, A. \& Reguera, B. (2018) Notes on the Cultivation of Two Mixotrophic Dinophysis Species and Their Ciliate Prey Mesodinium rubrum. Toxins 10, 505.

VAN DEN HofF, J. \& Bell, E. (2015) The ciliate Mesodinium rubrum and its cryptophyte prey in Antarctic aquatic environments. Polar Biology 38, 1305-1310.

Holt, J., Allen, J., Anderson, T.R., Brewin, R., Butenschön, M., Harle, J., Huse, G., Lehodey, P., Lindemann, C., Memery, L., SAlihoglu, B., Senina, I. \& Yool, A. (2014) Challenges in integrative approaches to modelling the marine ecosystems of the North Atlantic: Physics to fish and coasts to ocean. Progress in Oceanography 129, 285-313.

HolT, J. \& UMLAUF, L. (2008) Modelling the tidal mixing fronts and seasonal stratification of the Northwest European Continental shelf. Continental Shelf Research 28, 887-903.

Honda, D., Kawachi, M. \& INOUYe, I. (1995) Sulcochrysis biplastida gen. et sp. nov.: Cell structure and absolute configuration of the flagellar apparatus of an enigmatic chromophyte alga. Phycological Research 43, 1-16.

Hood, R.R., Zhang, X., Glibert, P.M., Roman, M.R. \& StOeCKER, D.K. (2006) Modeling the influence of nutrients, turbulence and grazing on Pfiesteria population dynamics. Harmful Algae 5, 459-479.

HoPPENRATH, M. \& LEANDER, B.S. (2007) Character evolution in polykrikoid dinoflagellates. Journal of Phycology 43, 366-377.

Huber, B.T., BiJMA, J. \& DARLing, K. (1997) Cryptic speciation in the living planktonic foraminifer Globigerinella siphonifera (d’Orbigny). Paleobiology 23, 33-62.

HunTER, M.D. \& PRICE, P.W. (1992) Playing chutes and ladders : heterogeneity and the relative roles of bottom-up and top- down forces in natural communities. Ecology 73, 724-732.

Hutchins, D., Mulholland, M. \& Fu, F. (2009) Nutrient cycles and marine microbes in a $\mathrm{CO}_{2-}$ enriched ocean. Oceanography 22, 128-145.

INOUYE, I., HORI, T. \& CHIHARA, M. (1990) Absolute configuration analysis of the flagellar apparatus of Pterosperma cristatum (prasinophyceae) and consideration of its phylogenetic position. Journal of Phycology 26, 329-344.

IOCCG (2014) Phytoplankton functional types from Space. In Reports of the International Ocean Color Coordinating Group (ed S. SATHYENDRANATH), p. 156. Dartmouth, NS: IOCCG.

IRIGOIEN, X., FlynN, K.J. \& HARRIS, R.P. (2005) Phytoplankton blooms: A 'loophole' in microzooplankton grazing impact? Journal of Plankton Research 27, 313-321.

Jacobson, D.M. \& Anderson, D.M. (1996) Widespread Phagocytosis of Ciliates and Other 
Protists By Marine Mixotrophic and Heterotrophic Thecate Dinoflagellates. Journal of Phycology 32, 279-285.

JAKOBSEN, H.H., HANSEN, P.J. \& LARSEN, J. (2000) Growth and grazing responses of two chloroplast-retaining dinoflagellates: Effect of irradiance and prey species. Marine Ecology Progress Series 201, 121-128.

JAKOBSEN, H.H. \& STROM, S.L. (2004) Circadian protist plankton cycles in growth and feeding rates of heterotrophic protist plankton. Limnology and Oceanography 49, 1915-1922.

Jang, S.H., JeOng, H.J., Kwon, J.E. \& LeE, K.H. (2017) Mixotrophy in the newly described dinoflagellate Yihiella yeosuensis: A small, fast dinoflagellate predator that grows mixotrophically, but not autotrophically. Harmful Algae 62, 94-103.

JaYAlaKshmi, K.J., SABU, P., DEVI, C.R.A. \& SANJEEVAN, V.N. (2015) Response of micro- and mesozooplankton in the southwestern Bay of Bengal to a cyclonic eddy during the winter monsoon, 2005. Environmental Monitoring and Assessment 187.

JEONG, H.A.E.J.I.N., KIM, J.H. \& YIH, W. (2004) Mixotrophy in the phototrophic harmful alga Cochlodinium polykrikoides (Dinophycean): prey species, the effects of prey concentration, and grazing impact. Journal of Eukaryotic Microbiology 51, 563-569.

JeOng, H.J., Kim, T.H., YoO, Y.D., YoOn, E.Y., Kim, J.S., SeOnG, K.A., KIM, K.Y. \& PARK, J.Y. (2011) Grazing impact of heterotrophic dinoflagellates and ciliates on common red-tide euglenophyte Eutreptiella gymnastica in Masan Bay , Korea. Harmful Algae 10, 576-588.

JeOnG, H.J., LEE, C.W., YIH, W.H. \& KIM, J.S. (1997) Fragilidium cf. mexicanum, a thecate mixotrophic dinoflagellate which is prey for and a predator on co-occurring thecate heterotrophic dinoflagellate Protoperidinium cf. divergens. Marine Ecology Progress Series 151, 299-305.

JeOng, H.J., OK, J.H., LiM, A.S., KwON, J.E., KIM, S.J. \& LEE, S.Y. (2016) Mixotrophy in the phototrophic dinoflagellate Takayama helix (family Kareniaceae): Predator of diverse toxic and harmful dinoflagellates. Harmful Algae 60, 92-106.

JeOng, H.J., Park, J.Y., Nho, J.H., PArk, M.O., Ha, J.H., SeOng, K.A., Jeng, C., SeOng, C.N., LEE, K.Y. \& YIN, W.H. (2005a) Feeding by red-tide dinoflagellates on the cyanobacterium Synechococcus. Aquatic Microbial Ecology 41, 131-143.

JeOng, H.J., SeOng, K.A., Kang, N.S., Yoo, Y. Du, Nam, S.W., PARK, J.Y., Shin, W., Glibert, P.M. \& JoHNS, D. (2010a) Feeding by raphidophytes on the cyanobacterium Synechococcus sp. Aquatic Microbial Ecology 58, 181-195.

JeOng, H.J., Shim, J.H., Kim, J.S., PARK, J.Y., LeE, C.W. \& LeE, Y. (1999) Feeding by the mixotrophic thecate dinoflagellate Fragilidium cf. mexicanum on red-tide and toxic dinoflagellates. Marine Ecology Progress Series 176, 263-277.

JeOng, H.J., YoO, Y. Du, Kim, J.S., SEONG, K.A., KANG, N.S. \& Kim, T.H. (2010b) Growth, feeding and ecological roles of the mixotrophic and heterotrophic dinoflagellates in marine planktonic food webs. Ocean Science Journal 45, 65-91.

JeOng, H.J., YoO, Y. Du, LeE, K.H., Kim, T.H., SeONG, K.A., KANG, N.S., LEe, S.Y., KiM, J.S., KIM, S. \& YIH, W.H. (2013) Red tides in Masan Bay, Korea, in 2004-2005: III. Daily variations in the abundance of mesozooplankton and their grazing impacts on red-tide organisms. Harmful Algae 30, S102-S113. 
JeOng, H.J., YoO, Y. Du, PARK, J.Y., SONG, J.Y., KIM, S.T., LEe, S.H., KIM, K.Y. \& YIH, W.H. (2005b) Feeding by phototrophic red-tide dinoflagellates : five species newly revealed and six species previ- ously known to be mixotrophic. Aquatic Microbial Ecology 40, 133-150.

JeOng, H.J., YoO, Y.D., KANG, N.S., LiM, A. S., SeONG, K. A., LEe, S.Y., LEE, M.J., LEe, K.H., KIM, H.S., SHIN, W., NAM, S.W., YIH, W. \& LEE, K. (2012) Heterotrophic feeding as a newly identified survival strategy of the dinoflagellate Symbiodinium. Proceedings of the National Academy of Sciences 109, 12604-12609.

JiAng, Y., XU, H., Hu, X., ZHU, M., Al-RASHEID, K.A.S. \& WARren, A. (2011) An approach to analyzing spatial patterns of planktonic ciliate communities for monitoring water quality in Jiaozhou Bay, northern China. Marine Pollution Bulletin 62, 227-235.

JIANG, Y., YANG, E.J., MIN, J.O., KIM, T.W. \& KANG, S.H. (2015) Vertical variation of pelagic ciliate communities in the western Arctic Ocean. Deep-Sea Research Part II: Topical Studies in Oceanography 120, 103-113.

JiaO, N., Herndl, G.J., HANSEll, D.A., Benner, R., KATtNer, G., Wilhelm, S.W., KirChMan, D.L., Weinbauer, M.G., LuO, T., Chen, F. \& AzAM, F. (2010) Microbial production of recalcitrant dissolved organic matter: Long-term carbon storage in the global ocean. Nature Reviews Microbiology 8, 593-599.

Jiao, N., Robinson, C., AZAM, F., ThOMAs, H., BALtar, F., DANG, H., HARdMAN-MOUNTFORd, N.J., Johnson, M., KirChMAN, D.L., KoCH, B.P., Legendre, L., LI, C., LIU, J., LuO, T., LUO, Y.W., ET AL. (2014) Mechanisms of microbial carbon sequestration in the ocean Future research directions. Biogeosciences 11, 5285-5306.

JOHNSON, M.D. (2011) The acquisition of phototrophy: adaptive strategies of hosting endosymbionts and organelles. Photosynthesis Research 107, 117-132.

JoHnson, M.D. (2015) Inducible Mixotrophy in the Dinoflagellate Prorocentrum minimum. Journal of Eukaryotic Microbiology 62, 431-443.

Johnson, M.D., BeAudoin, D.J., FradA, M.J., BRownLEE, E.F. \& STOECKER, D.K. (2018) High Grazing Rates on Cryptophyte Algae in Chesapeake Bay. Frontiers in Marine Science 5, 113.

Johnson, M.D., Oldach, D., Delwiche, C.F. \& Stoecker, D.K. (2007) Retention of transcriptionally active cryptophyte nuclei by the ciliate Myrionecta rubra. Nature 445, 426428.

Johnson, M.D. \& STOECKER, D.K. (2005) Role of feeding in growth and photophysiology of Myrionecta rubra. Aquatic Microbial Ecology 39, 303-312.

Johnson, M.D., StOECKER, D.K. \& MARshall, H.G. (2013) Seasonal dynamics of Mesodinium rubrum in Chesapeake Bay. Journal of Plankton Research 35, 877-893.

Johnson, P.T.J., Dobson, A., LAFFerty, K.D., Marcogliese, D.J., Memmott, J., ORlofske, S.A., Poulin, R. \& ThiEltges, D.W. (2010) When parasites become prey: Ecological and epidemiological significance of eating parasites. Trends in Ecology and Evolution 25, 362371.

Jolliff, J.K., Kindle, J.C., Shulman, I., Penta, B., Friedrichs, M.A.M., Helber, R. \& ARNONE, R.A. (2009) Summary diagrams for coupled hydrodynamic-ecosystem model skill assessment. Journal of Marine Systems 76, 64-82. 
JONES, H.L.J. (1997) A classification of mixotrophic protists based on their behaviour. Freshwater Biology 37, 35-43.

Jones, H.L.J., LeadBeATER, B.S.C. \& GREeN, J.C. (1993) Mixotrophy in marine species of Chrysochromulina (Prymnesiophyceae): ingestion and digestion of a small green flagellate. Journal of the Marine Biological Association of the United Kingdom 73, 283-296.

JONSSON, P. (1986) Particle size selection, feeding rates and growth dynamics of marine planktonic oligotrichous ciliates (Ciliophora: Oligotrichina). Marine Ecology Progress Series 33, 265-277.

JONSSON, P. (1987) Photosynthetic assimilation of inorganic carbon in marine oligotrich ciliates (Ciliophora, Oligotrichina). Marine Microbial Food Webs 2, 55-68.

JONSSON, P.R. (1994) Tidal rhythm of cyst formation in the rock pool ciliate Strombidium oculatum Gruber (Ciliophora, Oligotrichida): A description of the functional biology and an analysis of the tidal synchronization of encystment. Journal of Experimental Marine Biology and Ecology 175, 77-103.

Jost, C., Lawrence, C. A, CAmpolongo, F., van De Bund, W., Hill, S. \& DeAngelis, D.L. (2004) The effects of mixotrophy on the stability and dynamics of a simple planktonic food web model. Theoretical population biology 66, 37-51.

Jyothibabu, R., Madhu, N. V., Maheswaran, P.A., Jayalakshmy, K. V., NAIR, K.K.C. \& ACHUTHANKUTTY, C.T. (2008) Seasonal variation of microzooplankton $(20-200 \mu \mathrm{m})$ and its possible implications on the vertical carbon flux in the western Bay of Bengal. Continental Shelf Research 28, 737-755.

Kamiyama, T., ARIMA, S. \& Tsujino, M. (2003) Characteristics of the distribution of bacteria, heterotrophic nanoflagellates and ciliates in Hiroshima Bay in summer. Fisheries Science 69, 755-766.

Kang, N.S., JeOng, H.J., Moestrup, Ø., Shin, W., NAM, S.W., PARK, J.Y., De SAlas, M.F., KIM, K.W. \& NOH, J.H. (2010) Description of a new planktonic mixotrophic dinoflagellate Paragymnodinium shiwhaense $\mathrm{n}$. gen., n. sp. from the coastal waters off western Korea: Morphology, pigments, and ribosomal DNA gene sequence. Journal of Eukaryotic Microbiology 57, 121-144.

Kang, N.S., Jeong, H.J., Yoo, Y. Du, Yoon, E.Y., Lee, K.H., Lee, K. \& Kim, G. (2011) Mixotrophy in the newly described phototrophic dinoflagellate Woloszynskia cincta from western Korean waters: Feeding mechanism, prey species and Effect of prey concentration. Journal of Eukaryotic Microbiology 58, 152-170.

KARAgali, I., HøYer, J. \& DONLON, C. (2017) Using a 1-D model to reproduce the diurnal variability of SST. Journal of Geophysical Research:Oceans 122, 2945- 2959.

KAWACHI, M., InOUYE, I., MAEDA, O. \& ChIHARA, M. (1991) The haptonema as a food-capturing device: observations on Chrysochromulina hirta (Prymnesiophyceae). Phycologia 30, 563573.

KIM, M., KANG, M. \& PARK, M.G. (2018) Growth and Chloroplast Replacement of the Benthic Mixotrophic Ciliate Mesodinium coatsi. Journal of Eukaryotic Microbiology 0, 1-12.

KIM, M., KIM, K.Y., NAM, S.W., SHIN, W., YIH, W. \& PARK, M.G. (2014) The effect of starvation on plastid number and photosynthetic performance in the kleptoplastidic dinoflagellate Amylax triacantha. The Journal of eukaryotic microbiology 61, 354-363. 
KIM, M., NAm, S.W., ShIN, W., CoATs, D.W. \& PARK, M.G. (2012) Dinophysis caudata (Dinophyceae) sequesters and retains plastids from the mixotrophic ciliate prey Mesodinium rubrum. Journal of Phycology 48, 569-579.

KIM, S., KANG, Y.G., KIM, H.S., YIH, W., COATS, D.W. \& PARK, M.G. (2008a) Growth and grazing responses of the mixotrophic dinoflagellate Dinophysis acuminata as functions of light intensity and prey concentration. Aquatic Microbial Ecology 51, 301-310.

KIM, S., YoOn, J. \& PARK, M.G. (2015) Obligate mixotrophy of the pigmented dinoflagellate Polykrikos lebourae (Dinophyceae, Dinoflagellata). Algae 30, 35-47.

KiM, Y., HA, S. \& TANIGUCHI, A (2008b) Morphology and in situ sedimentation of the cysts of a planktonic oligotrich ciliate, Strombidium capitatum. Aquatic Microbial Ecology 53, 173179.

KIM, Y. \& TANIGUCHI, A. (1995) Excystment of the oligotrich ciliate Strombidium conicum. Aquatic Microbial Ecology 9, 149-156.

KIMOR, B., GORDON, N. \& NEORI, A (1992) Symbiotic associations among the microplankton in oligotrophic marine environments, with special reference to the Gulf of Aqaba, Red Sea 14, 1217-1231.

Kiørboe, T., Hansen, J.L.S., Alldredge, A.L., Jackson, G.A., Passow, U., Dam, H.G., DrAPEAU, D.T., WAITE, A. \& GARCIA, C.M. (1996) Sedimentation of phytoplankton during a diatom bloom: Rates and mechanisms. Journal of Marine Research 54, 1123-1148.

Kishi, M.J., Kashiwai, M., Ware, D.M., Megrey, B.A., Eslinger, D.L., Werner, F.E., Noguchi-Aita, M., Azumaya, T., Fujil, M., Hashimoto, S., Huang, D., IIzumi, H., ISHIDA, Y., KANG, S., KANTAKOV, G. A., ET AL. (2007) NEMURO-a lower trophic level model for the North Pacific marine ecosystem. Ecological Modelling 202, 12-25.

KiVI, K. \& SETALA, O. (1995) Simultaneous measurement of food particle selection and clearance rates of planktonic oligotrich ciliates (Ciliophora: Oligotrichina). Marine Ecology Progress Series 119, 125-138.

Koch, C., Brumme, B., Schmidt, M., Flieger, K., Schnetter, R. \& Wilhelm, C. (2011) The life cycle of the amoeboid alga Synchroma grande (Synchromophyceae, Heterokontophyta ) - highly adapted yet equally equipped for rapid diversification in benthic habitats $13,801-$ 808 .

KoIzUMI, Y., UCHIDA, T. \& HonJO, T. (1996) Diurnal vertical migration of Gymnodinium mikimotoi during a red tide in Hoketsu Bay, Japan. Journal of Plankton Research 18, 289294.

KoOIJMAn, S.A.L.M., DiJKstRA, H.A. \& KoOI, B.W. (2002) Light-induced mass turnover in a mono-species community of mixotrophs. Journal of Theoretical Biology 214, 233-254.

Kürten, B., Khomayis, H.S., Devassy, R., Audritz, S., Sommer, U., Struck, U., ElSHERBINY, M.M. \& AL-AIDAROOS, A.M. (2015) Ecohydrographic constraints on biodiversity and distribution of phytoplankton and zooplankton in coral reefs of the Red Sea, Saudi Arabia. Marine Ecology 36, 1195-1214.

LANCElOt, C., SPITZ, Y., GYPENS, N., RUdDick, K., BECQUEVORT, S., ROUSSEAU, V., LACROIX, G. \& BILLEN, G. (2005) Modelling diatom and Phaeocystis blooms and nutrient cycles in the Southern Bight of the North Sea: the MIRO model. Marine Ecology Progress Series 289 , 63-78. 
LANGER, M.R. \& LIPPS, J.H. (2003) Foraminiferal distribution and diversity, Madang Reef and Lagoon, Papua New Guinea. Coral Reefs 22, 143-154.

Laval-Peuto, M. \& Febvre, M. (1986) On plastid symbiosis in Tontonia appendiculariformis (Ciliophora, Oligotrichina). Biosystems 19 :137 - 158. Biosystems 19, 137-158.

Laval-Peuto, M. \& Rassoulzadegan, F. (1988) Autofluorescence of marine planktonic Oligotrichina and other ciliates. Hydrobiologia 159, 99 - 110.

LAVRENTYeV, P.J. \& FRANZÈ, G. (2017) CarbonBridgeMay 2014:Microzooplankton Biomass Distribution in the Waters Northwest of Spitsbergen. University of Akron, PANGAEA.

LAWS, E.A. (2013) Evaluation of in situ phytoplankton growth rates: a synthesis of data from varied approaches. Annual Review of Marine Science 5, 247-268.

LeE, H., PARK, K.T., LeE, K., JeOng, H.J. \& Yoo, Y. DU (2012) Prey-dependent retention of dimethylsulfoniopropionate (DMSP) by mixotrophic dinoflagellates. Environmental Microbiology 14, 605-616.

LEE, K.H., JeOng, H.J., JANG, T.Y., LiM, A.S., KANG, N.S., KIM, J.H., KIM, K.Y., PARK, K.T. \& LEE, K. (2014a) Feeding by the newly described mixotrophic dinoflagellate Gymnodinium smaydae: Feeding mechanism, prey species, and effect of prey concentration. Journal of Experimental Marine Biology and Ecology 459, 114-125.

LeE, K.H., JeOng, H.J., Kwon, J.E., KANG, H.C., Kim, J.H., JANG, S.H., PARK, J.Y., YoOn, E.Y. \& KIM, J.S. (2016) Mixotrophic ability of the phototrophic dinoflagellates Alexandrium andersonii, A. affine, and A. fraterculus. Harmful Algae 59, 67-81. Elsevier B.V.

LEE, M.J., JeONG, H.J., LEE, K.H., JANG, S.H., KIM, J.H. \& KIM, K.Y. (2015) Mixotrophy in the nematocyst-taeniocyst complex-bearing phototrophic dinoflagellate Polykrikos hartmannii. Harmful Algae 49, 124-134.

LeE, S.K., JeOng, H.J., Jang, S.H., LeE, K.H., KAng, N.S., LeE, M.J. \& PotVIN, É. (2014b) Mixotrophy in the newly described dinoflagellate Ansanella granifera: Feeding mechanism, prey species, and effect of prey concentration. Algae 29, 137-152.

LeEuwen, S.M. van, Le Quesne, W.F. \& PARKer, E.R. (2016) Potential future fisheries yields in shelf waters: A model study of the effects of climate change and ocean acidification. Biogeosciences 13, 441-454.

LEgendre, L., Rivkin, R.B., WeInBAUeR, M.G., Guidi, L. \& UiTZ, J. (2015) The microbial carbon pump concept: Potential biogeochemical significance in the globally changing ocean. Progress in Oceanography 134, 432-450.

LEGENDRE, P. \& LEGENDRE, L. (1998) Numerical Ecology Second edition. Elsevier, Amsterdam.

LEGRAND, C., Granél, E. \& CARLSSON, P. (1998) Induced phagotrophy in the photosynthetic dinoflagellate Heterocapsa triqueta. Aquatic Microbial Ecology 15, 65-75.

Leles, S.G., Mitra, A., Flynn, K.J., Stoecker, D.K., HAnsen, P.J., CAlbet, A., McManus, G.B., SANDERS, R.W., CARON, D.A., Not, F., HAllegraefF, G.M., PitTA, P., RAVEn, J.A., JOHNSON, M.D., GLIBERT, P.M., ET AL. (2017) Oceanic protists with different forms of acquired phototrophy display contrasting biogeographies and abundance. Proceedings of The Royal Society B 284, 20170664.

Leles, S.G., Mitra, A., Flynn, K.J., Tillmann, U., Stoecker, D., JeOng, H.J., BuRKholder, 
J.A., Hansen, P.J., Caron, D.A., Glibert, P.M., HallegraefF, G., Raven, J.A., SANDERS, R.W. \& ZuBKOV, M. (2019) Sampling bias misrepresents the biogeographical significance of constitutive mixotrophs across global oceans. Global Ecology and Biogeography 28, 418-428.

Leles, S.G., Polimene, L., Bruggeman, J., Blackford, J., Ciavatta, S., Mitra, A. \& FLYNN, K.J. (2018) Modelling mixotrophic functional diversity and implications for ecosystem function. Journal of Plankton Research 40, 627-642.

Leles, S.G., VAlentin, J.L. \& Figueiredo, G.M. (2016) Evaluation of the complexity and performance of marine planktonic trophic models. Anais da Academia Brasileira de Ciências 88, $1-24$.

LeRAy, M. \& KNOWLton, N. (2016) Censusing marine eukaryotic diversity in the twenty-first century. Philosophical Transactions of the Royal Society B: Biological Sciences 371.

LEVINSEN, H. \& NiElSEN, T.G. (2002) The trophic role of marine pelagic ciliates and heterotrophic dinoflagellates in arctic and temperate coastal ecosystems: A cross-latitude comparison. Limnology and Oceanography 47, 427-439.

LEVINSEN, H., NiElsen, T.G. \& HANSEN, B.W. (1999) Plankton community structure and carbon cycling on the western coast of Greenland during the stratified summer situation. II. Heterotrophic dinoflagellates and ciliates. Aquatic Microbial Ecology 16, 217-232.

LEVInsen, H., NiElsen, T.G. \& HANSEN, B.W. (2000) Annual succession of marine pelagic protozoans in Disko Bay, West Greenland, with emphasis on winter dynamics. Marine Ecology Progress Series 206, 119-134.

Lewis, K., Allen, J.I., Richardson, A.J. \& HolT, J.T. (2006) Error quantification of a high resolution coupled hydrodynamic-ecosystem coastal-ocean model: Part3, validation with Continuous Plankton Recorder data. Journal of Marine Systems 63, 209-224.

Lewitus, A.J., Glasgow, H.B. \& BurKholder, J.M. (1999) Kleptoplastidy in the Toxic Dinoflagellate Pfiesteria Piscicida (Dinophyceae). Journal of Phycology 35, 303-312.

Li, A., Stoecker, D.K. \& AdoLF, J.E. (1999) Feeding, pigmentation, photosynthesis and growth of the mixotrophic dinoflagellate Gyrodinium galatheanum. Aquatic Microbial Ecology 19, $163-176$.

Li, A., Stoecker, D.K., CoATs, D.W. \& AdAM, E.J. (1996) Ingestion of fluorescently labeled and phycoerythrin-containing prey by mixotrophic dinoflagellates. Aquatic Microbial Ecology 10, 139-147.

LI, C., YANG, G., NING, J., SUn, J., YANG, B. \& Sun, S. (2013) Response of copepod grazing and reproduction to different taxa of spring bloom phytoplankton in the Southern Yellow Sea. Deep-Sea Research Part II: Topical Studies in Oceanography 97, 101-108.

Lie, A.A.Y., Liu, Z., Terrado, R., Tatters, A.O., Heidelberg, K.B. \& CARON, D.A. (2018) A tale of two mixotrophic chrysophytes: Insights into the metabolisms of two Ochromonas species (Chrysophyceae) through a comparison of gene expression. PLOS ONE 13, 1-20.

Lim, A.S., JeOng, H.J., KIM, J.H., Jang, S.H., LEe, M.J. \& LEE, K. (2015) Mixotrophy in the newly described dinoflagellate Alexandrium pohangense: A specialist for feeding on the fastswimming ichthyotoxic dinoflagellate Cochlodinium polykrikoides. Harmful Algae 49, 1018. 
Lin, C.-H., Flynn, K.J., Mitra, A. \& Glibert, P.M. (2018) Simulating Effects of Variable Stoichiometry and Temperature on Mixotrophy in the Harmful Dinoflagellate Karlodinium veneficum. Frontiers in Marine Science 5, 1-13.

Lindemann, C., AKsnes, D.L., Flynn, K.J. \& Menden-Deuer, S. (2017) Editorial: Modeling the Plankton-Enhancing the Integration of Biological Knowledge and Mechanistic Understanding. Frontiers in Marine Science 4, 1-3.

Litchman, E. \& Klausmeier, C. A. (2008) Trait-Based Community Ecology of Phytoplankton. Annual Review of Ecology, Evolution, and Systematics 39, 615-639.

Litchman, E., Klausmeier, C. A, Schofield, O.M. \& Falkowski, P.G. (2007) The role of functional traits and trade-offs in structuring phytoplankton communities: scaling from cellular to ecosystem level. Ecology letters 10, 1170-1181.

LiU, H. \& HoPCROFT, R.R. (2006) Growth and development of Metridia pacifica (Copepoda: Calanoida) in the northern Gulf of Alaska. Journal of Plankton Research 28, 769-781.

LiU, H.X., HuANG, H.H., XU, S.N., DAI, M. \& SHEN, P.P. (2015) Planktonic community structure during a harmful bloom of Phaeocystis globosa in a subtropical bay, with special reference to the ciliate assemblages. Ecotoxicology 24, 1419-1429.

LIU, X., TANG, C.H. \& WonG, C.K. (2014) Microzooplankton grazing and selective feeding during bloom periods in the Tolo Harbour area as revealed by HPLC pigment analysis. Journal of Sea Research 90, 83-94.

LiU, Z., CAMPBell, V., Heidelberg, K.B. \& CARON, D.A. (2016) Gene expression characterizes different nutritional strategies among three mixotrophic protists. FEMS Microbiology Ecology 92, 1-11.

LÖDER, M.G.J., MEunier, C., Wiltshire, K.H., Boersma, M. \& ABERLE, N. (2011) The role of ciliates, heterotrophic dinoflagellates and copepods in structuring spring plankton communities at Helgoland Roads, North Sea. Marine Biology 158, 1551-1580.

LOEBL, M. \& VAN BeuSEKOM, J.E.E. (2008) Seasonality of microzooplankton grazing in the northern Wadden Sea. Journal of Sea Research 59, 203-216.

LOMbard, F., Renaud, F., SAinsbury, C., Sciandra, A. \& Gorsky, G. (2009) Appendicularian ecophysiology I. Food concentration dependent clearance rate, assimilation efficiency, growth and reproduction of Oikopleura dioica. Journal of Marine Systems 78, 606-616.

LONGHURST, A. (2007) Ecological Geography of the Sea. Academic Press, MA.

Longhurst, A., Sathyendranath, S., Platt, T. \& CAverhill, C. (1995) An estimate of global primary production in the ocean from satellite radiometer data. Journal of Plankton Research 17, 1245-1271.

LÓPEZ, E., VIESCA, L. \& ANADÓN, R. (2007) Seasonal variation in abundance and feeding rates of the first stages of copepods in a temperate sea. Marine Ecology Progress Series 352, 161175.

Lundgren, V.M., Glibert, P.M., Granéli, E., Vidyarathna, N.K., Fiori, E., OU, L., FlynN, K.J., Mitra, A., Stoecker, D.K. \& HAnsen, P.J. (2016) Metabolic and physiological changes in Prymnesium parvum when grown under, and grazing on prey of, variable nitrogen:phosphorus stoichiometry. Harmful Algae 55, 1-12. 
LYNN, D.H., ROFF, J.C. \& HoPCROFT, R.R. (1991) Annual abundance and biomass of aloricate ciliates in tropical neritic waters off Kingston, Jamaica. Marine Biology 110, 437-448.

Mackas, D.L., Greve, W., EdWards, M., Chiba, S., Tadokoro, K., Eloire, D., MaZzocchi, M.G., BAtTen, S., Richardson, A.J., Johnson, C., Head, E., Conversi, A. \& Peluso, T. (2012) Changing zooplankton seasonality in a changing ocean: Comparing time series of zooplankton phenology. Progress in Oceanography 97-100, 31-62.

MAFRA, L.L., TAVARES, C.P. DOS S. \& SCHRAMM, M.A. (2014) Diarrheic toxins in field-sampled and cultivated Dinophysis spp. cells from southern Brazil. Journal of Applied Phycology 26, $1727-1739$.

Malviya, S., Scalco, E., Audic, S., Vincent, F., Veluchamy, A., Poulain, J., Wincker, P., Iudicone, D., DE VARGas, C., BitTner, L., Zingone, A. \& Bowler, C. (2016) Insights into global diatom distribution and diversity in the world's ocean. Proceedings of the National Academy of Sciences 113, E1516-E1525.

Marasigan, A.N., Sato, S., FukuYo, Y. \& Kodama, M. (2001) Accumulation of a high level of diarrhetic shellfish toxins in the green mussel Perna viridis during a bloom of Dinophysis caudata and Dinophysis miles in Sapian Bay, Panay Island, the Philippines. Fisheries Science 67, 994-996.

MARGALEF, R. (1978) Life-forms of phytoplankton as survival alternatives in an unstable environment. Oceanol. Acta 1, 493-509.

Martin-JÉzÉquel, V., HiLdebrand, M. \& BrZezinsKi, M.A. (2000) Silicon metabolism in diatoms: Implications for growth. Journal of Phycology 36, 821-840.

Martin, A. \& Montagnes, D. (1993) Winter ciliates in a British Columbian fjord: six new species and an analysis of ciliate putative prey. Journal of Eukaryotic Microbiology 40, 535549.

Martiny, J.B.H., Bohannan, B.J.M., Brown, J.H., Colwell, R.K., Fuhrman, J.A., Green, J.L., Horner-Devine, M.C., KANE, M., Krumins, J.A., Kuske, C.R., Morin, P.J., NAEEM, S., ØvREÅs, L., ReYsenbaCH, A.L., SMITH, V.H., ET AL. (2006) Microbial biogeography: Putting microorganisms on the map. Nature Reviews Microbiology 4, 102-112.

MARUYAMA, S. \& KIM, E. (2013) A modern descendant of early green algal phagotrophs. Current Biology 23, 1081-1084.

MatTHEWS, S.G. (1991) The relative role of protozoans in the flux of phytoplankton nitrogen through pelagic food webs. University of Cape Town.

MCGILL, B.J., ENQUIST, B.J., WEIHER, E. \& WeSTOBY, M. (2006) Rebuilding community ecology from functional traits. Trends in Ecology and Evolution 21, 178-185.

McKenrie, C.H., Deibel, D., Paranjape, M.A. \& Thompson, R.J. (1995) The marine mixotroph Dinobryon balticum (Chrysophyceae): phagotrophy and survival in a cold ocean. Journal of Phycology 31, 19-24.

MCKIE-KrisberG, Z.M., GAST, R.J. \& SANDERS, R.W. (2015) Physiological Responses of Three Species of Antarctic Mixotrophic Phytoflagellates to Changes in Light and Dissolved Nutrients. Microbial Ecology 70, 21-29.

MCKIE-KRISBERG, Z.M. \& SANDERS, R.W . (2014) Phagotrophy by the picoeukaryotic green alga Micromonas: implications for Arctic Oceans. The ISME Journal 8, 1953-1961. 
McMahon, T., Raine, R., FAst, T., Kies, L. \& PAtching, J.W. (1992) Phytoplankton biomass, light attenuation and mixing in the shannon estuary, Ireland. Journal of the Marine Biological Association of the United Kingdom 72, 709-720.

McManus, G.B., Costas, B.A., Dam, H.G., Lopes, R.M., Gaeta, S.A., Susini, S.M. \& RoseTTA, C.H. (2007) Microzooplankton grazing of phytoplankton in a tropical upwelling region. Hydrobiologia 575, 69-81.

MCMAnus, G.B. \& FunRman, J. A (1986) Photosynthetic pigments in the ciliate Laboea strobila from Long Island Sound, USA. Journal of Plankton Research 8, 317-327.

McManus, G.B., SCHOENER, D.M. \& HABERLANDT, K. (2012) Chloroplast symbiosis in a marine ciliate: ecophysiology and the risks and rewards of hosting foreign organelles. Frontiers in microbiology $\mathbf{3}, 321$.

MCMANUS, G.B., ZHANG, H. \& LIN, S. (2004) Marine planktonic ciliates that prey on macroalgae and enslave their chloroplasts. Limnology and Oceanography 49, 308-313.

MCPARLAND, E.L. \& LEVINE, N.M. (2019) The role of differential DMSP production and community composition in predicting variability of global surface DMSP concentrations. Limnology and Oceanography 64, 757-773.

Menden-Deuer, S. \& Kiørboe, T. (2016) Small bugs with a big impact: linking plankton ecology with ecosystem processes. Journal of Plankton Research 38, 1036-1043.

MENDEN-DEUER, S. \& LESSARD, E.J. (2000) Carbon to volume relationships for dinoflagellates, diatoms, and other protist plankton. Limnology and Oceanography 45, 569-579.

Millette, N.C., Pierson, J.J., ACeves, A. \& Stoecker, D.K. (2017) Mixotrophy in Heterocapsa rotundata: A mechanism for dominating the winter phytoplankton. Limnology and Oceanography 62, 836-845.

Mironova, E., Telesh, I. \& Skarlato, S. (2013) Planktonic ciliates of the Neva Estuary (Baltic Sea): community structure and spatial distribution. Acta Protozoologica 52, 13-23.

MitRA, A. (2006) A multi-nutrient model for the description of stoichiometric modulation of predation in micro- and mesozooplankton. Journal of Plankton Research 28, 597-611.

Mitra, A., Castellani, C., Gentleman, W.C., Jónasdóttir, S.H., Flynn, K.J., Bode, A., Halsband, C., Kuhn, P., Licandro, P., Agersted, M.D., Calbet, A., Lindeque, P.K., Koppelmann, R., MøLler, E.F., GiSLASON, A., ET AL. (2014a) Bridging the gap between marine biogeochemical and fisheries sciences: configuring the zooplankton link. Progress in Oceanography 129, 176-199.

MitRA, A. \& FLYNN, K.J. (2005) Predator-prey interactions: Is 'ecological stoichiometry' sufficient when good food goes bad? Journal of Plankton Research 27, 393-399.

MitRA, A. \& FLYNN, K.J. (2006) Accounting for variation in prey selectivity by zooplankton. Ecological Modelling 199, 82-92.

MitRA, A. \& FLYNN, K.J. (2010) Modelling mixotrophy in harmful algal blooms: More or less the sum of the parts? Journal of Marine Systems 83, 158-169. Elsevier B.V.

Mitra, A., Flynn, K.J., Burkholder, J.M., Berge, T., CAlbet, A., RAVEN, J.A., Granéli, E., Glibert, P.M., Hansen, P.J., Stoecker, D.K., Thingstad, F., Tillmann, U., VÅGe, S., WILKEN, S. \& ZUBKOV, M. V. (2014b) The role of mixotrophic protists in the biological 
carbon pump. Biogeosciences 11, 995-1005.

Mitra, A., Flynn, K.J., Tillmann, U., Raven, J.A., Caron, D., Stoecker, D.K., Not, F., Hansen, P.J., HallegraefF, G., SAnders, R., Wilken, S., McManus, G., Johnson, M., PitTA, P., VÅGE, S., ET AL. (2016) Defining Planktonic Protist Functional Groups on Mechanisms for Energy and Nutrient Acquisition: Incorporation of Diverse Mixotrophic Strategies. Protist 167, 106-120.

Modigh, M. (2001) Seasonal variations of photosynthetic ciliates at a Mediterranean coastal site. Aquatic Microbial Ecology 23, 163-175.

Moeller, H. V. \& Johnson, M.D. (2018) Preferential Plastid Retention by the Acquired Phototroph Mesodinium chamaeleon. Journal of Eukaryotic Microbiology 65, 148-158.

Moeller, H. V., PeltomaA, E., Johnson, M.D. \& Neubert, M.G. (2016) Acquired phototrophy stabilises coexistence and shapes intrinsic dynamics of an intraguild predator and its prey. Ecology Letters 19, 393-402.

Montagnes, D., Lynn, D., ROFF, J. \& TAYLOR, W. (1988) The annual cycle of heterotrophic planktonic ciliates in the waters surrounding the Isles of Shoals, Gulf of Maine: an assessment of their trophic role. Marine Biology 99, 21-30.

Montagnes, D.J.S. \& Fenton, A. (2012) Prey-abundance affects zooplankton assimilation efficiency and the outcome of biogeochemical models. Ecological Modelling 243, 1-7.

Moon-VAn Der StaAy, S.Y., De Wachter, R. \& VAulot, D. (2001) Oceanic 18S rDNA sequences from picoplankton reveal unsuspected eukaryotic diversity. Nature 409, 607-610.

Moorthi, S., CARON, D. A., GAST, R.J. \& SANDERS, R.W. (2009) Mixotrophy: A widespread and important ecological strategy for planktonic and sea-ice nanoflagellates in the Ross Sea, Antarctica. Aquatic Microbial Ecology 54, 269-277.

Moorthi, S.D., Ptacnik, R., Sanders, R.W., Fischer, R., Busch, M. \& Hillebrand, H. (2017) The functional role of planktonic mixotrophs in altering seston stoichiometry. Aquatic Microbial Ecology 79, 235-245.

MyUnG, G., Kim, H.S., PARK, J.W., PARK, J.S. \& YIH, W. (2013) Sequestered plastids in Mesodinium rubrum are functionally active up to 80 days of phototrophic growth without cryptomonad prey. Harmful Algae 27, 82-87.

MYUNG, G., YIH, W., KIM, H.S., PARK, J.S. \& CHO, B.C. (2006) Ingestion of bacterial cells by the marine photosynthetic ciliate Myrionecta rubra. Aquatic Microbial Ecology 44, 175180 .

NÉZAn, E. \& ChOMÉRAT, N. (2009) Fragilidium duplocampanaeforme sp. nov. (Dinophyceae): A new phagotrophic dinoflagellate from the French Atlantic coast. European Journal of Protistology 45, 2-12.

Nishitani, G., Nagai, S., Hayakawa, S., Kosaka, Y., Sakurada, K., Kamiyama, T. \& GojobORI, T. (2012) Multiple Plastids Collected by the Dinoflagellate Dinophysis mitra through Kleptoplastidy. Applied and Environmental Microbiology 78, 813-821.

NyGAARD, K. \& TOBIESEN, A. (1993) Bacterivory in algae: A survival strategy during nutrient limitation. Limnology and Oceanography 38, 273-279.

OK, J.H., JEONG, H.J., LIM, A.S. \& LEE, K.H. (2017) Interactions between the mixotrophic 
dinoflagellate Takayama helix and common heterotrophic protists. Harmful Algae 68, 178191.

Olli, K., WeXels Riser, C., Wassmann, P., Ratkova, T., Arashkevich, E. \& Pasternak, A. (2002) Seasonal variation in vertical flux of biogenic matter in the marginal ice zone and the central Barents Sea. Journal of Marine Systems 38, 189-204.

Olson, M.B. \& STROM, S.L. (2002) Phytoplankton growth, microzooplankton herbivory and community structure in the southeast Bering Sea: Insight into the formation and temporal persistence of an Emiliania huxleyi bloom. Deep-Sea Research Part II: Topical Studies in Oceanography 49, 5969-5990.

OTA, S., Silver, T.D., ARCHIBALD, J.M. \& IsHidA, K.-I. (2009) Lotharella oceanica sp. nov. - a new planktonic chlorarachniophyte studied by light and electron microscopy. Phycologia 48, 315-323.

OTA, T. \& TANiguchi, A. (2003) Standing crop of planktonic ciliates in the East China Sea and their potential grazing impact and contribution to nutrient regeneration. Deep Sea Research Part II: Topical Studies in Oceanography 50, 423-442.

OWEn, R.W., GiAnESELlA-GALVÃO, S.F. \& KUTNER, M.B.B. (1992) Discrete, subsurface layers of the autotrophic ciliate Mesodinium rubrum off Brazil. Journal of Plankton Research 14, 97-105.

Painting, S., Moloney, C. \& LuCAS, M. (1993) Simulation and field measurements of phytoplankton-bacteria-zooplankton interactions in the southern Benguela upwelling region. Marine Ecology Progress Series 100, 55-69.

PARK, G.M., Kim, S., HyUnG, S.K., MYUnG, G., YI, G.K. \& YiH, W. (2006) First successful culture of the marine dinoflagellate Dinophysis acuminata. Aquatic Microbial Ecology 45, 101-106.

PARK, M.G. \& KIM, M. (2010) Prey Specificity and Feeding of the Thecate Mixotrophic Dinoflagellate Fragilidium Duplocampanaeforme. Journal of Phycology 46, 424-432.

PARK, M.G., KIM, M. \& KANG, M. (2013) A dinoflagellate Amylax triacantha with plastids of the cryptophyte origin: Phylogeny, feeding mechanism, and growth and grazing responses. Journal of Eukaryotic Microbiology 60, 363-376.

PARK, M.G., KIM, M. \& KIM, S. (2015) Phased cell division and facultative mixotrophy of the marine dinoflagellate Fragilidium duplocampanaeforme and its trophic interactions with the dinoflagellates Dinophysis spp. and a ciliate Mesodinium rubrum. Harmful Algae 43, 20-30.

Pawlowski, J., Holzmann, M., Fahrni, J.F., Pochon, X. \& Lee, J.J. (2001) Molecular identification of algal endosymbionts in large miliolid Foraminifera: 2. Dinoflagellates. The Journal of eukaryotic microbiology 48, 368-373.

PeltomaA, E. \& Johnson, M.D. (2017) Mesodinium rubrum exhibits genus-level but not species-level cryptophyte prey selection. Aquatic Microbial Ecology 78, 147-159.

PEREZ, M., DolAn, J. \& FUKAI, E. (1997) Planktonic oligotrich ciliates in the NW Mediterranean: growth rates and consumption by copepods. Marine Ecology-Progress Series 155, 89-101.

PÉREZ, M.T., DOLAN, J.R., VIDUSSI, F. \& FUKAI, E. (2000) Diel vertical distribution of planktonic ciliates within the surface layer of the NW Mediterranean (May 1995). Deep-Sea Research Part I: Oceanographic Research Papers 47, 479-503. 
Pinardi, N., Korres, G., Lascaratos, A., Roussenov, V. \& StaneV, E. (1997) Numerical simulation of the Mediterranean Sea upper ocean circulation. Geophysical Research Letters 24, 425-428.

Pitta, P. \& Giannakourou, A. (2000) Planktonic ciliates in the oligotrophic Eastern Mediterranean: Vertical, spatial distribution and mixotrophy. Marine Ecology Progress Series 194, 269-282.

Pitta, P., Giannakourou, A. \& Christaki, U. (2001) Planktonic ciliates in the oligotrophic Mediterranean Sea: Longitudinal trends of standing stocks, distributions and analysis of food vacuole contents. Aquatic Microbial Ecology 24, 297-311.

Polimene, L., Mitra, A., Sailley, S.F., Ciavatta, S., Widdicombe, C.E., AtKinson, A. \& ALLEN, J.I. (2015) Decrease in diatom palatability contributes to bloom formation in the Western English Channel. Progress in Oceanography 137, 484-497.

Polimene, L., SAilley, S., Clark, D., Mitra, A. \& Allen, J.I. (2017) Biological or microbial carbon pump? The role of phytoplankton stoichiometry in ocean carbon sequestration. Journal of Plankton Research 39, 180-186.

Polis, G.A., Myers, C.A. \& HolT, R.D. (1989) The Ecology and Evolution of Intraguild Predation: Potential Competitors That Eat Each Other. Annual Review of Ecology and Systematics 20, 297-330.

Polovina, J.J., Howell, E.A. \& ABECASSIS, M. (2008) Ocean's least productive waters are expanding. Geophysical Research Letters 35, 2-6.

PRIEDE, I.G. (2014) Biogeography of the Oceans: A Review of Development of Knowledge of Currents, Fronts and Regional Boundaries from Sailing Ships in the Sixteenth Century to Satellite Remote Sensing. Pure and Applied Geophysics 171, 1013-1027.

PRINCIOTTA, S.D.V., SMITH, B.T. \& SANDERS, R.W. (2016) Temperature-dependent phagotrophy and phototrophy in a mixotrophic chrysophyte. Journal of Phycology 52, 432-440.

Probert, I., Siano, R., Poirier, C., Decelle, J., Biard, T., Tuji, A., Suzuki, N. \& Not, F. (2014) Brandtodinium gen. nov. and B.nutricula comb. Nov. (Dinophyceae), a dinoflagellate commonly found in symbiosis with polycystine radiolarians. Journal of Phycology 50, 388399.

PUTT, M. (1990) Metabolism of photosynthate in the chloroplast-retaining ciliate Laboea strobila. Marine Ecology Progress Series 60, 271-282.

QIU, D., HuANG, L. \& LIN, S. (2016) Cryptophyte farming by symbiotic ciliate host detected in situ. Proceedings of the National Academy of Sciences 113, 12208-12213.

LE QUERE, C. (2006) Reply to Horizons Article 'Plankton functional type modelling: running before we can walk' Anderson (2005): I. Abrupt changes in marine ecosystems? Journal of Plankton Research 28, 871-872.

Le QuÉRÉ, C., Andrew, R.M., Friedlingstein, P., Sitch, S., Pongratz, J., Manning, A.C., Korsbakken, J.I., Peters, G.P., Canadell, J.G., Jackson, R.B., Boden, T.A., Tans, P.P., ANDREWS, O.D., ARORA, V.K., BAKKER, D.C.E., ET AL. (2018) Global Carbon Budget 2017. Earth System Science Data Discussions 10, 405-448.

Quevedo, M., ViescA, L., ANAdón, R. \& FernÁNDEZ, E. (2003) The protistan microzooplankton community in the oligotrophic north-eastern Atlantic: Large- and 
mesoscale patterns. Journal of Plankton Research 25, 551-563.

RAMETTE, A. \& TIEDJE, J.M. (2007) Biogeography: An emerging cornerstone for understanding prokaryotic diversity, ecology, and evolution. Microbial Ecology 53, 197-207.

RAVEN, J. A. (1997) Phagotrophy in phototrophs. Limnology and Oceanography 42, 198-205.

RAVEn, J. A., BeARdAll, J., FlynN, K.J. \& MABerly, S.C. (2009) Phagotrophy in the origins of photosynthesis in eukaryotes and as a complementary mode of nutrition in phototrophs: relation to Darwin's insectivorous plants. Journal of Experimental Botany 60, 3975-3987.

ReEs, A.P., HoPe, S.B., Widdicombe, C.E., Dixon, J.L., WoOdward, E.M.S. \& Fitzsimons, M.F. (2009) Alkaline phosphatase activity in the western English Channel: Elevations induced by high summertime rainfall. Estuarine, Coastal and Shelf Science 81, 569-574. Elsevier Ltd.

Reguera, B., Velo-Suárez, L., Raine, R. \& PARK, M.G. (2012) Harmful Dinophysis species: A review. Harmful Algae 14, 87-106.

REID, P. (1997) Mass encystment of a planktonic oligotrich ciliate. Marine Biology 95, 221-230.

Reygondeau, G., Longhurst, A., Martinez, E., Beaugrand, G., Antoine, D. \& Maury, O. (2013) Dynamic biogeochemical provinces in the global ocean. Global Biogeochemical Cycles 27, 1046-1058.

REYNOLDS, C. (1984) Phytoplankton periodicity: the interactions of form, function and environmental variability. Freshwater Biology 14, 111-142.

REYNOLDS, C. (1988) Functional morphology and the adaptive strategies of freshwater phytoplankton. In Growth and Reproductive Strategies of Freshwater Phytoplankton (ed C.D. SANDGREN), pp. 388-433. Cambridge University Press.

Richardson, A.J., Walne, A.W., John, A.W.G., JonAs, T.D., Lindley, J.A., Sims, D.W., STEVENS, D. \& WITT, M. (2006) Using continuous plankton recorder data. Progress in Oceanography 68, 27-74.

RILEY, G. (1946) Factors controlling phytoplankton populations on Georges Bank. Journal of Marine Research 6, 54-73.

RiobÓ, P., Reguera, B., Franco, J.M. \& Rodríguez, F. (2013) First report of the toxin profile of Dinophysis sacculus Stein from LC-MS analysis of laboratory cultures. Toxicon 76, 221224.

RokitTA, S.D., De NoOiJer, L.J., Trimborn, S., DE VArgas, C., Rost, B. \& John, U. (2011) Transcriptome analyses reveal differential gene expression patterns between the life-cycle stages of emiliania huxleyi (haptophyta) and reflect specialization to different ecological niches. Journal of Phycology 47, 829-838.

Romanova, N.D. \& SAZHIN, A.F. (2010) Relationships between the cell volume and the carbon content of bacteria. Oceanology 50, 522-530.

Rose, J.M. \& CARON, D.A. (2007) Does low temperature constrain the growth rates of heterotrophic protists? Evidence and implications for algal blooms in cold waters 52, 886895.

Rose, J.M., Caron, D.A., Sieracki, M.E. \& Poulton, N. (2004) Counting heterotrophic nanoplanktonic protists in cultures and aquatic communities by flow cytometry. Aquatic 
Microbial Ecology 34, 263-277.

RothHAUPT, K. (1996) Laboratorary Experiments with a Mixotrophic Chrysophyte and Obligately Phagotrophic and Photographic Competitors. Ecology 77, 716-724.

ROTHHAUPT, K.O. (1997) Nutrient turnover by freshwater bacterivorous flagellates: Differences between a heterotrophic and a mixotrophic chrysophyte. Aquatic Microbial Ecology 12, 6570 .

ROTHSCHILD, B.J. \& OSBORN, T.R. (1988) Small-scale turbulence and plankton contact rates. Journal of Plankton Research 10, 465-474.

RutTen, T.P.A., SandeE, B. \& Hofman, A.R.T. (2005) Phytoplankton monitoring by high performance flow cytometry: A successful approach? Cytometry Part A 64, 16-26.

RYCHERT, K., NAWACKA, B., MAJCHROWSKI, R. \& ZAPADKA, T. (2014) Latitudinal pattern of abundance and composition of ciliate communities in the surface waters of the Atlantic Ocean. Oceanological and Hydrobiological Studies 43, 436-441.

SAFI, K.A. \& HALL, J.A. (1999) Mixotrophic and heterotrophic nanoflagellate grazing in the convergence zone east of New Zealand. Aquatic Microbial Ecology 20, 83-93.

Sailley, S.F., Polimene, L., Mitra, A., Atkinson, A. \& Allen, J.I. (2015) Impact of zooplankton food selectivity on plankton dynamics and nutrient cycling. Journal of Plankton Research 0, 1-11.

Saltelli, A., Ratto, M., Andres, T., CAmpolongo, F., CARiboni, J., Gatelli, D., SAisana, M. \& TARANTOLA, S. (2008) Global Sensitivity Analysis. The Primer. In Global Sensitivity Analysis. The Primer p. John Wiley \& Sons Ltd, Chichester, UK.

Saltelli, A., Tarantola, S., Campolongo, F. \& Ratto, M. (2004) Sensitivity Analysis in Practice: A Guide to Assessing Scientific Models. John Wiley \& Sons, Ltd. John Wiley \& Sons, Ltd.

SANDERS, R.W. (1995) Seasonal distributions of the photosynthesizing ciliates Laboea strobila and Myrionecta rubra (= Mesodinium rubrum) in an estuary of the Gulf of Maine. Aquatic Microbial Ecology 9, 237-242.

SANDERS, R.W., BERninger, U.G., LIM, E.L., KEMP, P.F. \& CARON, D. A. (2000) Heterotrophic and mixotrophic nanoplankton predation on picoplankton in the Sargasso Sea and on Georges Bank. Marine Ecology Progress Series 192, 103-118.

SANDERS, R.W. \& GAST, R.J. (2012) Bacterivory by phototrophic picoplankton and nanoplankton in Arctic waters. FEMS Microbiology Ecology 82, 242-253.

Sankar, S., Polimene, L., Marin, L., Menon, N.N., SAmuelsen, A., Pastres, R. \& CiavatTA, S. (2018) Sensitivity of the simulated Oxygen Minimum Zone to biogeochemical processes at an oligotrophic site in the Arabian Sea. Ecological Modelling 372, 12-23.

SANTOFERRARA, L.F., GuIDA, S., ZHANG, H. \& MCMANUS, G.B. (2014) De novo transcriptomes of a mixotrophic and a heterotrophic ciliate from marine plankton. PLOS ONE 9, e101418.

SANTOFERRARA, L.F. \& MCMANUS, G.B. (2017) Integrating dimensions of biodiversity in choreotrichs and oligotrichs of marine plankton. European Journal of Protistology 61, 323330.

Sathyendranath, S., Stuart, V., Nair, A., OKa, K., Nakane, T., Bouman, H., Forget, 
M.H., MaAss, H. \& PlatT, T. (2009) Carbon-to-chlorophyll ratio and growth rate of phytoplankton in the sea. Marine Ecology Progress Series 383, 73-84.

SATO, M., ShiOZAKI, T. \& HASHIHAMA, F. (2017) Distribution of mixotrophic nanoflagellates along the latitudinal transect of the central North Pacific. Journal of Oceanography 73, 159168.

SCHANZ, F. (1985) Vertical light attenuation and phytoplankton development in Lake Zürich. Limnology and Oceanography 30, 299-310.

Schartau, M., Wallhead, P., Hemmings, J., LöPtien, U., Kriest, I., Krishna, S., Ward, B.A., Slawig, T. \& OschlieS, A. (2017) Reviews and syntheses: Parameter identification in marine planktonic ecosystem modelling. Biogeosciences 14, 1647-1701.

SCHMITT, R. (2018) The ocean's role in climate. Oceanography 31, 32-40.

Schmoker, C., Hernandez-Leon, S. \& CAlbet, A. (2013) Microzooplankton grazing in the oceans: impacts, data variability, knowledge gaps and future directions. Journal of Plankton Research 35, 691-706.

Schoener, D.M. \& MCManus, G.B. (2012) Plastid retention, use, and replacement in a kleptoplastidic ciliate. Aquatic Microbial Ecology 67, 177-187.

SCHOENER, D.M. \& MCMANUS, G.B. (2017) Growth, grazing, and inorganic C and N uptake in a mixotrophic and a heterotrophic ciliate. Journal of Plankton Research 39, 379-391.

Scholz, B., Guillou, L., Marano, A. V., Neuhauser, S., Sullivan, B.K., Karsten, U., KÜPPER, F.C. \& GLEASON, F.H. (2016) Zoosporic parasites infecting marine diatoms - A black box that needs to be opened. Fungal Ecology 19, 59-76.

SELIFONOVA, Z.P. (2011) Seasonal dynamics of micro and mesoplankton of the Temryuk estuarial pelagic ecosystem of the Sea of Azov. Inland Water Biology 4, 182-191.

Selosse, M.-A., Charpin, M. \& Not, F. (2017) Mixotrophy everywhere on land and in water: the grand écart hypothesis. Ecology Letters 20, 246-263.

SEONG, K.A., JEONG, H.J., KIM, S. \& KIM, G.H. (2006) Bacterivory by co-occurring red-tide algae, heterotrophic nanoflagellates, and ciliates. Marine Ecology Progress Series 322, 8597.

Setälä, O. \& KIVI, K. (2003) Planktonic ciliates in the Baltic Sea in summer: Distribution, species association and estimated grazing impact. Aquatic Microbial Ecology 32, 287-297.

SHAKED, Y. \& DE VARGAS, C. (2006) Pelagic photosymbiosis: rDNA assessment of diversity and evolution of dinoflagellate symbionts and planktonic foraminiferal hosts. Marine Ecology Progress Series 325, 59-71.

Sieburth, J.M., SmetaceK, V. \& Lenz, J. (1978) Pelagic Ecosystem Structure: Heterotrophic Compartments of the Plankton and Their Relationship to Plankton Size Fractions. Limnology and Oceanography 23, 1256-1263.

Sime-NGando, T., JuniPER, K. \& VEZINA, A. (1992) Ciliated protozoan communities over Cobb Seamount: increase in biomass and spatial patchiness. Marine Ecology Progress Series 89, $37-51$.

Simmons, A., Uppala, S., DeE, D. \& KobayAshi, S. (2007) ERA-Interim: New ECMWF reanalysis products from 1989 onwards. ECMWF Newsletter 110, 25-35. 
Sinha, B., Buitenhuis, E.T., Quéré, C. LE \& ANDERSON, T.R. (2010) Comparison of the emergent behavior of a complex ecosystem model in two ocean general circulation models. Progress in Oceanography 84, 204-224.

SkovgaARD, A. (1996) Mixotrophy in Fragilidium subglobosum (Dinophyceae): growth and grazing responses as functions of light intensity. Marine Ecology Progress Series 143, 247 253.

SKOVGAARD, A. (2000) A phagotrophically derivable growth factor in the plastidic dinoflagellate Gyrodinium resplendens (Dinophyceae). Journal of Phycology 36, 1069-1078.

SkovgaARD, A. \& LEgRAND, C. (2005) Observation of live specimens of Pseudotontonia cornuta (Ciliophora : Oligotrichida) reveals new distinctive characters. Journal of Marine Biological Association UK 85, 783-786.

SMALlEY, G.W. \& CoATS, W.D. (2002) Ecology of the Red-Tide Dinoflagellate. Journal of Eukaryotic Microbiology 49, 63-73.

SMAYDA TJ. (1997) Harmful algal blooms: their ecophysiology and general relevance to phytoplankton blooms in the sea. Limnology and Oceanography 42, 1137-1153.

SMITH, W.O. \& BARBER, R.T. (1979) Carbon budget for the auto- trophic ciliate Mesodinium rubrum. Journal of Phycology 15, 27-33.

SMYTh, T.J., Fishwick, J.R., Al-Moosawi, L., CummingS, D.G., HARris, C., Kitidis, V., ReES, A., MARTINEZ-VICENTE, V. \& WoODWARD, E.M.S. (2010) A broad spatio-temporal view of the Western English Channel observatory. Journal of Plankton Research 32, 585-601.

Sommer, U., Adrian, R., De Senerpont Domis, L., Elser, J.J., GAedKe, U., Ibelings, B., JePPESEN, E., LÜrling, M., Molinero, J.C., MoOIJ, W.M., VAN DONK, E. \& Winder, M. (2012) Beyond the Plankton Ecology Group (PEG) Model: Mechanisms Driving Plankton Succession. Annual Review of Ecology, Evolution, and Systematics 43, 429-448.

Sommer, U. \& Lengfellner, K. (2008) Climate change and the timing, magnitude, and composition of the phytoplankton spring bloom. Global Change Biology 14, 1199-1208.

SourniA, A. (1982) Form and Function in Marine Phytoplankton. Biological Reviews 57, 347 394.

Sousa, S.H.M., DE GodoI, S.S., Amaral, P.G.C., Vicente, T.M., MARTins, M.V.A., Sorano, M.R.G.S., Gaeta, S.A., PAssos, R.F. \& MAHIQUES, M.M. (2014) Distribution of living planktonic foraminifera in relation to oceanic processes on the southeastern continental Brazilian margin $\left(23^{\circ} \mathrm{S}-25^{\circ} \mathrm{S}\right.$ and $\left.40^{\circ} \mathrm{W}-44^{\circ} \mathrm{W}\right)$. Continental Shelf Research 89, 76-87.

SPERO, H. \& ANGEL, D. (1991) Planktonic sarcodines: microhabitat for oceanic dinoflagellates. Journal of Phycology 27, 187-195.

STEELE, J. (1959) The quantitative ecology of marine phytoplankton. Biological Reviews 34, 129 158.

Stickney, H.L., HoOD, R.R. \& STOECKER, D.K. (2000) The impact of mixotrophy on planktonic marine ecosystems. Ecological Modelling 125, 203-230.

StODEREGGer, K. \& HeRndL, G.J. (1998) Production and release of bacterial capsular material and its subsequent utilization by marine bacterioplankton. Limnology and Oceanography 43, 877-884. 
Stoecker, D., Hansen, P., CARon, D. \& Mitra, A. (2017) Mixotrophy in the marine plankton. Annual review of Marine Science 9, 311-335.

STOECKER, D.K. (1998) Conceptual models of mixotrophy in planktonic protists and some ecological and evolutionary implications. European Journal of Protistology 34, 281-290.

StOeCKER, D.K., GIFFORD, D.J. \& PUTT, M. (1994) Preservation of marine planktonic ciliates Losses and cell shrinkage during fixation. Marine Ecology Progress Series 110, 293.

StOECKER, D.K., GUSTAFSON, D.E. \& VERITY, P.G. (1996) Micro- and mesoprotozooplankton at $140^{\circ} \mathrm{W}$ in the equatorial Pacific: Heterotrophs and mixotrophs. Aquatic Microbial Ecology 10, 273-282.

Stoecker, D.K., Johnson, M.D., De Vargas, C. \& Not, F. (2009) Acquired phototrophy in aquatic protists. Aquatic Microbial Ecology 57, 279-310.

Stoecker, D.K. \& LaVRentyeV, P.J. (2018) Mixotrophic Plankton in the Polar Seas: A PanArctic Review. Frontiers in Marine Science 5, 292.

StOeCKeR, D.K. \& Michaels, A E. (1991) Respiration, photosynthesis and carbon metabolism in planktonic ciliates. Marine Biology 108, 441-447.

StOECKER, D.K., Michaels, A.E. \& DAVIS, L.H. (1987) Large proportion of marine planktonic ciliates found to contain functional chloroplasts. Nature 326, 790-792.

StOECKER, D.K., PUTT, M. \& MoISAN, T. (1995) Nano- and microplankton dynamics during the spring Phaeocystis sp. bloom in McMurdo Sound, Antarctica. Journal of the Marine Biological Association of the United Kingdom 75, 815-832.

StoeCKer, D.K. \& Silver, M.W. (1987) Chloroplast retention by marine planktonic ciliates. Annals of the New York Academy of Sciences 503, 562-565.

STOECKER, D.K. \& SILVER, M.W. (1990) Replacement and aging of chloroplasts in Strombidium capitatum (Ciliophora: Oligotrichida)*. Marine Biology 107, 491-502.

Stoecker, D.K., Silver, M.W., Michaels, A. E. \& DAVIS, L.H. (1988) Obligate mixotrophy in Laboea strobila, a ciliate which retains chloroplasts. Marine Biology 99, 415-423.

Stoecker, D.K., Silver, M.W., Michaels, A.E. \& DAVIS, L.H. (1989) Enslavement of algal chloroplasts by four Strombidium spp. (Ciliophora, Oligotrichida). Marine Microbial Food Webs 3, 79-100.

Stoecker, D.K., Weigel, A.C., Stockwell, D. A. \& Lomas, M.W. (2014) Microzooplankton: Abundance, biomass and contribution to chlorophyll in the Eastern Bering Sea in summer. Deep Sea Research Part II: Topical Studies in Oceanography 109, 134-144.

StOeCKeR ET AL (1991) Photosynthesis in Mesodinium rubrum: species specific measurements and comparison to community rates. Marine Ecology Progress Series 73, 245-252.

Stow, C.A., JollifF, J., MCGIllicuddy, D.J., Doney, S.C., Allen, J.I., FriedRICHS, M.A.M., Rose, K.A. \& WALlHEAD, P. (2009) Skill assessment for coupled biological/physical models of marine systems. Journal of Marine Systems 76, 4-15.

Stow, C.A., Roessler, C., Borsuk, M.E., Bowen, J.D. \& ReCKHOW, K.H. (2003) Comparison of Estuarine Water Quality Models for Total Maximum Daily Load Development in Neuse River Estuary. Journal of Water Resources Planning and Management 129, 307-314. 
STUKEL, M.R., LANDRY, M.R. \& SELPH, K.E. (2011) Nanoplankton mixotrophy in the eastern equatorial Pacific. Deep-Sea Research Part II: Topical Studies in Oceanography 58, 378386.

Su-Myat, Maung-Saw-Htoo-Thaw, Matsuoka, K., Khin-Ko-Lay \& KoiKe, K. (2012) Phytoplankton surveys off the southern Myanmar coast of the Andaman Sea: An emphasis on dinoflagellates including potentially harmful species. Fisheries Science 78, 1091-1106.

Sun, J., Feng, Y., Zhou, F., Song, S., JiAng, Y. \& Ding, C. (2013) Top-down control of spring surface phytoplankton blooms by microzooplankton in the Central Yellow Sea, China. DeepSea Research Part II: Topical Studies in Oceanography 97, 51-60.

Sunagawa, S., Coelho, L.P., Chaffron, S., Kultima, J.R., Labadie, K., Salazar, G., Djahanschiri, B., Zeller, G., Mende, D.R., Alberti, A., Cornejo-Castillo, F.M., Costea, P.I., Cruaud, C., D’Ovidio, F., Engelen, S., ET AL. (2015) Structure and function of the global ocean microbiome. Science 348.

SUZUKI, T. \& TANIGUCHI, A. (1998) Standing crops and vertical distribution of four groups of marine planktonic ciliates in relation to phytoplankton chlorophyll a. Marine Biology 132, $375-382$.

Suzuki, T., Yamada, N. \& TANiguchi, A. (1998) Standing crops of planktonic ciliates and nanoplankton in oceanic waters of the western Pacific. Aquatic Microbial Ecology 14, 4958.

SVERDRUP, H. (1953) On conditions for the vernal blooming of phytoplankton. ICES Journal of Marine Science 18, 287-295.

TAKAHAShI, O., MAYAMA, S. \& MATSUOKA, A. (2003) Host-symbiont associations of polycystine Radiolaria: Epifluorescence microscopic observation of living Radiolaria. Marine Micropaleontology 49, 187-194.

TARANGKoOn, W., HANSEN, G. \& HANSEN, P.J. (2010) Spatial distribution of symbiont-bearing dinoflagellates in the Indian Ocean in relation to oceanographic regimes. Aquatic Microbial Ecology 58, 197-213.

TARANGKoOn, W. \& HANSEN, P. (2011) Prey selection, ingestion and growth responses of the common marine ciliate Mesodinium pulex in the light and in the dark. Aquatic Microbial Ecology 62, 25-38.

TARRAN, G.A. \& BRUUN, J.T. (2015) Nanoplankton and picoplankton in the Western English Channel: Abundance and seasonality from 2007-2013. Progress in Oceanography 137, 446455 .

TAYLOR, F. (1982) Symbioses in marine microplankton. Annales de l'Institut Oceanographique de Paris 58, 61-90.

TEAM, R.C. (2016) R: A language and environment for statistical computing. R Foundation for Statistical Computing, Vienna, Austria.

Terrado, R., Pasulka, A.L., Lie, A.A.-Y., Orphan, V.J., Heidelberg, K.B. \& CAron, D.A. (2017) Autotrophic and heterotrophic acquisition of carbon and nitrogen by a mixotrophic chrysophyte established through stable isotope analysis. The ISME Journal 11, 2022-2034. Nature Publishing Group.

Terseleer, N., Bruggeman, J., LAnCElot, C. \& GyPens, N. (2014) Trait-based representation 
of diatom functional diversity in a plankton functional type model of the eutrophied southern north sea. Limnology and Oceanography 59, 1958-1972.

Thamatrakoln, K. \& HildeBrand, M. (2007) Analysis of Thalassiosira pseudonana silicon transporters indicates distinct regulatory levels and transport activity through the cell cycle. Eukaryotic Cell 6, 271-279.

Thingstad, T.F., Havskum, H., Garde, K. \& Riemann, B. (1996) On the strategy of 'eating your competitor': a mathematical analysis of algal mixotrophy. Ecology 77, 2108-2118.

Tillmann, U. (1998) Phagotrophy by a plastidic haptophyte, Prymnesium patelliferum. Aquatic Microbial Ecology 14, 155-160.

TitTel, J., Bissinger, V., ZiPPEL, B., GAEdKe, U., Bell, E., LORKE, A. \& KAMJUnKe, N. (2003) Mixotrophs combine resource use to outcompete specialists: implications for aquatic food webs. Proceedings of the National Academy of Sciences of the United States of America 100, 12776-12781.

Tomas, C.R. (1997) Identifying marine phytoplankton. Academic Press, San Diego, California.

TORRES, R., Allen, J.I. \& FigueIRAS, F.G. (2006) Sequential data assimilation in an upwelling influenced estuary. Journal of Marine Systems 60, 317-329.

Troost, T.A., KooI, B.W. \& KoolJMAn, S.A.L.M. (2005) Ecological Specialization of Mixotrophic Plankton in a Mixed Water Column. The American Naturalist 166, E45-E61.

TSAI, A.-Y., CHIN, W.-M. \& CHIANG, K.-P. (2009) Diel patterns of grazing by pigmented nanoflagellates on Synechococcus spp. In the coastal ecosystem of subtropical western pacific. Hydrobiologia 636, 249-256.

Tsai, A.Y., Gong, G.C., Sanders, R.W., Chen, W.H., ChaO, C.F. \& Chiang, K.P. (2011) Importance of bacterivory by pigmented and heterotrophic nanoflagellates during the warm season in a subtropical western Pacific coastal ecosystem. Aquatic Microbial Ecology 63, 918.

Tuerlinckx, F., Rijmen, F., VerbeKe, G. \& De BoecK, P. (2006) Statistical inference in generalized linear mixed models: A review. British Journal of Mathematical and Statistical Psychology 59, 225-255.

UnREIN, F., GASOL, J.M. \& MASSANA, R. (2010) Dinobryon faculiferum (Chrysophyta) in coastal Mediterranean seawater: Presence and grazing impact on bacteria. Journal of Plankton Research 32, 559-564.

UnRein, F., Gasol, J.M., Not, F., Forn, I. \& MassanA, R. (2014) Mixotrophic haptophytes are key bacterial grazers in oligotrophic coastal waters. The ISME Journal 8, 164-176.

UnRein, F., MASSANA, R., AlOnso-SÁEZ, L. \& Gasol, J.M. (2007) Significant year-round effect of small mixotrophic flagellates on bacterioplankton in an oligotrophic coastal system. Limnology and Oceanography 52, 456-469.

UTERMÖHL, H. (1958) Zur vervollkommnung der quantitativen phytoplankton-methodik. Mitteilungen Internationale Vereiningung fuer Theoretische und Angewandte Limnologie 9 , $1-38$.

VÅge, S., Castellani, M., Giske, J. \& Thingstad, T.F. (2013) Successful strategies in size structured mixotrophic food webs. Aquatic Ecology 47, 329-347. 
VAQuÉ, D., Blough, H.A. \& DuARTe, C.M. (1997) Dynamics of ciliate abundance, biomass and community composition in an oligotrophic coastal environment (NW Mediterranean). Aquatic Microbial Ecology 12, 71-83.

Vargas, C., Contreras, P. \& Iriarte, J. (2012) Relative importance of phototrophic, heterotrophic, and mixotrophic nanoflagellates in the microbial food web of a riverinfluenced coastal upwelling area. Aquatic Microbial Ecology 65, 233-248.

de Vargas, C., Audic, S., Henry, N., Decelle, J., Mahé, F., Logares, R., Lara, E., Berney, C., Bescot, N., Probert, I., Carmichael, M., Poulain, J., Romac, S., Colin, S., Aury, J.-M., ET AL. (2015) Eukaryotic plankton diversity in the sunlit ocean. Science 348, 1-11.

De Vargas, C., Bonzon, M., Rees, N.W., PAwlowski, J. \& ZANinetTi, L. (2002) A molecular approach to biodiversity and biogeography in the planktonic foraminifer Globigerinella siphonifera (d'Orbigny). Marine Micropaleontology 45, 101-116.

Velo-SuÁrez, L., GonZÁlez-Gil, S., PAZos, Y. \& Reguera, B. (2014) The growth season of Dinophysis acuminata in an upwelling system embayment: A conceptual model based on in situ measurements. Deep-Sea Research Part II: Topical Studies in Oceanography 101, 141151. Elsevier.

Verspecht, F., RipPeth, T.P., Howarth, M.J., SouZA, A.J., Simpson, J.H. \& Burchard, H. (2009) Processes impacting on stratification in a region of freshwater influence: Application to Liverpool Bay. Journal of Geophysical Research: Oceans 114, 1-12.

WANG, L., Lin, X., Goes, J.I. \& Lin, S. (2016) Phylogenetic Analyses of Three Genes of Pedinomonas noctilucae, the Green Endosymbiont of the Marine Dinoflagellate Noctiluca scintillans, Reveal its Affiliation to the Order Marsupiomonadales (Chlorophyta, Pedinophyceae) under the Reinstated Name Protoeuglena noctilucae. Protist 167, 205216.

WANG, Y., Zhang, W., Lin, Y., CAO, W., Zheng, L. \& YANG, J. (2014) Phosphorus, nitrogen and chlorophyll-a are significant factors controlling ciliate communities in summer in the Northern Beibu Gulf, South China Sea. PLoS ONE 9.

WARD, B. A, DutKIEWICZ, S., BARTON, A.D. \& Follows, M.J. (2011) Biophysical aspects of resource acquisition and competition in algal mixotrophs. The American naturalist 178, 98112.

WARD, B.A., DutKIEWICZ, S., JAHN, O. \& Follows, M.J. (2012) A size-structured food-web model for the global ocean. Limnology and Oceanography 57, 1877-1891.

WARD, B.A. \& Follows, M.J. (2016) Marine mixotrophy increases trophic transfer efficiency, mean organism size, and vertical carbon flux. Proceedings of the National Academy of Sciences 113, 2958-2963.

WAtKIns, J.M., MiX, A.C. \& WiLson, J. (1996) Living planktic foraminifera: Tracers of circulation and productivity regimes in the central equatorial Pacific. Deep-Sea Research Part II: Topical Studies in Oceanography 43, 1257-1282.

Weber, F., Anderson, R., Foissner, W., Mylnikov, A.P. \& JÜrgens, K. (2014) Morphological and molecular approaches reveal highly stratified protist communities along Baltic Sea pelagic redox gradients. Aquatic Microbial Ecology 73, 1-16.

Widdicombe, C.E., Eloire, D., Harbour, D., Harris, R.P. \& Somerfield, P.J. (2010) Longterm phytoplankton community dynamics in the Western English Channel. Journal of 
Plankton Research 32, 643-655.

Wilken, S., Huisman, J., NAus-Wiezer, S. \& VAn DonK, E. (2013) Mixotrophic organisms become more heterotrophic with rising temperature. Ecology Letters 16, 225-233.

WILKERSON, F.P. \& GRUNSEICH, G. (1990) Formation of blooms by the symbiotic ciliate Mesodinium rubrum: the significance of nitrogen uptake. Journal of Plankton Research 12, 973-989.

WiLliams, R.J. \& MARTineZ, N.D. (2000) Simple rules yield complex foodwebs. Nature 404, $180-183$.

WiSECAVER, J.H. \& HACKETT, J.D. (2010) Transcriptome analysis reveals nuclear-encoded proteins for the maintenance of temporary plastids in the dinoflagellate Dinophysis acuminata. BMC genomics 11, 366.

WiteK, M. (1998) Annual Changes of Abundance and Biomass of Planktonic Ciliates in the Gdansk Basin, Southern Baltic. International Review of Hydrobiology 83, 163-182.

WoOdward, E.M.S. \& REES, A.P. (2001) Nutrient distributions in an anticyclonic eddy in the northeast Atlantic ocean, with reference to nanomolar ammonium concentrations. Deep-Sea Research Part II: Topical Studies in Oceanography 48, 775-793.

Worm, B., Lotze, H.K., Hillebrand, H. \& Sommer, U. (2002) Consumer versus resource control of species diversity and ecosystem functioning. Nature 417, 848-851.

XU, H., YonG, J. \& XU, G. (2016) An approach to analyzing spatial patterns in annual dynamics of planktonic ciliate communities and their environmental drivers in marine ecosystems. Ecological Indicators 70, 297-303.

Yamaguchi, M., ItakuRa, S., NAgasaki, K., Matsuyama, Y., UChida, T. \& Imai, I. (1997) Effects of temperature and salinity on the growth of the red tide flagellates Heterocapsa circularisquama (Dinophyceae) and Chattonella verruculosa (Raphidophyceae). Journal of Plankton Research 19, 1167-1174.

YÁÑEZ, S., Hidalgo, P., RuZ, P. \& TANG, K.W. (2018) Copepod secondary production in the sea: Errors due to uneven molting and growth patterns and incidence of carcasses. Progress in Oceanography 165, 257-267.

YANG, E.J., JIANG, Y. \& LEE, S.H. (2016) Microzooplankton herbivory and community structure in the Amundsen Sea, Antarctica. Deep-Sea Research Part II: Topical Studies in Oceanography 123, 58-68.

YANG, J., LÖDER, M.G.J. \& WILTShIRE, K.H. (2014) A survey of ciliates at the long-term sampling station 'Helgoland Roads', North Sea. Helgoland Marine Research 68, 313-327.

Yelton, A.P., Acinas, S.G., Sunagawa, S., Bork, P., Pedrós-Alió, C. \& Chisholm, S.W. (2016) Global genetic capacity for mixotrophy in marine picocyanobacteria. ISME Journal 10, 2946-2957.

YIH, W., HyunG, S.K., HAE, J.J., MYUnG, G. \& YounG, G.K. (2004) Ingestion of cryptophyte cells by the marine photosynthetic ciliate Mesodinium rubrum. Aquatic Microbial Ecology 36, $165-170$.

YiH, W., Kim, H.S., Myung, G., PARK, J.W., Yoo, Y. Du \& JeOng, H.J. (2013) The red-tide ciliate Mesodinium rubrum in Korean coastal waters. Harmful Algae 30, S53-S61. 
Yoo, Y.Du, JEOng, H.J., KANG, N.S., SONG, J.Y., KIM, K.Y., LEE, G. \& KIM, J.H. (2010) Feeding by the newly described mixotrophic dinoflagellate Paragymnodinium shiwhaense: Feeding mechanism, prey species, and effect of prey concentration. Journal of Eukaryotic Microbiology 57, 145-158.

Yoo, Y. Du, SeOng, K.A., JeOng, H.J., Yih, W., Rho, J.R., NAM, S.W. \& Kim, H.S. (2017) Mixotrophy in the marine red-tide cryptophyte Teleaulax amphioxeia and ingestion and grazing impact of cryptophytes on natural populations of bacteria in Korean coastal waters. Harmful Algae 68, 105-117.

Yoo, Y. Du, Seong, K.A., Myung, G., Kim, H.S., Jeong, H.J., PAleniK, B. \& YiH, W. (2015) Ingestion of the unicellular cyanobacterium Synechococcus by the mixotrophic red tide ciliate Mesodinium rubrum. Algae 30, 281-290.

Yoo, Y., JeOng, H., Kim, M., KAng, N., Song, J., ShIn, W., KIM, K. \& LEe, K. (2009) Feeding by Phototrophic Red-Tide Dinoflagellates on the Ubiquitous Marine Diatom Skeletonema costatum. Journal of Eukaryotic Microbiology 56, 413-420.

Yool, A., Popova, E.E. \& ANDERSON, T.R. (2011) Medusa-1.0: a new intermediate complexity plankton ecosystem model for the global domain. Geoscientific Model Development 4, 381417.

Yool, A., Popova, E.E., Coward, A.C., Bernie, D. \& Anderson, T.R. (2013) Climate change and ocean acidification impacts on lower trophic levels and the export of organic carbon to the deep ocean. Biogeosciences 10, 5831-5854.

Yuasa, T., Horiguchi, T., Mayama, S., Matsuoka, A. \& TaKahashi, O. (2012) Ultrastructural and molecular characterization of cyanobacterial symbionts in Dictyocoryne profunda (polycystine radiolaria). Symbiosis 57, 51-55.

ZARAUZ, L. \& IRIGOIEN, X. (2008) Effects of Lugol's fixation on the size structure of natural nano-microplankton samples, analyzed by means of an automatic counting method. Journal of Plankton Research 30, 1297-1303.

Zendong, Z., Kadiri, M., Herrenknecht, C., NÉZAn, E., MAZzeO, A. \& Hess, P. (2016) Algal toxin profiles in Nigerian coastal waters (Gulf of Guinea) using passive sampling and liquid chromatography coupled to mass spectrometry. Toxicon 114, 16-27.

Zhang, Q., WARwick, R.M., MCNeILl, C.L., Widdicombe, C.E., Sheehan, A. \& WIDDICOMBE, S. (2015) An unusually large phytoplankton spring bloom drives rapid changes in benthic diversity and ecosystem function. Progress in Oceanography 137, 533545. Elsevier Ltd.

ZHANG, Q., YU, R., SONG, J., YAN, T., WANG, Y. \& ZHOU, M. (2011) Will harmful dinoflagellate Karenia mikimotoi grow phagotrophically? Chinese Journal of Oceanology and Limnology 29, 849-859.

Zhang, X., Anderson, J.T. \& Hood, R.R. (2003) Modeling Pfiesteria piscicida population dynamics: A new approach for tracking size and mass in mixotrophic species. Marine Ecology Progress Series 256, 29-44.

ZuBKov, M. V \& TARRAN, G.A. (2008) High bacterivory by the smallest phytoplankton in the North Atlantic Ocean. Nature 455, 224-226. 